$\mathrm{DOE} / \mathrm{BC} / 10842-19$

Distribution Category UC-122

FLOW IN POROUS MEDIA, PHASE AND ULTRALOW INTERFACIAL TENSIONS: MECHANISMS OF ENHANCED PETROLEUM RECOVERY

By

H. T. Davis

L. E. Scriven

July 1991
$\mathrm{DOE} / \mathrm{BC} / 10842--19$

DE91 002247

Work Per'ormed Under Contract No. AC19-85BC10842

Prepared for

U.S. Department of Energy

Assistant Secretary for Fossil Energy

Jerry Casteel, Project Manager

Bartlesville Project Office

P. O. Box 1398

Bartlesville, OK 74005

Prepared by

University of Minnesota

Department of Chemical Engineering and

Materials Science

Minneapolis, Minnesota 
FLOW IN POROUS MEDIA, PHASE

1 Oct 1988-30 Sept 1989

AND ULTRALOW INTERFACIAL

FINAL TECHNICAL REPORT

TENSIONS: MECHANISMS OF

DOE/DE-AC19-79BC10116

ENHANCED PETROLEUM RECOVERY

Department of Chemical Engineering and Materials Science

University of Minnesota

CONTENTS

Page No.

Research Highlights:1985-89 . . . . . . . . . . . . . . . . . . . . . . . i

Abstract . . . . . . . . . . . . . . . . . . . . . . . . 1

Introduction . . . . . . . . . . . . . . . . . . . . . . . . . . . . . . . . 2

Pore-space statistics and capillary pressure curves from volume-controlled

porosimetry . . . . . . . . . . . . . . . . . . . . 7

Fluid distribution and transport in porous media at low wetting phase saturations . . . . . . . . . . . . . . . . .50

Microscopic dynamics of flow in molecularly narrow pores . . . . . . . . . . . . 64

Molecular dynamics of the primitive model of 1-3 electrolytes . . . . . . . . . . 75

Density-functional free energy theory of the electrical double layer . . . . . . . . 88

A nonlocal free energy density functional approximation

for the electrical double layer . . . . . . . . . . . . . . . . . . . . . . . 103

Wetting transitions and surface critical phenomena at solid-fluid interfaces _ . . 126

Density distribution functions of confined Tonks-Takahashi fluids . . . . . . . . 171

A comparison of initial conditions for continuous flow systems . . . . . . . . . . 184

A comparison of synthetic boundary conditions for continuous flow systems . . . 207

Direct imaging of surfactant micelles, vesicles, discs and ripple phase structures by

cryo-transmission electron microscopy . . . . . . . . . . . . . . . . . . . 228

A low cost animation system applied to ray tracing in liquid crystals . . . . . . 252

Surfaces of constant mean curvature with prescribed contact angles . . . . . . . 265

Thesis titles . . . . . . . . . . . . . . . . . . . . . . . . . . . . 286

List of Publications . . . . . . . . . . . . . . . . . . . . . . . . . . . . 287 


\section{RESEARCH HIGHLIGHTS: 1985-89}

\section{Science of Porous Media and Flow Therein}

- Developed a computer simulations on theory to interpret the data generated in volumecontrolled mercury porosimetry. With the aid of the simulation, the Apparatus for Pore Examination, developed by Yuan and Swanson at Shell, can be a powerful tool for measuring pore siz' 'stributions in reservoir rock.

- From the study of capillary pressures at low saturations of wetting phase, found that the sizes of porespace asperities are fractally distributed, i.e., the sizes are distributed over many different decades of length scales. Showed that this fractal property implies universal dependence of capillary pressure, hydraulic conductance and capillary dispersion on saturation of wetting phase in the low saturation regime. The universal laws explained literature data on reservoir rocks and soils.

- With fast-freeze cold-stage scanning electron microscopy determined the distribution of oil and water in Berea sandstone and Prudhoe Bay reservoir rock as a function of wettabilities ranging from water-wet to mixed-wet to oil-wet. This most extensive microscopic study available today shows precisely where the oil is distributed as a function of wettability and local mineralogy and relates the capillary pressure curves to the oil and water distribution.

- Improved the method of analysis for determining the capillary pressure curve by the centrifuge method. The traditional approximate analysis developed by Hassler and Brunner places restrictions on sample size and mounting. The new analyses developed eliminate these restrictions and allow direct numerical evaluation of the capillary pressure without approximation of the theoretical equation or allow fast numerical evaluation with an approximation greatly superior to that of Hassler and Brunner.

- Developed an effective medium theory of anisotropic media. The theory is useful and economical for predicting conductance and permeabilities for multiple phases occupying anisotropic porous media. Such a theory did not previousiy exist. Monte Carlo computer simulations carried out on model systems showed that the theory is quite accurate.

- Carried out molecular simulations and constructed a statistical mechanical theory (density functional theory) to understand the solvation forces, disjoining pressure, adsorption, transport and flow of fluids in ultrathin films or in ultranarrow pore spaces. The results provide a molecular-level understanding of the effect of fluid-solid interaction on the wettability of pore surfaces and of the adsorption and transport of wetting fluid into tight spaces, such as between clay piatelets or near particle contacts of adsorbed or aggregated fines in reservoir rock. 


\section{Science of Microstructured Fluids: Phase behavior, Interfacial Tension and Rheology.}

- Applied a battery of experimental techniques to reveal the connection between the ultralow tensions enabling microemulsions to enhance oil recovery and the transport behavior, phase behavior and supramolecular microstructure of these oil, water surfactant solutions. Established the existence of bicontinuous microemulsions in which oil-rich and water-rich regions, separated by surfactant sheets, continuously span the solution. Related the ultralow tension microemulsions to their proximity of critical points in the phase diagram. By comparison with oil, water alcohol solutions, determined that the conditions for ultralow tensions require both proximity to the critical point and the existence of supramolecular microstructure. Showed that the transition from monocontinuous to bicontinuous microstructure can occur by percolation, i.e., random interspersion of oil-rich and water-rich regions, or can be driven by temperature controlled preferred mean curvature of the surfactant sheets.

- Invented and patented the controlled environment vitrification system (CEVS) enabling vitrification and viewing of thin (2000 angstroms) frozen samples of microstructure fluids in the transmission electron microscope (TEM). The CEVS has been installed in a dozen industrial and university laboratories around the world and has become the most unambiguous method for identifying the supramolecular microstructure of micellar solutions, liquid crystals and microemulsions. Cryo-TEM at Minnesota has been successful in (1) identifying the spherical to wormlike micelle transition as the reason some surfactant solutions have a $10^{4}$-fold increase in viscosity upon addition of less than a percent of salt, (2) finding a transition from a disk-like micellar solution to ripple phase liquid crystal (ripple phase consists of a periodic stack of corregated bilayers, $(3)$ determining the variety of liquid crystallites and vesicles formed by mechanical agitation of liquid crystal dispersions, and(4) exposing the bicontinuous structure of microemulsions.

- Developed the theory of imaging liquid crystallites with polarized light as a function of their shape and structure and invented a low cost system for simulating and animating liquid crystallites viewed with polarized light. The simulation results, available as a $16 \mathrm{~mm}$ movie, help identify liqquid crystallites and vesicles from micrographs taken with a polarized light microscope.

- Introduced an accurate, tractable molecular theory of the electric double layer which is so important in controlling the microstructure and phase behavior of many oil, water and surfactant solutions. By comparison with computer simulations, the theory was shown to be vastly superior to the traditional Gouy-Chapman theory of the electric double layer. 


\section{Supercomputer-Aided Mathematical Modelling}

- Developed an adaptive, finite element frontal displacement simulator in which initial and boundary conditions are handled efficiently and without irtroducing the usual numerical artifacts (oscillations) plaguing simulators. The simulator was tested to be accurate and economical by comparison with an analytically solvable model. The simulator was also used to study stability of two-phase frontal displacement in heterogeneous reservoirs and under conditions of favorable and unfavorable mobility ratios of the phases. It was shown that linear stability analysis fails for two-phase frontal displacement.

- Developed a mechanism-based simulator of two and three-phase frontal displacement. The model combines self-consistently the microscopic statistical network theory for computing capillary pressures and relative permeabiliiies of the advancing front and numerical solution of the Darcy-level frontal displacement equations. The model is potentially especially useful for predicting oil recovery in three-phase displacement processes because empirical correlations are rare for this case and will be expensive to generate because of the number of variables involved.

- Developed a three-dimensional, adaptive, finite element algorithm with automatic grid generation for studying the free energy, conduction and flow in periodic porous media. The algorithm and its associated supercomputer code has established the current state of the art in $3 \mathrm{D}$ computing. the algorithm has been used to calculate the free energy of bicontinuous liquid crystals. It has also been used to calculate flow and transport in several families of periodic porous media. Regions of recirculation flow, important in flow driven dispersive mixing in porous media, were found. The relationship between the form factor and the permeability of these porous media was investigated. The values of a characteristic transport length that can be deduced from the results agree with that proposed recently by physicists at Schlumberger. 


\title{
FLOW IN POROUS MEDIA, PHASE BEHAVIOR AND ULTRALOW INTERFACIAL TENSIONS: MECHANISMS OF ENHANCED PETROLEUM RECOVERY
}

\author{
Department of Chemical Engineering and Materials Science \\ University of Minnesota
}

\section{ABSTRACT: $\mathbf{1 9 8 5 - 8 9}$}

A major program of university research, longer-ranged and more fundamental in approach than industrial research, into basic mechanisms of enhancing petroleum recovery and into underlying physics, chemistry, geology, applied mathematics, computation, and engineering science has been built at Minnesota. The 1988-89 outputs of the interdisciplinary team of investigators were again ideas, instruments, techniques, data, understanding and skilled people:

- 76 scientific and engineering publications in leading journals.

- Ten Ph.D. theses, six authors going to industry. The other to a postdoctoral university position.

- Three M.S. theses. One author is now pursuing a Ph.D. degree in Chemical Engineering and the other is inactive.

- Numerous presentations to scientific and technical meetings, and to industrial, governmental and university laboratories in the U.S., Europe and South America.

- Vigorous program of research visits to and from Minnesota.

This report summarizes the papers and theses that emerged during the period 1 October 1988 to 30 September 1989 and features thirteen major accomplishments of the program during that year. The major accomplishments are reported in greater detail in the remainder of the report. The major accomplishments of the program of 198588 we described in three previous Annual reports and twelve Quarterly Reports. The major accomplishments during 1988-99 are reported in this report. However, the research highlights of the entire four years of the program are briefly outlined in the next section. 


\section{INTRODUCTION}

This report summarizes the outputs that emerged from the Minnesota research program during the period 1 October 1988 to 30 September 1989. The goals of the program, ideas, instruments, techniques, data, understanding, and skilled people for the longer term, doing so especially by elucidating basic mechanisms. For then the uncertainties in process design, particularly in scale-up, control and optimization, are reduced, and innovative process development is promoted.

The original focus was surfactant-based chemical flooding, but the approach taken were sufficiently fundamental that the research, longer-ranged than industrial efforts, has become quite multidirectional. Many current outputs of program are basic enough to pertain to petroleum recovery more broadly and to energy-related technologies as well.

\section{Research Highlights}

The emphasis of the research program is on understanding basic physical chemical mechanisms with the goal of transforming this knowledge into the concepts and mathematical formulations needed for engineering process design and analysis. In this section is presented highlights of the research progress described in detail in subsequent sections of the report.

Volume controlled porosimetry. A few years ago Yuan and Swanson introduced a new Apparatus for Pore Examination (APEX) for a new kind of porosimetry. Instead of setting the capillary pressure and measuring the increase in saturation, they inject a controlled amount of volume and measure the resulting capillary pressure. This volume-controlled porosimetry generates far more information about the morphology of the pore space. To use this information effectively one must, however, understand how capillarity and pore morphology jointly govern the capillary pressure curve observed in volume-controlled injection of a wetting phase.

Earlier Minnesota researchers (e.g. Mohanty et al. 1987, Heiba et al. 1982) established capabilities of simulating processes like mercury injection with mechanism-based, computer-facilitated models of pore-level displacements in the pore network. We bring these capabilities and some new features to bear on APEX to discover how much useful information about a porous medium can be extracted from volume-controlled mercury displacement. The disordered nature of porous media we reduce to decorated network approximations onto which any pore size distribution, pore structure, and topological feature can be mapped. Such networks can represent sandstones and carbonates closely, including pore systems that display bimodal size distributions, diagenetically altered shapes, random or correlated heterogeneities, and stratification. APEX mercury injection is quasi-static; so is our simulation. Displacement under these circumstances consist of smooth, reversible changes linked by jumps in capillary pressure, the sequence of which follows from the structure of the porous medium and the saturation history. Thus, careful examination of 
fluctuations in the capillary pressure provides detailed information about pore structure, notably distributions of pore size and pore volume. The results account quantitatively for the APEX mercury capillary pressure curves measured by Yuan and Swanson. In our work, sample size - an aspect not reported previously - is found to be a major factor in APEX response. By Monte Carlo simulation of APEX mercury injection we find the optimum size of specimen for examining pore space of given properties. By the same means we investigate added kinds of experiments that extend the capabilities of APEX mercury injection, namely withdrawal experiments, withdrawal after partial reinjection and full scanning loops. We also investigate the potential use of high pressure mercury porosimetry to characterize microporosity and surface roughness in reservoir rocks.

Fluid distribution and Transport in Porous media at low wetting phase saturation. Data on the capillary pressure, the hydraulic conductivity and the capillary dispersion coefficient as a function of saturation of wetting phase at low saturations can be used to deduce the distribution of sizes of the asperities of porespace and the disjoining pressure of the thin films of wetting phase. Katz and Thompson by electron microscopy observed that the asperities in the porespace of natural sandstone are fractally distributed. Davis showed that Melrose's capillary pressure at low water saturation implies the same fractal dimension. We have combined the theories of fractal geometry and thin film physics to derive power laws for the capillary pressure, hydraulic conductance and capillary dispersivity at low wetting phase saturation. The theory provides a basis for similar empirically derived laws for sandstones and soils.

Molecular dynamics of fluids in ultranarrow pores. Fluids in molecularly thin pores, e.g., water between clay platelets or oil in diatomacious earth, have no regions of homogeneous concentration. The pressure in such confined thin films of fluid is anisotropic and is very sensitive to pore size. To understand how the fluid molecules are distributed in confined thin films and how this density distribution affects pressure, diffusion and flow in ultrathin films, we have carried out molecular dynamics studies of simple fluids confined between flat solids. The density distribution and the diffusivity are not affected appreciably by the flow even though the shear rates are very high compared to laboratory rates.

However, the strong density variations across the pore render the usual dependence of the local viscosity on local density inappropriate. At separations greater than four molecular diameters flow can be described by a simple redefinition of local viscosity. In narrower pores a dramatic increase of effective viscosities is observed and is due to the inability of fluid layers to undergo the gliding motion of planar flow. This effect is partially responsibility for the strong viscosity increases observed experimentally in thin films that still maintain their fluidity

Molecular dynamics and molecular theory of wetting and adsorption. Van der Waals or dispersion forces and the electrical doubly layer interactions control the wetting and adsorption behavior of fluids contracting solid surfaces. As these forces operate at the molecular level an understanding of the mechanisms of wetting and adsorption can be 
achieved only through a molecular-level theory. The combination or density functional theory and molecular simulations has lead to considerable progress toward developing such a theory in recent years. Molecular dynamics of the primitive model electrolyte has provided much insight into the nature of the electrical double layer. We have developed a density functional free energy theory that predicts quantitatively the ion density and electrical potential profiles of a primitive electrolyte at a charged surface. The usual approach - a Stern layer plus a Gouy-Chapman.region - is wrong because it assumes the ions near the electrode form a dilute solution and that the Stern layer possesses a bulk-like dielectric constant. Density functional theory itself involves approximations, even though it is considerably better than the Gouy-Chapman theory. As a guide to help invent the most accurate, tractable density functional theories we have found exact solutions to simple one-dimensional fluids. Earlier we solved the hard-rod problem. In this report we present exact solutions for a 1D fluid whose particles interact with an arbitrary nearest neighbor potential. Density profiles and disjoining pressures are computed for square -well and triangular-well particles. We also show that the modern density functional theories which compare well with computer simulations, predict the same wetting transition behavior that older, less quantitative theories did.

New numerical methods to handle initial and boundary conditions in inumiscible displacement. The analysis of continuous flow situations requires identification of a 'system,' which is the region of particular interest, and its 'surroundings,' which are represented by conditions imposed at the boundary of the 'system.' General procedures for choosing boundary conditions at surfaces at which no phase boundary exists, i. e. 'synthetic' boundaries, seem to be lacking. Drawing on the example of immiscible displacement of oil by water in a one-dimensional, semi-infinite porous medium, we compare four types of synthetic downstream boundary conditions - Dirichlet (first kind), Neumann (second kind), Robin (third kind), and what is in essence none - to find which is the most efficient when predictions are to be computed from the solution of the governing equation set. The Robin-type condition proves best: it gives the most accurate solution at fixed cost or, alternatively, requires the least work to achieve a given accuracy. To represent faithfully the physics of the situation, the Dirichlet and Neumann conditions must be imposed farther downstream of the region of interest than the Robin condition. In addition, we explore the behavior of a 'pseudo-boundary condition,' which is in fact not a proper boundary condition at all, but discretization and truncation errors mask its redundant nature and allow it to perform well in cases where there is little upstream signalling. Although our findings are drawn from the displacement problem, they are more broadly applicable to analysis of transport phenomena.

An initial condition, which sets the state of a system at a particular time, should describe accurately the physics of the situation and should not create computational artifacts when the governing equations are solved numerically. Drawing on the example of two-phase flow in porous media, we show that unphysical oscillations can mar a solution should the initial condition violate an inflow boundary condition. Tracking these oscillations can increase by orders of magnitude the computer time needed to solve the equations 
of change. At an internal boundary between two different media, an initial condition that violates the steady-state equations of change produces features that might be equally undesirable. We propose a way to generate initial conditions that avoid these artifacts, viz. by splicing together a solution of the linearized governing equations in the region of change and solutions of the steady-state equations in regions of constancy. We demonstrate our proposal using the situation of two-phase flow in porous media. Our findings are broadly applicable because of the partial analogies among transport of mass, heat, and momentum.

Electron microscopy of surfactant fluid microstructure. A major goal of the Minnesota program has been to determine the microstructure of surfactant solutions and to relate the microstructure to the phase interfacial and rheological properties of surfactant solutions. In previous work we developed cryo-electron microscopy and holey polymer sample holders that enable one to visualize directly fluid microstructures as small as 50 angstroms. In this report, we present cryo-microscopy studies of surfactant microstructures in dilute aqueous solutions and dispersions - globular, swollen and cylindrical or wormlike micelles, discoid and ripple phase structures, and uni- and multi-lamellar vesicles - can be seen at high resolution by cryo-transmission electron microscopy (cryo-TEM) of thin vitrified sample films. Sample films are prepared within a chamber where temperature and chemical activities of the surrounding vapor are controlled, thereby preventing evaporation and temperature changes that could alter the microstructure in the labile systems. The thin liquid films are quenched by rapidly plunging them into liquid ethane. The resulting vitrified samples are mounted into a cold-stage and transferred into a TEM for direct observation. Monophasic solutions of cetyltrimethylammonium bromide (CTAB) show globular micelles that swell with added toluene or styrene to form swollen micelles. Wormlike micelles form in $\mathrm{CTAB}-\mathrm{NaBr}$ solutions. Dilute mixtures of dipalmitoylphosphatidylcholine (DPPC) and diheptanoylphosphatidylcholine (DHPC) show discoid structures above the main transition temperature of DPPC and $\Lambda$ and $\Lambda / 2$ ripple structures of the $\mathrm{P}_{\beta^{\prime}}$ phase at temperatures below the main transition temperature. A new model is proposed for the $\Lambda$ structure and the ripple structures are shown to exist as single bilayers. Biphasic dispersions of sodium 4-(1'-heptylnonyl)benzenesulfonate (SHBS or Texas \# 1) show spheroidal and tubular vesicles, and complex encapsulated vesicles and coiled tubules. Vesicle-like microstructures of SHBS persist at $90^{\circ} \mathrm{C}$. At the relatively low SHBS concentrations studied there is no evidence of the constant spacing characteristic of the lamellar phase at higher concentrations, suggesting that the structures observed may result from unbinding fluctuations that disrupt lamellar phases.

A low cost system for animating liquid crystallites viewed with polarized light. Animated movies of scientific graphics can be recorded on film with the low cost system of hardware and software described here. The hardware consists of a $16 \mathrm{~mm}$ camera, a stepper motor, and a simple camera-motor controller. The software is designed to produce bitmaps from graphical data, combine bitmaps into composite frames, and record frames onto film. The camera is fully controlled by the same graphics workstation that is used to display the images, so fades and dissolves can be performed in software with a camera not equipped for such special effects. The graphical data, generated on a supercomputer, is 
subsequently transferred to the workstation where it is stored and recorded frame by frame according to a configuration file. A variant of the software, which operates across local and wide area networks, makes use of network computing software to send computationally intensive tasks to a remote supercomputer or to other workstations in a distributed computing environment. We have used the system to simulate polarized light microscope images of liquid crystals according to a single-scattering ray-tracing theory.

Surfaces of constant mean curvature with prescribed contact angle. The relationships between the structures and properties of foams and emulsions are not yet fully understood. An emulsion or a foam is defined in general terms as a reasonably stable dispersion of one fluid phase in another, immiscible, fluid phase. Various additives may prevent the dispersion from separating for a period of time yet it is not thermodynamically stable. The relationships between physical structures and physical properties of such dispersions are of practical as well as theoretical interest. These relationships can be computed from threedimensional models of the structures. At volume fractions below some critical value, there is an 'internal' phase that is dispersed in a continuous 'external' phase and the former's form can be modeled as undistorted spheres. At volume fractions approaching unity, the 'internal' phase forms polyhedral structures and the 'external' phase is limited to thin films between polyhedral faces and channels at the polyhedral edges. At intermediate volume fractions, there are structures that are not well characterized, much less well understood. Physical properties cannot be accurately simulated without adequate A Galerkin weighted residual formulation of the Surface Divergence Theorem is used with finite element basis functions to compute geometric models of foam and emulsion structure. The models are surfaces of constant mean curvature arranged on a simple cubic lattice so that they meet the boundary planes of the unit cells at prescribed contact angles. Surfaces are computed for a variety of contact angles and mean curvatures. With contact angle between $5^{\circ}$ and $133^{\circ}$, the structure inverts as the mean curvature decreases, whereas with contact between $134^{\circ}$ and $180^{\circ}$, the structure fills the unit cell as the mean curvature decreases, passes through a turning point, and then increases. The models have implications to mercury porosimetry. 


\section{PORE-SPACE STATISTICS AND CAPILLARY PRESSURE CURVES FROM VOLUME-CONTROLLED POROSIMETRY}

\section{Synopsis}

Far more information about pore space of reservoir rock samples can be obtained from volume-controlled mercury porosimetry than from conventional pressure-controlled mercury porosimetry, as Yuan and Swanson (1986) demonstrated in landmark experiments with their new Apparatus for Pore Examination - APEX.

Earlier researches (e.g. Mohanty et al. 1987, Heiba et al. 1982) established capabilities of simulating processes like mercury injection with mechanism-based, computer-facilitated models of pore-level displacements in the pore network. We bring these capabilities and some new features to bear on APEX to discover how much useful information about a porous medium can be extracted from volume-controlled mercury displacement.

The disordered nature of porous media we reduce to decorated network approximations onto which any pore size distribution, pore structure, and topological feature can be mapped. Such networks can represent sandstones and carbonates closely, including pore systems that display bimodal size distributions, diagenetically altered shapes, random or correlated heterogeneities, and stratification.

APEX mercury injection is quasi-static; so is our simulation. Displacement under these circumstances consists of smuoth, reversible changes linked by jumps in capillary pressure, the sequence of which follows from the structure of the porous medium and the saturation history. Thus, careful examination of fluctuations in the capillary pressure provides detailed information about pore structure, notably distributions of pore size and pore volume.

The results account quantitatively for the APEX mercury capillary pressure curves so precisely measured by Yuan and Swanson. In our work, sample size - an aspect not reported previously - is found to be a major factor in APEX response. By Monte Carlo simulation of APEX mercury injection we find the optimum size of specimen for examining pore space of given properties.

By the same means we investigate added kinds of experiments that extend the capabilities of APEX mercury injection, namely withdrawal experiments, withdrawal after partial reinjection and full scanning loops. We also investigate the potential use of high pressure mercury porosimetry to characterize microporosity and surface roughness in reservoir rocks. 


\section{Introduction}

Mercury porosimetry, the forced intrusion of mercury into a porous material, has been used to characterize the microstruciure of the pore space since Washburn (1921) suggested how to obtain a 'pore size dist:ibution' from measurements of volume injected versus pressure applied. Ritter and Drake (1945) authored the first work fully devoted to mercury porosimetry, describing the construction and operation of the equipment, reporting many experimental duta and forming the basis of subsequent developments. In 1949 Purcell introduced the technique to the petroleum industry. Since then, mercury capillary pressure curves measured on reservoir rock samples (cores, chips, etc.) have been used routinely in connection with petroleum exploration and production. The goal is to get information on zelationships between petrophysical properties and the microstructure of the pore space, particularly information useful for predictions of porosity, permeability, relative permeabilities, and residual oil saturation of reservoir rocks.

In the classical experment, pressure or the mercury is raised in increments and the amount of mercury injected into a sample is measured at each step. This is pressurecontrolled measurement of a mercury capillary pressure curve. Commercially available mercury porosimeters offer a simple and rapid procedure to obtain a kind of 'fingerprint' of a reservoir rock. How much of the internal structure is represented by the fingerprint is not clear. Indeed, interpretation of the fingerprint is still the subject of active research. The basis of the method is the concept that in a previously evacuated specimen, mercury is forced !nto smaller and smaller pore segments against capillary forces as the mercury pressure is increased. Such measurement do not directly give the true pore size distribution. There may, for example, be large pore segments which could be filled at low pressure except that they connect with the mercury source only through smaller pore segments (cf. Everett 1958, Heiba 1985). The effect of these so-called ink-bottle pore segments is to assign too small a portion of the pore space to the large pore segments and too large a portion to the small pore segments, if the mercury injection data are taken at their face value. Porous media are irregular networks of pore space, the constrictions and enlarged junctions of which are usually termed pore throats and pore bodies. Thus, a pressurecontrolled capillary pressure curve, even if accurately measured (cf. Katz and Thompson 1987, Thompson et al. 1987), reveals the distribution of pore throat sizes in only a subset of the entire population of throats. It is possible for two porous media having different microstructures to give the same capillary pressure curves. This ambiguity is the main obstacle to correlating properties like permeability with pore structure as inferred from capillary pressure data.

Injection of mercury into a sample by a stepping-motor-driven positive displacement pump or a constant rate pump is another way of measuring capillary pressure curves. This is volume-controlled measurement of a capillary pressure curve. The mercury capillary pressure is monitored as the dependent variable. During the process, mercury menisci curve to accommodate to pore shapes and pressure differences. Because capillary pressure is inversely proportional to a mean radius of curvature of the mercury menisci, fluctuat: ons 
in curvature bring fluctuations in capillary pressure.

That pressure fluctuates during volume-controlled fluid displacement has been observed. Irreversible pressure fluctuations at the pore-level scale are evident from observations made by Haines (1930): during invasion of pore spaces, the development of unstable interface configurations leads to small oscillations in pressure. In 1959, Gates (as mentioned by Yuan and Swanson 1986) observed pressure fluctuations during mercury porosimetry of vuggy carbonates. Crawford and Hoover (1966) reported striking instances of fluctuations in capillary pressure curves recorded during injection of water into synthetic porous media. Morrow (1970) demonstrated that drying of a heap of tapered capillaries and a heap of spheres saturated with volatile liquid cannot be conducted smoothly and reversibly because of spontaneous changes in fluid configuration. In 1971 Gaulier described the first automatic device for exploiting pressure fluctuations to measure vugular porosity by constant rate porosimetry. In all these works, capillary pressures are measured in coarse-pored media. When capillary pressures are measured in fine-pored media, such as sandstones or clay-coated carbonates, fluctuations in pressure are generally too small to be observed or detected by available pressure transducers. Thus, experimental capillary pressure curves appear to be smooth and reversible.

Recently Yuan and Swanson (1986), using high-precision sputtered straingage pressure transducers and a high-precision stepping motor for controlled volume injection, successfully resolved very small pressure fluctuations as mercury was injected into sandstones and carbonates. These authors demonstrated in landmark experiments with their new Apparatus for Pore Examination (APEX) that far more information about the pore space of a reservoir rock sample can be obtained from volume-controlled mercury porosimetry than from conventional pressure-controlled mercury porosimetry.

APEX mercury injection is quasi-static. Displacement under these circumstances consists of smooth, reversible changes followed by spontaneous changes, generally irreversible ones, in capillary pressure at constant mercury saturation. The particular sequence of alternate reversible and spontaneous changes is determined by the structure of the porous medium and the saturation history. An understanding of this relationship is essential to convert the fluctuations in the capillary pressure into pore structure information. To this end, computer simulation of volume-controlled mercury porosimetry in a well defined model porous medium should be of great benefit through understanding Yuan and Swanson's experiment, and guiding extension of the capabilities of APEX. To our knowledge, volumecontrolled mercury porosimetry has not heretofore been simulated with mechanism-based. computer-facilitated models of pore-level displacement in networks of pore segments. We have developed such a simulation and here describe the method and report the results.

In the next section we synthesize two models of porous media, one of low size contrast between pore bodies and throats, the other of high size contrast. In the models the network of porespace is constructed by starting with a cubic lattice and pruning it down to a representative mean coordination. Pore segments are converging and diverging in 
character: biconical pores are chosen here to capture this essential feature. Sizes of pore segments are arrived at by drawing at random from a truncated log-normal distribution of pore throat radii. The size of a pore body is a multiple of the size of the largest adjacent pore throat. This produces correlation of size throughout the pore network.

Next we describe aspects of meniscus movement in the pore space that are taken into account in the analyses of injection and withdrawal. We consider low enough capillary number that menisci remain quasi-static most of the time, except when a meniscus becomes unstable and jumps under the combined effect of inertia and capillary forces. We restrict attention to the sequence of capillary equilibrium states that precede and succeed these jumps.

Next we give details of the computations from the theory. They constitute a 'Monte Carlo simulation' of volume-controlled mercury injection and withdrawal.

We conclude with a summary of results and a proposal of injection-withdrawal experiments to generate more information about pore space, thereby extending the power of APEX.

\section{Modeling Rock Morphology}

Capillarity-dominated flow is commonly interpreted in terms of simplified pore models. Simplification is necessary because nearly all porous media are so complex that their microstructure can be neither determined nor described completely. The simplification gained by treating the pores as though they were uniform cylinders, or the spaces between regularly or randomly packed spheres or cylinders had to be accepted in the past. As fuller understanding of the capillary processes in such idealized systems developed, a more realistic approach to these processes in real porous media emerged from statistical considerations of the disordered array of pore spaces of variable geometry and connectivity in sandstones and carbonates (Fatt 1956, Mohanty 1981, Heiba 1985).

Network modeling of the pore space is an active area of research with a corresponding large body of literature. The crudest model is that of a bundle of non-intersecting tubes, each of constant but different cross-section corresponding to some given pore size distribution. This 'parallel type' model is the basis of so-called 'hydraulic radius' theories used to describe single-phase transport in porous media (Scheidegger 1974). The opposite extreme model is obtained by assuming that all the pore space is serially lined up. Such model is referred to as 'serial type' model because capillaries of different pore radius are put together in series one after another (Scheidegger 1974, Dullien 1979). Such models fail to predict the salient features of capillary displacement, i.e. the known hysteresis between injection and withdrawal, and the trapping of displaced fluid.

It is clear that a more appropriate model of pore space should involve some kind of 
random network. Indeed such an approach, introduced by Fatt in 1956, has been widely used in the last two decades to simulate the petrophysical properties of rock formations and the processes that occur within it. The terms pore, pore body, pore throat, pore system, pore space distribution are used rather loosely however. The 'sticks and balls' model, which approximate the pore space as a network of tubes and spheres, has been frequently used. Sticks and balls seem simple because their singularities of pore wall curvatures invite definitions, e.g. of pore body and pore throat, that are nevertheless arbitrary at root. This model fails to capture the converging and diverging character of most porous media. Consequently, there cannot be stable interfaces in the midst of pore segments, an essential feature of volume-controlled displacement processes.

Mohanty et al. (1987) and Lin and Cohen (1982) have provided a conceptual framework - contraction mapping - by which pore spaces can be mapped into equivalent networks. The authors have identified the method of recording completely the geometry of porespace and composition of pore walls, etc. It is called decoration - i.e. 'decorating' the network map with information about local properties. Levels of decoration can be adopted rationally and systematically, for whatever purpose they are needed. To synthesize our model porous media, in the remainder we simply follow Mohanty et al. (1987) and Lin and Cohen (1982).

We reduce the disordered nature of porous media to a decorated network approximation onto which wide ranges of porosity, pore size distribution, pore structures and topological features can be mapped. The converging and diverging nature of pore segments we conveniently captured with biconical pore segments. We define a pore segment as a cubic body that opens onto a number $z$ of converging conical portions of pore space, each of which leads either to a dead end or to volumeless throat beyord which lies another segment. In this way, the common arbitrary practice of separating the pore space volume into pore bodies and pore throats is avoided. Figure 1 shows the cross-section of a typical pore segment of our networks. The volume $V_{p s}$ of a pore segment is given by

$$
V_{p s} \equiv V_{b}+\sum_{i}^{z} V_{c_{i}}
$$

where $V_{b}$ is the volume of a body of cube edge $2 r_{b}$, i.e.

$$
V_{b} \equiv 8 r_{b}^{3}
$$

and $V_{c_{i}}$ is the volume of the $i$-th converging conical portion of porespace of length $l_{c_{i}}$, which leads to a throat of size $r_{t_{i}}$,

$$
V_{c_{i}} \equiv \frac{1}{3} \pi l_{c_{i}}\left(r_{t_{i}}^{2}+r_{b} r_{t_{i}}+r_{b}^{2}\right)
$$

We construct a network of biconical pore segments as follows. 


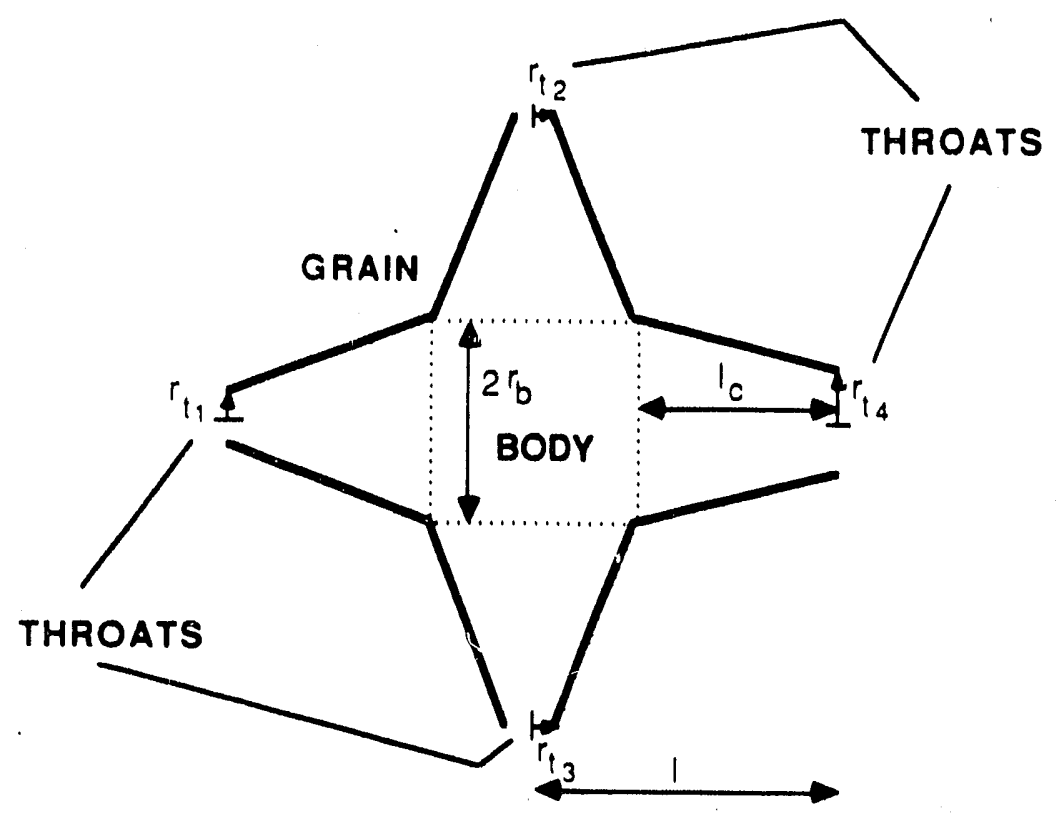

Figure 1. Idealization of a pore segment. Small scale roughness is not illustratrd.

(i) Choose an underlying lattice, i.e dimension and connectivity. Specify size and boundary conditions.

Notewithstanding two-dimensional networks are less expensive and easier to work with, we use a simple cubic lattice of nodes and branches as an underlying lattice to better account for the three-dimensional pore space of natural porous media. Actually, though this paper concentrates on the simple cubic lattice with a $N \times M \times L$ lattice size, the network generator can handle the two-dimensional square lattice equally well.

Although conceptually simple, the specification of boundary conditions we optimized to increase the versatility of the simulation. The boundary conditions are implemented as either open, closed, or periodic, depending on the displacement mode and the properties of the fluid/fluid pair.

To model volume-controlled porosimetry, the lateral sides of the pore network are connected to each other to form a cylindrical plug (periodic boundary condition). One side of the plug (of size $N \times M$ ) is connected to a source that supplies mercury (open boundary condition), while the opposite side (of size $N \times M$ also) is impermeable (closed boundary condition).

(ii) Prune the underlying lattice down to a representative mean coordination.

As a rule, the connectivity $z$ of a porous media has a probability distribution rather than a particular value. To obtain a representative mean coordination $\langle z\rangle$, we start with $z=6$, the connectivity of the underlying simple cubic lattice, and then randomly prune branches with probability $1-\langle z\rangle / 6$. The number of edges $\nu$ emanating from any vertex has then a binomial distribution with mean $\langle z\rangle$ and variance $\langle z\rangle(1-\langle z\rangle / 6)$ 
given by

$$
P_{\nu}=\left(\begin{array}{l}
6 \\
\nu
\end{array}\right)(<z>/ 6)^{\nu}(1-<z>/ 6)^{6-\nu}
$$

where

$$
\left(\begin{array}{l}
6 \\
\nu
\end{array}\right)=\frac{6 !}{\nu !(6-\nu) !}
$$

is the binomial coefficient.

(iii) Decorate the lattice with independent features.

Pore throat radii $\left(r_{t}\right)$ are randomly assigned according to a truncated log-normal distribution, which has the probability density function

$$
f\left(r_{t} ; \sigma\right)=\frac{\exp \left[-\frac{1}{2}\left(\frac{\ln r_{t}}{\sigma}\right)^{2}\right]}{r_{t} \sigma\left[\operatorname{erf}\left(\frac{\ln r_{t_{\max }}}{\sigma}\right)-\operatorname{erf}\left(\frac{\ln r_{\min }}{\sigma}\right)\right]} \quad r_{t_{\min }} \leq r_{t} \leq r_{t_{\max }}
$$

where $\sigma$ is the standard deviation of the parent normal distribution. Because the tails of the distribution are difficult to sample uniformly from one realization to the next, the full log-normal distribution was truncated (outside $\left[r_{t_{\min }}=0.1, r_{t_{\max }}=60\right] \mu \mathrm{m}$ ) and normalized to reduce the variance of the capillary pressure values.

We assign body size at $i$-th body according to $r_{b_{i}}=\rho r_{t}, \rho$ is a prescribed ratio. $r_{t}$ is the size of the largest throat adjacent to the $i$-body. This produces correlation of size throughout the pore network.

We ascribe a constant pore-center-to-pore-center distance $2 l$ (cf. Figure 1) so that linked to the other geometrical features, such as pore body size and pore throat radius, give a porosity $\phi$, consistent with that of the porous medium one wishes to mimic.

(iv) Deduce dependent features.

To each pore body we attached $z=6$ frustrums, each of right circular cone of radii $r_{b}, r_{t}$ (right circular cones if $r_{t}=0$ ) and height $l_{c}=l-r_{b}$ (cf. Figure 1). Semi-apical cone angles we obtain from $\alpha=\tan ^{-1}\left[\left(r_{b}-r_{t}\right) / l_{c}\right]$.

Figure 2 illustrates two-dimensional views of four networks generated according to this procedure. The network of Figure 2a, which has a high size contrast between pores bodies and pore throats, might represent a carbonate severely altered by diagenesis. Based on core analyses, such a rock can have sufficient porosity to make an attractive reservoir, but the pore segments are either poorly interconnected or else connected predominantly by small pore throats. Figure 2 b shows a network with low size contrast that might represent 
a consolidated sandstcne. Such a rock can have a relatively homogeneous pore system. Primary pores tend to Le destroyed with burial because of compaction and cementation. In this paper results are presented for the 'high aspect ratio' network of Figure $2 \mathrm{a}$ and for the 'low aspect ratio' network of Figure $2 \mathrm{~b}$, deferring other cases (Figure 2c and $2 \mathrm{~d}$ ) to a future publication.

\section{Characterization of Pore-Wall Roughness}

The geometry of pore walls in reservoir rocks varies from the smooth, crystalline surfaces of dolomites to the rough, pitted or clay-coated surfaces of sandstones and soils. Historically, studies of the physical propertits of porous media have focused on either the solid matrix or the pore space. Relatively little attentinn has been paid to the interface that uivides the solid space and the pore space. Recent efforts seek to characterize the roughness of pore walls and to understand its effects on the bulk properties of chaotic porous media. To this end fractal geometry should be a useful tool, as several have suggested (Katz and Thompson 1985, Wong et al. 1987!

For our purposes, what is important about fractal rnughness is that asperities are distributed over more than a few length scales. Even though real porous media resemble fractal objects, they do so only between lower and uppe; cutoff of scale. Katz and Thompson (1985) using microscopy and Wong et al. (1987) using neutron scattering, measured features of several sandstone, at the pore level. They found that the pore walls are surface fractals in a statistical sense on length scales between $1 \mathrm{~nm}$ and $100 \mu \mathrm{m}$ with Hausdorff dimensions between 2.57 and 2.87 .

It has been suggested (Davis 1989) that not only microscopy or neutron scattering, but also capillary pressure data at low saturation of wetting phase can be used to characterize pore-wall roughness over a range of length scales by a Hausdorff dimension. Our recent works (Novy et al. 1989, Toledo et al. 1989) support this view. High pressure mercury capillary pressure, such as those measured by Swanson (1985), should be equally useful.

The dependence of the inventory of pendular structures on capillary pressure can be measured in the laboratory, and conceptual models enable interpretations of these data. de Gennes (1985) examined two families of fractal extremes of pore-wall roughness: iterative pits (self-similar pits within pits) and iterative flocs (self- imilar grains fused to grains). Toledo et al. (1989) examined the case of the Menger sponge. In each case the following proportionality was obtained

$$
\left[1-V_{H g}\right] \propto \Delta p^{D-3}
$$

$V_{H g}$ is the volume of mercury occupying the pore space and $\Delta p=\left[p_{H g}-p_{v a c}\right]$, is the capillary pressure. High pressure mercury capillary pressure can be used to determine $D$, the Hausdorff dimension of the pore wails, $D$ being a measure of how the subject space fills the Euclidean space in which it is imbedded. 
(a)

(b)
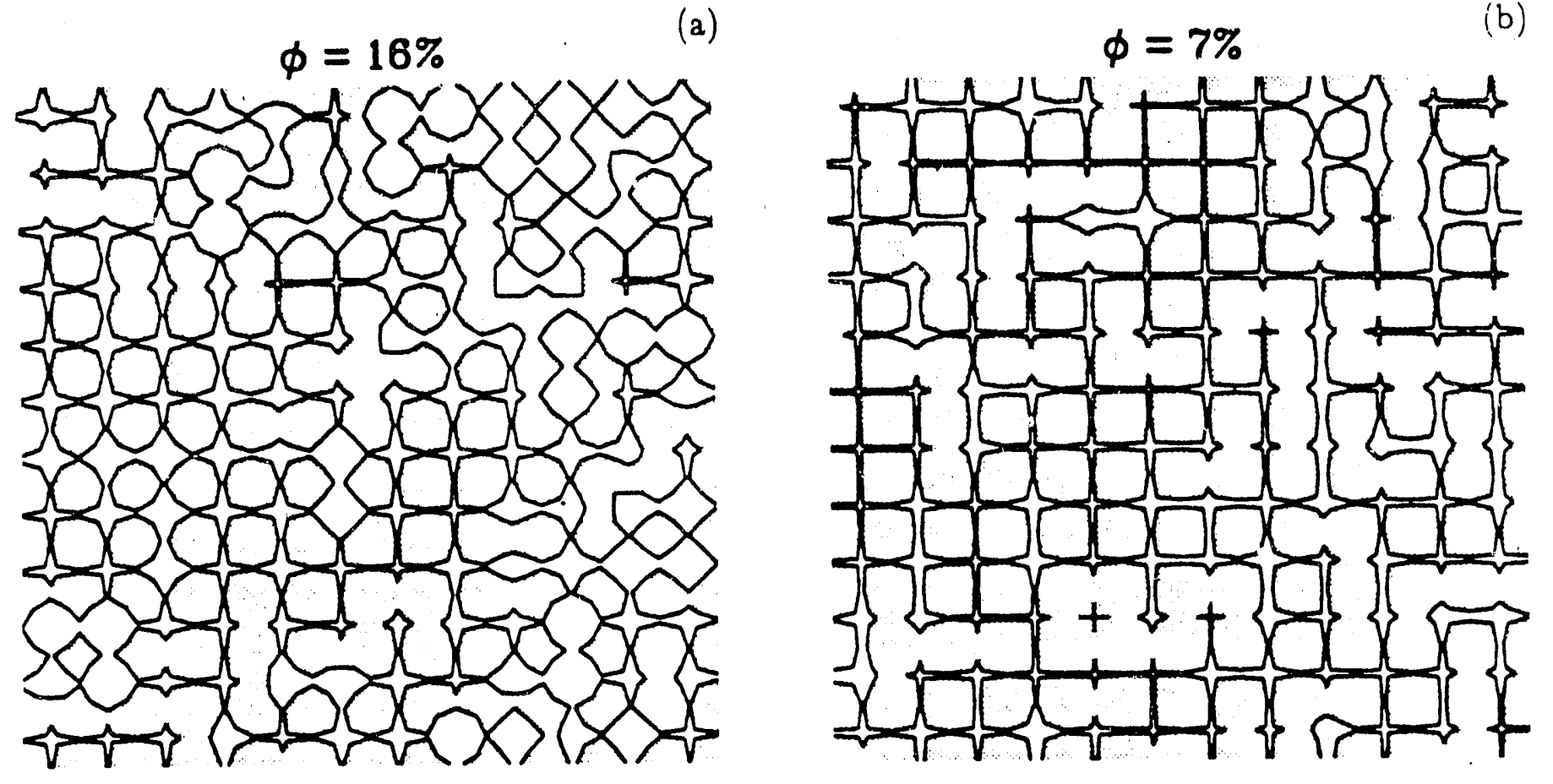

(c)
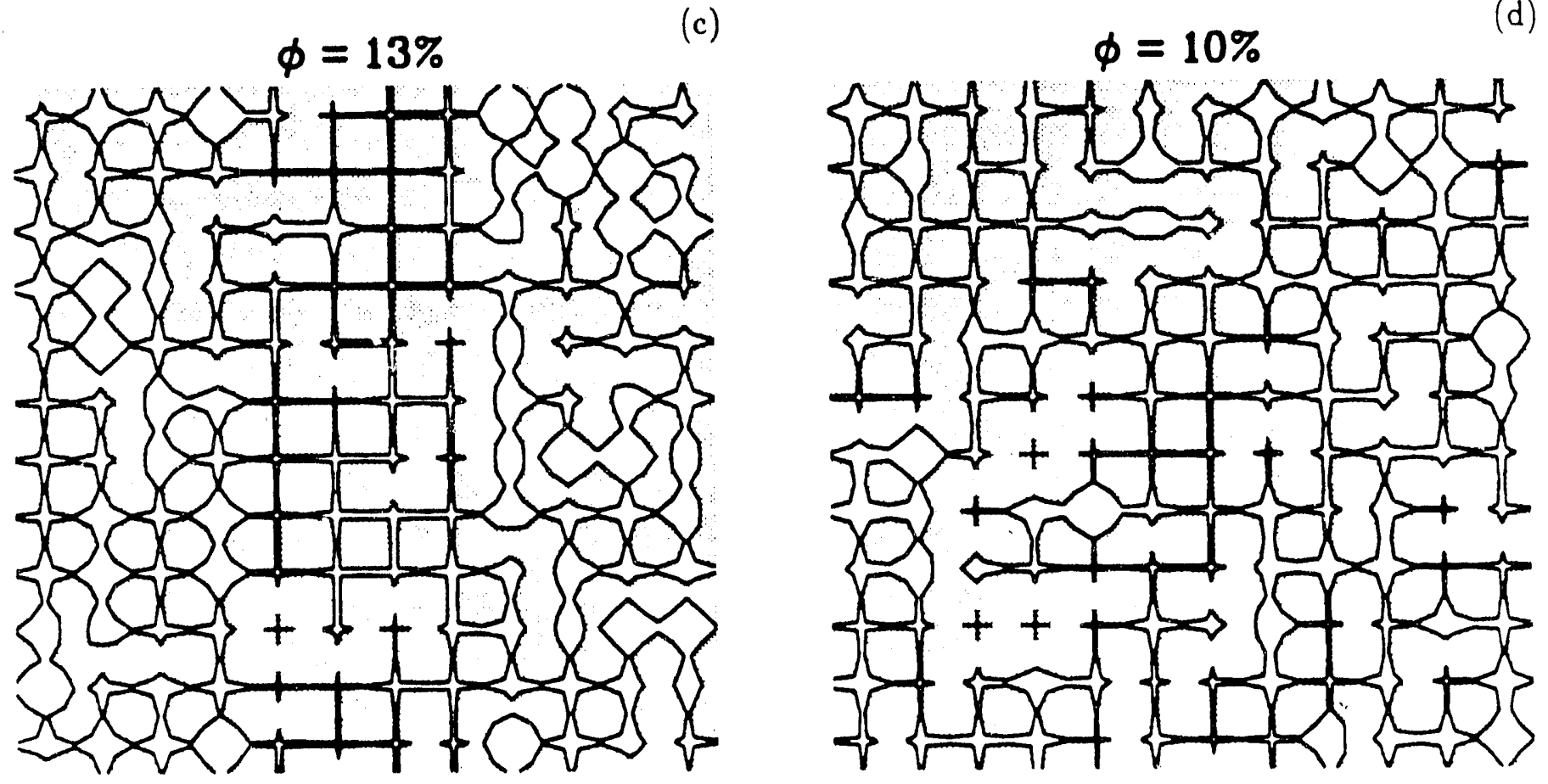

Figure 2. Computer-generated porous media. (a) 'High aspect ratio' network, representative of a carbonate rock with 'reconstructed' pore space, i.e. secondary porosity. (b) 'Low aspect ratio' network, representative of a consolidated sandstone. (c) and (d) Correlated and random heterogeneities, respectively. 
For the purpose of this work we used $\left[1-V_{H g}\right]=0.25 \Delta p^{0.45}$ (Davis 1989) to account for the inventory of pendular structures.

\section{Equilibrium and Stability of Static Interfaces in Porous Media}

The equilibrium and stability of interfaces constrained by solids play a central role in fluid distribution. Of primary interest here is to understand how capillary forces determine the distribution of fluid phases within the pore space of disordered porous media.

In the absence of external constraints (gravity or boundary conditions imposed by solid surfaces) an interface between two immiscible fluids tends to minimize its area by adopting a spherical shape. In the presence of a solid surface, however, the interface between two fluid masses assumes configurations, the equilibrium and stability of which depend on pore geometry, contact angle and externally imposed conditions (pressure, volume, etc.). A study of mercury porosimetry, is thus, in the most general case, a study of the nature of surfaces of constant mean curvature, subject to various boundary conditions.

It is only for constant curvature surfaces having radial or cylindrical symmetry, viz. the cylinder, unduloid, sphere, catenoid, and nodoid, that an exact analytical approach is possible (cf. Bolza 1909, Tyuptsov 1966, Huh 1969). For systems of lower symmetry, numerical approximation methods must be used. The goal of this section is to determine the equilibrium and stability of axisymmetric interfaces in axisymmetric biconical pore segments.

When mercury, a nonwetting phase, is present within an otherwise evacuated porous media, the complex mercury/vacuum interface consists of a number of menisci, i.e. surfaces that obey the Young-Laplace equation. According to Mohanty (1981), menisci are chiefly of two types: head menisci and neck menisci. Head menisci are interfaces with only positive elements of curvature, referred to as concave (toward the nonwetting phase) or clastic, i.e. surfaces with a positive Gaussian curvature. Neck menisci are interfaces with negative as well as positive curvatures, i.e. radii of curvature occur on both sides of an interface. This selloidal, saddle-shaped, or anticlastic interfaces have negative Gaussian curvature. Figure 3 iilustrates two such menisci in a biconical pore segment. Not shown in Figure 3 is the small scale surface roughness which is also present. The thread of nonwetting phase at the throat is called a neck. Accumulation of wetting phase around a nonwetting phase neck is called a collar. Collars are bounded by neck menisci and pore wall.

For an ensemble of menisci to be in stable equilibrium Gillette and Dyson (1974) and Mohanty (1981) showed that each meniscus must first be singly stable to volume-conserving shape perturbations. Consider the system mercury/vacuum. Let $[\Delta p]_{i}=\left[p_{H g}-p_{v a c}\right]_{i}=$ $\left[p_{H}\right]_{i}$ be the capillary pressure across the $i$-th meniscus, and $\left[V_{H g}\right]_{i}$ be the volume of mercury in the immediate neighborhood of the mercury/vacuum interface. At equilibrium, the capillary pressure $\Delta p$, acros: every meniscus of the ensemble, must be the same, 


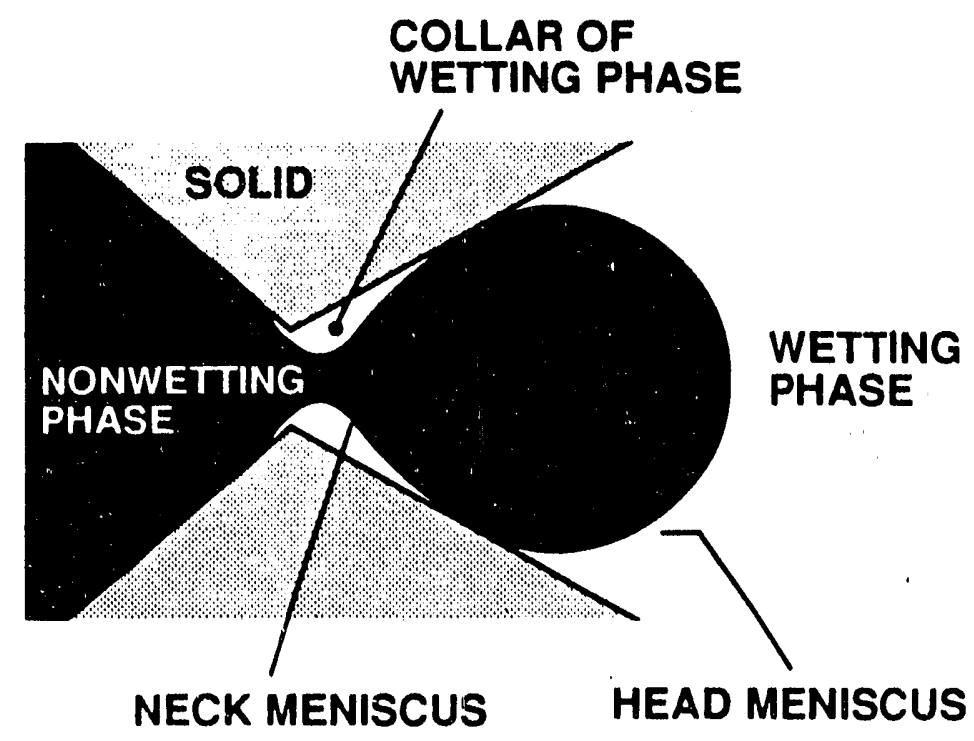

Tigure 3. A fluid/fluid interface in a biconical pore segment.

provided that pressure gradients and gravitational effects are negligibly small, then

$$
\Delta p_{i}=\Delta p_{j}=\cdots=\Delta p_{n}
$$

where $i, j, \ldots, n$ represent both neck menisci and head menisci.

For stable equilibrium, it is sufficient that (Melrose and Brandner 1974, Gillette and Dyson 1974)

$$
\left(\frac{d \Delta p}{d V_{H g}}\right)_{i}>0
$$

for every meniscus $i$ in the ensemble. Rücker (1887) recognized that Eq. (14) is necessary for stability of single interfaces.

While satisfying Eq. (5) by all menisci in the ensemble is sufficient, Gillette and Dyson (1974) found that this is not always necessary. If at most one meniscus violates Eq. (5), the rest of the ensemble can stabilize it if the following conditions are met

$$
\left(\frac{d \Delta p}{d V_{H g}}\right)_{i}>0 \quad \text { for all } i \neq j
$$

and

$$
\sum_{i}\left(\frac{d \Delta p}{d V_{H g}}\right)_{i}^{-1}<0
$$

Thus the ensemble stability conditions are given by either Eq. (5) or by Eqs. (6) and (7). Violating these conditions leads to instabilities and sudden movement of menisci. In what follows, we study the equilibrium and stability of neck menisci and head menisci in biconical pore segments. 


\section{Neck Menisci in Biconical Pore Segments}

As capillary pressure is lowered in a system similar to that in Figure 3, the curvature of the neck menisci changes to a critical value at which the fluid/fluid interface becomes unstable and suddenly ruptures. Prior to this event, the interface is stable and can be made to advance or retreat in response to small changes in the capillary pressure. Rupturing of a selloidal interface which has become unstable has been referred to as snap-off or choke-off, a well-understood phenomenon for systems with one fluid that completely wets the solid. Its occurrence depends on pore wall curvature and the pressure levels in the wetting and nonwetting phases. For zero contact angle, there is a critical ratio of pore radius $\left(r_{b}\right)$ to throat radius $\left(r_{t}\right)$, at which choke-off of a neck meniscus occurs. As a result the nonwettıng phase becomes disconnected and nonwetting phase is trapped in the medium. This is an important mechanism of trapping of oil in water-wet reservoir rocks during waterflooding (Stegemeier 1974, Mohanty 1981).

Breakage or choke-off when one of the fluids partially wets the solid, such as the case of mercury on silica surfaces, is a less-understood phenomenon, although it has been cited as a possible cause of hysteresis and trapping (van Brakel et al. 1981). Wardlaw (1976) found experimentally that if large pores are connected by narrow throats, mercury chokeoff occurs on pressure reduction at relatively high pressures. This result is at variance with the work of Mohanty (1981). Using pores with a variety of toroidal shapes, this last author demonstrated, theoretically and experimentally, that incomplete wetting adversely affects the tendency to choke-off. The conclusive work is yet to be done. If choke-off is a factor in mercury porosimetry, we doubt that the phenomenon can be captured with axisymmetric interfaces in axisymmetric pores. Other factors, such as pore segments of lower symmetry and pinning of the contact lines should be considered. In what follows we discuss the impact of a non-zero contact angle on the equilibrium and stability of neck menisci in biconical pore segments with two axes of symmetry and with one axis of symmetry. Our work closely follows the work of Huh (1969) and Mohanty (1981).

Figure 4 shows the volume of collars of wetting fluid (vacuum) as a function of capillary pressure, for $\theta=0^{\circ}, \theta=5^{\circ}$, and $\theta=35^{\circ}$, and semi-apical angles $\alpha\left[1^{\circ}<\alpha<30^{\circ}\right]$. Menisci represented by long broken lines are unstable to non-axisymmetric perturbations, whereas those represented by dotted and short dashed lines are unstable to axisymmetric perturbations. Selected equilibrium configurations in single conical pore segments are shown in Figure 5. Figure 6 shows equilibrium configurations in single non-symmetrical biconical pore segments. The contact angle is fixed, $\theta=0^{\circ}$ (Figure 6A) and $\theta=20^{\circ}$ (Figure $6 \mathrm{~B})$. Stability analysis to volume conserving shape perturbations indicate no region of stable neck menisci with $d \Delta p / d V_{H g}>0$ in symmetrical pores. Non-axisymmetry stabilizes some isolated menisci but they become unstable, i.e they do not exist when other menisci exist, thus hindering the choke-off process.

Our calculations, as those of Mohanty, only considered fixed contact angles, i.e. movable contact lines. Departure fron this situation brings in more hysteresis and pinning of 


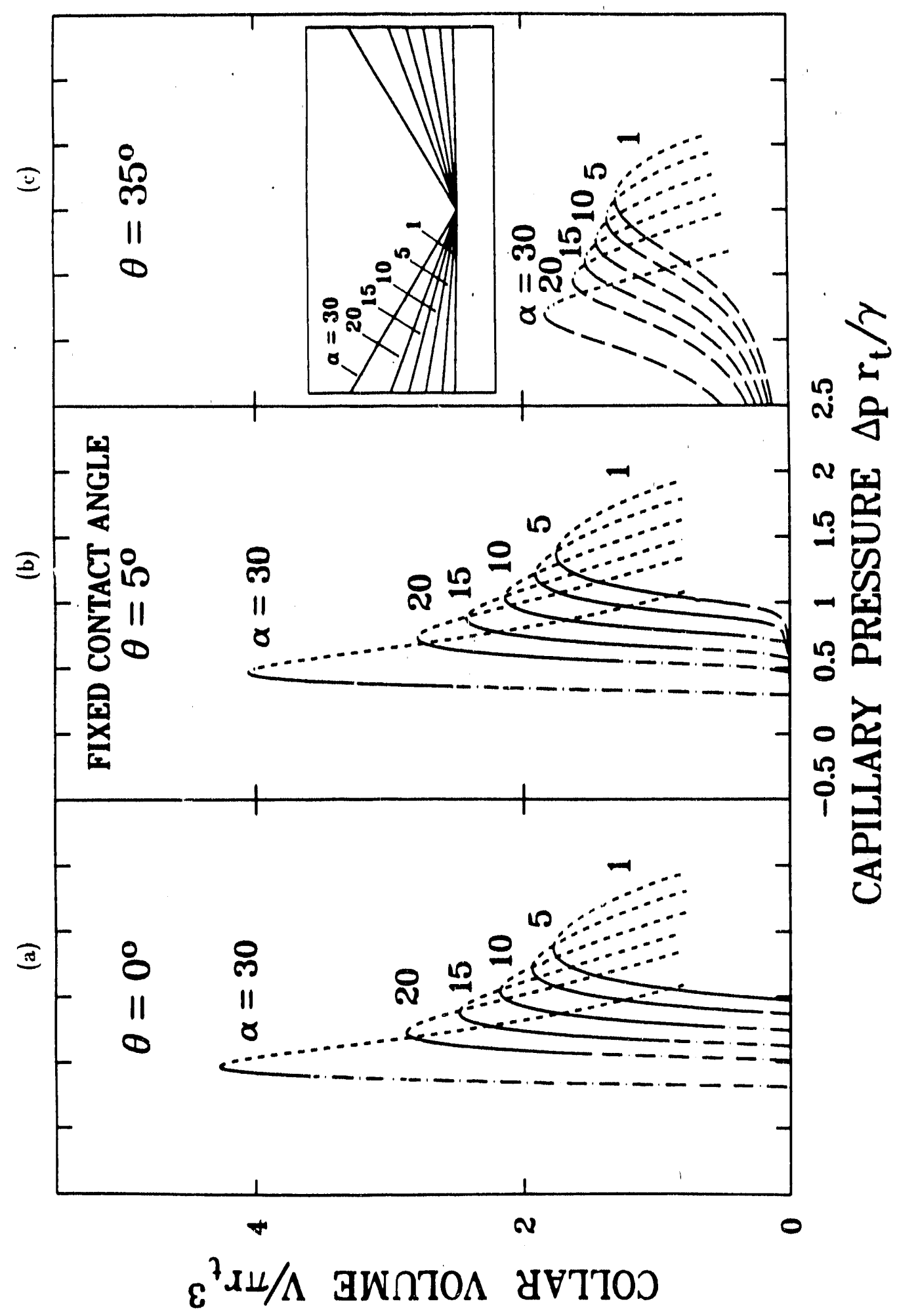

Figure 4. Equilibrium and stability of neck menisci to volume-conserving shape perturbations. Volume of vacuum collars in biconical pore segments (inset) as a function of capillary pressure. Fixed contact angle, movable contact lines. (a) $\theta=0^{\circ}$, (b) $\theta=5^{\circ}$, and (c) $\theta=35^{\circ}$. 


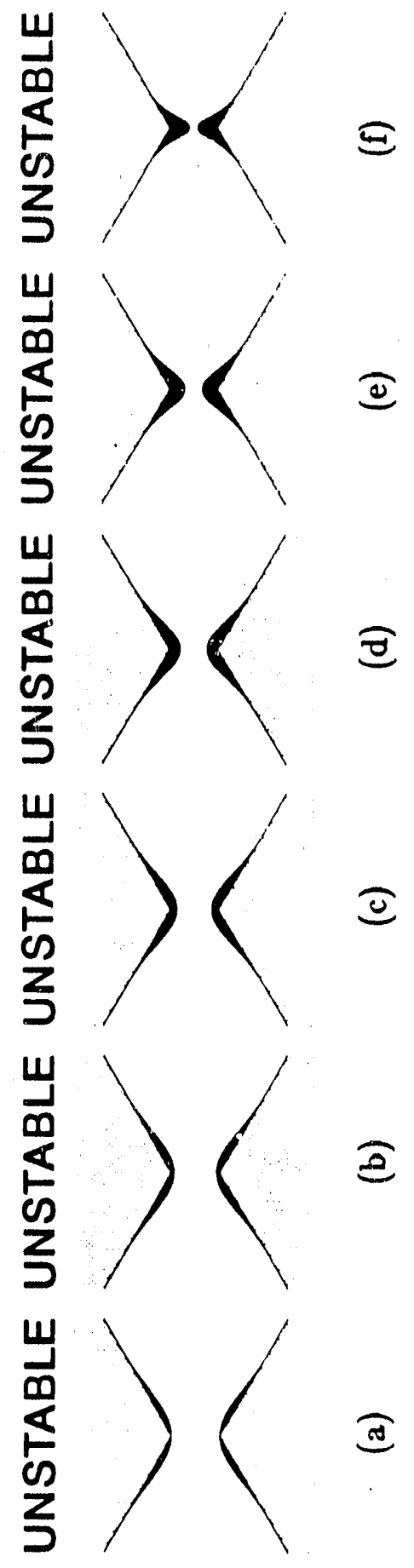

Figure 5. Isolated equilibrium configurations in biconical pores with reflective symmetry in the throat plane. Semi-apical angles $\alpha_{[l e f t]}=30^{\circ}, \alpha_{[r i g h t]}=30^{\circ}$. Fixed contact angle, movable contact lines. $\theta=0^{\circ}$. Non-dimensional capillary pressure $\Delta p r_{t} / \gamma$ : (a) 0.40 , (b) 0.42 , (c) 0.47 , (d) 0.53 , (e) 0.63 , and (f) 0.82 . 


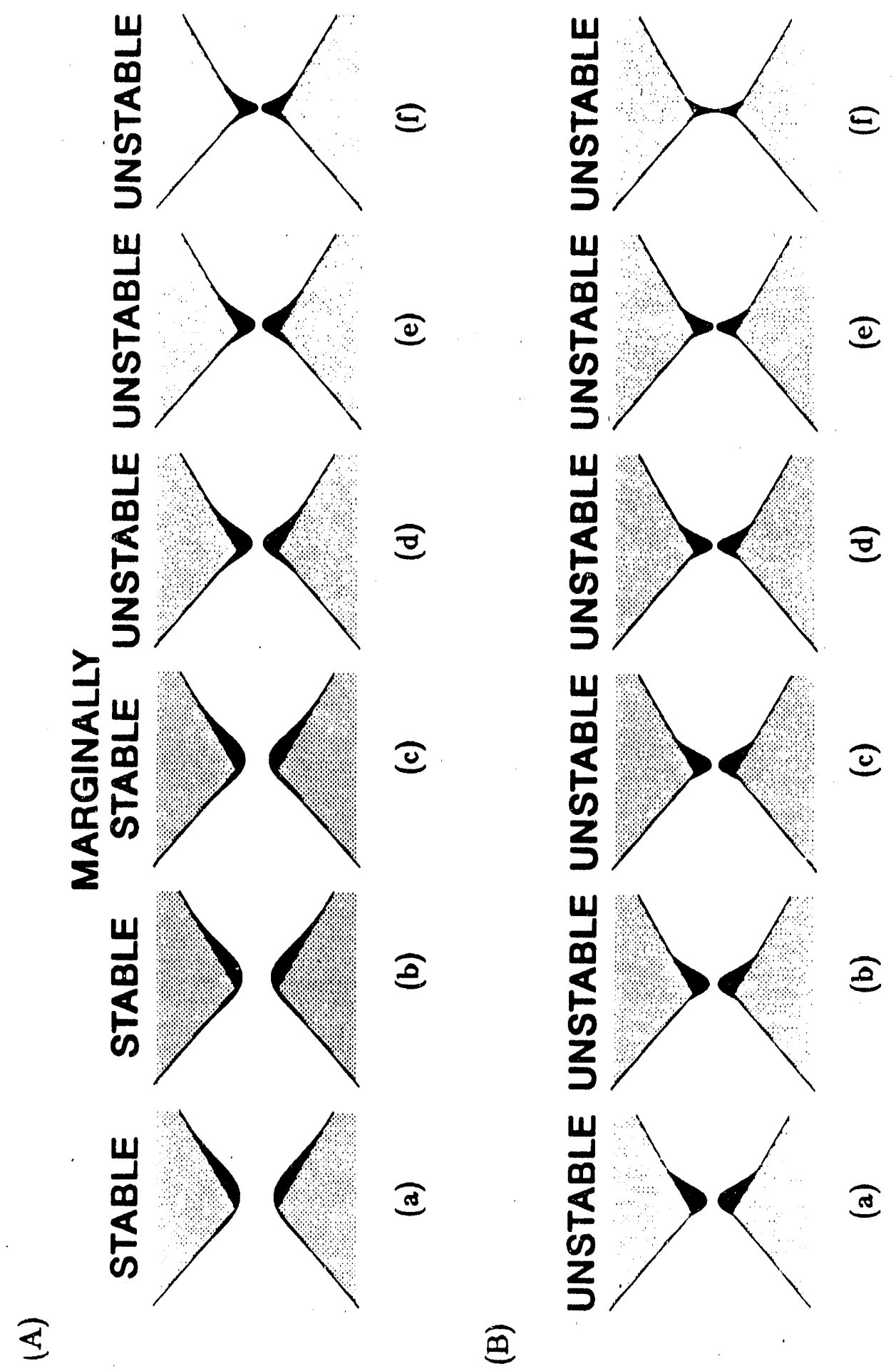

Figure 6. Isolated equilibrium configurations in biconical pores without reflective symmetry in the throat plane. Fixed contact angle, movable contact lines. Semiapical angles $\alpha_{[l e f t]}=40^{\circ}, \alpha_{[\text {right }]}=30^{\circ}$. Non-dimensional capillary pressure $\Delta p r_{t} / \gamma$ : (a) $\theta=0^{\circ}:$ (a) 0.36 , (b) 0.40 , (c) 0.43 , (d) 0.59 , (e) 0.68 , and (f) 0.81 . (B) $\theta=20^{\circ}$ : (a) 0.46 , (b) 0.51 , (c) 0.56 , (d) 0.61 , (e) 0.69 , and (f) $0 ; 86$. 
the contact lines and, in generai, adds a lot of physics to the analysis. Appropriate consideration to these factors rinay result in choke-off to be possible with incomplete wetting. A detailed study of neck "nenisci, their equilibrium and stability, in a variecy of model pores and subject to different boundary conditions will be published elsewhere. For the purpose of this work, choke-off is not considered.

\section{Head Menisci in Biconical Pore Segments}

Head menisci are surfaces of constant mean curvature, hence they obey the YoungLaplace equation. These interfaces are concave (toward the nonwetting phase) at all points. Figure 7 illustrates two such menisci for the mercury/vacuum system, one in the converging section of a biconical pore segment and the other one in the diverging section of a biconical pores segment. Both these menisci are stable when the volume of the fluids are held constant.

The stability condition for head menisci can be obtained directly because stability depends on how displacement changes interfacial curvature. Thus, $d\left[p_{H g}-p_{v a c}\right] / d V_{H g}$ is positive for menisci in a converging section and negative for menisci in a diverging section.

We consider the relationship between capillary pressure, $\Delta p=\left[p_{H g}-p_{v a c}\right]=P_{H g}$, and mercury volume, $V_{H g}$, for conical pore segments with semi-apical angles $\alpha\left[0^{\circ}<\alpha<45^{\circ}\right]$. The geometry of the system is shown in Figure 7.

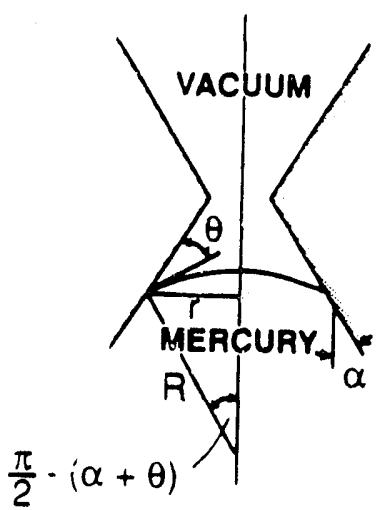

(a)

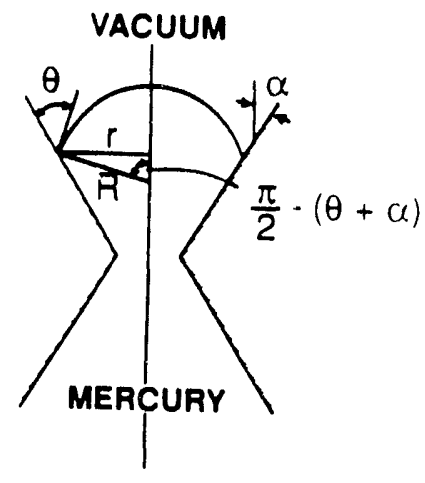

(b)

Figure 7. Geometrical relationships of head menisci in biconical pore segments. (a) Head meniscus in a converging section. (b) Head meniscus in a diverging section. 


\section{Converging Section}

The capillary pressure is given by

$$
\Delta p=\frac{2 \gamma}{r} \cos (\theta+\alpha)
$$

The volume of mercury, $V_{H g}$, up to a suitable constant, between the conical pore and the head meniscus is given by

$$
\frac{V_{H g}}{\pi r_{t}^{3}}=-\frac{8}{3}\left[\frac{\gamma}{\Delta p r_{t}}\right]^{3} F_{1}(\alpha, \theta)
$$

where

$$
F_{1}(\alpha, \theta) \equiv \cot \alpha \cos ^{3}(\theta+\alpha)-[2+\sin (\theta+\alpha)][1-\sin (\theta+\alpha)]^{2}
$$

and

$$
0<\frac{\Delta p}{\gamma / r_{t}} \leq 2 \cos (\theta+\alpha)
$$

In addition,

$$
\frac{V_{H g}}{\pi r_{t}^{3}}=-\frac{8}{3}\left[\frac{\gamma}{\Delta p r_{t}}\right]^{3} F_{2}(\alpha, \theta)
$$

where

$$
F_{2}(\delta) \equiv \cot \alpha \cos ^{3}(\delta)-[2+\sin (\delta)][1-\sin (\delta)]^{2}
$$

and

$$
\cos \delta=\frac{r_{b}}{R}
$$

and

$$
2 \cos (\theta+\alpha)<\frac{\Delta p}{\gamma / r_{t}} \leq 2
$$

\section{Diverging Section}

We distinguished three cases:

(i) $\alpha-\theta<0$

The capillary pressure is given by

$$
\Delta p=\frac{2 \gamma}{r} \cos (\theta-\alpha)
$$

The volume of mercury is given by

$$
\frac{V_{H g}}{\pi r_{t}^{3}}=\frac{8}{3}\left[\frac{\gamma}{\Delta p r_{t}}\right]^{3} F_{3}(\alpha, \theta)
$$


where

$$
F_{3}(\alpha, \theta) \equiv \cot \alpha \cos ^{3}(\theta-\alpha)+[2+\sin (\theta-\alpha)][1-\sin (\theta-\alpha)]^{2}
$$

and

$$
\frac{\Delta p}{\gamma / r_{b}} \leq 2 \cos (\theta-\alpha)
$$

In addition,

$$
\frac{V_{H g}}{\pi r_{t}^{3}}=\frac{1}{3} \cot \alpha\left[\frac{r_{b}}{r_{t}}\right]^{3}+\frac{8}{3}\left[\frac{\gamma}{\Delta p r_{t}}\right]^{3} F_{4}(\delta)
$$

where

$$
F_{4}(\delta) \equiv 4-[2+\sin \delta][1-\sin \delta]^{2}
$$

and

$$
\cos \delta=\frac{r_{b}}{R}
$$

and

$$
2 \cos (\theta-\alpha)<\frac{\Delta p}{\gamma / r_{b}} \leq 2 \cos \delta
$$

(ii) $\alpha-\theta>0$

The capillary pressure is given by

$$
\Delta p=\frac{2 \gamma}{r} \cos (\alpha-\theta)
$$

The volume of mercury is given by

$$
\frac{V_{H g}}{\pi r_{t}^{3}}=\frac{8}{3}\left[\frac{\gamma}{\Delta p r_{t}}\right]^{3} F_{5}(\alpha, \theta)
$$

where

$$
F_{5}(\alpha, \theta) \equiv \cot \alpha \cos ^{3}(\alpha-\theta)+4-[2+\sin (\alpha-\theta)][1-\sin (\alpha-\theta)]^{2}
$$

and

$$
\frac{\Delta p}{\gamma / r_{b}} \leq 2 \cos (\alpha-\theta)
$$

In addition, for

$$
2 \cos (\alpha-\theta)<\frac{\Delta p}{\gamma / r_{b}} \leq 2 \cos \delta
$$

$V_{H g}$ is given by Eq. (13). 
(iii) $\alpha-\theta=0$

The capillary pressure is given by

$$
\Delta p=\frac{2 \gamma}{r}
$$

The volume of mercury follows

$$
\frac{V_{H g}}{\pi r_{t}^{3}}=\frac{8}{3}\left[\frac{\gamma}{\Delta p r_{t}}\right]^{3} F_{6}(\alpha)
$$

where

$$
F_{6}(\alpha) \equiv 2+\cos \alpha
$$

and

$$
\frac{\Delta p}{\gamma / r_{b}} \leq 2
$$

In addition, for

$$
\frac{\Delta p}{\gamma / r_{b}} \leq 2 \cos \delta
$$

$V_{H g}$ is given by Eq. (13).

\section{Physics of Volume-Controlled Mercury Porosimetry}

The conditions for equilibrium and stability of interacting menisci in porous media have now been established. We consider next the pore-level physics of quasi-static, volumecontrolled mercury injection and withdrawal in the limit of vanishing mercury flow rate.

The crucial feature of these displacement processes is the strong menisci interaction. The interaction leads, for example, to simultaneous menisci coalescence, assisted jumps through a single pore segments or group of pore segments, concurrent local advance of a single meniscus and global retraction of all other existing menisci, etc. Careful consideration to these cooperative phenomena, the hallmark of volume-controlled displacement processes, distinguish our work from other studies of capillary displacements where, at each step, a single meniscus moves through the pore segment where the displacing force is largest.

Although here we concentrate in low capillary number mercury porosimetry, we can also account for waterflooding, i.e. oil displacement by water in reservoir rocks, where the interfaces move, on average, at $3 \mathrm{ft} /$ day.

A basic trait of fluid interface motion during volume-controlled displacement processes is the so-called Haines jump, resulting from unstable configurations (Haines 1930, Miller 
and Miller 1956, Melrose 1965). Jumps are followed by essentially smooth and reversible changes in pressure as mercury is constantly injected or withdrawn.

Haines jumps in volume-controlled fluid displacement are subject to two constraints: a volume constraint and a pressure constraint.

Volume constraint: The spontaneous redistribution of mercury within a porous medium takes place at constant mercury volume, i.e. the net flow of mercury vanished. The process can be described as concurrent local injection, i.e. one meniscus jumps, and global withdrawal, i.e. all other menisci recede.

When displacement is dominated by capillarity, the mercury volume withdrawn, $V_{H g}^{w}$, is exactly equal to the mercury volume injected into evacuated pore space, $V_{H g}^{i}$; thus

$$
V_{H g}^{w} \equiv V_{H g}^{i}
$$

Pressure constraint: Once an unstable configuration is reached, the interface moves rapidly to a new equilibrium position, i.e. the capillary pressure across the mercury/vacuum menisci is the same, i.e.

$$
\Delta p_{1}=\Delta p_{2}=\cdots=\Delta p_{n}
$$

where $n$ is the number of existing menisci.

Simultaneous solution of Eqs. (18) and (19) provides the new stable equilibrium menisci configuration after a Haines jump. Solution of Eqs. (18) and (19) indicates that the pore space spontaneously occupied by mercury in injection or evacuated in withdrawal can vary from a fraction of a single pore segment to an assembly of pores segments. Next, we discuss the physics of Haines jumps in volume-controlled mercury injection.

\section{Invasion of a single pore segment.}

Figure 8 shows the mechanism of volume-controlled mercury injection into an evacuated network of biconical pore segments together with capillary pressure vs. mercury injected. The directions of menisci movement are indicated by arrows. The process corresponds to a global injection of mercury with menisci advancing toward throats, grooves and pits (small scale roughness is not shown in Figure 8).

Mercury menisci initially advance from their equilibrium positions, at pressure $p_{1}$ (Figure 8a), driven by a slightly higher pressure as mercury arrives from the source. The injection of mercury continues quasi-statically. Figure 8a depicts an intermediate configuration at pressure $p_{2}$. These displacements are smooth and reversible with continuous increase of menisci curvature; hence capillary pressure as mercury invades the evacuated pore space. 
(a)
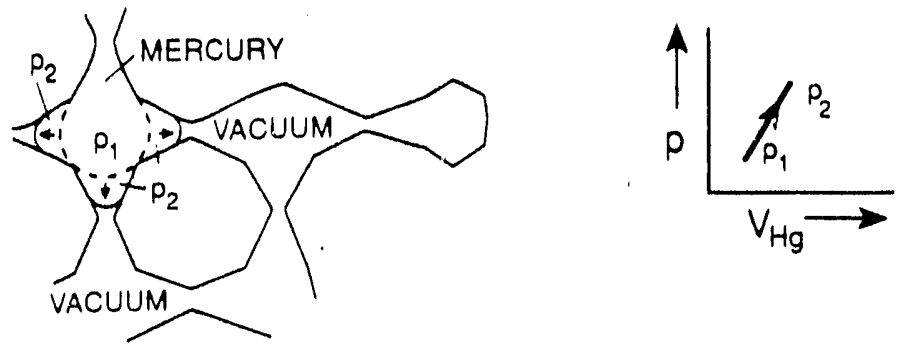

(b)
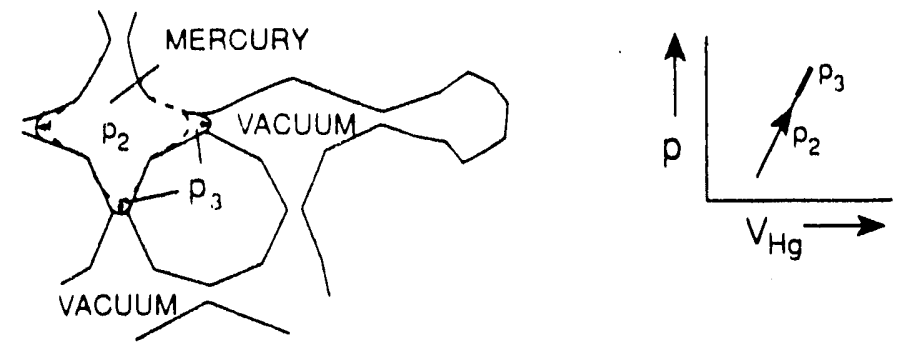

(c)
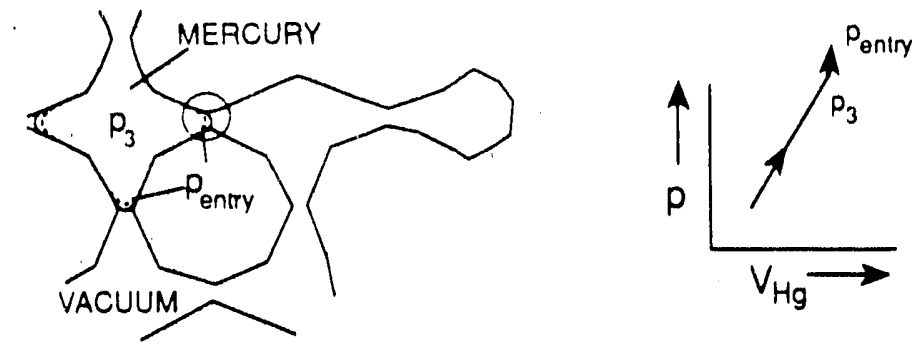

A) $\psi=\pi \cdot \theta+\left(\alpha_{1}-\alpha_{2}\right)$

(d)
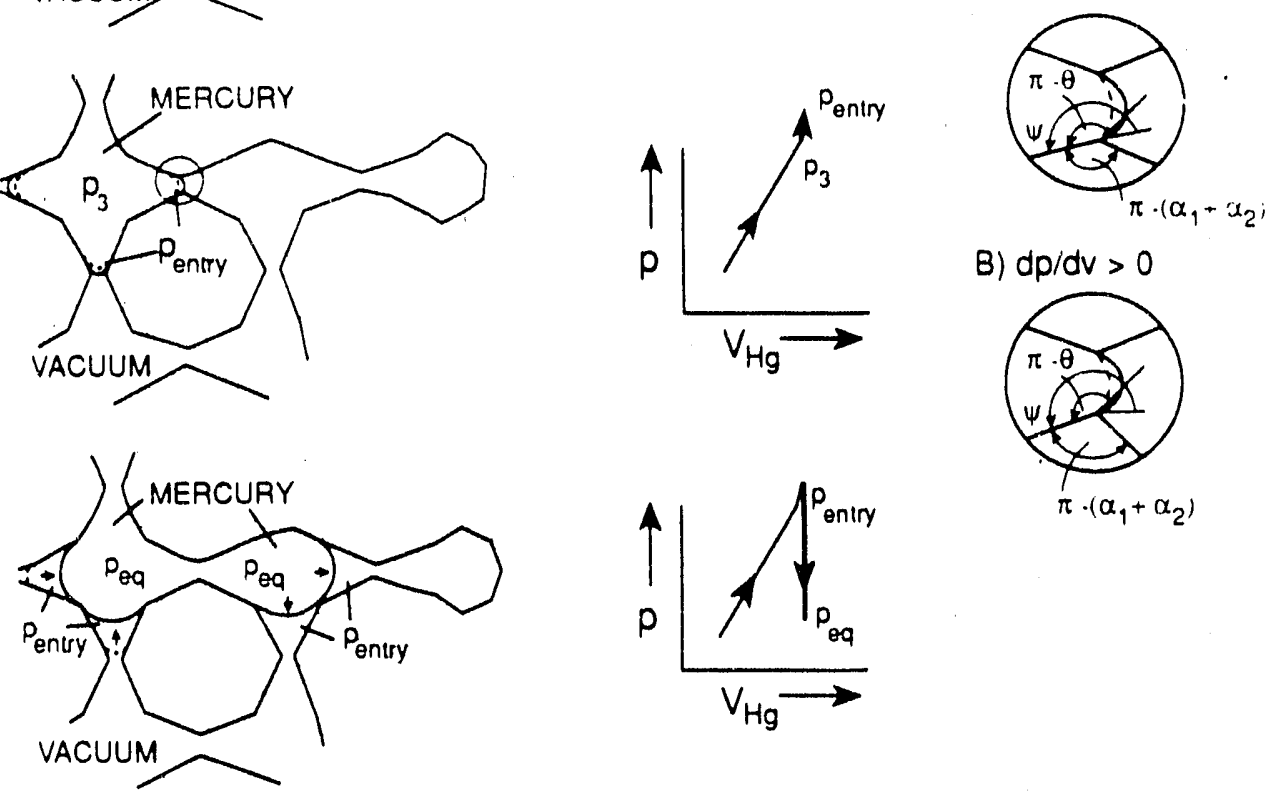

B) $d p / d v>0$

(e)
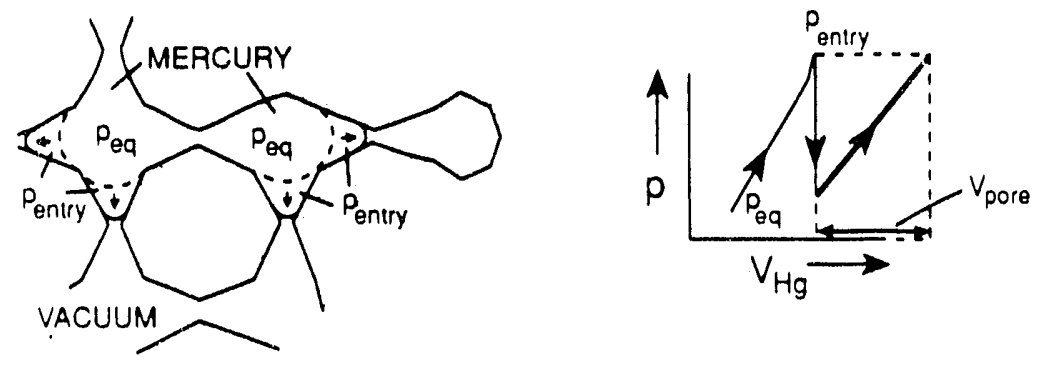

Figure 8. Mercury invasion of a single pore segment. (a) 'Global injection': menisci advance from equilibrium positions toward throats, grooves, and pits (small scale roughness is not shown). (b) One meniscus reaches largest of the throats. (c) Meniscus in (b) remains pinned at throat until one of the following cases occurs: (A) meniscus satisfies the contact angle in diverging section of a biconical pore, or (B) meniscus becomes unstable and jumps. (d) At jump, menisci move rapidly to a new equilibrium position and pressure drops suddenly. (e) Menisci have the same curvature as before the jump. Added volume after jump is a measure of pore segmer.ts' volume. 
Mercury menisci advance continuously until one meniscus reaches the largest of the accessible throats (Figure $8 \mathrm{~b}$ ), at which the capillary pressure is

$$
p_{3}=\frac{2 \gamma}{r_{t}} \cos (\theta+\alpha)
$$

where $r_{t}$ is the throat size.

The pore walls of natural porous media have edges, i.e. regions of extremely high curvature, such as the pore throats pictured here. The criterion for contact angle equilibrium given by the Young-Dupré equation does not apply at a throat. In contrast, for equilibrium, the angles at which the contact line can possibly meet the throat, cover a range which is determined by the angle subtended at the throat by the converging and diverging pore wall surfaces. This condition was first derived by Gibbs (1906/1961).

The range of angles through which the contact line can be observed at a throat, provided other requirements of stability are met is given by

$$
\pi-\theta \leq \psi \leq \pi-\theta+\left(\alpha_{1}+\alpha_{2}\right)
$$

where $\alpha_{1}$ and $\alpha_{2}$ are the semi-apical angles of a typical biconical pore segment. Clearly, the contact angle condition for equilibrium has been relaxed.

The crntact line is pinned until a value

$$
\psi=\pi-\theta+\left(\alpha_{1}+\alpha_{2}\right)
$$

is attained. If the condition represented by Eq. (22) is satisfied, the Young-Dupre equation is again valid and the menisci advance smoothly and reversibly as shown in the insert A of Figure 8c.

However, if the stability condition $d P / d V>0$ comes into force before the contact line satisfies Eq. (22), as shown in the insert $B$ of Figure 8c, then an unstable configuration is reached and the menisci move rapidly to a new equilibrium position accompanied by a sudden pressure drop (Figure 8d). The size of the throat through which the meniscus jumps is obtained from

$$
r_{t}=2 \gamma / p_{\text {entry }}
$$

In networks of biconical pore segments, such as those illustrated in Figure 2, the total volume recruited in the global withdrawal process $V_{H g}^{w}$ is given by

$$
i_{I g}^{w}=\frac{8}{3} \pi\left(\left[\frac{\gamma}{\Delta p_{f}}\right]^{3}-\left[\frac{\gamma}{\Delta p_{i}}\right]^{3}\right) \sum_{j}^{n(\Delta p)} F\left(\alpha_{j}, \theta\right)
$$

where $\Delta p_{i}$ and $\Delta p_{f}$ are respei ively the capillary pressure at the jump and after the jump. The summation includes all existing menisci, the number of which depends on the capillary 
pressure level, $n(\Delta p) \equiv n\left(\Delta p_{i}\right) \equiv n\left(\Delta p_{f}\right)$. The function $F$ is given by Eqs. (12), (13), (15) or (17) depending on the capillary pressure level, pore geometry, and location of the contact lines.

The volume injected into the new pore segment $V_{H g}^{i}$, follows from straightforward geometrical considerations

$$
\begin{aligned}
V_{H g}^{i} & =\frac{1}{3} \pi\left(r_{b_{j}}^{3}-r_{t_{j}}^{3}\right) \cot \alpha_{j}-\frac{2}{3} \pi r_{t_{j}}^{3}+V_{b}+ \\
& +\frac{\pi}{3} \sum_{\substack{i \\
i \neq j}}^{z-1} r_{b_{i}}^{3} \cot \alpha_{i}-\frac{8}{3} \pi\left[\frac{\gamma}{\Delta p_{f}}\right]^{3} \sum_{\substack{i \\
i \neq j}}^{z-1} F\left(\alpha_{i}, \theta\right)
\end{aligned}
$$

where $\Delta p_{f}$ is the capillary pressure after the jump. The summations take into account all new menisci, i.e. those created by the jump, that rest in converging sections of previously evacuated pore segments. In our model, $V_{b}=8 r_{b}^{3}$.

After a Haines jump has occurred, injection of mercury continues again under balanced, stable conditions. The displacement process is once more smooth and reversible and capillary pressure increases continuously as mercury is injected.

For networks of biconical pore segments, the total volume of injected mercury $V_{H g}^{a}$ is given by

$$
V_{H g}^{a}=\frac{8}{3} \pi\left(\left[\frac{\gamma}{\Delta p_{f}}\right]^{3}-\left[\frac{\gamma}{\Delta p_{i}}\right]^{3}\right) \sum_{j}^{n(\Delta p)} F\left(\alpha_{j}, \theta\right)
$$

where $\Delta p_{i}$ and $\Delta p_{f}$ are respectively the capillary pressure after the jump and before the next jump. The summation includes all menisci advancing toward throats, the number of which depends on the capillary pressure level, $n(\Delta p) \equiv n\left(\Delta p_{i}\right) \equiv n\left(\Delta p_{f}\right)$. The function $F$ is given by Eqs. (9) or (10) depending on the capillary pressure level, pore geometry, and the position of the contact lines.

When the capillary pressure returns to the value it had when the Haines jump occurred, the change in mercury volume corresponds to the filling of a new pore body (Figure $8 \mathrm{e}$ ). That volume is an excellent estimate of the volume of the pore segment, typically better than $80 \%$.

Invasion of an assembly of pore segments.

Menisci interaction can cause displacement in large group of pores during a single Haines jump. For example, Figure 9 shows that two pore segments are invaded by mercury before the constraints given by Eqs. (18) and (19) come into force. The discussion is now resumed immediately after an unstable configuration is attained (Figure 9a) and the 
menisci moves rapidly to a new equilibrium position (Figure $9 \mathrm{~b}$ ). In Figure $9 \mathrm{c}$ the pressure is back to the value it had before the jump. The volume of mercury injected is an excellent measure of the volume of the two pores segments as Figure $9 \mathrm{c}$ suggests.

Invasion of a portion of a pore segment.

Enforcing the constraints given by Eqs. (18) and (19) can result in an arrested or frustrated jump, as shown in Figure 10. The equilibrium configuration depicted in Figure 10a is stable, though $d \Delta p / d V_{H g}<0$ because the mercury displacement is infinitesimally slow.

Frustration arises when there are only a few menisci present. This is the case of small specimens, the start of injection, and when the viscous resistance to fluid redistribution is significant. Figure 10b shows that a Haines jump completes only as mercury arrives from the source. The volume of mercury injected after the jump and before the capillary pressure returns to the value it had before the jump is a measure of the pore segment's volume as indicated in Figure 10c.

\section{Menisci fusion and consequent invasion of a constriction of a pore segment.}

Figure 11 shows a situation in which the largest accessible throat holds two backto-back menisci (Figure 11a). The displacement is smooth and reversible with continuous increase in capillary pressure as mercury advances toward the throat (Figure 11b). At some critical capillary pressure these menisci come into contact and coalesce. As a consequerice menisci are lost and $d \Delta p / d V_{H g}$ increases as shown in Figure 11c.

Thus, the magnitude of the finite pressure change accompanying a Haines jump depends on both the size of the region spontaneously occupied by mercury and the capacity of the system to withdraw mercury by retraction of its menisci. We define this capacity as small when the menisci curvature decreases appreciable as to be measurable in the laboratory. In fine-pored media, here represented by a low aspect ratio network, there is a large number of menisci at any given capillary pressure and so the capacity of the system is large compared to the net volume redistributed by a Haines jump; the pressure change is small, hence difficult to resolve. On the contrary, small specimens hold fewer menisci at each capillary pressure. The capacity of the system to relinquish mercury is small so capillarity forces the menisci to move far back to the enlargements. Consequently the pressure change is large, hence accessible to pressure transducers. Should the specimen be too small, there is a possibility, ruled by Eqs. (18) and (19) for some of the menisci to take a jump backwards while being retracted. These are unwanted events because regions of pore space would be proved more than once and the statistics would be meaningless.

Thus, a new concept emerges out of this discussion: the concept of an 'optimum' 
(a)
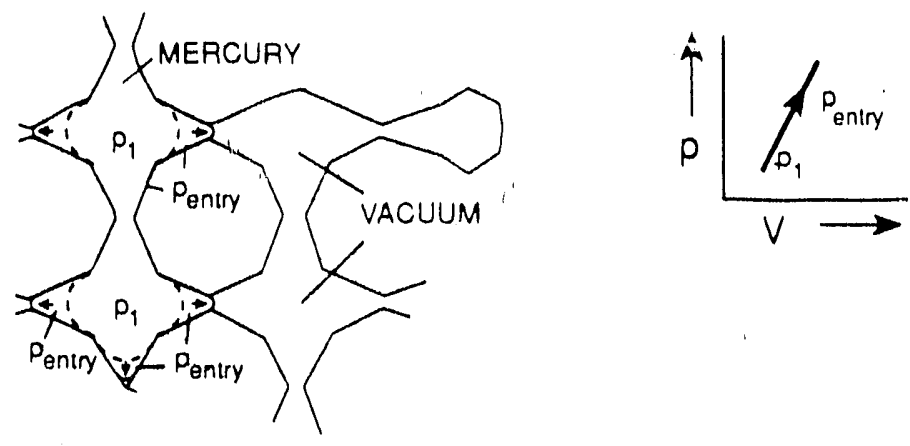

(b)
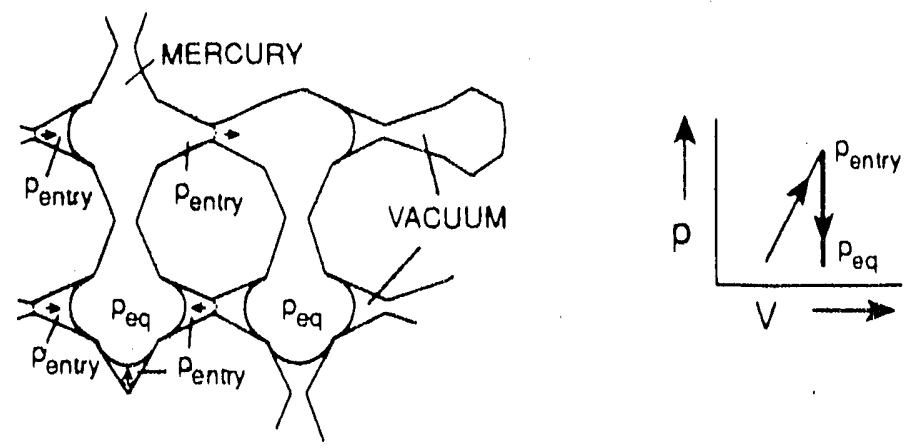

(c)
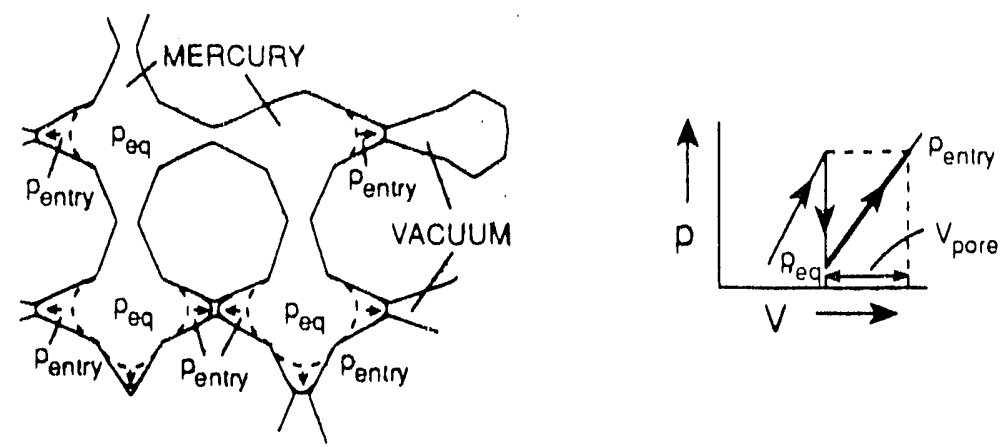

Figure 9. Mercury invasion of two pore segments. (a) One meniscus becomes unstable and jumps. (b) A new equilibrium position is attained only after invasion of two pore segments. (c) Volume added after jump is a measure of the volume of two pore segments. 
(a)
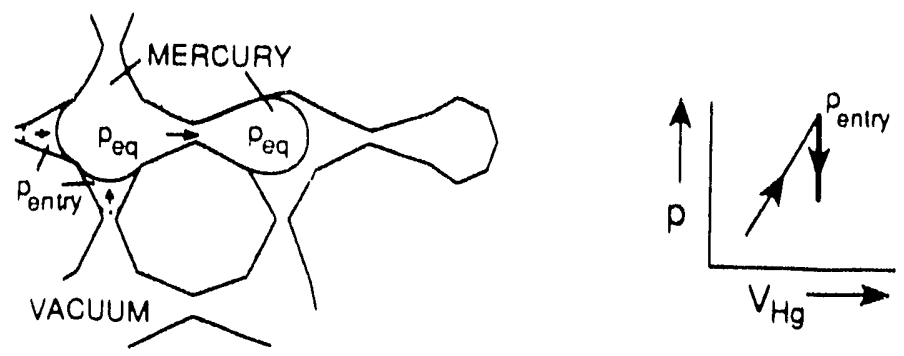

(b)
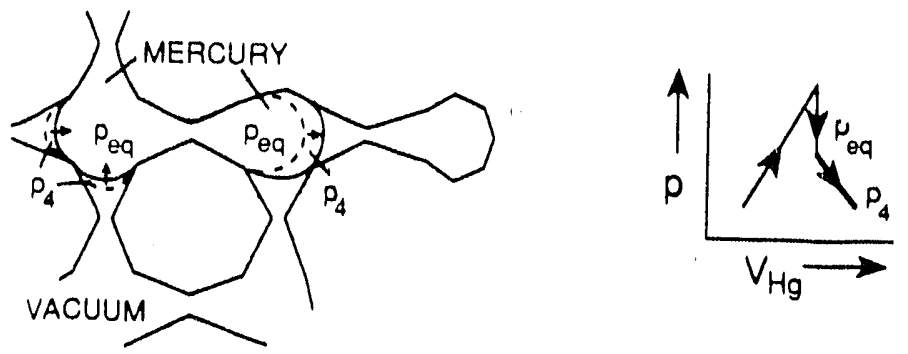

(c)
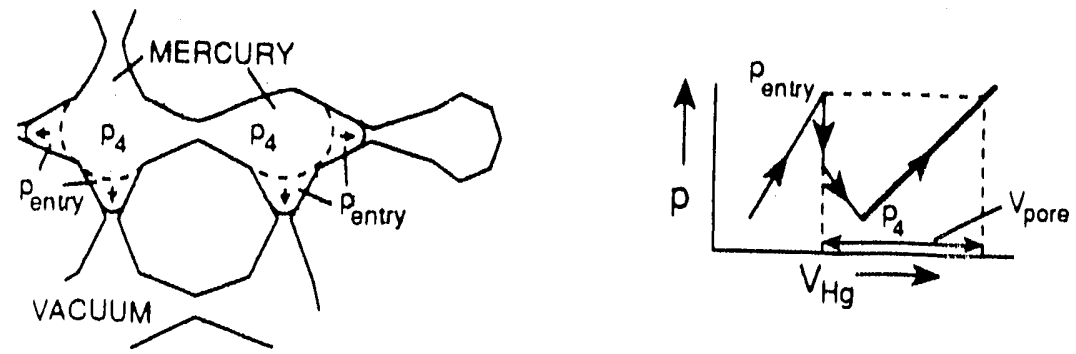

Figure 10. Mercury invasion of a portion of a pore segment. (a) Jump arrested by insufficient volume of mercury. (b) Jump completed as mercury arrives from source. (c) Menisci have the same curvature as before the jump. The volume of mercury added after the jump provides a measure of pore segment's volume. 
(a)
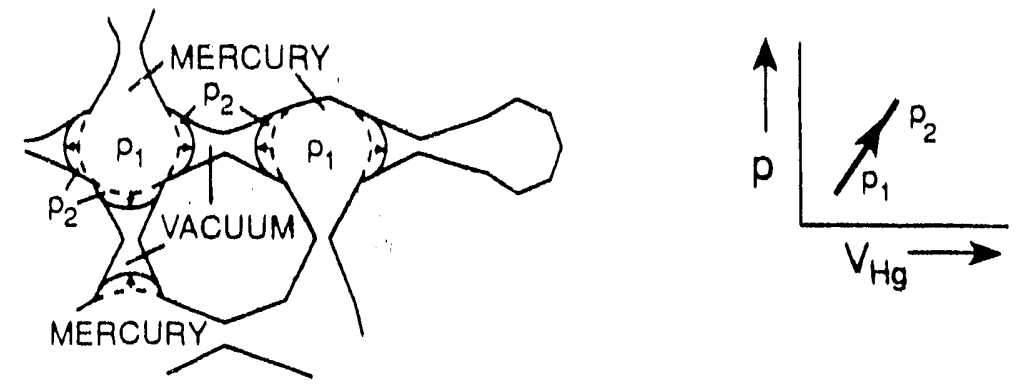

(b)
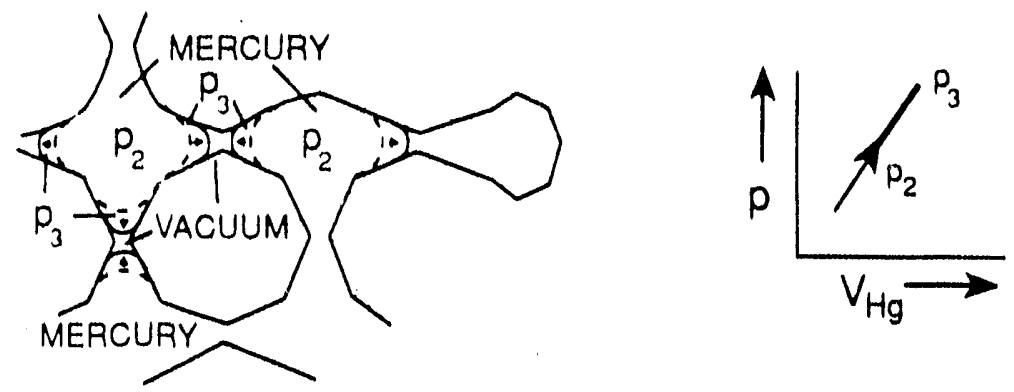

(c)
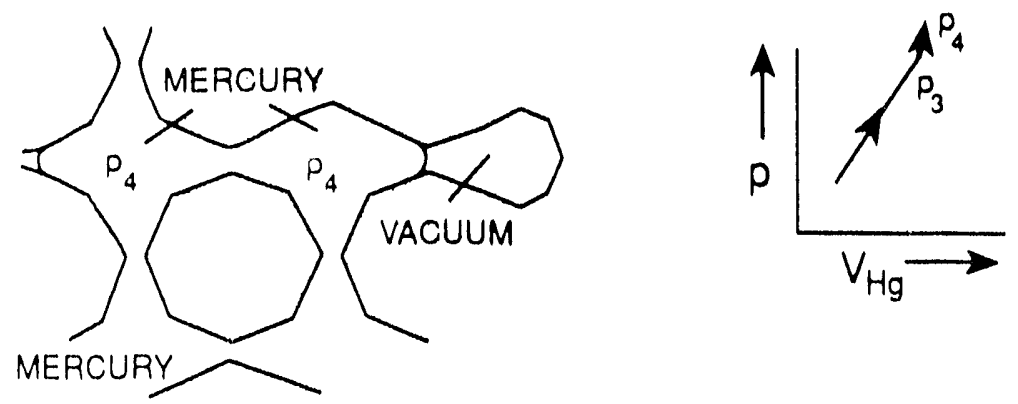

Figure 11. Fusion of menisci. (a) Largest throats hold back-to-back menisci. (b) Menisci advance toward throats, pits, and grooves. (c) Menisci come into contact and coalesce. Menisci are lost, so $d(\Delta p) / d V_{H g}$ increases. 
specimen size for examining pore space of given properties, large enough to give appreciable pressure changes and small enough to reduce duplication of information to a minimum. As a result, sample size becomes a major factor in volume-controlled porosimetry.

Upon completion of mercury injection, the direction of the pump can be reversed so that slow volume-controlled withdrawal begins. As in injection, mercury retraction is driven by the combined effect of capillarity and mercury flow rate (set by the pump). Although such an experiment has not been done, it is feasible and would provide pore space statistics not observable in the injection experiment. In what follows we iclentify the major physical events occurring in the withdrawal process.

\section{Incremental retreat.}

Figures $12 \mathrm{a}$ and $12 \mathrm{~b}$ depict stable equilibrium menisci configurations. The contact line is pinned at the edges delimiting a pore body, so the Young-Dupré equation is not enforced until Eq. (22) is satisfied. Then the menisci retract smoothly and reversibly.

The configurations are so stable that unpinning followed by displacement is possible only upon considerable pressure reduction, perhaps with mercury being in tension. Consequently, mercury retracts first from elsewhere. For networks of biconical pore segments, the total volume retracted in the global withdrawal process is given by the appropriate equations for head menisci in the diverging section of biconical pores.

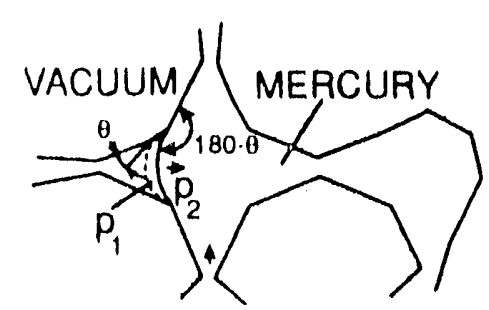

(a)
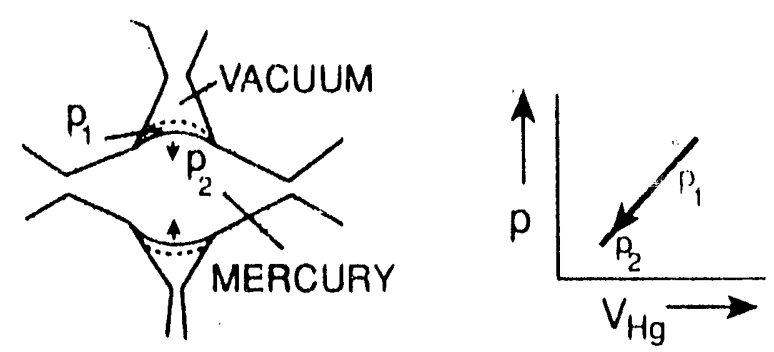

Figure 12. Incremental retreat of mercury. (a) and (b) represent two very stable equilibrium configurations. Upon reduction of pressure, menisci remain pinned at throats. 


\section{Retreat in a single pore segment.}

Figure 13 depicts the mechanism of volume-controlled mercury withdrawal together with the relationship capillary pressure vs. mercury ejected. The direction of menisci movement is indicated by arrows.

Mercury menisci advance continuously from capillary equilibrium position (Figure 13a). The process corresponds to a global withdrawal of mercury with menisci retracting toward the pore bodies. Mercury menisci retracts continuously until menisci fusion occurs in a pore body. As shown in Figure 13b, three menisci fuse into a single hemispherical meniscus that is no more stable. Infinitesimal mercury removal is not possible without increasing the pressure, i.e. $d \Delta p / d V_{H g}<0$. Menisci moves rapidly to a new equilibrium position, determined by Eqs. (18) and (19), accompanied by a sudden pressure increase (Figure 13b). The size of the pore body through which the meniscus jumps can be estimated from

$$
r_{b}=2 \gamma / p_{2}
$$

The process correspond to local withdrawal in a few pore segments and global injection with menisci advancing back to throats and pits.

After a Haines jump has occurred, retraction of menisci continues under balanced, stable conditions. The withdrawal process is once more smooth and reversible with capillary pressure decreasing continuously as mercury is withdrawn. When the pressure returns to the value it had before the jump, the change in mercury volume correspond to the evacuation of a pore segment (Figure 13c). That volume is as excellent a measure of the volume of the pore segment as that obtained from injection. Of course, cooperative effects can cause retraction in more than a single pore during a single Haines jump.

\section{Stepwise retreat in a single pore segment.}

Figure 14 shows mercury retraction from a pore segment when two menisci are present. This mechanism in square tubes was first studied by Lenormand and Zarcone (1084), experimentally and theoretically.

Mercury menisci continuously retract from capillary equilibrium position until menisci fusion occurs in a pore body, at which the capillary pressure is $p_{2}=2 \gamma / r_{b}$. As shown in Figure $14 \mathrm{a}$, two menisci fuse into a single meniscus, but contrary to the case of withdrawal from a single pore segment, the configuration is stable because removal of mercury is still possible without increasing the capillary pressure, i.e. $d \Delta p / d V_{H g}>0$. The contact line as shown in Figure 14b is pinned until the condition given by $\mathrm{Eq} .(22)$ is attained. Since here we are dealing with a complex three-dimensional menisci, we assume that the mercury volume withdrawn is $1 / 2$ of the volume of the pore seginent once the pressure decreases to $p_{3}=2 \gamma /\left(2 r_{b}\right)$, where $r_{b}$ is half the size of the pore body. Once the condition represented by Eq. (22) is satisfied, the Young-Dupré equation is again valid and the menisci retract 
(a)
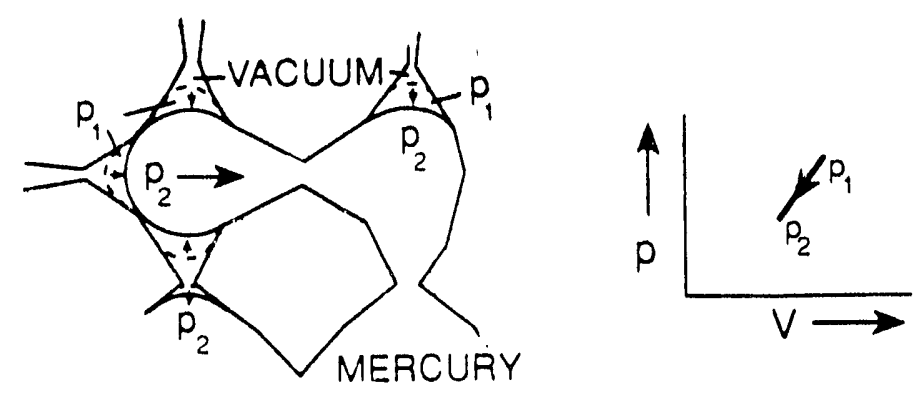

(b)

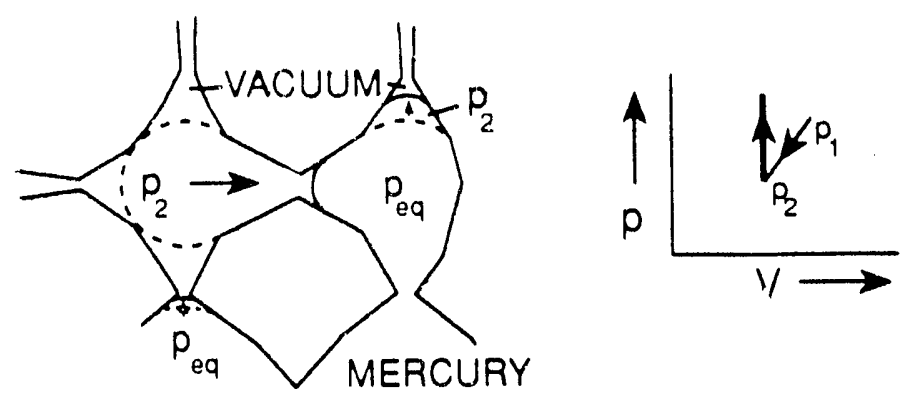

(c)
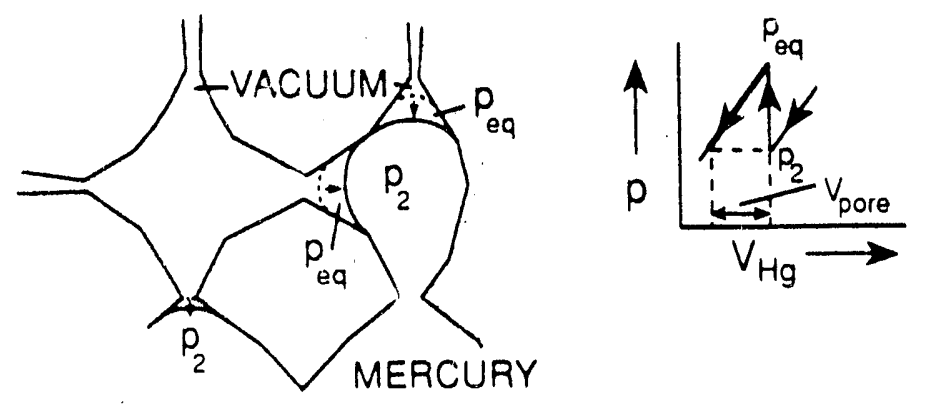

Figure 13. Retraction of mercury from a single pore segment. (a) Menisci retreat from an equilibrium position. Eventually, menisci fuse into an unstable hemispherical meniscus. (b) Menisci move rapidly to a new equilibrium position and pressure increases suddenly. (c) Menisci have the same curvature as before the jump. The volume of mercury withdrawn after jump is a measure of a pore segment's volume. 
(a)
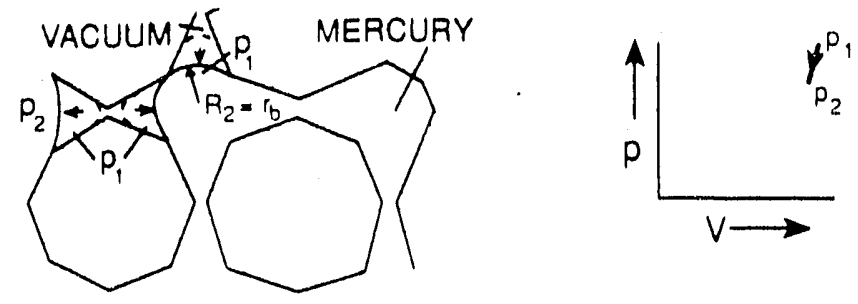

(b)
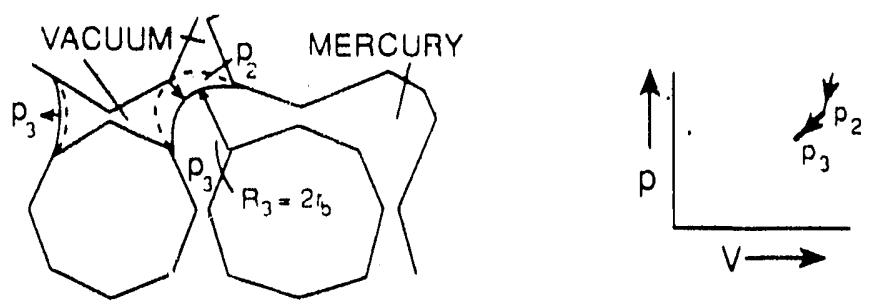

(c)
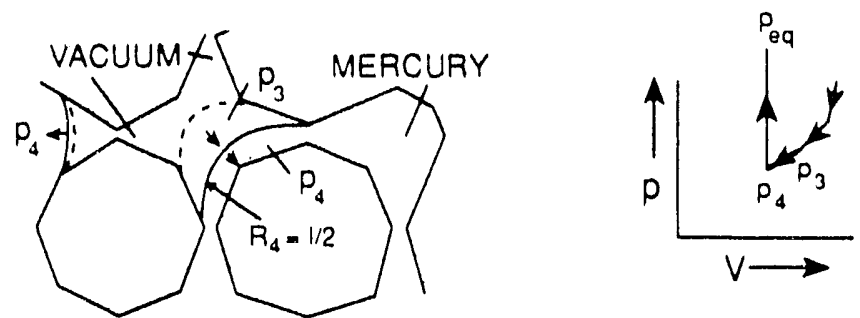

(d)
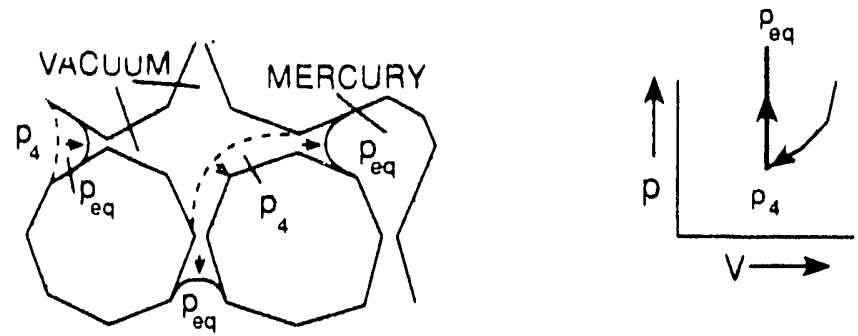

(e)
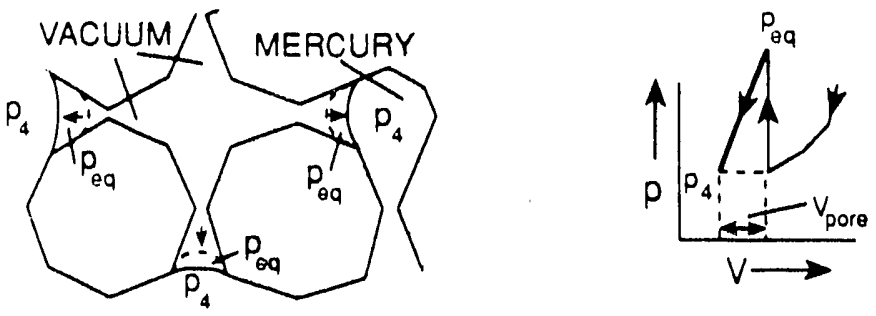

Figure 14. Stepwise retraction of mercury from a single pore segment. (a) Menisci fuse into a single stable meniscus. (b) The new meniscus remains pinned until contact angle is again satisfied. (c) Menisci retract smoothly and reversibly until the new meniscus is pinned again. (d) The new meniscus becomes unstable, fissicns, and jumps. (e) Menisci have the same curvature as before the jump. The volume of mercury withdrawn after the jump is a measure of a pore segment's volume. 
smoothly and reversibly until the large meniscus in Figure $14 \mathrm{c}$ is once more pinned. We assume the volume retracted is $3 / 4$ of that of the pore segment when capillary pressure decreases to

$$
p_{4}=2 \gamma /(l / 2)
$$

where $l$ is half the pore-body-to-pore-body distance. That the size, not the volume, of pore segments can be overestimated from Eq. (25) is shown in the section on results. At this point we assume the meniscus becomes unstable (Figure 14d) and the menisci moves rapidly to a new equilibrium position with a sudden pressure increase. The new position is determine by solving Eqs (18) and (19).

After the Haines jump, retraction continues under stable conditions with pressure decreasing as mercury is withdrawn. When the capillary pressure returns to the value it had before the jump, the change in mercury volume is a measure of the volume of the pore segment as shown in Figure 14e.

\section{Monte Carlo Simulation Details}

The computer simulation of volume-controlled mercury porosimetry proceeds as follows.

(i) Create a pore network of specified length $(L)$, width $(N)$, and thichness $(M)$, mean coordination number, and boundary conditions, according to the general procedure presented in the section PORE MORPHOLOGY.

(ii) Initialize a list of accessible pore segments, pore throats in injection and pore bodies in withdrawal, to consist of those pores connected to the mercury source.

(iii) Choose one of the accessible pores to be filled (injection) or evacuated (withdrawal) according to the criterion that mercury will flow through the largest accessible throat in injection, smallesi accessible body in withdrawal.

This choice nust be made at each step, hence on the order of $N \times N \times N$ times, where $N$ is the number of pores on a side of a cubic sample. A brute-force approach involves searching the entire list of accessible throats (of length at least order $N \times N$ ) each time step, and this approach therefore takes time proportional to at least $N^{3}$. We used a more sophisticated approach that involves sorting the pore throats (injection), pore bodies (withdrawal) by size as they are added to the list of accessible pores. This technique can reduce the time per step to order $\log N$ (Knuth 1973).

(iv) Find the menisci configuration after a Haines jump.

Determining the configuration of interfaces after a Haines jump is both concep- 
tually and computationally complex. The highly nonlinear Eqs. (18) and (19) are to be solved. It is not certain, a priori, if the jump will go through a single pore segment or a group of pore segments. This introduces the complexity because Eqs. (18) and (19) have to be modified accordingly, resulting in an entirely new problem. We used Newton-Raphson with a technique similar to first-order continuation. The initial state of the interfaces is given by the position attained immediately after the jump. This configuration is slightly perturbed, for example, increasing the volume in injection, or decreasing the volume in withdrawal. Equation (18) is then solved for the capillary pressure. A solution is accepted when all the menisci can be accommodated in the pore space and boundary conditions are satisfied (contact angle for instance).

(v) Add all of the nearest neighbors of the occupied pores (injection), evacuated pores (withdrawal) which are not already on the list of accessible pores.

(vi) In withdrawal, determine if any of the pores in the list of accessible pores are trapped. Remove each such pore (if any) from the list of accessible pores.

Determining which pores on the list have become trapped is also computationally difficult. In the brute-force approach, where a path is traced from each accessible pore to the mercury source, the time required is of order $N^{3}$ at each step. Since there are roughly $N^{3}$ steps, this leads to a time proportional to $N^{6}$ for the complete simulation. A classical breadth-first search (Aho et al. 1974) limits the time spent in this step to be proportional to the trapped mercury discovered at each step. Thus the total time is proportional to the amount of trapped mercury. In our simulations we found that the time complexity is roughly $N^{3}$. This step is the most costly in the simulation process.

(vii) If there are pores left on the list of accessible pores, return to step (iii) and repeat the procedure with the next-largest throat (injection) next-smallest body (withdrawal). If there are no pores on the list, the simulation for the sample size picked at step (i) is complete.

(viii) Evaluate the following least-squares objective function

$$
J=\sum_{i=1}^{N D}\left[f^{i n p u t}-f^{i n f e r r e d}\right]^{2}
$$

where $f$ is the pore throat size distribution and $N D$ is the number of discretizations of $r$. If $J$ is not a minimum return to step (i). If $J$ is minimum the simulation is complete. 


\section{Results and Discussion}

Figures 15 and 16 display computer simulation of volume-controlled mercury capillary pressure curves, respectively for a 'low aspect ratio' network of $30 \times 30 \times 30$ pore segments and for a 'high aspect ratio' network of $40 \times 40 \times 40$ pore segments. Envelopes corresponds to the classical pressure-controlled capillary pressure curves, i.e. primary injection curve, withdrawal curves, and reinjection curves. Simulation parameters are summarized in Table 1.

Table 1. Network parameters.

\begin{tabular}{ccccccc} 
Network & $\langle z\rangle$ & $r_{b} / r_{t}$ & $\begin{array}{c}L \\
(\mu \mathrm{m})\end{array}$ & St. Dev.* & $\begin{array}{c}\text { Mean Radius } \\
(\mu \mathrm{m})\end{array}$ & $\begin{array}{c}\text { Variance } \\
\left(\mu \mathrm{m}^{2}\right)\end{array}$ \\
\hline Low aspect ratio & 3.0 & 3.5 & 150 & $\sigma=1.5$ & 21.25 & 7106.69 \\
High aspect ratio & 3.0 & 2.0 & 200 & $\sigma=1.5$ & 21.25 & 7106.69 \\
\hline
\end{tabular}

*Standard deviation of the parent normal distribution

Several features are worth noting. We attempted to use realistic parameters in the calculations, but have not varied them extensively to obtain a quantitative fit of experimental data. Nonetheless, the envelopes of the volume-controlled capillary pressure curves reflect all the features of experimentally-measured capillary pressure curves (cf. Wardlaw et al. 1987). As shown by Yuan and Swanson (1986), the entry pressure for sandstones is lower than the entry pressure for carbonates, indicating that for samples they studied, sandstones have larger pore throat size than carbonates. Although we used the same pore throat size distribution to generate the networks (see Table 1), the feature that is changed is the size contrast between throats and bodies, the low aspect ratio network exhibit an entry pressure of approximately $50 \mathrm{kPa}$, lower than that of the high aspect ratio, $65 \mathrm{kPa}$. This result can be only explained by a sample size effect.

In this work, sample size is found to be a major factor in APEX response. By Monte C'arlo simulation of APEX mercury injection we find the 'optimum' size of specimen for exannining pore space of given properties; large enough to yield appreciable pressure drops and small enough to reduce duplication of information to a minimum. The optimum sample size for the low aspect ratio network is 35,000 (approximately $30 \times 30 \times 30$ pore segments and for the high aspect ratio network is 52,000 pores (approximately $40 \times 40 \times 40$ pore segments. A small optimum sample size for the low aspect ratio network results in larger pressure drops, accessible to accessible pressure transducers. A large optimum sample size for the high aspect ratio network reduces the possibility for some of the menisci to take jump backwards, consequently proving regions of pore space more than once is minimized.

Minimization of the least-squares objective function given by Eq. (29) is appropriate 
because the number of Haines jumps is maximized, i.e. an optimum number of pore throats is sampled. Proving all pore throats is of course impossible. Figures 17a and 18a show actual and inferred pore throat size distributions, respectively for the low aspect ratio network and for the high aspect ratio network, of optimum sizes. The errors bars on the inferred distributions are $95 \%$ confidence intervals around the mean of 11 realizations with the optimum network sizes. The actual distributions, input to the simulations, also have error bars because they change from realization to realization. The bars are typically of the same size or smaller than those for the inferred distributions. For clarity sake report only a typical actual distribution. The inferred distribution in Figures 17a and 18a are remarkable close to the actual distributions. After close examination, a pattern emerges out: small pore throats are underestimated and the larger throats are overestimated. Cooperative effects, even with an optimum sample size, can cause displacement in clusters of pores, interconnected by throats, during a single Haines jump. Therefore, the interconnecting throats are not detected. This should affect the statistics of small and large throats equally well. On the contrary, fusion of back-to-back menisci occurs mainly in the smaller throats. Although fusion is accompanied by a change in the slope in the capillary pressure curve, this may not be detected in the laboratory. Neglecting this information explain the pattern in Figures $17 \mathrm{a}$ and $18 \mathrm{a}$.

Figures $17 \mathrm{~b}$ and $18 \mathrm{~b}$ display pore body size distributions. The information is obtained from volume-controlled mercury withdrawal. As shown in these figures, withdrawal after complete primary injection leaves a number of pore segments, containing trapped mercury, inaccessible to measurement. Thus, below the residual mercury saturation, we use retraction after partial injection, as indicated by the scanning loops in Figures 15 and 16. To avoid sampling of pore bodies more than once, the maximum mercury saturation in a partial injection cycle is approximately equal to the residual mercury saturation left after the next cycle. Although the inferred pore body size distributions in Figures 17b and 18b are scattered, so are the actual distributions.

Figures $17 \mathrm{c}$ and $18 \mathrm{c}$ shows distribution of volume of pore segments obtained from volume-controlled mercury injection. As mentioned before, similar information can be obtained also from withdrawal, what would make an interesting comparison. Pore volume distributions covered approximately the volume range 0 to $15 \mathrm{nl}$, with a median of $5 \mathrm{nl}$ for the low aspect ratio network and 0 to $200 \mathrm{nl}$, with a median of $50 \mathrm{nl}$ for the high aspect ratio network. Although these results seem at variance with the statistical information reported by Yuan and Swanson (1986), this may not be so in view of the following discussion.

Yuan and Swanson focused on volume distribution of pore systems in Berea sandstone and San Andres dolomite. They found that distributions for both samples, of the same size apparently, covered approximately the same volume range, i.e. 1 to $500 \mathrm{nl}$. The information is deduced from APEX data acquired from volume-controlled mercury injection. In the process and after a Haines jump, the capillary pressure increases as mercury arrives from the source. Yuan and Swanson define a pore system as the pore space invaded by the mercury after the jump and before the capillary pressure returns to the value it had 


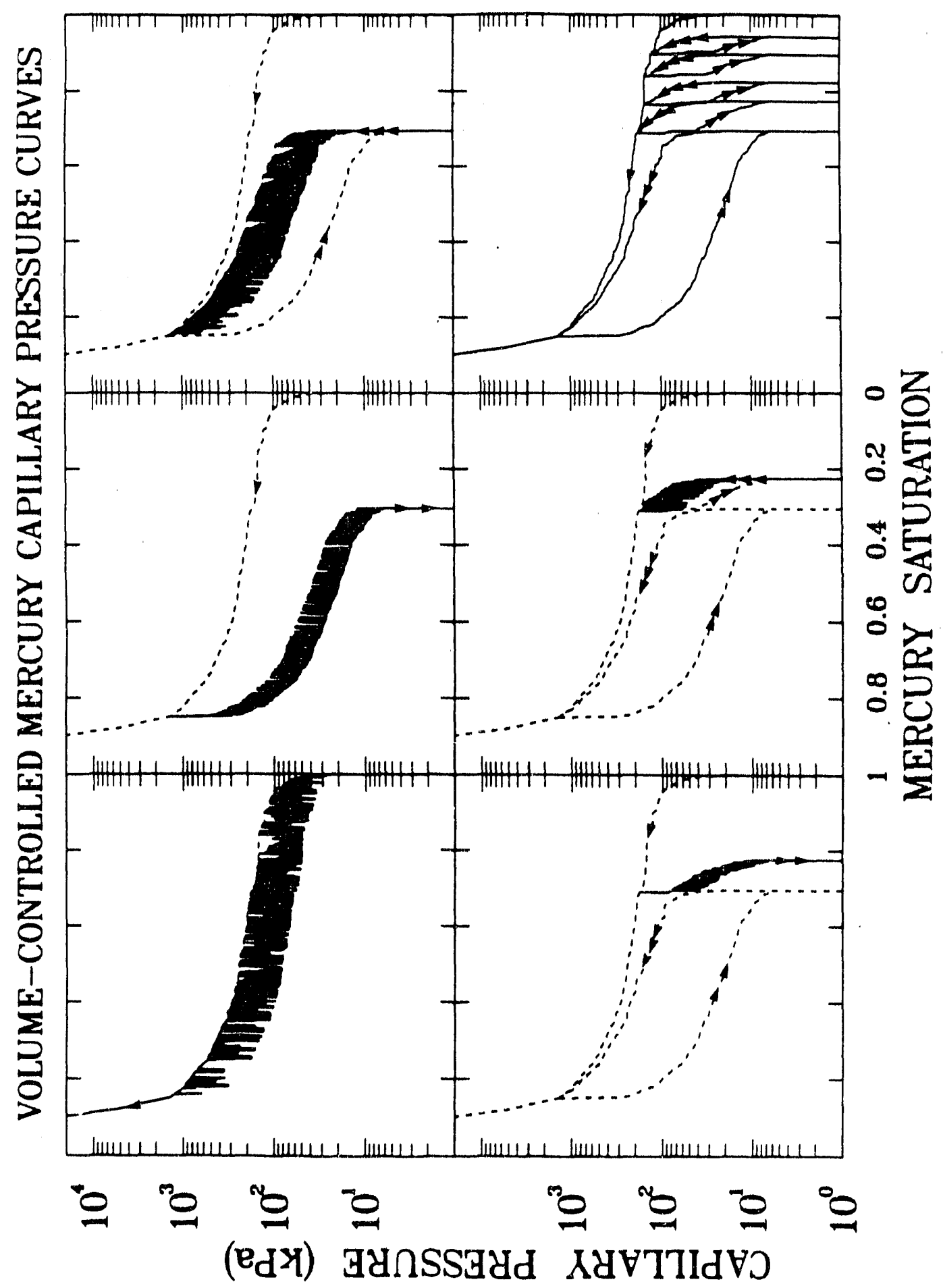

Figure 15. Computer simulation of 'volume-controlled mercury capillary pressure curves for a 'low aspect ratio' network of biconical pore segments. Envelopes correspond to classical pressure-controlled cap llary pressure curves. Primary injection curve (one arrow), withdrawal curves (two arrows), and reinjection curves (three arrows). 


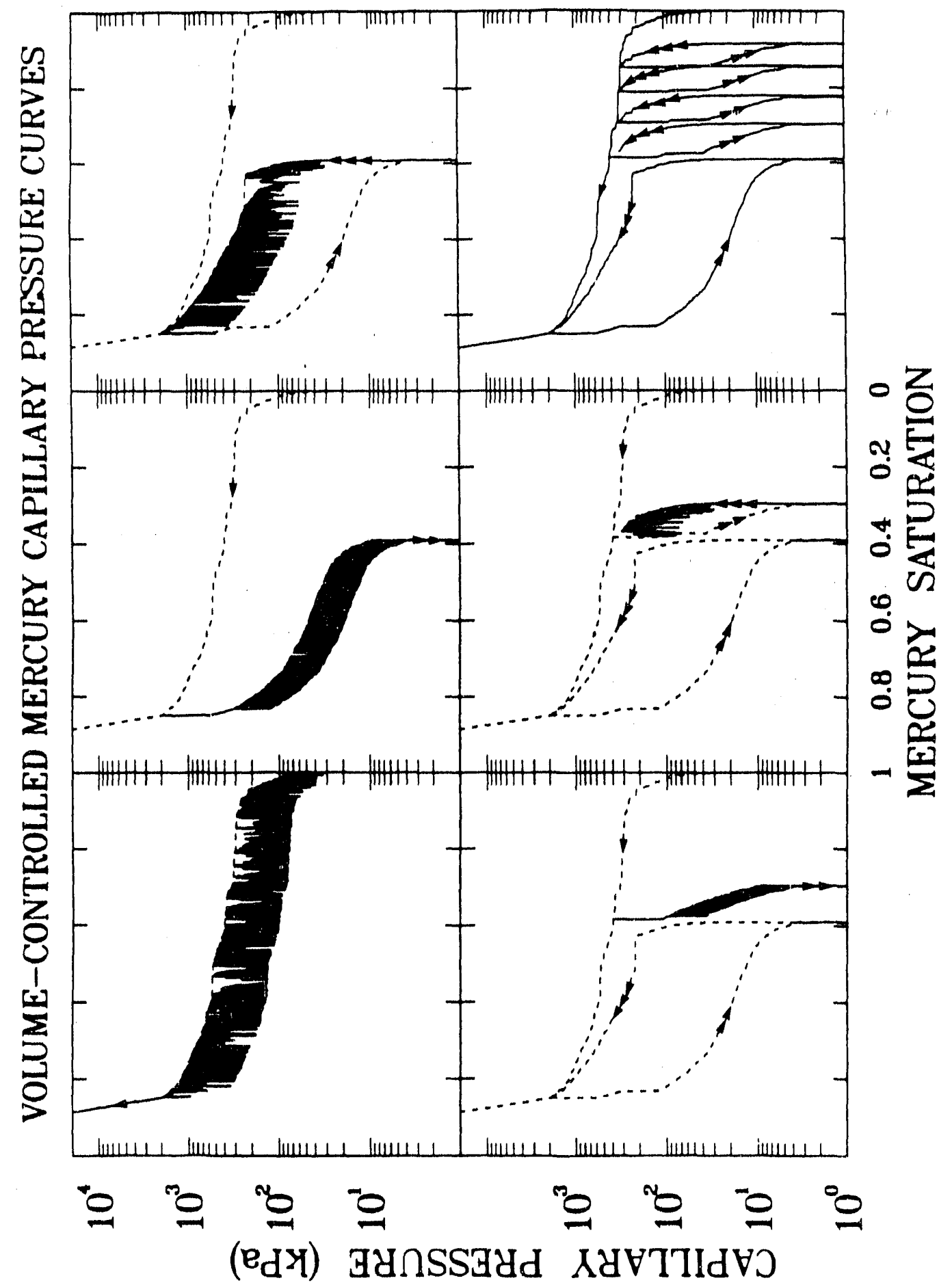

Figure 16. Computer simulation of volume-controlled mercury capillary pressure curves for a 'high aspect ratio' network of biconical pore segments. Envelopes correspond to classical pressure-controlled capillary pressure curves. Primary injection curve (one arrow), withdrawal curves (two arrows), and reinjection curves (three arrows). 


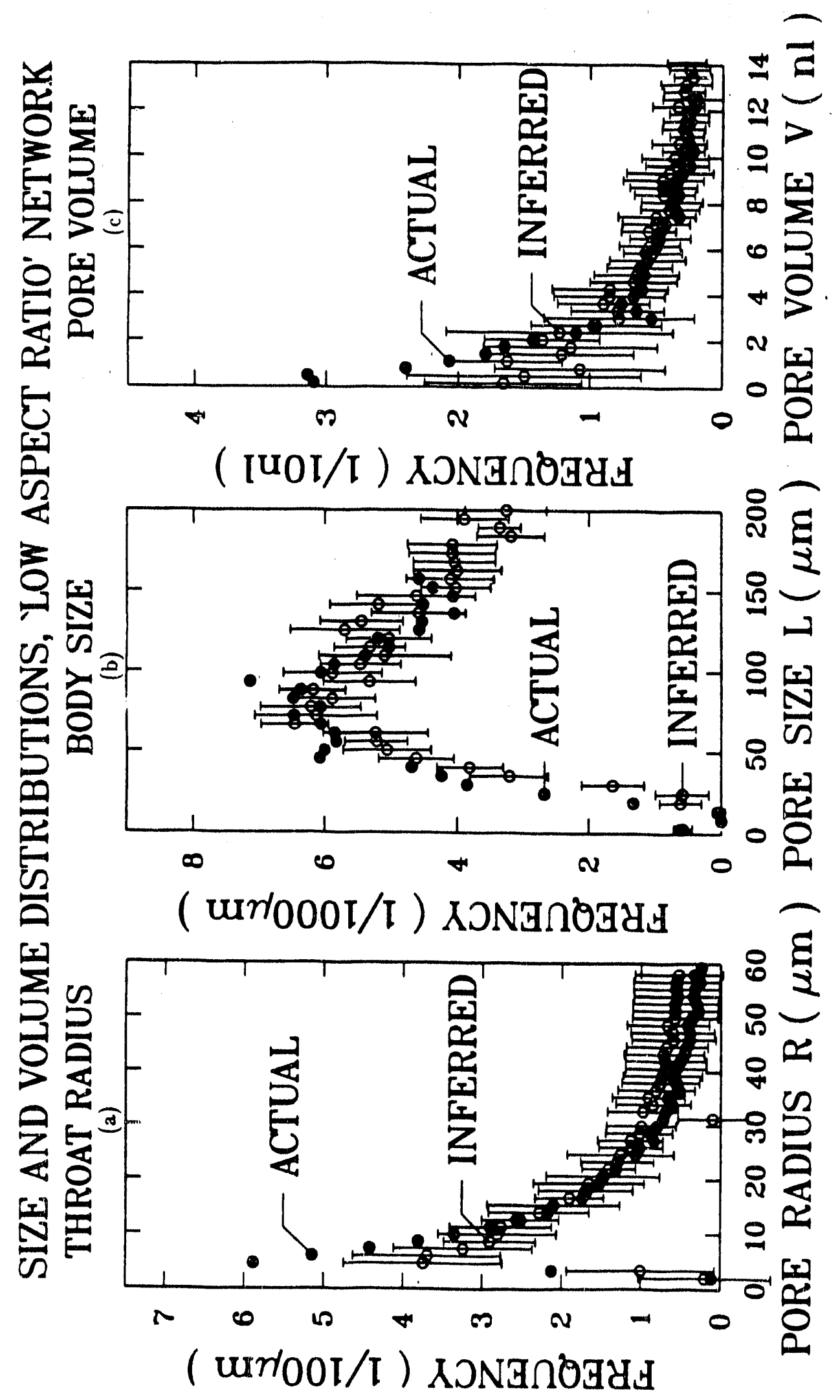

Figure 17. Pore size and volume distribution for a 'low aspect ratio' network (parameters in Table 1). Optimum sample size to deduce pore structure information is 35,000 pores. (a) Throat radius distribution, (b) body size distribution, and (c) pore volume distribution. Error bars are $95 \%$ confidence intervals around the mean from 11 realizations. 


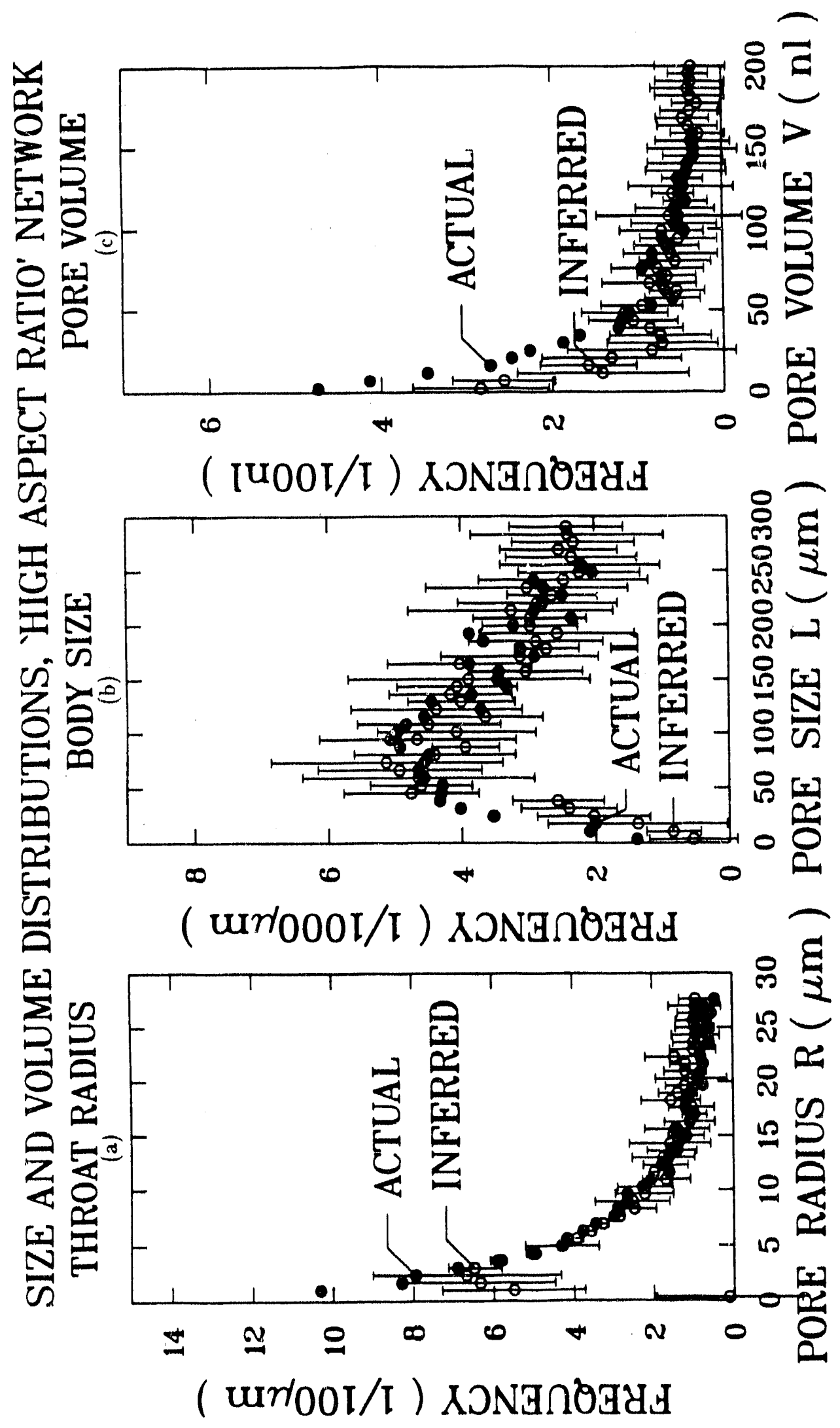

Figure 18. Pore size and volume distribution for a 'high aspect ratio' network (parameters in Table 1). Optimum sample size to deduce pore structure information is 35,000 pores. (a) Throat radius distribution, (b) body size distribution, and (c) pore yolume distribution. Error bars are $95 \%$ confidence intervals around the mean from 11 realizations. 
before the jump. Thus, a pore system is in the most general case a large group of pore segments connected through pore throats of larger size than the throat through which the jump occurs. We define a pore segment as the pore space occupied by mercury after the jump, when pressure is $p_{1}$, and immediately before the next jump, when pressure is $p_{2}$. Equilibrium and stability conditions can result in $p_{2}$ being lower than $p_{1}$. Actually, $p_{2}$ can be not only lower than $p_{1}$ but also lower than the pressure corresponding to the same saturation in the capillary pressure envelope. Cle:arly, the different definition of pore volume can explain the differences between the statistics of Yuan and Swanson and those displayed in Figures $17 \mathrm{c}$ and $18 \mathrm{c}$.

Another explanation is based on specimen size. In a large sample, larger than the optimum size defined above, Haines jumps are accompanied by small pressure drops. The amount of mercury redistributed is large and consequently, assemblies of pore segments of large volumes are occupied by mercury. This argument is consistent with the observations of Yuan and Swanson and explain the apparently large median pore system volume of 25 to $500 \mathrm{nl}$ in Berea sandstone.

The discrepancies however, are less pronounced if we compare the statistics for the high aspect ratio network and that for the San Andres dolomite. This suggests that specimen size in this case may be optimum in the sense we used it here.

Although interesting, Yuan and Swanson's statistics are available also from Katz and Thompson's 'Devil's staircase'. This is an accurate pressure-controlled experiment that yields stepwise capillary pressure curves. Each step represents a pore system with an associated volume and a threshold pressure which is inversely proportional to the size of the throat through which the pore system is invaded.

Yuan and Swanson suggest that APEX-measured prore systems represent potential traps for nonwetting phase and that the total pore system volume can be used to estimate the degree of trapping in a rock sample. To reconcile this argument with the observations of Chatzis et al. (1983 and 1984) is difficult. The work of Chatzis and coworkers suggests that residual oil in Berea sandstone occupies mainly single and double pore segments. We arrived to the same conclusion after analyzing results of Monte Carlo simulations of volumecontrolled liquid-liquid displacement in networks of biconical pore segments similar to those used here. Typically, choke-off disconnection of the nonwetting phase occurs before any wider region of the network's pore space relinquish any significant amount of nonwetting fluid. This results suggest that the trapping potential of a rock sample not only depends on pore structure but also on the wetting properties of the fluid/fluid pairs. Thus, although the statistics on pore system volume is readily available from the computer-generated data on volume-controlled mercury injection, we choose to resolve as many pressure fluctuations as practically possible and display the data as in Figures $17 \mathrm{c}$ and $18 \mathrm{c}$. It is shown that for the optimum sample size a remarkable recreation of the actual pore volume distribution is possible. The idea of using specimens of 'optimum size' in APEX experiments should now be clear: after a Haines jump, ideally, only single pore segments should be filled. 
Equilibrium and stability conditions indicate that this is not always possible. It is not surprising then, to observe if Figures $17 \mathrm{c}$ and $18 \mathrm{c}$ that pore volume of single pores is underestimated, and pore systems of a large volume are detected.

\section{Conclusions}

Monte Carlo simulation of volume-controlled mercury porosimetry in well-defined networks of biconical pore segments reveals that fluctuations in the mercury capillary pressure as mercury is injected provide detailed information about the pore space structure, notably pore throat size and volume distributions. The results reported in this paper confirm Yuan and Swanson's claim that far more information about the pore space of a reservoir rock sample can be obtained from volume-controlled porosimetry than from conventional pressure-controlled porosimetry.

Added kinds of volume-controlled experiments, namely withdrawal experiments, withdrawal after partial reinjection and full scanning loops can extcnd the capabilities of APEX mercury injection. That this is so is demonstrated here for statistical pore body size distributions.

Sample size, an aspect not reported previously, is found to be a major factor in APEX response. Smaller specimens are required for fine-pored media, such as sandstones and clay-coated carbonates. On the contrary, comparably larger specimens are needed for: coarse-pored media, such as carbonates severely altered by diagenesis.

The surface roughness of pore segments varies greatly among natural porous inedia. It is shown that high pressure mercury porosimetry and fractal geometry can be used to characterize microporosity and surface roughness in reservoir rocks.

This paper concentrates on weakly correlated, irregularly connected, fairly homogeneous three-dimensional networks of pore segments. It is anticipated that APEX examination of pore systems that display bimodal size distribution, random or correlated heterogeneities, and stratification will pave the way to correlating properties like permeability with pore structure as inferred from capillary pressure data.

\section{References}

Aho, A.V., Hopcroft, J.E., and Ullman, J.D., The Design und Analysis of Computer Algorithms, Addison-Wesley Reading, Massachusetts (1974).

Bolza, O., Vorlesungen über Variationsrechnung, Teubner, Leipzig (1909).

Chatzis, I., Morrow, N.R., and Lim, H.T., Soc. Pet. Eng. J. 311-326 (April 1983).

Crawford, F.W. and Hoover, G.M., J. Geophysical Res. 71, 2911 2920 (1966).

Davis, H.T., Europhys. Lett. 9, 629-632 (1989). 
de Gennes, P.G., Physics of Disordered Materials, D. Adler et al. (eds.), Plenum Press, New York (1985) pp. 227-241.

Dullien, F.A.L., Porous Media - Fluid Transport and Pore Structure, Academic Press, New York (1979).

Everett, D.H., Colston Papers, Vol. X, Structure and Properties of Porous Materials, D.H. Everett and F.S. Stone (eds.), Butterworths, London (1958).

Fatt, I., Trans. AIME 207 144-181 (1956).

Gaulier, C., paper SPE 3612 presented at the 1971 SPE Annual Technical Conference, New Orleans, Oct. 3-6.

Gibbs, J.W., The Scientific Papers, Thermodynamics, Vol. 1, New York, Dover (1906/1961).

Gillette, R.D. and Dyson, D.C., Arch. Rational Mech. Anal. 53, 150-177 (1974).

Haines, W.B., J. Agr. Sci. 20, 97-116 (1930).

Heiba, A.A., Ph.D. dissertation, University of Minnesota, Minneapolis, MN (1985).

Heiba, A.A., Sahimi, M., Davis, H.T. and Scriven, L.E., paper SPE 11015 presented at the 1982 SPE Annual Technical Conference, New Orleans,

Huh, C., PhD dissertation, University of Minnesota, Minneapolis, MN (1969).

Katz, A.J. and Thompson, A.H., Phys. Rev. Lett. 54, 1325-1328 (1985).

Katz, A.J. and Thompson, A.H., J. Geophysical Res. 92, 599-607 (1987).

Lin, S. and Cohen, M.H., J. Appl. Phys. 53, 4152-4165 (1982).

Melrose, J.C., Characterization of Porous Solids, K.K. Unger et al. (eds.), Elsevier, Amsterdam (1988) pp. 253-261.

Melrose, J.C., Soc. Pet. Eng. J. 259-271 (May 1965).

Melrose, J.C. and Brandner, C.F., J. Can. Pet. Tech. 54-62 (October 1974).

Miller, E.E. and Miller, R.D., Soil Sci. Soc. Am. Proc. 19, 267-271 (1955).

Mohanty, K.K., Ph.D. dissertation, University of Minnesota, Minneapolis, MN (1981).

Mohanty, K.K., Davis, H.T. and Scriven, L.E., SPERE 113-128 (February 1987).

Morrow N.R., Ind. Eng. Chem. 62, 32-56 (1970).

Novy, R.A., Toledo, P.G., Davis, H.T. and Scriven, L.E., Chem. Eng. Sci. 44, 1785-1797 (1989).

Purcell, W.R., JPT 1, 39-48 (1946).

Rücker, A.W., Phil. Mag. 23, 35-45 (1887).

Scheidegger, A.E., The Physics of Flow Through Porous Media, University of Toronto Press, Toronto $(1957 / 1960 / 1974)$.

Stegemeier, G.L., paper SPE 4754 presented at the 1974 Improved Oil Recovery Symposium of SPE-AIME, Tulsa, OK, April 22-24.

Swanson, B.F., SPWLA 1-17 (June 1985).

Thompson, A.H., Katz, A.J. and Raschle, R.A., Phys. Rev. Lett. 58 29-32 (1987).

Toledo, P.G., Novy, R.A., Davis, H.T. and Scriven, L.E., Soil Sci. Soc. Am. J. 54, 673-679 (1990).

Tyuptsov, A.D., Mekhanika Zhidkosti i Gaza 1, 78-85 (1966).

van Brakel, J., Powder Technol. 29, 1-12 (1981).

Wardlaw, N.C., AAPG Bull. 60, 245-257 (1976).

Wardlaw, N.C., Li, Y. and Forbes, D., Transport in Porous Media 2, 597-614 (1987). 
Washburn, E.W., Proc. Natl. Acad. Sci. 7, 115 (1921).

Wong, P.-Z., Physics and Chemistry of Porous Media II, J.R. Banavar et al. (eds.), AIP Conference Proceeding 154, AIP, New York (1087) pp. 304-318.

Yuan, H.H. and Swanson, B.F., paper SPE/DOE 14892 presented at the 1986 Symposium on EOR of SPE/DOE, Tulsa, OK, April 20-23. 


\title{
FLUID DISTRIBUTION AND TRANSPORT IN POROUS MEDIA AT LOW WETTING PHASE SATURATIONS
}

\begin{abstract}
Capillary pressure, hydraulic conductivity, and capillary dispersion coefficient have been observed to obey fnwer laws in the wetting phase saturation. We relate power-law behavior at low wetting phase saturations, i.e., at high capillary pressures, to the thinfilm physics of wetting phase and the fractal character of the porespace of natural porous media.
\end{abstract}

Recent displacement experiments show "anomalous" rapid spreading (hyperdispersion) of water during imbibition into a prewet porous medium. Either hyperdispersion or hypodispersion can occur according to our model; which one occurs depends on the disjoining pressure behavior of the thin films involved.

\section{Introduction}

The capillary pressure and the hydraulic conductivities (permeabilities) of wetting and nonwetting phases in a porous medium are complicated functions of wetting phase saturation $S_{w}$ (percent porespace occupied by wetting phase), the morphology (shape and curvature) and composition of porespace, and the process whereby wetting and nonwetting phases come to occupy the porous medium. Since oil recovery technology has driven much of the research on fluids in porous media, most of the studies have been on syster.s with appreciable amounts of wetting phase. It appears, however, that the capillary pressure and permeability behavior at low saturations of wetting phase provides information about the asperities or roughness of porespace and about the physics of thin films on pore surfaces that cannot be obtained from the high saturation studies. The behavior of wetting phase (water) at low saturation is of substantial interest to the soil scientist in connection with agricultural crops, the balance of aquifers and the migration of agricultural, chemical and nuclear contaminanis.

In this paper we describe recent work (Davis (1989), Toledo et al.(1990)) in which we have derived and tested scaling laws based on the hypotheses that porespace asperities or roughness are fractally distributed and that at sufficiently low saturation the hydraulic conductance of wetting phase is controlled by thin films.

\section{Porespace Morphology of Natural Sandstone}

A scanning electron micrograph of a fracture exposed surface of an oil reservoir sandstone is shown in Figure 1 (Suranto et al.1990). The major grains and pores of this clayey, consolidated sandstone are on the order of 100 microns in dimension (Figure 1a). Stacks 
of book like kaolinate clays (Figure 1b, 1e, 1f) are attached to the pore surfaces. These stacks sometimes extend hundreds of microns in width. The spacing between the booklets is about 10 microns and between the kaolinate platelets is less than a micron (Figure 1f). There are also aggregates of quartz crystallites (chert) on the pore surfaces (Figure 1c and 1d). The crystallites are a few tenths of a micron in diameter. Between the aggregates are porous regions of the order of a few microns to a micron in diameter. Within the chert aggregate are intergranular spaces of the order of a few hundredths of a micron in size.

It follows from the micrograph presented in Figure 1 that the porespace in this natural sandstone is highly disordered and that the characteristic sizes of the various cavities forming the porespace are distributed over at least four decades of length. This is consistent with scanning electron microscope (SEM) studies reported for several natural sandstones by Katz and Thompson (1985). They measured the number of features (asperities) versus size of the feature on several natural porous sandstone fracture surfaces and concluded that porespace is fractally distributed between a minimum dimension $\ell_{1}$ (of the order of 10 to $100 \AA$ ) and a maximum dimension $\ell_{2}$ (of the order of $100 \mu \mathrm{m}$ ). According to Katz and Thompson's argument the porosity $\phi$ of fractal sandstone is

$$
\phi=A\left(\frac{\ell_{1}}{\ell_{2}}\right)^{3-D}
$$

where $A$ is of the order of 1 and $D$ is the fractal or Hausdorff dimension, which is a measure of how fuly the subject space fills the Euclidean space in which it is imbedded. By plotting the number of geometric features versus size, they found values of $D$ ranging from 2.57 to 2.87 from SEM studies of five natural sandstones.

Roberts (1986) has criticized the conclusions of Katz and Thompson (1986), who countered that Roberts was ignoring the fact that sandstones are disordered media and that they are fractal only between the lengths $\ell_{1}$ and $\ell_{2}$.

If the volumes of the cavities formed by the various asperities illustrated in Figure 1 are fractally distributed, this has particular implications for the capillary pressure behavior at low saturations of wetting phase. In what follows we explain and report tests of these implications.

\section{The Capillary Pressure and the Saturation of Pendular Structures and Thin Films}

In this paper we are interested in fluid pairs in which one of the fluids strongly wets the porous medium. Thus, even at saturations so low that bulk wetting phase seemingly exists only as isolated regions or pendular structures, the wetting phase remains hydraulically connected through thin films. Common examples of such pairs are water/air and water/hydrocarbon in sandstones and soils. To achieve a given wetting phase saturation $S_{w}$, the pressures $P_{n w}$ in bulk wetting phase and $P_{w}$ in bulk nonwetting phase must satisfy 


\section{SURFACE TEXTURE OF RESERVOIR SANDSTONE}

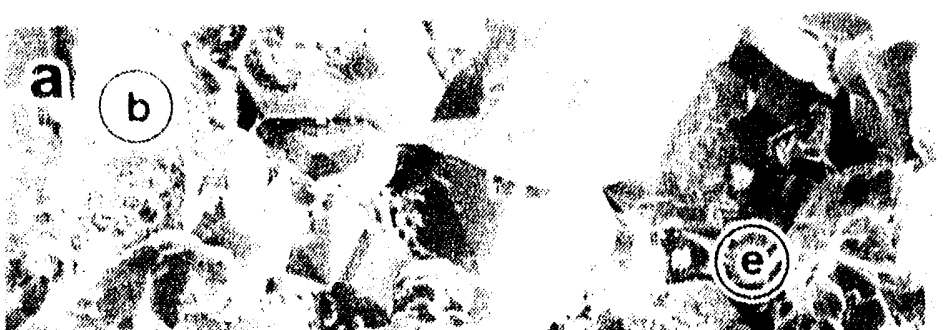

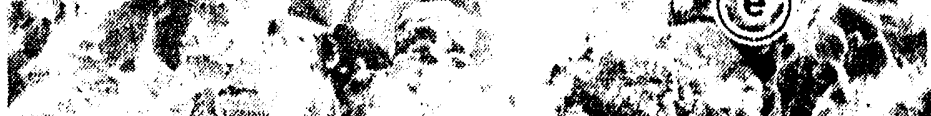
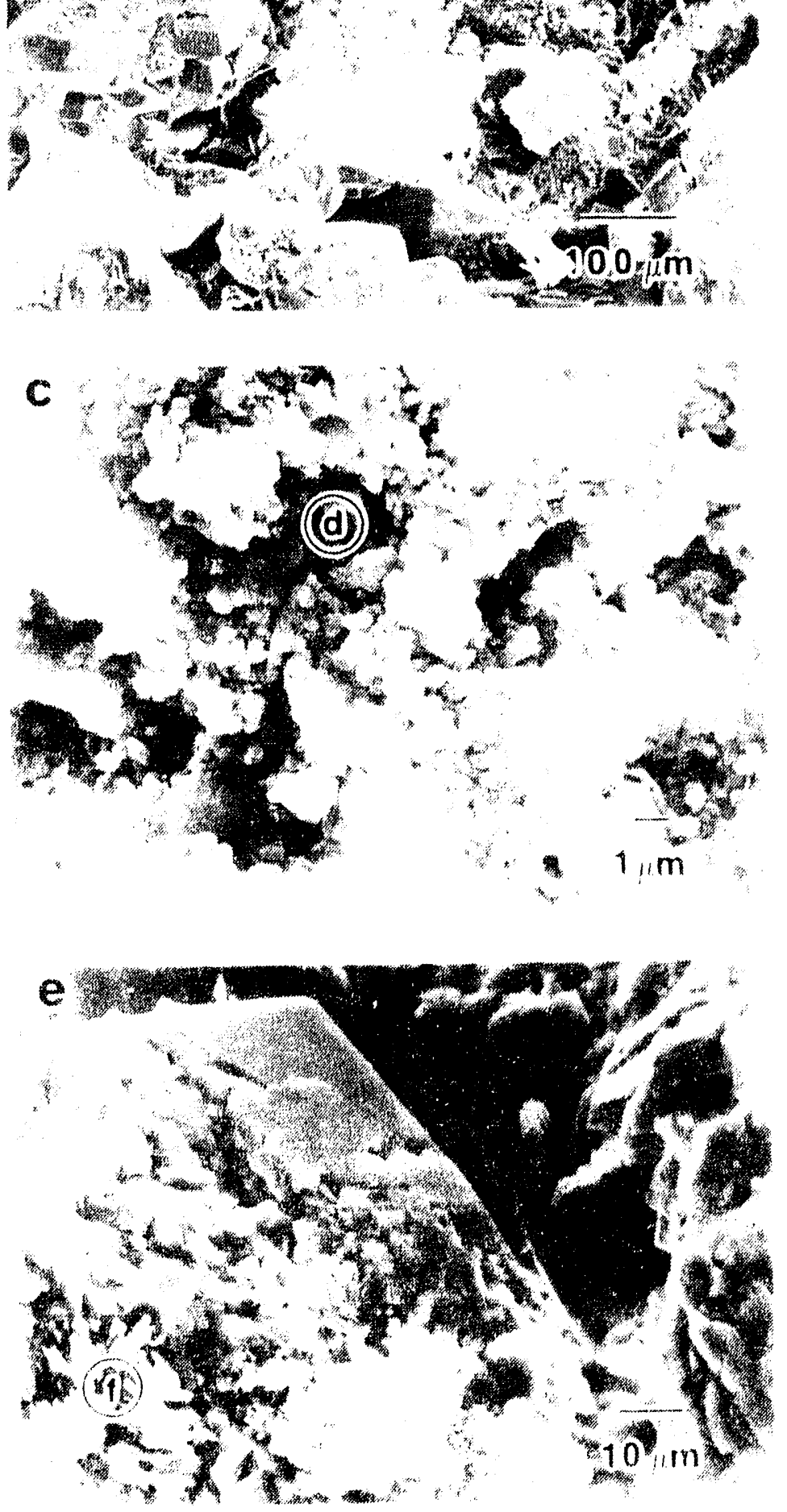
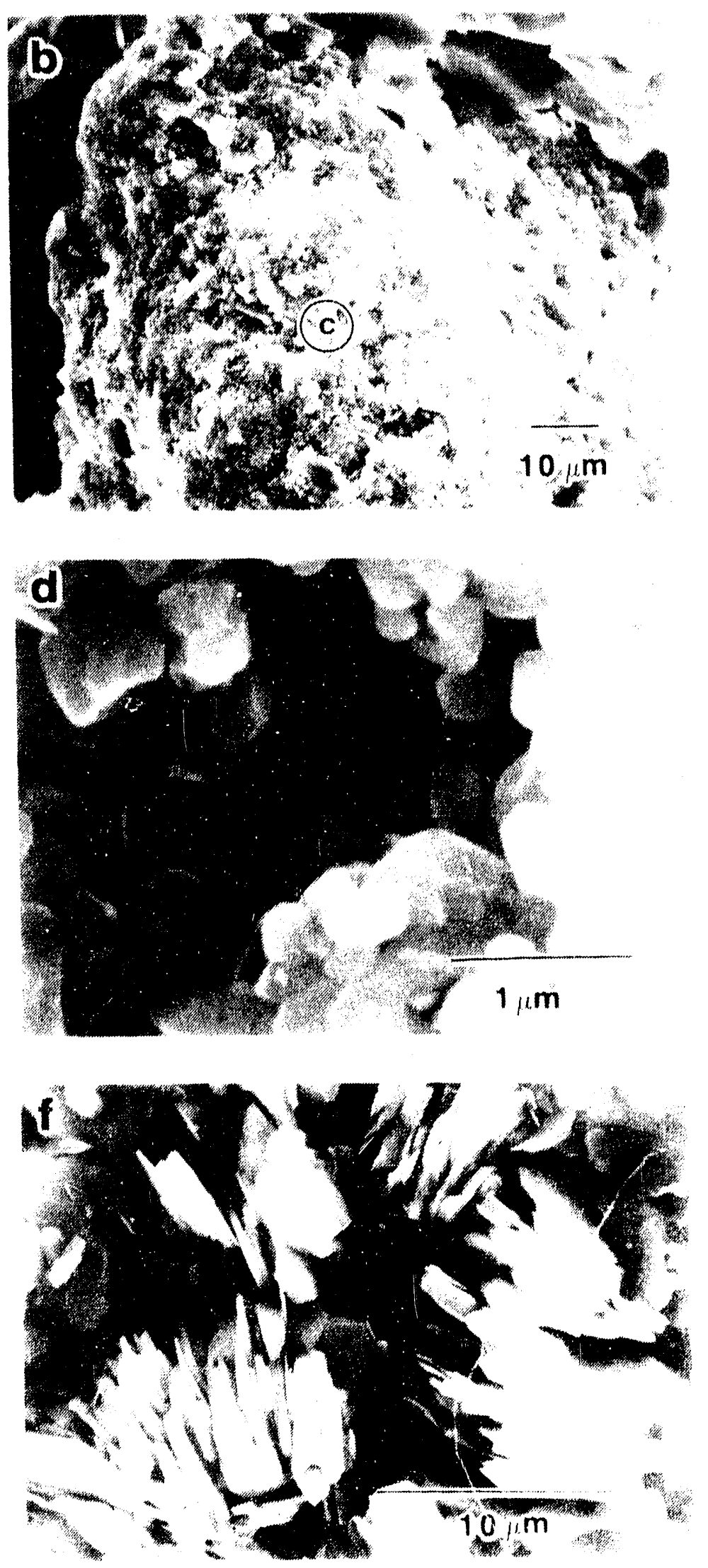
the Young-Laplace equation for the capillary pressure $P_{c}$, i.e.

$$
P_{c} \equiv P_{n w}-P_{w}=2 H \gamma
$$

where $H$ is the mean curvature and $\gamma$ is the interfacial tension of the meniscus between wetting and nonwetting phases. The mean curvature is related to the principal radii of curvature $R_{1}$ and $R_{2}$ of the meniscus by the expression

$$
H=\frac{1}{2}\left(\frac{1}{R_{1}}+\frac{1}{R_{2}}\right) .
$$

In a given displacement process, fixing the mean curvature of the menisci between wetting and nonwetting phases fixes the saturation $S_{w}$.

The Young-Laplace equation is applicable when both the wetting and nonwetting phases are present in bulk amounts. When the wetting phase is present as a thin film (thinner than a few thousand angstroms), the thin film pressure differs from that of bulk wetting phase by the disjoining pressure $\Pi(h)$ which depends on film thickness $h$. In this case the Young-Laplace equation must be replaced with the augmented Young-Laplace equation (Mohanty et al.1981).

$$
P_{c}=\Pi(h)+2 H \gamma .
$$

For water on clean quartz or glass the disjoining pressure has been shown (Pashley, 1980) to be well approximated by $\Pi(h)=41 h^{-1} \mathrm{dyn} / \mathrm{cm}$ for films thinner than $1000 \AA$ and by (Derjaguin et al.1976) $\Pi(h)=2 \times 10^{-7} h^{-2} d y n$ for films thicker than $1200 \AA$. For water in clayey soils the data of Viani et al. (1983) can be fit to $\Pi(h)=250 h^{-1 / 2} d y n / \mathrm{cm}^{3 / 2}$ for film thicknesses is in the range $175 \AA<h<375 \AA$. Fiims of nonpolar fluids obey the power law $\Pi(h)=A h^{-3}$ when $h$ is less than a few tenths of a micron, e.g., for octane on glass ${ }^{9}$ $\Pi(h)=9 \times 10^{-5} h^{-3} \mathrm{erg}$.

Equations (2) and (4) provide the basis for understanding how the capillary pressure sets the inventory of wetting fluid at saturations below the percolation threshold, in which case wetting phase exists as thin films or as "pendular structures" at intergranular contacts, i.e., isolated regions smaller-than-average porosity, or in nooks and crannies provided by the pore surface asperities. The pendular structures are hydraulically connected by thin films.

To understand the connection between capillary pressure and saturation, consider the pendular structures shown in Figure 2. The menisci in the conical and hemispherical pits are pieces of a sphere whose radius $r_{c}\left(H=r_{c}^{-1}\right)$ is given by

$$
r_{c}=2 \gamma / P_{c},
$$

whereas the menisci along the pore edges and between the fused spheres $\left(\rho>r_{c}\right)$ are pieces of a cylinder whose radius $r_{c}\left(H=\left(2 r_{c}\right)^{-1}\right)$ is given by

$$
r_{c}=\gamma / P_{c} \text {. }
$$




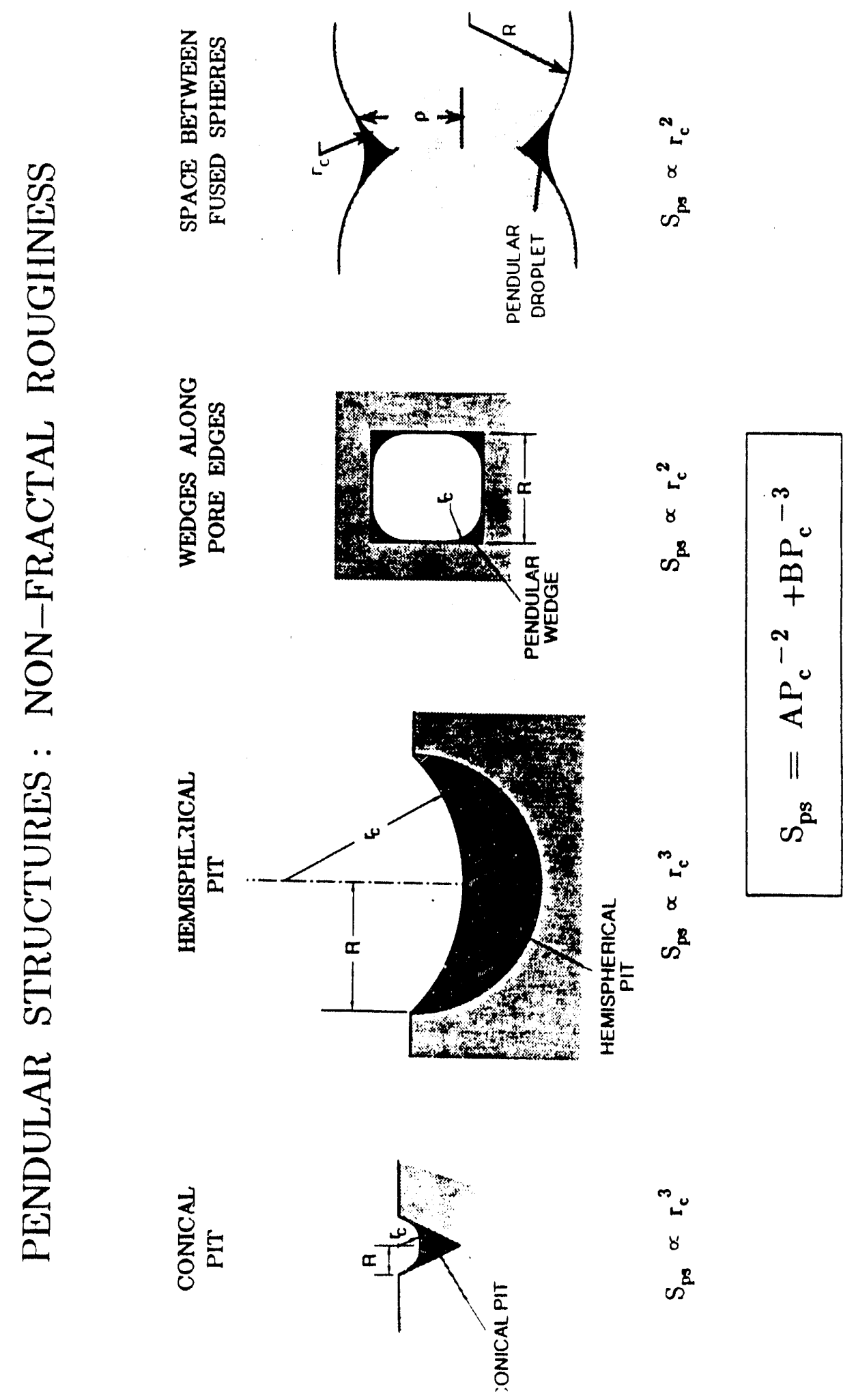

Figure 2. Wetting liquid present as pendular structures in conical and hemispherical pits, as wedges along pore edges and as collars between fused spheres. 
By elementary geometry, it follows that the volume of wetting phase in the conical and hemispherical pits scales as $r_{c}^{3}$, and so if all the pendular structures were in such pits and if their characteristic dimension $R$ were of the same length scale, the wetting phase saturation in pendular structures would scale as $S_{p s} \propto r_{c}^{3} \propto P_{c}^{-3}$.

Similarly, the volume of wedges and collars (with $\rho>>r_{c}$ ) scales as $r_{c}^{2}$, and so the saturation of pendular structures would scale as $S_{p s} \propto r_{c}^{2} \propto P_{c}^{-2}$ if all the edges and fused spheres were of the same geometric dimensions. Since pits, pore edges and fused grains are the most likely sites of pendular structures, we expect the scaling relation

$$
S_{p s}=A P_{c}^{-2}+B P_{c}^{-3}
$$

if the sites are nonfractal, i.e., if they are all of the same characteristic length scale.

If, on the other hand, the pendular structures are in sites fractally distributed according to Eq. (1), then at a capillary pressure $P_{c}$ all the porosity with dimension $\ell<r_{c}=2 \gamma / P_{c}$ would be filled with wetting phase and so $S_{p s} \propto r_{c}^{3-D}$, or

$$
S_{p s}=A P_{c}^{-(3-D)}
$$

Pendular structures in self-similar fractal media are depicted in Figure 3. Shortly after the appearance of the work of Katz and Thompson, deGennes (1985) pointed out that Eq. (8) holds in the cases of a porespace of self-similar iterative flocs of the type shown in Figure 3 and a porespace of self-similar iterative pits (self-similar pits within pits). The Menger sponge shown in Figure 3 is an interesting example because its fractal dimension can be easily determined. The sponge is generated by the following iterative process. A cube with sides of length $R$ is partitioned into 27 cubes the length of whose sides is $R / 3$. The seven cubes along the three axes of symmetry of the parent cube are removed. The partitioning and deletion process is iterated for each of the remaining cubes. The Menger sponge is made by indefinite continuation of this process. The resolution length $r$ is defined as the size of the smallest measurable feature. The Menger sponge in Figure 3 is depicted with a resolution length of $R / 81$. Holes smaller than $R / 81$ are not resolved in the figure. The number $N_{r}$ of solid cubes increases with resolution as

$$
N_{r}=\left(\frac{R}{r}\right)^{D}
$$

where $D$ is the fractal or Hausdorff dimension of the object. In the case of the Menger sponge 20 cubes are resolved at a resolution $r=R / 3$, and so $D=\log 20 / \log 3 \simeq 2.73$. In general, the Hausdorff dimension $D$ can range from 2 to 3 , depending on the geometry of the object.

As is obvious from Figure 1, a natural sandstone is not a regular self-similar fractal. Instead, consistently with the arguments given by Katz and Thompson (1986), we believe it is better viewed as a disordered assembly of matrix grains ranging in size from $\ell_{1 g}$ to 

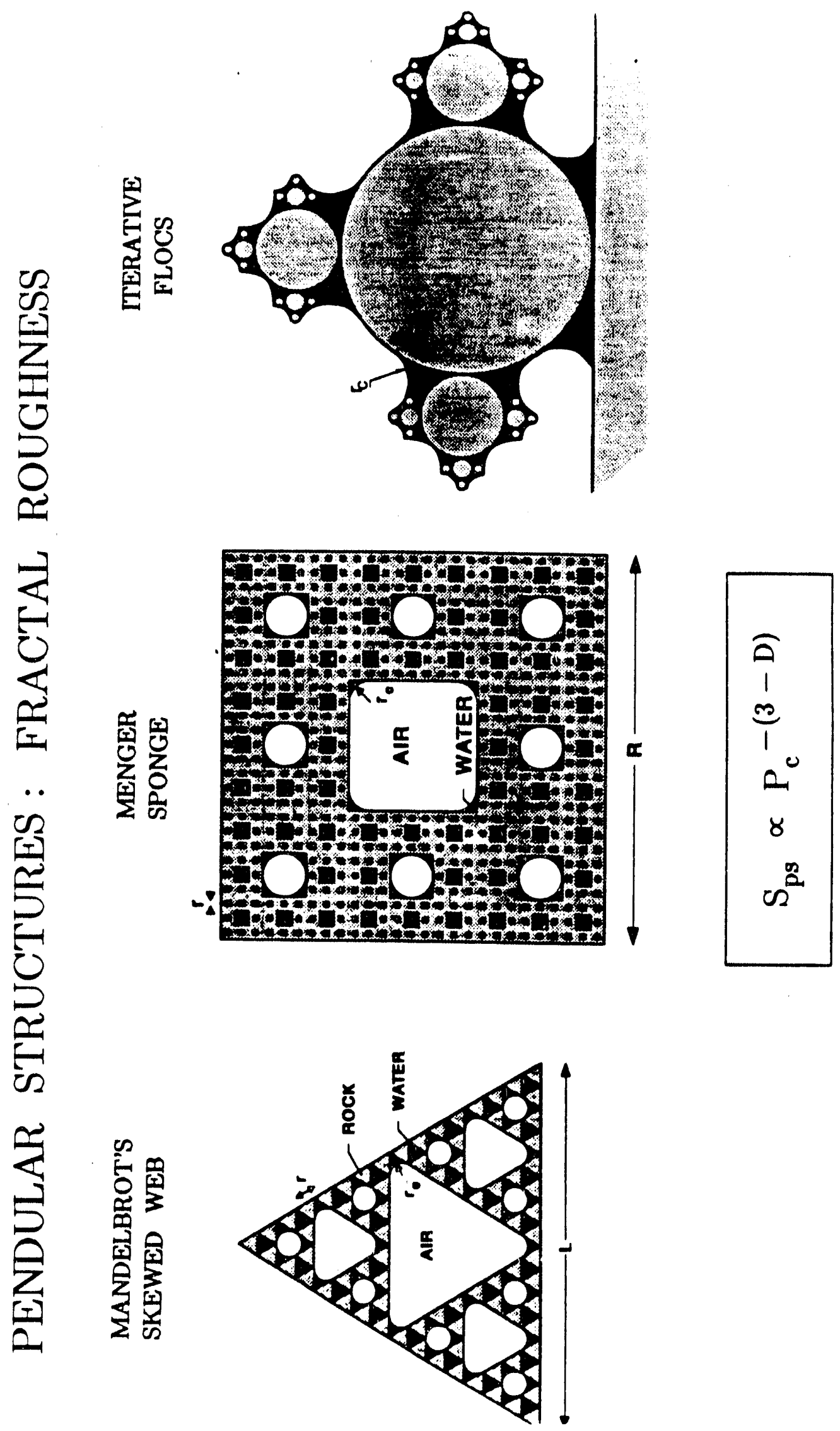

Figure 3. Wetting liquid present in fractally distributed pendular strutures. The examples given here are geometrically regular, self-similar fractal objects. 
$\ell_{2 g}$. The size distribution of pores will naturally be broad if that of the granular subparts is broad. It is the wide distribution of poresize and roughness that is the important distinction between Ergs. (7) and (8). If all of the pendular structures are about the same size, then, as indicated by Eq. (7), the saturation of wetting phase approaches zero as a strong function of capillary pressure (as $P_{c}^{-2}$ or $P_{c}^{-3}$ ). On the other hand, if the sizes are distributed broadly as in a fractal structure, the range of capillary pressure over which the pendular structtires are emptied is correspondingly broad. From Katz and Thompson's work we anticipate $D \simeq 2.5-2.8$, and so according to $\mathrm{Eq}$. (8) the pendular structure inventory approaches zero as $P_{c}^{-0.5}$ to $P_{c}^{-0.2}$, a much weaker function of $P_{c}$ than in the case of nonfractal structures.

As we commented at the outset, we are interested in the distribution of wetting phase at saturations below the wetting phase percolation threshold and so bulk wetting phase does not span the sample. Instead, wetting phase is present as isolated pendular structures hydraulically connected to one another through thin films. The thickness of these films is fixed by the capillary pressure through the augmented Young-Laplace equation, Eq. (4). Thin films tend to form in regions of porespace whose mean radius of curvature is large compared to film thickness $h$, that is in relatively smooth regions. Otherwisc, pendular structures will form instead of thin films. Thus, we expect the capillary contribution, $2 H \gamma$, to be negligible compared to the disjoining pressure contribution, i.e., we expect

$$
P_{c} \simeq \Pi(h)
$$

for thin films. The volume of wetting phase present as thin films is $V_{t f}=h A_{t f}$, where $A_{t f}$ is the area of porespace favoring thin films over pendular structures (i.e., the regions of relative smoothness - - these could include surfaces with large mean radius of curvature but with a small scale roughness, e.g., from chert, that lies underneath the meniscus of the thin film). Assuming that $A_{t f}$ is a weak function of the capillary pressure, we find that the saturation $S_{t f}$ of wetting phase present as thin films is proportional to film thickness. If the disjoining pressure obeys a power law of the form

$$
\Pi(h) \propto h^{-m}
$$

it then follows from Eq. (10) that

$$
S_{t f} \propto P_{c}^{-1 / m}
$$

The total saturation of wetting phase is given by $S_{w}=S_{t f}+S_{p s}$. However, under the conditions of the experiments to be discussed in a later section, the thin film saturation is generally negligible compared to that of pendular structures, and so the behavior of the capillary pressure versus wetting phase saturation can be used as a probe of porespace roughness independently of the disjoining pressure behavior. 


\section{Hydraulic Conductivity and Capillary Dispersivity}

The local flow of wetting phase at low saturations is very complicated because it involves flow in geometrically complex pendular structures and thin films. However, if the pendular structures do not span the sample, the major resistance to flow will be the thin films of thickness $h$ providing the hydraulic connectivity between the pendular structures. The hydraulic conductance of thin films is proportional to $h^{3}$ and inversely proportional to the viscosity $\mu_{w}$ of the wetting phase. The hydraulic conductance $\kappa_{u}$ of wetting phase is defined as $v_{w}=-\left(K_{w^{\prime}} / \mu_{w^{\prime}}\right) \Delta P_{w^{\prime}} / L$, where $v_{w}$ is the mean flow velocity in a sample of

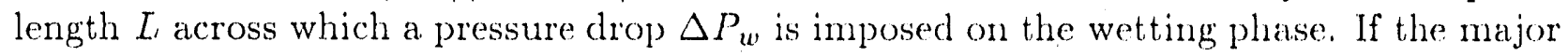
resistance to flow of wetting phase is provided by thin films, then $K_{u} \propto h^{3}$, and if the disjoining pressure obeys the power law (11), it follows from $\mathrm{Eq}$. (12) that

$$
I_{u} \propto P_{c}^{-3 / m}
$$

If, furthermore, the inventory of wetting phase is primarily that of pendular structures fractally distributed, Eq. (8), the hydraulic conductivity obeys the scaling law

$$
\Pi_{w} \propto S_{w}^{3 / m(3-D)}
$$

In this case, the saturation dependence of the hydraulic conductivity of wetting phase yields information regarding the disjoining pressure dependence on film thickness and the geometry of porespace.

If a sample porous medium at low wetting phase saturation contacts a reservoir of wetting phase as illustrated in Figure 4, it will spontaneously imbibe wetting phase according to a convection-dispersion equation

$$
\phi \frac{\partial S_{w}}{\partial t}+v \frac{\partial f}{\partial x}=\frac{\partial}{\partial x}\left[D_{c} \frac{\partial S_{w}}{\partial x}\right]
$$

where $\phi$ is porosity of the medium, $v$ is the net flow velocity, $f$ is the ratio of the flow velocity of wetting phase and the net flow velocity, and $D_{c}$, the capillary dispersivity, is approximated by

$$
D_{c}=-\frac{K_{w}^{*}}{\mu_{w}} \frac{d P_{c}}{d S_{w}}
$$

The fractional flow $f$ depends on saturation $S_{w}$ through the ratio $K_{w} / K_{n w}$ of the permeabilities of wetting and nonwetting phases.

The right hand side of $\mathrm{Eq}$. (15) has the form of a concentration-dependent diffusion equation. However, as pointed out by deGennes ((1985), the effect of capillary dispersion can be either hypodispersive, diffusive, or hyperdispersive depending on how $D_{c}\left(S_{u^{\prime}}\right)$ behaves with decreasing $S_{w}$. If $D_{c}\left(S_{w}\right)$ tends to zero as $S_{w}$ approaches zero, the invading front of wetting phase disperses less than in a diffusive front. If $D_{c}\left(S_{w}\right)$ tends to infinity 

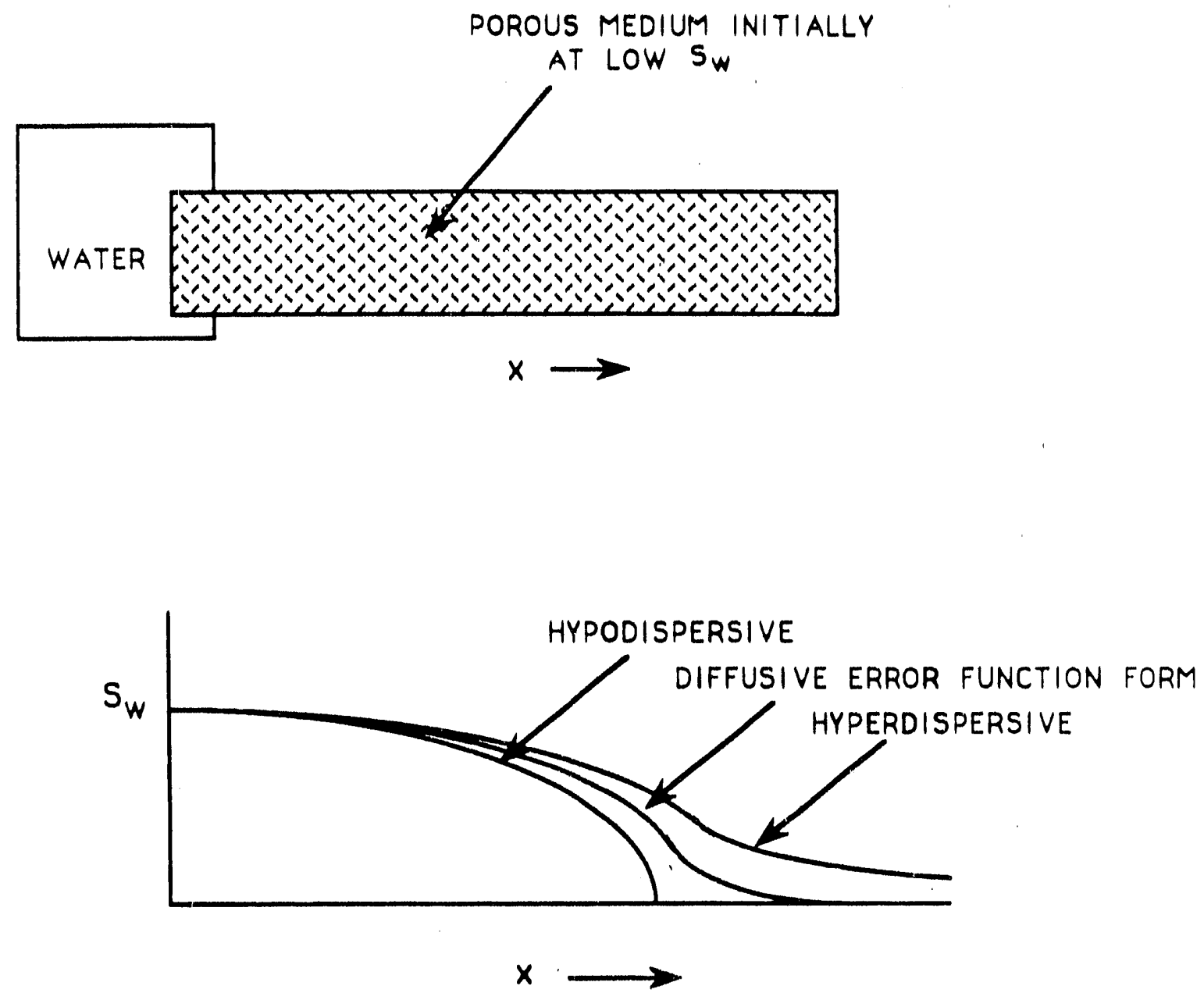

Figure 4. Illustration of hypodispersion $\left(D_{c} \rightarrow 0\right.$ as $\left.S_{u} \rightarrow 0\right)$, diffusion-like dispersion $\left(D_{c} \rightarrow\right.$ constant as $\left.S_{w} \rightarrow 0\right)$ and hyperdispersion $\left(D_{c} \rightarrow \infty\right.$ as $\left.S_{w} \rightarrow 0\right)$ in wetting phase invasion of a porous medium.

as $S_{w}$ approaches zero, the invading front disperses more broadly than in a diffusive front. If $D_{c}\left(S_{w}\right)$ tends to a constant value as $S_{w}$ approaches to zero, the front in the experiment indicated in Figure 4 spreads in a diffusive manner (i.e., or described by error function).

If $S_{w} \simeq S_{p s}$, if the pendular structures are fractally distributed [E(. (8)], and if thin films control hydraulic conductivity $\left[\mathrm{E}_{\mathrm{q}}\right.$. (14)], the cupillary dispersion coefficient obeys the scaling law

$$
D_{c} \propto S_{u}^{[3-m(4-D)] / m(3-D)}
$$

at low wetting phase saturations. Thus, the spreading will be hypodispersive if $m<$ $3 /(4-D)$, hyperdispersive if $m>3 /(4-D)$, and diffusion-like if $m=3 /(4-D)$. By changing wetting phase (e.g., water in air versus oil in air), one could expect to find both hypo and hyperdispersion in the same porous medium. 
Bacri et al.(1985) have observed hyperdispersion in oil/water flow in a porous medium. However, their experiments were conducted at saturations higher than those for which we expect the scaling laws given here to hold.

\section{Comparison of Theory and Experiment}

Few data exist on the behavior of a wetting phase at low saturation in a porous medium. In the soils literature, scaling laws of the form $P_{c} \propto S_{w}^{-a}, K_{w} \propto S_{w}^{b}$ and $D_{c} \propto$ $S_{u}^{c}$, where $a, b$ and $c$ are positive constants, have been observed empirically (Gardner et al. (1970), and Gardner (1960) for capillary pressure; Campbell (1974) and Gardner and Mayhugh (1955) for hydraulic conductance and Gardner and Mayhugh (1955)for capillary dispersivity).

We located and analyzed (Davis, 1989; Toledo et al.1990) three sets of carefully measured data on capillary pressure and hydraulic conductance of sandstones and clayey soils. Melrose (1988) carried out a series of measurements of the capillary pressure of water in the presence of air in Berea sandstone. As is illustrated in Figure 5, Davis (1989) found that the capillary pressure curve obeys the scaling law $P_{c} \propto S_{w}^{-2.23}$. Davis also showed that the nonfractal scaling relation, Eq. (7), fits the data poorly and is not appreciably improved by adding a term proportional to $P_{c}^{-1}$ to account for the thin film inventory (since II $\propto h^{-1}$ for thin films of water on quartz). Thus, the implication of Melrose's data is that in the saturation range $0.03<S_{w}<0.1$, the wetting phase inveritory is primarily pendular structures and that the sites holding the pendular structures are fractally distributed in size. According to Eq. (8), $P_{c} \propto S_{w}^{-1 /(3-D)}$. Since the slope of $\log P_{c}$ versus $\log S_{w}$ in Figure 5 is -2.23 , one obtains for the fractal dimension of the porespace roughness of Berea sandstone the value $D=2.55$. This result compares favorably with the range of values $2.57<D<2.87$ found by Katz and Thompson in SEM studies of other natural sandstones.

Nimmo and Akstin (1988) have reported recently measurements of the capillary pressure and hydraulic conductance of water at low saturations in the presence of air in several compacted samples of Oakley sands (a clayey soil). The data of Viani et al. (1983) on the disjoining pressure of clayey soils fit the formula $\Pi(h)=250 h^{-1 / 2} d y n / \mathrm{cm}^{3 / 2}$ ). We analyzed (Toledo et al.1990) their data in terms of the scaling laws

$$
P_{c} \propto S_{w}^{-1 /(3-D)} \text { and } K_{w} \propto S_{w}^{3 / m(3-D)}
$$

implied by the assumptions of negligible thin film inventory, fractally distributed pendular structures, thin film controlled hydraulic conduction, and a power law disjoining pressure $\Pi(h) \propto h^{-m}$. The capillary pressure data were used to obtain the fractal dimension $D$. We set $m=1 / 2$ in accordance with the results of Viani et al. and computed from $3 / m(3-D)$ the expronent of the scaling law for $K_{w}$. The results are summarized in Table 1 . The frictal (linensions deduced from the capillary pressure curves range from 2.09 to 2.62 with an average value of 2.45 . With the exception of sample 4 , the fractal dimensions of all the samples compare well to the value 2.55 of Berea sandstone. 


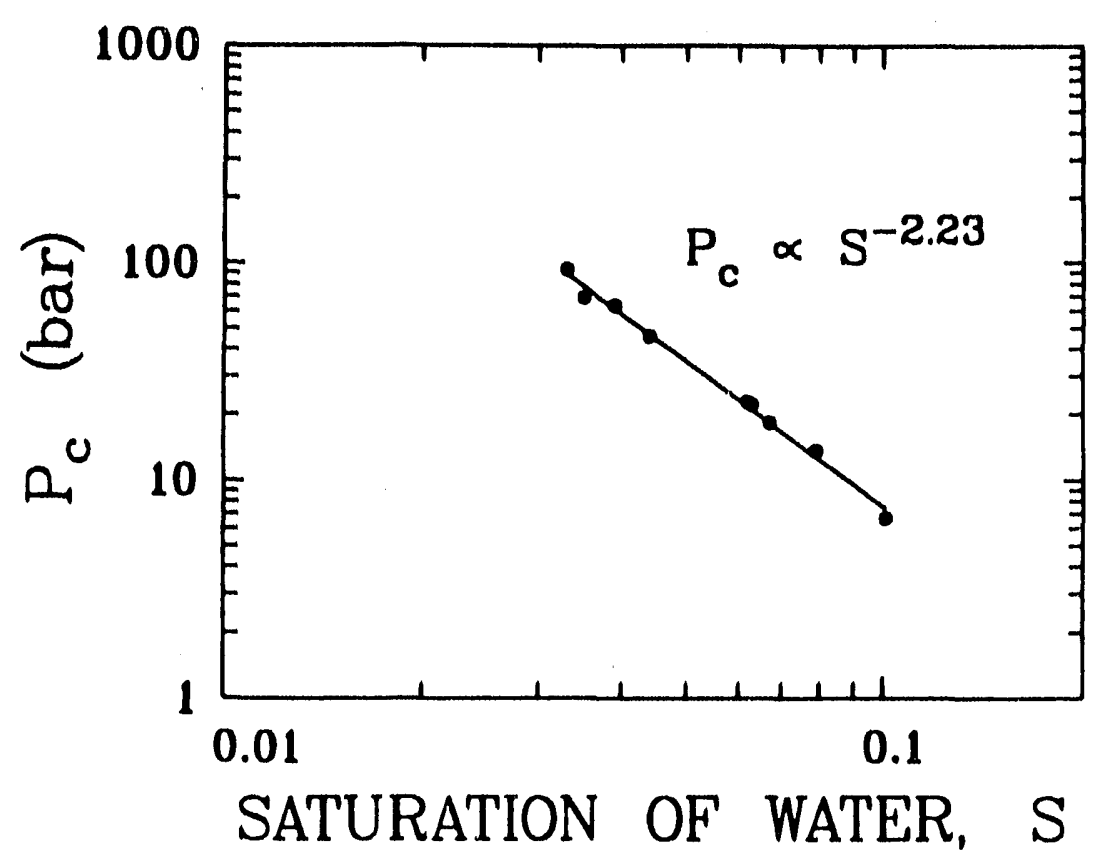

Figure J. Capillary pressure curve for water in Berea sandstone. (Data from Reference 18.)

The predicted exponent for $K_{w}$ agrees with the measured value to better than $24 \%$ for all samples except numbers 5 and 7 . Given the simplicity of the model, the agreement between theory and experiment as demonstrated in Table 1 is satisfactory.

As a matter of clarification, we point out that the assumption that film flow controls the hydraulic conductance does not require that most of the fiow paths be thin films. For example, suppose the conductance of a strip of film is 1000 times less than that of a strip of the same length of a pendular structure. Then if in a series of strips, only $1 \%$ are thin films, the net hydraulic resistance of the thin films in the series is ten times larger than the net hydraulic resistance of the pendular structures in the series.

Ward and Morrow (1987) determined the capillary pressure curves of water in the presence of air in a number of low permeability sandstones (natural gas reservoirs). Their results are plotted in Figure 6. In all cases the capillary pressure curve has two distinct regions. If Equation (18) is used to extract $D$, there is a low saturation region where the deduced value of $D$ is near 2 and there is a higher saturation region in which $D$ ranges from 2.61 to 2.89 . The values of $D$ in the higher saturation region are consistent with the findings of Katz and Thompson, but those in the low saturation region (especially those less than 2) do not fit the fractal model. We do not know much about the porespace of these low permeability sandstones, except that the mean pore size is significantly smaller than those of Berea and the sandstones examined by Katz and Thompson. It is possible that in the low saturation regime (where the capillary pressures are greater than 100 bars, the highest capillary pressure in Melrose's experiment was 93.6 bars) the pendular structures have been totally removed and only thin films remain. If the disjoining pressure of the thin films obeys the water on quartz law, $\Pi(h) \propto h^{-1}$, then $S_{t f} \propto P_{c}^{-1}$, and if $S_{w} \simeq S_{t f}$, 
then the expected scaling law would be $P_{c} \propto S_{w}^{-1}$. This law would be consistent with Eq. (18) if $D=2$. Thus, perhaps the low saturation region of the capillary pressure curves of Figure 6 is one in which most of the wetting phase occupies thin films and the higher saturation region is one in which the wetting phase samples a fractal distribution of pore sizis.
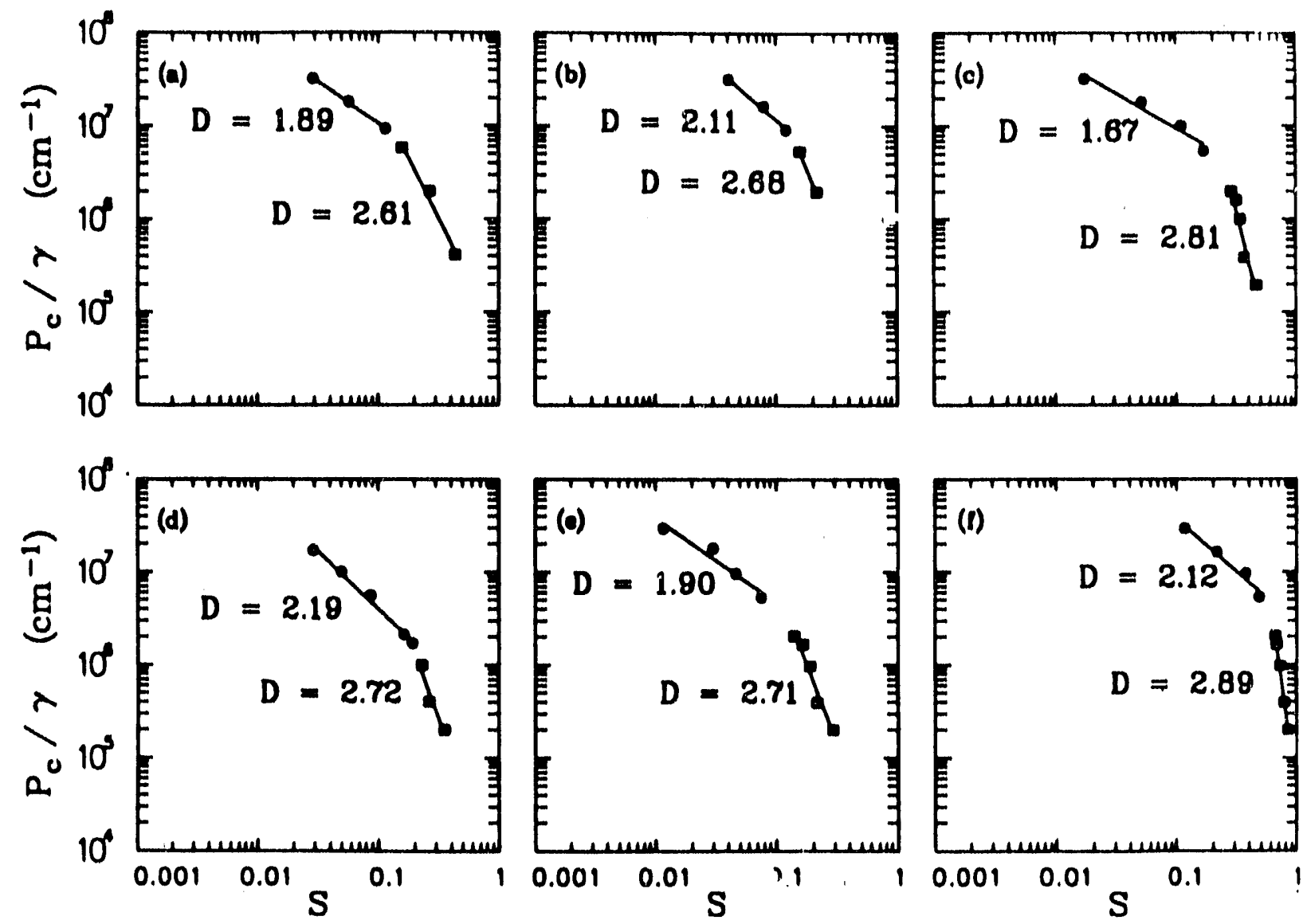

Figure 6. Capillary pressure curves for water in low permeability sandstones (data from Reference 20).

In closing, we would like to emphasize that the capillary pressure, permeability and capillary dispersivity of wetting phase at low saturations provide probes that can reveal geometric features of porespace and the nature of the disjoining forces on thin films of wetting phase. Under the assumptions that pendular structures occupy porespace of fractal dimension $D$, that the disjoining pressure obeys the power law $\Pi(h) \propto h^{-m}$, that the wetting phase inventory is primarily pendular structures, and that thin films control the hydraulic resistance of wetting phase, we deduce the power laws

$$
X \propto S_{w}^{ \pm \nu_{x}}, X=P_{c}, K_{w} \text { and } D_{c}
$$

where for capillary pressure $-\nu_{x}=1 /(3-D)$, for permeability $\nu_{x}=3 / m(3-D)$, and for capillary dispersion $\nu_{x}=[3-m(4-D)] / m(3-D)$. The experimental work analyzed above lends support to the scaling laws for $P_{c}$ and $K_{w}$ in the cases of natural sandstones 
and clayey soils. By choice of the wetting fluid the values of $m$ can be varied in the same porous sample to test the implications of the scaling laws for $K_{w}$ and $D_{c}$.

\section{References}

Bacri, J.-C., C. Leygnac and D. Salin, J. Phys. Lett. (Paris) 46, L467 (1985).

Brooks, R.H. and A.T. Corey, Hydraulic properties of porous media, Hydrology Paper No. 3, Colorado State University, Fort Collins, CO, 1964.

Campbell, G.S., Soil Sci. 117, 311 (1974).

Davis, H.T., Europhys. Lett. 8, 629 (1989).

deGennes, P.G., "Partial filling of a fractal structure by a wetting fluid," in Physics of Disordered Materials, edited by D. Adler, H. Fritzsche and S.R. Ovshinsky (Plenum Publ. Co., New York, NY) 1985, pp. 227-241.

Derjaguin, B.V., V.M. Sterov and N.V. Churaev, Kolloidnji Zhurnal 38 , 875 (1976).

Gardner, W.R. and M.S. Mayhugh, Soil Sci. Amer. Proc., 22, 197 (1958).

Gardner, W.R., UNESCO15, 37 (1960).

Gardner, W.R., D. Hillel and Y. Benyamini, Woter Resources Res, 6, 851 (1970).

Katz, A.J. and A.H. Thompson, Phys. Rev. Lett. 54, 1325 (1985).

Katz, A.J. und A.H. Thompson, Phys. Rev. Lett.56, 2112 (1986).

Melrose, J.C., "Characterization of reservoir rocks buy capillary pressure techniques," in

Characterization of Porous Solids, edited by K.F. Unger, J. Rouquerol, K.S.W. Sing and H. Kral (Elsevier Science Publishers B.V., Amsterdam) 1988, pp. 253-261.

Mohanty, K.K., H.T. Davis and L.E. Scriven, "Thin films and fluid distributions in porous media," in Surface Phenomena in Enhanced Oil Recovery, edited by D.O. Shah (Plenum Pub. Co., New York, NY) 1981, pp. 395-409.

Nimmo, J.R. and K.C. Akstin, Soil. Sci. Soc. Am. J.52, 303 (1988).

Pashley, R.M., J. Colloid Interface Sci. 78, 246 (1980).

Roberts, J.N., Phys. Rev. Lett. 56, 2111 (1986).

Sutanto, E., H.T. Davis and L.E. Scriven, "Liquid distributions in porous rock examined by cryo-scanning electron microscopy," paper SPE 20518 presented at the 1990 SPE Annual Technical Conference and Exhibition on Sept. 23-26, 1990 in New Orleans, LA.

Toledo, P.G., R.A. Novy, H.T. Davis and L.E. Scriven, Soil Sci. Soc. Am. J., 54, 673 (1990).

Viani, B.E., P.F. Low and C.B. Roth, J. Colloid Interface Sci. 96, 229 (1983) .

Ward, J.S. and N.R. Morrow, Soc. Pet. Eng. Form. Eval. September 1987, 345. 


\section{MICROSCOPIC DYNAMICS OF FLOW IN MOLECULARLY NARROW PORES}

\section{Synopsis}

Flow of fluids confined in molecularly narrow pores is studied by molecular dynamics. Strong density variations across the pore render the usual dependence of the local viscosity on local density inappropriate. At separations greater than four molecular diameters flow can be described by a simple redefinition of local viscosity. In narrower pores a dramatic increase of effec.ive viscosities is observed and is due to the inability of fluid layers to undergo the gliding motion of planar flow. This effect is partially responsibility for the strong viscosity increases observed experimentally in thin films that still maintain their fluidity.

\section{Introduction}

During the last two decades, molecular dynamics (MD) has been employed successfully for the simulation of macroscopic flows (Hoover, 1983). Recently, quite complex flows have been simulated (Marachel et al., 1987) and issues fundamental to fluid mechanics, sixch as the origin and the microscopic realization of the stick boundary conditions, were explored by MD simulations (Koplik et al.1988; Heinbuch and Fischer 1989). MD is ideally suited for the study of yet another class of flow phenomena, namely the flow of molecularly thin liquid films (and interfacial flows in general). What makes interfacial fluids fundamentally different from bulk fluids is the fact that their density varies considerably over microscopic (molecular) distances. A class of such strongly inhomogeneous fluids are fluids confined in very narrow spaces by solid barriers. In this case, the confined fluid minimizes its free energy by arranging its molecules in layers parallel to the solid walls. Despite the solid-like appearance of these layers, the medium preserves its fluidity, at least next to idealized smooth walls that lack molecular structure and, therefore, do not promote epitaxial crystallization.

Our understanding of the equilibrium properties of interfacial fluids (Nordholm et al. 1987; Fischer and Methfessel, 1980; Tarazona 1985) has advanced considerably, although much less has been accomplished towards rationalizing the effect of sharp density variations on a molecular scale on the flow and transport properties. The essence of the problem can be summarized as follows: the microscopic identification of thermal transport coefticients (Irving and Kirkwood, 1950) shows clearly that, on a microscopic scale, these quentities are nonlocal functionals of the density profile. This intrinsic nonlocality can be ignored when the density variaiions occur only on a macroscopic scale (regular fluids) but it cannot be neglected under any circumstances when the fluid density varies appreciably over molecular distances (interfacial fluids). Therefore, in the latter case, the usual constitutive equations are inappropriate and traditional hydrodynamics cannot be applied. These 
general considerations are supported by the MD results that we shall present in this paper, as well as by earlier MD simulations performed in our laboratory (Bitsanis et al.1987).

The most important finding in our earlier work (Bitsanis et al.1987) was that the flow behavior of the strongly inhomogeneous fluids simulated could be understood in terms of a local average density model (LADM). The idea is that the local transport coefficients can be approximated by the corresponding quantities of a homogeneous fluid, not at the local fluid density, but at an average fluid density inside a volume of molecular dimensions (in the work of Bitsanis et al.(1987) this volume was a sphere equal to the molecular size).

In this communication we present the result of a new, more extensive series of $M D$ simulations of strongly confined fluids undergoing simple flows. The conclusions of Bitsanis et al.(1987) are reconfirmed for all but the narrowest micropores. An important finding of this work, however, is that in films narrower than three to four fluid molecular diameters flow is dramatically different from that of bulk fluids and cannot be explained by a local average density model. As we shall discuss later, we think that this fundamentally different behavior originates from the inability of the molecules of the ultrathin film to undergo the types of systematic movements that would realize flow.

We simulated liquids consisting of spherically symmetric molecules interacting via pairwise additive forces of the Lennard-Jones (LJ) type. The fluids in our simulations were confined between planar, parallel 10-4-3 LJ solid walls. It should be emphasized that the atoms of the solid walls are smeared out, i.e., our walls lack the atomic roughness of actual solid surfaces. The smoothness of our walls suppresses the development of lateral fluid density variations, preserves the film's fluidity by ruling out the possibility of epitaxial crystallization and, consequently, does not enable us to answer the question of the origin of stick boundary conditions. Nevertheless, by eliminating all the above factors, our modeling can focus on one aspect of ultrathin film fluid rheology and transport, namely the effect of the sharp density variations over molecular distances on the molecular mechanisms of transport and on the "macroscopic" obserables of the film's flow and transport behavior.

All confined fluid films simulated had the same chemical potential. This was achieved by choosing the same temperature, pore average density and well potential as in the grand canonical ensemble Monte Carlo simulations of Snook and van Megen (1980).

We studied plane, simple shear (Couette) and pressure driven (Poiseuille) flows, employing the "reservoir" method of nonequilibrum (Ashurst and Hoover 1975). The application of this method to Conette flow simulations of micropore fluids was presented in Bitsanis et al.(1987). A straightforward modification of the original "reservoir" method, which consisted in the application of a uniform force field on the film's molecules while keeping the reservoirs stationary, was employed in the isothermal, Poiseuille flow simulations. Numerous tests on the reliability of the "reservoir" method, as well as the reasons for the choice of this particular method for isothermal flow simulations of strongly inhomogeneous fluids and the absence of any nonlinear effects because of the high shear rates 
present, are discussed in Bitsanis et al.(1987). Here we simply mention that the fluid-like reservoirs employed in our simulations are merely a means of imparting momentum and, therefore, of inducing flow in a fluid that has developed strong density variations because of the 10-4-3 potential, i.e., the smooth solid walls. Obviously, such smooth walls are incapable of driving flow; they would simply let the fluid film slide past them without shear.

Typically, the duration of our simulations was 50,000-100,000 time steps (which corresponds to $0.5-1.0 \mathrm{nsec}$ if the $\mathrm{LJ}$ parameters are given the Ar values). In what follows, all quantities are measured in a system of units with the LJ parameters as units of length and energy and the mass of the fluid molecule as unit mass. In these units, our time steps $0.00462\left(10^{-14} \mathrm{~s}\right.$ for $\left.\mathrm{Ar}\right)$ and the temperature and density of the bulk fluid that the micropore fluid were in equilibrium with was 1.2 and 0.5925 .

\section{Results and discussion}

A sample density profile determined from our simulations is shown in Fig. 1, for the pore width of 4.0. Since the zero of the solid-wall potential is placed at the center of mass of the fictitious solid particles (which have the same size as the fluid particles), the regions within a distance of 0.8 from the solid walls are depleted of fluid particles.

For all systems simulated, and for both rypes of flow, the density profiles under flow were identical with the equilibrium density profiles. This confirms an important conclusion, first drawn in Bitsanis et al.(1987), namely that the presence of flow seems to have no effect on the density profile even at the extremely high shear rates present in our simulations $\left(\approx 10^{10} s^{-1}\right)$. The insensitivity of the density profile on flow has considerable theoretical implication since it allows the decoupling of the problems of density profile determination and flow description.(Bitsanis et al., 1987; Bitsanis et al., 1988).

The Couette and Poiseuille flow velocity profiles of the same system are also shown in Fig. 1. These velocity profiles are different from those of a homogeneous fluid (linear for Couette flow and parabolic for Poiseuille flow). In both cases, the major deviation from the shape of the homogeneous fluid velocity profile is their flatness inside the regions occupied by the two outermost fluid monolayers. Effectively, the two outermost monolayers behave as a fluid of very high viscosity. This confirms the rough correlation between density and velocity profiles observed in Bitsanis et al(1987). Nevertheless, the deviations of the velocity profiles from the linear or parabolic shape are much weaker than those expected by usual hydrodynamics. According to usual hydrodynamics, the local viscosity is a point function of the local density. In view of the extreme density variations across the pore, such an approach would result in much stronger deviations of the velocity profiles from the linear or the parabolic shape (Bitsanis, et al., 1988). Despite their very viscous character, the two outermost monolayers remain fluid-like. This was confirmed by examining the radial distribution function and the mobilities (in all directions) of the molecules inside 


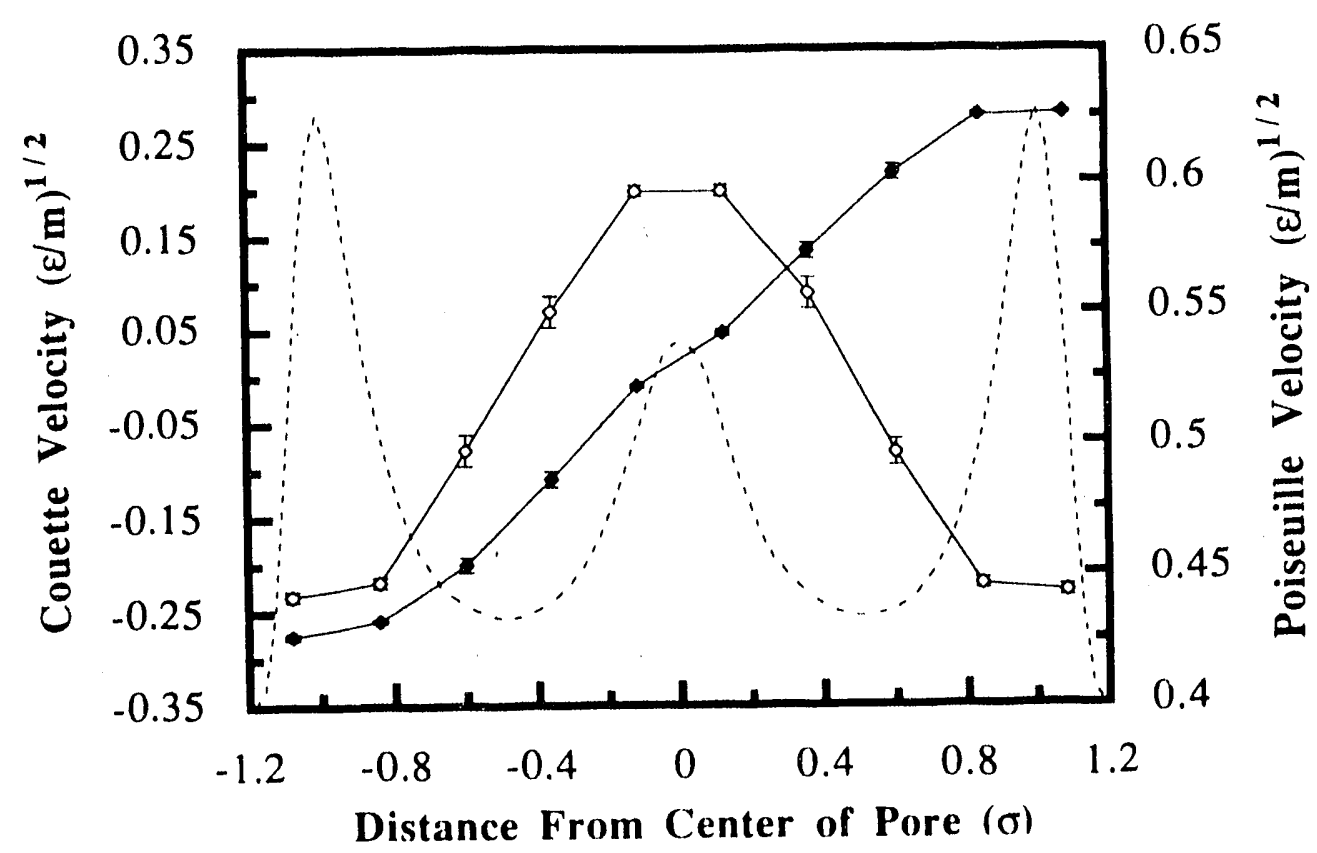

Figure 1. Density profile ( - - ) and Couette $(\bullet)$ and Poiseuille $(\diamond)$ flow velocity profiles for a pore of width $h=4.0 \sigma$. The velocity profile for Poiseuille flow is largest in the center of the pore. $\sigma$ is the diameter of a fluid molecule. Density and velocity are in units of $\sigma^{-3}$ and $(\epsilon / m)^{1 / 2}$. The pore average density of the fluid is 0.876 at this separation. Reservoir molecules are the same as fluid molecules and are at a density of 0.715 .

these layers.

It must be emphasized that slip was present in all our simulations. We do not draw any conclusions from this, since the coupling between fluid and the artificial reservoir walls is not representative of that with a realistic wall. The reservoir corresponding to Fig. 1 and 3 had particles identical to the fluid particles. Figure 2 reports results for a reservoir of molecules 20 times heavier than but otherwise identical to the fluid molecules. The heavier reservoir molecules reduce the slip. The shape of the velocity profile, however, is independent of the amount of slip and, therefore, reflects genuine flow features of the layered fluid.

For a fluid with variable density the effective viscosity is not a fluid property, but it depends on the type of flow as well. This is a consequence of simple hydrodynamics (any hydrodynamics for that matter), and it is equally true both for regular inhomogeneous fluids (density varies over macroscopic distances) and for interfacial fluids (density varies over molecular distances) (Bitsanis, et al.1988). For a particular type of fow, the effective viscosity is defined as the viscosity of a fictitious homogeneous fluid that would result in the same value of a certain macroscopic observable of the flow. If the macroscopic observable is selected in a sensible way the effective viscosity should be a good collective measure of 


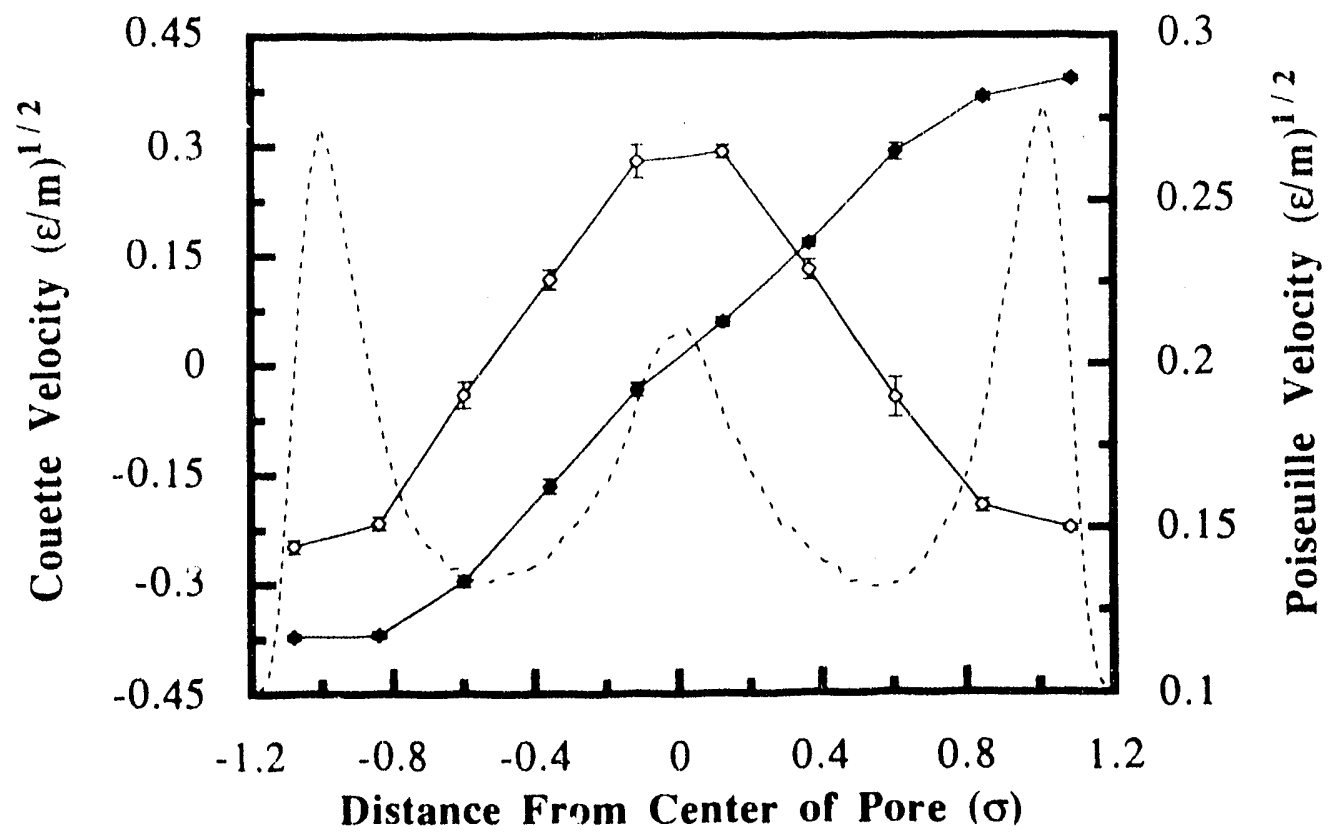

Figure 2. The same as in Fig. 1, except the reservoir molecules are twenty times heavier than the fluid molecules and are at a density 0.873 .

the inhomogeneous medium's resistance to flow.

The obvious macroscopic observable for the definition of the effective viscosity for Couette flow is the force on the wall or, equivalently, the shear stress which is constant throughout the fluid slab. Thus, the effective viscosity is given by

$$
\eta_{\text {eff }}^{c}=\frac{\tau_{z x}}{[v(0.8)-v(h-0.8)] /(h-1.6)}
$$

where $h$ is the pore width. All the quantities in Eq. (1) are determined directly from the simulation.

For Poiseuille flow, a proper macroscopic observable is the mass flow rate, so for the definition of viscosity we choose

$$
\eta_{e f f}^{p}=\frac{\rho_{\text {avg }}^{2} g(h-1.6)^{3}}{12 \int_{0.8}^{h-0.8} \rho(z) v(z) d z}
$$

where the pore average density is defined as:

$$
\rho_{a v g}=\frac{\int_{0.8}^{h-0.8} \rho(z) d z}{h-1.6}
$$




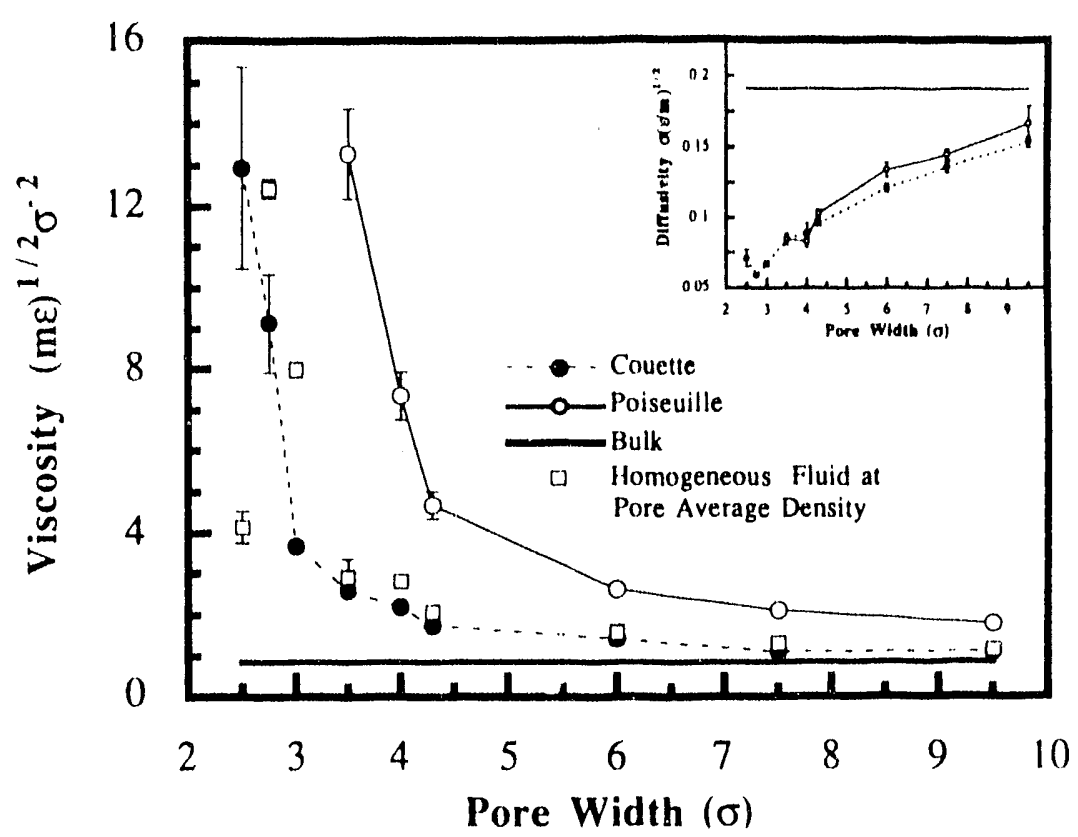

Figure 3. Effective viscosities in units of $(m \epsilon)^{1 / 2} / \sigma^{2}$ for Couette Poiseuille flows versus pore width in units of the fluid particle diameter. the viscosity of homogeneous fluid at the pore average density is also shown. Self diffusion coefficients in units of $\sigma(\epsilon / m)^{1 / 2}$ were computed from the velocity autocorrelation for diffusion parallel to the pore walls. The results are shown in the insert in the figure.

In the above equations, $v(z)$ is the flow velocity at a distance $z$ from the lower wall, $\tau_{z x}$ is the $z x$ component of the stress tensor, $h$ is the pore width, $\rho(z)$ the fluid density at $z$ and $g$ the pressure gradient that induces the Foiseuille flow. Note that for the definition of the effective viscosities an "effective pore width" $h-1.6$ has been used instead of $h$. There is some arbitrariness in this but we think that this is the correct quantity to use since there are no fluid particles within 0.8 of the origin of the wall potential (Fig. 1). For Poiseuille flow another effective viscosity can be defined in terms of the volumetric, rather than mass, flow rate. Obviously, the values of the two effective viscosities are not identical. Nevertheless, the volumetric flow and the mass flow rate effective viscosities exhibit a very sinilar dependence on the pore width which suggests that either is a physically meaningful quartitative measure of the fluid film's resistance to flow.

The Couette and Poiseuille flow effective viscosities determined from our simulations are plutted in Fig. 3. Since the effective viscosity of (both weakly and strongly) inhomogeneous fluids is a flow-dependent quantity. (Bitsanis, ot al.1988), it is no surprise that the effective viscosities of the same fluids are different for the two different flows.

Clearly, the most striking feature of the effective viscosity (Fig. 2) is the dramatic increase of both effective viscosities at very small pore widths (less than 4.0 for Poiseuille flow and less than 3.0 for Couette flow). This demonstrates the profoundly different nature 
of flow in ultrathin fluid films.

At larger pore widths the effective viscosities differ moderately from those of a homogeneous fluid with density equal to the pore average density. The Local Average Density Nodel (LADM) first presented in Reference 9, accounts for these differences very successfully. LADM, however, fails to predict the strong viscosity increase in ultrathin (less than $3.0-4.0)$ liquid filnss.

As we explained in section 1 , the constancy of the chemical potential leads to a variation of the pore average density with pore width. The overall trend is an increase of the pore average density with decreasing pore width. This will result in an increase of the effective viscosity, which has nothing to do with the layered fluid structure and is merely a reflection of the average density rise. Such an effect, although present, cannot account for the observed explosive increase of the effective viscosities, as shown in Fig. 3. The viscosities of the homogeneous fluids at the pore average density, which were determined by MD, increase much more slowly than the inhomogeneous fluid effective viscosities at small separations. Furthermore, the homogeneous fluid viscosity at very small separations oscillates with pore width following the oscillations of the pore average clensity. Hence, the dramatic increase of the inhomogeneous fluid effective viscosities is a result of fluid layering and not a consequence of the average density rise.

Despite the layering and the extreme resistance to flow, our systems retain their fluidity up ts smallest separations examin $d$. As a measure of fluidity, we have computed the self-diffusion coefficient from the velocity autocorrelation function in the direction parallel to the walls and perpendicular to the flow (for Couette flow the same result was found from the velocity autocorrelation function the direction of flow). The diffusivities decrease moderately with decreasing pore width but they retain liquid-like values at all pore wides (Fig. 3). Again, we would like to point out that the preservation of the filn's fluidity may be related to the smoothness of the solid walls. Structured solid surfaces can sometimes (not always) induce freezing (crystallization) of the thin film (Schoen, et 4.1.1988). We calculated, but do not report, diffusivities in the absence of flow. They agreed with those found in Conette flow to within the variances shown in Fig. 3. The higher values obtained for Poiseuille flow arise from the slightly higher temperature (abont nine percent higher) resulting in this flow.

Up to this point we have established that the observed increase of the effective viscosities observed in our simulations cannot be attributed to the increase of the pore average density to to some loss of fluidity (solidification). Irstead, as illustrated schematically in Fig. 4, this phenomenon is essentially an excluded volune effect on the glide of molecular layer required in a shearing flow. Even thongh the effective viscosity rises sharply with decreasing wall separation, the self-cliffusion coefficient by contrast decreases slowly (Fig. 3). Diffusive motion depends only on fluidity to individual molecular movement, not glide of an entire layer. 


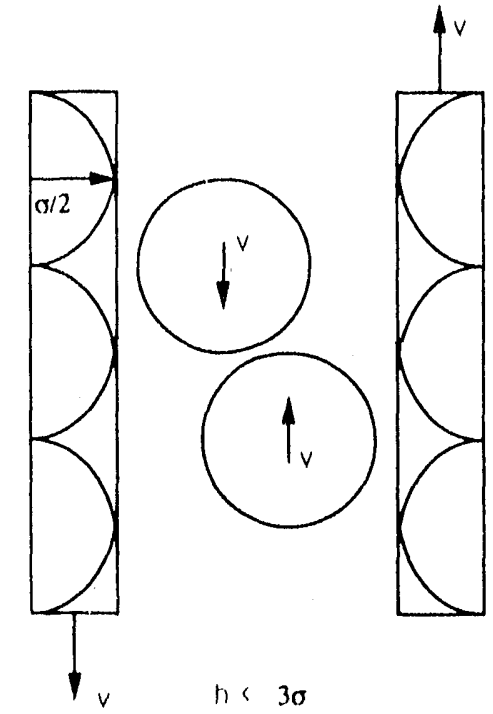

(a)

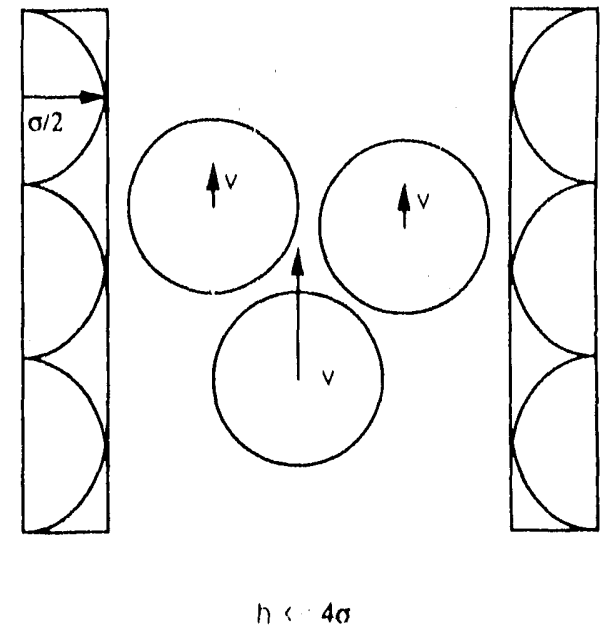

(b)

Figure 4. Schematic molecular explanation of the observed viscosity increase for both flows a) Conette flow; b) Poisenille flow. The wall molecules are smeared out in our simulations. They are drawn in the Figure only to indicate up to what distance from the zero of the wall potential (center of mass of solid molecules before they were smeared) fluid particles can approach.

To pursue the effect of molecular size on flow, consider Couette flow in fluid confined belween two 10-4-3 Lennard-Jones walls, less than 3 fluid molecular diameters apart (Fig. 4a). The space available to the fluid molecules is not sufficient to accommodate two ordered fluid monolayers. The layer of fluid molecules close to the left wall will have a tendency to move in the opposite direction of the layer close to the right wall. Because of the limited space, this requires a squeezing of the fluid molecules which is hardly possible due to the strength of the intermolecular repulsive forces. Such a situation will clearly result in a sharp increase of the Couette flow effective viscosity for $h<3.0$, as is actually observed in our simulations (Fig. 3). A similar situation arises for Poiseuille flow but now three fluid layers are needed for the realization of Poiseuille flow (Fig. 4b) (e.g. the middle layer must now glide between the two outer ones with a systematically higher velocity than that of the two outermost layers). This will lead to a similar increase of the Poiseuille flow effective viscosity for $h<4.0$, again in agreement with the simulation results. Of course, particle 1 (Fig. 4a) can certainly pass particle 2 by means of their thermal (random) motion. This, however, would require a temporary exchange of their layer identities. It will not produce flow since it will contribute to the the systematic momentum transfer which accompanies flow.

Finally, we would like to commment on the relation between our simulation findings and several measurements of the rheological response of ultrathin liquid films that have been carried out in the last four to five years (Chan, et al.1985; Israelachvili and McGuig- 
gan 1988; Van Alsten and Granick, 1988). These studies determined that the continuum description of the thin film breaks down for films thinner than approximately ten molecular diameters. Of course the origin of this breakdown of continuum mechanics is the development of fluid layering, a manifestation of the molecular nature of the fluid when the film thickness becomes molecularly small. Our current simulations (and those of Reference 9) reveal how these initial deviations from continuum hydrodynamics occur. The fluid film now has a variable density and the ability of molecules to move or exchange momentum varies from point to point. These effects can be quantified adequately by means of properly defined local transport coefficients. The frequency of the collisions experienced by a molecule with its center of mass at a position $\mathbf{r}$ from the wall is related to the average density in the neighborhood of $\mathbf{r}$ and not with the density exactly at $\mathbf{r}$. This is the un. derlying idea of LADM, and this is the mechanism by which the strong density variations effect transport and flow properties.

Before attempting a more cletailed comparison with the experimental observations one has to acknowledge the very significant differences between our molecular model and the systems studied experimentally. First of all, it should be noted that the experiments were carried out at constant load (i.e., constant normal stress) whereas our computer simulations were carried out at fixed pore widths and with average pore densities set by the constant chemical potential simulations of Snook and van Megen (1980). Perhaps the most important difference between experiment and simulation is the lack of atomic roughness of our 10-4-3 walls. As mentioned farlicr, atomically structured walls can promote cpitaxial crystallization (Schoen, et al.1988) and they are responsible for the rich behavior that has been observed in molecular simulations (Schoen, et al.1989). It is very likely that these effects are partially responsible for the rheological behavior observed experimentally, and more specifically for the solid-like, elastic response seen in films one to three (or five, depending on the fluid) molecular diameters wide. Our simulations cannot explain (and they were not designed to explain) the solid-like response observed experimentally. Instead, our aim was to focus on the viscous response of a strongly inhomogeneous fluid that is gluaranteed (by the wall smoothness) to maintain its fluidity.

Slightly wider films (three or five to six or ten molecular diameters, depending on the material) were found experimentally to exhibit a viscous (fluid-like) behavior but with an apparent viscosity much higher than the bulk viscosity. We think that this behavior is similar to the sharp increase of the viscosity observed in our simulations and that the physical origin of the phenomenon is basically the same in both cases (the inability of the layers to glide past each other). Nevertheless, significant differences still exist. First of all, the viscosity rise was observed experimentally in significantly thicker films, and it was not as stcep as in our simulations. Speculating on the origin of these differences, we note that the presence of one (or two) epitaxially crystallized fluid layers (a possibility that cannot be dismissed in the experiments) would effectively decease the wicl th of the flowing thin film by two (or four) molecular diameters. Furthermore, the chain molecules that were mostly studied experimentally cannot simply pack their monomers on top of each other for obvious steric reasons. This implies that a film, say six monomer dianeters 
thick, is not likely to have six monomers layers but rather a smaller number of more diffuse, more complex and wider periodic structures ("layers"). A decrease in separation will be accompanied by a gradual change in shape of the interior monomer "layers", and it will result in a smoother viscosity rise as the chains arlopt more and more deformed configurations in order to accommodate their monomers in the confined space.

Furthermore, our simple LJ particles cannot model hydrocarbons, but they are reasonable representations of fluids of more compact molecules (e.g. OMCTS and cyclohexane). The high shear rates employed in our simulations may have important consequences (melting of epitaxial layers), but only if epitaxy (or crystallization) is indeed a possibility. As we have mentioned several times up to now, phenomena like the above simply do not take place next to smooth walls.

As a further consideration, a pressure increase at the separations slightly larger than 3 for Couette flow or 4 for Poiseuille might diminish the already limited available free volume which will make the layer gliding from marginally possible to virtually impossible. This might be related to the extreme pressure sensitivity of the viscosity observed in Reference 16.

\section{Summary}

Summarizing, the strongly layered nature of ultrathin liquid films confined between solid walls results in a flow behavior profoundly different from that of homogeneous fluids. The differences originate directly from the fluid layering and manifest the inability of fluid layers consisting of finite volume particles to the collective gliding plane motion necessary for the realization of flow. The segregation of fluid molecules into layers, and the resulting dynamic restrictions described above are, we believe, one of the major causes of the dramatic viscosity increases seen experimentally (Van Alsten and Granick 1988; and Schoen et al.1989) in thin films that still exhibit a viscous (fluid-like) response.

\section{References}

Ashurst, W.T., W.G. Hoover, Phys. Rev. A 11, 658 (1975).

Bitsanis, I., J.J. Magda, M. Tirrell, H.T. Davis, J. Chem. Phys. 87, 1733 (1987).

Bitsanis, I., T.K. Vanderlick, M. Tirrell, H.T. Davis, J. Chem. Phys. 89, 3152 (1988).

Chan, D.Y.C. , R.G. Horn, J. Chem. Phys. 835311 (1985).

Fischer, J., M. Methfessel, Phys. Rev. A 22, 2836 (1980).

Hoover, W.G., Ann. Rev. Phys. Chein. 34, 103 (1933).

Irving, J.H., J.G. Kirkwood, J. Chem. Phys. 18, 817 (1950).

Israelachvili, J.N., P.M. McGuiggan, A.M. Homola, Science 241, 795 (1988).

Koplik, J., J.R. Banavar, J.F. Willemsen, Phys. Rev. Lett. 60, 1282 (1988).

Maraschal, M., E. Kestemont, Nature 329, 427 (1987); D. Heyes, Nature 329, 390 (1087).

Nordholm, S., M. Johnson, B.C. Freasier, Aust. J. Chem.'33, 2139 (1980). 
Shoen, M., J.H. Cushman, D.J. Diestler, C.L. Rhykaerd Jr., J. Chem. Phys, 88, 1394 (1988).

Shoen, M., C.L. Rhykaerd Jr., D.J. Diestler, J.H. Cushman, Science 245, 1223 (1989),

Snook, I.K., W. van Megen, J. Chem. Phys, 72, 2907 (1980).

Bitsanis, I., Some:s, S.A., Davis, H.T., M. Tirrell, J. Chem. Phys. 93, 3427 (1090).

Tarazona, P., Pliys. Rev. A 31, 2072 (1985).

Van Alsten, J. and S. Granick, Phys, Rev, Lett, 61, 2570 (1988). 


\section{MOLECULAR DYNAMICS STUDY OF THE PRIMITIVE MODEL OF 1-3 ELECTROLYTE SOLUTIONS}

\section{Synopsis}

Molecular dynamics simulations at constant temperature have been carried ont for the primitive model of $1-3$ electrolyte solutions. 'Thermodynamics, pair distribution functions, and self-diffusion coefficients were computed to examine the electrostatic effects on the structural and dynamical properties. The simulation results were used to evaluate various theoretical equations, namely, the exponential form of Debye-Huckel theory, the mean spherical approximation, and the hypernetted chain approximation. As has been observed for symmetrical electrolytes, the latter turns out to be the best approximation. For asymmetrically charged 1-3 electrolytes, it wis found that ionic aggregation significantly influenced the dynamical properties of electrolytes. Coherent motion between highly charged negative ions and positive ions surrounding them was deduced from the time dependence of the velocity autocorrelation functions, particularly at concentrations between 0.4 and 4 total molar.

\section{Introduction}

The wettability of reservoir porespace determines how oil and water are distributed in oil bearing reservoirs and affects the displacement of oil in a recovery process. Wettability, in turn, is affected by adsorption and double layer forces at the fluid-solid surfaces of the reservoir. Understanding adsorption and double layer forces at the molecular level will enhance our ability to design enhanced oil recovery formulations that reduce unwanted wettability behavior or to modify the wettability in a beneficial way.

During the last couple of decades much progress has been made in our understanding and interpretation of the physical properties of electrolyte solutions. A number of different theoretical approximations have been used to evaluate the thermodynamic and structural properties of bulk electrolytes. One of the simplest but most commonly used models in these theoretical approaches is the so-called "primitive model electrolyte solution" (Outhwaite 1975; Friedman and Dale 1977) in which the charged hard-sphere ions are immersed in a continuum solvent represented only as a uniform dielectric constant of the medium.

Reliable and unambiguous results, in turn, have become increasingly necessary to eliminate any underlying uncertainties involved in theoretical approximations. However, the present level of modeling, particularly the use of a solvent continuum assumption, is far too crude to allow the direct comparison with real laboratory experiment. Consequently, machine experiments (computer simulations), which provide essentially "exact" experimental data for precisely defined model systems, have proven to be an extremely useful diagnostic tool to investigate such systems. The great advantage of computer simulations over experiment lies in the possibility of obtaining detailed physical information, which may be very difficult or impossible to realize in the laboratory. The best known example is the measuroment of pair distribution functions as a function of distance at the molecular level. 
There are in general two classes of computer experiment: stochastic Monte Carlo (MC) and deterministic molecular dynamics (MD) simulations. In the MD calculations, the actual trajectories of molecules are evaluated by the numerical integration of Newton's equations of motion. In addition to static equilibrium properties, time-dependent transport properties can be also obtained by the MD method. For the system of primitive model electrolytes, however, the discontinuous nature of hard-sphere repulsions combined with continuous electrostatic interactions introduces some technical difficulties into the traditional MD algorithm. Recently, implementations have been made to extend the MD method to the system of hardcores with soft attractive potentials. In the work of Heyes (1982) for the restricted primitive model of 1:1 electrolytes, two different forms of MD were combined. The hard-sphere collisions were allowed to take place while the forces and velocity changes due to continuous electrostatic interactions were evaluated. The agreement with the previous $\mathrm{MC}$ calculations was excellent even at higher concentrations.

Almost all simulations for the primitive electrolytes have been carried out using the MC simulation method (Valleau, 1970, 1980, ; van Megen and Snook 1990; Rogde and Hafskjold 1983; Ciccariello and Gazzillo 1983). Much of the earlier work on the MC calculations in a variety of different ensembles has been summarized by Levesque et al (1984). Valleau and his collaborators $(1970,1980)$ have extensively reported the canonical and the grand canonical MC results of such systems. In their studies, various theoretical approaches were also discussed and compared with the results obtained from their MC simulations. They adopted the minimum image convention to evaluate the Coulombic part of potentials for each configuration, and the resulting energy was linearly extrapolated as a function of $1 / \mathrm{N}$ to estimate the values of an infinite system. These data should be accepted with some care because the method with as few as 200 ions the method could yield results depending on system size. This is particularly true for higher concentrations and for higher charged systems. Explicit results for osmotic pressure were not published in the previous works.

In the MD simulations, better statistics can be achieved. For instance, in order to calculate the virial contribution to the equation of state for the hard-core system, the MC computations require an accurate estimation of pair distribution functions at the contact point (Freasier, 1980). For the system of ionic solutions, pair functions may change rapidly near contact distances due to the formation of ionic aggregates (see Fig. 4a). The extrapolation of pair functions to the contact value lead to large uncertainties in this case of ionic solutions. For this reason the MC results for osmotic pressure coefficients are known to be less certain than those for energy calculations.

In the present paper, we report computer simulation results for the asymmetrically charged system of 1-3 electrolyte solutions via molecular dynamics simulation techniques. This asymmetric system is of special interest because it provides a strong test of the applicability of approximate theories available in the literature. Computational details of the method employed here are discussed in the next section. In section 3, the thermodynamic and structural properties obtained from our MD simulations are compared with various theoretical predictions, namely, the exponential form of Debye-Huckel theory (Valleau, et al. 1970,1980: the mean spherical approximation (Waisman and Lebowitz, 1972), and the hypernetted chain approximation (Rasaiah and Friedman, 1968; Rasaiah, 1972). We also report 
in this section the dynamical properties including the velocity autocorrelation functions and the self-diffusion coefficients. The dynamical properties of the primitive model electrolytes are of course not comparable to those of electrolyte solutions since solvent dynamics are totally absent in this model. However, the results are an inexpensive by-product of the simulation and are interesting to the extent that they yield insight in to the interplay between short range repulsive and long range forces and provide an indication of the distribution of ion cluster formation.

\section{Model and Computations}

The MD calculations were carried out with a system containing 216 charged hard-spheres with equal diameter, $d$, of $0.425 \mathrm{~nm}$ and mass, $\mathrm{m}$, of 100 a.m.u. (There are 162 cations with charge +1 and 54 anions with charge -3.) Usual periodic boundary conditions were applied in a cubic fundamental cell to approximate an infinite system.

The pair potential energy between ions $i$ and $j$ is (in S.I. units)

$$
U_{i j}(r)= \begin{cases}\infty & \text { if } x<d \\ \frac{z_{i} z_{j} q^{2}}{4 \pi \epsilon_{0} \epsilon_{r} r} & \text { if } x>d\end{cases}
$$

where $z_{i}$ and $z_{j}$ are the valence of ion $i$ and $j$, and $q$ is the unit of electronic charge. $\epsilon_{r}$ is the uniform dielectric constant of the medium relative to the permittivity of free space, $\epsilon_{0}$. As used in most other studies, the relative dielectric constant, $\epsilon_{r}$, was chosen to be 78.356 corresponding to those for water at room temperature, $298.16 \mathrm{~K}$.

The long-ranged interactions in ionic systems give a configurational energy that converges slowly with the increasing system size. Use of a spherical cut-off or a minimum image convention has shown to be inappropriate, particularly for highly charged dense systems (Adams, 1983; Adams and Debye, 1987). 'The resulting configurational energy for such systems must take account for the ion pairs not only with the nearest images in a fundamental cell but also with all images in other periodic cells. The Coulombic potentials or forces, in this study, were calculated using the Ewald sum technique (1921), which is a well-known method for evaluating the electrostatic interactions in ionic crystals.

The Ewald transformation is expressed by two convergent sums. One in real space of a short-ranged potential, $U_{\mathbf{n}}$,

$$
U_{\mathbf{n}}=\frac{1}{2} \sum_{i=1}^{N} \sum_{j=1}^{N} \sum_{\mathbf{n}}^{1} \frac{z_{i} z_{j} q^{2}}{4 \pi \epsilon_{o} \epsilon_{r}} \frac{\operatorname{erfc}\left(\alpha r_{i j_{\mathbf{n}}}\right)}{r_{i j_{\mathbf{n}}}}
$$

and the other in reciprocal lattice space of periodic Fourier domains, $U_{\mathbf{h}}$,

$$
U_{\mathrm{h}}=\frac{1}{2 \pi L} \sum_{i=1}^{N} \sum_{j=1}^{N} \sum_{\mathrm{h} \neq 0} \frac{z_{i} z_{j} q^{2}}{4 \pi \epsilon_{o} \epsilon_{r}} \exp \left(-\frac{\pi^{2} \mathbf{h}^{2}}{\alpha^{2} L^{2}}\right) / \mathbf{h}^{2} \cos \left(\frac{2 \pi}{L} \mathbf{h} \cdot \mathbf{r}_{i j}\right)
$$

where $L$ is the box length, $\alpha$ is an arbitrary parameter, typically set to $5 / L$, and $e r f c$ is the complementary error function. Note that $\mathbf{h}$ is a reciprocal wave vector in units such that its 
components are integers. In the latter case of reciprocal space, the order of computations can be reduced by the method suggested by Singer and described in Ref. [18]. In this method, the double sum over particles $i$ and $j$ is simplified into two single sums over particle $i$. Total of 6.8 wave vectors were computed using a recurrence relationship of complex arithmetic to avoid repeated calculations.'

The system of a hard-core repulsion with continuous attractive interactions gives rise to problems for MD computer simulations. Computational techniques for trajectory calculations are totally different between the discontinuous and the continuous MD method. In the hard-core MD program , troduced in the pioneering work of Alder and Wainwright (1959), th.: collision equations between all possible colliding pairs must be solved before advancing their trajectories. In contrast, in the MD for continuous potentials, the time evolution of phase space is followed by solvire the equations of motion suitably discretized in time.

Two distinct algorithms can be combined into the same MD program by returning to the hybrid "step-by-step" approach described elsewhere (Heyes, 1982; McNeil and Madden, 1982, Heyes and Woodcock, 1986). The time step interval is of urder of femto seconds which is very small compared to the average time between hard-sphere collisions. The first step is identical to the system of conventional continuous potentials. The system trajectories are adiaced from the current positions to the next positions only under the influence of continuous forces without imposing the hard-core constraints. In this step, any possible hard-core collision occurring during a fixed time interval is ignored, and this may result in unrealistic hard-core penetration. The next step is then to check whether or not the pair distances are closer than a hard-sphere contact diameter, and the resulting configuration is resolved for the overlapping pairs. Because the time interval is so small, the majority of particles proceed without colliding with each outher. When an overlap is found in a time step, the step is repeated, but this time the particle volocities are assumed to be constant. Under this assumption, the particles are advanced until the overlapping pair collides and the algebraic equations of colliding hard-spheres are used to find the postcollision positions and velocities. The algorithm then returns to the continuous potential MD sequence. This apprcach has been applied for the various systems of model fluids and did not show any measırable inaccuracy for the thermodynamic and transport properties of such systems (Heyes, 1982; McNeil and Madden, 1982, Heyes and Woodcock, 1986). A logic flow chart for this MD method is illustrated in Figure 1.

For the computational efficiency, it is tppropriate to eliminate any redundant calculations and this can be done by the construction of the collider table to speed up the searching routine. Before entering the force loop in the first step, the maximum velocity was scanr ${ }^{\circ} \mathrm{d}$ to decide the maximum cut-off distance for the collider table. The table was updated at ei: $h$ step to ensure possible colliding pairs. Only the pairs within this maximum distance were considered to examine the colliding pairs in the next step, rather than all possible pairs.

The equations of motion were integrated using the leapfrog version of Verlet algorithm (1967), and the velocities were scaled at $e . c h$ time step to maintain constant temperature in the manner described by Berendsen et al. (1984) In addition, the initial configuations were generated by randomly irserting particles to assist in the equilibration of the system. 
Step 1: Evaluate new positions under the influence of continuous forces only.

Step 2 : Examine the resulting configuration for overlaps. The next steps 3 to 5 are executed for overlapping pairs.

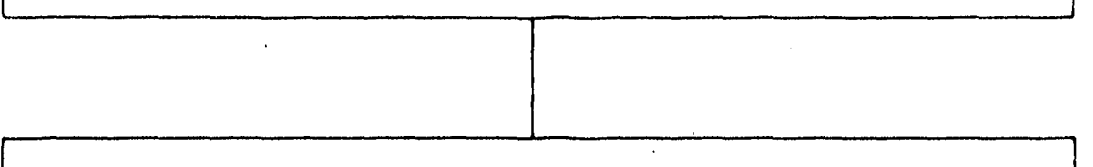

Step 3 : Determine the hard-core collision times.

Step 4 : Scan the minimum collision time and advance particles during this time.

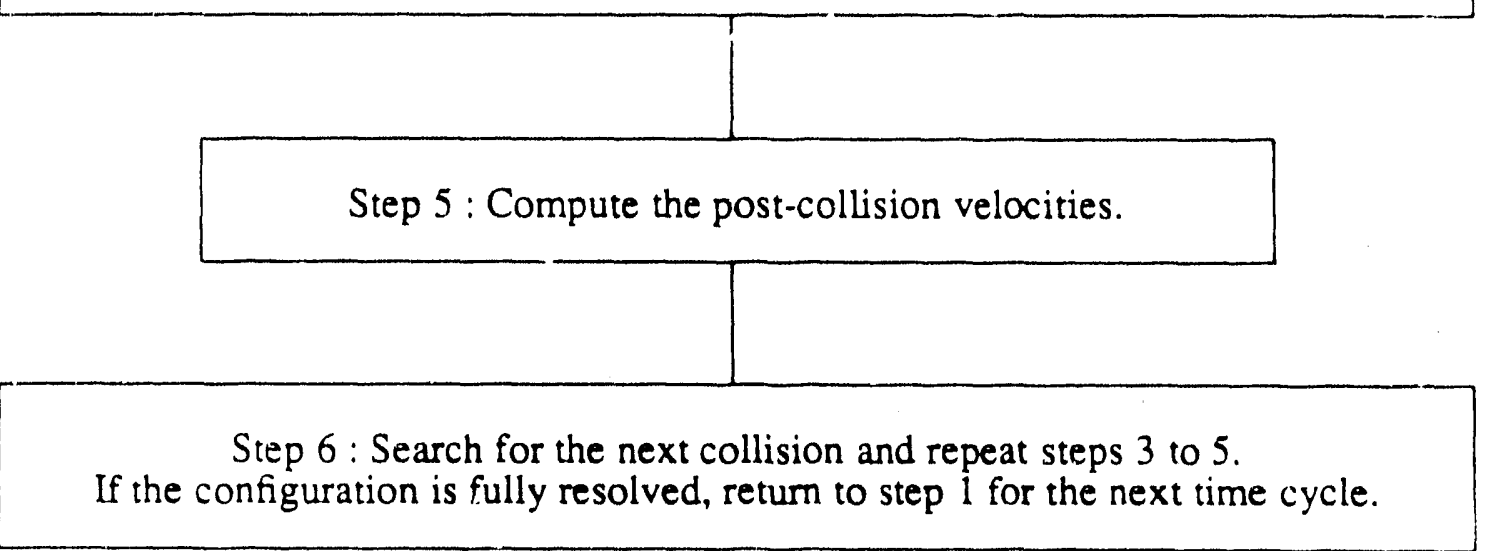

Figure 1. Schernatic of the molecular drisamics proceduie.

Configurations were aged, or equilibrated, for 6,000 steps before accumulating data and the resulting ensemble averages were obtained during the final 40,000 steps. The MD algorithm implemented here has been tested in a number of ways. When the ionic charges were assigned to a value of zero, the results faithfully reproduced the thermodynamic and transport properties of the pure hard-sphere system available in the literature (Alder et al. , 1970). The results obtained from the MD simulations for a few selected runs of $1-1$ electrolytes were also compared to the MC (Valleau, et al 1970; 1980) and MD (Heyes, 1982Ileyes, 1982 The good arreement with the previous data again confirmed the quaiity of our MD method. $A_{1} l l$ production calculations were performed on a Cray-2 supercomputer at Minnesota Supercomputer (enter. Extensive use was made of vectorization and optimization. 


\section{Results and Discussion}

The thermodynamic and transport properties of 1-3 electrolyte solutions obtained from the MD simulations are presented in Table 1 . The range of concentration investigated here was from $0.02 \mathrm{Mt}$ to $6 \mathrm{Mt}$, where $\mathrm{Mt}$ is the total ion concentration in units of mole per liter, but not the stoichiometric concentration, $M s$. (Note that $M t=4 M s=21.6318 n d^{3}$, where $n d^{3}$ is the total number density.) The self-diffusion coefficients were calculated from the integration of the velocity autocorrelation function using the Green-Kubo relations and from the slope of the mean-square displacement versus time using the Einstein equations. The two methods gave results in good agreement, typically less than $3 \%$ difference, and the self-diffusion coefficients in Table 1 are averages of the values found by the two methods. In this table, we also report the ion/ion collision frequencies in columns 7 to 9 .

Table 1. System characteristics and MD results for 1-3 Primitive Model Electrolytes

Table 1. System Characteristics and MD Results for 1-3 Primitive Model Electrolytes

\begin{tabular}{cccccccc}
\hline $\begin{array}{c}M t \\
(\mathrm{moles} / \mathrm{l})\end{array}$ & $-U / N k T$ & $P V / N k^{\prime} \Gamma$ & $\begin{array}{c}D_{+} \\
\left(10^{-4} \mathrm{~cm}^{2} / \mathrm{s}\right)\end{array}$ & $\begin{array}{c}D_{-} \\
\left(10^{-4} \mathrm{~cm}^{2} / \mathrm{s}\right)\end{array}$ & $\begin{array}{c}\omega_{++} \\
\left(10^{10} \mathrm{~s}^{-1}\right)\end{array}$ & $\begin{array}{c}\omega_{--} \\
\left(10^{10} \mathrm{~s}^{-1}\right)\end{array}$ & $\begin{array}{c}\omega_{-+} \\
\left({ }^{1} 0^{10} \mathrm{~s}^{-1}\right)\end{array}$ \\
0.02 & $0.5169(0.0437)^{*}$ & 0.8558 & 315.21 & 113.84 & 0.068 & 0.000 & 1.929 \\
0.2 & $1.1983(0.0543)$ & 0.7433 & 57.42 & 22.26 & 1.042 & 0.000 & 11.119 \\
0.5 & $1.5010(0.0495)$ & 0.7239 & 28.54 & 12.94 & 2.801 & 0.000 & 17.185 \\
1.0 & $1.7423(0.0495)$ & 0.7370 & 17.24 & 8.817 & 5.977 & 0.007 & 23.603 \\
2.0 & $2.0022(0.0469)$ & 0.8110 & 9.574 & 5.553 & 13.142 & 0.014 & 33.174 \\
4.0 & $2.2978(0.0438)$ & 1.0293 & 5.151 & 3.288 & 31.182 & 0.019 & 50.895 \\
6.0 & $2.4957(0.0405)$ & 1.3495 & 3.490 & 1.699 & 55.679 & 0.167 & 70.319 \\
\hline
\end{tabular}

*The values in parentheses indicate uncertainties in the MD simulations.

In Figures 2 and 3 we illustrate the concentration dependence of the reduced configurational energy, $U / N k T$, and the reduced osmotic pressure, $P V / N k T$, respectively. Also shown in these figures are the results obtained with the exponential form of Debye-Huckel theory (DHX) [Valleau et ai., 1970,1980; Debye and Huckel, 1923), the mean spherical approximation (MSA) (Waisman and Lebowitz, 1972), and those reported in Ref. [4] for the hypernetted chain theory (HNC). The thermo tynamic properties can be expressed in terms of pair distribution furctions, $g_{i j}(r)$, between isnic species $i$ and $j$.

The configurational energy of the fluid is

$$
\frac{U}{N k T}=\frac{2 \pi n}{k^{\prime}} \sum_{i} \sum_{j} \int_{d}^{\infty} U_{i j}(r) g_{i j}(r) r^{2} d r,
$$

and, the virial expression for the osmotic pressure is

$$
\frac{P V}{N k T}=1+\frac{U}{3 N k T}+\frac{2 \pi n d^{3}}{3} \sum_{i} \sum_{j} X_{i} X_{j} g_{i j}(d),
$$




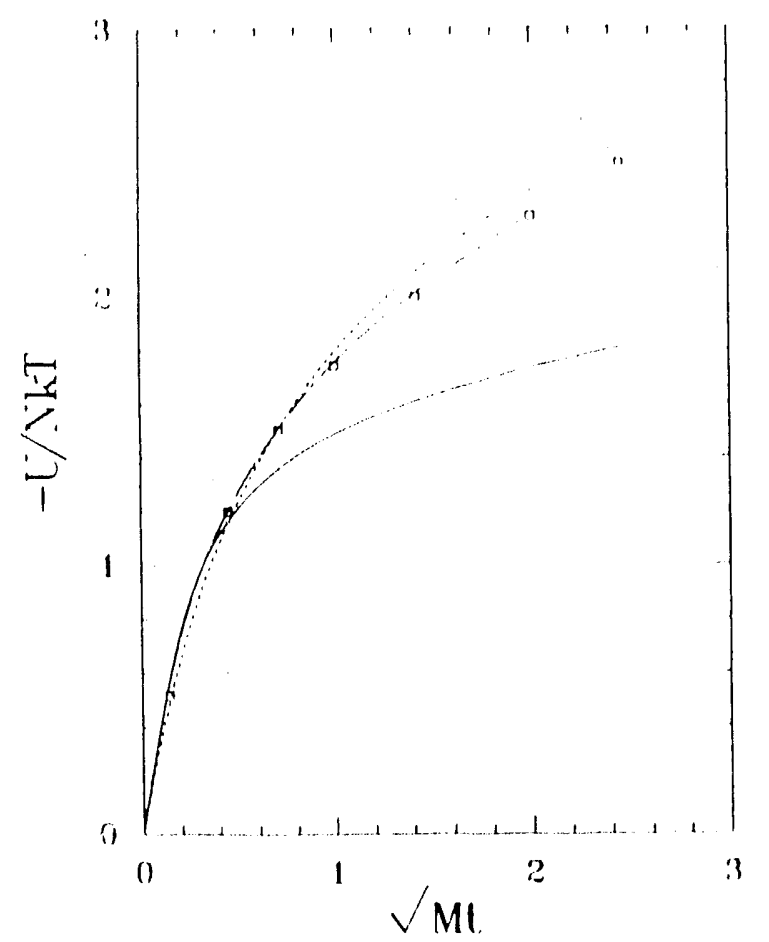

Figure 2. Reduced configurational energy of 1-3 primitive model electrolytes as a function of concentration. $\square M D$ results; -DHX; - MSA; - - HNC[4].

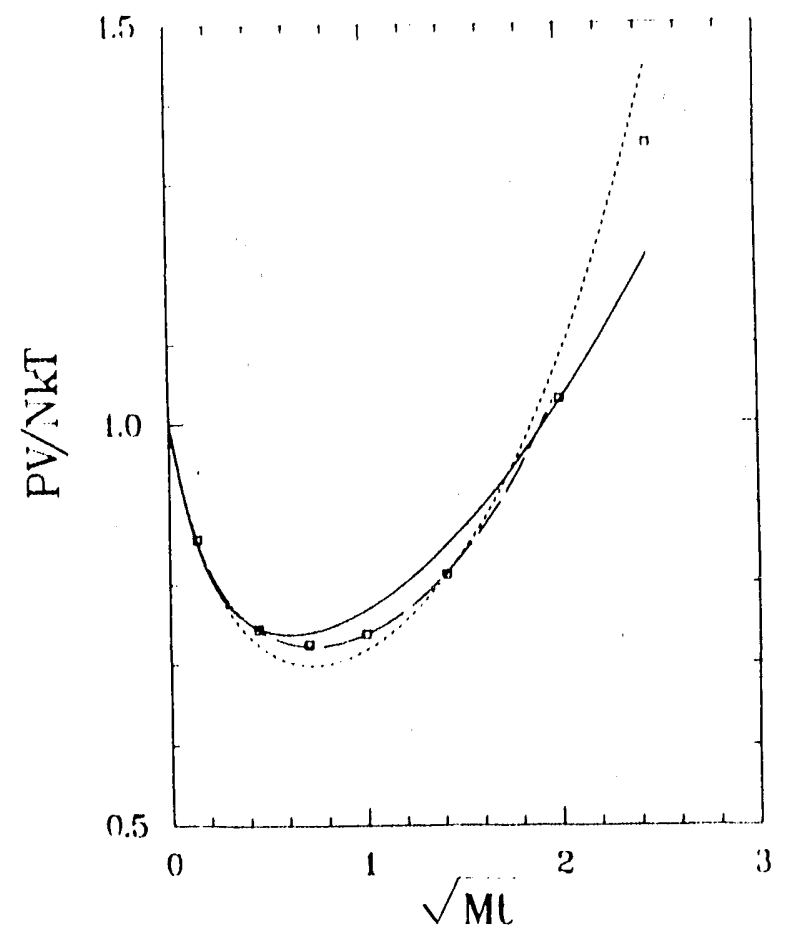

Figure 3. Reduced osmotic pressure of $1-3$ primitive model electrolytes as a function of concentration. The symbols have the same meaning as in Figure 2.

where $X_{i}$ is the mole fraction of species $i$ and $g_{i j}(d)$ is the contact value of the pair function for species $i$ and $j$.

We first focus our attention on the results obtained from the DHX theory. This simple approximation is inadequate over most of the range of the concentrations investigated in this work. The discrepancy is gradually amplified with increasing concentration. Although the poor results are observed here, we point out that for the 1-1 electrolyte system and $M t<2.0$ the DHX energy predictions are in reasonable qualitative agreement with previous simulation results [8]. The main failure of the DHX approximation is that it misses the structure of the pair distribution functions. The oscillatory behavior occurring at higher concentrations due to the hard-sphere exclusion volume is totally ignored in this theory. The contact values of the pair distribution functions of the DHX theory are also inaccurate.

This last point is illustrated in Figures $4 \mathrm{a}$ and $4 \mathrm{~b}$. Pair distribution functions were computed by sorting the relative pair distances into the equal radial increments of width $0.01 \mathrm{~d}$. Even at the low concentration of $M t=0.2$ (Figure $4 \mathrm{a}$ ), slightly more +- and ++ ion pairings at the short range of distances were found in the MD results compared with the DHX pair functions. The sharp peak near the contact point in $g_{+-}$indicates the strong tendency to the formation of unlike-pairs. As seen in Figure $4 b(M t=4.0)$, however, the DHX model significantly overestimates-pairings in $g_{--}$while the underestimation of the Coulombic attraction between unlike-pairs results in much lower value in $g_{+}$. In the $\mathrm{MC}$ 
calculations for the $2-2$ electrolytes $[9,11]$, the presence of linear ion triplets was manifested by a local maximum in like-pair functions near $r=2 d$. Similar results were observed, but, in the case of our 1-3 system, the maximum peak in g-_ was shifted to approximately $r=2.5 \mathrm{~d}$. The salient feature displayed here is a noticeable trend to the formation of larger ionic clusterings for higher charged systems.
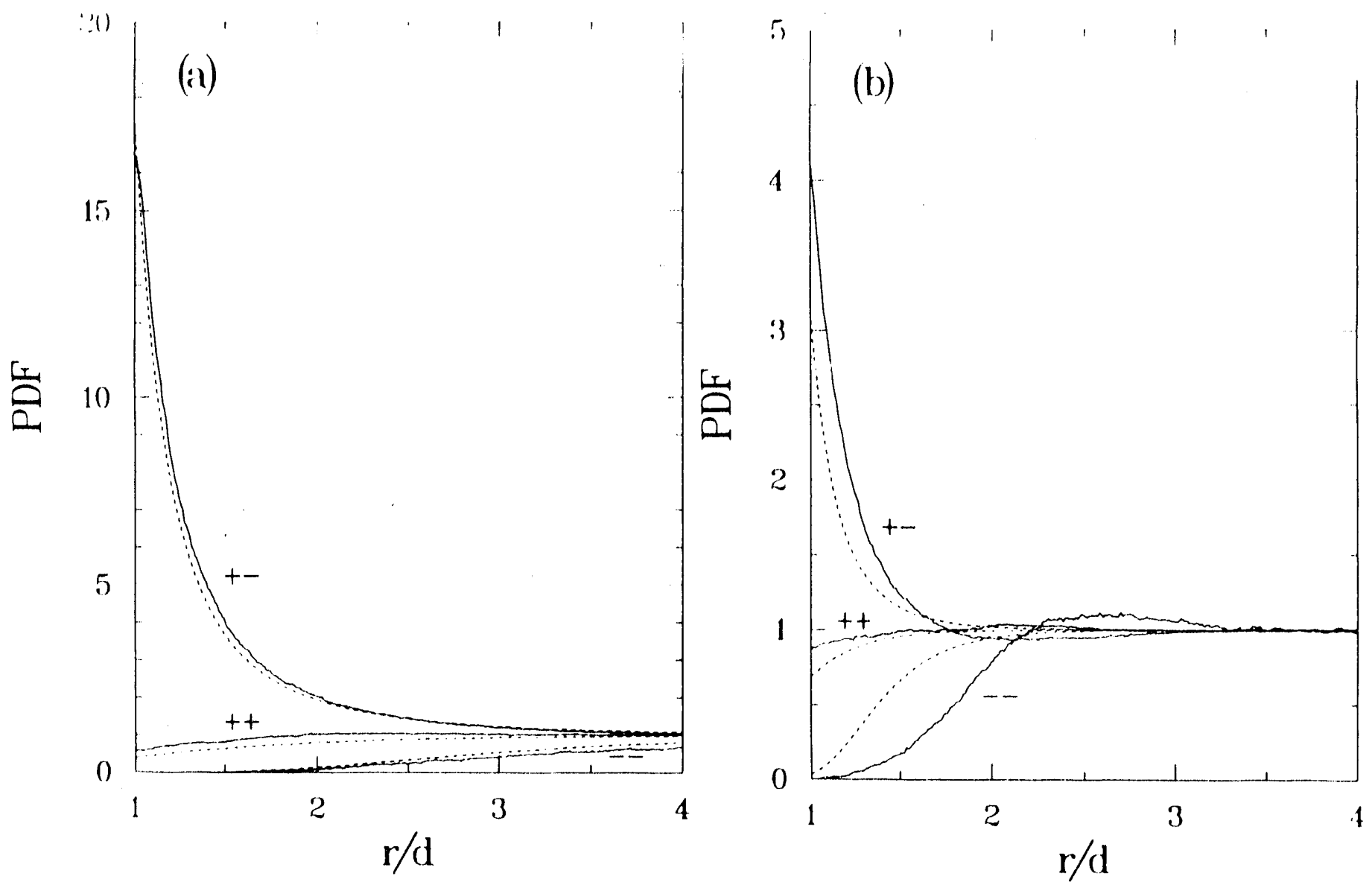

Figure 4. Pair distribution functions for like-pairs and unlike-pairs. (a) $M T=0.2$; (b) $M t=4.0$. Also shown as dotted curves are DHX predictions.

The MSA and HNC theories are both based on the Ornstein-Zernike integral equation along with specific closure relationships. The MSA model has a great advantage; it is the only theory that can be solved analytically for the system of charged hard-spheres [3]. As an extension to the Debye-Huckel theory, in the MSA theory, the hard-sphere oscillations due to the ion-corcs have been taken into account. In the limit of point charges, the MSA becomes identical to the Debye-Huckel theory. As illustrated in Figure 2 and 3, the MSA results for the energy and for the osmotic pressure are in relatively good agreement with the MD data. However, the MSA pair distribution functions are known to be poor. Particularly for a range of low concentration, the linearized nature of the MSA model leads to the unphysical negative values among the like-pair functions as in the Debye-Huckel theory. The MSA pair functions are only symmetrically disposed around those for the hard-sphere pair function. 
The better agreement for pressure than for energy in the DHX model seems to be coincidental and caused by a cancellation of errors between energy and collision contributions (the second and the third term in the virial pressure in eq. (4), respectively). For $M t=2.0$, for instance, an underestimation in $g_{+-}(d)$ by about $30 \%$ is almost equally balanced by an overestimate in energy. The HNC results for the system of 1-3 electrolytes [4] predict the configurational energy and the osmotic pressure in wry good agreement to the MD results within statistical errors. Although special iterative teringigues are necessary in order to reach convergence [4], HNC the ory has also shown to be "uccossful for the $1: 1$ and $2: 2$ electrolytes $[9,11]$.

The velocity autocorrelation functions (VACF) can provide useful insights into ion dynamics and transport. The normalized VACF is defined as a function of time, $t$, by

$$
\mathrm{VACF}=\frac{1}{N} \sum_{i=1}^{N}<\mathbf{v}_{i}(0) \cdot \mathbf{v}_{\boldsymbol{i}}(t)>/<\mathbf{v}_{i}^{2}(0)>
$$

where the symbol $\langle>$ denotes the average over an equilibrium ensemble. Those time correlation functions are, in principle, measurable quantities from inelastic scattering experiments.

In Figures 5a to 5c, we plot the normalized VACFs for a few selected runs to illustrate the manner in which those functions change with increasing concentration. The solid curves correspond to the VACFs for positively charged ions, VACF $(+)$, and, respectively, the dotted curves, for negatively charged ions, VACF $(-)$. The VACFs for lower concentration exhibit a stronger velocity autocorrelation than those for higher concentration. The primary mechanism of decay of the time correlation functions is the hard-sphere collision, in which colliding particles rapidly forget their initial velocities through successive collision. In the high concentration regime, the hard-core repulsive collisions are expected to play a dominant role in determining the dynamical properties of the system. In agreement with this, the resulting $\mathrm{VACF}(+) \mathrm{s}$ exhibit the exponential behavior which characterizes the dynamics of hard-sphere fluids [24].

However, as shown by the $\operatorname{VACF}(-) \mathrm{s}$ in Figure 5 , there are substantial differences between the motion of the positive charges and the highly charged negative ions. The $\mathrm{VACF}(-)$ s decay more rapidly than the VACF $(+)$ s. The VACF $(-)$ s exhibit non-exponential behavior. At low or intermediate concentration, the long-ranged Coulombic potential increasingly influences the collective motion of ionic fluids by the acceleration or retardation of velocities between colliding pairs. The electrostatic force enhances correlations between unlike-pairs, and decorrelation between like-pairs.

The most striking feature of the $\mathrm{VACF}(-) \mathrm{s}$ is the peculiar peak displayed in the range of intermediate concentration. The peak occurs at relatively the short time de ay between 5 ps and 10 ps. At the very low concentration regime, the $\operatorname{VACF}(-)$ decays monotonically. However, a weakly oscillatory behavior starts to emerge in the VACF $(-)$ for $M t=0.5$ (Figure 5a), and is clearly apparent for $M t=1.0$ (Figure $5 \mathrm{~b}$ ). This can be explained in terms of the coherent motion of a highly charged negative ion, and the positive ions surrounding it. The coherent motion in this range of concentration results in an increased persistence of velocity of the negative ions. Because the cluster tends to move coherently over a period of time longer than the mean collision time between ions in the cluster, this 

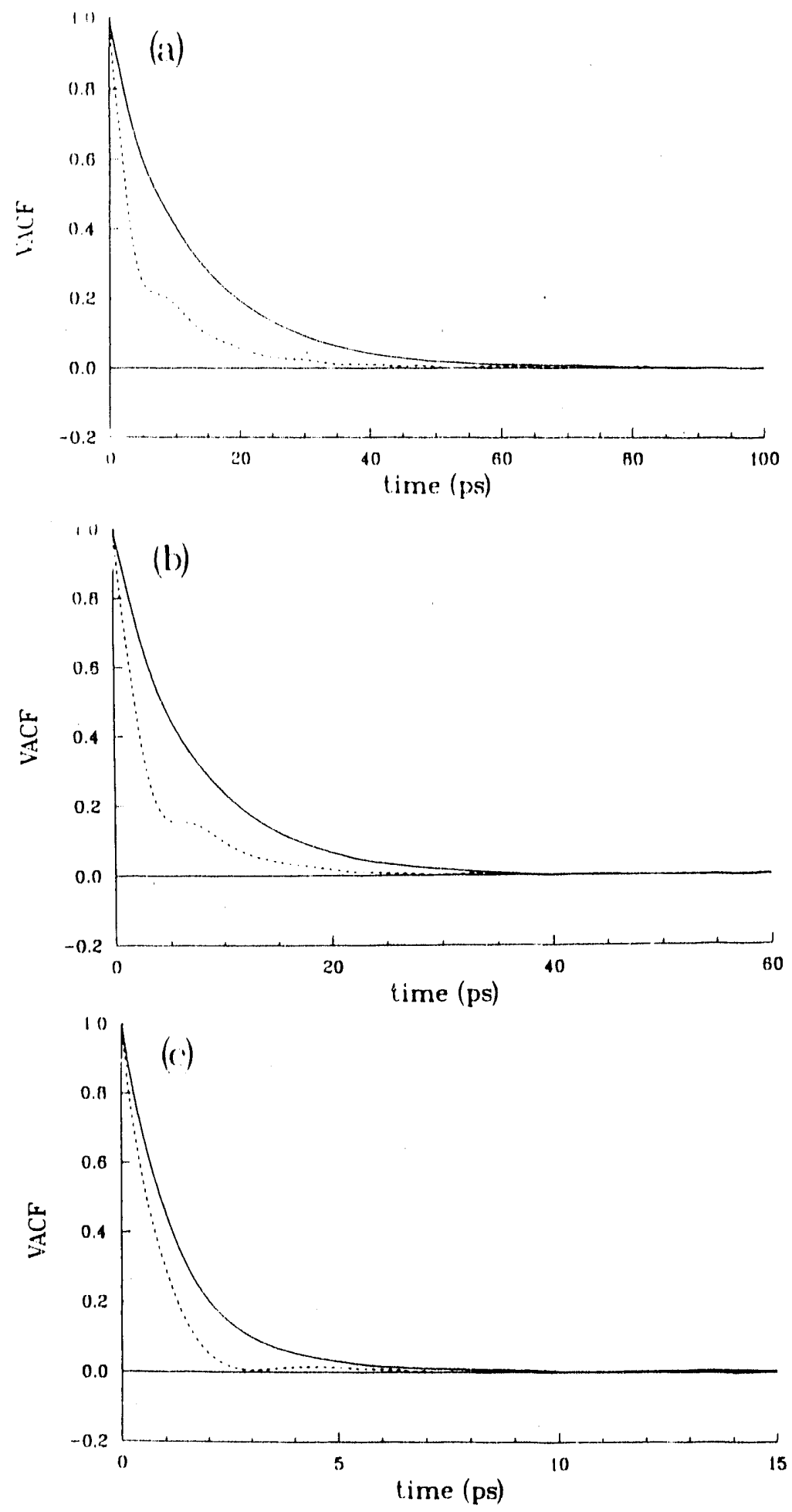

Figure 5. Normalized velocity autocorrelation function vs. time at three different concentrations: a) $M t=0.5$, b) 1.0 , and c) 6.0. The solid curves correspond to the velocity autocorrelation function of positive ions $(\mathrm{VACF}(+))$ and dotted curves to that of negative ions $(\operatorname{VACF}(-))$. 
collective motion conducts the negative ion in the direction of the net drift of the entire cluster. In the cases presented in Figures 5a and 5b, the bump in tho VACF (-) is located at nearly twice the mean collision time between negative and positive particles. This fact suggests that, the mechanism responsible for this velocity persistence is a double collision. First, a collision occurs between the negative central ion and the positive ions in front of it. Then, a second collision occurs when the same negative ion is reached by the positive ions which the clectrostatic attraction drag behind it. In contrast, as seen in Figure 5c, these bumps are reduced at the higher concentration of $M t=6.0$. In this case, there is perhaps not enough free volume for the ionic clusters to move coherently over appreciable distances.

In the previous MD simulations [8] for the 1-1 electrolytes, the modified Enskog theory of hard-sphere was shown to predict the self-diffusion coefficients reasonably accurately. It is also interesting to note that the diffusion constants of positive ions for the 1-3 electrolytes here (Table 1) are close to those for the 1-1 electrolytes except for the low concentration limit of $M t<0.2$. However, the self-diffusion coefficients of negative ions are smaller by a factor of two or three than those of positive ions. An interpretation of this observation is that the free motion of negative ions is likely to be restricted by the formation of ionic aggregates.

For a hard-sphere system [25], the collision frequencies, $\omega_{i j}$, are expressed in terms of the contact values of pair distribution functions,

$$
\omega_{i j}=4 n_{i} d^{2}(\pi k T / m)^{1 / 2} g_{i j}(d)
$$

.sp where $\omega_{i j}$ is the number of collisions per particle of component $j$, per unit time between particles of component $i$ and $j$. The collision frequencies determined from the MD simulation are plotted in Figure 6. For the purpose of comparison with the corresponding hard-sphere system, the predictions using the MD contact values in eq. (6) are also shown in this figure. The MD results are seen to be in excellent agreement with theory. This suggests that the microscopical behavior of the primitive electrolytes is much like to the hard-sphere dynamics. In this sense, the transport properties of the primitive model electrolytes are, at least qualitatively, related to those for the system of hard-sphere fluids.

\section{Conclusion}

In the present paper we have reported the MD simulations for the thermodynamic and transport properties of 1-3 electrolyte solutions. The results obtained from computer simulations have been used to assess the applicabilities of theoretical equations, namely, the exponential form of Debye-Huckel theory, the mean spherical approximation, and the hypernetted chain theory. The HNC theory is shown to be the best approximation compared with the simulation results both for energy and for osmotic pressure. For asymmetrically charged 1-3 bulk electrolytes, the presence of ionic clustering was observed in pair distribution functions. The local maximum in $g_{-}$- was shifted to $r=2.5 d$.

The MD results presented in this paper indicate that the diffusion processes in primitive model electrolytes are strongly dependent on concentration. In contrast, experimental diffusion coefficients in aqueous solutions are nearly concentration independent [26]. This result 


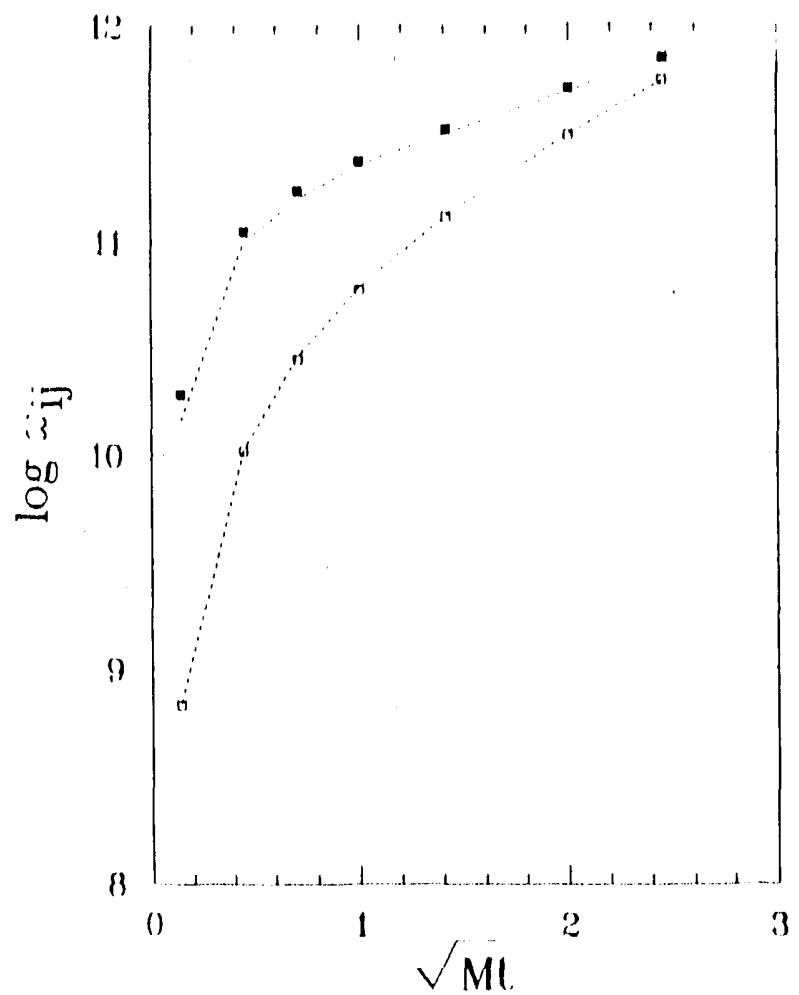

Figure 6. Semilogarithmic plot of collision frequency as a function of concentration. $\square M D$ results for $\omega_{++} ; M D$. results for $\omega_{-+}$. The dotted curves are predictions by Eq. (6) using the MD contact values of pair distribution functions.

is to be expected since solvent-ion collisions are absent in primitive model electrolytes. 'This simple model is inadequate to describe the ionic structures and dynamics in aqueous electrolyte solutions. However, the primitive model electrolyte shows solution thermodynamic behavior in qualitative agreement with experimental results on simple aqueous electrolytes [9]. Moreover, at sufficiently high ionic densities the model would give a reasonable first approximation to the equilibrium and dynamical behavior of molten salts.

\section{References}

Adams, D.J. , J. Chem. Phys., 782585 (1983).

Adams, D.J. and G.S. Dubey, J. Comp. Phys., 72156 (1987).

Alder, B.J. and T.E. Wainwright, J. Chem. Phys., 31459 (1959).

Alder, B.J. D.M. Gass and T.E. Wainwright, J. Chem. Phys., 533813 (1970).

Andersen, H.C. , P. Chandler and J.D. Weeks, J. Chem. Phys., 572626 (197\%).

Berendsen, H.J.C. , J.P.M. Postma, W.F. van Gunsteren, A. DiNola and Card, D.N. and J.P. Valleau, J. Chem. Phys., 526232 (1970);

Ciccariello, S. and D. Gazzillo, Molec. Phys., 481369 (1983).

Croxton, T.L. and D.A. McQuarrie, J. Phys. Chem., 831840 (1979).

Chapman, S. and T.G. Cowling, The Mathematical Theory of Non-Uniform Gases (Cambridge Univ. Press, 1970).

Debye, P. and E. Huckel, Physik Z., 24 195, 305 (1923). 
Ewald, P.P., Ann. Phys. Leipzig, 64253 (1921).

Freasier, B.C., Molec. Phys., 391273 (1980).

Friedman, II.L. and W.D.T. Dale, in: Modern Theoretical Chemistry Vol. 5, Statistical Mechanics, ed. B.J. Berne (Plenum Press, New York, 1977).

Haak, J.R., J. Chem. Phys, 813684 (1984).

Hansen, J.P., F. Joly and I.R. McDonald, Physica, 132A 472 (1985).

Heyes, D.M., Chem. Phys., 69155 (1982).

Heyes, D.M. and L.V. Woodcock, Molec. Phys., 591369 (1986).

Levesque, D., J.J. Weis and J.P. Hansen, in: Topics in Current Physics Vol. 36, Applicatiuns of the Monte Carlo Method in Statistical Physics, ed. K. Binder (Springer, Berlin, 1984).

McNeil, W.J. and W.G. Madden, J. Chem. Phys., 76 6221 (1982).

Medina-Noyola, M., D.A. McQuarrie and W. Olivares, Chem. Phys. Lett., 58351 (1978); M. Medina-Noyola, J. Chem. Phys., 815059 (1984).

Outhwaite, C.W., in: Statistical Mechanics Vol. 2, Specialist Periodical Reports, ed. K. Singer (The Chemical Society, London, 1975).

Rasaiah, J.C. and H.L. Friedman, J. Chem. Phys., 482742 (1968); J.C. Rasaiah, ibid, 56 3071 (1972).

Rogde, S.A. and B. Hafskjold, Molec. Phys., 481241 (1983).

Sangster, M.J.L. and M. Dixon, Adv. Phys., 25247 (1976).

Tyrrell, H.J.V. and K.R. Harris, Diffusion in Liquids. A Theoretical and Experimental Study (Butterworths, London, 1984).

Valleau, J.P. and K. Cohen, J. Chem. Phys., 725935 (1980); J.P. Valleau, K. Cohen and D.N. Card, J. Chem. Phys., 725942 (1980).

van Megen, W. and I. Snook, Molec. Phys., 391043 (1980).

Verlet, L., Phys. Rev., 15998 (1967).

Waisman, E. and J.L. Lebowitz, J. Chem. Phys., 56 3086, 3093 (1972). 


\section{DENSITY FUNCTIONAL FREE ENERGY THEORY OF THE ELECTRICAL DOUBLE LAYER 2:1 ELECTROLYTES}

\section{Synopsis}

This paper reports the predictions of a nonlocal free energy density functional theory of the ion density and the mean electrostatic potential profiles of the restricted primitive model of the electrical double layer. Results for unsymmetrical 2:1 electrolytes predicted by three different nonlocal free energy density functional approximations are compared with those of Monte Carlo simulations and of the modified Gouy-Chapman theory. The diffuse layer potentials are also compared with those of some recent theories of the electrical double layer. In the free energy density functional theory, the hard-sphere repulsive contribution to the Helmholtz free energy is represented by nonlocal density functional models which have appeared in previous works. The mean spherical approximation for the bulk electrolyte is used to model the electrostatic part of the nonuniform ion-ion correlations present in the interface. In the case of singly charged counterions the agreement between the free energy density functional theory and the Monte Carlo simulations is within a few percent at the electrolyte concentrations and surface charges studied. In the case of doubly charged counterions, however, the agreement is poor at low electrolyte concentrations although good at high concentrations. The density functional theory correctly predicts the existence of an extremum in the diffuse layer potential as a function of the surface charge density.

\section{Introduction}

The wettability of reservoir porespace determines how oil and water are distributed in oil bearing reservoirs and affects the displacement of oil in a recovery process. Wettability, in turn, is affected by adsorption and double layer forces at the fluid-solid surfaces of the reservoir. Understanding adsorption and double layer forces at the molecular level will enhance our ability to design enhanced oil recovery formulations that reduce unwanted wettability behtivior or to modify the wettability in a beneficial way.

Recently, a nonlocal frce energy density functional theory for the restricted primitive model (RPM) of the electrical double layer was presented and the results for symmetrical 1:1 and 2:2 electrolytes were reported (Mier y Teran et al. (1990), Tang et al. (1990)). An electrical double layer is the separation of charge which occurs in an electrolyte in the p) esence of an electrode. In the RPM of the electrulyte, the ions are modeled as charged hi rd spheres of equal diameter $d$ and the solvent is represented by a uniform dielectric $m$ edium with dielectric constant $\epsilon$. At the same time, the electrode is taken to be an infinite, planar, uniformly charged, hard wall with surface charge densiiy $\sigma$. 
In Mier y Teran et al. (1990) the grand potential functional for the RPM of the electrical double layer was constructed and minimized to obtain integral equations for the ion density distribution functions. The equations are equivalent to those obtained by functional differentiation of the Helmholtz free energy with respect to component density to derive expressions for the chemical potential of each species in the inhomogeneous electrolyte, as was done in Tang et al. (1990). In the free energy density functional theory, the hard-sphere repulsive contribution to the free energy is represented by a nonlocal generic density functional proposed by Percus (1981) and extended to hard sphere mixtures by Vanderlick et al. (1989b). It has been shown by Vanderlick et al. (1989a) that this generic functional can be used as a generating functional of several nonlocal approaches including the generalized van der Waals (GVDW) model (Nordholm et al. (1981)), the generalized hard-rod model (GHRM) (Robledo and Varea (1981); Fischer and Heinbuch (1988)), and the Tarazona (TRZ) model (Tarazona (1985)). In addition, we have used the analytical solution of the mean spherical approximation (MSA) (Waismann and Lebowitz (1972)) for the bulk electrolyte to approximate the electrostatic part of the nonuniform OrnsteinZernike direct correlation function of the inhomogeneous electrolyte in the interface.

The results of previous papers (Mier y Teran et al. (1990); Tang et al. (1990)) can be summarized as follows. Comparison of the density functional theory with the Monte Carlo (MC) simulations of Torrie and Valleau (Torrie et al. (1980)) shows that, when combined with the Carnahan-Starling equation of state, the free energy density functional theory is successful in predicting the behaviors of thr: ion density and the mean electrostatic potential profiles for symmetrical electrolytes. The theory predicts correctly the layering effect of the counterions and the chaige inversion phenomenon. The results predicted by the GHRM (Mier y Teran et al. (1990)), the GVDW, and the TRZ models (Tang et al. (1990)) are in close agreement with each other at low concentrations and low surface charge densities but the differences between them increase when the charge or the concentration is increased. Comparison with the MC values of the diffuse layer potential as a function of the surface charge density shows that the GHRM is superior to the other two models at high surface charge densities for highly concentrated 1:1 electrolytes (Tang et al. (1990). We believe this is due to a fortuitous cancellation of errors in the calculations when the GHRM is combined with the MSA solution of the electrostatic correlation, since the TRZ model, which is a better approximation than the GHRM for inhomogeneous hard-sphere system (Vanderlick et al. (1989a)), better represents the layering effect of the counterions at high charge densities. For 2:2 electrolytes, on the other hand, all these three models predict results which are only in qualitative agreement with the MC data for the diffuse layer potential.

In this paper we report results of the density functional theory for 2:1 electrolytes next to both positively and negatively charged electrodes. As in Tang et al. (1990), we present a comparison between the GHRM, the GVDW, and the TRZ models. We also compare our results with those of the modified Gouy-Chapman (MGC) theory (Gory (1910); Chapman (1913); Stern (1924)) and of some recent theories of the electrical double layer. These includt- the most refined version of the modified Poisson-Boltzmann theory (MPB5) 
(Outhwaite and Bhuiyan (1983)), the hypernetted chain/mean spherical approximation (HNC/MSA) theory (Lozada-Cassou and Henderson (1983)), and the Yvon-Born-Green (YBG) theory with closure introduced by Caccamo et al. (1986). In addition, we also compare our results with those of Plischke and Henderson (1989), who solved the inhomogeneous Ornstein-Zernike (OZ) equation for the pair correlation function together with the Lovett-Mou-Buff-Wertheim equation and the hypernetted chain closure (OZ/LMBW), whenever the latter are available. As pointed out previously by others (Torrie and Valleau (1982); Lozada-Cassou and Henderson (1983)), results for 2:1 electrolytes are interesting not only because their practical applications but also because the MGC theory exhibits both qualitative agreement or disagreement with the MC simulations, depending upon whether the counterion is monovalent or divalent.

The rest of the paper is arranged as follows. In Sec. II we review briefly the free energy density functional theory of the RPM of the electrical double layer. We report our results in Sec. III and conclude in Sec. IV with a disscusion.

\section{Theory}

With the the same notations as those in Tang et al. (1990) the Helmholtz free energy density functional $F(\{\mathbf{n}\})$ for a mixture of charged particles in an external field of potential $v_{\alpha}(\mathbf{r})$ can be written as

$$
\begin{aligned}
F(\{\mathbf{n}\})= & \sum_{\alpha} \int d^{3} r n_{\alpha}(\mathbf{r}) v_{\alpha}(\mathbf{r})+k T \sum_{\alpha} \int d^{3} r n_{\alpha}(\mathbf{r})\left[\ln \left(\Lambda_{\alpha}^{3} n_{\alpha}(\mathbf{r})\right)-1\right] \\
& +k T \sum_{\alpha \beta} \iint d^{3} r d^{3} r^{\prime} n_{\alpha}(\mathbf{r}) n_{\beta}\left(\mathbf{r}^{\prime}\right) \int_{0}^{1} d \lambda \int_{0}^{\lambda} d \lambda^{\prime} c_{\alpha \beta}\left(\mathbf{r}, \mathbf{r}^{\prime} ; \lambda^{\prime}\right)
\end{aligned}
$$

where $k$ is Boltzmann's constant, $T$ the absolute temperature, and $\Lambda_{\alpha}$ the de Broglie wavelength of particles of species $\alpha$. The first two terms in Eq.(2.1) correspond to the external field and the ideal gas contributions to the free energy, respectively. The third term represents the contribution from the ion-ion pair interactions. The path integration characterized by the parameter $\lambda$ is along a linear density path from the initial state $n_{\alpha}^{i}(\mathbf{r})=0$ to the final state $n_{\alpha}(\mathbf{r})$, with $c_{\alpha \beta}\left(\mathbf{r}, \mathbf{r}^{\prime} ; \lambda\right)$ being the Ornstein-Zernike direct correlation function of a nonuniform electroiyte at a particular point, $n_{\alpha}(\mathbf{r} ; \lambda)=\lambda n_{\alpha}(\mathbf{r})$ $(0 \leq \lambda \leq 1)$, on the integration path.

Althuugh $c_{\alpha \beta}\left(\mathbf{r}, \mathbf{r}^{\prime}\right)$ is not in general known for inhomogeneous electrolytes, it can be separated formally into three parts:

$$
c_{\alpha \beta}\left(\mathbf{r}, \mathbf{r}^{\prime}\right)=-\frac{q_{\alpha} q_{\beta}}{\epsilon k T\left|\mathbf{r}-\mathbf{r}^{\prime}\right|}+c_{\alpha \beta}^{h s}\left(\mathbf{r}, \mathbf{r}^{\prime}\right)+\Delta c_{\alpha \beta}\left(\mathbf{r}, \mathbf{r}^{\prime}\right) .
$$

Here $q_{\alpha}$ is the charge of ions of species $\alpha$. The first term on the right side of $E_{1} \cdot(2.2)$ is simply the Coulombic interaction between charged particles, whereas the second term 
is the hard-sphere correlation due to the finite size of the particles, which is discussed in more detail later in this section. The third term is the residual part of the Ornstein-Zernike direct correlation function, which must be prescribed by some means. We choose here to approximate this nonuniform residual correlation function by the counterpart of a neutral bulk electrolyte. The latter is further approximated by the analytical solution given by Waismann and Lebowitz (1972) when the MSA is used for the RPM electrolytes. This solution, $\Delta c_{\alpha \beta}\left(\left|\mathbf{r}-\mathbf{r}^{\prime}\right|\right)$, depends only on the relative distance between two particles. The explicit expression for $\Delta c_{\alpha \beta}\left(r^{\prime}\right)$ can be found in Mier y Teran et al. (1990).

By functional differentiation of the free energy density functional $F(\{\mathbf{n}\})$ with respect to the density distribution function $n_{\alpha}(\mathbf{r})$ we obtain the chemical potential $\mu_{\alpha}$ of particles of species $\alpha$. The chemical potential is everywhere constant and so $\mu_{\alpha}$ can be eliminated from the general expression for the double layer by subtracting from it the corresponding equation for the coexisting bulk electrolyte. This yields the following integral equation for the ion density distribution function:

$$
\begin{aligned}
\ln \left(n_{\alpha}(\mathbf{r}) / n_{\alpha}^{0}\right)= & -\frac{1}{k T}\left(v^{h s}(\mathbf{r})+q_{\alpha} \psi(\mathbf{r})\right) \\
& +\sum_{\beta} \int d^{3} r^{\prime}\left(n_{\beta}\left(\mathbf{r}^{\prime}\right)-n_{\beta}\right) \Delta c_{\alpha \beta}\left(\mathbf{r}, \mathbf{r}^{\prime}\right) \\
& -\frac{\delta F^{\text {excess }}(\{\mathbf{n}\})}{\delta n_{\alpha}(\mathbf{r})}+\frac{\partial F^{\text {excess }}\left(\mathbf{n}^{0}\right)}{\partial n_{\alpha}^{0}}
\end{aligned}
$$

where $n_{\alpha}^{0}$ is the number density of particle $\alpha$ in the bulk electrolyte. In Eq.(2.3), $v^{h s}(\mathbf{r})$ is the interaction potential between an ion and an uncharged hard wall,

$$
\begin{aligned}
v^{h s}(\mathbf{r}) & =\infty, & & x<d / 2 \\
& =0, & & x>d / 2
\end{aligned}
$$

where $x$ is the ion's distance to the wall. On the other hand, $\psi(\mathbf{r})$ is the mean electrostatic potential, due to both the ionic charges in the electrolyte and the uniformly distributed charges on the surface, which can be expressed in terms of the ion density distribution function by solving Poisson's equation. The result is

$$
\psi(x)=\frac{4 \pi}{\epsilon} \int_{x}^{\infty} d x^{\prime}\left(x-x^{\prime}\right) \sum_{\alpha} q_{\alpha} n_{\alpha}\left(x^{\prime}\right)
$$

where the condition of overall electroneutrality,

$$
\int_{0}^{\infty} d x^{\prime} \sum_{\alpha} q_{\alpha} n_{\alpha}\left(x^{\prime}\right)+\sigma=0
$$

has,been imposed. 
The crucial step leading to Eq.(2.3) is introducing the excess hard-sphere free energy functional $F^{\text {excess }}(\{\mathbf{n}\})$ to incorporate the hard-core repulsive contribution to the free energy as we did in previous studies (Mier y Teran et al. (1990); Tang et al. (1990). We use the generic functional proposed by Percus (1981), which is the three-dimensional generalization of the Helmholtz free energy of an inhomogeneous one-dimensional hard rod system. For hard sphere mixtures this functional can be written as in Vanderlick et al. (1989a)

$$
F^{\text {excess }}(\{\mathbf{n}\})=\sum_{\alpha} \int d^{3} r \bar{n}_{\alpha}^{\nu}(\mathbf{r}) F_{0}\left(\overline{\mathbf{n}}^{\top}(\mathbf{r})\right),
$$

where $F_{0}(n)$ is the excess free energy per particle of a homogeneous hard sphere fluid of density $n$; this function can be derived from the hard-sphere equation of state. In our calculations, we chose the Carnahan-Starling formula for $F_{0}(n)$, i.e.,

$$
F_{0}(n)=k T \frac{y(4-3 y)}{(1-y)^{2}}
$$

where $y \equiv \pi n d^{3} / 6$.

In the expression for the excess hard-sphere free energy density functional, Eq.(2.7), $\bar{n}_{\alpha}^{\nu}(\mathbf{r})$ and $\bar{n}_{\alpha}^{\tau}(\mathbf{r})$ are coarse-grained densities defined as spatial averages of the local density $n_{\alpha}(\mathbf{r})$ over certain small domains:

$$
\begin{aligned}
& \bar{n}_{\alpha}^{\nu}(\mathbf{r}) \equiv \int d^{3} r^{\prime} \nu_{\alpha}\left(\mathbf{r}-\mathbf{r}^{\prime} ;\{\mathbf{n}\}\right) n_{\alpha}\left(\mathbf{r}^{\prime}\right), \\
& \bar{n}_{\alpha}^{\tau}(\mathbf{r}) \equiv \int d^{3} r^{\prime} \tau_{\alpha}\left(\mathbf{r}-\mathbf{r}^{\prime} ;\{\mathbf{n}\}\right) n_{\alpha}\left(\mathbf{r}^{\prime}\right) .
\end{aligned}
$$

The weighting functions $\nu_{\alpha}\left(\mathbf{r}-\mathbf{r}^{\prime} ;\{\mathbf{n}\}\right)$ and $\tau_{\alpha}\left(\mathbf{r}-\mathbf{r}^{\prime} ;\{\mathbf{n}\}\right)$ are, in the most general case, functionals of the density distribution functions. By changing the specific form of the weighting functions, different density functional models can be generated (Vanderlick et al. (1989a). In this work we solved Eq.(2.3) for the three model functionals mentioned before: the GVDW model, the GHRM, and the TRZ model. The weighting functions $\nu_{\alpha}\left(\mathbf{r}-\mathbf{r}^{\prime} ;\{\mathbf{n}\}\right)$ and $\tau_{\alpha}\left(\mathbf{r}-\mathbf{r}^{\prime} ;\{\mathbf{n}\}\right)$ for these three models have been given explicitly in Tang et al. (1990).

\section{Results}

In this work we carried out calculations for 2:1 electrolytes at bulk concentrations of $0.005,0.05$, and $0.5 \mathrm{M}$. For the latter two concentrations we considered both positive and negative surface charge densities $\sigma$. For concentration of $0.005 \mathrm{M}$ the MC data reported (Torrie and Valleau (1982)) are for negative values of $\sigma$ only; therefore we restricted our calculations to the same range of $\sigma$. For positive surface charges the counterions are singly charged, whereas for negative surface charges the counterions are doubly charged. 
Due to the planar symmetry of the problem, Eq.(2.3) can be reduced to a onedimensional integral equation by integrating over the $y-z$ plane, which is parallel to the charged surface. In order to solve this one-dimensional integral equation we employed the finite element method described elsewhere (Mier y Teran et al. (1982)). As in Mier y Teran et al. (1990) and Tang et al. (1990), we used quadratic Lagrange interpolating polynomials as basis functions for the GHRM and linear functions for the GVDW and the TRZ rnodels. We generated solutions in the domain $d / 2 \leq x \leq L$, where $L$ was the cutoff distance from the wall used as the upper limit in the integrals. A uniform mesh of $N$ points was used in all cases. Both $N$ and $L$ depended strongly on the concentration of the bulk electrolyte.

The Newton iterative scheme was used to solve the system of nonlinear algebraic equations for the nodal values of the reduced density: $g_{\alpha i}=n_{\alpha}\left(x_{i}\right) / n_{\alpha}^{0}$. The iteration was continued until the Euclidean norm of the updates after $k$ iterations became less than $10^{-10}$, i.e.,

$$
\left[\frac{\sum_{\alpha} \sum_{i}\left(g_{\alpha i}^{(k+1)}-g_{\alpha i}^{(k)}\right)^{2}}{2 N}\right]^{\frac{1}{2}}<10^{-10}
$$

As a second test of the numerical accuracy of our calculations we also checked the agreement of the overall electroneutrality condition, Eq.(2.6). This equation was satisfied to at least five significant figures in all the calculations.

Dimensionless quantities proved convenient. The dimensionless surface charge density is $\sigma^{*}=\sigma d^{2} / e$ with $e$ being the magnitude of the electronic charge. The dimensionless mean electrostatic potential is $\psi^{*}(x)=\beta e \psi(x)(\beta=1 / k T)$. The distance $x$ is reduced by the hard sphere diameter $d$. As in our previous work we fixed the plasma parameter to $\Gamma^{*} \equiv \beta e^{2} / \epsilon d=1.6809$ in order to compare our results with MC data (Torrie and Valleau (1982)) This value of $\Gamma^{*}$ corresponds to $T=298 \mathrm{~K}, \epsilon=78.5$, and $d=4.25 \AA$.

Our results of the dimensionless diffuse layer potential, $\psi^{*}(0)=e \psi(0) / k T$, at several values of the surface charge density $\sigma^{*}$ for $2: 1$ electrolytes are summarized in Table 1 . For comparison, in the same table we also display the MC values (Torrie and Valleau (1982), the results of the MGC (Gouy (1910); Chapman (1913); Stern (1924)), the MPB5 (Outhwaite and Bhuiyanb (1989)), the HNC/MSA (Lozada-Cassou and Henderson (1983)), and the YBG (Caccamo et al. (1986)) theories. For positive values of $\sigma^{*}$, the agreement between the results of the density functional theory and the $\mathrm{MC}$ data is within a few percent, which is comparable with the statistical uncertainty in the MC data. In this case the GHRM is slightly superior to the other two density functional models. For negative surface ch fes, the agrement between our results and MC simulation is only fair at low concentrations $131 \%$ high for the GHRM and $25 \%$ high for the GVDW and the TRZ models) but improves at the higher concentration of $0.5 \mathrm{M}(22 \%$ high for all the three models). The GVDW and the TRZ models are both slightly better than the GHRM for the range of negative values of $\sigma^{*}$ investigated in this work. 
TABLE 1. Diffuse layer potential, $\psi^{*}(0)=e \psi(0) / k T$, for 2:1 electrolytes

\begin{tabular}{|c|c|c|c|c|c|c|c|c|}
\hline$\sigma^{*}$ & $\mathrm{MC}^{a}$ & MGC & $B G Y^{b}$ & $\mathrm{MPB5}^{\mathrm{C}}$ & $\mathrm{HNC} / \mathrm{MSA}^{d}$ & GHRM & GVDW & TRZ \\
\hline \multicolumn{9}{|c|}{$0.005 \mathrm{M}$} \\
\hline-0.01 & $-0.91(0.02)$ & -0.959 & - & -0.865 & - & -0.947 & -0.947 & -0.947 \\
\hline-0.02 & $-1.37(0.01)$ & -1.518 & - & -1.28 & - & -1.485 & -1.484 & -1.484 \\
\hline-0.05 & $-1.87(0.03)$ & -2.381 & - & -1.79 & - & -2.233 & -2.228 & -2.228 \\
\hline \multicolumn{9}{|c|}{$0.05 \mathrm{M}$} \\
\hline-0.05 & $-1.05(0.01)$ & -1.315 & -1.11 & -0.999 & -1.158 & -1.160 & -1.156 & -1.156 \\
\hline-0.0975 & $-1.21(0.06)$ & -1.916 & -1.476 & -1.22 & -1.511 & -1.522 & -1.505 & -1.505 \\
\hline-0.126 & $-1.26(0.03)$ & -2.161 & -1.739 & -1.27 & -1.577 & -1.596 & -1.568 & -1.569 \\
\hline-0.20 & $-1.18(0.03)$ & -2.612 & -1.739 & -1.29 & -1.497 & -1.551 & -1.481 & -1.485 \\
\hline-0.284 & $-1.02(0.03)$ & -2.959 & -1.74 & -1.28 & -1.176 & -1.288 & -1.153 & -1.164 \\
\hline 0.04294 & $1.73(0.02)$ & 1.740 & 1.705 & 1.69 & 1.89 & 1.675 & 1.672 & 1.672 \\
\hline 0.18 & $3.99(0.05)$ & 4.324 & 4.04 & 3.92 & 3.89 & 4.005 & $3 ; 922$ & 3.922 \\
\hline \multicolumn{9}{|c|}{$0.5 \mathrm{M}$} \\
\hline-0.05 & $-0.40(0.02)$ & -0.547 & -0.372 & -0.373 & -0.311 & -0.317 & -0.311 & -0.312 \\
\hline-0.099 & $-0.50(0.01)$ & -0.951 & -0.509 & -0.463 & -0.462 & -0.476 & -0.462 & -0.463 \\
\hline-0.1704 & $-0.46(0.01)$ & -1.379 & -0.574 & -0.459 & -0.482 & -0.518 & -0.480 & -0.484 \\
\hline-0.24 & $-0.35(0.03)$ & -1.683 & -0.557 & -0.410 & -0.351 & -0.425 & -0.351 & -0.360 \\
\hline 0.0989 & $1.04(0.02)$ & 1.308 & 0.97 & 0.985 & 0.966 & 0.988 & 0.965 & 0.967 \\
\hline 0.20 & $1.94(0.03)$ & 2.364 & 1.875 & 1.85 & 1.806 & 1.914 & 1.814 & 1.831 \\
\hline
\end{tabular}
a. G. M. Torrie and J. P. Valleau (1982). Statistical uncertainty is shown in parenthesis.
b. C. Caccamo, G. Pizzimenti, and L. Blum (1983).
c. C. W. Outhwaite and L. B. Bhuiyan (1983).
d. M. Lozada-Cassou and D. Henderson (1983).

A comparison of the results of the three density functional models with the MGC theory and the MC data is presented in Fig. 1. This figure shows the dimensionless diffuse layer potential as a function of the electrode charge density $\sigma^{*}$ at concentrations of 0.005 , 0.05 , and $0.5 \mathrm{M}$. The MGC theory, which neglects the exclusive effects due to the finite size of the ions, overestimates the thickness of the double layer and predicts a monotonic rise 


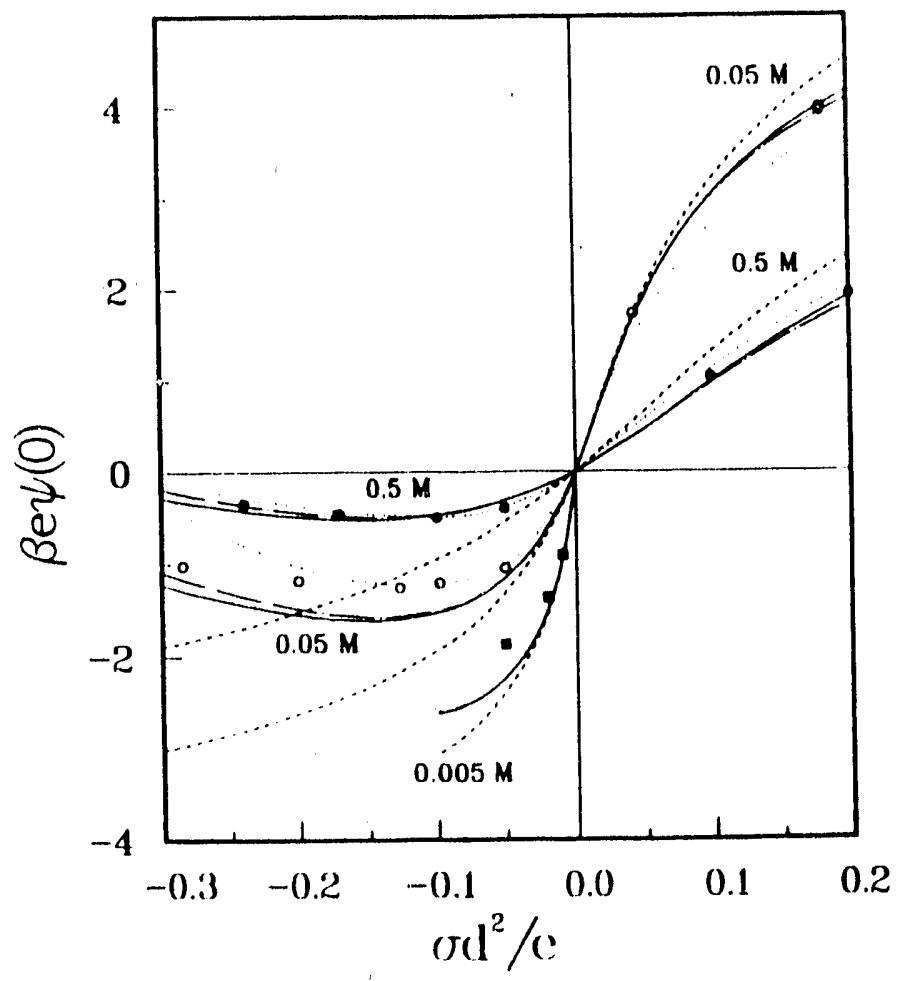

Figure 1. Reduced diffuse layer potential, $\beta e \psi(0)(\beta=1 / k T)$, as a function of the charge density, $\sigma d^{2} / e$, for $2: 1$ electrolytes. Solid curves represent the results of the GHRM, dot-dashed curves the results of the GVDW and the TRZ models. The results displayed are at concentrations of $0.005,0.05$, and $0.5 \mathrm{M}$. Solid squares, open circles, and solid circles are the corresponding $\mathrm{MC}$ results. The dashed curves are the results of the MGC theory and the dot curves are the results of the OZ/LMBWW theory (Plischke and Henderson (1989)).

of the diffuse layer potential as the magnitude of $\sigma^{*}$ increases. As has been pointed out by other authors (Torrie and Valleau (1982)), for positive surface charges the behavior of the diffuse layer potential as a function of $\sigma^{*}$ is reminiscent of that of $1: 1$ electrolytes. For negative surface charges, the monotonic behavior of the MGC theory disagrees significantly with $\mathrm{MC}$ simulations, since the simulation results present a minimum in the diffuse layer potential as a function of $\sigma^{*}$. The density functional theory predicts the presence of that minimum. Because the results of the GVDW and the TRZ models differ by no more than $1 \%$ and are indistinguishable in our plots, we show only one curve for these two approximations. At $c=0.005 \mathrm{M}$, the results of all the three density functional models 

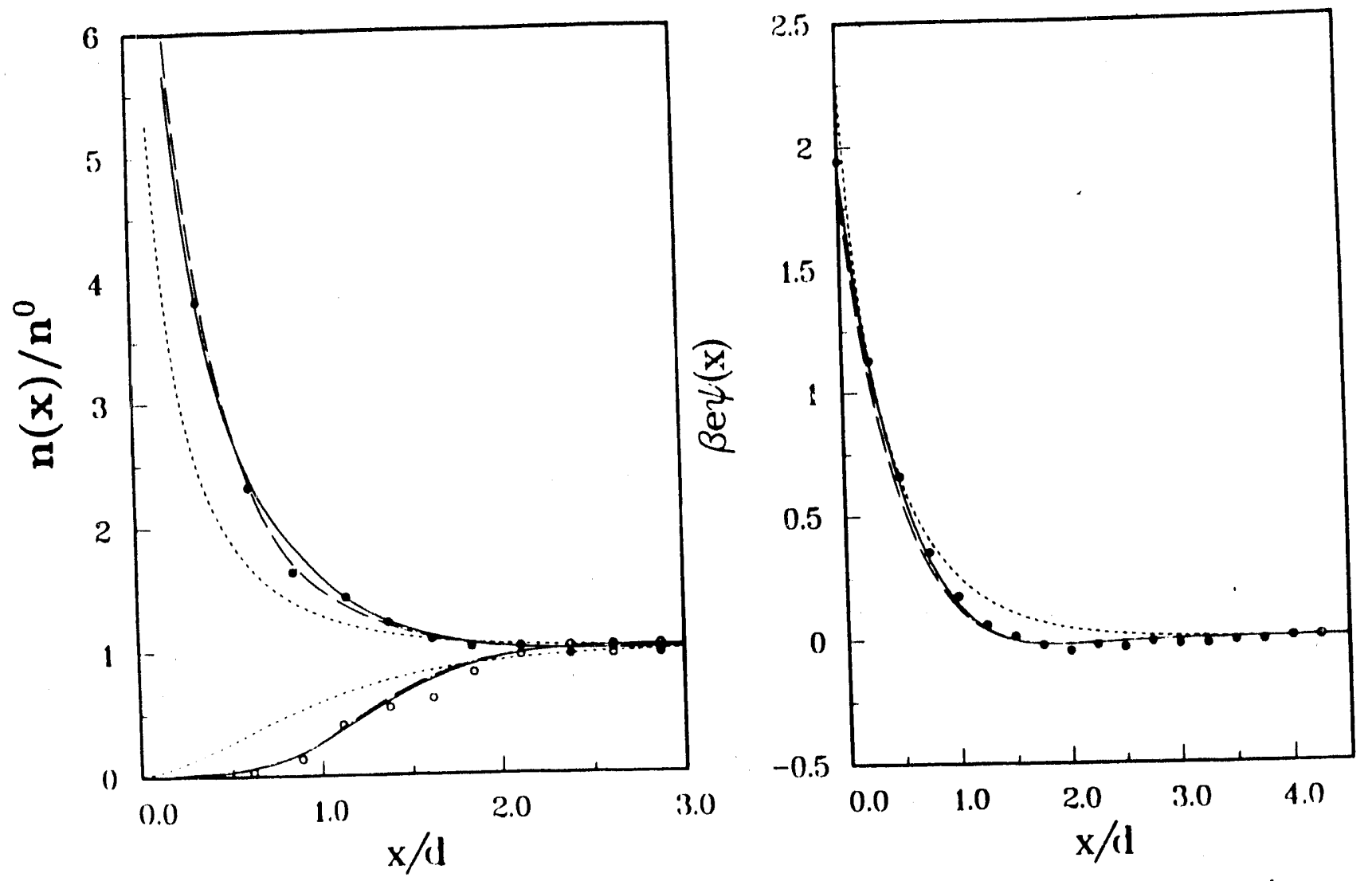

Figure 2. Reduced density profiles, $n(x) / n^{0}$, Figure 3. Reduced mean electrostatic poof a 2:1 electrolyte at $c=0.5 \mathrm{M}$ and $\sigma^{*}=$ tential profile, $\beta e \psi(x)$, of a $2: 1$ electrolyte 0.2 . The dots are the $\mathrm{MC}$ results. The at $c=0.5 \mathrm{M}$ and $\sigma^{*}=0.2$ All symbols as dashed curves correspond to the MGC the- in Fig. 2.

ory, the solid curves to the GHRM, and the dot-dashed curves to the GVDW and the TRZ models. Note that the charged surface is at $x=-d / 2$.

are indistinguishable (within 1\%) in the plots. In this figure we also present the results of the OZ/LMBW theory of Plischke and Henderson (1989) at concentrations of 0.5 and 0.05 $\mathrm{M}$; the results at lower concentration of $0.05 \mathrm{M}$ are not available. The $\mathrm{OZ} / \mathrm{LMBW}$ theory predicts the most accurate results currently available; large deviation from $M C$ values at $c=0.05 \mathrm{M}$ and $\sigma^{*}<-0.2$ was believed to be due to numerical difficulties. Our results compete in accuracy with those of OZ/LMBW.

We proceed now to examine the information contained in the ion density profiles and the mean electrostatic potential profiles. When the surface is positively charged and the electrolyte concentration is low, the behavior of the double layer is qualitatively similar to that found in 1:1 electrolytes. The behavior at higher concentrations is more interesting. In Fig. 2 we display the ion density profiles at $0.5 \mathrm{M}$ and $\sigma^{*}=0.20$. The solid curves 


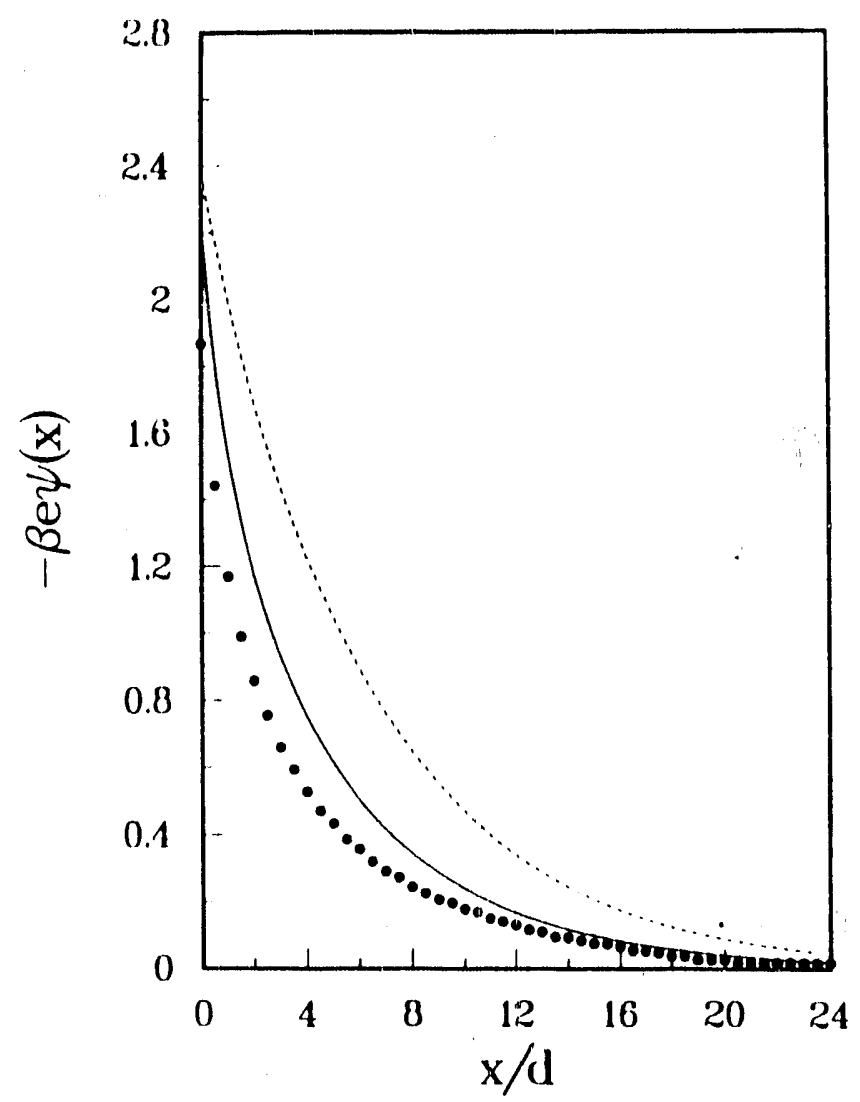

Figure 4. Reduced mean electrostatic potential profile, $-\beta e \psi(x)$, of a 2:1 electrolyte at $c=0.005 \mathrm{M}$ and $\sigma^{*}=-0.05$. The dots are the $\mathrm{MC}$ results. The dashed curve correspond to the MGC theory, and the solid curve to the density functional approximations. In this case, the results of the three density functional approximations are undistinguishable in the figure.

correspond to the results of the GHRM, whereas the dot-dashed curves correspond to those of the GVDW and the TRZ models. The circles represent the MC data (Torrie and valleau (1982)). The dashed curves are the MGC density profiles calculated with the analytical solution for 2:1 RPM electrolytes found by Grahame (1953). As shown in Fig. 2 , each of the three density functional approximations predicts a counterion profile with a 


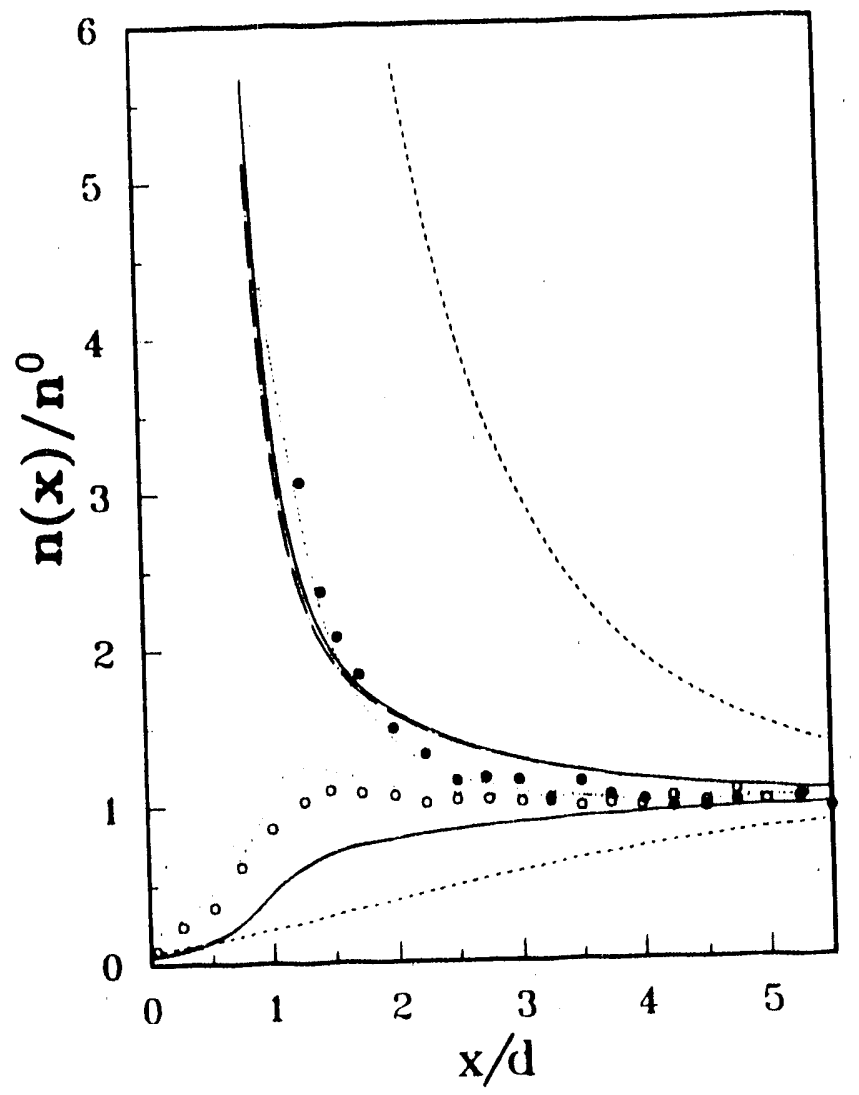

Figure 5. Reduced density profiles, $n(x) / n^{0}$, of a $2: 1$ electrolyte at $c=0.05 \mathrm{M}$ and $\sigma^{*}=$ -0.2. All symbols as in Fig. 2 except that the dot curves are the results of the OZ/LMBW theory (Plischke and Henderson (1989).

shallow minimum around $x=2.5 d$. To this minimum corresponds a maximum in the coion density profile. The position of the charged surface is shifted to $x=-0.5 d$ in this figure and in all the figures to be presented. The MC results seem to show the same oscillatory behavior, but the oscillation is partially hidden by the statistical uncertainty of the data. As usual, the MGC theory predicts monotonic density profiles. The oscillatory behavior is also apparent in MC profile of the mean electrostatic potential. In Fig. 3 we see the MC data of the function $\psi(x)$ have a change in sign around $x^{t}=1.5 d$ and present a shallow 


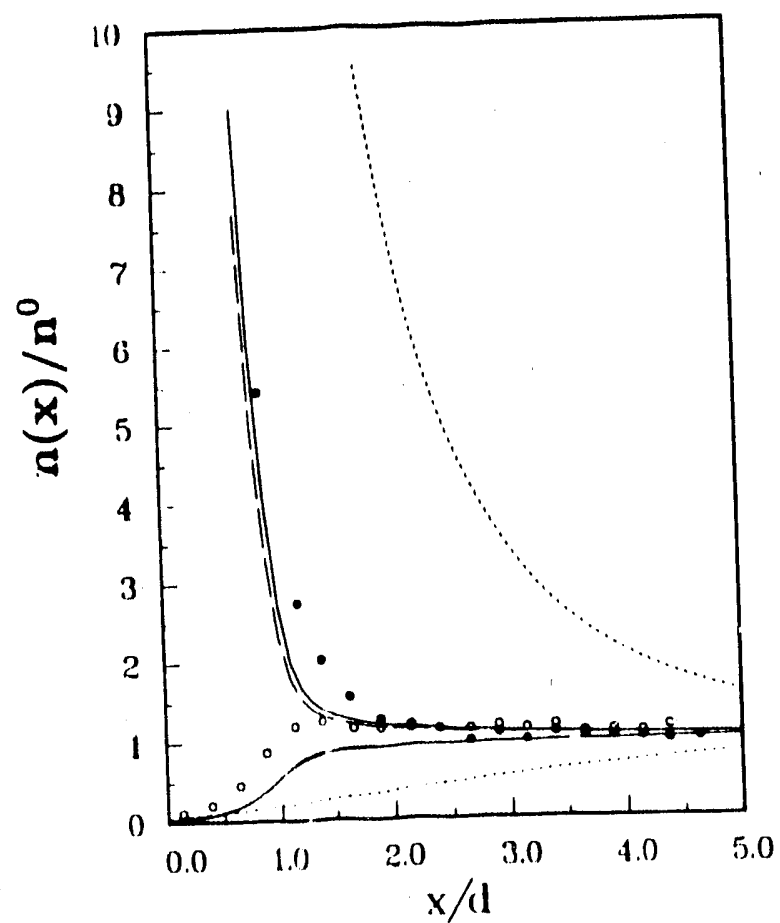

Figure 6. Reduced density profiles, $n(x) / n^{0}$, of a 2:1 electrolyte at $c=0.05 \mathrm{M}$ and $\sigma^{*}=$ -0.284 . All symbols as in Fig. 2.

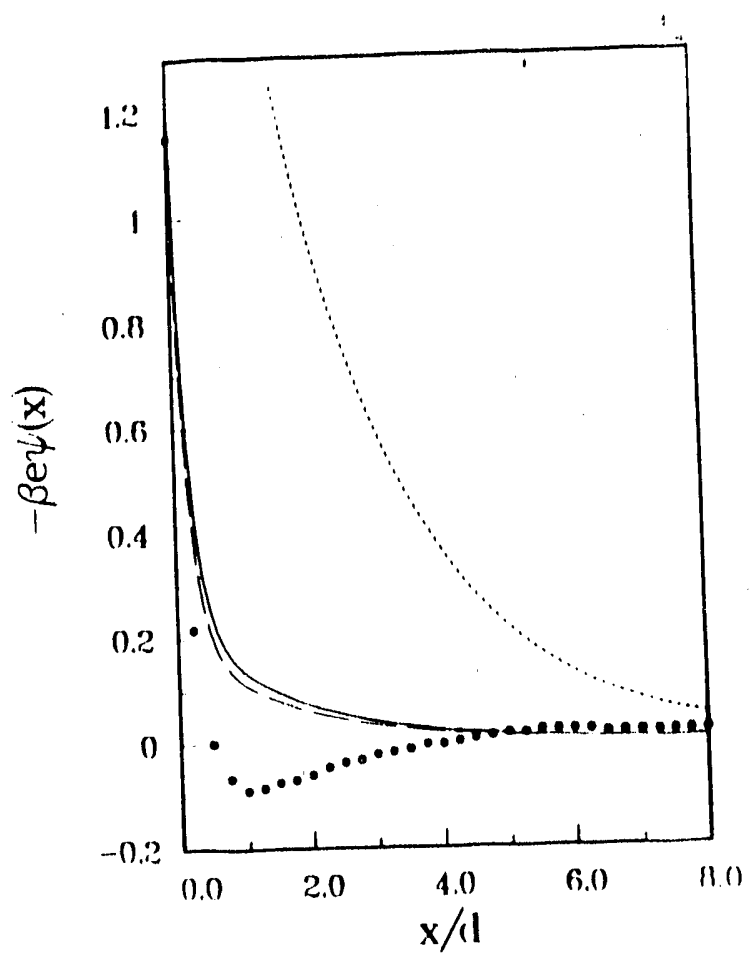

Figure 7. Reduced mean electrostatic potential profile, $-\beta e \psi(x)$, of a 2:1 electrolyte at $c=0.05 \mathrm{M}$ and $\sigma^{*}=-0.284$. All symbols as in Fig. 2.

minimum. The three density functional models predict quite well the location and the size of this minimum in $\psi(x)$.

For 2:1 electrolytes next to a negatively charged surface, the counterions are doubly charged. In these systems, the density and the potential profiles are monotonic at low surface charges. This is the case for $c=0.005 \mathrm{M}$ and $\sigma^{*}=-0.05$ : see Fig. 4 , where the results of the three density functional models for the potential profile $\psi(x)$ coincide up to the scale used in our plot. The density functional theory predicts results which represent considerable improvements over the MGC theory, but it still overestimates the thickness of the double layer.

At higher concentrations and surface charges the $\mathrm{MC}$ density profiles are no longer monotonic. In Fig. 5 we present the density profiles at $c=0.05 \mathrm{M}$ and $\sigma^{*}=-0.20$. The coion density profile obtained in the MC simulation has a peak around $x=1.5 d$. The results obtained by Plischke and Henderson (1989) show such a peak and agree quite well with the MC simulation. The density profiles predicted by the density functional theory are still monotonic but show a tendency towards an oscillatory behavior. As before, the difference between the GVDW and the TRZ models is negligible. Differences between the GHRM and the other two density functional approximations are also small in this case. These three approximations produce coion density profiles which are indistinguishable in the figure. At the larger surface charge of $\sigma^{*}=-0.284$ and the same concentration $c$ $=0.05 \mathrm{M}$, the $\mathrm{MC}$ data show a small charge inversion between $x=2.5 d$ and $4.5 d$ : see 


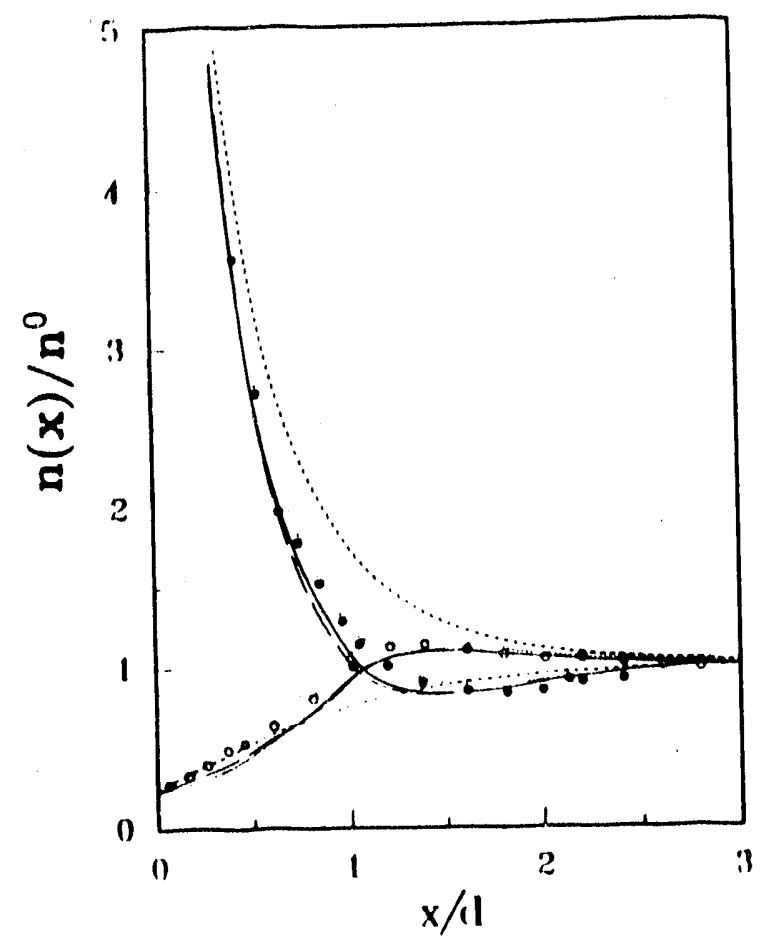

Figure 8. Reducer! density profiles, $n(x) / n^{0}$, of a 2:1 electrolyte at $c=0.5 \mathrm{M}$ and $\sigma^{*}=$ -0.1704 . All symbols as in Fig. 2 except that the dot curves are the results of the $\mathrm{OZ} / \mathrm{LMBW}$ theory (Plischke and Henderson (1989))

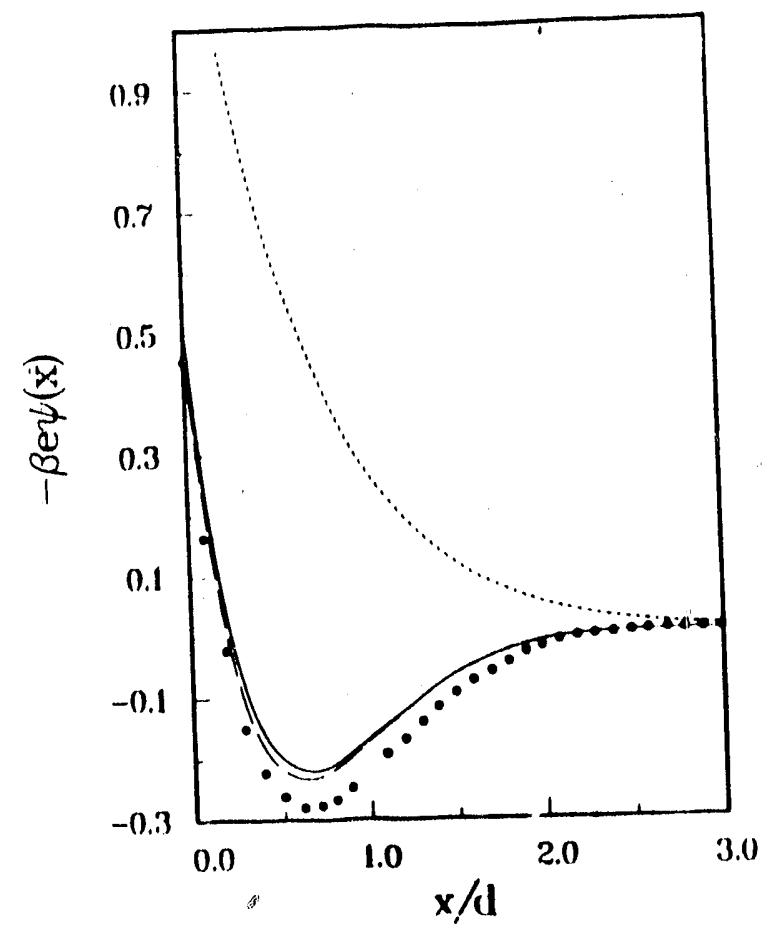

Figure 9. Reduced mean electrostatic potential profile, $-\beta e \psi(x)$, of a $2: 1$ electrolyte at $c=0.5 \mathrm{M}$ and $\sigma^{*}=-0.1704$. All symbols as in Fig. 2.

Fig. 6. The profiles predicted by the density functional models are still monotonic and the differences between them continue to be small. The MGC density profiles are also monotonic. The charge inversion presents in the MC data at these conditions produces an oscillation in the mean electrostatic potential profile with a minimum around $x=d$ : see Fig. 7. The free energy density functional approximations, however, fail to predict this minimum in their potential profiles.

The oscillatory behaviors of the density and the mean electrostatic potential profiles are more evident at higher concentrations. In Fig. 8 we present a comparison of the MC density profiles with those of the density functional theory and those of OZ/LMBW at $c=0.5 \mathrm{M}$ and $\sigma^{*}=-0.1704$. For comparison, in the same figure we also display the results of the MGC theory, which show no oscillation. The charge inversion is pronounced and clearly seen in the profiles predicted by the density functional theory. The results of the GHRM and the GVDW (and the TRZ) model are distinguishable within some ranges, but the difference between them is still small. The results of $\mathrm{OZ} / \mathrm{LMBW}$ agree a little better with the MC profiles than our results do. The charge inversion found in this case produces a relatively deep minimum in the mean electrostatic potential profile. In Fig. 9 we compare our results of the potential profile with the MC data. The density 
functional theory predicts correctly the oscillation of the mean electrostatic potential, but the deepness of the minimum is underestimated.

\section{Summary}

We have applied three different nonlocal free energy density functional approximations to the electrical double layer consisting of a 2:1 RPM electrolyte in the presence of a planar charged surface. The approach we have used in this work is the same as that in previous works (Meir y Teran et al. (1990); Tang et al. (1990)). For 2:1 electrolytes, the free energy density functional theory predicts correctly the behavior of the diffuse layer potential as a function of the surface charge density: the asymmetry with respect to the sign of the surface charge and the existence of an extremum.

When the counterions are singly charged, the density functional theory is quantitatively accurate in predicting the ionic density profiles and the mean electrostatic potential profiles of 2:1 electrolytes. Our values of the diffuse layer potential agree within a few percent with the Monte Carlo (MC) data at the densities and surface charges explored. The generalized hard-rod model (GHRM) is slightly better than the generalized van der Waals (GVDW) model and the Tarazona (TRZ) model in this case; this is the same conclusion as for 1:1 electrolytes (Tang et al. (1990)). The differences between the results of theory and the simulations grow slowly when the surface charge density increases.

When the counterions are doubly charged, the density functional theory predicts results which agree only qualitatively with the $\mathrm{MC}$ simulations. At low concentrations, the theory predicts correctly the monotonic behaviors of the density and the mean electrostatic potential profiles at small surface charges, but fails to predict the oscillatory behaviors present in the $\mathrm{MC}$ results when the surface charge is increased. At high concentrations, the density and the mean electrostatic potential profiles obtained in this work show the charge inversion phenomenon in agreement with the $\mathrm{MC}$ simulations.

The GVDW model is the zeroth-order approximation of the TRZ model. Thus, the small differences found between the predictions of these two models within the intervals of concentration and surface charge density $\sigma$ studied, for both positive and negative values of $\sigma$, show that the zeroth-order term in the weighting function of the TRZ model completely overshadows the other two terms in this regime. This feature makes the GVDW model more attractive than the TRZ model in applications to more complicated systems when the fluid inhomogeneity is not very large, because the GVDW model is almost as accurate as the TRZ model in this case and it can be generalized (to mixtures of hard spheres of different sizes, for example) and numerically implemented much more easily. 


\section{References}

Caccamo, C., Pizzimenti, G., and Blum, L., J. Chem. Phys., 84, 2327, 1986.

Chapman, D. L., Phil. Mag., 25, 475, 1913.

Fischer, J., and Heinbuch, U., J. Chem. Phys., 88, 1909, 1988.

Gouy, G., J. Phys., 9, 451, 1910.

Grahame, D. C., J. Chem. Phys., 21, 1054, 1953.

Lozada-Cassou, M., and Henderson, D., J. Phys. Chem., 87, 2821, 1983.

Mier-y-Teran, L., Falls, A. H., Scriven, L. E., and Davis, H. T., Proceeding 8th Symposium of Thermophysical Properties, ed. Sengers, J. V., American Society of Mechanical Engineers, New York, Vol. 1, pp. 45-56 1982.

Mier-y-Teran, L., Suh, S. H., White, H. S., and Davis. H. T., J. Chem. Phys. 92, 5087, 1990.

Nordholm, S., Johnson, M., and Freasier, B. C., Aust. J. Chem., 33, 2139, 1980; Johnson, M., and Nordholm, S., J. Chem. Phys., 75, 1953, 1981.

Outhwaite, C. W., and Bhuiyan, L. B., J. Chem. Soc. Faraday Trans. 2, 79, 707, 1983;

J. Chem. Phys., 85, 4206, 1986.

Percus, J. K., J. Chem. Phys., 75, 1316, 1981.

Plischke, M., and Henderson, D., J. Chem. Phys., 90, 5738, 1989.

Robledo, A., and Varea, C., J. Stat. Phys., 26, 513, 1981.

Stern, O., Z. Electrochem., 30, 508, 1924.

Tang, Z., Mier-y-Teran, L., Davis, H. T., Scriven, L. E., and White, H. S., Molec. Phys., accepted. (1990).

Tarazona, P., Phys. Rev. A, 31, 2672, 1985; Tarazona, P., Marini Bettolo Marconi, U., and Evans, R., Molec. Phys., 60, 573, 1987.

Torrie, G. M., and Valleau, J. P., J. Chem. Phys., 73, 5087, 1980; Torrie, G. M., Valleau, J. P., and Patey, G. N., ibid., 76, 4615, 1982; Valleau, J. P., and Torrie, G. M., ibid., 76, $4623,1982$.

Torrie, G. M., and Valleau, J. P., J. Phys. Chem., 86, 3251, 1982.

Vanderlick, T. K., Davis, H. T., and Percus, J. K., J. Chem. Phys., 91, 7136, 1989b.

Vanderlick, T. K., Scriven, L. E., and Davis, H. T., J. Chem. Phys., 90, 2422, 1989a.

Waismann, E., and Lebowitz, J. L., J. Chem. Phys., 56, 3086; 1972, ibid., 56, 30931972. 


\section{A NONLOCAL FREE ENERGY DENSITY FUNCTIONAL APPROXIMATION FOR THE ELECTRICAL DOUBLE LAYER.}

\section{Synopsis}

We construct a free energy density functional approximation for the primitive model of the electrical double layer. The hard sphere term of the free energy functional is based on a nonlocal generic model functional proposed by Percus. This latter model functional, which is a generalization of the exact solution for the non-uniform hard rod model, requires as input the free energy of a homogeneous hard-sphere mixture. We choose the extension of the Carnahan-Starling equation of state to mixtures. The electrostatic part of the non-uniform fluid ion-ion correlations present in the interface, is approximated by that of an homogeneous bulk electrolyte. Using the mean spherical approximation for a neutral electrolyte, we apply the theory to symmetrical $1: 1$ and $2: 2$ salts in the restricted primitive model. We present comparisons of density profiles and diffuse layer potentials with GouyChapman theory and Monte Carlo data. We also compare our results with data from other recent theories of the double layer. For highly charged surfaces, the profiles show the layering of counterions and charge inversion effects, in agreement with Monte Carlo data.

\section{Introduction}

Understanding the behavior of charged particles near charged surfaces is an important problem in physical chemistry. Separation of charge in response to the field of the charged surface is referred to as the electrical double layer. Double layers are present in electrochemistry in the form of the electrode/electrolyte interface, and they often play a major role in the stability of soap films, colloidal dispersions, and biological membranes. As a result of the occurrence of double layers in numerous situations, there has been a considerable effort to describe them theoretically. The early theory that met with significant success was that of Gouy (1910) and Chapman (1913) and was based on the PoissonBoltzmann equation. More recently, theory has been built on more rigorous methods of the statistical mechanics of the liquid state (Carnie and Torrie, 1984). Because the physical systems in which double layer occur are generally quite complicated (for a recent review see Schmickler and Henderson, 1986), theoretical efforts have been directed towards determination of the properties of greatly simplified models. The Gouy-Chapman theory (Gouy, 1910; Chapman, 1913) was developed for a model of point charges next to a uniformly charged planar surface, for example. A later modification of this theory by Stern (Stern, 1924), known as the modified Gouy-Chapman theory (MGC), is based on the same model. This description is quite accurate provided the real ionic radius is not too large compared to ionic spacing and the charge on the surface and on the particles are relatively small (low density-weakly coupled systems). At higher densities or for highly coupled systems, the core interaction becomes important. Thus, to take into account of the finite size of the charged particles, many authors focused their attention on a model electrical double layer composed of charged hard spheres at a hard, planar, polarizable, 
uniformly charged suriace. The ions cre assumed to be immersed in a continuum with a dielectric constant which may be different from that of the charged wall. This model is known as the primitive model (PM) of the double layer.

There is a considerable body of recent work on the PM double layer. For testing theory, the Monte Carlo (MC) simulations by Valleau and co-workers (Valleau, et al. 1980-1982) are especially significant. There is the work on the modified Poisson-Boltzmann (MPB) approximation (Outhwaite, et al. 1978-1986), which is based on the Kirkwood (Carnie and Torrie, 1984) hierarchy. In that approach the effects of the wall on the ion-ion correlations are handled in a natural way. On the other hand, the work based on the singlet OrnsteinZernike (OZ) equation (Hendorson et al.,1976) with the mean spherical approximation (MSA) or the hypernetted chain approximation (HNC) stresses a more carefu! treatment of the effects due to the finite size of the ions while the direct ion-ion correlation functions near the surface as a approximated by the functions calculated in the bulk solution (Blum. 1977) There is also work based on the Born-Green-Yvon (BGY) equation (Henderson, et al, 1981; Bruno, $\epsilon$ al al 1987) for the inhomogeneous pair correlation functions as closures for the BGY equation, this latter method emphasizes the importance of properly handling the ion-ion correlation functions near the wall. The BGY equation exactly satisfies the contact theorem (Carnie and Torrie, 1984). This is especially important in the high density-high coupling regime. The recent work by Forstmann and collaborators (Daguanno, et al., 1986; Alts, et al., 1987) in which the ion-ion direct correlation functions are computed using the MSA at a local non-neutral concentration (HNC/LMSA), is also aimed at building into the theory good pair correlation functions. Similar in spirit to the work of Forstmann and collaborators is that of Kjellander and Marcelja (Kjeilander, et al, 1985, 1986) in which the double layer interaction between two uniformly charged surfaces immersed in an electrolyte solution is calculated. Perhaps the most accurate recent work is that of Plischke and Henderson (1989). In that work, the inhomogeneous $\mathrm{OZ}$ equation with the $\mathrm{HNC}$ and MSA closures was solved together with the Lovett-Mou-Buff-Wertheim equation (Lovett, et al., $1976)$ for the density profiles of the : ons (OZ/LMBW).

Double layers are good examples of strongly inhomogeneous systems. In an electrolyte, especially at elevated surface charges, the density variations near the electrode are extremely large. The situation of an ion near the wall is tota" $y$ different from that of a similar ion in the neutral bulk. Ideally, a double layer theory should take into account the correlations arising from both the hard-core repulsion and the electrostatic interactions. Because these correlations are strongly dependent on the distance from the wall, only a few theories are able to handle properly the ion packing near the electrode. According to MC results for 1:1 electrolytes (Torrie, et al., 1982) at high electrode charges the counterions start the formation of a second layer before the first layer is densely packed. Of the theories mentioned above, only the HNC/LMSA, the BGY, the Kellander and Marcelja and the $\mathrm{OZ} / \mathrm{LMBW}$ theories are able to predict the formation of the second layer of counterions.

Parallel to the development of the double layer theories, the last de de has seen a great deal of activity in. the study of non-uniform fluids using free energy density func- 
tional theories. This method, which originated with van der Waals (1908), requires the construction of an expression for the free energy of the inhomogeneous system. Even though the rigorous statistical mechanics formalism of density functional theory was established more than twenty years ago (Stell, 1964;Percus 1964), the reduction of the exact results to tractable accurate approximations has been the goal of many, more recent investigations (Ebner, et al., 1976; Davis and Scriven, 1982; Evans, 1979; Nordholm, et al, 1982; Tarazona, 1985; Curtin and Ashcroft, 1985; Meister and Kroll, 1985). Treatments based on local density approximations have proven useful to describe weakly structured systems, like fluid-fluid interfaces (Ebner, 1976; Davis and Scriven, 1982; Evans, 1979), or fluids in weak external fields, but are not applicable to the strong inhomogeneities characteristic of fluid-solid interfaces. In order to handle the strong inhomogeneities present in fluid-solid interfaces, a nonlocal approach was introduced by Nordholm and coworkers (Nordholm, et al, 1982) in their generalized van der Waals theory (GVDW). Since then, systematic improvement. in the method in which finite size effects are considered have been published (Tarazona, 1985; Meister and Kroll, 1985) of nonlocal theories include the studies of the structure of confined fluids (Heffelfinger, et al, 1989; Ball and Evans, 1988; Vanderlick et al., 1990; Vanderlick, et al., 1989), capillary condensation (Tarazona, et al., 1987; Peterson, et al, 1988), layering transitions (Ball and Evans, 1988), and the planar electrical double layer (Boyle, et al., 1987). Very recently, the nonlocal smoothed-density approach (SDA) due to Tarazona (Tarazona, 1985), was extended to binary hard sphere mixtures with arbitrary size ratio (Tan, et al., 1989).

In this work, we present a theory for the electrical double layer in which the effects due to the finite size of the particles are considered within the framework of a generic nonlocal functional proposed by Percus (Percus, 1981) and generalized to multicomponent fluids by Vanderlick et al.((1990). This generic functional can be used to generate the functionals of several known nonlocal approaches (Vanderlick et al., 1989). These include the GVDW, the SDA, and a functional proposed by Robledo and Varea (Robledo and Varea, 1981), and by Fischer and Heinbuch (Fischer and Heinbuch, 1988), as a generalization of Percus' exact solution of the one dimensional hard-rod system (Percus, 1976). We use the latter, termed here generalized hard-rod model (GHRM), to construct a model functional for the electrical interface. Its advantage over the OZ/LMBW theory used by Plischke and Henderson is that its working equations are much cheaper to evaluate.

The article is as follows. The PM of a planar double layer is described in Sec. II. The general free energy density functional formalism for the electrical double layer is presented in Sec. III. In Sec. IV we report our results for the density profiles and electrostatic potential and compare these with the $\mathrm{MC}$ results of Valleau and co-workers (Torrie et al., 1982 ) and with results of some of the theories mentioned above.

\section{Primitive model}

In the primitive model of the electrical double layer, the electrolyte is assumed to 
be a fluid of charged hard spheres of charge $q_{\alpha}$ and diameter $d_{\alpha}$ immersed in a dielectric continuum of dielectric constant $\epsilon$. Separating the Coulombic and short-range repulsive contributions to the pair interaction, we have

$$
u_{\alpha \beta}\left(\mathbf{r}, \mathbf{r}^{\prime}\right)=q_{\alpha} q_{\beta} u^{c}\left(\left|\mathbf{r}-\mathbf{r}^{\prime}\right|\right)+u_{\alpha \beta}^{\mathrm{r}}\left(\left|\mathbf{r}-\mathbf{r}^{\prime}\right|\right),
$$

where

$$
u^{c}(r)=1 / \epsilon r \quad
$$

and

$$
\begin{aligned}
u_{\alpha \beta}^{r}(r) & =\infty, & & r<\left(d_{\alpha}+d_{\beta}\right) / 2 \\
& =0, & & r>\left(d_{\alpha}+d_{\beta}\right) / 2 .
\end{aligned}
$$

The electrode is considered to be an infinite flat hard wall with a uniform charge density $\sigma$. This impenetrable hard wall produces a repulsive potential, for particles of species $\alpha$, of the form

$$
\begin{aligned}
v_{\alpha}^{r}(x) & =\infty, & x<d_{\alpha} / 2, \\
& =0, & x>d_{\alpha} / 2,
\end{aligned}
$$

where $x$ is the ion's distance to the plate. On the other hand, the uniform surface charge density gives rise to a Coulombic potential of the following form

$$
v^{c}(x)=-2 \pi \sigma|x| / \epsilon+C
$$

where $C$ is a constant which depends on the choice of the point of zero potential.

In order to eliminate image charges, the dielectric constant is also taken to be $\epsilon$ in the region $x \leq d_{\alpha} / 2$. The total external potential can now be written as

$$
v_{\alpha}(x)=q_{\alpha} v^{c}(x)+v_{\alpha}^{r}(x) \text {. }
$$

A quantity of an enormous importance in the electrical double layer theory is the mean electrostatic potential. The mean electrostatic potential $\psi(\mathbf{r})$ at a point $\mathbf{r}$ is related to the density distribution functions $n_{\alpha}(x)$ in the following way

$$
\psi(\mathbf{r})=v^{c}(x)+\int d^{3} r^{\prime} u^{c}\left(\left|\mathbf{r}-\mathbf{r}^{\prime}\right|\right) \sum_{\infty} q_{\alpha} n_{\alpha}\left(x^{\prime}\right) .
$$

The formal solution to Poisson's equation yields the following expression for the mean electrostatic potential

$$
\psi(x)=\frac{4 \pi}{\epsilon} \int_{x}^{\infty} d x^{\prime}\left(x-x^{\prime}\right) \sum_{\alpha} q_{\alpha} n_{\alpha}\left(x^{\prime}\right) .
$$

The boundary conditions used in arriving at Eq.(2.8) are $\psi(\infty)=0$, and

$$
\left.\frac{d \psi(x)}{d x}\right|_{x=0}=\frac{-4 \pi \sigma}{\epsilon}
$$


In the derivation of Eq. (2.8) we required

$$
\int_{0}^{\infty} d x^{\prime} \sum_{\alpha} q_{\alpha} n_{\alpha}\left(x^{\prime}\right)=-\sigma
$$

which is the constraint of overall electroneutrality of the system. From Eq. (2.8) we can observe why the mean electrostatic potential evaluated at the closest appronch distance is frequently used as a measure of the charge scparation in the double layer.

\section{Density functional free energy theory}

\section{General formalism}

We start our study of the double layer problem with a discussion of the grand canonical density functional formalism for a mixture of ionic species in an external field. In this work we adopt the general approach due to Morita and Hiroike (Morita and Hiroike, 1961), De Dominicus (DeDominicus, 1962), Stillinger and Buff (1962) and to Lebowitz and Percus (1963), and used later by many investigators (Ebner, et al, 1976, Davis and Scriven, 1982; Saam and Ebner, 1977). The particle-particle direct correlation functions of a non-uniform fluid which appear in the formalism allow us to write an exact expression for the density distribution functions. The same formalism has been used by Forstmann and collaborators (1987) as the starting point of their HNC/LMSA theory of the double layer. This approach, which is due to Mermin (Mermin, 1965) and was employed by Hohenberg and Kohn (1964) for the inhomogeneous electron gas, is naturally expressed in the language of the grand canonical ensemble.

The properties of an interface and its coexisting bulk fluid are determined by the constancy of the chemical potentials, $\mu_{\alpha}$, and temperature, $T$, throughout the system. The free energy appropriate to the grand canonical ensemble is the grand potential. For a mixture of particles of different kinds $(\alpha=1, \ldots, \mathrm{c})$, the grand potential functional is

$$
\Omega=-k T \ln \Xi
$$

where $\Xi$ is the grand partition function and $k$ is Boltzmann's constant. The equilibrium density distribution is an unconstrained minimum of the grand potential functional, $\Omega$, where

$$
\Omega(\{\mathbf{n}\})=F(\{\mathbf{n}\})-\sum_{\alpha} \int d^{3} r \mu_{\alpha} n_{\alpha}(\mathbf{r}) .
$$

Here, $F(\{\mathbf{n}\})$ is the Helmholtz free energy functional of the system and $\{\mathbf{n}\}$ denotes the functional dependence of $\Omega$ and $F$ on the particle densities, $n_{\alpha}(\mathbf{r}), \alpha==1, \ldots, c$.

For a mixtıre of charged particles of species at absolute tempera ure $T$ in the field of 
an external potential $v_{\alpha}(\mathbf{r})$, the grand potential functional can be written as

$$
\begin{aligned}
\Omega(\{\mathbf{n}\}) & =\sum_{\alpha} \int d^{3} r n_{\alpha}(\mathbf{r})\left(v_{\alpha}(\mathbf{r})-\mu_{\alpha}\right) \\
& +k T \sum_{\alpha} \int d^{3} r n_{\alpha}(\mathbf{r})\left[\ell n\left(\Lambda_{\alpha}^{3} n_{\alpha}(\mathbf{r})\right)-1\right]-\phi(\{\mathbf{n}\}) .
\end{aligned}
$$

The second term on the right of Eq.(3.3) is the ideal gas contribution to the Helmholtz free energy and $\Lambda_{\alpha}$ is the thermal de Broglie wavelength of particles $\alpha$. The term $-\phi$ in the same equation corresponds to the interparticle interaction contribution to the free energy.

The grand potential functional $\Omega(\{\mathbf{n}\})$ is minimized, for fixed $v_{\alpha}(\mathbf{r})$ and $u_{\alpha \beta}\left(\mathbf{r}, \mathbf{r}^{\prime}\right)$, when $n_{\alpha}(\mathbf{r})$ takes its equilibrium value. In that case, $\Omega$ corresponds to the equilibrium grand potential function. The functional $-\phi$, on the other hand, can be used as a generating functional for $n$-body correlation functions, in particular, from the first functional derivative we obtain:

$$
\frac{1}{k T} \frac{\delta \phi(\{\mathbf{n}\})}{\delta n_{\alpha}(\mathbf{r})}=c_{\alpha}(\mathbf{r} ;\{\mathbf{n}\})
$$

while the second functional derivative of $\phi(\{\mathbf{n}\})$ defines the Ornstein-Zernike direct correlation function

$$
\frac{1}{k T} \frac{\delta^{2} \phi(\{\mathbf{n}\})}{\delta \mathbf{n}_{\alpha}(\mathbf{r}) \delta \mathbf{n}_{\beta}(\mathbf{r})}=c_{\alpha \beta}\left(\mathbf{r}, \mathbf{r}^{\prime} ;\{\mathbf{n}\}\right) .
$$

The equilibrium condition can then be expressed as

$$
\frac{\delta \Omega(\{\mathbf{n}\})}{\delta n_{\alpha}(\mathbf{r})}=k T \ln \left(n_{\alpha}(\mathbf{r}) / \zeta_{\alpha}\right)+v_{\alpha}(\mathbf{r})-k T c_{\alpha}(\mathbf{r} ;\{\mathbf{n}\})=0
$$

where $\zeta_{\alpha}=\Lambda_{\alpha}^{-3} \exp \left(\beta \mu_{\alpha}\right)$ is the fugacity of component $\alpha$ in the mixture and $\beta=1 / k T$.

By functional integration between an initial state $\mathbf{n}^{i}$, and a final state $\mathbf{n}$, it is possible to obtain

$$
\begin{aligned}
\phi(\{\mathbf{n}\}) & =\phi\left(\left\{\mathbf{n}^{i}\right\}\right)+k T \sum_{\alpha} \int d^{3} r\left[n_{\alpha}(\mathbf{r})-n_{\alpha}^{i}(\mathbf{r})\right] c_{\alpha}\left(\mathbf{r} ;\left\{\mathbf{n}^{i}\right\}\right) \\
& +k T \sum_{\alpha \beta} \iint d^{3} r d^{3} r^{\prime}\left[n_{\alpha}(\mathbf{r})-n_{\alpha}^{i}(\mathbf{r})\right] \\
& \times\left[n_{\beta}\left(\mathbf{r}^{\prime}\right)-n_{\beta}^{i}\left(\mathbf{r}^{\prime}\right)\right] \int_{0}^{1} d \lambda \int_{o}^{\lambda} d \lambda c_{\alpha \beta}\left(\mathbf{r}, \mathbf{r}^{\prime} ; \lambda\right)
\end{aligned}
$$

In order to obtain this result, the linear density path,

$$
n_{\alpha}(\mathbf{r} ; \lambda)=n_{\alpha}^{i}(\mathbf{r})+\lambda\left[n_{\alpha}(\mathbf{r})-n_{\alpha}^{i}(\mathbf{r})\right]
$$


was used for the integration. The parameter $\lambda$ can take values in the interval $0 \leq \lambda \leq 1$. Equations (3.3), (3.6) and (3.7) can now be employed to write the following expression for the grand potential functional:

$$
\begin{aligned}
\Omega(\{\mathbf{n}\}) & =\Omega\left(\left\{\mathbf{n}^{i}\right\}\right)+\sum_{\alpha} \int d^{3} r n_{\alpha}(\mathbf{r})\left[v_{\alpha}(\mathbf{r})-v_{\alpha}^{i}(\mathbf{r})\right] \\
& +k T \sum_{\alpha} \int d^{3} r n_{\alpha}(\mathbf{r}) \ln \left(n_{\alpha}(\mathbf{r}) / n_{\alpha}^{i}(\mathbf{r})\right) \\
& -k T \sum_{\alpha} \int d^{3} r\left[n_{\alpha}(\mathbf{r})-n_{\alpha}^{i}(\mathbf{r})\right] \\
& -k T \sum_{\alpha \beta} \iint d^{3} r d^{3} r^{\prime}\left[n_{\alpha}(\mathbf{r})-n_{\alpha}^{i}(\mathbf{r})\right]\left[n_{\beta}\left(\mathbf{r}^{\prime}\right)-n_{\beta}^{i}\left(\mathbf{r}^{\prime}\right)\right] \\
& \times \int_{0}^{1} d \lambda \int_{0}^{\lambda} d \lambda^{\prime} c_{\alpha \beta}\left(\mathbf{r}, \mathbf{r}^{\prime} ; \lambda^{\prime}\right) .
\end{aligned}
$$

When dealing with long-ranged Coulombic potentials it is convenient to define a shortrange part of the direct correlation function $c_{\alpha \beta}^{S R}\left(\mathbf{r}, \mathbf{r}^{\prime}\right)$ by

$$
c_{\alpha \beta}\left(\mathbf{r}, \mathbf{r}^{\prime}\right)=-\beta q_{\alpha} q_{\beta} u^{c}\left(\left|\mathbf{r}-\mathbf{r}^{\prime}\right|\right)+c_{\alpha \beta}^{S R}\left(\mathbf{r}, \mathbf{r}^{\prime}\right)
$$

The short-range correlation function, $c_{\alpha \beta}^{S R}\left(\mathbf{r}, \mathbf{r}^{\prime}\right)$, can be further separated by subtracting from it the hard-sphere contribution, $c^{H S}\left(\mathbf{r}, \mathbf{r}^{\prime}\right)$. This is

$$
\Delta c_{\alpha \beta}\left(\mathbf{r}, \mathbf{r}^{\prime}\right)=c_{\alpha \beta}\left(\mathbf{r}, \mathbf{r}^{\prime}\right)-c_{\alpha \beta}^{H S}\left(\mathbf{r}, \mathbf{r}^{\prime}\right) .
$$

These definitions allow us to rewrite the grand free energy functional as

$$
\begin{aligned}
\Omega(\{\mathbf{n}\}) & =\Omega\left(\left\{\mathbf{n}^{i}\right\}\right)+\sum_{\alpha} \int d^{3} r n_{\alpha}(\mathbf{r})\left[v_{\alpha}(\mathbf{r})-v_{\alpha}^{i}(\mathbf{r})\right] \\
& +k T \sum_{\alpha} \int d^{3} r n_{\alpha}(\mathbf{r}) \ln \left(n_{\alpha}(\mathbf{r}) / n_{\alpha}^{i}(\mathbf{r})\right) \\
& -k T \sum_{\alpha} \int d^{3} r\left[n_{\alpha}(\mathbf{r})-n_{\alpha}^{i}(\mathbf{r})\right] \\
& +\frac{1}{2} \sum_{\alpha \beta} \iint d^{3} r d^{3} r^{\prime}\left[n_{\alpha}(\mathbf{r})-n_{\alpha}^{i}(\mathbf{r})\right]\left[n_{\beta}\left(\mathbf{r}^{\prime}\right)-n_{\beta}^{i}\left(\mathbf{r}^{\prime}\right)\right] q_{\alpha} q_{\beta} u^{c}\left(\left|\mathbf{r}-\mathbf{r}^{\prime}\right|\right) \\
& -k T \sum_{\alpha \beta} \int \hat{d} d^{3} r d^{3} r^{\prime}\left[n_{\alpha}(\mathbf{r})-n_{\alpha}^{i}(\mathbf{r})\right]\left[n_{\beta}\left(\mathbf{r}^{\prime}\right)-n_{\beta}^{i}\left(\mathbf{r}^{\prime}\right)\right] \\
& \times \int_{0}^{1} d \lambda \int_{0}^{\lambda} d \lambda^{\prime} \Delta c_{\alpha \beta}\left(\mathbf{r}, \mathbf{r}^{\prime} ; \lambda^{\prime}\right)+\Delta F^{H S}(\{\mathbf{n}\}) .
\end{aligned}
$$


The last term on the right side of $\mathrm{Eq} .(3.11)$ represents the excess free energy change, between the initial state $\mathbf{n}^{i}$, and the final state $\mathbf{n}$, produced by the hard sphere interaction exclusively (in the presence of the other interactions).

U'sing the equilibrium condition, Eq.(3.6), we can obtain the following formal exprsssion for the equilibrium density profiles $n_{\alpha}(\mathbf{r})$ :

$$
\begin{aligned}
k T \ell n\left(n_{\alpha}(\mathbf{r}) / n_{\alpha}^{i}(\mathbf{r})\right)=-\left(v_{\alpha}^{r}(\mathbf{r})-v_{\alpha}^{r^{i}}(\mathbf{r})\right)-q_{\alpha}\left(\psi(\mathbf{r})-\psi^{i}(\mathbf{r})\right) \\
+k T \sum_{\beta} \int d^{3} r^{\prime}\left[n_{\beta}\left(\mathbf{r}^{\prime}\right)-n_{\beta}^{i}\left(\mathbf{r}^{\prime}\right)\right] \int_{0}^{1} d \lambda \Delta c_{\alpha \beta}\left(\mathbf{r}, \mathbf{r}^{\prime} ; \lambda\right) \\
\quad-\frac{\delta \Delta F^{H S}(\{\mathbf{n}\})}{\delta n_{\alpha}(\mathbf{r})} .
\end{aligned}
$$

In the derivation of $\mathrm{Eq}(3.12)$ ust has been made of the definition of the mean electrostatic potential, Eq. (2.7).

\section{Generalized hard-rod model}

The formalism presented above must be completed by specification of a model functional for the excess free energy of a hard-sphere mixture. Generalizing the exact result for an inhomogenecus system of hard-: ods, Percus (1981) and Vanderlick et al.(1990) have defined a generic free energy functional for th: inhomogeneour hard sphere fluid. In three dimensions, the free energy function of a hard sphere mixture is (Vanderlick, et al., 1990)

$$
F^{\text {excess }}=\sum_{\alpha} \int d^{3} r \bar{n}_{\alpha}^{\nu}(\mathbf{r}) F_{o}\left(\left\{\overline{\mathbf{n}}^{\top}(\mathbf{r})\right\}\right)
$$

where $F_{0}(\{n(\mathbf{r})\})$ is the excess free energy per pricle of a homogeneous mixture of hard spheres evaluated at the position $\mathbf{r}$ and $\bar{n}^{\nu}(\mathbf{r})$ and $n^{\tau}(\mathbf{r})$ are coarse grain densities. Each one of these densities is defined by a weighting function of the relative position to the hard sphere center, and, in the most general case, is also a functional of the density distribution,

$$
\begin{aligned}
& \bar{n}_{\alpha}^{\nu}(\mathbf{r})=\int d^{3} r^{\prime} \nu_{\alpha}\left(\mathbf{r}-\mathbf{r}^{\prime} ;\{\mathbf{n}\}\right) n_{\alpha}\left(\mathbf{r}^{\prime}\right), \\
& \bar{n}_{\alpha}^{\tau}(\mathbf{r})=\int \mathrm{d}^{3} \mathrm{r}^{\prime} \tau_{\alpha}\left(\mathbf{r}-\mathbf{r}^{\prime} ;\{\mathbf{n}\}\right) \mathrm{n}_{\alpha}\left(\mathbf{r}^{\prime}\right) .
\end{aligned}
$$

If these are to apply to a uniform fluid mixture they must satisfy the following normalization conditions:

$$
\int d^{3} r \nu_{\alpha}\left(\mathbf{r}-\mathbf{r}^{\prime} ;\{\mathbf{n}\}\right)=\int d^{3} r \tau_{\alpha}\left(\mathbf{r}-\mathbf{r}^{\prime} ;\{\mathbf{n}\}\right)=1
$$

In order to establish a theory for strongly inhomogereous fluids based on Eqs.(3.12)-(3.16), particular forms for the weighting functions $\nu$ and $\tau$ must be specified. The assignment 
of weighting functions generates different model density functionals. A discussion of how different appropriate selections of weighting functions generate several important model functionals can be found in the work of Vanderlick et al.(1989). Of particular importance for this work are the forms of $\nu(\mathbf{r})$ and $\tau(\mathbf{r})$ proposed by Robledo and Varea (1081), and by Fischer and Heinbuch (1988) as three dimensional generalizations of the hard-rod model. This model, termed here Generalized Hard Rod Model (GHRM), is characterized by the following weighting functions:

$$
\begin{gathered}
\nu_{\alpha}\left(\mathbf{r}-\mathbf{r}^{\prime}\right)=\delta\left(\left(d_{\alpha} / 2\right)-\left|\mathbf{r}-\mathbf{r}^{\prime}\right|\right) /\left(4 \pi\left(d_{\alpha} / 2\right)^{2}\right), \\
\tau_{\alpha}\left(\mathbf{r}-\mathbf{r}^{\prime}\right)=H\left(\left(d_{\alpha} / 2\right)-\left|\mathbf{r}-\mathbf{r}^{\prime}\right|\right) /\left(4 \pi\left(d_{\alpha} / 2\right)^{3} / 3\right),
\end{gathered}
$$

where $d_{\alpha}$ is the diameter of the particles in the fluid, $\delta(r)$ is the Dirac delta function, and $H(r)$ is the Heaviside step function:

$$
\begin{aligned}
H(r) & =1, \quad r \geq 0 \\
& =0, \quad r \leq 0
\end{aligned}
$$

In this model, the coarse grain density $\bar{n}_{\alpha}^{\nu}(\mathbf{r})$ is the average density of species over the surface of a sphere of radius $d_{\alpha} / 2$. The coarse-grain density $\bar{n}_{\alpha}^{\tau}(\mathbf{r})$ is the average density of species inside a sphere of radius $d_{\alpha} / 2$.

The functional in Eq. (3.13) allows us to determine an expression for the free energy change $\Delta F^{H S}(\{\mathbf{n}\})$ appearing in Eq.(3.11). It is

$$
\Delta F^{H S}(\{\mathbf{n}\})=\sum_{\alpha} \int d^{3} r \int_{0}^{1} d \lambda \frac{\partial}{\partial \lambda}\left(\bar{n}_{\alpha}^{\nu}(\mathbf{r} ; \lambda) F_{o}\left(\left\{\overline{\mathbf{n}}^{\tau}(\mathbf{r} ; \lambda)\right\}\right)\right) .
$$

Using this expression we can finally rewrite our equation for the equilibrium density profiles, Eq.(3.12), as

$$
\begin{aligned}
k T \ell n\left(n_{\alpha}(\mathbf{r}) / n_{\alpha}^{i}(\mathbf{r})\right) & =-\left(v_{\alpha}^{r}(\mathbf{r})-v_{\alpha}^{r^{i}}(\mathbf{r})\right)-q_{\alpha}\left(\psi(\mathbf{r})-\psi^{i}(\mathbf{r})\right) \\
& +k T \sum_{\beta} \int d^{3} r^{\prime}\left[n_{\beta}\left(\mathbf{r}^{\prime}\right)-n_{\beta}^{i}\left(\mathbf{r}^{\prime}\right)\right] \int_{0}^{1} d \lambda \Delta c_{\alpha \beta}\left(\mathbf{r}, \mathbf{r}^{\prime} ; \lambda\right) \\
& -\sum_{\beta} \int d^{3} r^{\prime} \int_{0}^{1} d \lambda \frac{\partial}{\partial \lambda}\left[\frac{\delta \bar{n}_{\beta}^{\nu}\left(\mathbf{r}^{\prime} ; \lambda\right)}{\delta n_{\alpha}(\mathbf{r})} F_{o}\left(\left\{\overline{\mathbf{n}}^{\tau}\left(\mathbf{r}^{\prime}\right) ; \lambda\right\}\right)\right] \\
& -\sum_{\beta} \int d^{3} r^{\prime} \int_{0}^{1} d \lambda \frac{\partial}{\partial \lambda}\left[\bar{n}_{\beta}^{\nu}(\mathbf{r}) \sum_{\gamma} \frac{\partial F_{o}\left(\left\{\tilde{\mathbf{n}}^{\top}\left(\mathbf{r}^{\prime} ; \lambda\right)\right\}\right)}{\partial \bar{n}_{\gamma}^{\tau}\left(\mathbf{r}^{\prime}\right)} \frac{\delta \bar{n}_{\gamma}^{\tau}\left(\mathbf{r}^{\prime} ; \lambda\right)}{\delta n_{\alpha}(\mathbf{r})}\right] .
\end{aligned}
$$

To study a bulk fluid in equilibrium with a planar electrode we now identify the initial state $\left\{\mathbf{n}^{i}\right\}$ with the neutral bulk electrolyte. This corresponds to an homogeneous 
solution in which no external forces are present. Since we are considering an infinite plane with uniform charge density, local densities vary only in the direction $x$ normal to the wall. Additionally, Eq.(3.21) requires the knowledge of inhomogeneous direct correlation function in excess over the hard sphere, $\Delta c_{\alpha \beta}\left(\mathbf{r}, \mathbf{r}^{\prime}\right)$, for all the possible positions $\mathbf{r}$ and $\mathbf{r}^{\prime}$ across the interface. Since these correlation functions are not known, we approximate the function $\Delta c_{\alpha \beta}\left(\mathbf{r}, \mathbf{r}^{\prime}\right)$ with the function $\Delta c_{\alpha \beta}\left(\left|\mathbf{r}-\mathbf{r}^{\prime}\right|\right)$ of the homogeneous neutral bulk electrolyte in equilibrium with the interface; this is

$$
\Delta c_{\alpha \beta}\left(\mathbf{r}, \mathbf{r}^{\prime} ; \lambda\right) \cong \Delta c_{\alpha \beta}\left(\mathbf{r}, \mathbf{r}^{\prime} ; \lambda=0\right)=\Delta c_{\alpha \beta}\left(\left|\mathbf{r}-\mathbf{r}^{\prime}\right|\right)
$$

It is convenient to emphasize here that $\Delta c_{\alpha \beta}\left(\left|\mathbf{r}-\mathbf{r}^{\prime}\right|\right)$ is a pair correlation function for a neutral bulk electrolyte whereas the interface is locally non-neutral. With this approximation, and by using $\mathrm{F}_{\text {q.s }}$ (3.14) and (3.15) for the GHRM, Eq.(3.21) can be rewritten, for a planar symmetry, as

$$
\begin{aligned}
n_{\alpha}(x) / n_{\alpha} & =\exp \left\{-\beta q_{\alpha} \psi(x)\right. \\
& -\int_{0}^{\infty} d x^{\prime} \nu_{x \alpha}\left(x-x^{\prime}\right) \beta F_{o}\left(\left\{\overline{\mathbf{n}}^{r}\left(x^{\prime}\right)\right\}\right)+\beta F_{o}(\mathbf{n}) \\
& -\sum_{\beta} \int_{0}^{\infty} d x^{\prime} \bar{n}_{\beta}^{\nu}\left(x^{\prime}\right) \frac{\partial \beta F_{o}\left(\left\{\overline{\mathbf{n}}^{r}\left(x^{\prime}\right)\right\}\right)}{\partial \bar{n}_{\alpha}^{r}\left(x^{\prime}\right)} \tau_{x \beta}\left(x-x^{\prime}\right)+\sum_{\beta} n_{\beta} \frac{\partial \beta F_{o}(\mathbf{n})}{\partial \mathbf{n}_{\alpha}} \\
& \left.+\sum_{\beta} \int d^{3} r^{\prime}\left[n_{\beta}\left(x^{\prime}\right)-n_{\beta}\right] \Delta c_{\alpha \beta}\left(\left|\mathbf{r}-\mathbf{r}^{\prime}\right| ;\{\mathbf{n}\}\right)\right\} \\
& \text { for } x>d_{\alpha} / 2,
\end{aligned}
$$

and

$$
n_{\alpha}(x)=0, \text { for } x<d_{\alpha} / 2 .
$$

For the planar symmetry the coarse grain densities $\bar{n}_{\alpha}^{\nu}(x)$ and $\bar{n}_{\alpha}^{\tau}(x)$ can be written as

$$
\tilde{n}_{\alpha}^{\nu}(x)=\int \nu_{x \alpha}\left(x-x^{\prime}\right) n_{\alpha}\left(x^{\prime}\right) d x^{\prime}
$$

and

$$
\bar{n}_{\alpha}^{\tau}(x)=\int \tau_{x \alpha}\left(x-x^{\prime}\right) n_{\alpha}\left(x^{\prime}\right) d x^{\prime},
$$

where the reduced weighting functions $\nu_{x \alpha}$ and $\tau_{x \beta}$, are defined by

$$
\begin{aligned}
& \nu_{x \alpha}(x)=\iint \nu_{\alpha}(\mathbf{r}) d y d z \\
& \tau_{x \alpha}(x)=\iint \tau_{\alpha}(\mathbf{r}) d y d z
\end{aligned}
$$


After integration over coordinates $y$ and $z$, we obtain, for the GHRM,

$$
\begin{gathered}
\nu_{x \alpha}(x)=H\left(\left(d_{\alpha} / 2\right)-|x|\right) / d_{\alpha}, \\
\tau_{x \alpha}(x)=6 H\left(\left(d_{\alpha} / 2\right)-|x|\right)\left(\left(d_{\alpha} / 2\right)^{2}-x^{2}\right) / d^{3} .
\end{gathered}
$$

We approximate now the excess free energy per particle $F_{o}$ of the homogeneous hardsphere fluid by the Carnahan-Starling (1970) equation of state. This equation was generalized to a mixture of hard spheres of different sizes by Mansoori, et al.(1971). Thr expression for the free energy is

$$
\begin{aligned}
\frac{F_{o}}{k T}= & \frac{-3}{2}\left(1-y_{1}+y_{2}+y_{3}\right)+\left(3 y_{2}+2 y_{3}\right)(1-\xi)^{-1} \\
& +\frac{3}{2}\left(1-y_{1}-y_{2}-\frac{1}{3} y_{3}\right)(1-\xi)^{-2}+\left(y_{3}-1\right) \ln (1-\xi)
\end{aligned}
$$

where

$$
\xi=\sum_{\alpha} \xi_{\alpha}, \quad \xi_{\alpha}=v_{\alpha} n_{\alpha}, \quad v_{\alpha}=\frac{\pi}{6} d_{\alpha}^{3} .
$$

The variables $y_{1}, y_{2}$, and $y_{3}$ are defined as follows:

$$
\begin{gathered}
y_{1} \equiv \frac{1}{2} \sum_{\alpha \beta} \Delta_{\alpha \beta}\left(d_{\alpha}+d_{\beta}\right) /\left(d_{\alpha} d_{\beta}\right)^{1 / 2}, \\
y_{2} \equiv \frac{1}{2} \sum_{\alpha \beta} \Delta_{\alpha \beta}\left(d_{\alpha} d_{\beta}\right)^{\frac{1}{2}} \sum_{\gamma} \xi_{\gamma} /\left(\xi d_{\gamma}\right), \\
y_{3} \equiv\left[\sum_{\alpha}\left(\xi_{\alpha} / \xi\right)^{\frac{2}{3}}\left(n_{\alpha} / n\right)^{\frac{1}{3}}\right]^{3},
\end{gathered}
$$

and

$$
\Delta_{\alpha \beta} \equiv\left(v_{\alpha} v_{\beta}\right)^{\frac{1}{2}} n_{\alpha} n_{\beta}\left(d_{\alpha}-d_{\beta}\right)^{2} /\left(\xi n d_{\alpha} d_{\beta}\right) .
$$

In the last two equations, $n$ is the total number density given by $\sum_{\alpha} n_{\alpha}$.

In their study of fluids confined between planar walls, Vanderlick et al. (1989) compared three different approximate density functional free energy theories of inhomogeneous fluids for hard spheres and Lennard-Jones potentials. Their study included the GHRM and the SDA due to Tarazona (1985). The results of that study show that whereas the GHRM is quantitatively inferior to the SDA, it is qualitatively correct. Since the GHRM captures the qualitative behavior of confined hard-sphere and Lennard-Jones fluids and retains enough mathematical simplicity we are encouraged to apply it to more complicated systems. 


\section{MSA Approximation for $\Delta \mathbf{c}_{\alpha \beta}\left(\left|\mathbf{r}-\mathbf{r}^{\prime}\right|\right)$}

It follows from Eq.(3.23) that our description of the electrical planar interface is still not complete without a prescription for bulk phase direct correlation functions $\Delta c_{\alpha \beta}$

$\left(\left|\mathbf{r}-\mathbf{r}^{\prime}\right|\right)$. Several choices can be immediately invoked from bulk electrolyte theory. A simple choice is to use the direct correlation functions of the MSA. The MSA is a relatively accurate approximation which generates analytic expressions for the direct correlation function of several important model potentials (Barker and Henderson, 1976). Waismann and Lebowitz (1972) showed that the integral equation resulting from the Ornstein-Zernike equation, has an analytical solution when the MSA closure for the restricted primitive model(RPM) is employed. The RPM is a still simpler model in which all ions have the same size; $d_{\alpha}=d$. For the RPM, the MSA provides the following expression for the function $\Delta c_{\alpha \beta}\left(\left|\mathbf{r}-\mathbf{r}^{\prime}\right|\right)$ :

$$
\begin{aligned}
\Delta c_{\alpha \beta}(s) & =\frac{-\beta q_{\alpha} q_{\beta}}{\epsilon}\left[(2 B / d)-(B / d)^{2} s-1 / s\right], s<d \\
& =0 \quad s>d,
\end{aligned}
$$

where $s=\left|\mathbf{r}-\mathbf{r}^{\prime}\right|$ and

$$
B=\left[\varphi+1-(1+2 \varphi)^{\frac{1}{2}}\right] / \varphi,
$$

and $\varphi=\kappa_{D} d$. The quantity $\kappa_{D}$ is the inverse Debye screening length given by

$$
\kappa_{D}^{2}=(4 \pi \beta / \epsilon) \sum_{\alpha}^{c} n_{\alpha} q_{\alpha}^{2} .
$$

The solution given by Eq.(3.36) holds for an arbitrary number, $c$, of ionic species provided global charge neutrality is maintained,

$$
\sum_{\alpha}^{c} n_{\alpha} q_{\alpha}=0
$$

The MSA direct correlation function is an important piece in the formulation of the HNC/MSA theory of the double layer (Blum, 1977) In that theory, the approximation

$$
c_{\alpha \beta}\left(\mathbf{r}, \mathbf{r}^{\prime} ; \lambda\right) \cong c_{\alpha \beta}\left(\mathbf{r}, \mathbf{r}^{\prime} ; \lambda=0\right)=c_{\alpha \beta}\left(\left|\mathbf{r}-\mathbf{r}^{\prime}\right|\right)
$$

is made; thus, the effect of the external potential and the inhomogeneities of the interface on $c\left(\mathbf{r}, \mathbf{r}^{\prime}\right)$ are entirely neglected. We believe that this approximation is more severe than the similar approximation of Eq.(3.22) used in this work.

\section{Results}

In this section we present our results for the density profiles, mean electrostatic potential profile, and diffuse layer potential drop for solutions containing symmetrical $1: 1$ and 
2:2 electrolytes. The results are compared with existing MC data and, when possible, with results obtained from several other approximations. The calculations were performed by means of the method of subdomains, finite element basis functions, collocation weighted residuals, (Strang and Fix, 1973) and Newton iteration with initialization chosen by parametric continuation (Mier y Teran et al.1982). We choose quadratic Lagrange interpolating polynomials as basis functions. This numerical technique was applied before to the solution or PY, HNC (Mier y Teran et al, 1982) and MSA (Mier y Teran, et al., 1985) integral equations for bulk simple fluids and was extended by Mier y Teran et al. (Blum, 1977) for solving the $\mathrm{HNC} / \mathrm{MSA}$ integral equation for the double layer problem. A detailed comparative discussion about the application of this method to the solution of $\mathrm{HNC} / \mathrm{MSA}$ equation for the double layer RPM and its efficiency and accuracy can be found in Mier y Teran et al., (1989).

With the algorithm mentioned above, we reduced the set of Eqs.(3.23) to a system of algebraic equations for the values of reduced density profiles at the nodes: $g_{\alpha i}=n_{\alpha}\left(x_{i}\right) / n_{\alpha}$. This nonlinear set of equations is solved by Newton's method. The iterative process is continued until the Euclidean norm of the updates after iteration $k+1$ becomes less than $10^{-10 .}$

$$
\left[\sum_{\alpha}^{2} \sum_{i=1}^{N}\left(g_{\alpha i}^{(k+1)}-g_{\alpha i}^{(k)}\right)^{2} / 2 N\right]^{\frac{1}{2}} \leq 10^{-10}
$$

where $N$ is the number of nodes in the domain $d / 2<x<R$, and $R$ is the cutoff value for the integrals in Eqs.(3-23). Both the number of nodes $N$ and the value of $R$ depend on concentration. We used a uniform mesh in the domain $d / 2<x<R$.

Either the charge density, $\sigma$, or the electrostatic potential at the electrode, $\psi_{e}$, can be specified and the equations solved. At very low charge densities, $\sigma$, or potentials, $\psi_{e}$, we found it convenient to us: the MGC density profiles as an initial guess. Once a solution for certain values of the parameters is found, initial estimates at other values for the parameters can be found easily by a first-order continuation technique. Typically, three to five Newton iterations are needed to reach convergence. After convergence was attained, the value of $\sigma$ or the value of $\psi_{e}$, depending on which quantity was used as the parameter, were computed using Eq.(2.8) or Eq.(2.10) respectively. The agreement with the value of $\sigma$ or $\psi_{e}$ originally used to solve the equations gives an indication of the accuracy of the numerical method. Except for the very low concentration regime, Eq.(2.8) or (2.10) was satisfied to at least five significant figures. In the most dramatic case treated; 1:1 electrolyte at $0.01 \mathrm{M}$, the dimensionles, Debye distance, $\varphi^{-1}$, becomes very large and Eq.(2.8) or (2.10) was satisfied to four siguiticant figures only.

In our calculations we have used dimensionless parameters. We express all lengths in units of the diameter $d$. The dimensionless surface charge is $\sigma^{*}=\sigma d^{2} / e$, where $e$ is the magnitude of the electronic charge. Similarly the dimensionless potential profile is 
$\psi^{*}(x)=\beta e \psi(x)$. In order to compare with the MC data of Valleau and co-workers, (Torrie et al., 1982) we fixed the value of the plasma parameter to $\Gamma=\beta e^{2} / \epsilon d=1.6809$. This value corresponds to

$$
\epsilon=78.5, \quad T=298 K^{\circ} \text { and } d=4.25 \AA \text {. }
$$

\section{$1: 1$ electrolytes}

We solved $\mathrm{Eq}(3.23)$ for electrolytes ranging from $0.01-2 \mathrm{M}$ and surface charge ranging from 0.05-0.9. In Table 1 we list the dimensionless diffuse layer potential, $\beta e \psi(0)$, where $\psi(0)$ is the potential drop between the point of closest approach to the surface and infinity. Note that the position of the wall has been shifted to $x=-d / 2$. In Table 1 we also display the MC results (Torrie et al., 1982), those of MGC, BGY (Bruno et al., 1987), MPB5 (Outhwaite et al., 1986) theories and the OZ/LMBW results obtained recently by Plischke and Henderson (1989) using the HNC closure. The general agreement of our results with the $\mathrm{MC}$ data is quite good. A clearer comparison of our results for 1:1 electrolytes with MC data is given by Fig. 1 where we plot the diffuse layer potential $\psi^{*}(0)$ as a function of the reduced charge density $\sigma^{*}$. As reported before by other authors (Bruno et al., 1987; Plischke and Henderson, 1989), in the low concentration regime, density profiles become very long ranged and special numerical difficulties appear. We believe that the discrepancies between our results and the MC data at $c=0.01 \mathrm{M}$ can be attributed, at least in part, to this cause. The crosses shown in Fig. 1 are the results of Plischke and Henderson (1989).

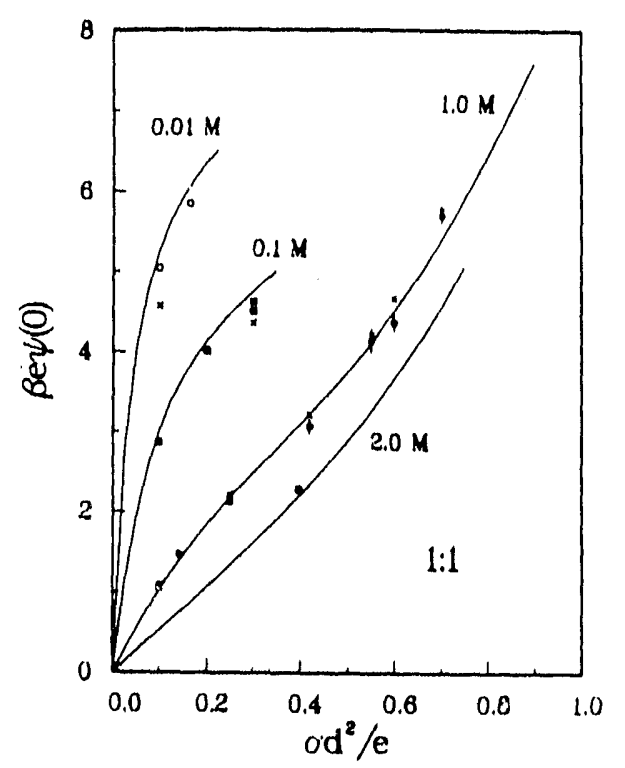

Fig. 1 Reduced diffuse layer potential, $\beta e \psi(0)$, as a function of the charge density, $\sigma d^{2} / e$, for 1:1 electrolytes. Solid lines represent the results of the GHRM functional density theory presented here for $0.01 \mathrm{M}, 0.1 \mathrm{M}, 1 \mathrm{M}$ and $2 \mathrm{M}$. Open circles, solid squares, solid circles and open squares are the corresponding $\mathrm{MC}$ results. The crosses $(\mathrm{x})$ are the results of the OZ/LMBW theory with the $\mathrm{HNC}$ closure.

The classical MGC theory, which neglects the finite size of the ions, predicts an in- 
TABLE 1. Diffuse layer potential

\begin{tabular}{|c|c|c|c|c|c|c|c|}
\hline $\mathrm{C}$ & $\sigma^{*}$ & MGC & $M C^{a}$ & $B G Y^{b}$ & $M P B 5^{c}$ & $P H^{d}$ & This w \\
\hline \multicolumn{8}{|c|}{$1: 1$ electrolytes } \\
\hline $0.01 \mathrm{M}$ & 0.10 & 5.44 & $5.05(0.05)$ & - & 5.08 & 4.58 & 5.26 \\
\hline $0.1 \mathrm{M}$ & 0.30 & 5.34 & $4.63(0.03)$ & 5.0 & 4.74 & 4.37 & 4.76 \\
\hline \multirow[t]{6}{*}{$1 \mathrm{M}$} & 0.10 & 1.4 & $1.09(0.06)$ & 1.055 & 1.03 & 1.06 & 1.03 \\
\hline & 0.25 & 2.79 & $2.13(0.05)$ & 2.31 & 2.10 & 2.22 & 2.18 \\
\hline & 0.42 & 3.74 & $3.08(0.1)$ & 3.46 & 3.02 & 3.23 & 3.23 \\
\hline & 0.55 & 4.26 & $4.15(0.15)$ & 4.21 & - & 4.22 & 4.12 \\
\hline & 0.60 & 4.43 & $4.38(0.11)$ & 4.48 & - & 4.68 & 4.52 \\
\hline & 0.70 & 4.74 & $5.71(0.14)$ & 5.02 & - & 5.76 & 5.41 \\
\hline \multirow[t]{2}{*}{$2 \mathrm{M}$} & 0.396 & 2.99 & $2.29(0.09)$ & 2.303 & - & 2.29 & 2.19 \\
\hline & \multicolumn{7}{|c|}{$2: 2$ electrolytes } \\
\hline $0.05 \mathrm{M}$ & 0.20 & 2.61 & $1.33(0.02)$ & 1.81 & 1.36 & 1.18 & 1.59 \\
\hline $0.5 \mathrm{M}$ & 0.1704 & 1.36 & $0.63(0.04)$ & 0.64 & 0.537 & 0.69 & 0.57 \\
\hline
\end{tabular}

a G. M. Torrie and J.P. Valleau,; 1980 and 1982. Statistical uncertainty is shown in parenthesis.

$b$ C. Carcamo, G. Pizzimenti, and L. Blum,; 1986.

c C.W. Outhwaite and L.B. Bhuiyan, ; 1986.

d M. Plischke and D. Henderson,1988.

Table 1. Diffuse Layer Potential

terfacial thickness which is greater than that obtained by $\mathrm{MC}$ simulations for low surface charge densities, and smaller than that of the MC data for large $\sigma^{*}$. This phenomenon is evident at $1 \mathrm{M}$ concentration. In Fig. 2 we plot the diffuse layer potential as a function of surface charge density for $\mathrm{c}=1 \mathrm{M}$. In that figure we also display the $\mathrm{MC}$ data, and the results obtained from the approximations listed in Table 1. The agreement of our results with $M C$ is very good and in some cases of comparable accuracy with those obtained by Plischke and Henderson (1989) with the OZ/LMBW and the HNC closure. The data available for the MPB5 theory show an excellent agreement with MC data. Unfortunately the data are for low values of $\sigma^{*}$ only, and because of the secondary role played by the excluded volume effects in the MPB5 theory, it is not expected that the theory can be applied at higher surface charges where the size effects are very important. The BGY 
theory of Bruno et al.(1987), which is very good for low $\sigma^{*}$ fails to predict the change iu curvature showe's by the $\mathrm{MC}$ results at intermediate charge densities.

The classical theory of Gouy-Chapman always predict monotonic variation for the density profiles of both co-ions and counterions. In contrast, for 1:1 electrolytes, the MC results of Valleau and collaborators (1982) for the structure of the RPM double layer exhibit interesting layering effects for high surface charges. In Fig. 3 we present a comparison of our results for the density profiles of a double layer for a bulk density of $c=0.1 \mathrm{M}$ and $\sigma^{*}=0.30$, with those corresponding to MC simulation and the MGC theory. Our results agree quite well with the MC results. All the profiles showed are monotonic in this case. In Fig. 4 we plot the mean electrostatic profile which correspond to the same condition presented in Fig. 3. Again we obtain good agreement with the MC results. The MGC theory is relatively successful in describing both density profiles and mean electrostatic potential at $c=0.1 M$ and $\sigma^{*}=0.30$.

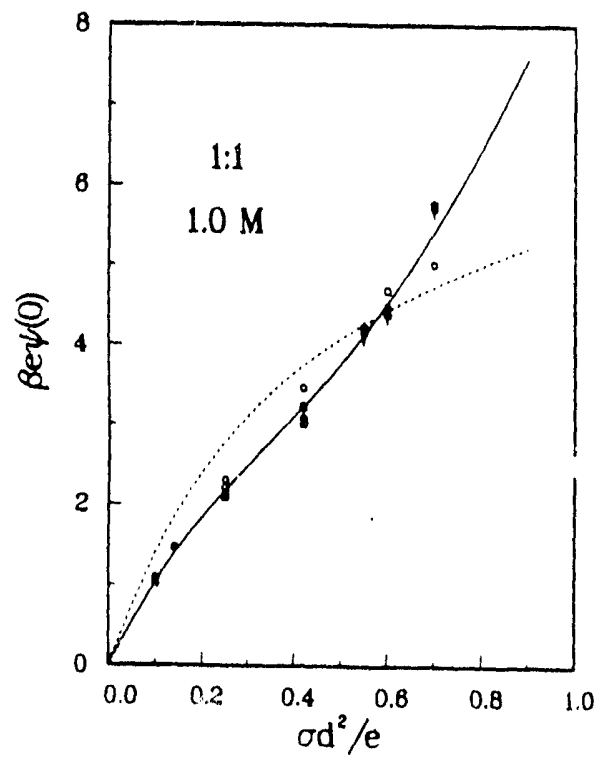

Fig. 2 Reduced diffuse layer potential, $\beta e \psi(0)$, as a function of charge density, $\sigma d^{2} / e$, for 1 M, 1:1 electrolytes. The solid line represents results of the functional density theory. Solid circles are the $\mathrm{MC}$ results of Valleau and collaborators, Solid squares correspond to the MPB5 theory, open circles to the BGY theory, and open squares to the $\mathrm{OZ} / \mathrm{LMBW}$ theory with the HNC closure.

In Fig. 5 we present, with solid lines, the counterion and co-ion density profiles obtained in this work for $c=1 M$ and $\sigma^{*}=0.42$. For comparison, in the same figure we show the $M C$ and $M G C$ results. Also shown in the figure are the results obtained by Plischke and Henderson for the OZ/LMBW theory with the MSA closure. It is important to mention at this point that, for this concentration and surface charge, the results of the $\mathrm{O} Z / \mathrm{IMBW}$ theory with the MSA closure are in very good agreement with those of the same theory when the HNC closure is einployed (Plischlie and Henderson, 1989). The 


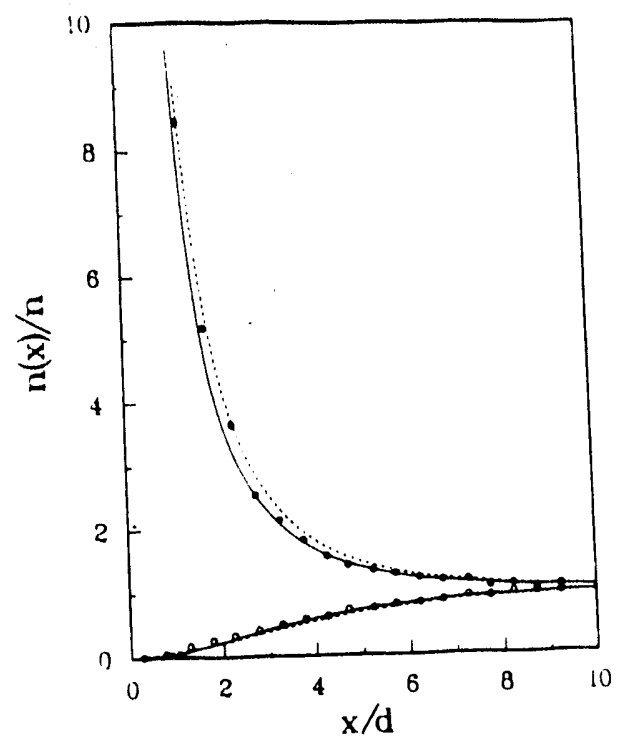

Fig. 3. Reduced density profiles, $n(x) / n$, for a $1: 1$ electrolyte at $c=0.1 M$ and $\sigma^{*}=$ 0.30 . The dots are the $\mathrm{MC}$ results. The dashed lines correspond to the MGC theory and the solid lines to this work. Note that the wall is at $x=-d / 2$.

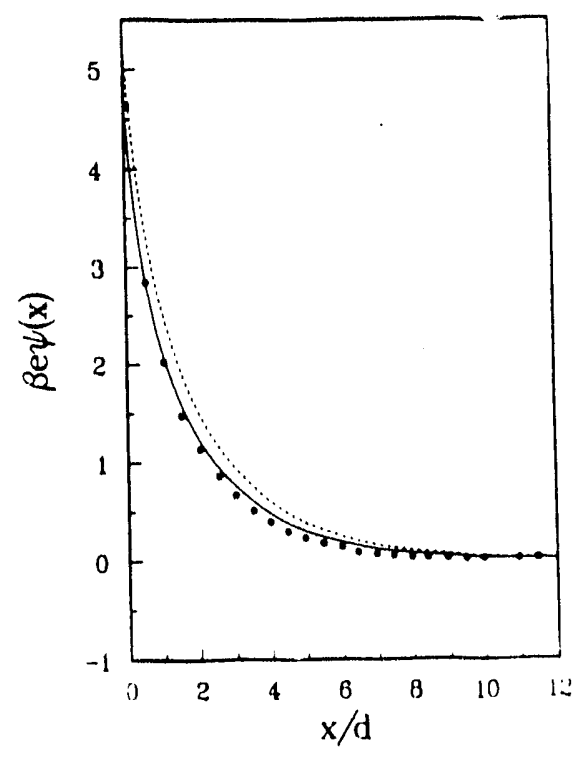

Figure 4. Reduced mean electrostatic potential profile for a $1: 1$ electrolyte at $c=$ $0.1 M$ and $\sigma^{*}=0.3$. All symbols as in Fig. 3.

$\mathrm{MC}$ results clearly show the onset of the formation of a second layer of counterions near $x / d=1$. Since the MGC theory is a point charge theory, it does not predict the layering phenomenon. On the other hand, the OZ/LMBW theory accurately follows the behavior of the MC data. It is interesting to see that the density functional theory presented in this paper is also able to predict the formation of the second layer of counterions. However, the position of the second layer is clearly shifted towards the electrode. The theory also exaggerates the size of the second peak. The co-ion density profile predicted by the density functional theory agrees quite well with $\mathrm{MC}$ data and is almost indistinguishable from that of the OZ/LMBW theory.

At a bulk concentration of $c=1 M$ and a charge density $\sigma^{*}=0.7$, a second layer of counterions is clearly formed. In Fig. 6 we compare the density profiles predicted by MGC, OZ/LMBW, and density functional theories with the MC results for that conditions. Again the $\mathrm{NGC}$ theory predicts monotonic profiles while the OZ/LMBW theory very well predicts both the position and magnitude of the second layer. Again the density functional theory overemphasize the value of the density of the second layer. As in Fig. 5, the position of this layer is shifted towards the electrode. This can be a consequence of the way in which the GHRM takes into account the hard-core effects. The GHRM predicts, for a hard-sphere fluid near a hard wall, a density profile with a second peak shifted towards the wall when 


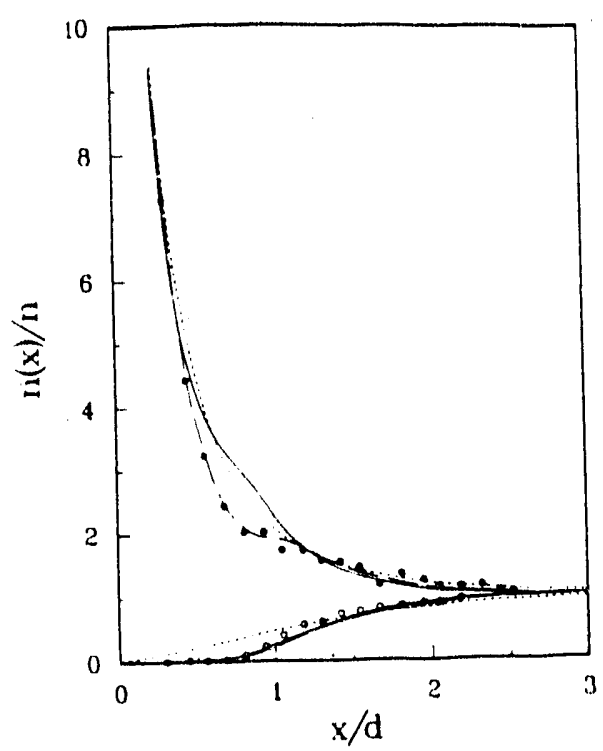

Fig. 5 Reduced density profiles, $n(x) / n$, for a 1:1 electrolyte at $c=1 M, \sigma^{*}=0.42$. The dots are the MC results. The dashed lines correspond to the $\mathrm{MGC}$ theory, the dot dashed lines to the OZ/LMBW theory with the MSA closure, and the solid lines to this work.

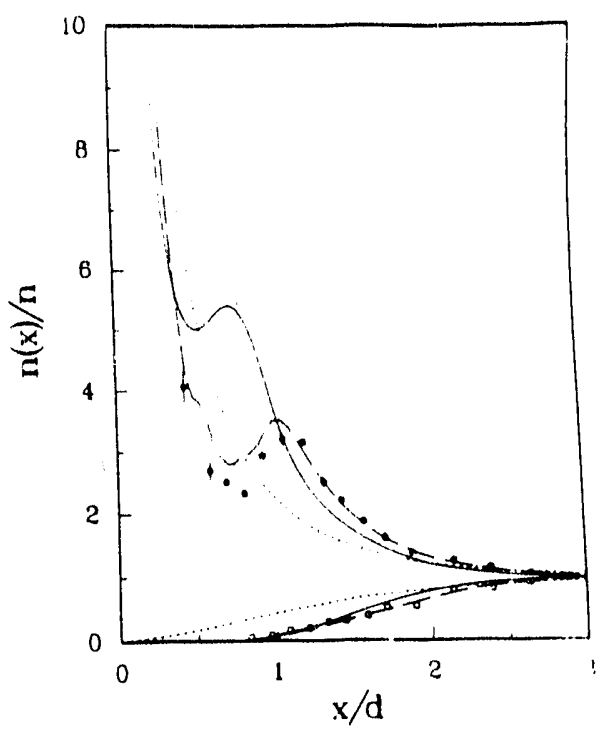

Fig. 6 Reduced density profiles, $n(x) / n$, for a $1: 1$ electrolyte at $c=1 M$ and $\sigma^{*}=0.7$. All symbols as in Fig. 5.

compared with simulation results, (Vanderlick et al. 1989).

The mean electrostatic potential profile which corresponds to the last conditions presented is shown in Fig. 7. The agreement between the density functional theory and the $\mathrm{MC}$ results is good. The density functional theory is able to predict the presence of a very shallow minimum in this function. A similar minimum is present in the MC results. The mean electrostatic potential function is not very sensitive to the details in the structure of the double layer.

\section{$2: 2$ electrolytes}

We have computed results of our density functional theory for two concentrations: 0.05 and $0.5 \mathrm{M}$. In the lower part of Table 1 we display some results of this work for the diffuse layer potential and compare with those of the theories previously mentioned. We find reasonable agreement with $\mathrm{MC}$ data.

In the case of divalent electrolytes, the $\mathrm{MC}$ results show the interesting phenomenon of charge inversion. This phenomenon, which is a result of both hard-core and electrostatic 


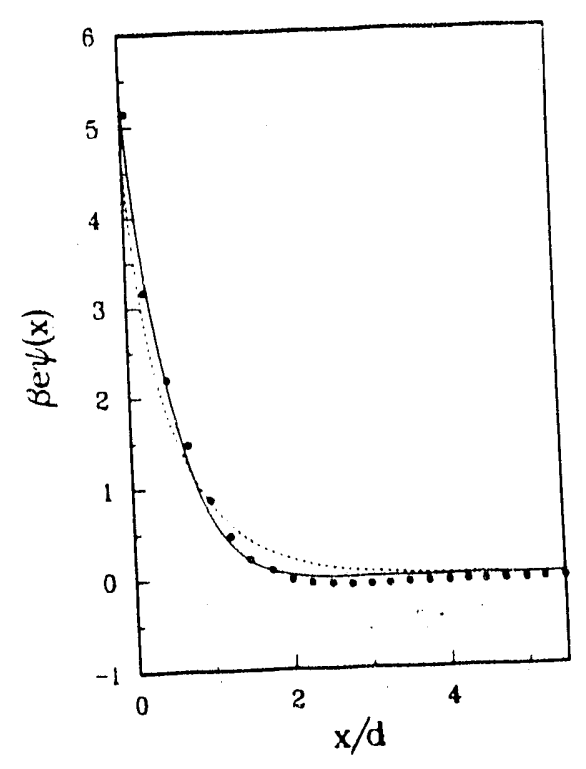

Figure 7. Reduced electrostatic potential profile for a $1: 1$ electrolyte at $c=1 M$ and $\sigma^{*}=0.7$. The dots are the $\mathrm{MC}$ results. The dashed lines correspond to the MGC theory and the solid line to this work.

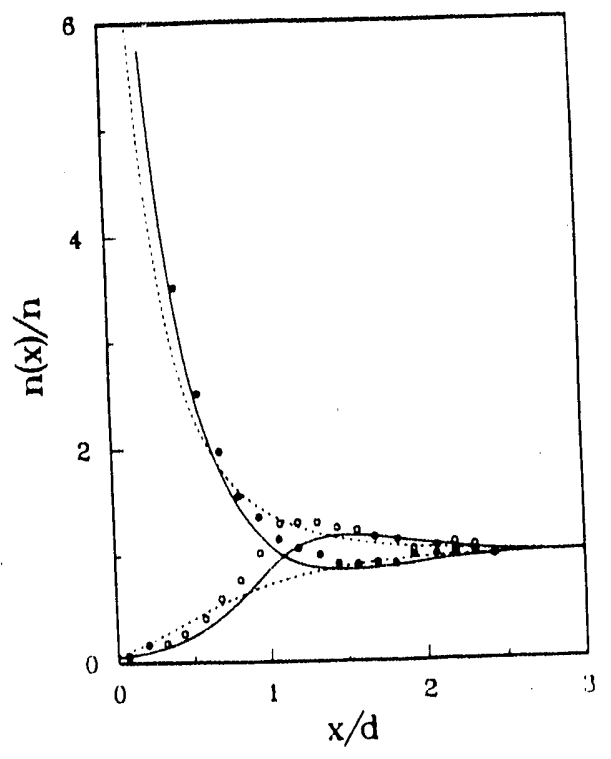

Fig. 8. Reduced density profiles, $n(x) / n$, for a $2: 2$ electrolyte at $c=0.5 M$ and $\sigma^{*}=$ 0.1704 . The dots are the $\mathrm{MC}$ results. The dashed lines correspond to the MGC theory and the solid lines to this work.

interactions, consists in the formation of a second layer of co-ions next to the first layer of counterions. In Fig. 8 we plot density profiles for a double layer at $c=0.5 \mathrm{M}$ and $\sigma^{*}=0.1704$. Nearly all the counterion charge is concentrated into a thin layer next to the wall. The response of the system to this dipole layer is the formation of a layer of co-ions within $x=d$ and $x=2 d$ approximately. As can be seen in Fig. 8, the density functional theory predicts the charge inversion phenomenon. We obtained a counterion density profile in reasonable agreement with MC data. On the other hand, our theory underestimates the magnitude of the maximum in the co-ion profile. As expected, the MGC theory totally misses the charge inversion.

In Fig. 9 we show the mean electrostatic potential profile for the same conditions presented in the previous figure. The MC simulations result in a potential profile which is oscillatory with a minimum of about -0.2 just beyond one diameter from the wall. Our density functional theory predicts the oscillatory behavior and is in good agreement with the $\mathrm{MC}$ data.

\section{Summary}

We presented a nonlocal free energy density functional theory for the electrical double layer. Within the frame of the grand canonical formalism, we construct a free energy 


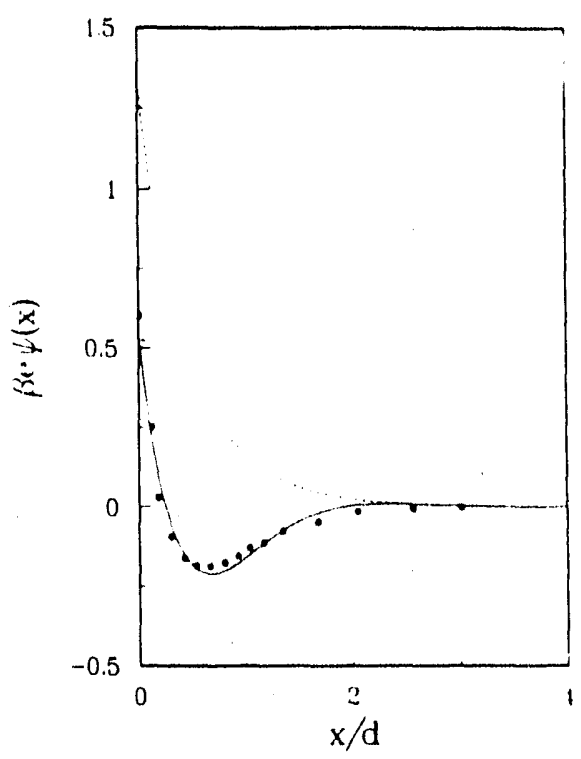

Figure 9. Reduced mean electrostatic potential profile for a $2: 2$ electrolyte at $c=0.5 \mathrm{M}$ and $\sigma^{*}=0.1704$. The dots are the $\mathrm{MC}$ results. The dashed line corresponds to the MGC theory and the solid line to this work.

functional of the density distribution. We then separate the short ranged part of the inhomogeneous direct correlation functions which appear in the formalisin into a hardsphere term and a residual term. The residual term contains the correlations arising from the Coulombic interactions between particles in the fluid. The hard-sphere part of the free energy functional is then approximated by a generic functional proposed by Percus (1981) as a three dimensional generalization of an inhomogeneous hard-rod system. We used its extension to mixtures due to Vanderlick et al.(1.990). This generic nonlocal functional requires the specification of two coarse- grain densities. In this work we choose to use the weighting functions proposed by Robledo and Varea (1981) and by Fischer and Heinbuch (1988) to generate a GHRM functional for the free energy of an inhomogeneous hard-sphere system. In our calculations we approximate the free energy of a bulk hardsphere mixture with the Carnahan-Starling (1970) expression. The residual inhomogeneous direct correlation functions are approximated with those corresponding to the neutral bulk electrolyte which is in equilibrium with the interface. Use is made of the analytical solutions of the MSA (Waismann and Lebowitz, 1972).

The GHRM free energy density functional theory described in Sec.III correctly describes the physical features presented by the MC simulations for 1:1 and 2:2 RPM electrolytes. For 1:1 electrolytes the theory predicts the layering of counterions which occurs when the charge of the electrode is increased. Although the theory exaggerates the magnitude of the counterion layering, predicts a diffuse layer potential which is in very good agreement with the $\mathrm{MC}$ data. For 2:2 electrolytes, the theory predicts the charge inversion phenomenon and ${ }^{\prime \prime l l u e s}$ of the diffuse layer potential which are in good agreement with 
$\mathrm{MC}$ results. In general, there are small quantitative rather than qualitative differences between the $\mathrm{MC}$ results for the density profiles and mean electrostatic potential and those obtained in this work.

Even when calculations with the GHRM density functional theory are relatively simple, the theory competes in accuracy with the more sophisticated OZ/LMBW theory (Plischke and Henderson, 1989). Because of its simplicity, the GHRM theory requires only a small fraction of the computing time used to solve the OZ/LMBW theory. For the 1:1 electrolyte at $c=1 M$, our code requires only $240 \mathrm{~s}$ of a Cray $2 \mathrm{CPU}$ time to calculate solutions and mean electrostatic potential profiles at 15 different values of reduced charge density, $\sigma^{*}$, with a uniform mesh of 241 nodes, for example.

From a density functional formulation similar to that presented here, Forstmann and collaborators (1987) used in the interface ion-ion correlation functions of homogeneous electrolytes with non-neutral compositions. Instead, in our work we are employing a nonlocal GHRM functional for the hard sphere part of the free energy and neutral bulk electrolyte correlation functions for the residual electrostatic part. Use of non-neutral composition residual electrostatic correlations is left for future work.

The results of the GHRM for a hard-sphere system near a hard-wall, (Vanderlick et al. 1989), show a poor quantitative agreement with MC results. Since a very good agreement between the functional theory and the $\mathrm{MC}$ results for the planar double layer is reported in Sec. IV, one can naturally ask if a fortuitous cancellation of error is occurring when we combine the hard-sphere free energy functional with the MSA solutions for the electrostatic part of direct correlation functions. The answer to this question probably can be given by solving the theory for a more accurate functional for the hard-sphere contribution to the free energy. The SDA of Tarazona (1985) seems to be a good option for this purpose. We hope to contribute to the solution of this question in the near future.

\section{Rieferences}

Alts, T., P. Nielaba, B. Daguanno, and F. Forstmann, Chem. Phys. 111, 223 (1987).

Ball, P. C., and R. Evans, J. Chem. Phys. 89,4412 (1988).

Ball, P. C., and R. Evans, Mol. Phys. 63, 159 (1988).

Barker, J.S. and D. Henderson, Rev. Mod. Phys. 48, 587 (1976).

Blum, L., J. Phys. Chem. 81, 136 (1977); D. Henderson and W. R. Smith, J. Stat. Phys. 19, 191 (1978); D. Henderson and L. Blum, J. Chem. Phys. 69, 5441 (1978); D. Henderson, L. Blum, and W. R. Smith, Chem. Phys. Lett. 63, 381 (1979); D. Henderson and L. Blum, J. Electroanal. Chem. 111, 217 (1980); S. L. Carnie, D. Y. C. Chan, D. J. Mitchell, and B. W. Ninham, J. Chem. Phys. 74, 1472 (1981); M. Lozada-Cassou, R. Saavedra-Barrera, and D. Henderson, ibid. 77, 5150 (1982); M. Lozada-Cassou and D. Henderson, J. Phys. Chem. 87, 2821 (1983); J. Barojas, D. Henderson, and M. LozadaCassou, ibid. 87, 4547 (1983); ibid. 88, 2926 (1984); S. L. Carnie, Mol. Phys. 54i, 509 
(1985); L. Mier y Teran, E. Diaz-Herraza, M. Lozada-Cassou, and D. Henderson, J. Phys. Chem. 92, 6408 (1988).

Bongiono, V. and H. T. Davis, Fhys. Rev. A 12, 2213(1975); Bongiono, V., L. E. Scriven, and H. T. Davis, J. Colloid Interf. Sci. 57, 462(1976); H. T. Davis and L. E. Scriven, Adv. Chem. Phys. 49, 357 (1982).

Boyle, E.J., L. E. Scriven, anci H. T. Davis, J. Chem. Phys. 86, 2309 (1987).

Caccamo, C., G. Pizzinsenti, and L. Blum, Phys Chem. Liquids 14, 311 (1985); J. Chem. Phys. 84, 3327 (1986); E. Biuno, C. Caccano, and G. Pizzimenti, ibid. 86, 5101 (1987). Carnahan, N. F. and K. E. Starling, J. Chem. Phys. 51, 635 (1969); ibid 53, 600) (1970). Carnie, S. L. and G. M. Torrie, Advan. Chem. Phys, 56, 141 (1084).

Chapman, D. L., Philos. Mag. 25, 475 (1913).

Croxton, T. L., D. A. McQuarrie, Chem. Phys. Lett. 68, 489 (1979); Mol. Phys. 42, 141 (1081); D. Henderson, L. Blum, L. B. Bhuiyan, Mol. Phys. 43, 1185 (1981); L. Blum, J. Hernando, and J. L. Lebowitz, J. Phys. Chem. 81, 2825 (1983).

Curtin, W. A. and N. W. Ashcroft, Phys. Rev. A 32, 2909 (1985); Phys. Rev. Lpett. 56, $2775(1986)$.

DeDominicus, C., J. Math Phys. 3. 983 (1962).

Ebner, C., W. F. Saam, and D. Stroud, Phys. Rev. A 14, 2264 (1976).

Evans, R., Adv. in Phys. 28, 143 (1979).

Fischer, J. and U. Heinbuch, J. Chem. Phys. 88, 1909 (1988).

Gouy, G., J. Phys. 9, 451 (1910).

Heffelfinger, G. S., Z. Tan, U. Marini Bettolo Marconi, F. van Swol, and Gubbins, K. E., Molecular Simulation 2, 393 (1989).

Honenberg, P. and W. Kohn, Phys. Rev. B 136, 864 (1964).

Kjellander, R. and S. Marcelja, Chem. Phys. Lett. 112, 49 (1984); J. Chem. Phys. 82, 2122 (1985); Chem. Phys. Lett. 127, 462 (1986).

Lovett, R.. C. Y. Mou, and F. P. Buff, J. Chem. Phys. 65, 570 (1076); M. Wertheim, ibid. $65,2377(1976)$.

Lebowitz, J. L. and J. K. Percus, J. Math. Phys. 4, 116 (1963).

Mansoori, G.A., N. F. Carnahan, K. E. Starling, and T. W. Leland, J. Chem. Phys. 54, $1.523(1971)$.

Meister, T. F. and D. M. Kroll, Phys. Rev. A 31, 4055 (1985).

Mermin, N. D., Phys. Rev. A 137, 1441 (1965).

Mier y Teran, L., A. H. Falls, L. E. Scriven, and H. T. Divis, Proceedings 8 th Symposium of Thermophysical Properties, ed. J. V. Sengers, American Society of Mechanical Engineers, New York,(1982) 1, pp. 45-56.

Mier y Teran, L., E. Fernandez-Fassnacht, and S. Quinones, Phys. Lett. 107A, 329 (1985); L. Mier y Teran, E. Fernandez-Fassnacht, Rev. Mex. Fis. 32, S241 (1986); Phys. Lett. $117 \mathrm{~A}, 43(1986)$.

Mier y Teran, L., E. Diaz-Herrera, M. Lozada-Cassou, and R. Saavedra-Barrera, J. Comput. Phys. 84, 326 (198:).

Morita, T. and K. Hiroike, Prog. theor. Phys. 25, 537 (1961).

Niclaba, P. and F. Forstmann, Chem. Phys. Lett. 117, 46 (1985); P. Nielaba, T. Alts, B. Daguanno, and F. Forstmann, Phys. Rev. A 34, 1505 (1986); B. Daguanno, P. Niclaba, 
T. Alts, and F. Forstmann, J. Chem. Phys. 85, 3476 (1986).

See, e.g., S. Nordholm, J. Gibson, and M. A. Hooper. J. Stat. Phys. 28, 391 (1982).

Outhwaite, C. W., J. Chem. Soc. Faraday Trans. 2 74, 1214 (1978); S. Levine and C. W. Outhwaite, ibid. 74, 1670 (1978); L. B. Bhuiyan, C. W. Outhwaite, and S. Levine, Chem. Phys. Lett. 66, 321 (1979); C. W. Outhwaite, L.B. Bhuiyan, and S. Levine ibid. 67, 150 (1979); C. W. Outhwaite, L. B. Bhuiyan, and S. Levine, J. Chem. Soc. Faraday Trans. 2 76,1388 (1980); S. Levine, C. W. Outhwaite, and L. B. Bhuiyan, J. Electroanal. Chem. 123, 105 (1981); C. W. Outhwaite, L. B. Bhuiyan, and S. Levine, Chem. Phys. Lett. 78, 413 (1981); L. B. Bhuiyan, C. W. Outhwaite, and S. Levine, Mol. Phys. 42, 1271 (1981); C. W. Outhwaite and L. B. Bhuiyan, J. Chem. Soc. Faraday Trans. 2 78, 775 (1982); C. W. Outhwaite and L. B. Bhuiyan, J. Chem. Soc. Faraday Trans. 2 79, 707 (1983); C. W. Outhwaite, ibid. 79, 1315 (1983); C. W. Outhwaite and L. B. Bhuiyan, J. Chem. Phys. 85, 4206 (1986). D. Henderson, F. F. Abraham, and J. A. Barker, Mol. Phys. 31, 1291 (1976).

Percus, J. K., J. Stat. Phys. 15, 505 (1976).

Percus, J. K., J. Chem. Phys. 75, 1316 (1981).

Percus, J. K. in "The Equilibrium Theory of Classical Fluids", edited by Frisch, H. L. and J. Lebowitz, (Benjamin, New York, 1964), pp. II30-II70.

Peterson, B. K., K. E. Gubbins, G. S. Heffelfinger, U. Marini Bettolo Marconi, and F. van Swol, J. Chem. Phys. 88, 6487 (1988).

Plischke, M. and D. Henderson, J. Chem. Phys. 88, 2712 (1988); 90, 5738 (1989).

Robledo, A. and C. Varea, J. Stat. Phys. 26, 513 (1981).

Saam, W. F. and C. Ebner, Phys. Rev. A 15, 2566 (1977).

Schmickler, W. and D. Henderson, Progr. Surf. Sci. 22, 323 (1986).

Strang, G. and G. Fix, An Analysis of the Finite Element Method (Prentice Hall, Englewood Cliffs. New Jersey, 1973).

Stell, G. in "The Equilibrium Theory of Classical Fluids", edited by H. L. Frisch and J. L. Lebowitz, (Benjamin, New York, 1964), pp. II71-II226.

Stern, O., Z. Electrochem. 30, 508 (1924).

Stillinger, F. H. and F.P. Buff, J. Chem. Phys., 37, 1(1962).

Tarazona, P., Phys. Rev. A 31, 2672 (1985); 32, 3148 (1985).

Tarazona, P., U. Marini Bettolo Marconi, and R. Evans, Mol. Phys. 60, 573, (1987).

Tan, Z., U. Marini Bettolo Marconi, F. van Swol, and K. E. Gubbins, J. Chem. Phys. 90, 3704 (1989).

Torrie, G. M. and J. P. Valleau, J. Chem. Phys. 73,5807 (1980); G. M. Torrie, J. P. Valleau and G. N. Patey, ibid. 76,4623 (1982); G. M. Torrie and J. P. Valleau, J. Phys. Chem. 86, 3251 (1982).

Vanderlick, T. K., H.T. Davis and J.K. Percus, J. Chem. Phys. 91, 11 (1989).

Vanderlick, T. K., L. E. Scriven and H. T. Davis, J. Chem. Phys. 90, 2422 (1989).

van der Waals, J. D. and Ph. Kohnstamm, Lehrbuch der Thermodynamik, (Maas and van Suchtelen, Leipzig, 1908), 1.

Waismann, E. and J. L. Lebowitz, J. Chem. Phys. 56, 3086 (1972); 56, 3093 (1972). 


\section{WETTING TRANSITIONS \\ AND SURFACE CRITICAL PHENOMENA \\ AT SOLID-FLUID INTERFACES}

\section{Synopsis}

The van der Waals density functional model of inhomogeneous fluids introduced by Teletzke et al. (1982b) is applied to construct global phase diagrams that display wetting transition points, thin to thick thin-film transition points, and accompanying surface critical, tricritical, and multicritical points of a solid-fluid interface.

The model is based on mean field theory, yields an integral equation for density distributions, can be solved analytically, and predicts both first- and second-order wetting transitions depending on location in field variable space. Here we show that the model, although mathematically simple, predicts thin to thick thin-film transition and surface critical, tricritical, and multicritical phenomena. The various transition and critical points are obtained directly by solution of algebraic equations derived from the model.

The global phase diagrams have as coordinates the temperature and chemical potential and an interaction potential parameter $W$ which is the ratio of characteristic energy of two-body solid- fluid interaction to fluid-fluid interaction. Diagrams are constructed for three values of the three- body interaction potential energy parameter, $W_{3}=1 / 3,1$, and 3 , corresponding to the three phase diagrams of Nakanishi and Fisher (1982) who inferred the diagram structure from scaling studies of a magnetic system obeying the LandauGinzburg free energy model. Our results agree with their critical exponents $\Delta$ and $\Delta_{1}$ for the "ordinary," "special," and "extraordinary" multicritical points. However, we find a quantitative difference between our results and the parameter range over which $N K$ suggested one of the scaling relations holds.

\section{Introduction}

Interest in wetting transitions with associated surface critical phenomena, important in science and technology, has grown rapidly since the seminal paper of Cahn (1977). Several reviews of the subject have appeared (see for instance, Binder (1983), Davis et al. (1984), Diehl (1986), Sullivan and Telo da Gama (1986), and Kung (1989)). Wetting transitions often occur when a pair of fluids contacting a solid have an ultralow interfacial tension. As surfactant-enhanced oil recovery often involves oil-rich and water-rich phases exhibiting ultralow tensoins against a microemulsion phase it is important to understand the general patterns of wetting transitions. These are investigated herein on the basis of a simple, but qualitatively general, molecular model. 
In this paper we restrict our attention to three phase systems in which at least two of the phases have a mutual critical point. The three phases could be solids, licpuid $(L)$, and vapor $(V)$. If at a given temperature and pressure a drop of liquid placed on the solid surface in the presence of its vapor does not spread indefinitely and the contact angle made by the $L-V$ interface at the solid surface is not zero then the liquid is said not to wet the solid surface. As the system temperature, pressure or composition is changed such that $I$ and $V$ approach their mutual critical point a condition is reached at which the contact angle goes to zero and, if enough of the liquid phase is available, a macroscopir layer of the liquid completely wets the solid surface. This transition between nonwetting and wetting by the liquid phase is called the wetting transition (Heady and Cahn, 1972; Cahn, 1977).

Theory predicts that the wetting transition can be either first-order (Cahn, 1977; Ebner and Saam, 1977) or second-order (Sullivan, 1979). Extensive research that used mean field theories of inhomogeneous fluids had shown that for the solid-fluid interface where the system temperature and pressure are above the fluid's triple point the order of the wetting transition depends on at least the following factors: (1) the asymptotic behavior of the interaction potentials which has been assumed to be either an exponential (Sullivan, 1979) or power-law decay (Ebner and Saam,1977); (2) ratio of characteristic energy of solid-fluid interaction to that of fluid-fluid interaction for both exponential potentials (Sullivan, 1979) and power-law potentials (Ebner, 1980; Teletzke et al., 1982a); (3) the ratio of the characteristic length of solid-fluid interaction to that of fluid-fluid interaction for both exponential potentials (Hauge and Schick, 1983; Tarazona and Evans, 1983) and power-law potentials (Teletzke et al., 1982a); and (4) Higher-order effects that involve three- body solid-fluid interactions of exponential potentials (Teletzke et al., 1982b) and retarded interactions of power-law potentials (Dietrich and Schick, 1985, 1986; see also Kroll and Meister, 1985). These works indicate that in systems with exponential potentials both first-order and secondorder wetting transitions can occur depending on the last three factors indicated above. However, in systems with power-law potentials first-order wetting transition should be far more common than second-order wetting transitions (de Gennes, 1983; 1985; Privman, 1984). Investigations into the effects of fluctuation indicate that second-order wetting transition cannot occur for systems with power-law potentials (Nightingale et al., 1983, 1984).

The existence of first-order wetting transition has been inferred from experiments (Schmidt and Moldover, 1983; Pohl and Goldberg, 1982; Moldover and Cahn, 1980). If the wetting transition is first-order, then a surface phase transition can also occur in two phase systems. For a solid-fluid interface, with the fluid in the vapor single phase region of the temperature-density phase diagram, Cahn (1977) showed that above the wetting transition temperature there exists a temperature-density path along which two different liquid-like thin films coexist. The surface phase transition occurring when this path is crossed is known as the thin to thick thin-film transition or prewetting transition (Cahn, 1977; Teletzke et al., 1982a). With increasing temperature the thin-film coexistence curve on the bulk temperature- density phase diagram eventually terminates at a critical point called the surface critical point. At the present time experimental evidence for the existence of such a thin-film transition remains inconclusive (Schmidt and Moldover, 1986). 
Surface multicritical points, which occur only for special values of the interaction potential energy and range, were first explored in another system that was modeled by equations similar in form to the gradient theory approximation (see e.g. Davis and Scriven, 1982) of density functional theory: namely, the ferromagnetic free surface (Mills, 1971; Kaganov and Omel'yanchuk, 1971; Binder and Hohenberg, 1972 and 1974; Kumar, 1974; Lubensky and Rubin, 1975; see also review of Binder, 1983). The connection between surface phase transitions at ferromagnetic free surfaces and wetting transitions at solid-fluid interfaces was discussed by Nakanishi and Fisher (1982).

The order of the wetting transition, the thin to thick thin-film transition, and the surface multicritical points can be conveniently displayed together in global phase diagrams whose coordinate axes are field variables which include thermodynamic variables and parameters of the interaction potentials. Such a global phase diagram was first introduced by Pandit et al. (1982) who studied a lattice-gas model of solid-fluid interface with nearest-neighbor interactions. Their work showed that the point where the wetting transition turns from first-order to second-order is similar to a bulk fluid tricritical point. Nakanishi and Fisher (1982) used gradient theory with effectively exponential interaction potentials and extended the range of parameter space and were able to infer the structure of two more global phase diagrams. The global phase diagrams of Nakanishi and Fisher occupy an important place in the theory of wetting transitions. These diagrams have been further discussed in three recent review articles (Binder, 1983; Diehl, 1986; Sullivan and Telo da Gama, 1986). Furthermore, work has been done to obtain global phase diagrams in other systems as well: binary fluid-fluid interfaces (Costas et al., 1983); systems with more than two stable bulk phases (Lipowsky, 1983); and systems with long-range forces (Ebner and Saam, 1987a,b). However the solid-fluid interface with exponential interaction potentials remains the simplest and one of the more basic systems for studies of wetting transition; it is therefore worthwhile to obtain the diagrams of this system by direct computation. The diagrams of Nakanishi and Fisher were based on gradient theory with a polynomial free energy. It appears from their paper that Nakanishi and Fisher deduced the global phase diagrams without actually computing transition and critical points.

Recently a version of mean field integral theory has received attention in the literature because it has all of the attractive features of gradient theory yet it is not derived from a gradient approximation so that it gives rise to correlation functions that are more realistic than those of gradient theory (Bellare, 1988). In particular with the proper choice of exponential interaction potentials this integral theory is exactly solvable for a wide variety of systems such as: solid.fluid interface (Sullivan,1979,1981), fluid between two solid walls (Evans et al.,1986), binary fluid interface (Sullivan, 1982), and solid-binary fluid interface (Telo da Gama and Evans, 1983). However this model predicts only second-order wetting transition at solid-fluid interfaces; Teletzke et al.(1982b) generalized the interaction potentials introduced by Sullivan(1979) so that the new model is still solvable exactly but predicts both second-order and first-order wetting transitions depending on location in field variable space. 
of solutions to the density functional equations is exploited to see better the physical origins of the patterns of wetting and surface-state transitions. The graphical construction is presented in Section B2 and the equations that govern first-order wetting transition, thinfilm transition, surface critical point are outlined in Sections B3 and B4. Results are given iti Section $\mathrm{C}$ where predicted global phase diagrams are compared to those of Nakanishi and Fisher (1982).

\section{The Mean Field Model With Exponential Potentials}

In a closed, isothermal, $\chi$-component system, equilibrium states correspond to absolute minima in the grand potential $\Omega=F-\sum_{i} \mu_{i} N_{i}$. Here $F$ is the Helmholtz free energy, $\mu_{i}$ the ith component chemical potential which is constant throughout the system, $N_{i}$ is the total number of ith component particles, and the summation includes all components. For a solid-fluid system where the solid interacts with the fluid only through long-range van der Waals forces the grand potential can be written as

$$
\Omega=-p V+\gamma A
$$

in which $p$ is the bulk pressure, $V$ the volume, $\gamma$ the solid-fluid tension, and $A$ the surface area.

The van der Waals model of the grand potential of inhomogeneous fluid is a functional of the number density profiles $n_{i}(r)$, and is a function of the temperature $T$ and of the chemical potentials $\mu_{i}$

$$
\begin{aligned}
\Omega(\{\mathbf{n}\}, T, \mu) & =\int d^{3} r f^{h}(\mathbf{n}(\mathbf{r})) \\
& +1 / 2 \sum_{i} \sum_{j} \iint d^{3} r d^{3} r^{\prime} V_{i j}\left(\mathbf{r}, \mathbf{r}^{\prime}\right) n_{i}(\mathbf{r}) n_{j}\left(\mathbf{r}^{\prime}\right) \\
& +\sum_{i} \int d^{3} r\left[U_{i}(\mathbf{r})-\mu_{i}\right] \mathbf{n}_{\mathbf{i}}(\mathbf{r})
\end{aligned}
$$

Here, $f_{h}(\mathbf{n}(\mathbf{r}))$ is the free energy of a homogeneous fluid with density distributions $\mathbf{n}=$ $\left\{n_{1}, \ldots, n_{x}\right\}$ whose molecules only interact via hard-core potential. $V_{i j}\left(\mathbf{r}, \mathbf{r}^{\prime}\right)$ is the longranged part of the interaction energy between a molecule of species $i$ at $r$ and a molecule of species $j$ at $r^{\prime}, U_{i}(\mathbf{r})$ is the interaction energy between the solid and a fluid molecule of species $i, \mu_{i}$ is the chemical potential of species $i$. The summations range over the components $1,2, \ldots, \chi$ in the fluid.

The condition of thermodynamic equilibrium is that the functional derivative of $\Omega$ with respect to each component density is zero:

$$
\delta \Omega / \delta n_{i}(\mathbf{r}) \equiv \delta F / \delta n_{i}(\mathbf{r})-\mu_{i}=0, \quad i=1, \ldots, \chi
$$


where the symbol " $\delta / \delta n_{i}(r)$ " denotes functional differentiation with respect to the density $n_{i}$. For systems with a planar solid-fluid interface the above equations are equivalent to

$$
\delta \Omega / \delta n_{i}(r)=A \delta \gamma / \delta n_{i}(r)=0, \quad i=1, \ldots, \chi,
$$

where $\gamma$ is the excess free energy of the solid-fluid interface. The higher-order functional derivatives of $\Omega$ determine thermodynamic stability. The equations at (3a) are the usual starting point for theoretical investigation of inhomogeneous fluid wherein one first solves for the density profiles and afterwards substitutes the density profiles into the appropriate formula to get the surface tension. In this paper we find that in studying wetting transition and critical phenomena the equivalent equations at $(3 b)$ have a very simple interpretation in the mean field model with exponential potentials of Sullivin $(1979,1981)$ and Teletzke et al. (1982b). In what follows we consider only planar interfaces, and so clensities depend only on the distance $x$ from the solid surface.

\section{Chemical Potential Equations And Surface Tension Expression}

With the exponential interaction model introduced by Teletzke et al. (1982b), Equation (3a) becomes

$$
\begin{aligned}
\mu_{k}^{h}(\mathbf{n}(x))-\mu_{k} & =\left(1+W_{3}\right) W_{2, k} \exp \left[-x / s_{k}\right] \\
& +1 / 2 \sum_{j} a_{k j} / r_{k j} \int_{0}^{\infty}\left\{\exp \left[-\left|x-x^{\prime}\right| / r_{k j}\right]\right. \\
& \left.+W_{3} \exp \left[-\left(x+x^{\prime}\right) / r_{k j}\right]\right\} n_{j}\left(x^{\prime}\right) d x^{\prime} ; k=1, \ldots, \chi
\end{aligned}
$$

where

$$
\begin{array}{ll}
\mathbf{n} & \equiv\left(n_{1}, \ldots, n_{\chi}\right) \equiv \text { set of component densities, } \\
\mu_{k}^{h}(\mathbf{n}(x)) & \equiv \text { chemical potential of component } \mathrm{k} \text { in a hard sphere fluid at local } \\
& \text { composition } n(x), \\
\mu_{k} & \equiv \text { chemical potential of component } k, \\
s_{k} & \equiv \text { characteristic range of solid-fluid two-body potential for component } k, \\
r_{k j} & \equiv \text { characteristic range of fluid-fluid potential between components } k \text { and } j, \\
a_{k j} & \equiv \text { characteristic energy of interaction between components } k \text { and } j \text { relative } \\
W_{2, k} & \text { to that of the first component, } \\
W_{3} & \text { ratio of solid-fluid interaction strength of kth component to fluid-fluid } \\
\text { interaction strength of first component, }
\end{array}
$$

In the computational results presented below the following units are used: $\left[a_{k j}\right]=a_{11}$, $\left[\mu_{k}\right]=a_{11} r_{11}^{-3},\left[n_{k}\right]=r_{11}^{-3},\left[W_{2, k}\right]=a_{11} r_{11}^{-3}$. Teletzke et al. generalized Sullivan's model by introduction of exponential terms with the dimensionless coefficient $W_{3}$ in both the solid-fluid and fluid-fluid interaction potentials. These interactions can be interpreted as a measure of three-body interaction strength among the solid and two fluid molecules (Davis et al., 1984). Sullivan applied his model, for which $W_{3}=0$, to solid-fluid interfaces but found only 
second-order wetting transition." Teletzke et al. studied the range $0 \leq W_{3} \leq 1$ and found first-order wetting transitions when the absolute value of the pair-interaction parameter $\left|W_{2}\right|$ is sufficiently large. In this paper we extend the range of $W_{3}$ to $W_{3}>1$; in addition we perform computations to construct global phase diagrams that allow us to make comparison with the scaling results of Nakanishi and Fisher (1982).

The system of $x$ chemical potential integral equations Eqs.(4) reduces to one differential equation if the characteristic ranges of solid-fluid and fluid-fluid interactions are equal, i.e., $r_{k j}=s_{k} \equiv s$ for $k=1, \ldots, \chi$ and $j=1, \ldots, \chi$, and if $W_{2, k}$ satisfy the following equations

$$
W_{2, k}=\sqrt{a_{k}} W_{2,1}, \quad \text { for } k=2, \ldots, \chi .
$$

Then the chemical potentials satisfy

$$
\mu_{k}^{h}-\mu_{k}=\sqrt{a_{k}}\left(\mu_{1}^{h}-\mu_{1}\right) ; \quad \text { for } k=2, \ldots, \chi .
$$

and the new differential equation is

$$
\frac{d^{2} \mu_{1}^{h}}{d x^{2}}=\mu_{1}^{h}-\mu_{1}-\sum_{j} \sqrt{a_{1 j}} n_{j}(x) \equiv \frac{\partial \Psi}{\partial \mu_{1}^{h}}
$$

where

$$
\Psi\left(\mu_{1}^{h} ; T, \mu_{1}\right) \equiv\left(\mu_{1}^{h}\right)^{2}-2 \mu_{1} \mu_{1}^{t_{t}}-2 p^{h}+\text { constant }
$$

and $p^{h}$ is the pressure of a homogeneous fluid of hard spheres at the given local $\mu_{1}^{h}$. The first boundary condition is

$$
d \mu_{1}^{h} / d x=0 \text { for } x \rightarrow \infty,
$$

since the fluid becomes honogeneous far from the wall. The second boundary condition is

$$
d \mu_{1}^{h} /\left.d x\right|_{x=0}=\beta\left(\mu_{1}^{h}(0)-\mu_{1}\right)-2 W_{2,1} \equiv Z\left(\mu_{1}^{h}(0)\right)
$$

where

$$
\beta \equiv\left(1-W_{3}\right) /\left(1+W_{3}\right)
$$

The surface tension is given by (Sullivan, 1981; Teletzke et al., 1982b)

$$
\begin{aligned}
\gamma & =\beta\left(\mu_{1}^{h}(0)-\mu_{1}\right)^{2} / 2-2 W_{2,1}\left(\mu_{1}^{h}(0)-\mu_{1}\right)+2\left[W_{2,1}\right]^{2} /(1+\beta) \\
& +\int_{\mu_{1}^{h}(0)}^{\mu_{1}^{h}(\infty)}[ \pm \sqrt{\Psi(y)}] d y .
\end{aligned}
$$

In what follows we restrict ourselves to single component fluids. The simpler notation $W$ will be used in place of $W_{2}$ in equations to follow. We use the lattice-gas model (Lee and Yang, 1952) for which the hard-sphere contribution to the equations of state are given by

$$
\begin{gathered}
\mu^{h}(n)=k_{B} T \ln [n /(1-n b), \\
p^{h}(n)=-\left(k_{B} T / b\right) \ln (1-n b),
\end{gathered}
$$


where $k_{B}$ is Boltzman's constant and $T$ is the absolute temperature. The critical temperature of the bulk fluid is $T_{c}=0.25 a / b k_{B}$. In what follows the units of temperature and pressure are $[T]=T_{c}$ ard $[p]=a_{11} / r_{11}^{6}$. Also, $r_{11}$ is chosen such that $b=r_{11}^{3}$ and so $b=1$ in the units $\left[r_{11}^{3}\right]$.

\section{The Method of Graphical Construction}

For the purpose of computing wetting and thin-film transition points it is not necessary to know the entire profile $\mu^{h}(x)$. A method of graphical construction due to Cahn (1977) can be applied to Eqs.(6-10) (Sullivan (1981) and Teletzke et al. (1982b)) to demonstrate the existence of first or second-order wetting transition, thin to thick thin-film iransition, and surface critical point without actually solving for density profiles. Here we will illustrate the method only for first-order wetting transition.

The second-order differential equation Eq.(6) is first integrated to give

$$
d \mu^{h} / d x= \pm \sqrt{\Psi\left(\mu^{h} ; T, \mu\right)}
$$

In the above equation the boundary condition for $x \rightarrow \infty$, Equation (8), has been applied so that

$$
\Psi \equiv\left(\mu^{h}-\mu\right)^{2}-2\left(p^{h}-p\right)
$$

where $p$ is the fluid pressure far from the interface. Thus the profile $\mu^{h}(x)$ can be obtained by simple quadrature:

$$
x-x_{o}=\int_{\mu^{h}\left(x_{0}\right)}^{\mu^{h}(x)} \frac{d y}{[ \pm \sqrt{\Psi(y)}]} .
$$

The sign convention in the above equations is as follows: If the surface and bulk densities are such that $\mu^{h}(0)<\mu_{B}^{h}$ then $\mu^{h}(x)$ increases monotonically with $x$ and the positive sign of $\pm \sqrt{\Psi(y)}$ of $\pm \sqrt{\Psi}$ must be taken. If $\mu^{h}(0)>\mu_{B}^{h}$ then $\mu^{h}(x)$ decreases monotonically with $x$ and the negative sign must be taken. $\mu_{B}^{h}$ is the hard-sphere chemical potential of the stable bulk phase.

Since the profile can be obtained by quadrature, it remains to determine $\mu^{h}(0)$, the hardsphere chemical potential at $x=0$. Elimination of $d \mu^{h}(0) / d x$ between Eq.(12) evaluated at $x=0$ and the boundary condition (9) gives the following algebraic equation for $\mu^{h}(0)$ :

$$
Z\left(\mu^{h}(0)\right)= \pm \sqrt{\Psi\left(\mu^{h}(0)\right)} .
$$

It turns out that the solution space of Eq.(15) holds all the information needed to locate wetting transitions and critical points.

Figure la shows a typical plot of the functions $Z\left(\mu^{h}(0)\right),+\sqrt{\Psi\left(\mu^{h}(0)\right)}$, and $-\sqrt{\Psi\left(\mu^{h}(0)\right)}$; the fluid, indicated in the figure by "V", is in the vapor single phase region very close to $\mathrm{L}-\mathrm{V}$ 
coexistence region. For the lattice-gas model the vapor phase has a hard sphere chemical potential $\mu_{B}^{h}$ and the liquid phase, with which this vapor phase is nearly in coexistence, has hard sphere chemical potential $\approx-\mu_{B}^{h}$. To satisfy the sign convention, segments are taken from the curves $+\sqrt{\Psi\left(\mu^{h}(0)\right)}$ and $-\sqrt{\Psi\left(\mu^{h}(0)\right)}$ to form the aggregate curve or function $\pm \sqrt{\Psi\left(\mu^{h}(0)\right)}$ in the algebraic equation (Eq.15); Fig.1(b) displays this aggregate function. Also shown in this Figure are the linear function $Z\left(\mu^{h}(0)\right)$ and the areas A1 and A2 bounded by the curves $\pm \sqrt{\Psi}$ and $Z$; these are discussed later.

From the algebraic equation (15) it is clear that any intersection between the two functions $Z$ and $\pm \sqrt{\Psi}$ locates a value of $\mu^{h}(0)$ that satisfies the boundary condition; the solution $\mu^{h}(x)$ with this boundary value can then be constructed by quadrature from Eq.(14). Figure $1 b$ depicts a situation where there are three possible solutions to the algebraic equation (Eq.15). Of the three solutions the ones that satisfy $\mu_{B}^{h}<\mu^{h}(0)<-\mu_{B}^{h}$ give composition profiles that describe nonwetting situations; the ones that satisfy $\mu^{h}(0)>-\mu_{B}^{h}$ give composition profiles that describes a wetting situation. The physical solution is that which has the smallest value of surface tension. Consider a temperature $T$ sufficiently low such that $T<T_{w}$ where $T_{w}$ is the wetting transition temperature. Then as the temperature is increased with the system kept infinitesimally outside the $\mathrm{L}-\mathrm{V}$ coexistence region, there comes a point where not just one but two of the solutions to the algebraic equation possess the same minimum value of tension. This is the point of wetting transition. Due to the equality of surface tension, at such a point the two areas designated A1 and A2 in Figure $1 \mathrm{~b}$ beccme identical. This is reminiscent of Maxwell's equal area construction for liquidvapor bulk transitions. This method of graphical construction applies in similar manner to thin-film transition and surface critical point.

3. An Alternate Method for Locating the First-Order Wetting Transition, the Thin to Thick Thin-Film Transition, and the Critical Points

In this section we present a new method for locating a first-order wetting transition, a thin to thick thin-film transition, and surface critical point; the method uses graphical analysis. In what follows its motivation is explained, it is compared with the methods of Teletzke et al. (1982a) and Tarazona and Evans (1983), and the equations used to locate the various transition and critical points are discussed.

A primary goal in this work is to obtain the global phase diagrams of the exponential model of Teletzke et al. (1982a). A natural way is to apply iteratively the graphical method discussed in Section 2. For instance, to determine for given $\left(W_{3}, W\right)$ the wetting transition temperature $T_{w}$ of first-order wetting: (i) start with a sufficiently low temperature $T<T_{w}$ and decide on a step size $\Delta T$ for incrementally increasing temperature, (ii) solve 


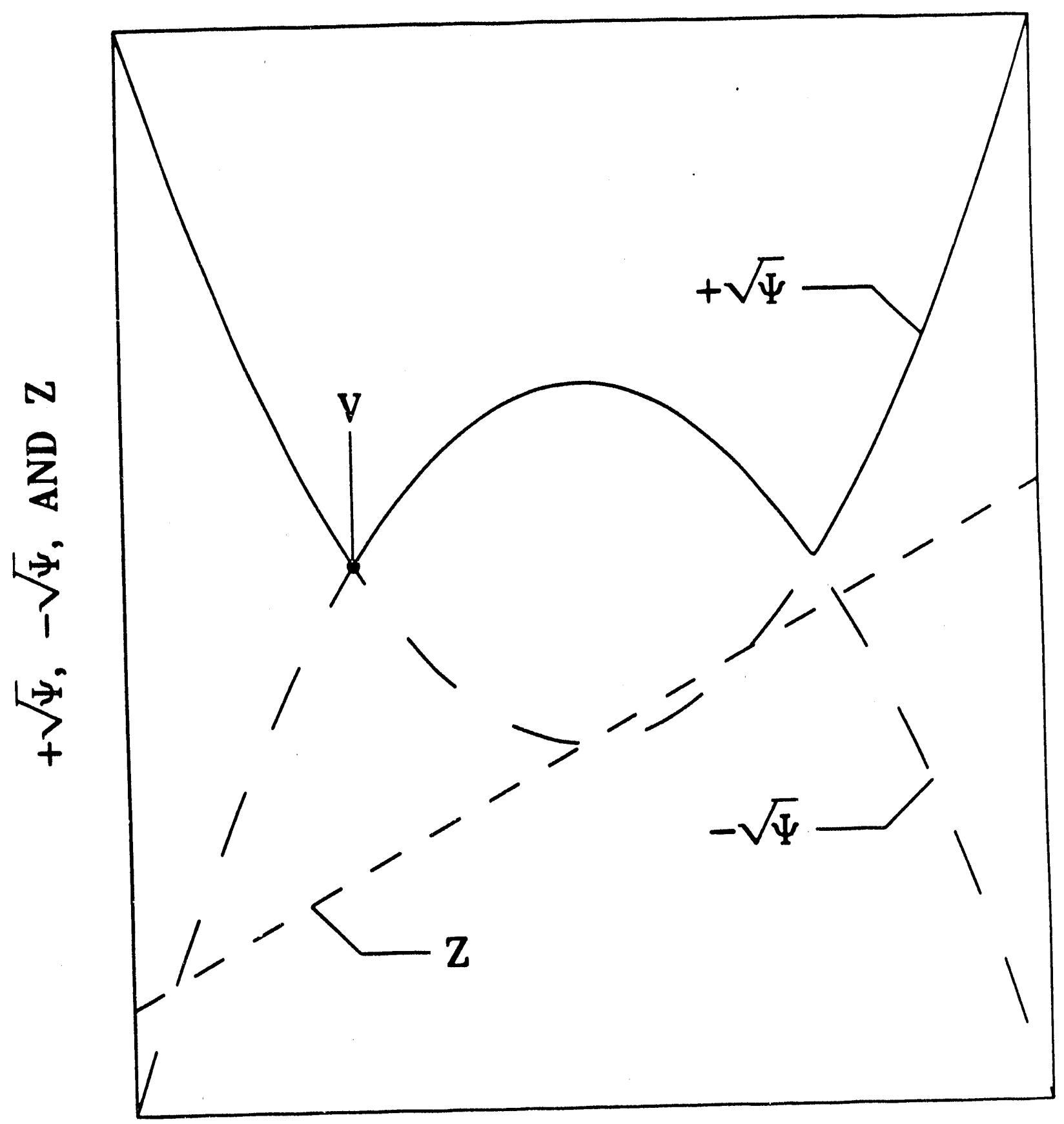

\section{HARD-SPHERE CHEMICAL POTENTIAL}

1:iqure 1a. Schematic representation of the dependence of the functions $+\sqrt{\Psi},-\sqrt{\Psi}, \pm \sqrt{\Psi}$. and $Z$ on $\mu^{h}$. Bulk fluid is in vapor single phase region near liquid-vapor binodal; its location is indicated by " $V$." (a) The solid curve is $+\sqrt{\Psi}$, dashed curve is $-\sqrt{\Psi}$, and dashed curve with shorter dashes is $Z$. 


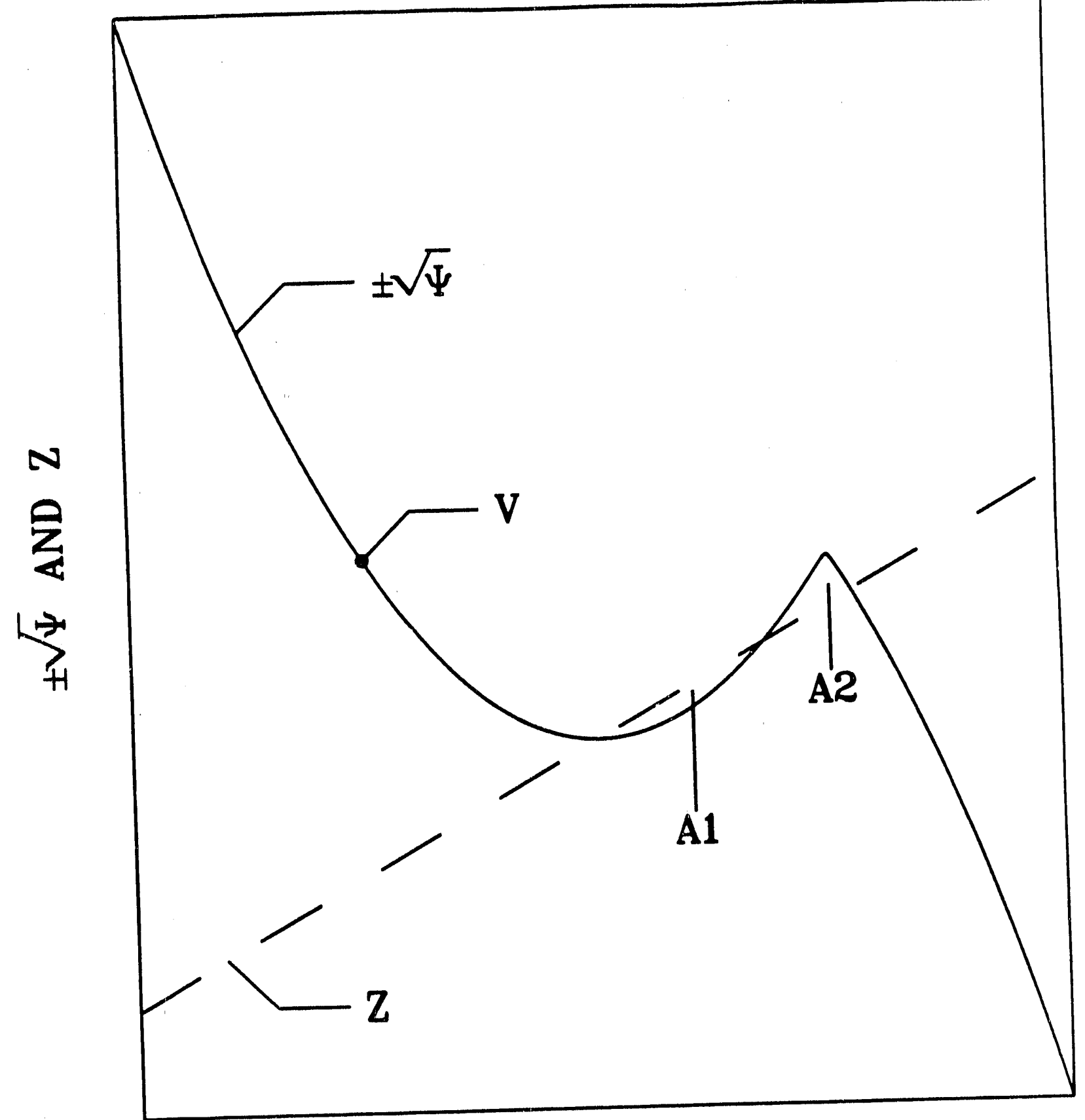

\section{HARD-SPHERE CHEMICAL POTENTIAL}

Figure 1b. The solid curve is $\pm \sqrt{\Psi}$ which is composed with segments of $+\sqrt{\Psi}$ and $-\sqrt{\Psi}$ that satisfy the sign convention discussed in section $\mathrm{B} 2$, the dashed curve is $Z$, and $A 1$ and $A 2$ are two areas bounded by $\pm \sqrt{\Psi}$ and $Z$. 
Eq.(15) for the three candidate solutions, (iii) compute the surface tension for each using Eq.(11), (iv) test whether the wetting transition temperature has been reached or exceeded; if the candidate with the lowest surface tension has the smallest value of $\mu^{h}(0)$ so that the adsorption at the interface is finite then increment the temperature and repeat steps (ii) through (iv); otherwise the wetting transition has been passed and the wetting transition temperature is bounded by the temperatures of the preceding and current iterations. Clearly this method can only give $T_{w} \pm \Delta T$ where $\Delta T$ is the temperature step size. Construction of the global phase diagram that corresponds to a given value of $W_{3}$ requires the wetting transition temperature for a set of $W$ values. To distinguish between or resolve the wetting transition temperature of two adjacent values of $W$ requires $\Delta T$ to be sufficiently small. Thus $\Delta T$ is a function of the set $\{W\}$ and also of $W_{3}$. Similarly for surface critical points $\left\langle T_{c s}, \mu_{c s}\right)$ the method only gives $T_{c s} \pm \Delta T$ and $\mu_{c s} \pm \Delta \mu$ and both $\Delta T$ and $\Delta \mu$ are functions of $\{W\}$ and $W_{3}$. Improper choice of $\Delta T$ and $\Delta \mu$ cause jagged edges to appear in the resulting global phase diagrams. To circumvent this difficulty we expressed the graphical construction in algebraic form and used Newton iteration to solve these sets of equations directly for the different transition and critical points. The errors $\Delta T_{w}, \Delta T_{c s}$, and $\Delta \mu_{c s}$ are then controlled by the size of the tolerance in the convergence criterion of the Newton iteration.

For arbitrary mean field models there are at least two ways to locate the first-order wetting transition, thin to thick thin-film transition, and surface critical points. The first method, due to Teletzke et al. (1982a), uses the adsorption isotherm while the second method, due to Tarazona and Evans (1983), uses the dependence of nonequilibrium surface tension on the Gibbs adsorption. It is useful to describe these two methods in further detail. Table 1 is an outline of the method used by Teletzke et al., Tarazona and Evans, and this work. The most important difference among the three is shown in the last three rows of Table 1; whereas the methods of Teletzke et al. and Tarazona and Evans require for instance an entire series of density profile solutions to locate a thin to thick thin-film transition point the method used in this work obtains the desired transition point directly; the price we pay being a larger equation set.

In the first method Teletzke et al. solved the chemical potential equation (Eq.3a):

$$
\delta \Omega / \delta n(x) \equiv \delta F / \delta n(x)-\mu=0 .
$$

using the Galerkin finite element method and Newton iteration to get the density profile $n(x)$ and the Gibbs adsorption $\Gamma$ at the disjoining potential:

$$
\Delta \mu \equiv \mu(h ; T)-\mu(h=\infty ; T)=\mu-\mu(\text { sat })
$$

where $\mu(h ; T)=\mu$ is the chemical potential of the system at the temperature $T$, film thickness $h \equiv \Gamma / n^{L}$ with $n^{L}$ the density of saturated bulk liquid at $T$, and $\mu\left(h=\infty ; T^{\prime}\right)=\mu($ sat $)$ is the chemical potential of the vapor at the temperature $T$ when it is in coexistence with the liquid phase at which point the film thickness $h$ becomes infinite. They then used continuation techniques to trace out the adsorption isotherm. The existence of multiple solutions at a given value of disjoining potential indicates a first-order wetting transition in which the solution having the smallest value of surface tension is the physical solution. The set of equations 


\section{TABLE 1}

Comparison of methods for locating first-order wetting transition, thin to thick thin-film transition, and surface critical point of a solid-pure fluid interface. m Three methods are given for comparison, that used by Teletzke et al. (1982a); that used by Tarazona and Evans (1983); and that used in this work for the exponential model of Teletzke et al. (1982b).

\begin{tabular}{|c|c|c|c|}
\hline \multicolumn{2}{|c|}{ Teletzke et al. } & Tarazona and Evans & This work \\
\hline Equations solved & $\begin{array}{l}\text { Chemical potential } \\
\text { equation }\end{array}$ & $\begin{array}{l}\text { Chemical potential } \\
\text { surface tension }\end{array}$ & $\begin{array}{l}\text { Equation for } \\
\text { and its derivatives }\end{array}$ \\
\hline Equation type & Ord. differential eqn. & Integral equation & Algebraic equation \\
\hline Numerical method & $\begin{array}{l}\text { Galerkin finite } \\
\text { element method and } \\
\text { Newton iteration }\end{array}$ & Picard iteration & Newton iteration \\
\hline Immediate result & Density profile $n(x)$ & Density profile $n(x)$ & $\begin{array}{l}\text { Wetting transition or } \\
\text { critical points. }\end{array}$ \\
\hline $\begin{array}{l}\text { Construct used to } \\
\text { locate transition } \\
\text { critical points }\end{array}$ & Adsorption isotherm & $\begin{array}{l}\text { Nonequilibrium } \\
\text { surface tension } \\
\text { as function of film } \\
\text { thickness } \gamma_{n e}\left(h_{n e} ; T\right)\end{array}$ & Not needed \\
\hline $\begin{array}{l}\text { Criterion for } \\
\text { first-order wetting } \\
\text { transition and thin- } \\
\text { film transition }\end{array}$ & $\begin{array}{l}\text { Multiplicity } \\
\text { adsorption isotherm }\end{array}$ & $\begin{array}{l}\text { Multiple minima in } \\
\gamma_{n e}\left(h_{n e} ; T\right)\end{array}$ & Not needed \\
\hline $\begin{array}{l}\text { Criterion for } \\
\text { surface critical point }\end{array}$ & $\begin{array}{l}\text { Continue increment- } \\
\text { ing } T \text { until multiple } \\
\text { solutions merge }\end{array}$ & $\begin{array}{l}\text { Continue increment } \\
\text { ing } T \text { until multiple } \\
\text { minima merge }\end{array}$ & $\begin{array}{l}\text { Not needed } \\
\text { for model of } \\
\text { Teletzke } \\
\text { et al. }(1982 \mathrm{~b})\end{array}$ \\
\hline
\end{tabular}

that they solved to get first-order wetting transition, thin to thick thin-film transition, and surface critical point are shown in Table 2. Here the letters a and b designate the coexisting thin-film states; the letters $\alpha$ and $\beta$ designate the coexisting bulk phases, which in this case are liquid and vapor. For the first-order wetting transition, the first two equations represent the fact that the two film states $n_{\alpha}(x)$ and $n_{\beta}(x)$ that coexist at the wetting transition are extremals of the grand potential; the third equation expresses the equality of surface tension between the coexisting $\alpha$ and $\beta$ film states; and the last two equations express the thermodynamic coexistence between the bulk vapor and liquid phases. For the thin to thick 


\section{TABLE 2}

Comparison of equaiions for locating first-order wetting transition, thin to thick thin-film transition, and surface critical point of a solid-pure fluid interface. Here $F$ is Helmholtz free energy of inhomogeneous fluids, $\mu$ is the chemical potential, $p$ is the fluid pressure, $n(x)$ is the density profile, $\gamma$ is the equilibrium surface tension, $\alpha$ and $\beta$ are the coexisting film states, $a$ and $b$ are the coexisting liquid and vapor states.

\begin{tabular}{|l|l|l|l|}
\hline \hline $\begin{array}{l}\text { Transition or } \\
\text { critical point }\end{array}$ & Teletzke et al. & Tarazona and Evans & This work \\
\hline First-order wetting & $\delta F / \delta n(x)]_{\alpha}-\mu=0$, & $\delta F / \delta n(x)]_{\alpha}-\mu=0$, & $\left.\partial \gamma / \partial \mu^{h}(0)\right]_{a}=0$ \\
transition & $\delta F / \delta n(x)]_{\beta}-\mu=0$, & $\delta F / \delta n(x)]_{\beta}-\mu=0$, & $\left.\partial \gamma / \partial \mu^{h}(0)\right]_{\beta}$ \\
& $\alpha=\beta$, & $\gamma_{a}=\gamma_{\beta}$ & $\gamma_{\alpha}=\gamma_{\beta}$ \\
& $\mu_{a} \equiv \mu_{b}$, & $\mu_{a} \equiv p_{b}$ & $\mu_{a} \equiv \mu_{b}$ \\
\hline Thin to thick & $\delta F / \delta n(x)]_{\alpha}-\mu=0$, & $\delta F / \delta n(x)]_{\alpha}-\mu=0$, & $\left.\partial \gamma / \partial \mu^{h}(0)\right]_{\alpha}=0$ \\
thin-film transition & $\delta F / \delta n(x)]_{\beta}-\mu=0$, & $\delta F / \delta n(x)]_{\beta}-\mu=0$, & $\left.\partial \gamma / \partial \mu^{h}(0)\right]_{\beta}=0$ \\
& $\gamma_{\alpha}=\gamma_{\beta}$. & $\gamma_{\alpha}=\gamma_{\beta}$. & $\gamma_{\alpha}=\gamma_{\beta}$ \\
\hline Surface critical & Obtained by conti- & Obtained by conti- & $\partial \gamma / \partial \mu^{h}(0)=0$ \\
point & nuation along thin- & nuation along thin- & $\partial^{2} \gamma / \partial \mu^{h}(0)^{2}=0$ \\
& film transition line & film transition line & $\partial^{3} \gamma / \partial \mu^{h}(0)^{3}=0$ \\
\hline \hline
\end{tabular}

thin-film transition the equations are similar to those for first-order wetting transition except that since the transition occurs in the single-phase region the liquid-vapor bulk equilibrium equations are no longer needed. For the surface critical point Teletzke et al. located this by continuation to the end point of the thin-film transitions where the two coexisting film states become identical in structure.

In the second method, also outlined in Table 1, Tarazona and Evans (1983) employed a feature of Picard iteration, when applied to the chemical potential equation to obtain the nonequilibrium surface tension as a function of film thickness $\gamma_{n e}\left(h_{n e} ; T\right)$ where $\gamma_{n e}$ is a nonequilibrium surface tension, $h_{n e}$ is the film thickness proportional to the Gibbs adsorption, and $T$ is the temperature. The desired equilibrium film state corresponds to the absolute minimum in $\gamma_{n e}\left(h_{n e} ; T\right)$. The equations that they solve are shown in Table 2 . At a first-order wetting transition the curve $\gamma_{n e}\left(h_{n e} ; T\right)$ as a function of $h_{n e}$ has two identical absolute minima: one minimum occurs at $h_{1}<\infty$ and the other minimum occurs at $h_{2}=\infty$ with a local maximum in between the two. At the thin to thick thin-film transition the curve is similar to that for first-order wetting transition except that both of the absolute minima 
have finite $h$ values. At the surface critical point the two minima and the maximum merge together resulting in a curve that is everywhere concave upwards.

In the fourth column of Table 1 we present our method. Here the equations for locating first-order wetting transition, thin-film transition, and surface critical points are used from the beginning. The advantage is the elimination of an intermediate construction such as the adsorption isotherm or the nonequilibrium surface tension as a function of film thickness, both of which demand the computation of at least several density profiles for sufficient accuracy in locating transition and critical points, For more complicated mean field models the equation set listed under column one of Table 2 can be used. Either the method of Teletzke et al. or Tarazona and Evans (1983) can be used to provide a suitable initial estimate but intermediate constructions are not necessary for the rest of the computations.

In the fourth column of Table 2 are the equations that we solve for locating first-order wetting transition, thin-film transition, and surface critical points. These equations are discussed in more detail in the next section.

\section{Equations Governing Wetting Transitions And Critical Phenomena}

The discussion of the method of graphical construction shows that any profile $\mu^{h}(x)$ that satisfies the chemical potential equation (Eqs. 6 and 7) must satisfy Eq.(15), i.e., where $\mu^{h}(0)$ is the value of $\mu^{h}(x)$ at the interface. This equation can be directly derived from the surface tension expression $Z\left(\mu^{h}(0)\right)= \pm \sqrt{\Psi\left(\mu^{h}(0)\right)}$, Eq. 13, by differentiating the surface tension with respect to $\mu^{h}(0)$ :

$$
\partial \gamma / \partial \mu^{h}(0)=Z\left(\mu^{h}(0)\right)-\left[ \pm \sqrt{\Psi\left(\mu^{h}(0)\right)}\right]=0 .
$$

In subsequent sections this equation enables us to express the graphical solution of the first-order wetting transition, the thin to thick thin-film transition, and the surface critical point in terms of the surface tension and its derivatives. The concept of surface tension derivative in the context of wetting transitions is not new; Nakanishi and Pincus (1983) used the vanishing of the second derivative of surface tension to define their surface spinodal points. Here we show that defining equations can also be written for the first-order wetting transition, the thin to thick thin-film transition, and the surface critical point. In addition equations that do not utilize the derivatives of surface tension also exists for second-order wetting transition, surface tricritical point, and multicritical point.

First - order wetting transition. The graphical method implies that at the first-order wetting transition the two coexisting film states should satisfy three conditions: (1) tension is a minimum, (2) equality of surface tension, and (3) the thermodynamic coexistence between the bulk fluid phases. These conditions translate into the following equations which are also 
listed in Table 2:

$$
\begin{gathered}
\partial \gamma /\left.\partial \mu^{h}(0)\right|_{\alpha}=0 \\
\partial \gamma /\left.\partial \mu^{h}(0)\right|_{\beta}=0, \\
\gamma_{\alpha}=\gamma_{\beta}, \\
\mu_{a}=\mu_{b}, \\
p_{a}=p_{b},
\end{gathered}
$$

where the letters $\alpha$ and $\beta$ designate the coexisting film states; the letters a and b designate the coexisting bulk phases (liquid and vapor in this case). Substitution of Eq. 11 into Eqs. $19 \mathrm{a}-19 \mathrm{e}$ yields

$$
\begin{gathered}
\beta\left(y_{\alpha}-\mu\right)-2 W-\left[ \pm \sqrt{\Psi\left(y_{a}\right)}\right]=0 \\
\beta\left(y_{\beta}-\mu\right)-2 W-\left[ \pm \sqrt{\Psi\left(y_{\beta}\right)}\right]=0 \\
\beta\left(y_{\alpha}^{2}-y_{\beta}^{2}\right) 2-(\beta \mu+2 W)\left(y_{\alpha}-y_{\beta}\right)+\int_{y_{\alpha}}^{y_{\beta}}\left[ \pm \sqrt{\Psi\left(y^{\prime}\right)}\right] d y^{\prime}=0, \\
\mu_{a}-\mu_{b}=0, \\
p_{a}-p_{b}=0 .
\end{gathered}
$$

Here $y_{\alpha}$ and $y_{\beta}=\mu^{h}(0)$ for surface phases $\alpha$ and $\beta$. Solution of Eqs.(20) for different values of $W$ and $W_{3}$ enables construction of the first-order wetting transition curves in the global phase diagrams. These will be discussed in Section C.

Thin to thick thin - film transition. The equations are similar to those of first-order wetting transition except that since it occurs when the fluid is in either the liquid or the vapor singlephase region the equations for bulk phase coexistence should be removed. Therefore

$$
\begin{gathered}
\gamma_{\alpha}=\gamma_{\beta}, \\
{\left[\partial \gamma / \partial \mu^{h}(0)\right]_{\alpha}=0,} \\
{\left[\partial \gamma / \partial \mu^{h}(0)\right]_{\beta}=0 .}
\end{gathered}
$$

With substitution of the equation for $\gamma$ the above equations become

$$
\begin{gathered}
\beta\left(y_{\alpha}^{2}-y_{\beta}^{2}\right) / 2-(\beta \mu+2 W)\left(y_{\alpha}-y_{\beta}\right)+\int_{y_{\alpha}}^{y_{\beta}}\left[ \pm \sqrt{\Psi\left(y^{\prime}\right)}\right] d y^{\prime}=0 \\
\beta\left(y_{\alpha}-\mu\right)-2 W-\left[ \pm \sqrt{\Psi\left(y_{\alpha}\right)}\right]=0 \\
\beta\left(y_{\beta}-\mu\right)-2 W-\left[ \pm \sqrt{\Psi\left(y_{\beta}\right)}\right]=0
\end{gathered}
$$

Solution of Eqs.(22) for different values of $W$ and $W_{3}$ yields the sets of thin-film transition curves in the global phase diagrams. These are discussed in Section (. 
Surface critical point. At the surface critical point the two coexisting film states not only share the same value of surface tension but also become completely identical in structure. Hence there is no need to state the equality of surface tension. However the film state should still satisfy Eq.(18) so that the first derivative of the surface tension with respect to $\mu^{h}(0)$ must still vanish. In addition higher-order derivatives must also vanish since in analogy to bulk criticality the critical point is not only the confluence point of binodal curves but also, as previously noted by Nakanishi and Pincus (1983), of the spinodal curves. Thus the equations for surface critical point are

$$
\begin{gathered}
\partial \gamma / \partial \mu^{h}(0)=0 \\
\partial^{2} \gamma / \partial \mu^{h}(0)^{2}=0 \\
\partial^{3} \gamma / \partial \mu^{h}(0)^{3}=0,
\end{gathered}
$$

Upon substitution of the equation for $g$ the above equations become

$$
\begin{gathered}
\beta(y-\mu)-2 W-[ \pm \sqrt{\Psi(y)}]=0 \\
\beta-[ \pm \sqrt{\Psi(y)}]=0 \\
\partial[ \pm \sqrt{\Psi(y)}] / \partial y=0
\end{gathered}
$$

where $y$ denotes the value of $\mu^{h}(0)$ at the surface critical point. Solution of Eqs.(24) for different values of $W$ and $W_{3}$ yields the curves of surface critical point in the global phase diagrams. 'These are discussed in section $\mathrm{C}$.

Surface tricritical point and second - order wetting transition As pointed out by Pandit et al. (1982), in appropriate regions of parameter space a surface tricritical point $\left(W_{t s}, T_{t s}, \Delta \mu_{t s}=0\right)$ can be defined such that for $\Delta \mu=0$ and $|W|>W_{t s}$ the wetting transition is first-order, while for $|W|<W_{t s}$, the transition is second-order. This is in direct analogy to the bulk case where the bulk tricritical point is the termination of a line of critical points and beyond this point the transition becomes first-order (Griffiths, 1970). For our mean field integral theory such surface tricritical points occur for $0<W_{3}<1$ or $1>\beta>0$.

The equations for the surface tricritical point cannot be obtained by analogy to bulk tricriticality because of the singularity at the wetting transition so that all derivatives of $\gamma$ with respect to $\mu^{h}(0)$ of order higher than or equal to two diverge at $\Delta \mu=0$. The same situation holds for wetting transitions. Nevertheless equations for the surface tricritical point and second-order wetting transitions can still be defined in another way that is valid only for the mean field model of Teletzke et al. (1982b). The transition equation is (Sullivan, 1979):

$$
Z\left(\mu^{h}(0)\right)=\beta\left(\mu^{h}(0)-\mu\right)-2 W=0
$$

where $\mu^{h}(0)=\mu_{L}^{h}$ and there is $L-V$ coexistence. 
Multicritical points. The density functional model of Teletzke et al. (1982b) is mathematically equivalent to gradient theory used by Cahn (1977) in that the second. order differential equation $\mathrm{Eq} .(6)$ is of the same form as the chemical potential equation of gradient theory. It turns out that gradient theory with parameters equivalent to $W=-\beta \mu / 2$ has already been studied extensively by workers interested in surface phase transitions at, ferromagnetic free surfaces which occur at the bulk critical temperature $T_{c}$ (Mills, 1971; Kaganov and Omel'yanchuk, 1971; Binder and Hohenberg, 1972 and 1974; Kumar, 1974; Lubensky and Rubin, 1975; see also reviews of Binder, 1983 and Kung, 1989). They found that at conditions that correspond to $L-V$ coexistence the surface phase transitions are multicritical points. Four types were identified depending on the value of $\beta$ :three of these, the so-called "ordinary," "special," and "extraordinary" multicritical points turn out lo correspond to wetting transitions at different values of $\beta$; while the fourth one, the "surface" multicritical point, corresponds to a surface critical point (Nakanishi and Fisher, 1982). The appropriate $\beta$-values at which the first three multicritical points occur are listed in Table 3 along with other information.

\section{Results of Computations}

This section presents solutions of the equations, discussed in Section B3, that govern first-order wetting transition, thin to thick thin-film transition, surface critical point, secondorder wetting transition, and surface tricritical point in the mean field model with exponential potentials. These equations are nonlinear algebraic equations and were solved by Newton iteration. The initial estimate to the solution was obtained by either searching in the appropriate parameter space, as for instance searching in temperature to locate wetting transition, searching in chemical potential at given temperatures to locate thin to thick thinfilm transition; or by zeroth-order continuation from another solution with a similar set of parameter values if such a solution was available. The convergence criteria were

$$
\left\|\mathbf{F}^{(k)}\right\|<10^{-5} \text { and }\left\|\mathbf{y}^{(k)}-\mathrm{y}^{(k-1)}\right\|<10^{-5}
$$

where $\left\|\mathbf{F}^{(k)}\right\|$ is the Euclidean norm of the vector equation set at the $k^{\text {th }}$ iterate and $\left\|y^{(k)}-y^{(k-1)}\right\|$ is the Euclidean norm of the difference between the estimate to the solution at the $k^{\text {th }}$ and $(k-1)^{\text {th }}$ iterate. In general the average number of Newton iterations nocded is much less than 10 even when near a surface critical point. 'The only exceptions are the calculations for $\beta=1 / 2$ of the surface critical points in the vicinity of the surface tricritical point at $W_{t s}=0.2028, T_{t s}=0.8538, \Delta \mu_{t s}=0$ for which it took much more than 10 iterations to converge. Due to this difficulty the surface critical point temperatures 'I's reported in Table 4 for $\beta=1 / 2$ for $W=0.23,0.22,0.21$ were not obtained from solving the set of nonlinear equations ( $\mathrm{Eq} .24)$ but rather from a search in the $(T, \Delta \mu)$ parameter space in the same manner as described above for the initial estimates. We turn now to the results. 


\section{First-Order Wetting Transition, Thin To Thick Thin-Film Transition, And Surface Critical Point}

We solved for the wetting transition, thin film transition, and surface critical point for about $20 W$-values in the vicinity of $W=-\beta \mu / 2$ for each of the cases $\beta=1 / 2,0,-1 / 2$ or equivalently $W_{3}=1 / 3,1,3$ (see Tables 4,5, and 6). Of the 60 or so $W$-values reported here, about 50 correspond to first-order wetting transition and therefore have thin-film transitions and surface critical points.

\section{TABLE 3}

Summary of results on the order of the wetting transition at solid-fluid interfaces for the mean field integral theory with exponential potentials. Here $W_{3}$ is the three-body interaction strength relative to the two-body interactions; $\beta \equiv\left(1-W_{3}\right) /\left(1+W_{3}\right) ; W$ is the ratio of solid-fluid to fluid-fluid interaction energies; $W^{*}$ is a positive constant that depends on $W_{3}$; $\mu$ is the constant chemical potential of the system; and the last column lists where possible equivalent surface-phase transitions are predicted to exist for the ferromagnetic free surface

\begin{tabular}{|c|c|c|c|c|}
\hline$W_{3}$ & $\beta$ & $W$ & Order & $\begin{array}{l}\text { Ferromagnetic } \\
\text { free surface }\end{array}$ \\
\hline 0 & 1 & All & Second & \\
\hline $0<W_{3}<1$ & $1>\beta>0$ & $\begin{array}{c}-\beta \mu / 2 \\
<W^{*} \\
W^{*} \\
>W^{*}\end{array}$ & $\begin{array}{l}\text { Second } \\
\text { Second } \\
\text { Tricritical } \\
\text { First }\end{array}$ & "Ordinatry transition" \\
\hline 1 & 0 & $\begin{array}{c}0 \\
>0\end{array}$ & $\begin{array}{l}\text { Multicritical } \\
\text { First }\end{array}$ & "Special transition" \\
\hline$>1$ & $<0$ & $\begin{array}{c}-\beta \mu / 2 \\
>-\beta \mu / 2\end{array}$ & $\begin{array}{l}\text { Multicritical } \\
\text { First }\end{array}$ & "Extraordinary transition" \\
\hline
\end{tabular}

Figures $2(\mathrm{a}-\mathrm{c})$ are plots of $\partial \gamma / \partial \mu^{h}(0)$ as a function of $\mu^{h}(0)$ near a first-order wetting transition, a thin to thick thin-film transition, and a surface critical point respectively wherein the interaction potentials have parameters $\beta=0$ and $W=0.004$. The horizontal line is $\partial \gamma / \partial \mu^{h}(0)=0$; intersections between the curve and the horizontal line are values of $\mu^{h}(0)$ that extremizes the surface tension and satisfies Eq.(18) or equivalently Eq.(17). The first two plots show van der Waals-like loops that connect coexisting film states; the third plot show critical isotherm-like curve near the surface critical point. However, the analogy to bulk transitions and critical phenomena is incomplete due to the singularity at the wetting transition point. 


\section{TABLE 4}

Properties of the thin to thick thin-film transition line from the mean field integral theory with exponential interaction potentials and $\beta=0.5$. The third column gives the length of the thin-fitm transition line; the fourth column gives the distance frora bulk coexistence.

\begin{tabular}{cccc}
\hline$W$ & $T_{w w} / T_{c}$ & $\left(T_{c s}-T_{w}\right) / T_{c}$ & $\left(\mu_{c s}-\mu\right) / k_{B} T_{c}$ \\
\hline 0.3000 & 0.2802 & 0.2488 & 0.0870 \\
0.2900 & 0.3764 & 0.1821 & 0.0680 \\
0.2800 & 0.4532 & 0.1367 & 0.0509 \\
0.2700 & 0.5198 & 0.1020 & 0.0366 \\
0.2600 & 0.5799 & 0.0744 & 0.0250 \\
0.2500 & 0.6352 & 0.0520 & 0.0159 \\
0.2400 & 0.6869 & 0.0340 & 0.0091 \\
0.2300 & 0.7354 & 0.0156 & 0.0031 \\
0.2200 & 0.7813 & 0.0087 & 0.0014 \\
0.2100 & 0.8247 & 0.0013 & - \\
0.2028 & 0.8538 & - & - \\
0.1900 & 0.9022 & - & - \\
0.1800 & 0.9317 & - & - \\
0.1700 & 0.9552 & - & - \\
0.1600 & 0.9733 & - & - \\
0.1500 & 0.9865 & - & - \\
0.1400 & 0.9952 & - & - \\
0.1300 & 0.9994 & & - \\
0.1250 & 1.0000 & - & - \\
\hline \hline
\end{tabular}

Figure $2\left(\right.$ a) shows $\partial \gamma / \partial \mu^{h}(0)$ as a function of $\mu^{h}(0)$ near the first-order wetting transition. From the figure there are three values of $\mu^{h}(0)$ that satisfy $\partial \gamma / \partial \mu^{h}(0)=0$, the profiles $\mu^{h}(x)$ that correspond to these values of $\mu^{h}(0)$ are therelore candidate film-states. The two outer points are relevant while the middle one has a higher tension value than the other two. At the wetting transition the $5 \mathrm{~h}$ where there is a sharp change in slope becomes singular. Apart from the existence of a near singular point the curve exhibits all the familiar characteristics of a vall der Waals loop in bulk liquid-vapor systems. The singular point marks the nearby existence of a second stable bulk phase and implies that the film state with the largest value of $\mu^{h}(0)$ have infinite or macroscopic thickness at $\Delta \mu=0$. From Eq.(18)

$$
\partial \gamma / \partial \mu^{h}(0)=Z\left(\mu^{h}(0)\right)-\left[ \pm \sqrt{\Psi\left(\mu^{h}(0)\right)}\right]=0
$$

Hence just as in the method of graphical construction discussed in Section 32 the equality of surface tension between the two outermost film states results in an equal-areas construction for the van der Waals-type loop. 
Properties of the thin to thick thin-film transition line from the mean field integral theory with exponential interaction potentials and $\beta=0$. The third column gives the length of the thin-film transition line; the fourth column gives the distance from bulk coexistence.

\begin{tabular}{cccc}
\hline \hline$W$ & $T_{w} / T_{c}$ & $\left(T_{c s}-T_{w}\right) / T_{c}$ & {$\left[\left(\mu_{c s}-\mu\right) / k_{B} T_{c}\right]$} \\
\hline \hline 0.0040 & 0.9808 & 0.0117 & 0.0018 \\
0.0038 & 0.9817 & 0.0111 & 0.0016 \\
0.0036 & 0.9827 & 0.0105 & 0.0015 \\
0.0034 & 0.9836 & 0.0099 & 0.0014 \\
0.0032 & 0.9846 & 0.0094 & 0.0012 \\
0.0030 & 0.9856 & 0.0087 & 0.0011 \\
0.0028 & 0.9865 & 0.0082 & 0.0010 \\
0.0026 & 0.9875 & 0.0076 & 0.0009 \\
0.0024 & 0.9885 & 0.0070 & 0.0008 \\
0.0022 & 0.9894 & 0.0064 & 0.0007 \\
0.0020 & 0.9904 & 0.0058 & 0.0006 \\
0.0018 & 0.9914 & 0.0052 & 0.0005 \\
0.0016 & 0.9923 & 0.0046 & 0.0004 \\
0.0014 & 0.9933 & 0.0041 & 0.0004 \\
0.0012 & 0.9942 & 0.0035 & 0.0003 \\
0.0010 & 0.9952 & 0.0029 & 0.0002 \\
0.0008 & 0.9962 & 0.0023 & 0.0002 \\
0.0006 & 0.9971 & 0.0017 & 0.0001 \\
0.0004 & 0.9981 & 0.0012 & 0.0001 \\
\hline \hline
\end{tabular}

As the temperature increases above the wetting temperature, the surface-phase transition moves into the vapor single-phase region and at the appropriate value of the bulk chemical potential there is a thin to thick thin-film transition. Figure $2(b)$ shows $\partial \gamma / \partial \mu^{h}(0)$ as a function of $\mu^{h}(0)$ near the thin-film transition at $T=0.985$. Here the singular point of Figure 2(a) has disappeared and this figure now has all the familiar properties of bulk liquidvapor phase transition. Again the coexisting film-states are those that satisfy the equation $\partial \gamma / \partial \mu^{h}(0)=0$ and the equality of tension is equivalent to an equal-areas construction for the van der Waals-type loop.

As the temperature is increased further the thin-film transition moves further and further 


\section{TABLE 6}

Properties of the thin to thick thin-film transition line from the mean field integral theory with exponential interaction potentials and $\beta=0.5$. The third column gives the length of the thin-film transition line; the fourth column gives the distance from bulk coexistence.

\begin{tabular}{cccc}
\hline \hline$W$ & $T_{w} / T_{c}$ & $\left(T_{c s}-T_{w}\right) / T_{c}$ & {$\left[\left(\mu_{c s}-\mu\right) / k_{B} T_{c}\right]$} \\
\hline-0.0650 & 0.3270 & 0.9800 & 0.6528 \\
-0.0700 & 0.4096 & 0.9017 & 0.5408 \\
-0.0800 & 0.4776 & 0.8377 & 0.4856 \\
-0.0850 & 0.5381 & 0.7808 & 0.4308 \\
-0.0900 & 0.5942 & 0.7279 & 0.3768 \\
-0.0950 & 0.6477 & 0.6774 & 0.3218 \\
-0.1000 & 0.6996 & 0.6280 & 0.2677 \\
-0.1050 & 0.7508 & 0.5788 & 0.2139 \\
-0.1100 & 0.8024 & 0.5288 & 0.1602 \\
-0.1150 & 0.8558 & 0.4766 & 0.1067 \\
-0.1200 & 0.9139 & 0.4192 & 0.0534 \\
-0.1210 & 0.9267 & 0.4065 & 0.0427 \\
-0.1220 & 0.9403 & 0.3929 & 0.0320 \\
-0.1230 & 0.9552 & 0.3781 & 0.0213 \\
-0.1240 & 0.9723 & 0.3610 & 0.0107 \\
-0.1242 & 0.9762 & 0.3571 & 0.0085 \\
-0.1246 & 0.9852 & 0.3481 & 0.0043 \\
-0.1248 & 0.9907 & 0.3426 & 0.0021 \\
\hline \hline
\end{tabular}

away from the bulk $\mathrm{L}-\mathrm{V}$ binodal and the two coexisting film states become more and more similar in structure until a critical point is reached where these two states become identical. This is the surface critical point $(T \mathrm{cs}, 5 \mathrm{cs})$. Figure 2(c) shows $\partial \gamma / \partial \mu^{h}(0)$ as a function of $\mu^{h}(0)$ near the surface critical point. The analogy with bulk critical point is again evident though such analogy is incomplete due to a singularity at the wetting transition point.

\section{Field-Density Phase Diagrams}

Before discussing the global phase diagrams let us consider field variable-density phase diagrams and field variable-field variable phase diagrams. For examples of such diagrams see Cahn(1977) and Teletzke ei al. (1982a). Figurc 3 is' a section of a bulk fluid field 


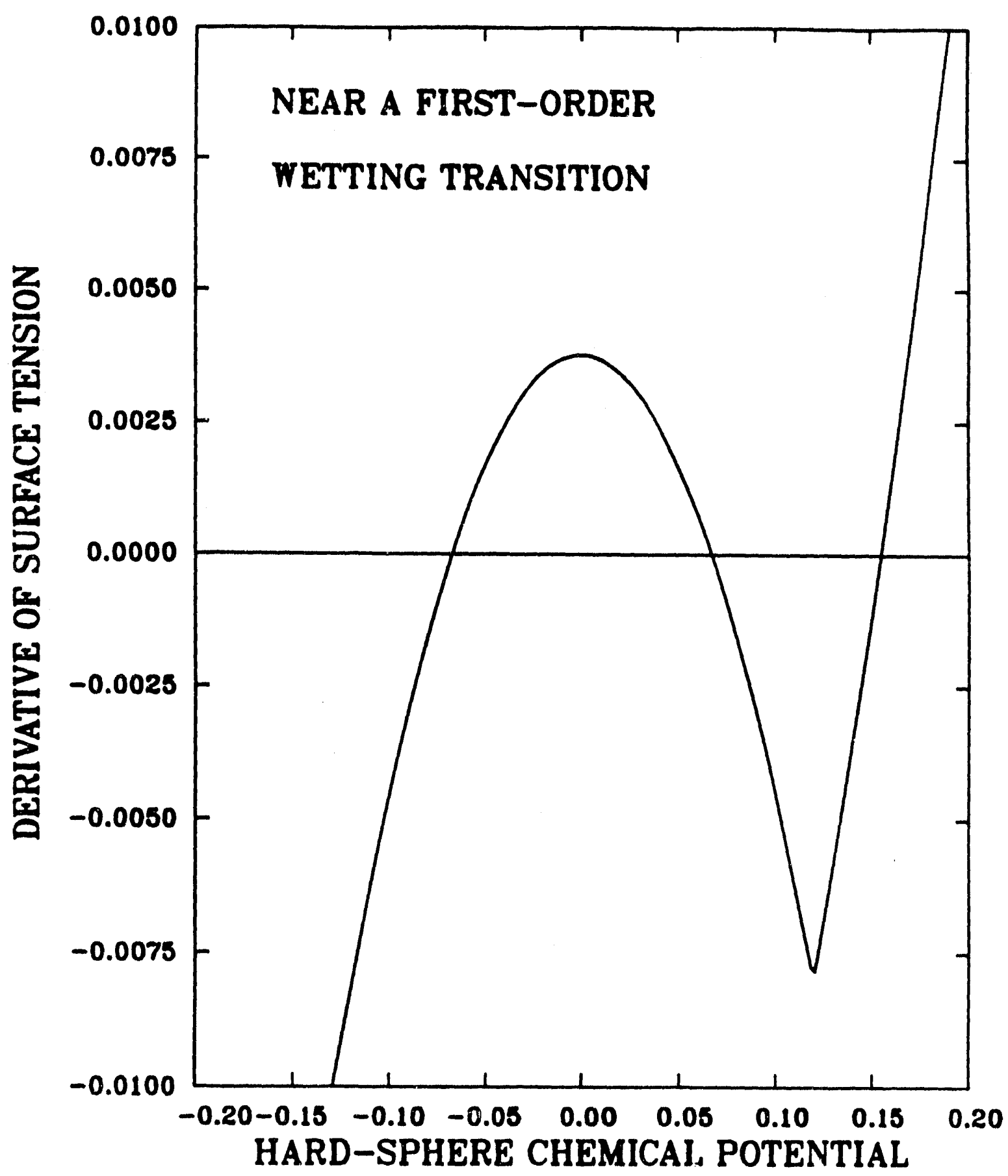

Figure 2a. The derivative of the surface tension: $\partial \gamma / \partial \mu^{h}(0)$ as a function of the hard-sphere chemical potential $\mu^{h}(0)$ at the interface. The interaction potential has parameter values: $\beta=0$ and $W=0.004$. The solid-fluid interface is close to the first-order wetting transition; 


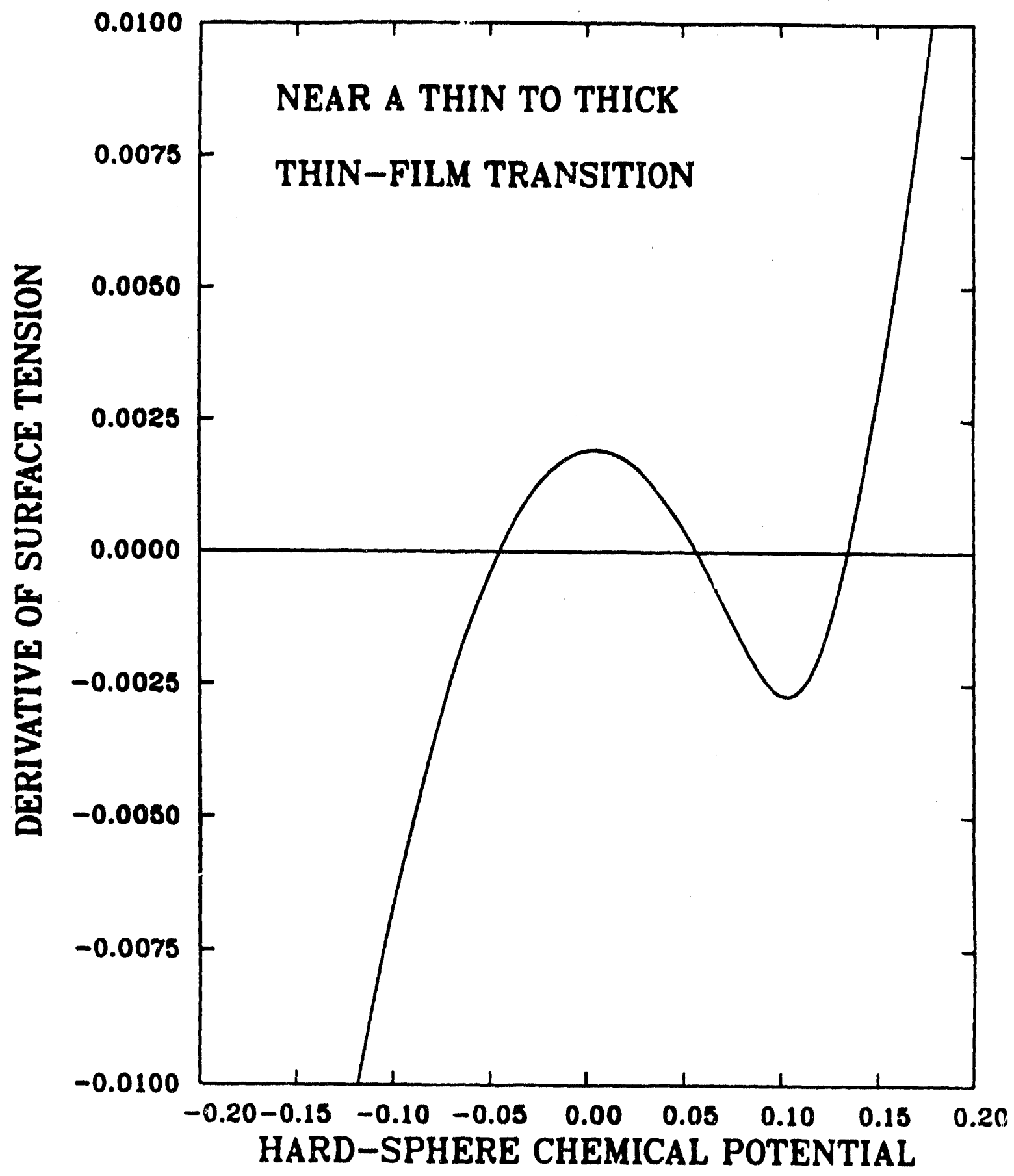

Figure $2 b$. The derivative of the surface tension: $\partial \gamma / \partial \mu^{h}(0)$ as a function of the hard sphere chemical potential $\mu^{h}(0)$ at the interface. The interaction potential has parameter values: $\beta=0$ and $W=0.004$. The solid-fluid interface is close to the thin to thick thin-film transition at $T=0.985$. 


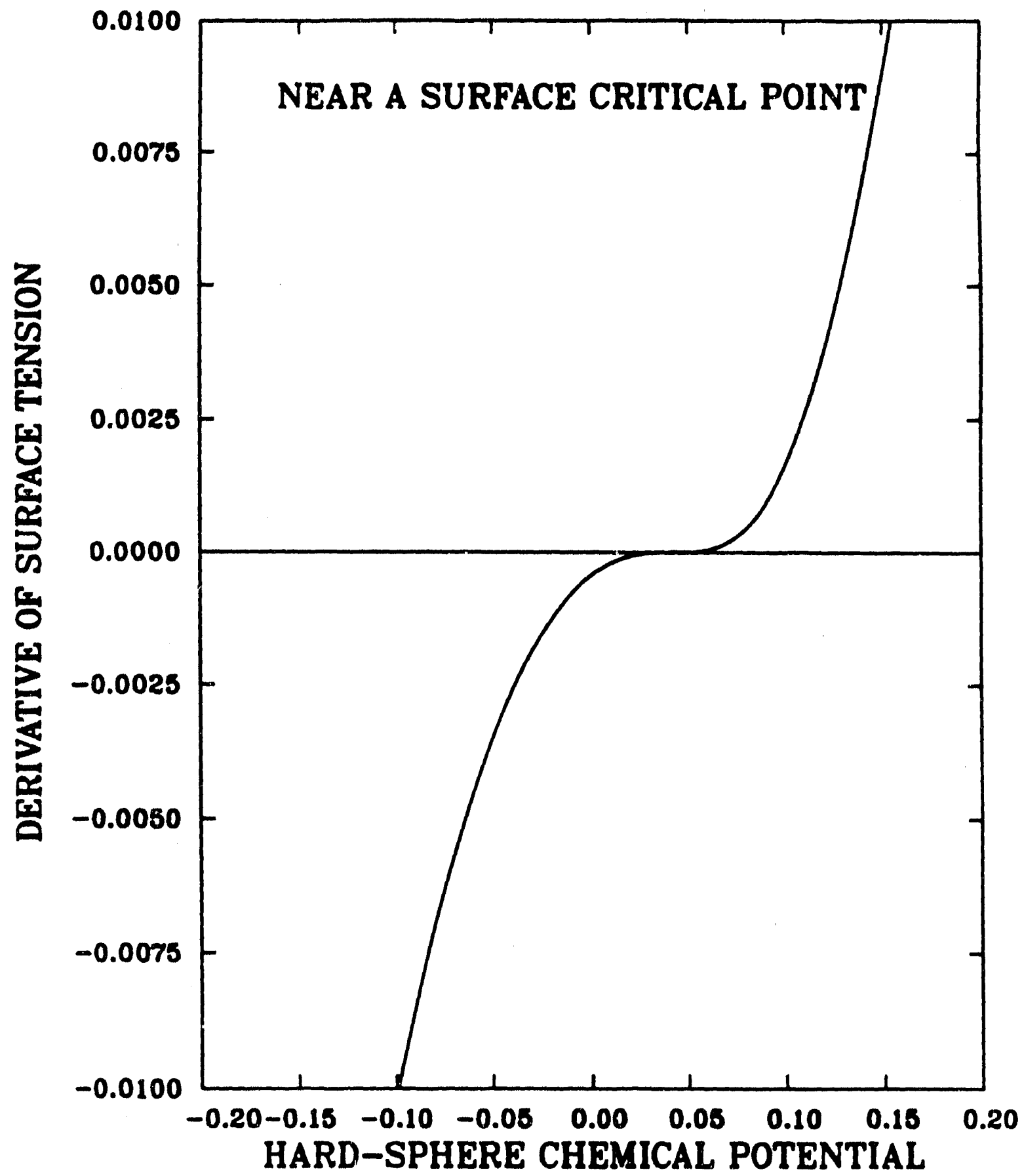

Figure 2c. The derivative of the surface tension: $\partial \gamma / \partial \mu^{h}(0)$ as a function of the hard-sphere chemical potential $\mu^{h}(0)$ at the interface. The interaction potential has parameter values: $\beta=0$ and $W=0.004$. The solid-fluid interface is close to the surface critical point. 


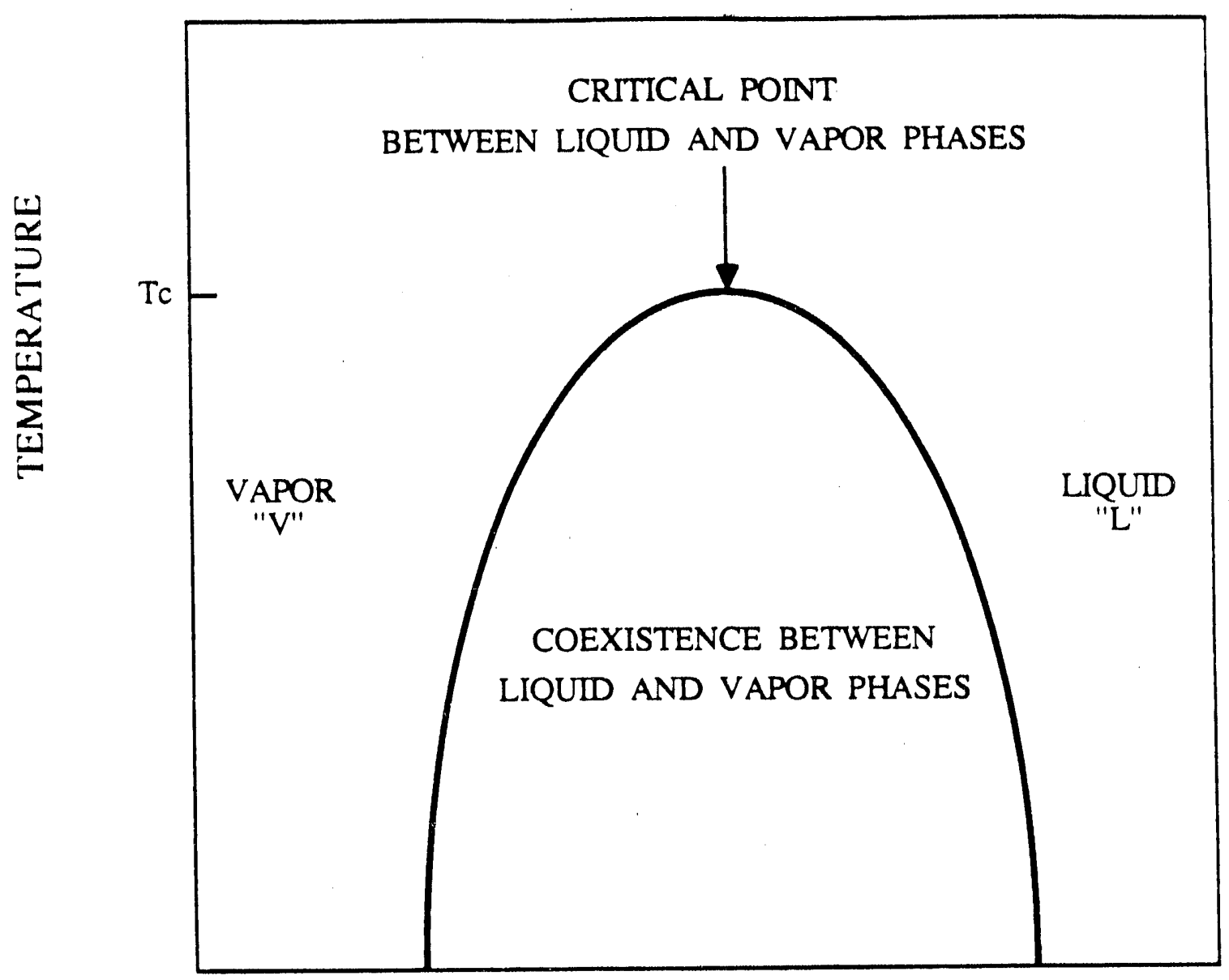

\section{DENSITY}

Figure 3. A schematic representation of a section of a fluid field-density phase diagram.

variable-density phase diagram in schematic form showing three regions: liquid phase, vapor phase, and a two-phase region where the two coexists. Wetting transitions occur on the bulk coexistence line while thin to thick thin-film transitions occur inside the single phase region. Figure 4 pictures what happens at the solid- fluid interface depending on where the bulk fluid is on the phase diagram and whether the wetting transition is second-order (Fig.4a) or first-order (Fig.4b). The solid line inside the $\mathrm{L}-\mathrm{V}$ two-phase region marks the wetting transition. The four capsules on either sides of the diagram pictures the solid-fluid interface depending on where the bulk fluid is on the phase diagram. The meaning for each of the three patterns used inside the capsules are explained in the legend above the diagram. The straight lines inside the two lower capsules measure the contact angle made by the $\mathrm{L}-\mathrm{V}$ interface through the liquid phase.

Figure 4(a) shows that at a temperature $T<T_{w}$ and near the vapor binodal of the two-phase region, a drop of liquid placed on the solid surface in the presence of its vapor does not spread indefinitely and the contact angle made by the $\mathrm{L}-\mathrm{V}$ interface at the solid 


\begin{tabular}{|c|c|c|}
\hline & LEGEND: & \\
\hline VAPOR & INERT SOLID & LIQUID \\
\hline
\end{tabular}

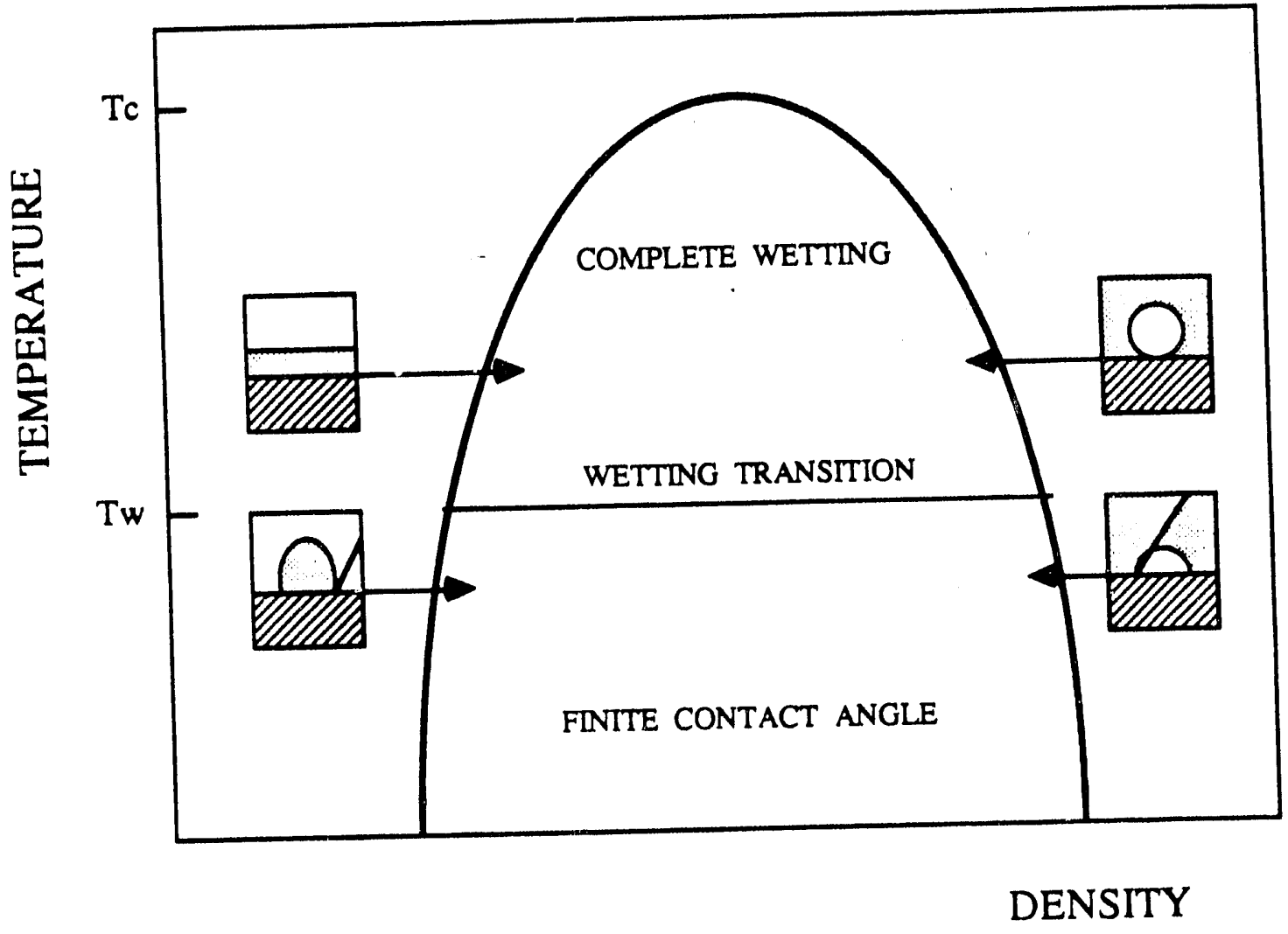

Figure 4a. A schematic representation of a field-density (temperature-density) phase diagram for second-order wetting transition

surface is not zero so that the liquid is said not to wet the solid surface. At $T \geq T_{w}$ and near the vapor binodal the contact angle goes to zero and, if enough of liquid is available, a macroscopic layer of the liquid completely wets the solid surface. This transition between 


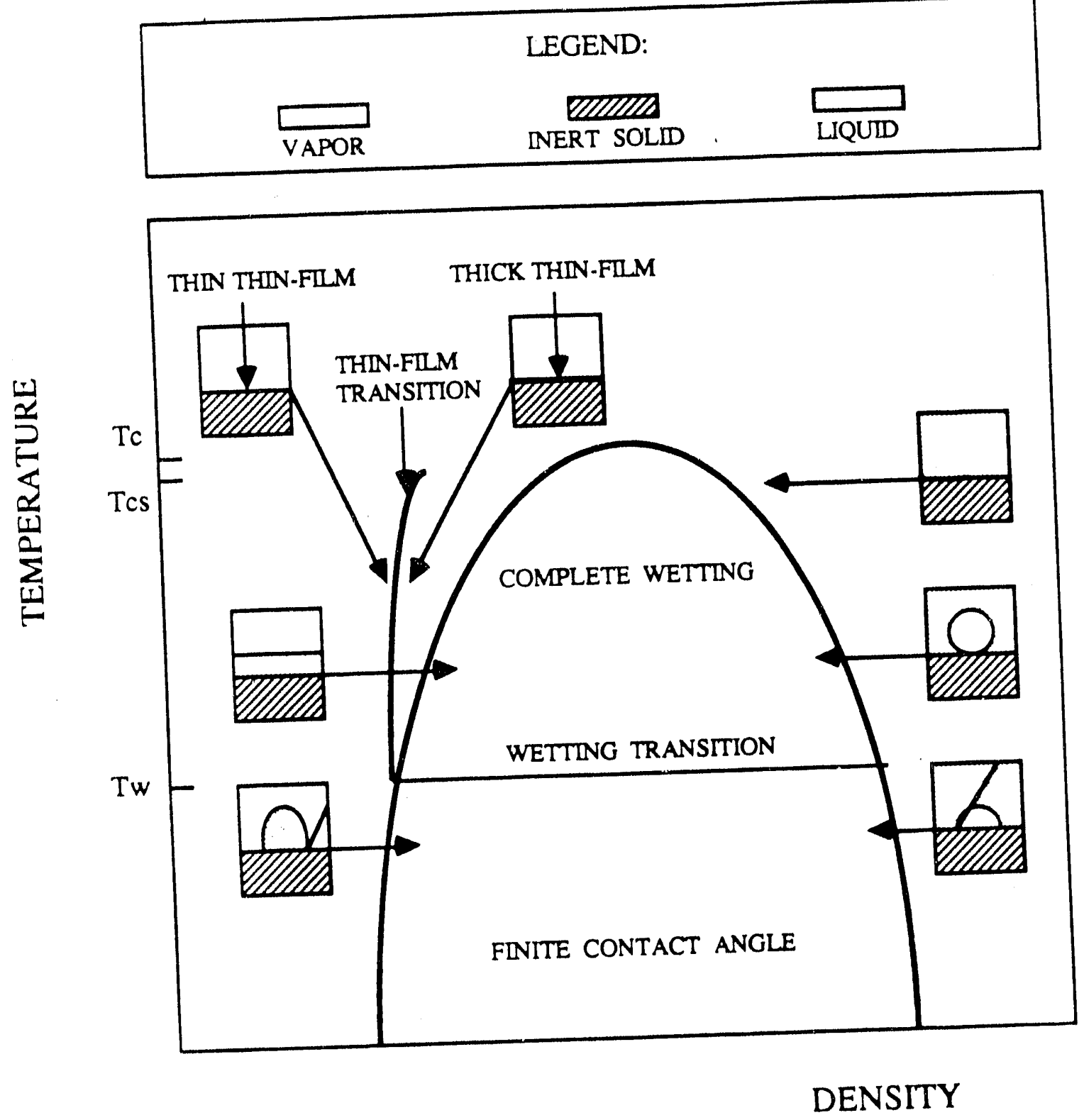

Figure 4b. A schematic representation of a field-density (temperature-density) phase liagralll for first-order wetting transition.

nonwetting and wetting by the liquid is called the wetting transition (Heady and Cahn, 1972; (ahn, 1977). What is shown here is a second-order wetting transition which is distinguished by the lack of thin-film transitions in the single-phase region. 
If the wetting transition is first-order (Fig. $4 \mathrm{~b}$ ), then in the vapor single phase region of the temperature-density phase diagram and above the wetting transition temperature there exists a temperature-density path along which two different liquid-like thin films coexist. The surface phase transition occurring when this path is crossed is known as the thin to thick thin-film transition or prewetting transition (Cahn, 1977; Teletzke et al., 1982a). With increasing temperature the thin-film coexistence curve on the bulk temperature- density phase diagram eventually terminates at a critical point called the surface critical point.

For our global phase diagram it is convenient to represent wetting transitions and critical points on field variable-field variable phase diagrams (Pandit et al. 1982). Figure 5 shows the transformation. The top figure is a field-density phase diagram; it is equivalent to the field-field phase diagram on the bottom left. In the figure on the bottom right the bulk chemical potential has been subtracted from the vertical y-axis so that bulk coexistence line is $y=0$. Thus far both the field-density and field-field diagrams are for given values of the interaction potential parameters $\left(W, W_{3}\right)$. In the next section we consider the effect of changing these parameter values on the wetting transition and critical points.

\section{Global Phase Diagrams}

The dependence of the order of the wetting transition, the thin to thick thin-film transition, and the surface multicritical points on interaction strength can be conveniently displayed together in global phase diagrams whose coordinate axes are field variables which include temperature, chemical potential, and an interaction potential parameter $W$ where $W$ is the ratio of characteristic energy of two-body solid-fluid interaction to fluid-fluid interaction. Three such diagrams were constructed for the following values of the three-body interaction potential energy parameter: $W_{3}=1 / 3,1$, and 3 ; corresponding to the three phase diagrams of Nakanishi and Fisher (1982; hereafter designated as NF) who inferred the diagram structure from scaling studies of a magnetic system. A comparison between the two sets of results are given at the end of this section.

Figure 6 demonstrates the evolution of the field-field phase diagram as $W$ increases for the case $W_{3}=1$. As the solid becomes more attractive to the fluid molecules, that is $W$ becomes more positive, the wetting transition goes from first-order on liquid side (top two figures), to first-order on vapor side (lowest two figures). In between is the "special" multicritical point. Putting these together we get Fig. 7, which is the global phase diagram for $W_{3}=1$. Figures 8 and 9 are the global phase diagrams for $W_{3}=1 / 3$ and 3 respectively.

By describing the evolution of the field-field phase diagram as $W$ increases we are actually describing the cross sections of the global phase diagram given by equations $W=$ constant. We now consider in greater detail this and other cross sections that contain the multicritical 

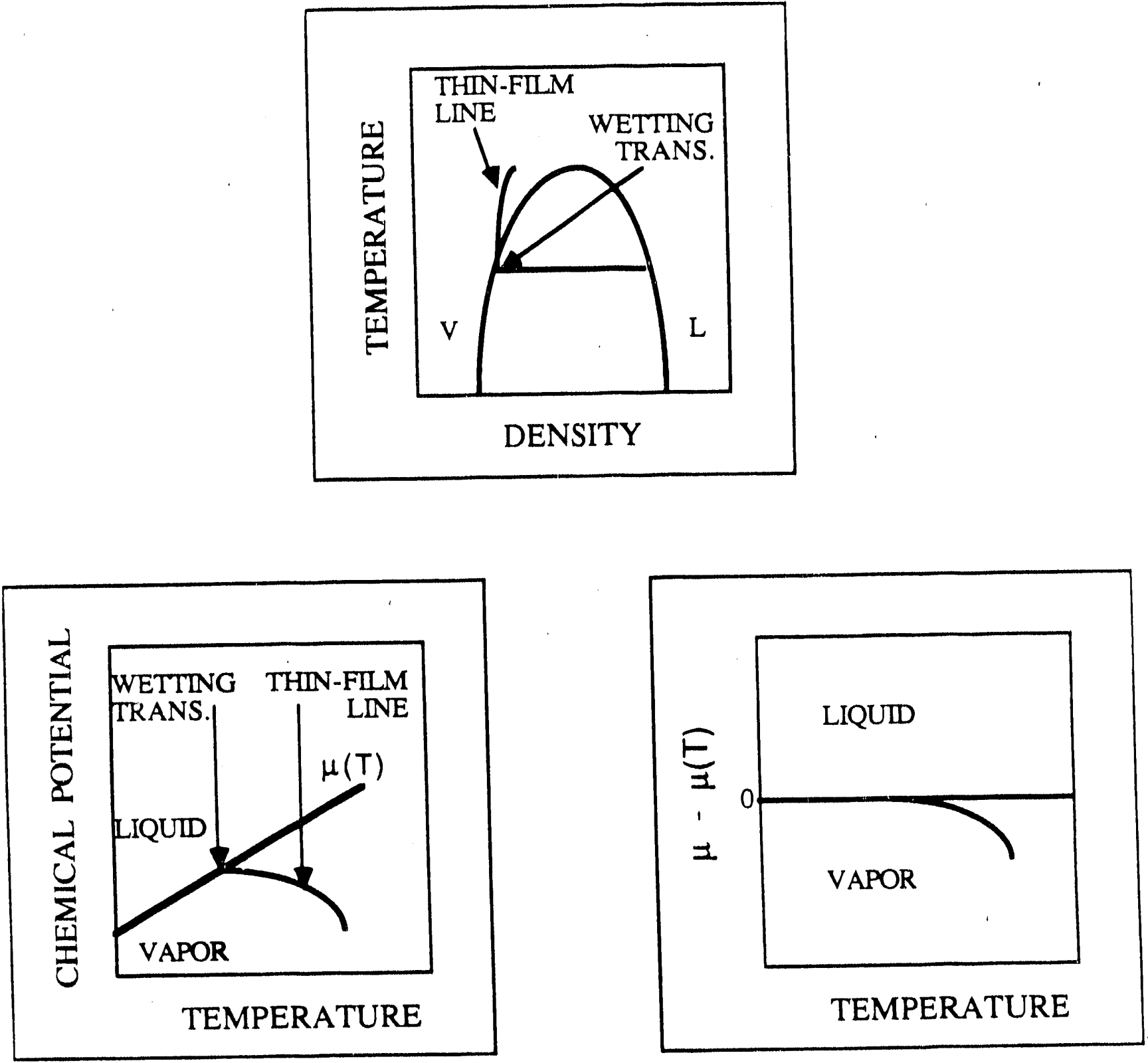

Figure 5. Illustration of the transformation from field density phase diagram to field-field phase diagram. 


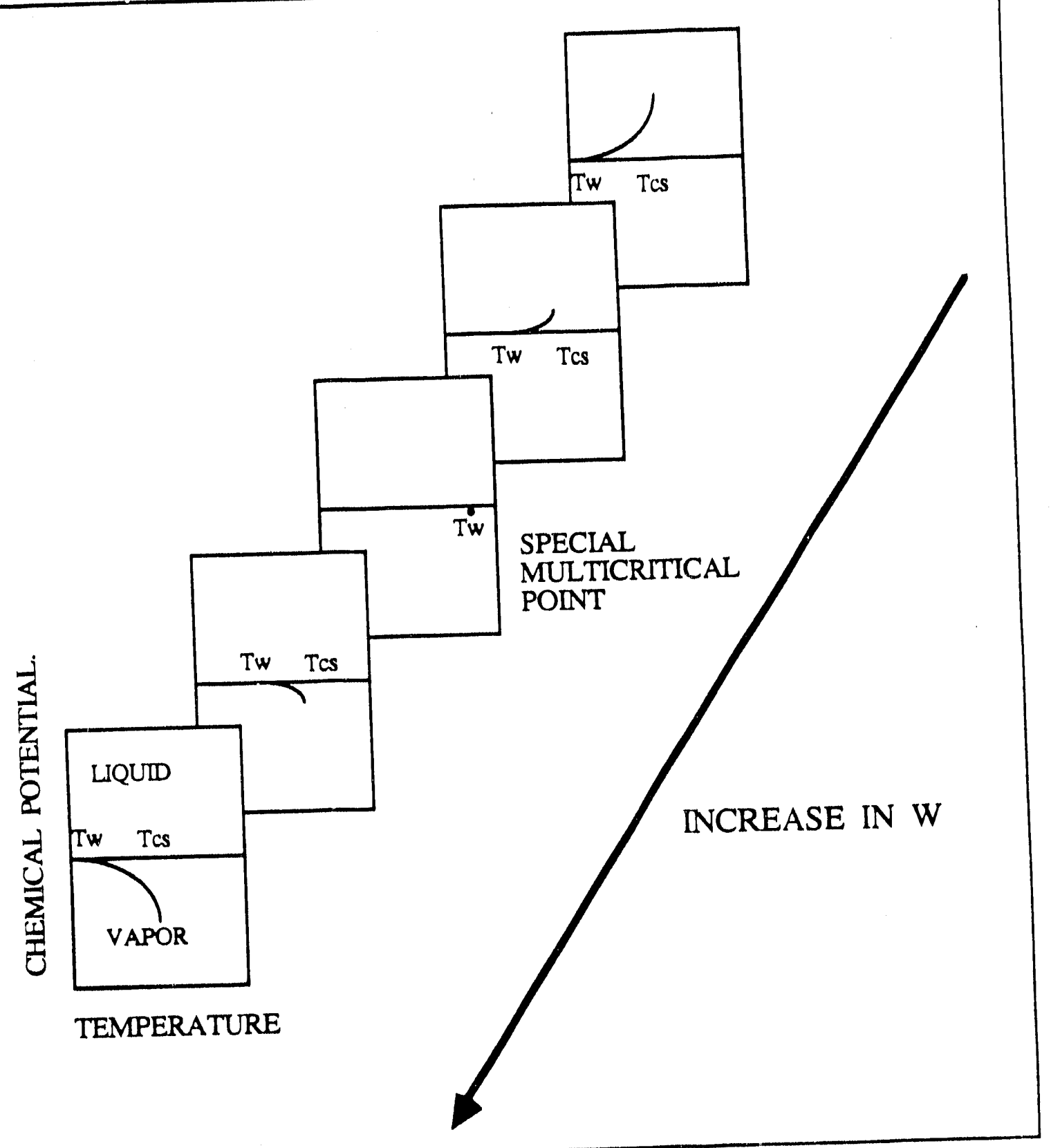

ligure 6. Schematic diagram of the evolution of the field-field phase diagram as $W$ increases for the case $W_{3}=1$. 


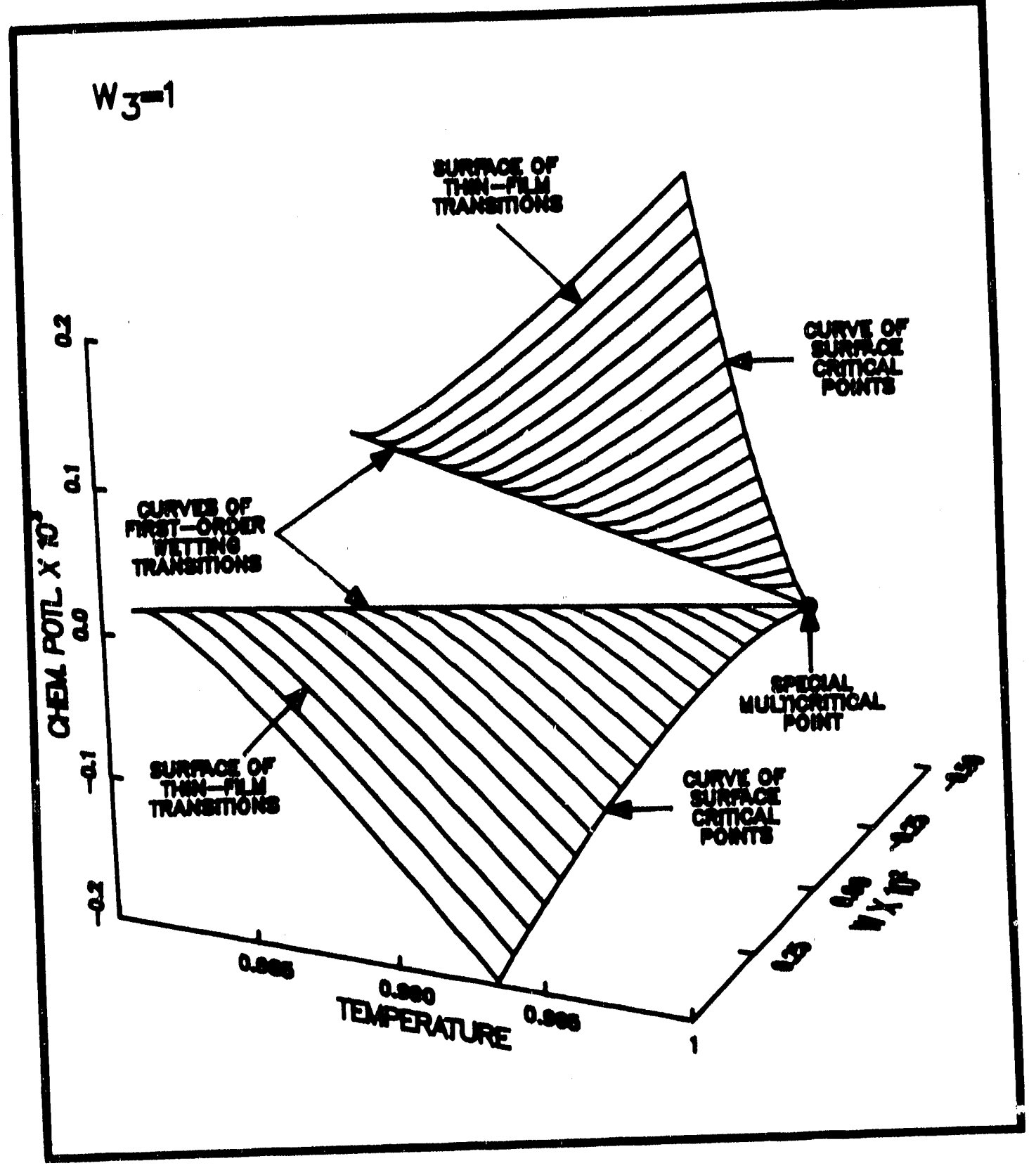

Figure 7. Global phase diagram for $W_{3}=1$. 
$w_{3}=1 / 3$

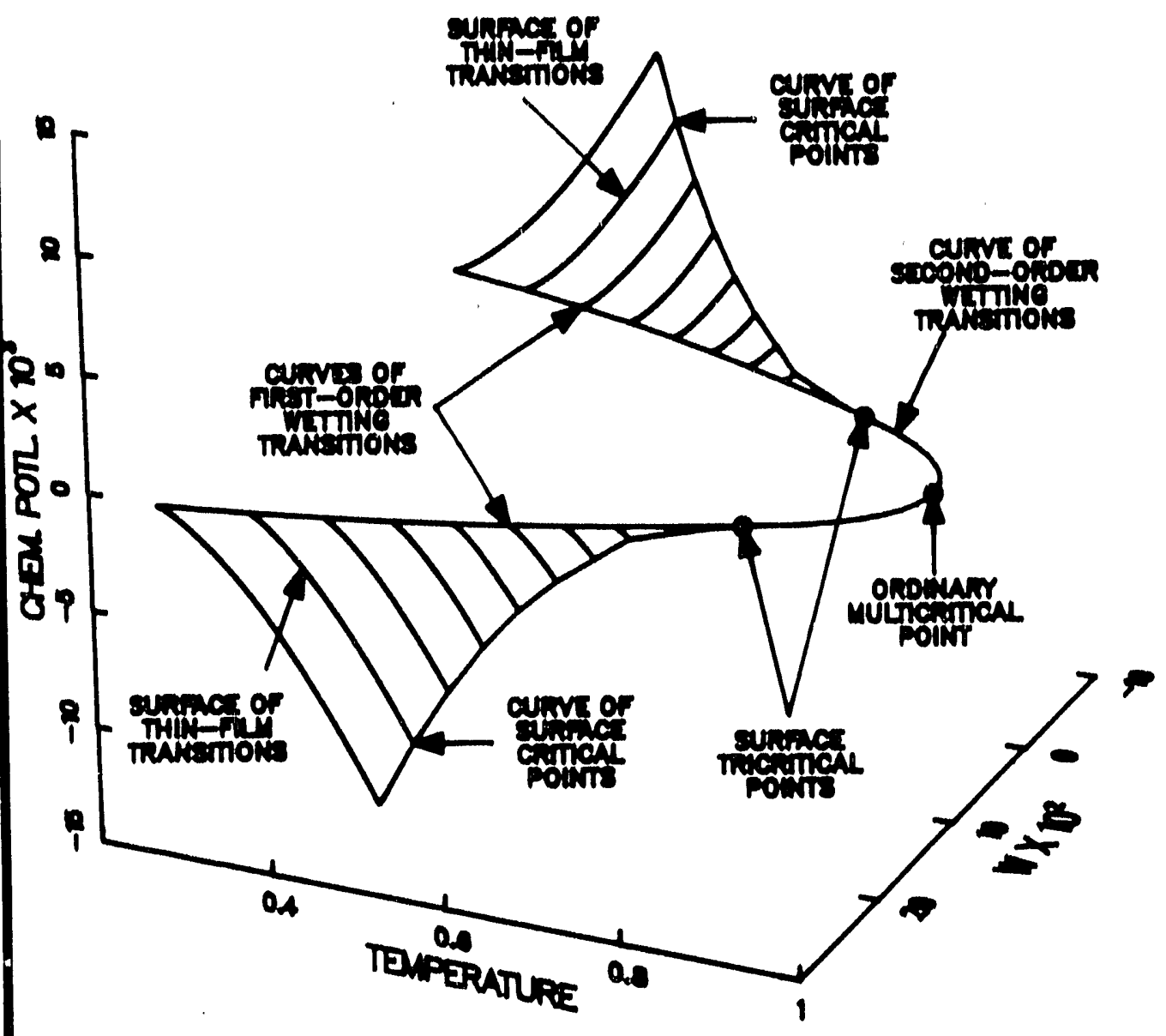

Figure 8. Global phase diagram for $W_{3} \doteq 1 / 3$. 


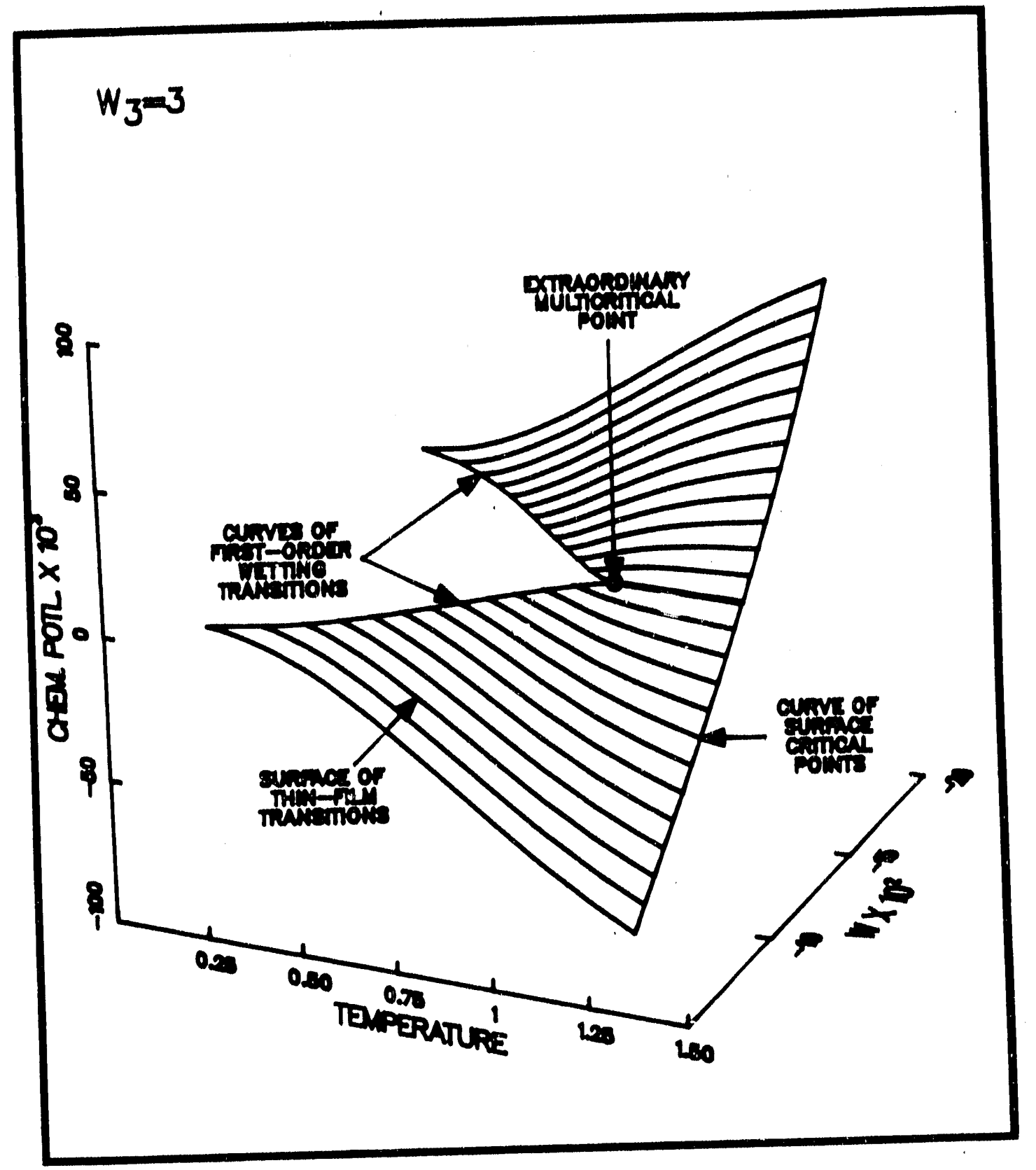

Figure 9. Global phase diagram for $W_{3}=3$. 
point located at $(W, T, \Delta \mu)=(-\beta \mu / 2,1,0)$, in which $T=1$ is the bulk critical temperature, for different values of $\beta$ (or $\left.W_{3}\right)$. We also present our results on how the wotting transition, thin to thick thin-fitm transition, and surface critical points scale in the vicinty of these multicritical points and compare ont exponents with those of NF. 'The exponents that we ohtain are combinations of the $\Delta$ and $\Delta_{1}$ exponents of $\mathrm{NF}$.

We begin with the case $\beta=1 / 2$ or $W_{3}=1 / 3$ (Figure 8). The coordinate axes are the ratio of solid-fluid to fludd-flud interaction chergies $W$, temperature th in units of $T_{c}$ where $T_{c}$ is the $L-V$ critical temperature, and chenical potential $\Delta \mu_{1}: \mu-\mu^{B}$ where $\mu^{B 3}$ is the chemical potential at l, $-\mathrm{V}$ coexistence. 'Therefore the half-plane defined by $\Delta \mu=0$ and $T \leq 1$ is a $L-V$ coexistence plane. On the $L-V$ coexistence plane are points of firstorder wetting transition, points of second-order wotting transition, two surface tricritical points, and one multicritical point. The multicritical point has coordinates $]^{\prime}=1$ and $W=-\beta \mu / 2=0.125$ and is an "ordinary" multicritical point (see e.g., Lubensky and Rubin, 1975). The two other points on this plane that are symmetrically disposed in $\mathrm{W}$ about the multicritical point are surface tricritical points with coordinates given by $\left(W_{t s}, T_{t, 4}, \Delta \mu_{t, s}\right)=(0.2028,0.8538,0)$. The solid curves on the coexistence plane bounded by the two surface tricritical points and passed through the multicritical point are points of second-order wetting transition. 'The curves on the same plane that joins the tricritical points at one end and proceed to very low temperatures are curves of first-order wetting transitions. The "wing"-like structures are surfaces of thin to thick thin-film transition. Each individual curve on these surfaces is a line of thin-film transition which joins the coexistence plane at a point of first-order wetting transition and terminates at the other end in a surface critical point. Finally the surface critical points are joined together in a curve that forms the edge of the two "wings" and joins the coexistence plane at the tricritical points. Therefore surface tricritical points are the confluence points for the line of first-order wetting transitions, the line of surface critical points, and the line of second-order wetting transitions. The entire diagram is symmetric about the line passing through the multicritical point and given by the intersection of the planes $W=-\beta \mu / 2$ and $\Delta \mu=0$ because lattice-gas model possesses a symmetrical binodal. According to $\mathrm{NF}$, near the multicritical point the distance between the second-order wetting transition temperature $T_{w}$ and the bulk critical temperature $T_{c}$ should scale with the distance from the "ordinary" multicritical point.

$$
1-\left(T_{w} / T_{c}\right) \approx(W+\beta \mu / 2)^{1 / \Delta_{1}} \approx(W+\beta \mu / 2)^{2}
$$

where the exponent $\Delta_{1}=1 / 2$ for the "ordinary" multicritical point. In Figure 10 the quantity $1-\left(T_{w} / T_{c}\right)$ is plotted as a function of $W+\beta \mu / 2$ for $W+\beta \mu / 2>0$. The straight, line is the above scaling relation of $N F$ and the filled circles are our computed results; there is good agreement between the two over a wide range of parameters.

Figure 7 is the global phase diagram of $\beta=0$ or $W_{3}=1$. The multicritical point on the $L-V$ coexistence plane has coordinates $T=1$ and $W=-\beta \mu / 2=0$ and is called the "special" multicritical point (Binder and Hohenberg, 1974; Kumar, 1974). All second-order wetting transitions have now disappeared and the solid curves on the coexistence plane that 


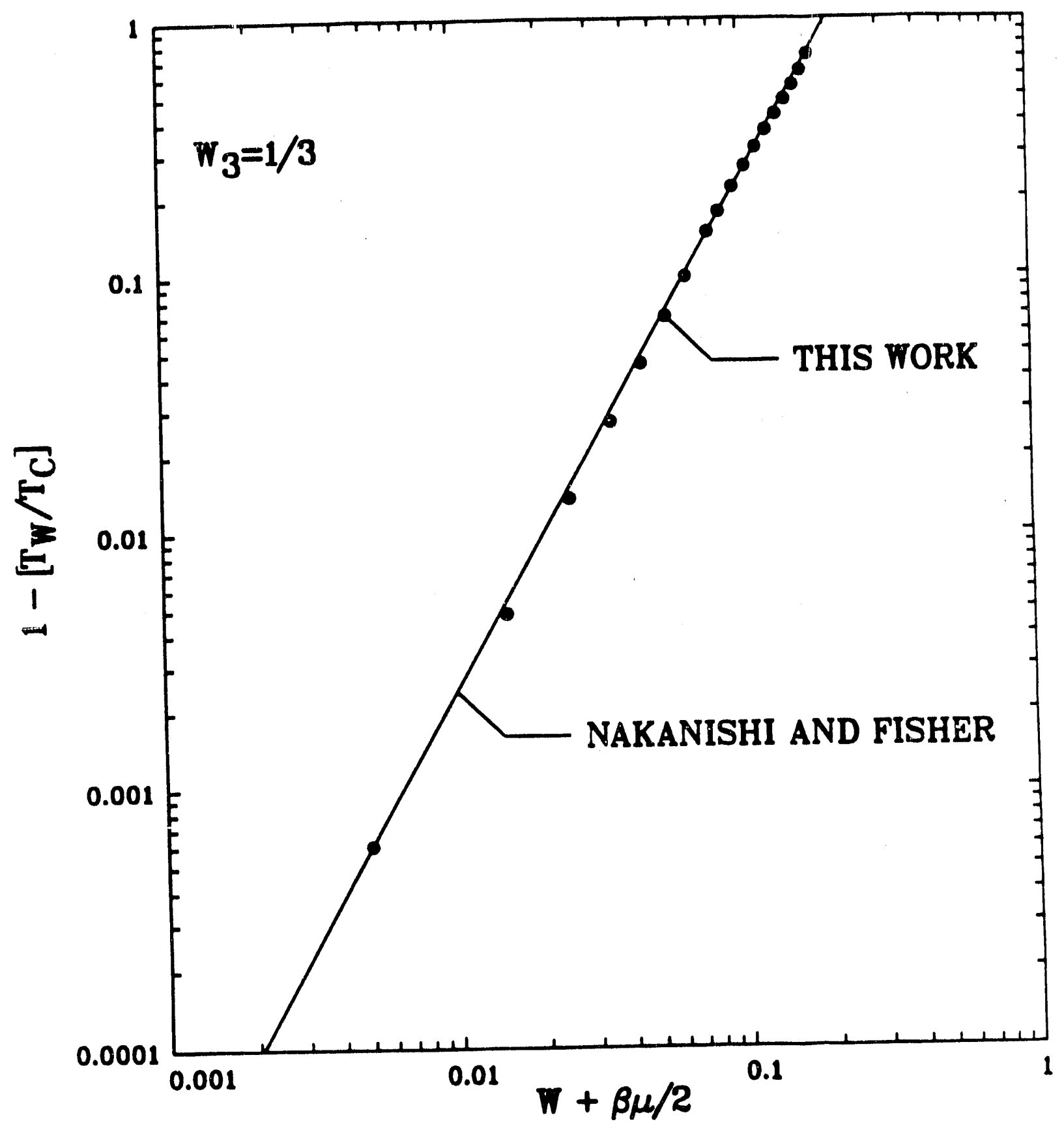

Figure 10 Plot of the distance between the second-order wetting $\operatorname{tr} ı$ sition temperature $T_{w}$ and the bulk critical temperature $T_{c}$ as a function of the distance from the "ordinary" multicritical point measured in terms of $W+\beta \mu / 2$. Here $W$ is the ratio of solid-fluid to fluid-fluid interaction energies; $\beta \equiv\left(1-W_{3}\right) /\left(1+W_{3}\right)$, and $\mu$ is the fluid chemical potential. 
pass through the multicritical point are points of first-order wetting transition. The "wing"like structures are again surfaces of thin to thick thin-film transition. The edges of the two "wings" are surface critical points which joined the coexistence plane at the multicritical point. According to NF, near the "special" multicritical point the distance between the first-order wetting transition temperature $T_{w}$ and the bulk critical temperature $T_{c}$ should scale with the distance from the "special" multicritical point as $(\beta \mu=0)$

$$
1-\left(T_{w} / T_{c}\right) \approx W \approx W^{1 / \Delta_{1}}
$$

where the exponent $\Delta_{1}=1$ for the "special" multicritical point. The distance between the surface critical point and the bulk critical point in terms of temperatures: $1-\left(T_{c s} / T_{c}\right)$ should scale in the same manner as that of the wetting temperature

$$
1-\left(T_{c s} / T_{c}\right) \approx W \approx W^{1 / \Delta_{1}}
$$

and the distance between the surface critical point and the bulk critical point measured in terms of chemical potential $\Delta \mu_{c s}$ should scale as $(\beta \mu=0)$

$$
\Delta \mu_{c s} \approx W^{\Delta / \Delta_{1}} \approx W^{3 / 2} .
$$

where the exponent $\Delta=3 / 2$ so that $\Delta / \Delta_{1}=3 / 2$. In Figures 11-13 the above three scaling relations are plotted together with our computed results. Again the scaling law holds over a wide range of parameters.

Figure 7 is the global phase diagram for $\beta=-1 / 2$ or $W_{3}=3$. The multicritical point on the $\mathrm{L}-\mathrm{V}$ coexistence plane has coordinates $T=1$ and $W=-\beta \mu / 2=-0.125$ and is called the "extraordinary" multicritical point (Lubensky and Rubin, 1975). The solid curves on the coexistence plane that pass through the multicritical point are again points of first-order wetting transition. The "wing"-like structures are again surfaces of thin to thick thin-film transition. The edges of the two "wings" are surface critical points which joined the coexistence plane at a point of higher temperature $(T>1)$ than that of the multicritical point. According to NF, near the "extraordinary" multicritical point the distance between the first-order wetting transition temperature $T_{w}$ and the bulk critical temperature $T_{c}$ should scale with the distance from the "extraordinary" multicritical point as $(\beta \mu=1 / 4)$

$$
1-\left(T_{w} / T_{c}\right) \approx(W+\beta \mu / 2)^{1 / \Delta} \approx(W+\beta \mu / 2)^{2 / 3} .
$$

In Figure 14 the above scaling relation is plotted with our computed results. There is good agreement for $W+\beta \mu / 2<0.01$, or, because the wetting temperatures are symmetric about $W=-\beta \mu / 2$, the above scaling relation is valid only for $\Delta W_{w} \equiv|W+\beta \mu / 2|<0.01$. Furthermore, from the global phase diagram (Fig.9), the range in $W$ over which the surface critical point temperature exceeds the bulk critical temperature, i.e., $T_{c g}>T_{c}$, is given by at least $\Delta W_{c s} \neq|W+\beta \mu / 2| \leq 0.06$. Hence $\Delta W_{c s}$ is larger than $\Delta W_{w}$. This differs from the corresponding global phase diagram of $\mathrm{NF}$ in which the reverse is portrayed. 


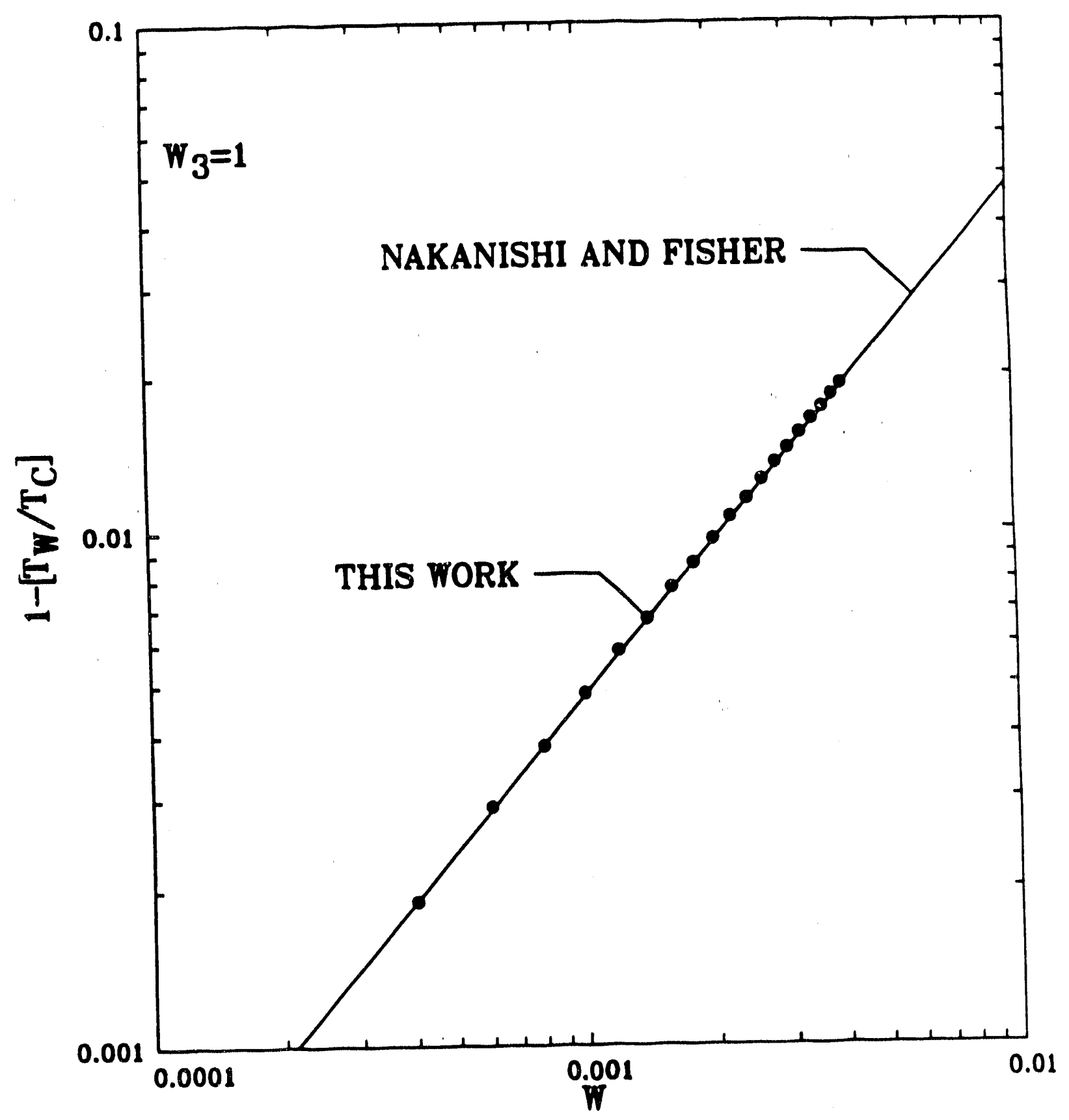

Figure 11. Plot the distance between the first-order wetting transition temperature $T_{w}$ and the bulk critical temperature $T_{c}$ as a function of the distance $W$ from the "special" multicritical point. 


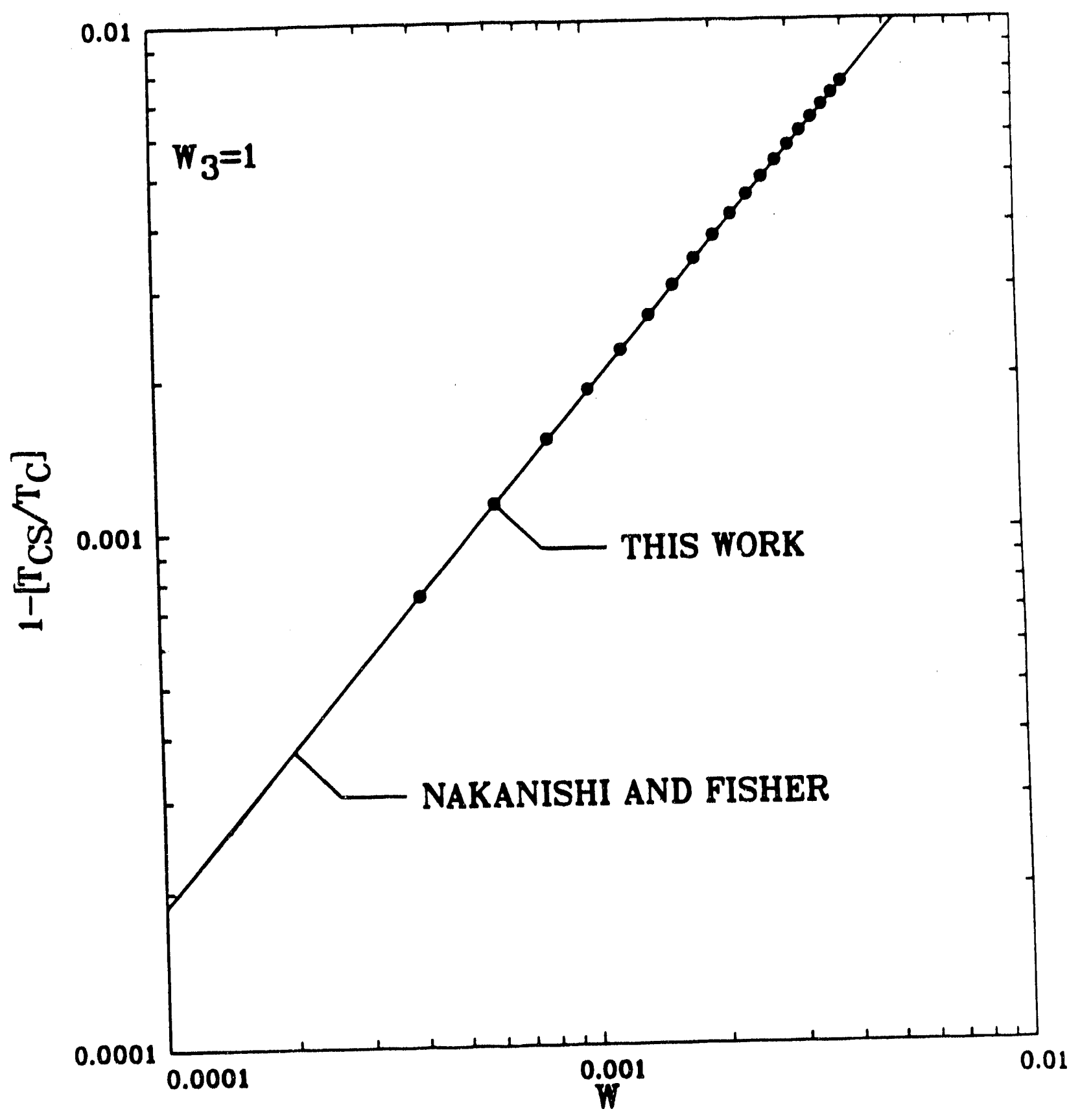

ligure 12. Plot of the distance between the surface critical point $T_{c s}$ and the bulk critical point $T_{c}$ as a function of the distance $W$ from the "special" multicritical point. 


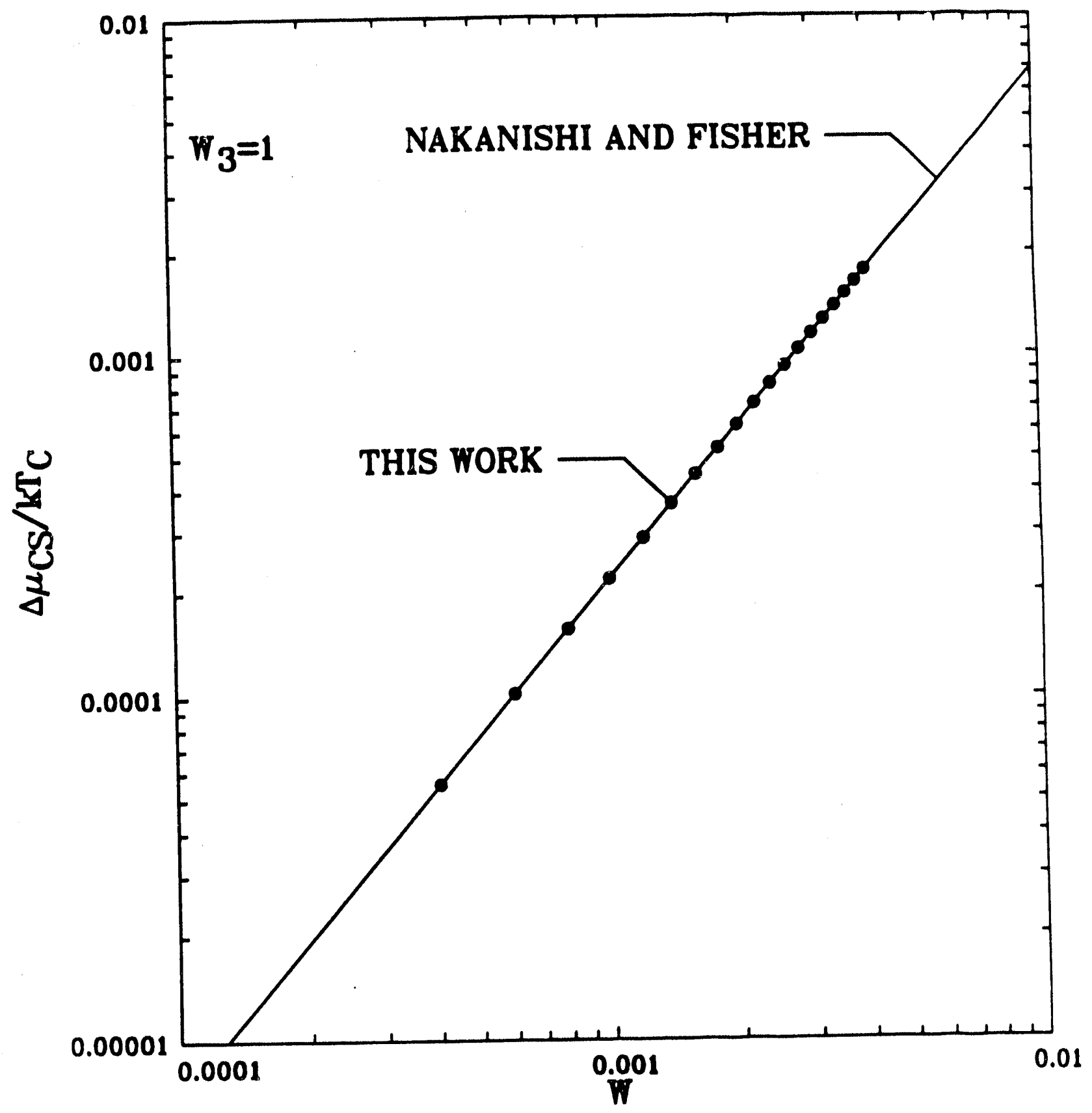

Figure 13. Plot of the distance between the surface critical point and the bulk critical point measured in terms of chemical potential $\Delta \mu_{c s} \equiv \mu_{c s}-\mu_{c}$, in units of $k_{B} T_{c}$, as a function of the distance $W$ from the "special" multicritical point. 


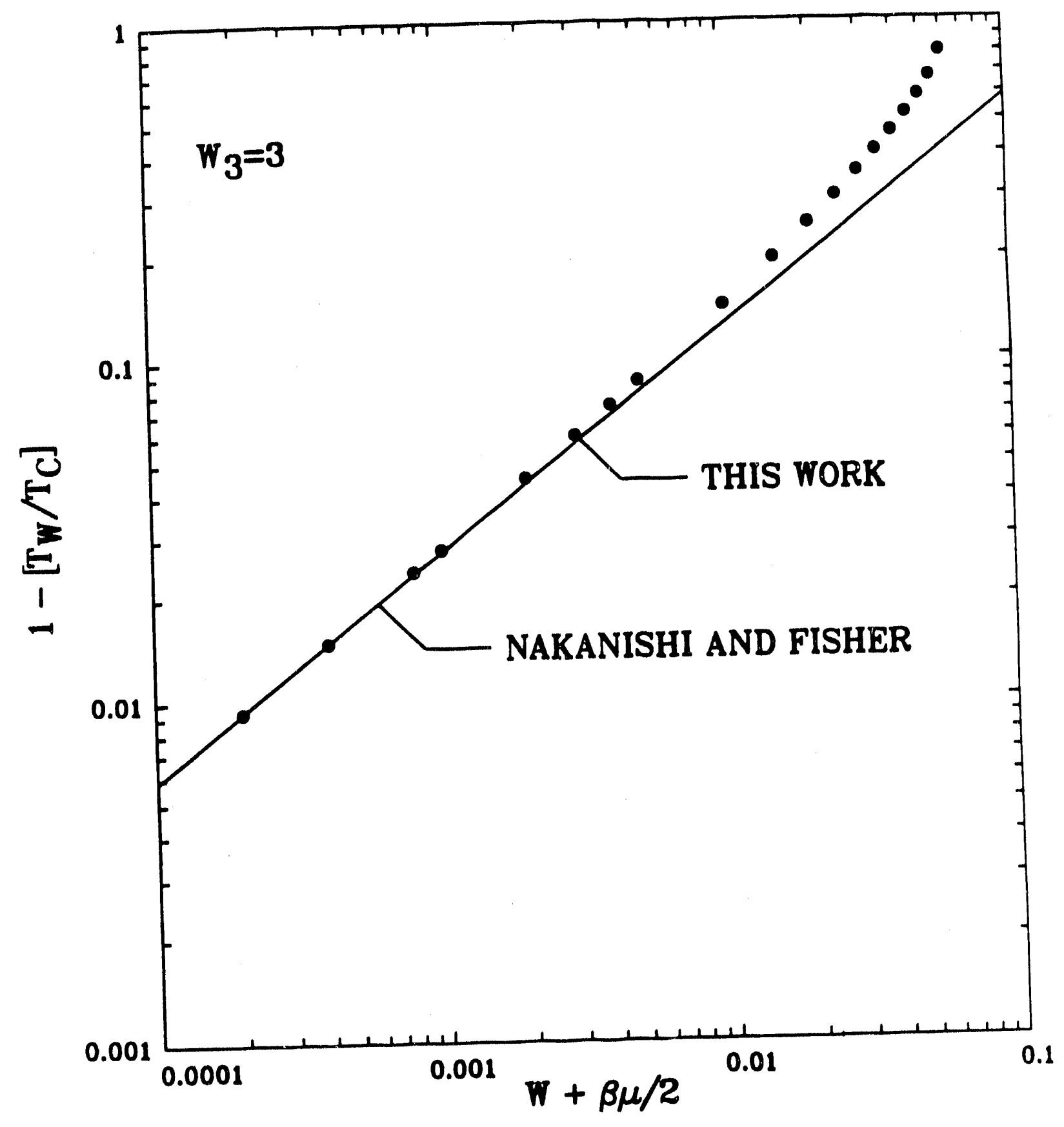

Figure 14. Plot of the distance between the first-order wetting transition temperature $T_{w}$ and the bulk critical temperature $T_{c}$ as a function of the distance $W+\beta \mu / 2$ from the "extraordinary" multicritical point. 
(a) Nakanishi and Fisher (1982)
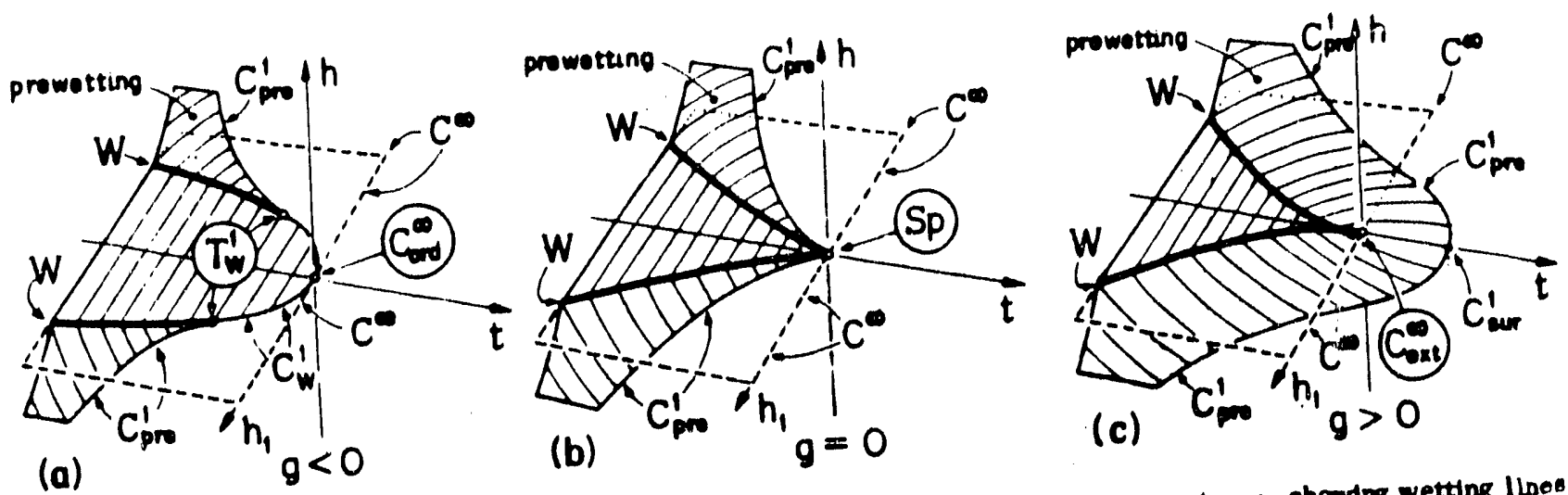

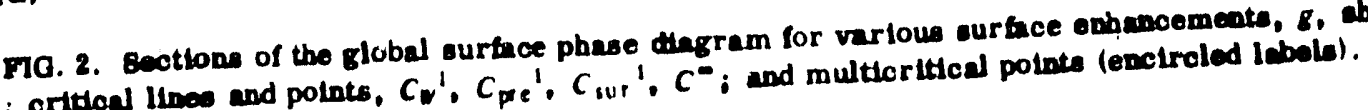

(b) This work
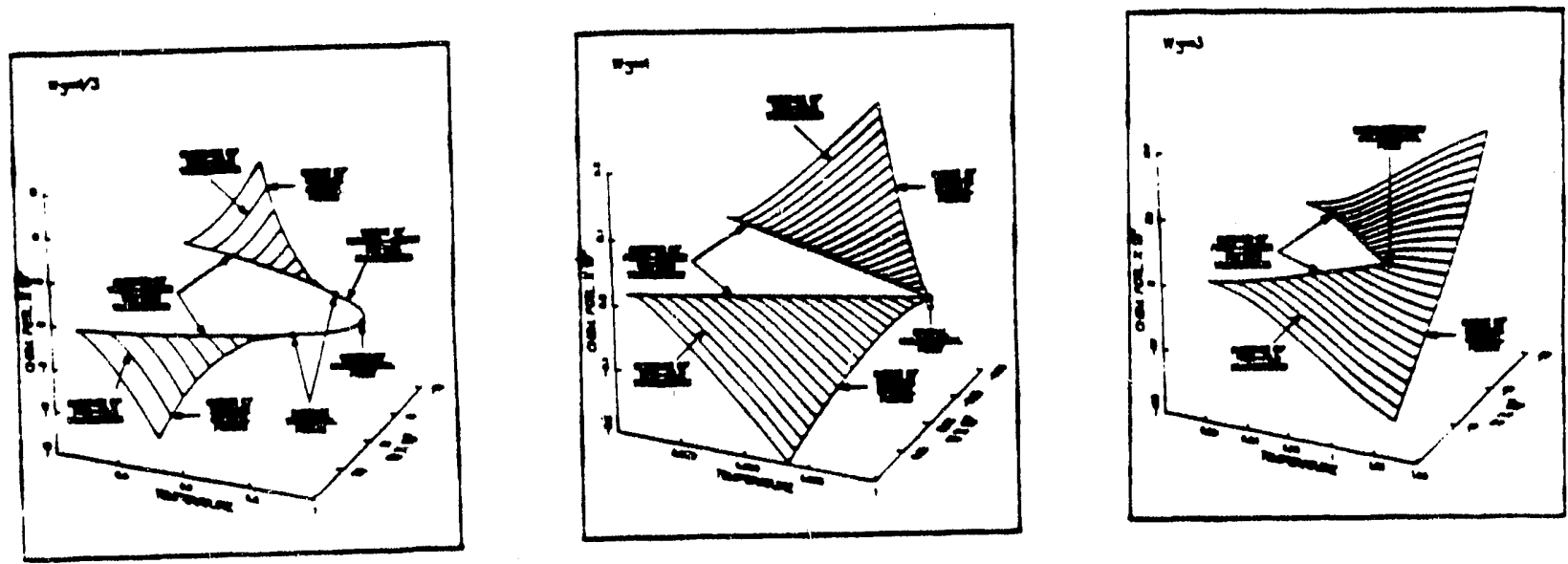

Figure 15. Comparison between the global phase diagrams of(a) Nakanishi and Fisher (1982) and (b) the present work (Figs. 7-9). 
Figure 15 shows the three global phase diagrams of NF and the corresponding diagrams that we obtained by computation (Figures 7-9) with the model of Teletzke et al. For $W_{3}=1 / 3$ and $W_{3}=1$, which correspond to NF's $\gamma<0$ and $\gamma=0$, the diagrams agree in all qualitative features. And we already saw that where comparisons can be made the agreement in terms of critical exponents is also quantitative. For $W_{3}=3$, which corresponds to NF's $\gamma>0$, there is agreement as to the exponent for the wetting temperature; however, there are sorne discrepancies as to the range in $W$ over which the critical exponent for the extraordinary multicritical point remains valid relative to the range in $W$ over which we have $T_{c s} \geq T_{c}$. In particular the NF diagram implies that the critical exponent still applies when $T_{c s}<T_{c}$ for all values of $W_{3}>1$; however this does not agree with our result.

\section{Conclusions}

1. A generalization of the model of Teletzke et al. (1982b) to multicomponent systems is presented in section B1. With appropriate interaction potential parameter values the multicomponent version is still solvable analytically.

2. A new method for locating first-order wetting transitions, thin to thick thin-film transitions, and surface critical points is presented in section B3-4. At the core of the method are sets of equations - which can be integral, differential, or algebraic depending on the mean field model used - that govern the transitions and critical points. The essential idea is to obtain the transition and critical points directly through solution of these equations rather than indirectly through constructions of either the adsorption isotherm (Teletzke et al., 1982a) or nonequilibrium surface tension as function of adsorption (Evans and Tarazona, 1983). The price paid for this convenience is a larger equation set. However the benefit is that the error incurred in locating the transition and critical points inside global phase diagrams is easier to control.

3. The equations that govern the various transition and critical points of the van der Waals model of Teletzke et al. (1982b) are shown in section B4. In this model the equations reduce to algebraic equations. These equations are solved by Newton iteration and the results presented in terms of global phase diagrams $(T, \Delta \mu, W)$ that show both bulk phase conditions $(T, \mu)$ and two-body interaction strength $(W)$ for three different values of a parameter $W 3$ that measures three-body interaction strength: $W_{3}=1 / 3,1$, and 3 .

4. The structure of such global phase diagrams was first inferred by Nakanishi and Fisher (1982) from scaling studies. Our results are in accord with their critical exponents $\Delta$ and $\Delta_{1}$ for the "ordinary," "special," and "extraordinary" multicritical points. However, for $W_{3}=3$ where the distance between the first-order wetting transition temperatures $T_{w}$ and the bulk critical temperature $T_{c}$ should scale with the distance from the 
"extraordinary" multicritical point as

$$
1-\left(\left(T_{w} / T_{c}\right) \approx(W+\beta \mu / 2)^{1 / \Delta_{1}} \approx(W+\beta \mu / 2)^{2 / 3}\right.
$$

with $\beta \equiv\left(1-W_{3}\right) /\left(1+W_{3}\right)$ and $\mu$ the fluid chemical potential, the range in $W$ over which the above scaling relation holds is not as wide as the diagram of Nakanishi and Fisher would suggest.

\section{References}

Bellare, J. (1988) Ph.D. Thesis, University of Minnesota.

Binder, K. (1983) Critical behavior at surfaces. Phase Transitions and Critical Phenomena, 8. Edited by C.. Domb and M. Green. Academic Press, London.

Binder, K. and Hohenberg, P. (C. (1972) Phase transitions and static spin correlations in Ising models with free surfaces. Physical Review B 6, 3461-87.

Binder, K. and Hohenberg, P. C. (1974) Surface effects on magnetic phase transitions. Physical Review B 9, 2194-214.

Cahn, J. W. (1977) Critical point wetting. Journal of Chemical Physics 66, 3667-72.

Costas, M. E., Varea, C., and Robledo, A. (1983) Global phase diagram for the wetting transition at interfaces in fluid mixtu'es. Physical Review Letters 51, 2394-7.

Davis, H. T., Benner, R. E., Jr., Scriven, L. E., and Teletzke, G. F. (1984) "Statistical Mechanics of Adsorption and Wetting Transitions." Paper presented at the 5th International Symposium on Surfactants in Solution, Bordeaux, France.

Davis, H. T. and L. E. Scriven (1982) Stress and structure in fluid interfaces. Advances in Chemical Physics. 49, 357.

De Gennes, P. (1983) Physique des surfaces et des interfaces. C. R. Acad. Sc. Paris.297:9-12. De Gennes, P. (1985) Wetting: statics and dynamics. Reviews of Modern Physics 57, 827-63. Dietrich, S., and Schide, M. (1985) Critical wetting of surfaces in systems with long-range forces. Physical Review B 31, 4718-20.

Diehl, H. W. (1986) Field-theoretic approach to critical behavior at surfaces. Phase Transitions and Critical Phenomena volume 10. Edited by C. Domb and J. L. Lebowitz. Academic Press, London.

Diehl, H.W., and Dietrich, S'. (1981) Field-theoretical approach to multicritical behavior near free surfaces. Physical Review B 24, $2878-80$.

Dietrich, S., and Schick, M. (1986) Order of wetting transitions. Physical Review B33, $4952-68$.

Ebner, C., and Saam, W.F. (1977) New phase-transition in thin argon films. Physical Review Letters 38, 1486-89.

Ebner, C., and Saam, W. F. (1987a) Effect of long-range forces on wetting near bulk critical temperatures: an ising-model study. Physical Review B 35, 1822-34.

Ebner, C., and Saam, W. F. (1987b) New' reentrant wetting phenomena and critical behavior near bulk critical points. Physical Review letters 58, 587-90.

Evans, R., Marconi, U. M. B., and Tarazona, P. (1986) Fluids in narrow pores. Journal of Chemical Physics 84, 2376-99.

Griffiths, R. B. (1970) Physical Review Letters 24, 715. 
Hauge, E. H., and Schick, M. (1983) Continuous and first-order wetting transition from the van der Waals theory of fluids. Physical Review B 27, 4288-301.

Heady, R. B. and Cahn, J. W. (1972) Experimental test of classical nucleation theory in a liquid-liquid miscibility gap. Journal of Chemical Physics 58, 896-910.

Kaganov, M. I. and Omel'yanchuk, A. N. (1971) Zh. Eksp. Teor. Fiz. 61, 1979 [Soviet Physics JETP 34, 895 (1972)]

Kroll, D. M., and Meister, T. F. (1985) Wetting transitions at solid-gas interfaces. Physical Review B 31. 392-402.

Kumar, P. (1974) Magnetic phase transition at a surlace: mean-field theory. Physical Review B 10:2928-33 [erratum: Physical Review B 12, 3984 (1975)].

Kung, W.C. (1989) Ph.D. Thesis, University of Minnesota.

Lee, T. D. and Yang, C. N. (1952) Physical Review 87, 410.

Lipowsky, R. (1983) Surface induced disordering at first-order bulk transitions. Z. Phys, B 51, 165-172.

Lubensky, T. C. and Rubin, M. H. (1975) Critical phenomena in semi-infinite systems II mean-field theory. Physical Review B 12, 3885-901. Mills, D. L. (1971) Surface effects in magnetic crystals near the ordering temperature. Physical Review B 3, 3887-95.

Moldover, M. R. and Cahn, J. W. (1980) An interface phase transition: complete to partial wetting. Science 207, 1073-5.

Nakanishi, H., and Fisher, M. E. (1982) Multicriticality of wetting, prewetting, and surface transitions. Physical Review Letters 49, 156.5-68.

Nakanishi, H., and Pincus, P. (1983) Surface spinodals and extended wetting in fluids and polymer solutions. Journal of Chemical Physics 79, 997-1003.

Nightingale, M. P., Saam, W. F., and Schick, M. (1983) Absence of critical wetting in systems with long-range forces. Physical Review Letters 51, 1275-78.

Nightingale, M. P., Saam, W. F., and Schick, M. (1984) Wetting and growth behaviors in adsorbed systems with long-range forces. Physical Review B 30, 3830-40.

Pandit, R., Schick, M., and Wortis, M. (1982) Systematics of multilayer adsorption phenomena on attractive substrates. Physical Review B 26, 5112-40.

Pohl, D. W. and Goldburg, W. I. (1982) Physical Review Letters 48, 1111.

Privman, V. (1984) Wetting phenomena with long-range forces. Journal of Chemical Physics 81. 2463-67.

Schmidt, J. W. and Moldover, M. R. (1983) First-order wetting transition at a liquid-vapor interface. Journal of Chemical Physics 79. 37.9-87.

Schmidt, J. W. and Moldover, M. R. (1986) A search for the prewetting line. Journal of Chemical Physics 84. 4563-8.

Sullivan, D. E. (1979) Van der Waals model of adsorption. Physical Review B 20, 39914000.hfil

Sullivan, D. E. (1981) Surface tension and contact angle of a liquid-solid interface. J. Chem. Phys. 74, 2604-15.

Sullivan, D. E. (1982) J. Chem. Phys. 77, 2632.

Sullivan, D. E. and Telo da Gama, M. M. (1986) Wetting transitions and multilayer adsorption at fluid interfaces. Fluid Interfacial Phenomena edited by C. A. Croxton. John Wiley and Sons, Chichester.

Tarazona, P., and Evans, R. (1983) Wetting transitions at models of a solid.gas interface. Molecular Physics 48, 799-831. Teletzke, G. F., Scriven, L. E., and Davis, H. T. (1982a) 
Gradient theory of wetting transitions, Journal of Colloid and Interface Science 87, 550-71. Teletzke, G. F., Scriven, L. E., and Davis, H. T. (1982b) Wetting transitions: first order or second order? J. Chem. Phys. 77, 5794-98.

Telo da Gama, M. M. and Evans, R. 1983. Adsorption and wetting transitions o at a model of the interface between a solid and a binary fluid mixture. Molecular Physics 48, 687-714. 


\section{DENSITY DISTRIBUTION FUNCTIONS OF CONFINED TONKS-TAKAHASHI FLUIDS}

\section{Synopsis}

The density distribution functions of a confined one-dimensional fludd of particles obeying the Tonks-Takaliashi nearest neighbor two-body potential are reduced to simple functions of the grand canonical ens mble partition function. The resulting formulas are analogous to those found by Robledo and Rowlinson for a hard-rod fluid. In the absence of an external field the partition functions can be evaluated by the method of Laplace transforms. The dependence of the pressure $P$ on the separation $L$ of the confining walls is investigated for three model potentials: (i) hard-rod, (ii) square-well, and (iii) triangle-well. $P$ is an oscillating function of $L$ in all three cases. The oscillations arise from the ondering effect of the repulsive forces between particles. The attractive interactions of the trianglewell potential reinforces the ordering whereas those of the square-well potential diminishes the ordering. Results for semi-confined and homogeneous fluids are also presented.

\section{Theory}

We consider the Tonks-Takahashi fluid, which is a one-dimensional fluid of particles obeying the nearest neighbor two body potential (Lieb and Mattis, 1966).

$$
\begin{aligned}
u\left(x_{i j}\right) & =\infty, \quad\left|x_{i j}\right|<a \\
& =\psi\left(x_{i j}-a\right), \quad a<\left|x_{i j}\right|<a_{2} \\
& =0 \quad, \quad\left|x_{i j}\right|>a_{2},
\end{aligned}
$$

where $a_{2} \leq 2 a$ and $\psi$ is an arbitrary function of $x_{i j}$. Suppose $N$ particles are confined to the box of length $L=x_{N+1}-x_{o}-a$, as shown in Figure 1. The walls of the box are a pair of particles fixed at $x_{o}$ and $x_{N+1}=L+a+x_{o}$ respectively. We assume there is also an external potential $v\left(x_{i}\right)$ acting on each of the $\mathrm{N}$ particles.

The configuration partition function of the system can be expressed in the form

$$
\begin{aligned}
& Z_{N}\left(x_{o}, x_{N+1}\right)=N ! \int_{x_{o}}^{x_{N}+1} d x_{N} \int_{x_{o}}^{x_{N}} d x_{N-1} \ldots \int_{x_{o}}^{x_{2}} d x_{1} \times \\
& e^{-\beta \sum_{i>j}^{N} u\left(x_{i}-x_{j}\right)} e^{-\beta \sum_{i}^{N} v\left(x_{i}\right)} e^{-\beta r\left(x_{1}-x_{o}\right)-\beta u\left(x_{N+1}-x_{N}\right)}
\end{aligned}
$$




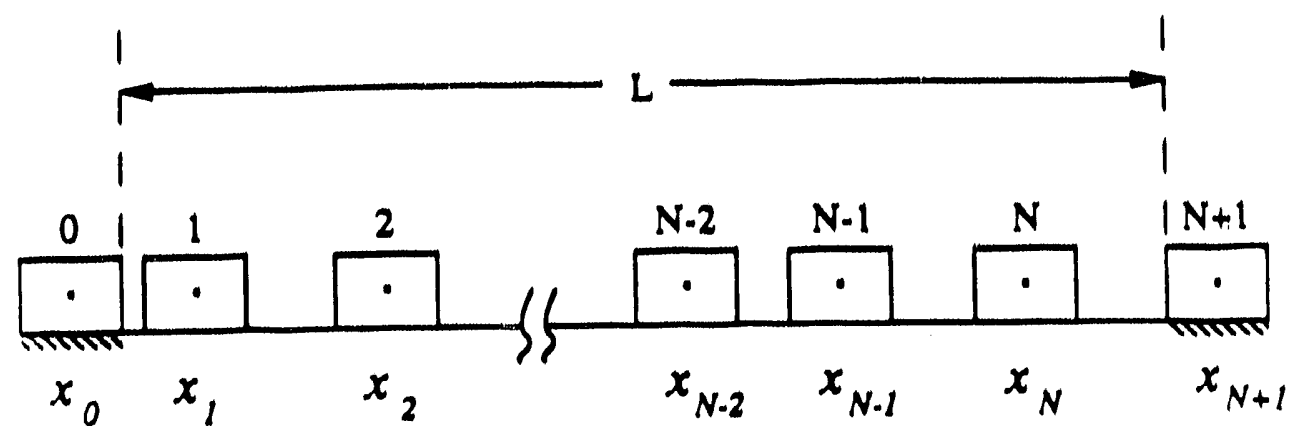

Figure 1. Tonks-Takahashi gas of $N$ particles confined to a subregion $L=x_{N+1}-x_{0}-a$ by a pair of the same kind of particles fixed at positions $x_{0}$ and $x_{N+1}$.

where $x_{o} \leq x_{1} \leq x_{2} \ldots \leq x_{N} \leq x_{N+1}$ and $\beta=1 / k T . k$ is Boltzmann's constant and $T$ is the absolute temperature. Taking the functional derivative with respect to $v(x)$, we find

$$
\begin{aligned}
\frac{1}{N !} \frac{\delta Z_{N}}{\delta v(x)}\left(x_{0}, x_{N+1}\right) & =-\beta e^{-\beta v(x)} \sum_{k=1}^{N} \int_{x_{0}}^{x_{N+1}} d x_{N} \ldots \int_{x_{0}}^{x_{k+2}} d x_{k+1} \times \\
& e^{-\beta u\left(x_{N+1}-x_{N}\right)} e^{-\beta u\left(x_{k+1}-x\right)} e^{-\beta \sum_{i>j=k+1}^{N} u\left(x_{i j}\right)-\beta \sum_{i=k+1}^{N} v\left(x_{i}\right)} \times \\
& \int_{x_{0}}^{x} d x_{k-1} \ldots \int_{x_{0}}^{x_{2}} d x_{1} e^{-\beta u\left(x-x_{k}-1\right)} e^{-\beta u\left(x_{1}-x_{0}\right)} \times \\
& e^{-\beta \sum_{i>j=1}^{k-1} u\left(x_{i j}\right)-\beta \sum_{i=1}^{k-1} v\left(x_{i}\right)} \\
& =-\beta e^{-\beta v(x)} \sum_{k=1}^{N} \frac{Z_{k-1}\left(x_{0}, x\right)}{(k-1) !} \frac{Z_{N-k}\left(x, x_{N+1}\right)}{(N-k) !}
\end{aligned}
$$

The grand canonical ensemble partition function of the system is

$$
\Xi\left(x_{o}, x_{N+1}\right)=\sum_{N=0}^{\infty} \frac{e^{\beta N \mu}}{N ! \Lambda^{N}} Z_{N}
$$

where $\mu$ is the chemical potential and $\Lambda$ is the de Broglie thermal wavelength. The density distribution $n(x)$ can be computed from the formula

$$
n(x)=-k T \frac{\delta \ln \Xi\left(x_{o}, x_{N+1}\right)}{\delta v(x)}
$$

Substitution of Eq. (3) into this expression and use of the transformation of summations

$$
\sum_{N=1}^{\infty} \sum_{k=1}^{N} f_{N-k} g_{k-1}=\sum_{k^{\prime}=0}^{\infty} \sum_{N^{\prime}=0}^{\infty} f_{N^{\prime}} g_{k^{\prime}}
$$



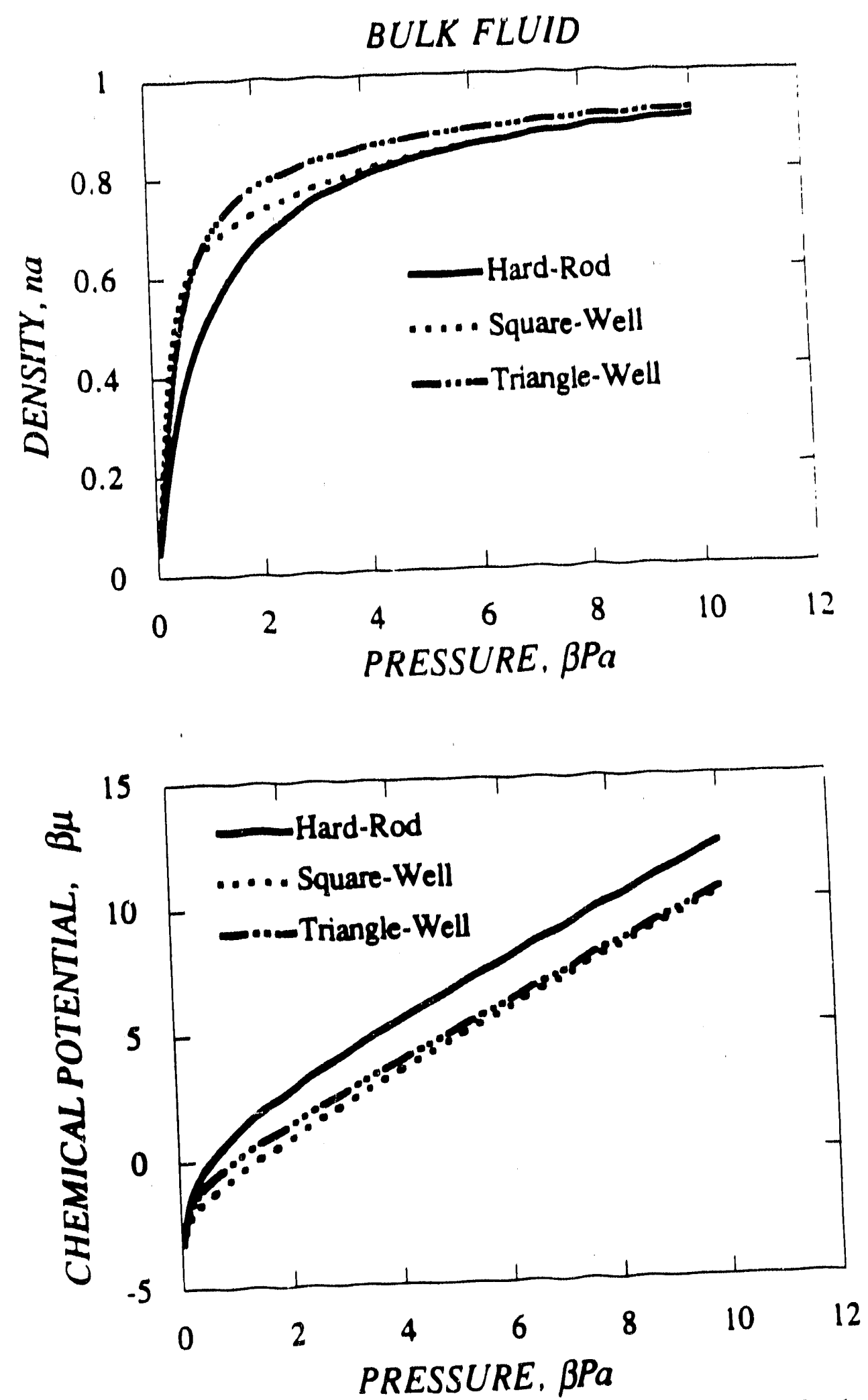

Figure 2. Derisity versus pressure and chemical potential versus pressure, for homogeneous fluids. Units of density, chemical potential and pressure are $a^{-1}, \beta^{-1}$ and $\left(\beta^{\prime}()^{-1}\right.$. For the square-well and triangle-well fluids $\beta \epsilon=2$. 
lead to the result

$$
\frac{\delta \Xi}{\partial v(x)}\left(x_{o}, x_{N+1}\right)=-\beta \frac{e^{\beta(\mu-v(x))}}{\Lambda} \Xi\left(x_{o}, x\right) \Xi\left(x, x_{N+1}\right),
$$

from which it follows that

$$
n(x)=\frac{e^{\beta(\mu-v(x))}}{\Lambda} \frac{\Xi\left(x_{o}, x\right) \Xi\left(x, x_{N+1}\right)}{\Xi\left(x_{0}, x_{N+1}\right)} .
$$

The k-body density distribution function for the present system can similarly be shown to be given br

$$
n^{(k)}\left(x_{1}, \ldots, x_{k}\right)=\frac{e^{\beta k \mu}}{\Lambda^{k}} \frac{e^{-\beta \sum_{i=1}^{k} v\left(x_{k}\right)}}{\Xi\left(x_{o}, x_{N+1}\right)} \Xi\left(x_{o}, x_{1}\right) \prod_{l=2}^{k} \Xi\left(x_{l-1}, x_{l}\right) \Xi\left(x_{k}, x_{N+1}\right),
$$

where $x_{o} \leq x_{1} \leq x_{2} \ldots \leq x_{k} \leq x_{N+1}$. Equations (7) and (8) are for the Tonks-Takahashi model, an extension of the results derived earlier by Robledo and Rowlinson (1986) for hard-rods $(\psi=0)$ in the absence of an external field $(v=0)$.

Although Eqs. (7) and (8) represent closed form solutions for the density distribution fuictions, one is still faced with the problem of evaluating the grand canonical ensemble partition function. This is not easy to do in general. However, in the special case that the external potential is zero $(v=0)$ the Luplace transform can $\mathrm{b}^{\prime}$; used to evaluate the partition function. With the variable change $y_{j}=x_{j}-j a-x_{o}, j=1, \ldots, N+1$, the canonical ensemble partition function of the Tonks-Takahashi gas becomes

$$
\begin{aligned}
Z_{N}\left(y_{N+1}\right)= & N ! \int_{0}^{y_{N+1}} d y_{N} e^{-\beta \psi\left(y_{N+1}-y_{N}\right)} \int_{0}^{y_{N}} d y_{N-1} e^{-\beta \psi\left(y_{N}-y_{N-1}\right)} \ldots \times \\
& \int_{0}^{y_{3}} d y_{2} e^{-\beta \psi\left(y_{3}-y_{2}\right)} \cdots \int_{0}^{y_{2}} d y_{1} e^{-\beta \psi\left(y_{2}-y_{1}\right)-\beta \psi\left(y_{1}\right)}
\end{aligned}
$$

Note that $y_{N+1}=L-N a$. Because Eq. (9) is in the form of an iterated convolution integral, the metk $d$ of Laplace transform can be applied to obtain (Lieb and Mattis, 1966), Salsburg, et al. 1953)

$$
Z_{N}\left(y_{N+1}=L-N a\right)=\frac{N !}{2 \pi i} \int_{-i \infty+\tau_{0}}^{i \infty+\tau_{0}} e^{(L-N a) s}[K(s ; j), N+1
$$

where $\tau_{o}>0$ and

$$
K(s)=\int_{0}^{\infty} e^{-y y-\beta \psi(y)} d y
$$


Choosing a coordinate system such that $x_{0}=-a / 2$ and $x_{N+1}=L+a / 2$, we obtain the following results for the density distribution functions

$$
n(x)=\frac{e^{\beta \mu}}{\Lambda} \Xi_{L-x-\frac{a}{2}} \Xi_{x-\frac{a}{2}} / \Xi_{L}
$$

and

$$
n^{(k)}\left(x_{1}, \ldots, x_{k}\right)=\frac{e^{\beta k \mu}}{\Lambda^{k} \Xi_{L}} \Xi_{x_{1}-\frac{a}{2}} \prod_{l=2}^{k} \Xi_{x_{l}-x_{l-1}-a} \Xi_{L-x_{k}-\frac{a}{2}},
$$

where

$$
\Xi_{y} \equiv \sum_{N=0}^{\infty} \frac{e^{N \beta \mu}}{\Lambda^{N}} \int_{-i \infty+\tau_{0}}^{i \infty+\tau_{0}} \frac{e^{(y-N a) s}}{2 \pi i} \cdot[K(s)]^{N+1} d s
$$

The pressure $P$ of the confined fluid can be computed from the r rmula

$$
P=k T\left(\partial \ln \Xi_{L} / \partial L\right)
$$

Next let us consider a semi-confined fluid, which is defined as a bulk fluid in contact with a wall at $x_{0}$. In this case, $L \rightarrow \infty, \Xi_{L-x-\frac{a}{2}} / \Xi_{L} \rightarrow e^{-\beta P\left(x+\frac{a}{2}\right)}$ and so

$$
n(x)=\frac{e^{\beta \mu}}{\Lambda} e^{-\beta P\left(x+\frac{a}{2}\right)} \Xi_{x-\frac{a}{2}}
$$

The pressure $P$ of a bulk Tonks-Takahashi fluid at chemical potential $\mu$ obeys the relation (Lieb and Mattis, 1966).

$$
\beta \mu=\beta P a-\ln K(\beta P)+\ln \Lambda \text {. }
$$

We can also derive an expression for the pair correlation function in bulk fluid by considering the limits that $x_{2}$ and $L$ approach infinity, but $x_{2}-x_{1}$ is finite. With the aid of the properties $n\left(x_{i}\right) \rightarrow n$ as $x_{i} \rightarrow \infty, n$ being the bulk phase density, and $\Xi\left(x_{2}, x_{N+1}\right) / \Xi\left(x_{1}, x_{N+1}\right) \rightarrow \exp \left[-\beta P\left(x_{2}-x_{1}\right)\right]$ as $x_{N+1} \rightarrow \infty$, we find

$$
n^{(2)}\left(x_{1}, x_{2}\right)=n \frac{e^{\beta \mu}}{\Lambda} e^{-\beta P\left(x_{2}-x_{1}\right)} \Xi_{x_{2}-x_{1}-a}
$$

Then, the pair correlation function, which is defined as $g^{(2)}\left(x_{2}-x_{1}\right) \equiv n^{(2)}\left(x_{1}, x_{2}\right) / n^{2}$, can be expressed as

$$
g^{(2)}\left(x_{2}-x_{1}\right)=n\left(x_{2}-x_{1}-a / 2\right) / n,
$$

where $n(x)$ is given by Eq. (17).

While this manuscript was under review, the author received a preprint of an article by Monson (1990). In the paper he presents the singlet density distribution functions for a hinary mixture of square-well Tonks-Takahashi particles confined by nearest neighbor 
square-well particles of arbitrary strength. His work suggests an immediate generalization of the results presented above.

Suppose the wall particles at $x_{0}$ and $x_{N+1}$ interact with the fluid particles according to the nearest neighbor potential

$$
\begin{aligned}
u^{w}(|x|) & =\infty, \quad|x|<a \\
& =\psi^{w}(|x|-a), \quad a<|x|<a_{2}^{w} \\
& =0, \quad|x|>a_{2}^{w},
\end{aligned}
$$

where $a_{2}^{w} \leq 2 a$. The wall particles at $x_{0}$ and $x_{N+1}$ can in general be different from one another. The $k$-body density distribution function can then be shown to be given by

$$
n^{(k)}\left(x_{1}, \ldots, x_{k}\right)=\frac{e^{\beta k \mu}}{\Lambda^{k}} \frac{e^{-\beta \sum_{i=1}^{k} v\left(x_{k}\right)}}{\Xi^{w 1, w 2}\left(x_{o}, x_{N+1}\right)} \Xi^{w 1}\left(x_{o}, x_{1}\right) \prod_{l=2}^{k} \Xi\left(x_{l-1}, x_{l}\right) \Xi^{w 2}\left(x_{k}, x_{N+1}\right),(2
$$

where $\Xi$ denotes the partition function for a Tonks-Takahashi fluid confined by wall particles that are the same as the fluid particles. In $\Xi^{w 1}\left(x_{1}-x_{0}\right)$ the confining particle at $x_{0}$ is a Tonks-Takahashi particle interacting with the fluid via the potential $u^{w 1}\left(x_{1}-x_{0}\right)$. Similarly, in $\Xi^{w 2}\left(x_{k}, x_{N+1}\right)$ the wall particle at $x_{N+1}$ interacts with the fluid via the potential $u^{w 2}\left(x_{N+1}-x\right)$ and in $\Xi^{w 1, w 2}\left(x_{0}, x_{N+1}\right)$ the walls at $x_{0}$ and $x_{N+1}$ have the potentials $u^{w 1}$ and $u^{\prime \prime 2}$, respectively.

In the external field-free case, we obtain

$$
n^{(k)}\left(x_{1}, \ldots, x_{k}\right)=\frac{e^{\beta k \mu}}{\Lambda^{k} \Xi_{L}^{w 1, w 2}} \Xi_{x_{1}-\frac{a}{2}}^{w 1} \prod_{l=2}^{k} \Xi_{x_{l}-x_{l-1}-a} \Xi_{L-x_{k}-\frac{a}{2}}^{w 2},
$$

where

$$
\Xi_{y}^{w i}=e^{-\beta \Psi^{w i}(y)} \sum_{N=1}^{\infty} \frac{e^{N \beta \mu}}{\Lambda^{N}} \int_{-i \infty+\tau_{0}}^{i \infty+\tau_{0}} \frac{e^{(y-N a) s}}{2 \pi i} K^{w i}(s)[K(s)]^{N} d s, \quad i=1 \text { and } 2
$$

and

$$
\Xi_{L}^{w 1, w 2}=e^{-\beta \Psi(L)^{w 1, w 2}}+\sum_{N=1}^{\infty} \frac{e^{N \beta \mu}}{\Lambda^{N}} \int_{-i \infty+\tau_{0}}^{i \infty+\tau_{0}} \frac{e^{(L-N a) s}}{2 \pi i} K^{w 1}(s) K^{w 2}(s)[K(s)]^{N-1} d s
$$

where

$$
K^{w i}(s) \equiv \int_{0}^{\infty} e^{-y s-\beta \psi^{w i}(y)} d y
$$


The partition function $\Xi_{y}$ is given by Eq. (15).

The semi-confined fluid density profile for this case is given by

$$
n(x)=\frac{e^{\beta \mu}}{\Lambda} e^{-\beta P\left(x+\frac{a}{2}\right)} \Xi_{x-\frac{a}{2}}^{w 1}
$$

where $P$ is given by Eq. (18).

\section{Applications}

In this section we present comparisons of the confining pressure and the density profiles for three one-dimensional model fluids in the external field-free case $(v(x)=0)$.

These are

(i) hard-rod fluid:

$$
\psi(y)=0, \quad y>0
$$

(ii) square-well fluid: $a_{2}=2 a$

$$
\begin{aligned}
\psi(y) & =-\epsilon, \quad 0<y<a \\
& =0, \quad y>a
\end{aligned}
$$

(iii) triangle-well fluid: $a_{2}=2 a$

$$
\begin{aligned}
\psi(y) & =\epsilon(y-a) / a, \quad 0<y<a \\
)) & =0, \quad y>a .
\end{aligned}
$$

In terms of the full pair potential these models read $u\left(x_{i j}\right)=\infty,\left|x_{i j}\right|<a$ and $(i) u\left(x_{i j}\right)=$ $0,\left|x_{i i}\right|>a ;($ ii $) u\left(x_{i j}\right)=-\epsilon, a<\left|x_{i j}\right|<2 a, u\left(x_{i j}\right)=0,\left|x_{i j}\right|>2 a$; and $(i i i) u\left(x_{i j}\right)=$ $\epsilon\left(\left|x_{i j}\right|-a\right) / a, a<\left|x_{i j}\right|<2 a, u\left(x_{i j}\right)=0,\left|x_{i j}\right|>2 a$.

The Laplace transforms of $e^{-\beta \psi}$ for the three models are

$$
K(s)=\frac{1}{s}
$$

$$
K(s)=\frac{e^{\beta \epsilon}}{s}+\left(1-e^{\beta \epsilon}\right) \frac{e^{-s a}}{s}
$$

(iii)

$$
K(s)=\frac{e^{\beta \epsilon}-e^{-s a}}{s+(\beta \epsilon / a)}+\frac{e^{-s a}}{s} .
$$


The equations of state (see Eq. (18)) of the homogeneous phases $(L \rightarrow \infty)$ of these fluids are, respectively (Lieb and Mattis, 1966),

$$
\begin{aligned}
\beta \mu & =\beta P a+\ln (\beta P a)+\ln (\Lambda / a), \\
n^{-1} & =a+\frac{1}{\beta P},
\end{aligned}
$$

(ii)

$$
\begin{aligned}
\beta \mu & =\beta P a+\ln (\beta P a)-\ln \left\{e^{\beta \epsilon}+\left(1-\epsilon^{\beta \epsilon}\right) e^{-\beta P a}\right\}+\ln (\Lambda / a) \\
n^{-1} & =a+\frac{1}{\beta P}+\frac{a\left(1-e^{\beta \epsilon}\right) e^{-\beta P a}}{e^{\beta \epsilon}+\left(1-e^{\beta \epsilon}\right) e^{-\beta P a}}
\end{aligned}
$$

and

$$
\begin{aligned}
\beta \mu & =\beta P a+\ln [\beta P a(\beta P a+\beta \epsilon)]-\ln \left[\beta P a e^{\beta \epsilon}+\beta \epsilon e^{-\beta P a}\right]+\ln (\Lambda / a), \\
n^{-1} & =a+a \frac{2 \beta P a+\beta \epsilon}{(\beta P a)^{2}+(\beta \epsilon)(\beta P a)}-a \frac{e^{\beta \epsilon}-\beta \epsilon e^{-\beta P a}}{\beta P a e^{\beta \epsilon}+\beta \epsilon e^{-\beta P a}}-
\end{aligned}
$$

$P$ and $n$ are the bulk pressure and density of the fluid at chemical potential $\mu$ at temperature $T(\beta \equiv 1 / k T)$. The density-pressure equation of state is derived from the chemical potential-pressure equation with the Gibbs-Duhem equation, $n^{-1}=(\partial \beta \mu) /(\partial \beta P)$. For each of these models $\beta \mu$ and $n a$ are monotonic functions of $\beta P a$, reflecting the well-known fact that one-dimensional fluids of particles interacting with pair potentials of finite range cannot experience a phase transition. The isotherms for these fluids for $\beta \epsilon=2$ are shown in Figure 2. The datum for the chemical potential is $\ln (\Lambda / a)$.

At sufficiently high $\beta P a$, the equations of state of the square-well and triangle well fluids approach the hard-rod formulas. And as expected, at a given pressure, the density of the hard-rod fluid is the lowest of the three fluids. Overall similarities among the equations of state are more striking than the differences when compared with the behavior of the corresponding three-dimensional fluids.

To see whether the similarities among the models persists in molecularly narrow pores, we have inverted the Laplace transforms analytically, using the residue theorem, to calculate $\Xi_{y}$ in the field free case $v(x)=0$. The results are given in the Appendix. We chose to compare confined fluids that are at equilibrium with homogeneous phases at nearly the same bulk density, namely, $n a=3 / 4$. In particular, we set $\beta \epsilon=2$ and used the following bulk phase properties: 


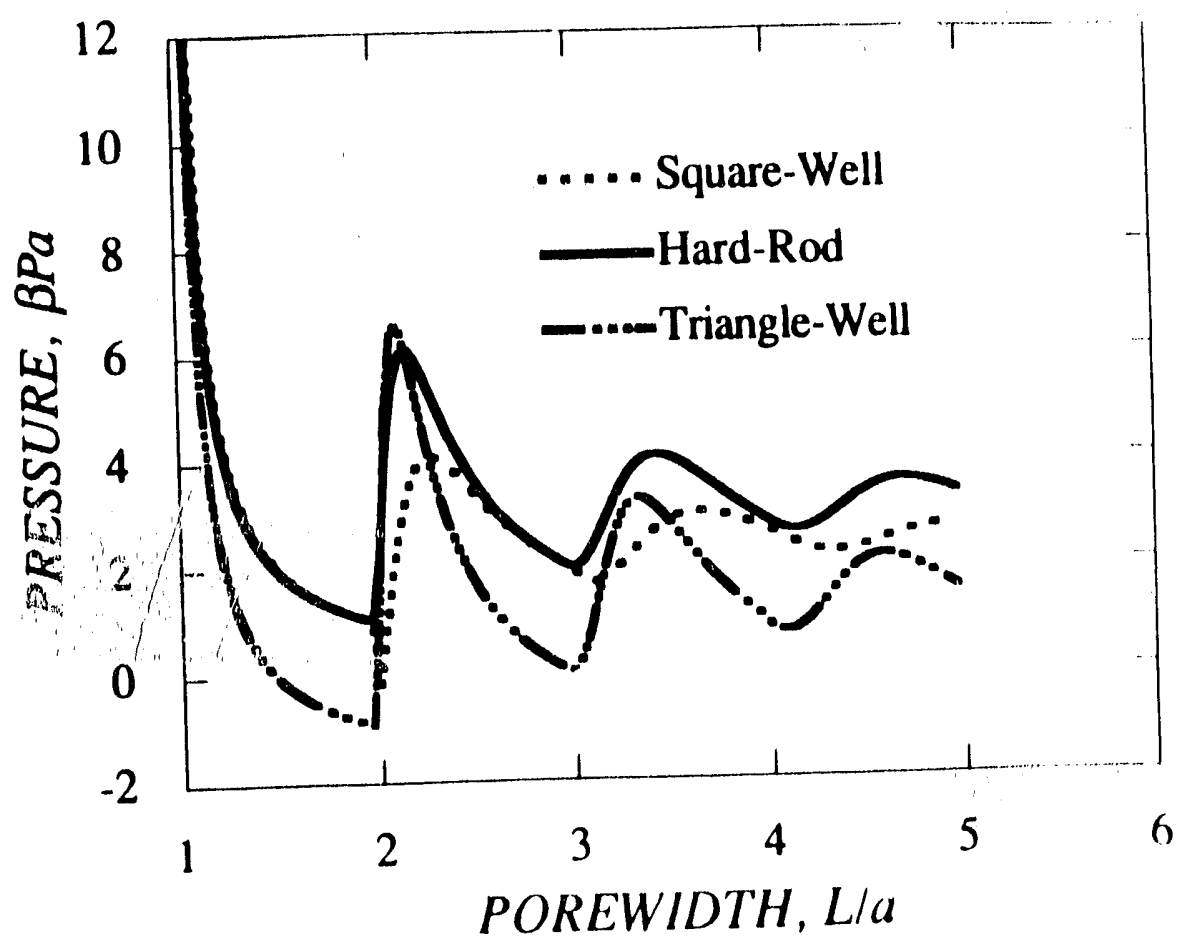

Figure 3. Pressure of confined fluids versus confining wall separation ("porewidth") $L$. $\beta \epsilon=2$.

(i) hard-rod fluid

$$
\beta P a=3, \quad \beta \mu=4.0986, \quad n a=3 / 4
$$

(ii) square-well fluid

$$
\beta P a=2.4, \quad \beta \mu=1.3572, \quad n a=0.7510
$$

(iii) triangle-well fluid

$$
\beta P a=1.6, \quad \beta \mu=0.8474, \quad n a=0.7511 .
$$

It seems to us more sensible to compare the behavior of confined fluids in equilibrium with bulk phases at the same temperature and density instead of at the same temperature and pressure or chemical potential. The choice, nevertheless, is somewhat arbitrary.

The dependence of pressure on pore width $L$ is sh wn in Fig. 3. The behavior is qualitatively similar to that of the confining pressures observed in real fluids.(Horne and Israelachvili (1981)) In accordance with previous conclusions from computer simulations,(van Megen and Snook, (1979)) the hard-rod model(Vanderlick et al.(1989)) and 
approximate molecular theories of three dimensional fluids, (Davis et al.(1987)) the oscillations in the pressure as a function of porewidth correspond to the degree of order (layering) in the density distribution of the confined fluid. The pressure minima occur near integral values of $L / a$. To see the qualitative pattern consider the density profiles shown in Fig. 4 . At the width $L=3.5 a$ as many as 3 particles can occupy the pore, but the particles have only a half a particle diameter of free volume (length) to distribute among themselves. Thus, the density peaks are narrow and high, resulting in a relatively high pressure. At $L=4 a$, however, there is still only room for three particles, but they now have a full particle diameter of free volume to distribute among themselves. The result is the density peaks are broader and shorter, resulting in a lower pressure. At $L=4.5 a$, four particles can occupy the pore but have only a half a particle diameter of free volume, and so again the density peaks are sharper and the pressure higher.

It is interesting that the continuous triangle-well potential shifts the peaks in $P$ to smaller $L$ but the discontinuous square-well potential shifts the peaks to larger $L$. This coincides with the fact that the triangle-well potential increases the layering tendency relative to the hard-rod potential whereas the square-well potential decreases the layering tendency. This behavior is indicated in Fig. 4 by the magnitudes of the peaks in the density profiles. The peaks, which represent strong ordering or layering, are largest for triangle-well fluid and are smallest for the square-well fluid. Thus, it appears that the square-well attraction competes with the excluded volume ordering effect and the trianglewell attraction enhances the effect.

Consider the case of a semi-confined fluid, for which the density profile is given by $\mathrm{Eq}$. (17). The density profiles of the three semi-confined fluids at the bulk conditions of Eqs. (40)-(42) are shown in Figure 5. Relative to the hard-rod fluid, the triangle-well attraction sharpens the density maxima and minima and shifts them towards the wall. The square-well attraction has the opposite effect.

The extent to which the two confining walls sup rimpose their effects on the density profile can be seen by comparing Figures 4 and 5 . At the separation $L=4.5 a$, the density distributions between the walls and $x=1.5 a$ are quite similar to those of a semi-confined fluid. From $x=1.5 a$ to the pore center at $x=2.25 a$, the profiles of the confined fluid differ greatly from those of the semi-co fined fluid. The effect of opposed walls on this case is stronger for the square-well fluid than for the other fluids. The density profiles for $L=3.5 a$ are have a relatively flat region between $x=a$ and $x=1.5 a$ that does not occur in the semi-confined fluid.

In closing we note that according to Eq. (19b) it follows that the pair correlation function of homogeneous fluid as a function of particle separation follows immediately from Fig. 5 by shifting the density profile to the right by amount $a / 2$ and rescaling the 

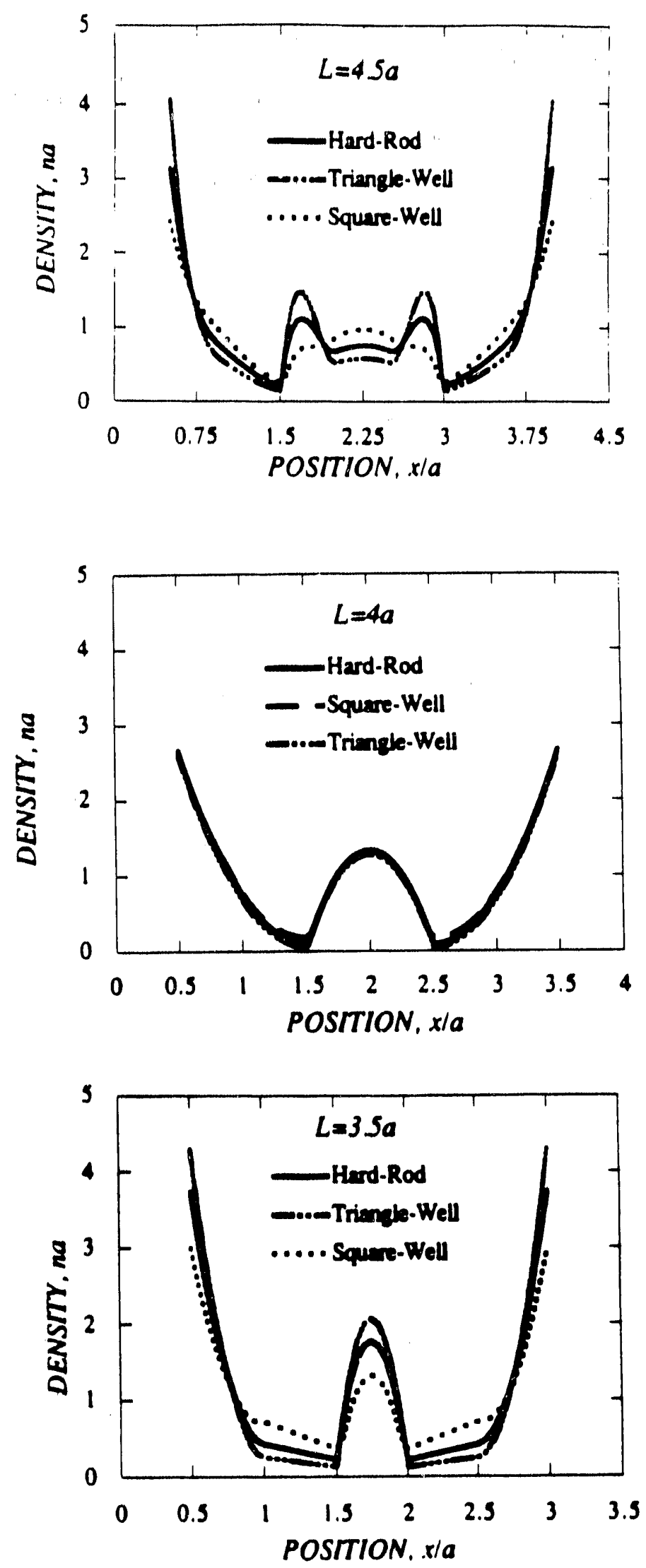

Figure 4. Density of confined fluids versus distance from the pore wall for porewidths $L=$ $3.5 a, 4 a$ and $4.5 a . \beta \epsilon=2$. 


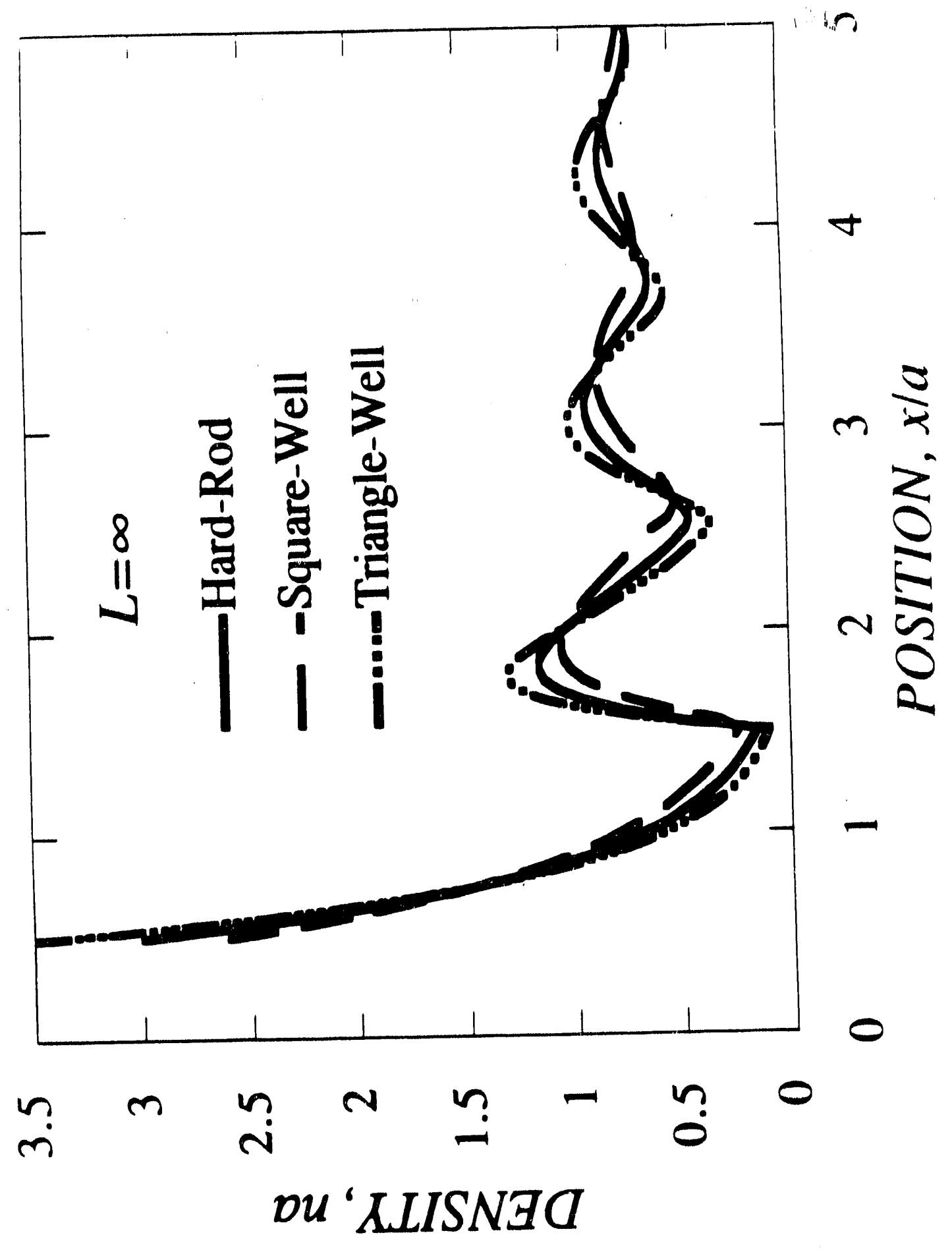

Figure 5. Density of semiconfined fluids $(L=\infty)$ versus distance from the pore wall. $\beta \epsilon=2$. 


\section{Reference}

Davis, H.T., I. Bitsanis, T.K. Vanderlick and M. Tirrell, in Supercomputer Research in Chemistry and Chemical Engineering, eds. K.S. Jensen and D.G. Truhlar, ACS Symposium Series 352, ACS (1987).

Horne, R.G. and J.N. Israelachvili, J. Chem. Phys. 75, 1400 (1981).

Lieb, E.H. and D.C. Mattis, Mathematical Physics in One Dimension (Academic Press, N.Y., 1966), pp. 3-9

Lieb, E.H. and D.C. Mattis, Mathematical Physics in One Dimension (Academic Press, N.Y., 1966), pp. 25-27.

Monson, P.A., Molecular Physics XXX 1990.

Robledo, A. and J.S. Rowlinson, Mol. Phys. 58, 711 (1986).

Salsburg, Z., R. Zwanzig and J. Kirkwood, J. Chem. Phys. 21, 1098 (1953).

Vanderlick, T.K., H.T. Davis and J.K. Percus, J. Chem. Phys. 91, 7136 (1989).

van Megen, W. and I.K. Snook, J. Chem. Soc., Faraday Trans. II 75, 1095 (1979).

\section{Appendix: Evaluation of Eq. (15) for three models}

(i) Hard-rod fluid:

$$
\Xi_{y}=\sum_{N=0}^{\infty} \frac{e^{N \beta \mu}}{N ! \Lambda^{N}}(y-N a)^{N} \eta(y-N a)
$$

(ii) Square-well fluid:

$$
\Xi_{y}=\sum_{N=0}^{\infty} \sum_{k=0}^{N+1} \frac{e^{N \beta \mu}}{N ! \Lambda^{N}} \frac{(N+1) !}{(N+1-k) ! k !} e^{(N+1-k) \beta \epsilon}\left(1-e^{\beta \epsilon}\right)^{k}(y-N a-k a)^{N} \eta(y-N a-k a) .
$$

(iii) Triangle-well fluid:

$$
\begin{gathered}
\Xi_{y}=\sum_{N=0}^{\infty} \frac{e^{N \beta \mu}}{\Lambda^{N}} \sum_{k=1}^{N+1} \frac{(N+1) ! \alpha^{k}}{(N+1-k) ! k !} \eta(y-N a-k a) e^{(N+1-k) \beta \epsilon} \times \\
\left\{\frac{(-1)^{k}}{N !} \sum_{j=0}^{N}(y-N a-k a)^{N-j} \frac{N !}{(N-j) ! j !} \frac{(k+j-1) !}{(k-1) !} \frac{e^{-(y-N a-k a) \alpha}}{\alpha^{k+j}}\right. \\
\left.+\sum_{0}^{k-1}(-1)^{j}(y-N a-k a)^{k-1-j} \frac{1}{(k-1-j) ! j !} \frac{(N+j) !}{N !} \frac{1}{\alpha^{N+1+j}}\right\} \\
\quad+\sum_{N=0}^{\infty} \frac{\epsilon^{N \beta \mu+(N+1) \beta \epsilon}}{\Lambda^{N} N !} \eta(y-N a)(y-N a)^{N} c^{-(y-N a) \alpha}
\end{gathered}
$$

where $\alpha \equiv \beta \epsilon / a . \eta(y)$ is the Heaviside function, i.e., $\eta(y)=0$ when $y<0$ and $=1$ when $y>0$. 


\section{A COMPARISON OF INITIAL CONDITIONS \\ FOR CONTINUOUS-FLOW SYSTEMS}

\section{Synopsis}

An initial condition, which sets the state of a system at a particular time, should describe accurately the physics of the situation and should not create computational ar. tifacts when the governing equations are solved numerically. Drawing on the example of two-phase flow in porous media, we show that unphysical oscillations can mar a solution should the initial condition violate an inflow boundary condition. Tracking these oscillations can increase by orders of magnitude the computer time needed to solve the equations of change. At an internal boundary between two different media, an initial condition that violates the steady-state equations of change produces features that might be equally undesirable. We propose a way to generate initial conditions that avoid these artifacts, viz. by splicing together a solution of the linearized governing equations in the region of change and solutions of the steady-state equations in regions of constancy. We demonstrate our proposal using the situation of two-phase flow in porous media. Our findings are broadly applicable because of the partial analogies among transport of mass, heat, and momentum.

\section{Introduction}

The analysis of continuous-flow situations requires the definition of a 'system,' which is a region of particular interest, and the 'surroundings,' which are the remainder of the universe. Equations of change that describe the behavior of the system derive from laws of conservation of mass, momentum, energy, and so forth and equally from constitutive relations that represent material response. A boundary defines the region or domain of interest by separating it from the rest of the universe. Boundary conditions must express accurately the interaction between the system and its surroundings. In the case of a dynamic, or evolving, system, the state of the entire system at a particular time must be specified by an 'initial' condition. Together, boundary conditions and an initial condition single out a particular solution of the equations of change.

Two earlier papers (Novy et al., 1990a,b) examine the issues of locating boundaries and choosing boundary conditions; they also lay out a systematic way of proceeding should a 'synthetic' boundary, i.e one that does not coincide with a physical boundary, be drawn. The goals of this paper are, first, to show the computational difficulties that can follow from initial conditions that do not satisfy all boundary conditions and steady equations of change and, second, to propose a method for generating initial conditions that do. Because an initial condition can be considered a 'boundary' condition in time, concepts discussed in our first two papers are relevant here.

Closed-form solutions of equations of change often depend on initial conditions that 
do not satisfy all boundary conditions. For example, a condition at the boundary might require a step change in the solution from its initial value, as does diffusion of heat or mass from a well-stirred fluid into a semi-infinite slab, which admits a closed-form solution in terms of an error function (see e.g., Crank, 1975, pp. 20-21). A survey of the books by Crank (1975) and Carslaw and Jaeger (1959) reveals other situations in which there is a step change at a boundary immediately after the initial state. Such abrupt changes can be treated as limiting processes and pose no difficulty in cases where a solution is known in terms of standard mathematical functions.

Digital computers now enable the study of systems that are described by equations of change that do not have closed-form solutions so far as is known or expected. Two schemes of computer-aided analysis in wide use are finite difference approximation and Galerkin's method with finite element basis functions, which is also known as the Galerkin finite element method (GFEM). These methods are, however, sensitive to step changes in conditions, and unphysical oscillations in space, or 'wiggles,' can result, signalling that the discretization of space is too coarse to resolve developing gradients accurately. Wiggles can appear in solutions computed with either finite difference schemes (Roache, 1972) or the Galerkin finite element method (Gresho and Lee, 1981). One way to suppress wiggles is by 'upwinding,' which unphysically enhances dispersion in order to smooth abrupt changes. Gresho and Lee (1981) argue against upwinding and recommend that wiggles should be prevented by local refinement of the discretization of space. We point out another way to avoid those wiggles that are associated with initial conditions which do not satisfy all boundary conditions. Our method neither degrades the solution by adding artificial dispersion nor requires an excessively fine discretization of space.

The context here is immiscible displacement of oil by a mixture of water and oil in a one-dimensional porous medium, which is either homogeneous or composed of two media with different flow properties. The homogeneous medium lets us show the artifacts that can follow from incompatible initial and inflow boundary conditions. The composite medium does likewise, but also illustrates what can happen when an initial condition violates the steady equations of change downstream. Because two-phase flow in porous media can be described in terms of convecuion and dispersion, the partial analogies among transport of mass, heat, and momentum make our findings more broadly applicable.

\section{Theory}

In this section, we derive the equations of change, describe our porous media of interest, specify inflow and outflow boundary conditions, and explain how to compute an initial condition that avoids computational artifacts.

Equations of Change

Two-phase flow in a porous medium (see Figure 1) is described by the equation of 
continuity of each phase $i$

$$
\phi \frac{\partial S_{i}}{\partial t}=-\nabla \cdot \mathbf{q}_{i}
$$

and the linear constitutive equation called Darcy's law, which relates the superficial flow velocity $\mathbf{q}_{i}$ and the gradient of the mechanical potential $\left(P_{i}+\rho_{i} g z\right)$ of phase $i$ :

$$
\mathbf{q}_{i}=-\frac{k k_{r i}}{\mu_{i}} \nabla\left[P_{i}+\rho_{i} g z\right]
$$

Flow in our examples is horizontal, so we ignore gravity. $\phi(\mathbf{x})$ is the porosity or void fraction of the bulk medium at position $\mathbf{x}, S_{i}$ is the local saturation or fractional void volume of phase $i, t$ is time, $k(\mathbf{x})$ is the absolute permeability of the medium, $k_{r i}\left(S_{i}\right)$ is the relative permeability of phase $i$, and $\mu$ is viscosity. In general, porosity, absolute permeability, and even the relation between relative permeability and saturation can vary with position. The two phases can be thought of as oil (o) and water (w).

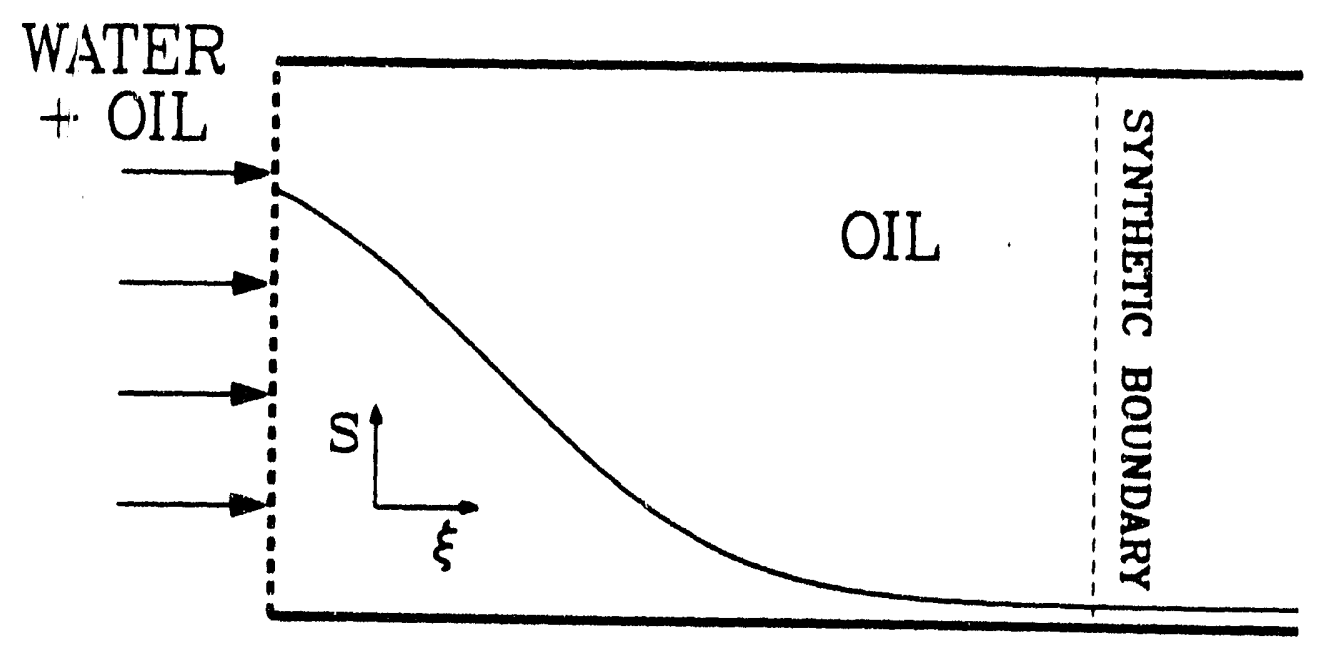

Figure 1. Displacement of oil in a porous medium with variation in one direction only:

Substituting Darcy's law of each phase into its respective continuity equation and then both subtracting and adding the results give a pair of partial differential equations. These equations can be reduced to non-dimensional form as follows. Porosity and absolute permeability are divided by suitable characteristic values so that $\phi^{*}(\mathbf{x}) \equiv \phi(\mathbf{x}) / \phi_{\text {char }}$ and $k^{*}(\mathbf{x}) \equiv k(\mathbf{x}) / k_{\text {char }}$. Capillary pressure $P_{c}\left(S_{u^{\prime}}\right) \equiv P_{0}-P_{u^{\prime}}$, average pressure $P_{a} \equiv$ $\left(P_{o}+P_{w}\right) / 2$, and units of length $U_{l}$, time $U_{t}$, and pressure $U_{p}$ are so defined that

$$
\begin{aligned}
\nabla & \equiv \nabla^{*} / U_{l}=C_{a} \sqrt{\phi_{\text {rhar }} / k_{\text {char }}} \nabla^{*} \\
t & \equiv U_{t} \tau=\frac{\phi_{\text {char }}}{q C^{\prime} a} \sqrt{k_{\text {char }} / \phi_{\text {char }}} \tau \\
P & \equiv U_{p} P^{*}=\sigma \sqrt{\phi_{\text {char }} / k_{\text {char }}} P^{*}
\end{aligned}
$$


where $C a$ is the capillary number $q \mu_{w} / \phi_{c h a r} \sigma, q$ is the total superficial flow velocity $\left(q_{o}+q_{w}\right), \sigma$ is the oil-water interfacial tension, and the asterisk denotes non-dimensional quantities. The equations to be solved for the capillary pressure $P_{c}^{*}$ and the average pressure $P_{a}^{*}$ then are

$$
\begin{aligned}
\nabla^{*} \cdot\left[k^{*}\left(M k_{r o}-k_{r w}\right) \nabla^{*} P_{a}^{*}\right] & +\frac{1}{2} \nabla^{*} \cdot\left[k^{*}\left(M k_{r o}+k_{r u}\right) \nabla^{*} P_{c}^{*}\right] \\
& =-2 \phi^{*} \frac{\partial P_{c}^{*}}{\partial \tau}\left[\frac{d P_{c}^{*}}{d S}\right]^{-1}
\end{aligned}
$$

and

$$
\nabla^{*} \cdot\left[k^{*}\left(M k_{r o}+k_{r w}\right) \nabla^{*} P_{a}^{*}\right]+\frac{1}{2} \nabla^{*} \cdot\left[k^{*}\left(M k_{r w}-k_{r w}\right) \nabla^{*} P_{c}^{*}\right]=0
$$

$S \equiv S_{w}$ and $M$ is the viscosity ratio $\mu_{w} / \mu_{o}$. Equations (6) and (7) are usually called the saturation equation and the pressure equation. Peaceman (1977) derived these equations and alternative corms, including the so-called 'velocity formulation, which we use below.

\section{Porous Media of Interest}

Our first system of interest is a homogeneous porous medium, i.e. one in which porosity, absolute permeability, and the dependence on saturation of the relative permeabilities and capillary pressure do not vary with position. In this case, our choices of relative permeabilities $k_{r i}(S)$ and capillary pressure $P_{c}^{*}(S)$ allow the one-dimensional forms of Eqs. (6) and (7) to have the closed-form solution derived by Yortsos and Fokas (1983). The ratio of the relative permeabilities must obey

$$
\frac{k_{r u}(S)}{k_{r o}(S)}=\frac{S-S_{u r}}{1-S-S_{o r}}
$$

where $S_{o r}$ and $S_{u r}$ are the so-called 'residual' or 'irreducible' saturations of oil and water in the porous medium. In general, $S_{u r} \leq S \leq 1-S_{o r}$. In one simple case, Eq. (8) implies linear relative permeabilities, which are unrealistic but adequate for our purposes here:

$$
\begin{aligned}
& k_{r o}(S)=\frac{1-S-S_{o r}}{1-S_{o r}-S_{u r}} \\
& k_{r u}(S)=\frac{1-S-S_{u r}}{1-S_{o r}-S_{u r r}} .
\end{aligned}
$$

Integration of the derivative of capillary pressure $d P_{c}^{*} / d S$ used by Yortsos and Fokas gives, to an additive constant $C$,

$$
\begin{aligned}
P_{c}^{*}(S) & =\frac{(M-1)^{2}}{1-S_{o r}-S_{w r}} \ln \left[1-S_{o r}-S\right]-\frac{(M-1)^{3}}{M\left(1-S_{o r}-S_{u r}\right)} \times \\
& \times \ln \left|\frac{M\left(1-S_{o r}-S\right)+S-S_{u r}}{M-1}\right|-\frac{(M-1)^{2}}{M\left(1-S_{o r}-S_{u r}\right)} \ln \left[S-S_{w r}\right]+C
\end{aligned}
$$


Figure 2 shows the homogeneous porous medium and its capillary pressure curve, which was computed with $C=0, S_{w r}=0.05, S_{o r}=0.1$, and $M=1.1$.

Figure 3 depicts our second system of interest, which is a composite porous medium made up of two different homogeneous media: one upstream, which has larger pores, higher porosity, and higher absolute permeability, and one downstream, which has smaller pores, lower porosity, and lower absolute permeability. Yortsos and Chang (1990) found, in closed form, the steady-state profile of saturation in the case of a step change in absolute permeability alone. Their solution extends easily, however, to the case of step changes in both absolute permeability and porosity. To admit this closed-form solution at steady state, the system may have any functions of relative permeability $k_{r i}(S)$ throughout, and we choose for convenience the linear relations used in the entirely homogeneous medium, i.e. Eqs. (9) and (10). Capillary pressure, on the other hand, is allowed to vary with position according to

$$
P_{c}(S ; \xi)=\sigma J(S) \sqrt{\phi(\xi) / k(\xi)}
$$

or, in non-dimensional form,

$$
P_{c}^{*}(S ; \xi)=J(S) \sqrt{\phi^{*}(\xi) / k^{*}(\xi)} .
$$

$J(S)$ is the so-called 'Leverett J-function.' Leverett (1941) guessed from dimensional analysis that the function $J$ of Eq. (12) might depend only on saturation. He found that dividing values of measured capillary pressure by $\sigma \sqrt{\phi / k}$ reduced data from several liquid/liquid pairs in unconsolidated sands to a roughly common curve called $J(S)$. Figure 3 shows the two capillary pressure curves of our composite porous medium, in which we use the right side of Eq. (11) with $C=0.1$ for $J(S)$ throughout the medium and Eq. (13) for $P_{c}^{*}(S)$ in each part.

\section{Boundary Conditions}

In order to have a unique solution. Eqs. (6) and (7) require two boundary conditions each in $P_{c}^{*}$ and $P_{a}^{*}$ and an initial profile of $P_{c}^{*}$. We apply the same boundary conditions to both the homogeneous medium and the composite medium.

At the injection boundary, a water-oil mixture with a volume fraction of water $F_{w}^{i n j}$ enters the porous medium with a superficial velocity $q$. Because the boundary cannot accumulate mass,

and

$$
q F_{u^{\prime}}^{i n j}=q_{w}(0, \tau)
$$

$$
q F_{o}^{i n j}=q\left(1-F_{w}^{i n j}\right)=q_{o}(0, \tau)
$$

Substitution of Darcy's law [Eq. (2)] for $q_{w}$ and $q_{o}$ and solution for the gradient of capillary pressure $\left[\nabla^{*} P_{0}^{*}-\nabla^{*} P_{w^{\prime}}^{*}\right]$ give one boundary condition at the injection face:

$$
\nabla^{*} P_{c}^{*}(0 . \tau)=-\frac{F_{w}^{i n j}-f_{w}(S)}{D^{*}(S)} \frac{d P_{c}^{*}}{d S}(S)
$$




\section{SYNTHETIC OUTFLOW BOUNDARY}

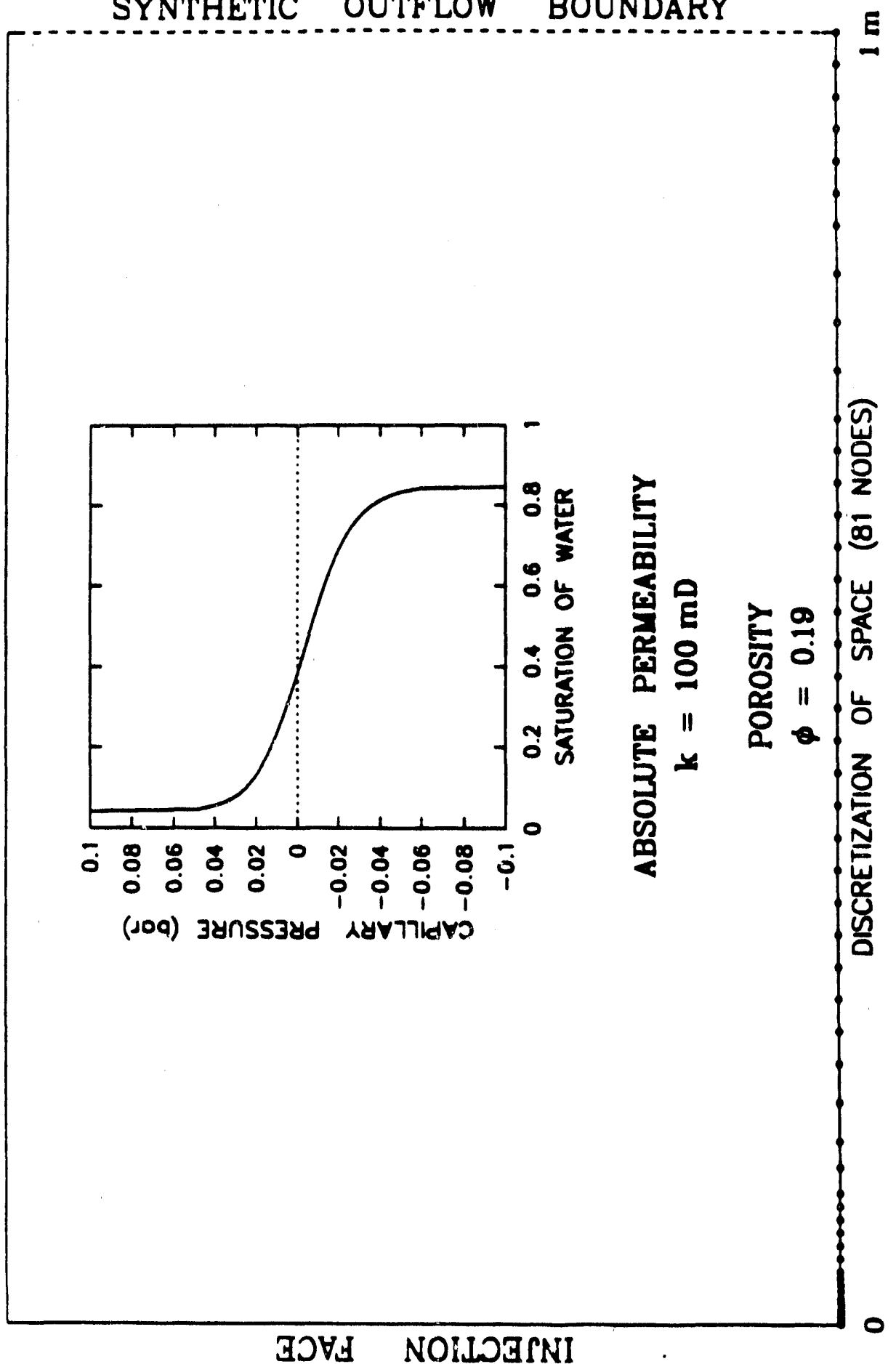

Figure 2. Homogeneous porous medium and its flow properties. 


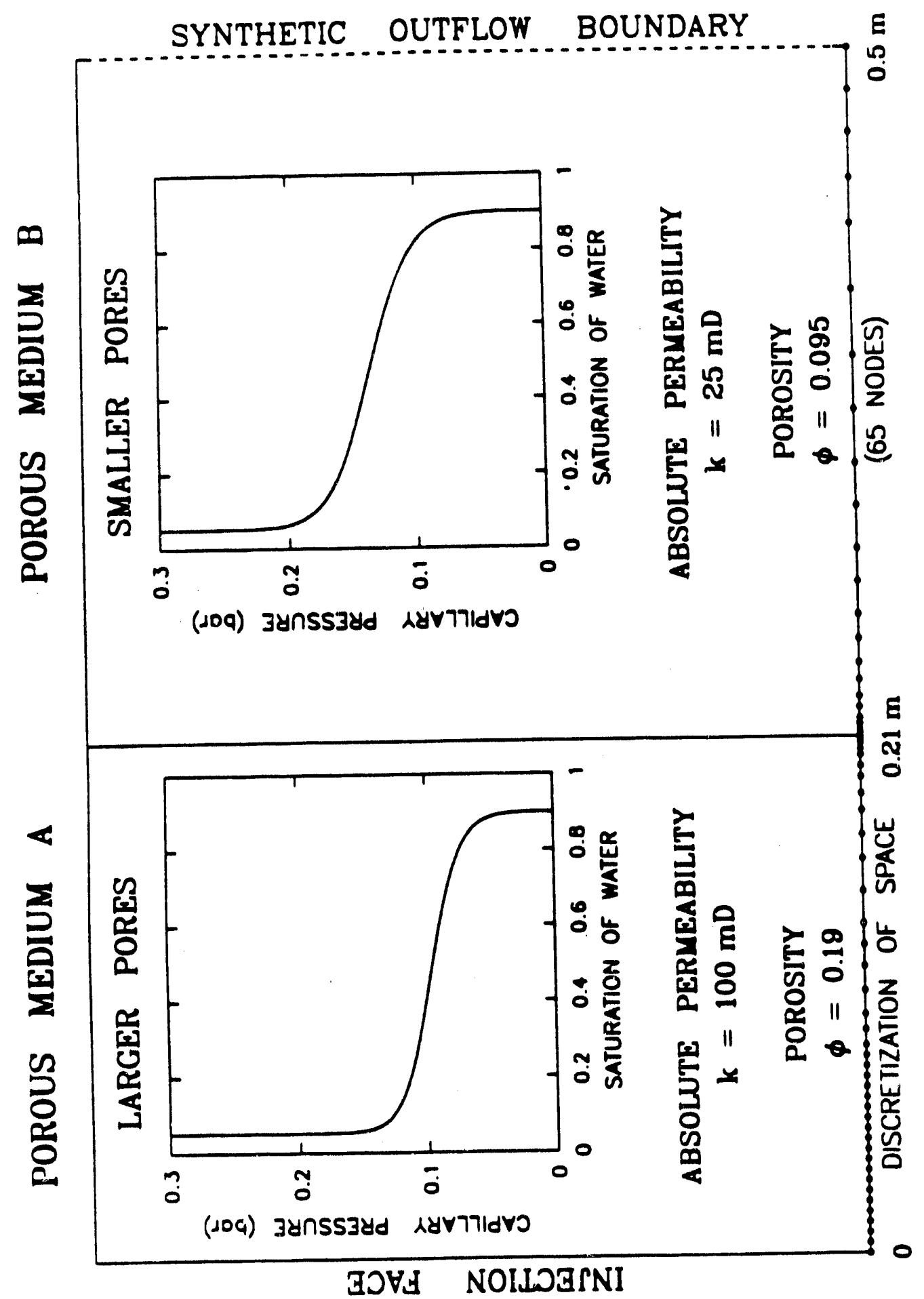

Figure 3. Composite porous medium and its flow properties. 
where $f_{w}(S)$ is the fractional flow of water

$$
f_{w}(S) \equiv \frac{k_{r w}}{M k_{r o}+k_{r w}}
$$

and $D^{*}(S)$ is the capillary dispersion coefficient

$$
D^{*}(S) \equiv-\frac{M k^{*} k_{r o} k_{r w}}{M k_{r o}+k_{r w}} \frac{d P_{c}^{*}}{d S}
$$

Figures 4 and 5 show how $f_{w}(S)$ and $D^{*}(S)$ vary given our choices of relative permeabilities [Eqs. (9) and (10)], residual saturations $\left(S_{o r}=0.1 ; S_{w r}=0.05\right)$, and viscosity ratio $(M=1.1)$. The curves shown in Figure 4 are atypical, for most fructional flow curves have an inflection point. Equation (16) can be rearranged to show that it is, in fact, a mass balance across the injection boundary:

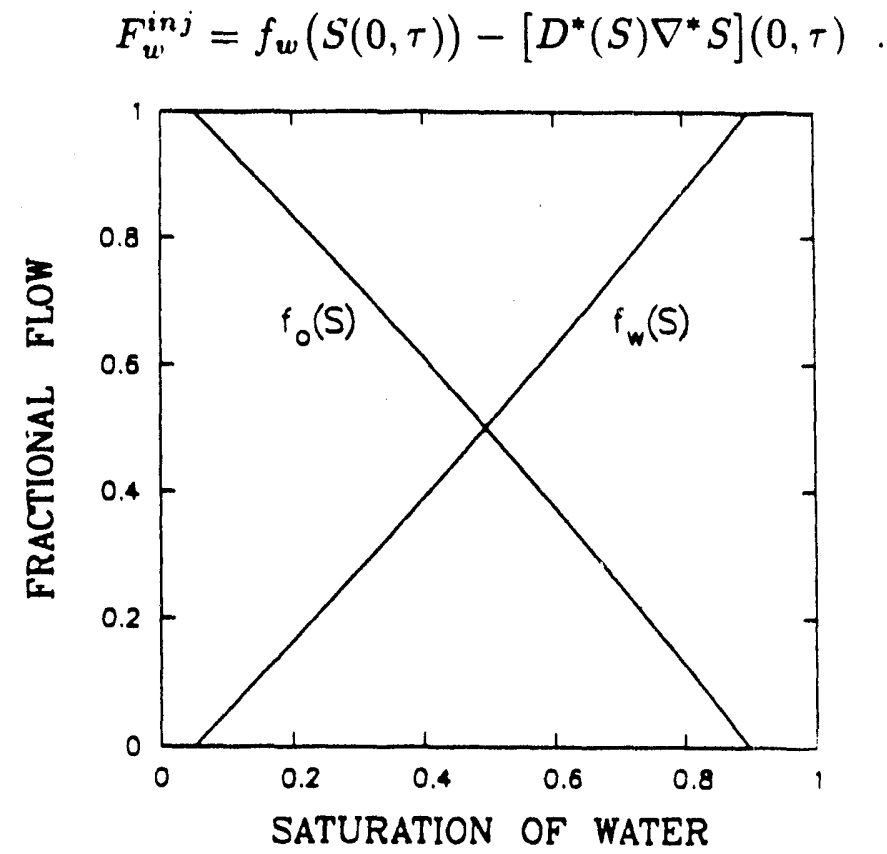

Figure 4. Curves of fractional flow of oil and water used in all examples.

Water flows in bulk toward the boundary as part of a water-oil mixture and is carried into the porcus medium by convection, which is represented by the fractional flow, and by net capillary dispersion, which appears as would Fickian diffusion with a concentrationdependent diffusion coefficient.

Substituting Darcy's law for $q_{w}$ and $q_{0}$ in Eqs. (14) and (15) and solving for the gradient of average pressure $\left[\left(\nabla^{*} P_{o}^{*}+\nabla^{*} P_{w}^{*}\right) / 2\right]$ give a second boundary condition at the injection face:

$$
\nabla^{*} P_{a}^{*}(0, \tau)=\frac{F_{w}^{i n j} f_{o}(S)+F_{o}^{i n j} f_{w}(S)}{2 D^{*}(S)} \frac{d P_{c}^{*}}{d S}(S)
$$




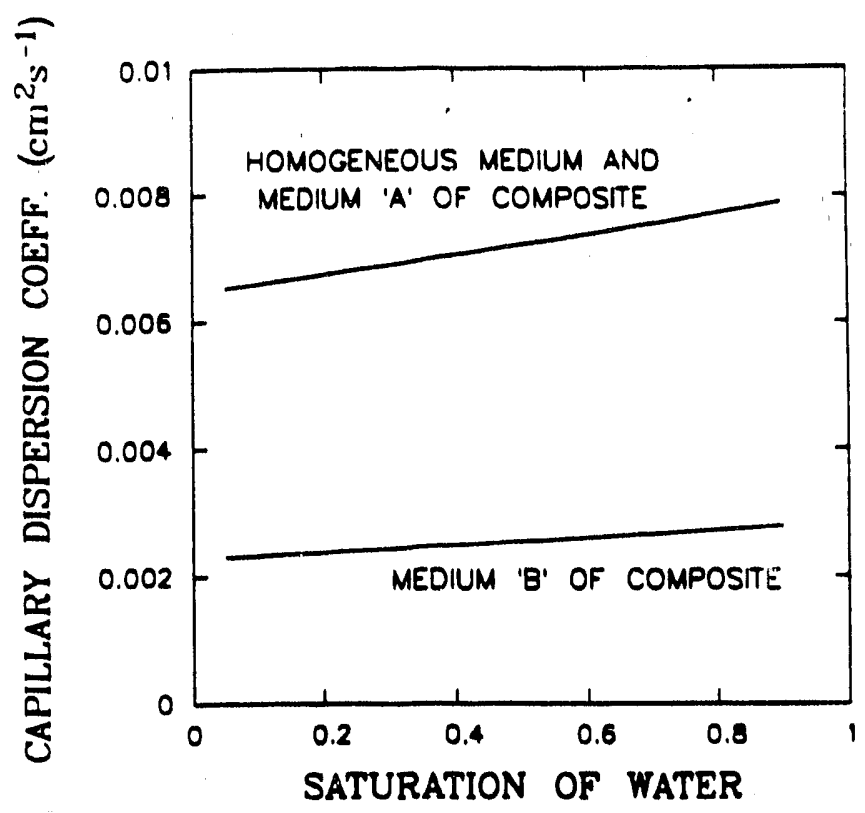

Figure 5. Curve of $D^{*}(S)$ used in each porous medium.

where $f_{o}(S)=1-f_{w}(S)$ is the fractional flow of oil. Relatively large gradients of average pressure are needed to inject a mixture of mostly water $\left(F_{w}^{i n j}\right.$ large) into a region that contains mostly oil ( $f_{o}$ large), and vice versa.

Both the homogeneous medium and the composite medium studied here are taken to be of such great extent that they are semi-infinite. Consequently, we mark the downstream end of the computational domain with a so-called 'synthetic' boundary, i.e. an edge at which there is no physical boundary, at a position $\xi_{S}$ and use the outflow boundary conditions on $P_{c}^{*}$ and $P_{a}^{*}$ discussed in an earlier paper (Novy et al., 1990b). As described there, the outflow boundary condition on capillary pressure is derived by solving the partial differential equation that describes the flow with its coefficients evaluated at the saturation far downstream, i.e. at $S_{\infty}$ :

$$
\nabla^{*} P_{c}^{*}\left(\xi_{S}, \tau\right)=-\frac{\frac{d P_{c}^{*}}{d S} \exp \left[\frac{-\left(\xi_{S}-f_{w}^{\prime}\left(S_{\infty}\right) \tau / \phi^{*}\right)^{2}}{4 D^{*}\left(S_{\infty}\right) \tau / \phi^{*}}\right]}{\sqrt{\pi D^{*}\left(S_{\infty}\right) \tau / \phi^{*}} \operatorname{erfc}\left[\frac{\xi_{S}-f_{w}^{\prime}\left(S_{\infty}\right) \tau / \phi^{*}}{\sqrt{4 D^{*}\left(S_{\infty}\right) \tau / \phi^{*}}}\right]} \times\left[S-S_{\infty}\right]
$$

Finally, because only the gradient of average pressure appears in Eqs. (6) and (7), a pressure datum must be set. This is done at the synthetic boundary:

$$
P_{a}^{*}\left(\xi_{S}, \tau\right)=0 \text {. }
$$

Equation (22) acts as a Dirichlet boundary condition on average pressure.

In contrast to our study of synthetic boundary conditions (Novy et al., 1990b), we are not concerned here with optimizing the location of the boundary to obtain at the lowest cost a solution on a finite medium that is arbitrarily close to the solution on a semi-infinite 
medium. Instead, we locate the synthetic boundary so that moving it farther downstream has a negligible effect on the solution in the regions of greatest interest.

\section{Choice of Initial Condition}

Specification of an initial profile of capillary pressure completes the problem statement. One choice is a distribution of capillary pressure that is uniform:

$$
P_{c}^{*}(\xi, 0)=P_{c, 0}^{*}
$$

In the case of a homogeneous medium, this condition implies either no flow or a steadystate flow such that the fraction of water in the injected liquid equals the total flux of water throughout the medium. In the case of a composite or heterogeneous medium, the capillary pressure can have a finite, uniform value only if there is no flow. This foreshadows the fact that using Eq. (23) as an initial condition leads to computational difficulties, such as wiggles, because the flow rate or, alternatively, the fraction of water in the injected liquid increases immediately at $\tau=0^{+}$to its steady-state value. We show and disçuss the difficulties below. One way to avoid them is to raise stepwise $q$ or $F_{w}^{i n j}$, perhaps linearly or exponentially, to its steady value over a small time interval. 'Ramping up' one of these quantities, however, alters the situation under study. In addition, because time is discretized, a ramp is in fact equivalent to a sequence of small jumps, each of which could yet lead to artifacts. We propose instead a technique for generating initial conditions that satisfy the boundary conditions and steady equations of change under the condition of flow and thus avoid the above computational difficulties.

\section{Initial Condition in Regions of Constancy}

At small times, the capillary pressure (or saturation) and average pressure are changing only near the injection face. Accordingly, we compute the initial condition as follows. First, we find the steady state at which the total flux of water $\left(f_{w}-D^{*} \nabla^{*} S\right)$ at each point in the medium equals the flux far downstream $\left(f_{w, \infty}\right)$. In the case of a homogeneous porous medium, this implies $S(\xi)=S_{\infty}$. Second, we compute in the region of change, which is adjacent to the injection boundary, an approximate solution that satisfies the inflow boundary condition. Third, we splice these two solutions together to get an initial condition that does not cause oscillations in the evolving profiles. Figure 6 shows these steps.

The profile of $P_{c}^{*}$ such that $f_{w} \quad D^{*} \Gamma^{*} S=f_{u^{\prime}, \infty}$ everywhere and the associated profile of $P_{a}^{*}$ are found by solving the equations of change and boundary conditions at a steady state such that $F_{u^{\prime}}^{i n j}=f_{u^{\prime}, \infty}$ :

$$
\nabla^{*} \cdot\left[k^{*}\left(M k_{r o}-k_{r w}\right) \nabla^{*} P_{a}^{*}\right]+\frac{1}{2} \nabla^{*} \cdot\left[k^{*}\left(M k_{r o}+k_{r w}\right) \nabla^{*} P_{c}^{*}\right]=0 \quad \text { [from Eq. 6] }
$$




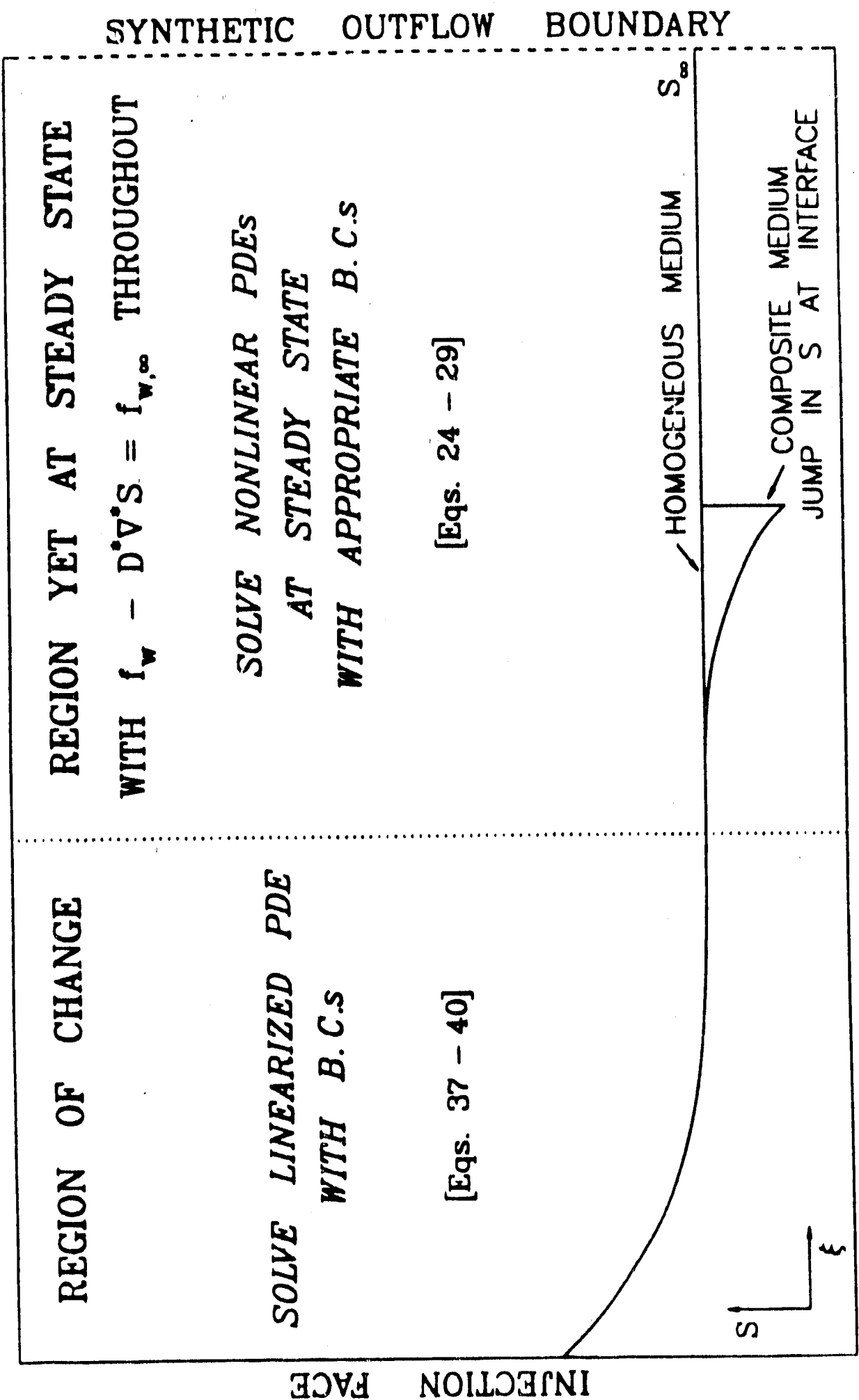

Figure 6 . Cieneration of an initial condition that satisfies the inflow boundary condition upstream and steady equations of change downstream. 


$$
\begin{array}{cc}
\nabla^{*} \cdot\left[k^{*}\left(M k_{r o}+k_{r w}\right) \nabla^{*} P_{a}^{*}\right]+\frac{1}{2} \nabla^{*} \cdot\left[k^{*}\left(M k_{r o}-k_{r w}\right) \nabla^{*} P_{c}^{*}\right]=0 & {[\text { from Eq. 7] }} \\
\nabla^{*} P_{c}^{*}(0)=0 & \text { [from Eq. 16] } \\
\nabla^{*} P_{a}^{*}(0)=\frac{f_{w}\left(S_{\infty}\right)\left[1-f_{w}\left(S_{\infty}\right)\right]}{D^{*}\left(S_{\infty}\right)} \frac{d P_{c}^{*}}{d S}\left(S_{\infty}\right) & \text { [from Eq. 20] } \\
P_{c}^{*}\left(\xi_{S}\right)=P_{c, \infty}^{*} & \\
P_{a}^{*}\left(\xi_{S}\right)=0 & \text { [from Eq. 22] }
\end{array}
$$

The value of $S_{\infty}$ is that which satisfies $f_{w}\left(S_{\infty}\right)=f_{w, \infty}$. A condition on the gradient of capillary pressure at the outflow boundary would leave Eqs. (24)-(25) with an infinity of solutions. We use the Dirichlet boundary condition (28) instead.

In the case of a homogeneous medium, the desired solution of Eqs. (24)-(29) is the uniform profile of capillary pressure (or saturation)

$$
P_{c}^{*}(\xi)=P_{c, \infty}^{*} \quad\left[S(\xi)=S_{\infty}\right]
$$

and the linear profile of average pressure

$$
P_{a}^{*}(\xi)=\frac{f_{w}\left(S_{\infty}\right)\left[1-f_{w}\left(S_{\infty}\right)\right]}{D^{*}\left(S_{\infty}\right)} \frac{d P_{c}^{*}}{d S}\left(S_{\infty}\right) \times\left[\xi-\xi_{S}\right]
$$

In the case of a composite medium, we solve Eqs. (24)-(29) by the Galerkin finite element method. Figure 6 shows a typical profile of $S$ in the case of a composite medium. The shape of the profile of saturation near the interface between 'porous medium A' and 'porous medium B' (see Figure 3) can be explained as follows. At any particular capillary pressure, the saturation of water in medium B is greater than that in medium A. Because the capillary pressure must be continuous across the interface (otherwise flow would instantly make it so), there is a jump in saturation from medium A to medium B. This implies that $f_{w}(S)$ immediately to the left of the interface is smaller than $f_{u^{\prime}}(S)$ to the right of the interface. To maintain a uniform total flux of water, the net dispersive flux on the upstream side must assume a non-zero, positive value, i.e. the gradient of saturation must be negative there. Upstream of the interface, the magnitude of this gradient decreases to zero as the saturation of water increases to $S_{\infty}$, and the fractional flow of water increases to $f_{w, \infty}$. Should the capillary pressure curve of medium B lie below that of medium A, the 
prcfile of $S$ would increase to a maximum at the interface between the media, rather than decrease to a minimum.

\section{Initial Condition in Region of Change}

What remains is to compute an initial condition on capillary pressure near the inflow boundary, where there is change (see Figure 6). For this purpose, we use the so-called velocity formulation of the situation, which has explicit convective and dispersive terms. A different combination of the equation of continuity and Darcy's law for each phase yields in non-dimensional form (cf. Peaceman, 1977)

$$
\phi^{*} \frac{\partial S}{\partial r}+f_{w}^{\prime}(S) \frac{\partial S}{\partial \xi}=\frac{\partial}{\partial \xi}\left[D^{*}(S) \frac{\partial S}{\partial \xi}\right]
$$

where the prime denotes differentiation with respect to $S$. This equation is to be solved approximately with the initial condition

$$
S(\xi, 0)=S_{0}
$$

and the boundary conditions

$$
\begin{array}{r}
\frac{\partial S}{\partial \xi}(0, \tau)=-\frac{F_{w}^{i n j}-f_{w}(S)}{D^{*}(S)}(0, \tau) \\
\frac{\partial S}{\partial \xi}(\infty, \tau)=0
\end{array}
$$

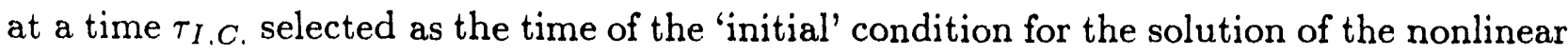
partial differential equations of change (6) and (7). The method of solving Eqs. (32)--(35) must be immune to the difficulties caused by the incompatibility of the initial condition (33) and the inflow boundary condition (34), i.e. the initial condition specifies a zero gradient throughout but the right side of Eq. (34) is nonzero at $S=S_{0}$.

We compute an approximate solution of Eqs. (32)-(35) by evaluating the flow properties $f_{u}(S), f_{u}^{\prime}(S)$, and $D^{*}(S)$ in Eqs. (32) and (34) at a particular position and time and then solving the resulting linear system. Expanding $D^{*}(S)$ in a Taylor series around a position $\xi_{i}$ and a time $\tau_{j}$ gives

$$
\begin{aligned}
D^{*}(S(\xi, \tau)) & =D^{*}\left(S\left(\xi_{i}, \tau_{j}\right)\right)+\left[\xi-\xi_{i}\right] D^{* \prime} S^{\prime}\left(S\left(\xi_{i}, \tau_{j}\right)\right) \frac{\partial S}{\partial \xi}\left(\xi_{i}, \tau_{j}\right)+ \\
& +\left[\tau-\tau_{j}\right] D^{* \prime}\left(S\left(\xi_{i}, \tau_{j}\right)\right) \frac{\partial S}{\partial \tau}\left(\xi_{i}, \tau_{j}\right)+\cdots
\end{aligned}
$$

The series for $f_{w}(S)$ and $f_{w}^{\prime}(S)$ are similar. Defining $S^{*} \equiv S-S_{0}$, inserting the Taylor series for $f_{w}(S), f_{w}^{\prime}(S)$, and $D^{*}(S)$ into Eqs. (32) and (34), and neglecting terms above 
zero order in position and time give a system that is linear in $S^{*}$ :

$$
\begin{aligned}
\phi^{*} \frac{\partial S^{*}}{\partial \tau}+f_{w}^{\prime}\left(S\left(\xi_{i}, \tau_{j}\right)\right) \frac{\partial S^{*}}{\partial \xi} & =D^{*}\left(S\left(\xi_{i}, \tau_{j}\right)\right) \frac{\partial^{2} S^{*}}{\partial \xi^{2}} \\
S^{*}(\xi, 0) & =0 \\
\frac{\partial S^{*}}{\partial \xi}(0, \tau) & =-\frac{F_{w}^{i n j}-f_{w}(S)}{D^{*}(S)}\left(0, \tau_{j}\right) \\
\frac{\partial S^{*}}{\partial \xi}(\infty, \tau) & =0 .
\end{aligned}
$$

Laplace transformation converts this system to a boundary-value problem of second order that is readily solved for the Laplace transform of $S^{*}$. At a particular time $\tau$, we invert the Laplace transform of $S^{*}$ at a set of positions $\xi_{i}$ by means of the computer software routine INLAP of the International Mathematics and Statistics Libraries on a CRAY-2 supercomputer.

One way to compute an initial condition on $P_{c}^{*}$ (or $S$ ) in the region of change (see

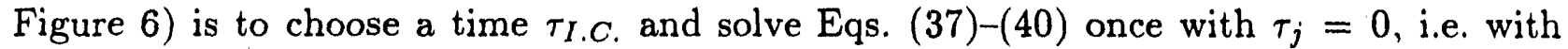
the flow properties evaluated at $S=S_{0}$. A better way, which takes into account the variation of $f_{w}(S)$ and $D^{*}(S)$ as the profile of saturation evolves, is to reach $\tau_{I . C}$. by solving Eqs. (37)-(40) at a sequence of $N$ times $\left\{\tau_{I . C .} / N, 2 \tau_{I . C .} / N, \ldots, \tau_{I . C .}\right\}$, using the solution computed at time $\tau_{j}$ to evaluate the flow properties for the system at time $\tau_{j+1}$. We call this scheme forward marching. The positions $\xi_{i}$ at which the solution is found are fixed. The number of time steps, $N$, is raised until the computed solution at time $\tau_{I, C}$. changes by no more than a desired amount.

As $\tau_{I . C}$. increases, however, the final solution of the sequence of linearized equation sets (37)-(40) diverges from the solution of the nonlinear set (32)-(35) because nonlinearity becomes important. We judge whether or not a solution of the linear system (37)-(40) approximates well the solution of the nonlinear system (32)-(35) by computing the magnitude of the single nonlinear term that does not tend to zero as $N \rightarrow \infty$, i.e. as $\tau \rightarrow \tau_{j}$ at each time step. Inserting the Taylor series for $f_{u}^{\prime}(S)$ and $D^{*}(S)$ into the nonlinear equation (32) and letting $\xi \rightarrow \xi_{i}$ and $\tau \rightarrow \tau_{j}$ give

$$
\phi^{*} \frac{\partial S^{*}}{\partial \tau}+f_{u}^{\prime}\left(S\left(\xi_{i}, \tau_{j}\right)\right) \frac{\partial S^{*}}{\partial \xi}-D^{*}\left(S\left(\xi_{i}, \tau_{j}\right)\right) \frac{\partial^{2} S^{*}}{\partial \xi^{2}}=D^{* \prime}\left(S\left(\xi_{i}, \tau_{j}\right)\right)\left[\frac{\partial S^{*}}{\partial \xi}\right]^{2}
$$

On the left side are the terms of the linearized equation (37). On the right side is the one nonlinear term that remains as $N \rightarrow \infty$. Computing the relative magnitudes of the largest linear term and the nonlinear term, i.e.,

$$
r=\log \left[\left(\max \left\{\left|\phi^{*} \frac{\partial S^{*}}{\partial \tau}\right|,\left|f_{w}^{\prime} \frac{\partial S^{*}}{\partial \xi}\right|,\left|D^{*} \frac{\partial^{2} S^{*}}{\partial \xi^{2}}\right|\right\}\right) / D^{*}\left(\frac{\partial S^{*}}{\partial \xi}\right)^{2}\right]
$$


allows us to assess qualitatively, at each position and time, the proximity of the solution of the linear system to the solution of the nonlinear system. In the examples st!?died here, we find that keeping $\tau_{I . C}$. small enough that $r>1.5$ gives us an initial condition in the region of change that differs from the closed-form solution of the nonlinear system by less than $2 \%$ along the entire profile.

Table 1 summarizes the equations of change and boundary conditions that we solve with an initial condition that represents either a flow or a no-flow situation. We use Galerkin's method with piecewise quadratic finite element basis functions and the adaptive, fully-implicit time integration scheme of Gresho et al. (1980). Figures 2 and 3 show the discretizations of space used. Details of the solution technique are not crucial to this paper. Strang and Fix (1973) explain well the mathematical basis of the Galerkin finite element method (GFEM), and Zienkiewicz (1977), among others, describes its use.

\section{Results and Discussion}

We compare solutions with an initial condition that either satisfies or violates the inflow boundary condition upstream and the steady equations of change downstream. Table 2 lists the parameters used in each type of porous medium. Injecting a mixture of oil and water $\left(F_{w}^{i n j}=0.8\right)$ into the composite medium leads to a steady state other than a trivial, uniform distribution of water. We do not report the solutions of average pressure because they do not alter the conclusions drawn from the profiles of saturation and capillary pressure alone.

\section{Homogeneous Porous Medium}

In the case of a homogeneous porous medium (see Figure 2), Figure 7 shows a set of four solutions that each use an initiai condition that satisfies the inflow boundary condition (16). These initial conditions were computed by solving a sequence of linearized systems (37)-(40) as explained above. To the right of each of the saturation profiles is the distribution of relative error, as compared to the closed-form solution of the nonlinear equations of change given by Fokas and Yortsos (1982) and Yortsos and Fokas (1983). The error in the 'initial' condition falls with the time $t_{I . C}$. chosen for it. Figure 7 gives, in each case, the lower bound on the value of $r$ [defined in Eq. (42)], which equals the number of decades that the one neglected nonlinear term is below the largest term in the linearized PDE

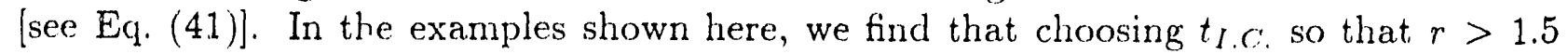
gives relative errors in the initial condition below $2 \%$. As $t_{I . C}$. increases, the nonlinear term that we neglect to compute the initial condition becomes more important, and as the lower bound of $r$ approaches 1 , the relative error in the initial condition in the region of change exceeds $10 \%$. In each case of $t_{I . C}$, however, the error in the initial onndition does decay as the displacement proceeds. This may not be true of other types of systems. An analysis of sensitivity to initial clata would be prudent should our method be applied to 


\section{Equations of Change}

$$
\begin{aligned}
& \nabla^{*} \cdot\left[k^{*}\left(M k_{r o}-k_{r w}\right) \nabla^{*} P_{a}^{*}\right]+\frac{1}{2} \nabla^{*} \cdot\left[k^{*}\left(M k_{r o}+k_{r w}\right) \nabla^{*} P_{c}^{*}\right]=-2 \phi^{*} \frac{\partial S}{\partial \tau} \\
& \nabla^{*} \cdot\left[k^{*}\left(M k_{r o}+k_{r w}\right) \nabla^{*} P_{a}^{*}\right]+\frac{1}{2} \nabla^{*} \cdot\left[k^{*}\left(M k_{r w}-k_{r w}\right) \nabla^{*} P_{c}^{*}\right]=0
\end{aligned}
$$

\section{Boundary Conditions}

$$
\begin{gathered}
\nabla^{*} P_{c}^{*}(0, \tau)=-\frac{F_{w}^{i n j}-f_{w}(S)}{D^{*}(S)} \frac{d P_{c}^{*}}{d S}(S) \\
\nabla^{*} P_{a}^{*}(0, \tau)=\frac{F_{w}^{i n j} f_{o}(S)+F_{o}^{i n j} f_{w}(S)}{2 D^{*}(S)} \frac{d P_{c}^{*}}{d S}(S) \\
\nabla^{*} P_{c}^{*}\left(\xi_{S}, \tau\right)=K^{\prime}\left(\xi_{S}, \tau\right) \times\left[S-S_{\infty}\right] \frac{d P_{c}^{*}}{d S}(S) \\
P_{a}^{*}\left(\xi_{S}, \tau\right)=0
\end{gathered}
$$

Initial Condition

$$
P_{c}^{*}(\xi, 0)=P_{c, 0}^{*}
$$

or

A computed initial condition $P_{c}^{*}(\xi, 0)$ that satisfies both the inflow boundary condition upstream and steady equations of change downstream. 
Table 2. Values of Parameters in Cases of Homogeneous and Composite Media

\begin{tabular}{lccc}
\multicolumn{1}{c}{ Parameter } & Symbol & Homogeneous & Composite \\
\hline Characteristic absolute permability & $k_{c h a r}$ & $100 \mathrm{mD}$ & $100 \mathrm{mD}$ \\
Characteristic porosity & $\phi_{c h a r}$ & 0.19 & 0.19 \\
Residual saturation of water & $S_{w r}$ & 0.05 & 0.05 \\
Residual saturation of oil & $S_{o r}$ & 0.1 & 0.1 \\
Fraction of water in injected liquid & $F_{w}^{i n j}$ & 1 & 0.8 \\
Fractior al flow of water far downstream & $f_{w, \infty}$ & 0.0001 & 0.082 \\
Total superficial velocity & $q$ & $0.85 \mathrm{~m} / \mathrm{d}$ & $0.28 \mathrm{~m} / \mathrm{d}$ \\
Oil-water interiacial tension & $\sigma$ & $72 \mathrm{mN} / \mathrm{m}$ & $72 \mathrm{mN} / \mathrm{m}$ \\
Viscosity of water & $\mu_{w}$ & $1 \mathrm{cP}$ & $1 \mathrm{cP}$ \\
Viscosity of oil & $\mu_{o}$ & $0.91 \mathrm{cP}$ & $0.91 \mathrm{cP}$ \\
Capillary number & $C a$ & $7.2 \times 10^{-7}$ & $2.4 \times 10^{-7}$ \\
Units of length [Eq. (3)] & $U_{1}$ & $1 \mathrm{~m}$ & $3 \mathrm{~m}$ \\
Units of time [Eq. (4)] & $U_{t}$ & $5.4 \mathrm{~h}$ & $48 \mathrm{~h}$ \\
Units of pressure [Eq. (5)] & $U_{p}$ & $1 \mathrm{bar}$ & $1 \mathrm{bar}$ \\
\hline
\end{tabular}

$\ddagger 1$ Darcy $=9.87 \times 10^{-13} \mathrm{~m}^{2}$

systerns of different character.

We did attempt solution wi h the initial condition of uniform capillary pressure, which implies no flow and violates the inflow boundary condition. The resuiting shock at the injection face, however, prevented convergence of the Newton iterations at the first time step regardless of our initial guess. Fortunately, we were able to compute solutions with the no-flow initial condition in the case of the cumposite medium.

\section{Composite Porous Medium}

Figure 8 shows the evolving profiles of saturation and cipillary pressure in the case of a composite porous medium (see Figure 3 ) with the initial condition of unifurm capillary pressure, which implies a step change in saturation at the interface between the media (see Figure 8a). This initial condition violates both the inflow boundary condition and, at the internal interface, the steady equations of change. The inflow boundary condition (16) does not require an instantaneous change in saturation itself, but in the gradient of saturation. This too is sufficient to cause computational artifacts. In Fig. $8 d$ the capillary pressure profile shows a large oscillation: it has fallen at the face but has soared one node inward. This large wiggle decays over the next few time steps. By this time, however, 


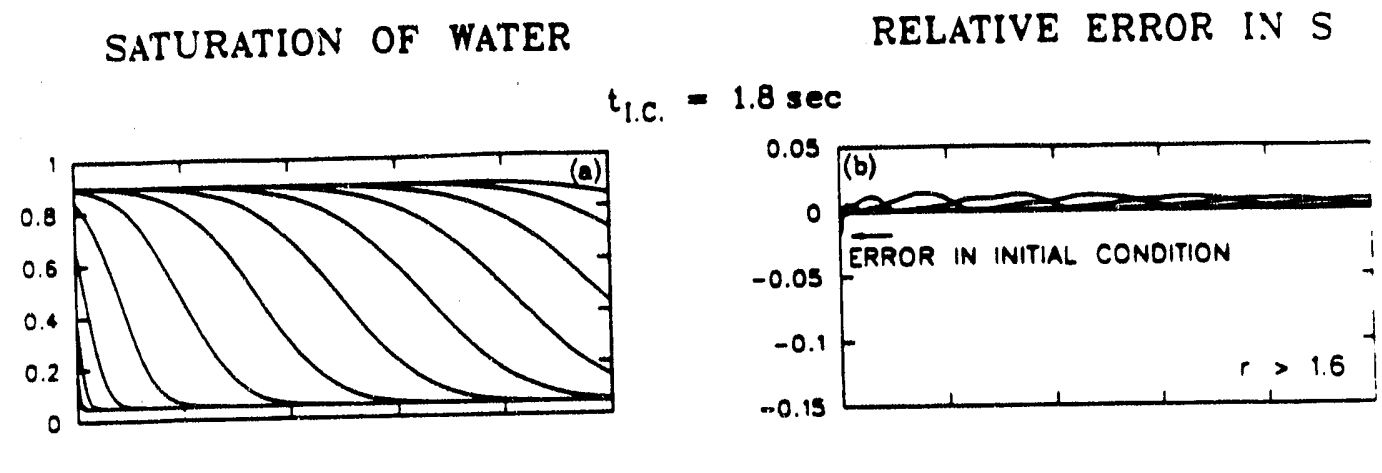

$t_{\text {t.c. }}=3.6 \mathrm{sec}$
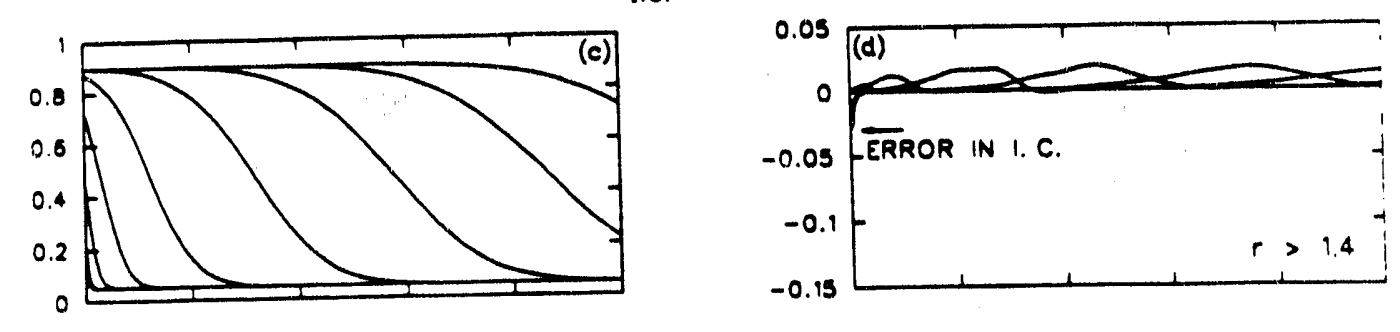

$t_{1 . c .}=18 \mathrm{sec}$
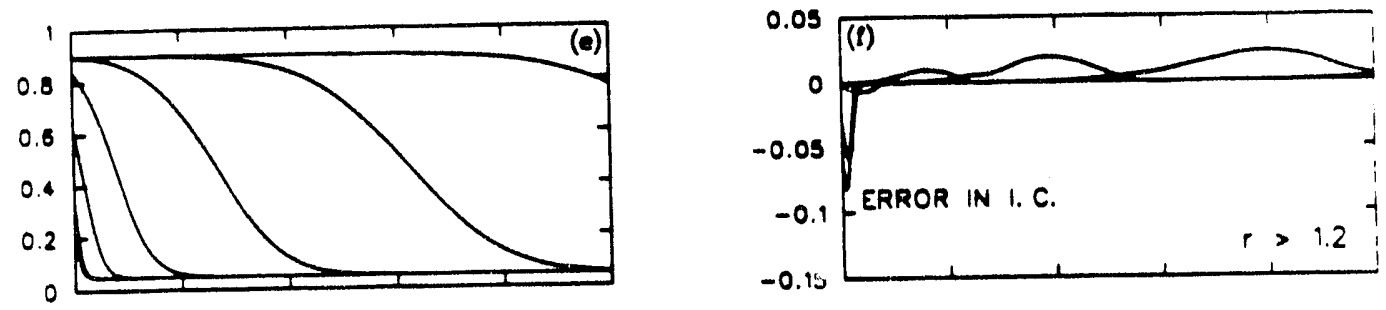

$t_{\text {l.c. }}=36 \mathrm{sec}$
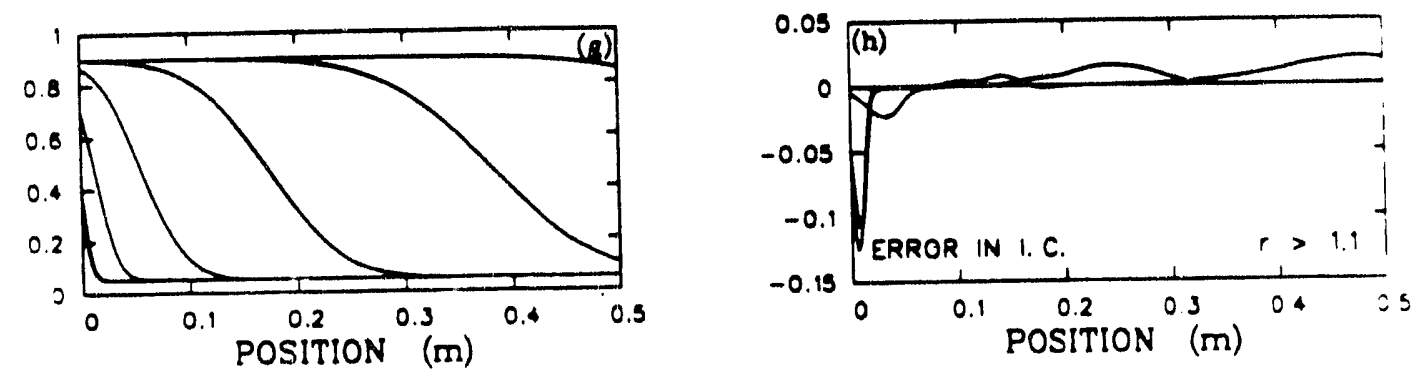

Figure 7. Computed profiles of saturation and relative error $\left(S_{G F E M}-S_{\text {closed form }}\right) /$ $S_{\text {closed form }}$ in the homogeneous medium with 4 flow-compatible initial conditions. Profiles are plotted after every 10 time steps. 
a disturbance (more visible in the plot of capillary pressure) has begun to grow at the interface between the media.

This disturbance appears because the initial condition does not satisfy the steadystate equations of change at the internal interface when there is flow. The saturation upstream of this interface is lower than the saturation downstream; thus, the fractional flow of water, which is also the total flux of water because $\nabla^{*} S=0$ on each side, is lower on the left side than on the right side. To conserve mass, gradients form that cause a net dispersive flux toward the interface from both sides. This feature can also be explained in terms of what the equations of change require with the condition $\nabla^{*} P_{c}^{*}=0$. With a uniform distribution of capillary pressure, the pressure equation (7) requires that

$$
k^{*} \frac{\partial P_{a}^{*}}{\partial \xi}=-\frac{c}{M k_{r o}+k_{r w}},
$$

where $c$ is constant with respect to position and positive (flow is in the direction of increasing $\xi$ ). Setting $\nabla^{*} P_{c}^{*}=0$ in the saturation equation (6) and applying Eq. (43) give

$$
2 \phi^{*} \frac{\partial S}{\partial \tau}=c \frac{\partial}{\partial \xi}\left[\frac{M k_{r o}-k_{r w}}{M k_{r o}+k_{r w}}\right] \text {, }
$$

or, in terms of fractional flows,

$$
2 \phi^{*} \frac{\partial S}{\partial \tau}=c \frac{\partial\left[f_{o}-f_{w}\right]}{\partial \xi}
$$

The gradient on the right side of Eq. $(45)$ is zero on each side of the interface between the media. At the interface itself, however, this gradient is negative. Thus, the equations of change demand a decrease in saturation, i.e. an increase in capillary pressure, at the interface. Figures $8 \mathrm{c}-\mathrm{f}$ show this.

The use of either coarse discretizations of space or upwinding might suppress the growth of such a cusp in capillary pressure at a boundary between two porous media that have different relations between capillary pressure and saturation. Modellers of flow in fractured petroleum reservoirs (which have such boundaries) use initial conditions of uniform capillary pressure but do not mention seeing any immediate changes at the internal boundaries as shown in Figures 8c-f (see, for example, Douglas et al., 1989; and Gilman and Kazemi, 1983).

The cusp in capillary pressure decays once injected water reaches it. Figures $8 \mathrm{~g}, \mathrm{~h}$ show the evolution of the profiles during this stage. Finally, Figures 8i,j depict the approach to steady state, at which the total flux of water equals $F_{w}^{i n j}$ throughout the medium. Barely visible as dashed lines are the closed-form solutions of $S(\xi)$ and $P_{c}^{*}(\xi)$ at steady state, which are straightforward extensions of the work of Yortsos and Chang (1990). The Galerkin finite element solution approaches the correct steady state. 
INITIAL CONDITION
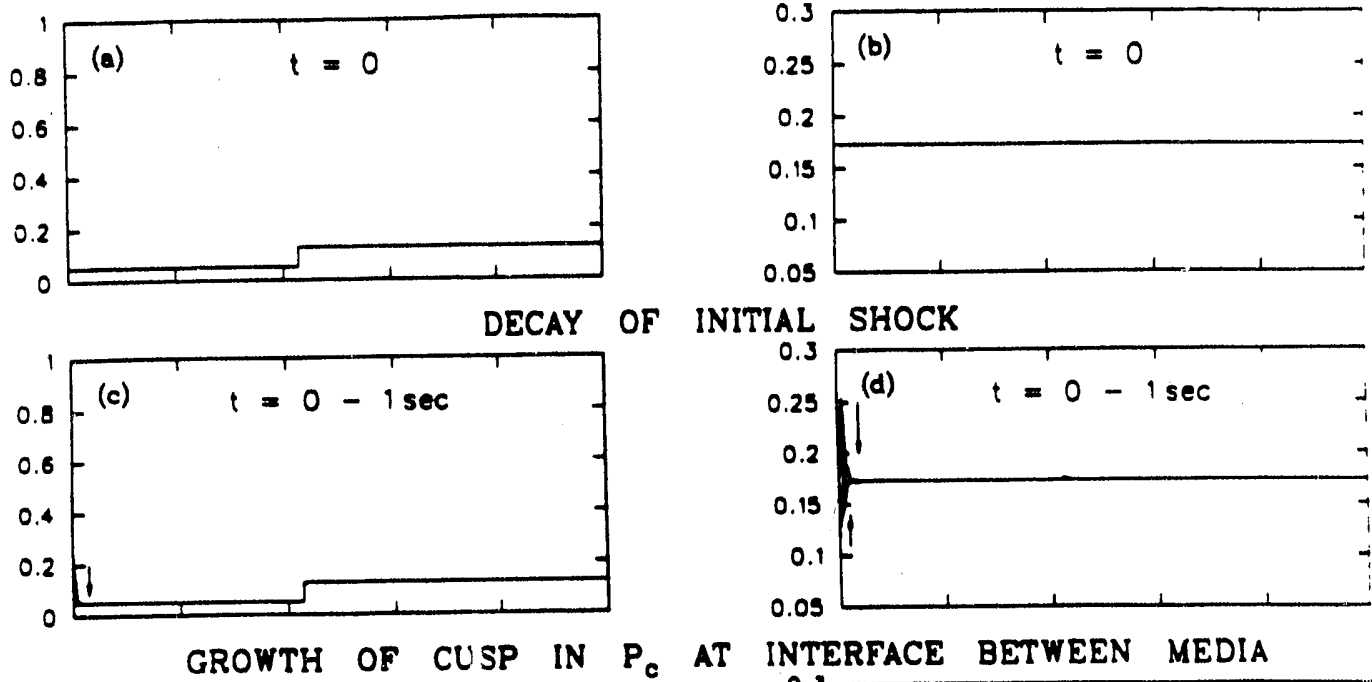

INITIAL SHOCK
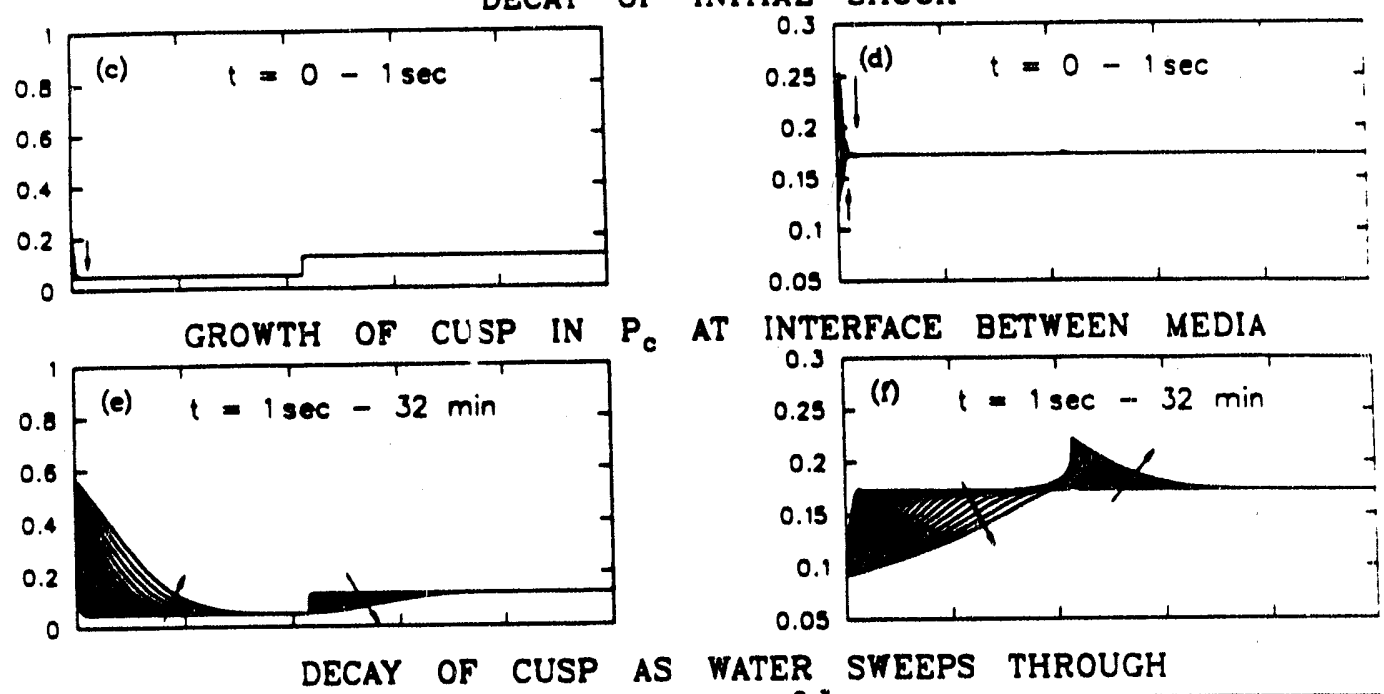

AT

INTERFACE BETWEEN MEDIA
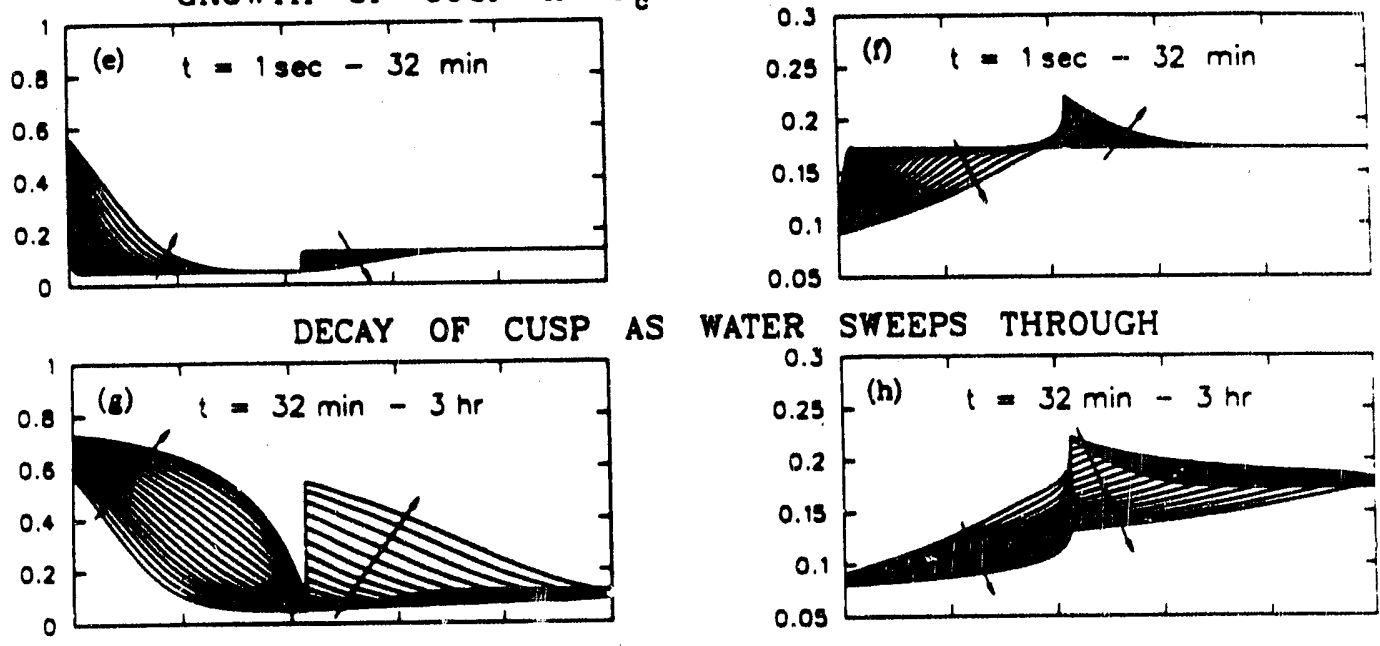

WATER SWEEPS THROUGH

APPROACH TO
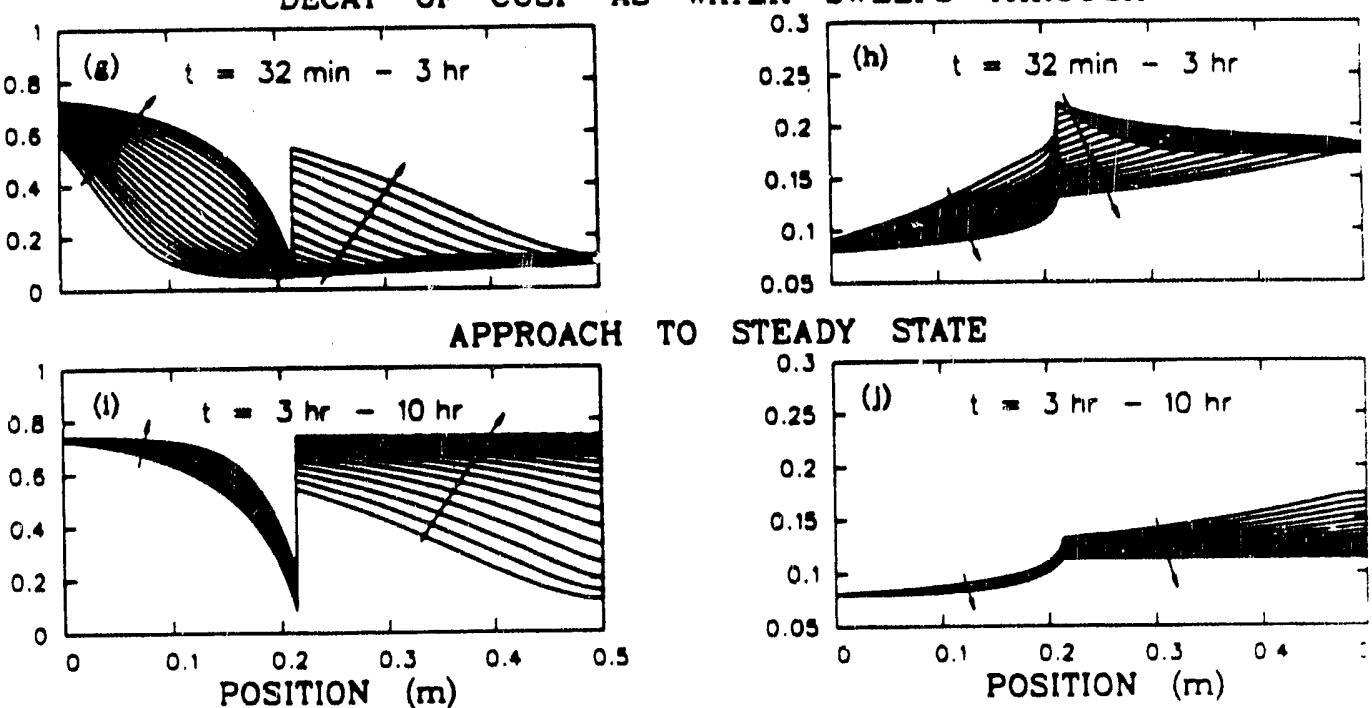

TTEADY STATE

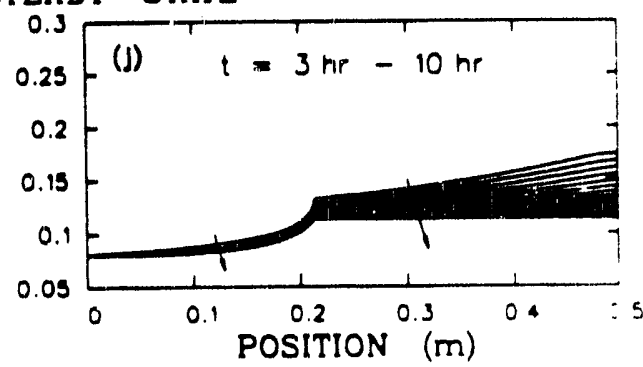

Figure 8. Computed profiles of saturation and capillary pressure in the composite medium with an initial condition that violates the inflow boundary condition upstream and steady equations of change downstream. Profiles are plotted after each time step. 
Figure 9 shows the evolving profiles of the same cisplacement, but with an initial condition that was computed as shown in Figure 6 and detailed above. This initial condition satisfies both the inflow boundary condition and the steady-state equations of change near the interface between the media. The solution evolves without artifacts and approaches the correct steady state. Because the e are no abrupt changes or oscillations in capillary pressure or saturation, the adaptive scheme of time integration sets larger time steps, and accurate predictions of capillary pressure and average pressure allow the Newton iterations at each time step to converge quickly. The total computer time needed to reach $t=10 \mathrm{~h}$ with the flow-compatible initial condition is $40 \%$ of that needed with the no-flow initial condition (uniform $P_{c}^{*}$ ) shown in Figure 8.

In both the homogeneous and composite media, using a smaller initial time step and a finer discretization of space near the injection boundary can eliminate the oscillations in saturation and capillary pressure caused by the initial condition of uniform $P_{c}^{*}$ (see Figure 8). One difficulty that remains, however, is permanent oscillation from one time level to the next in the time derivatives of these quantities. These oscillations lead to poor predictions of $S$ and $P_{c}^{*}$ at each time level, and the adaptive predictor-corrector scheme of time integration thus sets time steps that are unacceptably small. For example, in the case of the composite porous medium, reducing the initial time step to $10 \%$ of the value used in Figure 8 and adding 5 finite elements at the injection boundary eliminate the wiggles in $S$ and $P_{c}^{*}$ at the inlet. Because of the oscillations in the time derivatives of $S$ and $P_{c}^{*}$, however, the adaptive time step levels off at nearly $0.001 \mathrm{~h}$. Thus, approximately 10,000 time steps would be needed to reach $t=10 \mathrm{~h}$. Our initial condition (see Figure 9) required 36 .

\section{Conclusions}

An initial condition, which sets the state of a system at a particular time, should describe accurately the physics of the situation and should not create computational artifacts. Drawing on the example of two-phase flow in porous media, we have shown that unphysical oscillations can mar a solution should the initial condition violate an inflow boundary condition. Tracking these unwanted features significantly increases the amount of computer time needed to solve the equations of change. In the case of a composite medium, an initial condition that violates the steady-state equations of change under flow conditions produces features in the solution that might be equally undesirable.

We have proposed and tested a way to avoid these ills by constructing an initial condition that satisfies the inflow boundary condition upstream and the steady equations of change downstream. In the region of change, the governing nonlinear equations are linearized and solved (using a method immune to wiggles, such as Laplace transformation) at a small value of time such that the solution is as close as desired to that of the nonlinear equations. In the region of the system that is yet at a steady state, the steady equations of change are solved with the appropriate boundary conditions. Splicing together the 
SATURATION OF WATER

CAPILLARY PRESSURE (bar) INITIAL CONDITION
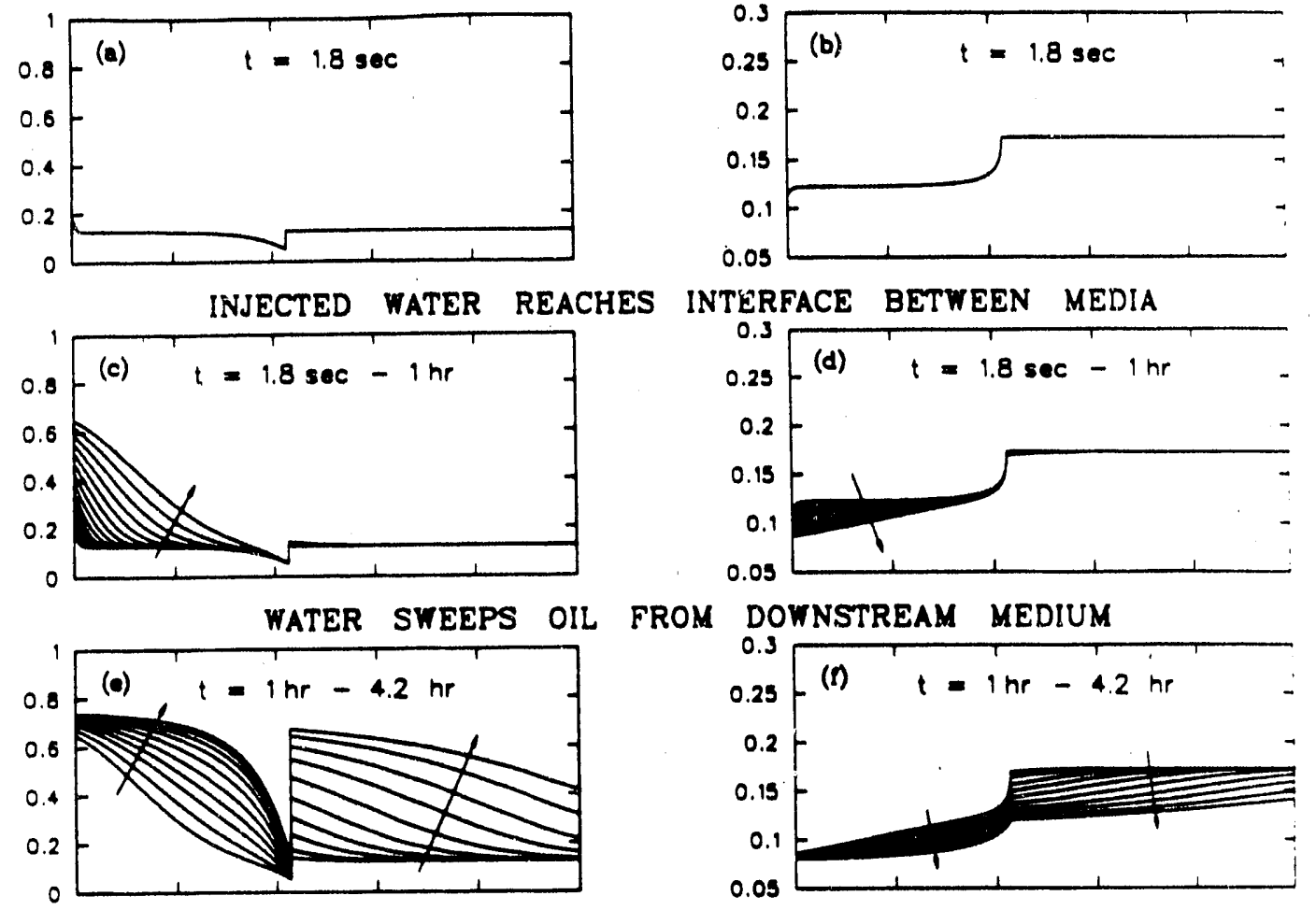

APPROACH TO

STEADY STATE
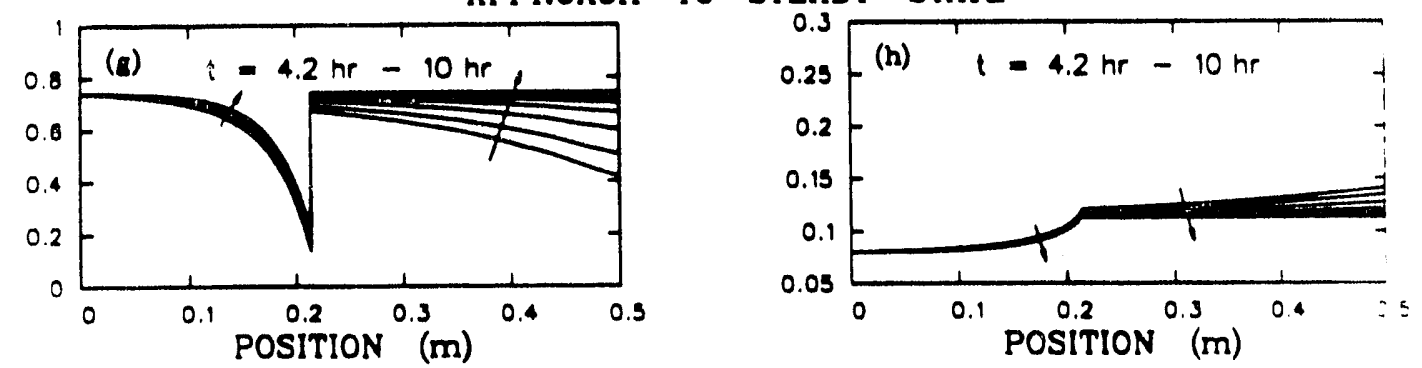

Figure 9. Computed profiles of saturation and capillary pressure in the composite medium with an initial condition that satisfies the inflow boundary condition upstream and steady equations of change downstream. Profiles are plotted after each time step. 
solutions in these two regions gives the desired initial condition. Computing the evolution of a system from this initial condition is more efficient and free of artifacts.

Although we examined the particular case of two-phase flow in porous media, our findings relate to a variety of situations in mass, heat, and momentum transport. Initial conditions are a vital - but sometimes overlooked - component of the description of continuous-flow systems.

\section{References}

Carslaw, H.S. and Jaeger, J.C., Cionduction of Heat in Solids, 2nd edn., Oxford University Press, Oxford (1959).

Crank, J., The Mathematics of Diffusion, 2nd edn., Oxford University Press, Oxford (1975).

Douglas, J. Jr., iarbogast, T. and Paes Leme, P.J., SPE 18425, presented at the Tenth SPE Symposium on Reservoir Simulation, Houston, TX, February 6-8 (1989).

Fokas, A.S. and Yortsos, Y.C., SIAM J. Appl. Math. 42, 318-332 (1982).

Gilman, J.R. and Kazemi, H., Soc. Pet. Engrs. J. 23, 695-707 (1983).

Gresho, P.M. and Lee, R.L. Comput. Fluids 9, 223-253 (1981).

Gresho, P.M., Lee, R.L. anu Sani, R.L., In Recent Advances in Numerical Methods in Fluids (Edited by Taylor, C. and Morgan, K.), Vol. 1, Pineridge Press, Swansea (1980) pp. 27-79.

International Mathematics and Statistics Libraries, version 10. IMSL, Inc., Housto... (1987).

Leverett, M.C., Trans. AIME 142, 152-169 (1941).

Novy, R.A., Davis, H.T. and Scriven, L.E., Chem. Engng. Sci. 45, 1515-1524 (1990a).

Novy, R.A., Davis, H.T. and Scriven, L.E., Chem. Engng. Sci. (in press) (1990b).

Peaceman, D.W., Fundamentals of Numerical Reservoir Simulation. Elsevier, NY (1977). Roache, P.J., Computational Fluid Dynamics, Hermosa, Albuquerque, NM (1972) pp. 161162.

Strang, G. and Fix, G.J., An Analysis of the Finite Element Method. Prentice-Hall, Englewood Cliffs, NJ (1973).

Yortsos, Y.C. and Chang, J., Transp. Por. Media (in press) (1990).

Yortsos, Y.C. and Fokas, A.S., Soc. Pet. Engrs. J. 23, 115-123 (1983).

Zienkiewicz, O.C., The Finite Element Method, 3rd edn. McGraw-Hill, NY (1077). 


\section{A COMPARISON OF SYNTHETIC BOUNDARY CONDITIONS FOR CONTINUOUS-FLOW SYSTEMS}

\section{Synopsis}

The analysis of continuous-flow situations requires identification of a 'syslem,' which is the region of particular interest, and its 'surroundings,' which are represented by conditions imposed at the boundary of the 'system.' General procedures for choosing boundary conditions at surfaces at which no phase boundary exists, i.e. 'synthetic' boundaries, seem to be lacking. Drawing on the example of immiscible displacement of oil by water in a onedimensional, semi-infinite porous medium, we compare four types of synthetic downstream boundary conditions - Dirichlet (first kind), Neumann (second kind), Robin (third kind), and what is in essence none - to find which is the most efficient when predictions are to be computed from the solution of the governing equation set. The Robin-type condition proves best: it gives the most accurate solution at fixed cost or, alternatively, requires the least work to achieve a given accuracy. To represent faithfully the physics of the situation, the Dirichlet and Neumann conditions must be imposed farther downstream of the region of interest than the Robin condition. In addition, we explore the behavior of a 'pseudo-boundary condition,' which is in fact not a proper boundary condition at all, but discretization and truncation errors mask its redundant nature and allow it to perform well in cases where there is little upstream signalling. Although our findings are drawn from the displacement problem, they are more broadly applicable to analysis of transport phenomena.

\section{Introduction}

The analysis of continuous-flow situations requires the definition of a region of particular interest, which is commonly termed 'the system,' and the remainder of the universe, which is traditionally called 'the surroundings.' Equations of change that describe the behavior of the system derive from laws of conservation of mass, momentum, energy, charge, and so forth and equally from constitutive relations that represent material behavior. A boundary, i.e. an idealization that consists of a closed mathematical surface, defines the region or domain of interest by separating it from the rest of the universe. Boundary conditions must both single out a particular solution of the equations of change within the system and, more importantly, express accurately the interaction between the system and. its surroundings.

In some simple cases, the solutions of the equations of change of a particular system can be written in terms of standard mathematical functions. These systems usually have regular domains, e.g. of rectangular, cylindrical, spherical, or other symmetry, and transport coefficients that do not vary with location. Moreover, they usually are not com-

plicated by nonlinear coupling of the transport of one quantity, e.g. heat, with the transport 
of another, e.g. momentum. Digital computers now enable the study of systems of greater complexity that are described by equations that do not have closed-form solutions so far as is known or expected. Two schemes of computer-aided analysis in wide use are finite difference approximation and Galerkin's method with finite element basis functions.

Even though all real continuous-flow situations are finite, the current tools of computer-aided analysis usually limit the size of the region that we can study in detail. To mark the region of interest where there are no phase boundaries, so-called 'synthetic boundarie' are often drawn. How to select the most efficient boundary conditions at surfaces that do not coincide with phase boundaries is a vital, and largely uncodified, issue.

In an earlier paper (Novy et al., 1990), we explored this issue in the context of the transport of a chemical species in a continuous-flow reactor, i.e. the celebrated problem of Danckwerts (1953), who ignited debate by suggesting the outflow boundary conditions that now bear his name (see especially Wehner and Wilhelm, 1956; Pearson, 1959; and Bischoff, 1960, 1961). We concluded that the lengths of finite entrance and exit sections that abut a reactor can have an impact on predictions of reactor behavior: for example, using the solution of Danckwerts (or, equivalently, of Wehner and Wilhelm) to predict reactant conversion at steady state in a perfectly-mixed reactor equal in length to its entrance section leads to an error of over $15 \%$. Also, we proposed and tested two boundary conditions for the synthetic boundary at the downstream end of the exit section, namely instant mixing and instant removal, which bracket the range of concentration gradients that may be imposed in the transient situation.

The goal of this paper is to compare four types of synthetic boundary conditions Dirichlet (first kind), Neumann (second kind), Robin (third kind), and what is in essence none - and to find which yields the most accurate solution at a given computational cost. A Dirichilet condition sets the value of the unknown function itself, whereas a Neumann condition sets the gradient of the function in a direction normal to the boundary. Named after the 19th century French mathematician Gustave Robin, whose researches included distribution of electrostatic potential in unbounded regions (Robin, 1886), the Robin boundary condition sets the value of a combination of the unknown function and its normal gradient that is linear in the unknown function (e.g. Iordanidis, 1973). A Robintype condition on temperature arises in the transport of heat across the interface between a solid and a moving fluid: at the solid/fluid boundary, the conductive flux of heat in the solid, which is proportional to the gradien $\iota$ of temperature in the solid, must equai the convective flux of heat in the fluid, which, from Newton's law of cooling, is proportional to the difference between the temperature at the boundary and the ambient temperature of the fluid. In his celebrated treatise on the transport of heat, Fourier (1822) equated these fluxes and may have been the first to derive a boundary condition of the third kind. In fact, hydrogeologists, among others, name the boundary condition of the third kind in honor of Fourier, not Robin (de Marsily, 1986). 
The context here is immiscible displacement of oil by water in a one-dimensional porous medium that is long enough to be considered semi-infinite and thus requires a synthetic boundary downstream. The particular equation set that we study has a closedform solution (Fokas and Yortsos, 1982; Yortsos and Fokas, 1983), which makes comparison easy. In addition to comparing synthetic boundary conditions in the case of a flow with planar symmetry, we propose Robin boundary conditions that are suita', le for cylindrically and spherically symmetric flows. Although we draw on the example of two-phase flow in porous media, the pariial analogies among transport of mass, heat, momentum, and charge make our findings more broadly applicable.

\section{Theory}

Two partial differential equations that are often used to describe two-phase flow in a porous medium can be derived from the equation of continuity of each phase $i$

$$
\phi \frac{\partial S_{i}}{\partial t}=-\nabla \cdot \mathbf{q}_{i}
$$

and the linear constitutive equation called Darcy's law, which relates the superficial flow velocity $\mathrm{q}_{i}$ and the gradient of the pressure $P_{i}$ of phase $i$ :

$$
\mathbf{q}_{i}=-\frac{k k_{r i}}{\mu_{i}} \nabla P_{i} .
$$

$\phi$ is the porosity or void fraction of the bulk medium, $S_{i}$ is the saturation or fractional void volume of phase $i, t$ is time, $k$ is the absolute permeability of the medium, $k_{r i}\left(S_{i}\right)$ is the relative permeability of phase $i$, and $\mu$ is viscosity. In this paper, the two phases can be thought of as oil $(o)$ and water $(w)$ in a porous medium that is preferentially wet by water.

Substituting Darcy's law of each phase into its respective continuity equation and then both subtracting and adding the results give a pair of partial differential equations. Defining the capillary pressure $P_{c}\left(S_{w}\right) \equiv P_{o}-P_{w}$, the average pressure $P_{a} \equiv \frac{1}{2}\left(P_{o}+P_{w}\right)$, and the units of length, time, and pressure such that

$$
\begin{aligned}
\nabla & \equiv C a \sqrt{\phi / k} \nabla^{*} \\
t & \equiv \frac{\phi}{q C a} \sqrt{\phi / k} \tau \\
P & \equiv \sigma \sqrt{\phi / k} P^{*}
\end{aligned}
$$

where $C a$ is the capillary number $q \mu_{w} / \phi \sigma, q$ is the total superficial flow velocity $\left(q_{o}+q_{w}\right)$, $\sigma$ is the oil-water interfacial tension, and the asterisk denotes non-dimensional quantities, yields the final equations to be solved for the saturation of water $S \equiv S_{w}$ and the average 
pressure $P_{a}^{*}$ :

$$
\begin{aligned}
& \nabla^{*} \cdot\left[\left(M k_{r o}-k_{r w}\right) \nabla^{*} P_{a}^{*}\right]+\frac{1}{2} \nabla^{*} \cdot\left[\left(M k_{r o}+k_{r w}\right) \frac{d P_{c}^{*}}{d S} \nabla^{*} S\right]=-2 \frac{\partial S}{\partial \tau} \\
& \nabla^{*} \cdot\left[\left(M k_{r o}+k_{r w}\right) \nabla^{*} P_{a}^{*}\right]+\frac{1}{2} \nabla^{*} \cdot\left[\left(M k_{r o}+k_{r w}\right) \frac{d P_{c}^{*}}{d S} \nabla^{*} S\right]=0 .
\end{aligned}
$$

$M$ is the viscosity ratio $\mu_{w} / \mu_{o}$. We use $M=1.1$ in all of our examples. Equations (6) and (7) are usually called the saturation equation and the pressure equation. Peaceman (1977) gives derivations of these equations and alternative forms, including the so-called 'velocity formulation,' which we use below.

Our choices of relative permeabilities $k_{r i}(S)$ and capillary pressure $P_{c}^{*}(S)$ are those that are necessary in order for Eqs. (6) and (7) to have the closed-form solution derived by Yortsos and Fokas (1983). The ratio of the relative permeabilities must obey

$$
\frac{k_{r w}(S)}{k_{r o}(S)}=\frac{S-S_{w r}}{1-S_{o r}-S}
$$

where $S_{o r}$ and $S_{w r}$ are the residual saturations of oil and water in the porous medium. In general, $S_{w r} \leq S \leq 1-S_{o r}$. We use the values $S_{o r}=0.15$ and $S_{w r}=0.0375$ in all of our examples. In one simple case, Eq. (8) implies linear relative permeabilities, which are unrealistic but adequate for our purposes here. Integration of the derivative of capillary pressure $d P_{c}^{*} / d S$ used by Yortsos and Fokas gives, to an additive constant $C$,

$$
\begin{aligned}
P_{c}^{*}(S)+C & =\frac{(M-1)^{2}}{1-S_{o r}-S_{w r}} \ln \left[1-S_{o r}-S\right]-\frac{(M-1)^{3}}{M\left(1-S_{o r}-S_{w r}\right)} \times \\
& \times \ln \left|\frac{M\left(1-S_{o r}-S\right)+S-S_{u r}}{M-1}\right|-\frac{(M-1)^{2}}{M\left(1-S_{o r}-S_{u r}\right)} \ln \left[S-S_{w r}\right] .
\end{aligned}
$$

The value of $C$ is irrelevant here, for only the derivative of $P_{c}^{*}(S)$ appears in the set of governing equations. Figure 1 depicts the capillary pressure curve with $C=0, S_{w r}=$ $0.0375, S_{\text {or }}=0.15$, and $M=1.1$.

Figure $2 \mathrm{a}$ shows our region of interest. To displace oil, water is injected through an open face into a homogeneous, isotropic porous medium of great extent. We are interested in computing accurately and efficiently the evolution of the profiles of saturation and average pressure.

Equations (6) and (7) require two boundary conditions each in $S$ and $P_{a}^{*}$ and an initial saturation profile. Choosing the boundary conditions at the injection face is straightforward. At this face, only water is flowing, i.e. $q_{w} / q=1$. This implies that the gradient of pressure in the oil is zero at the injection face. Using this fact, one can deduce from Darcy's law (Eq. 2) for water and from the definition of capillary pressure the following condition on saturation:

$$
\nabla^{*} S(0, \tau)=\left[k_{r w} \frac{d P_{c}^{*}}{d S}\right]^{-1}
$$




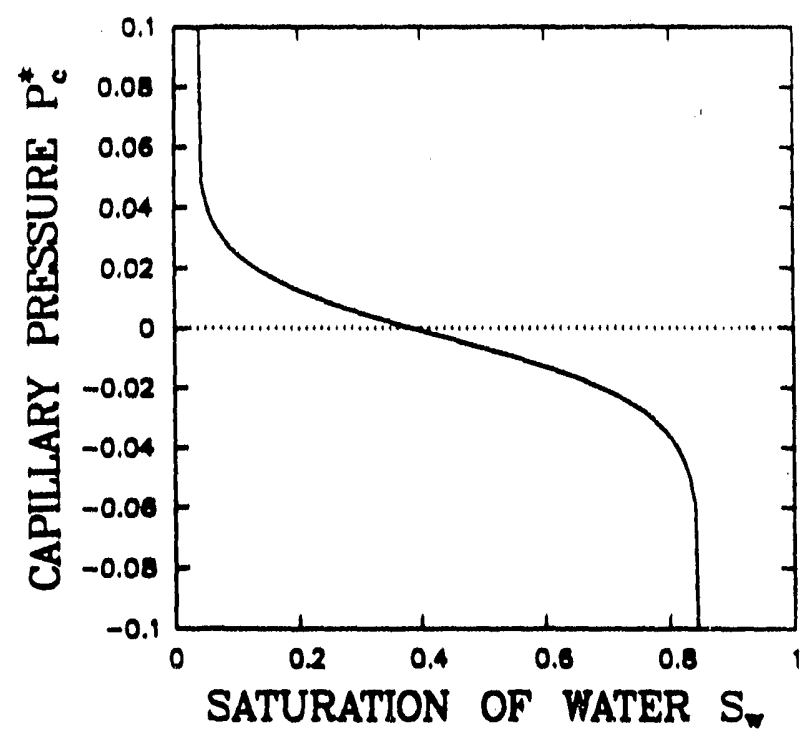

Figure 1. Capillary pressure curve of Yortsos and Fokas with $S_{w r}=0.0375, S_{o r}=0.15$, $M=1.1$, and additive constant $C=0$.

The boundary condition on average pressure derives similarly from Durcy's law for water and the definition of average pressure:

$$
\nabla^{*} P_{a}^{*}(0, \tau)=-\frac{1}{2 k_{r w}}
$$

Selecting two more boundary conditions is not as easy. The medium can be regarded as semi-infinite, because the downstream boundary is distant. Yet we do not have the resources to study the entire domain: a syuthetic boundary is needed downstream. Because only the gradient of average pressure appears in Eqs. (6) and (7), a pressure datum must be set. This is done at the synthetic boundary:

$$
P_{a}^{*}\left(\xi_{S}, \tau\right)=0 \text {. }
$$

Equation (12) acts as a Dirichlet boundary condition on average pressure.

A second boundary condition on $S$ is needed. Table 1 lists three types of conditions that can be chosen at a synthetic boundary. Systematic comparisons of the effects of these boundary conditions on computed solutions are few. Bixler (1982), in his research on slot and knife coating flows, con . $\mathrm{u}$ ded that a Robin-type boundary condition provides the greatest accuracy per unit computational cost. Basaran (1984) reached the same conclusion in his study of the statics and stability of charged drops. In general, the synthetic boundary must be placed farther downstream with the Dirichlet and Neumann conditions than with the Robin condition. The constant of proportionality $K$ of the Robin condition and an estimate of where to locate the synthetic boundary can often be obtained by solving the governing equation set in the asymptotic regime far downstream.

Instead of using Eqs. (6) and (7) for this purpose, we employed the boundary condition obtained from the so-called velocity formulation of this problem, which has convective and 


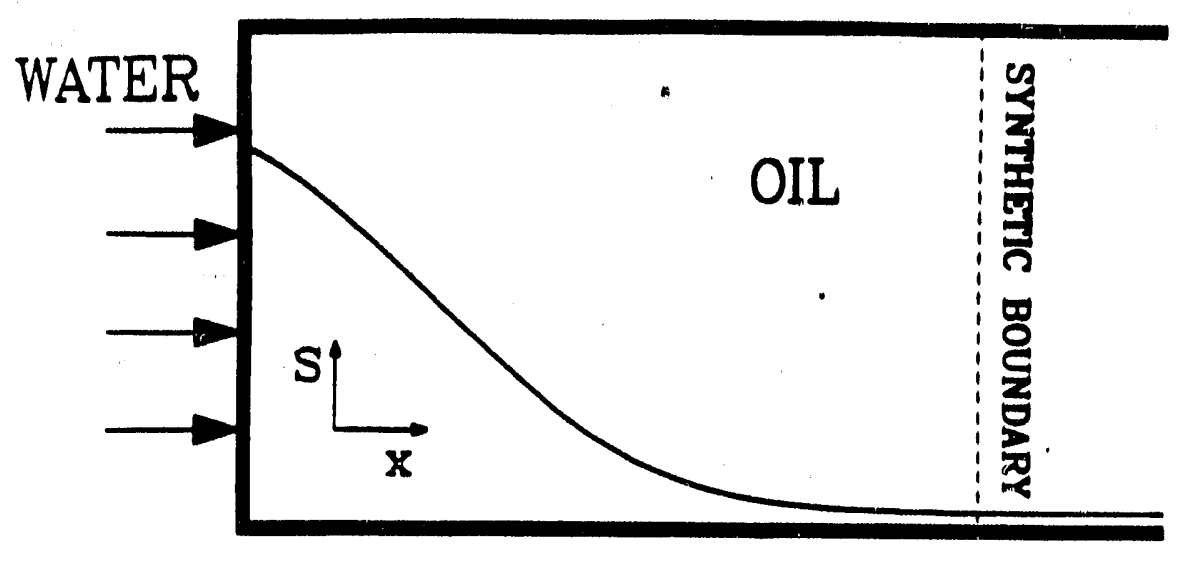

(a)

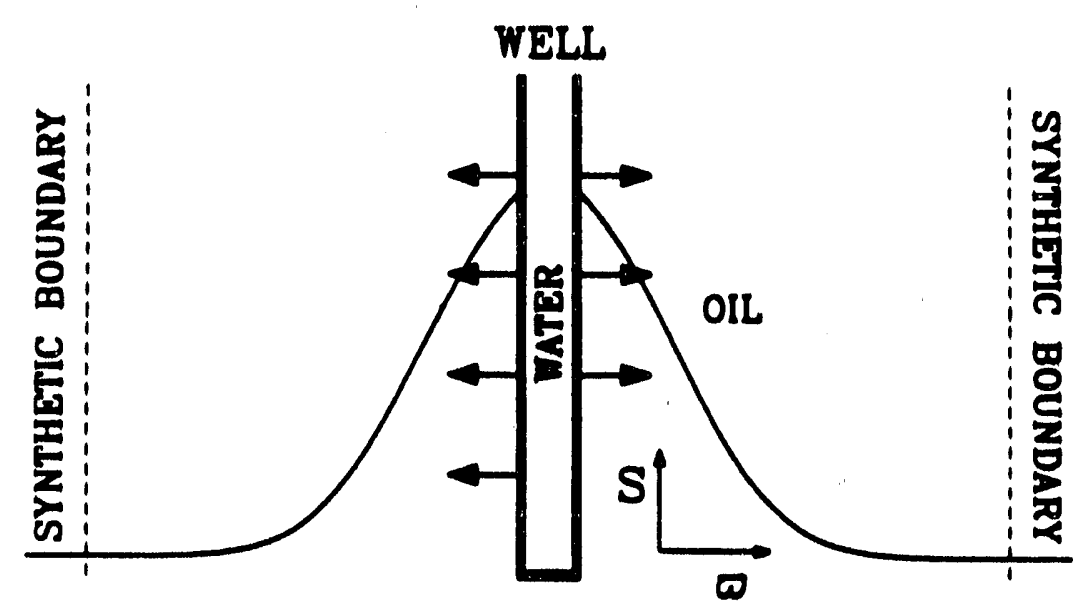

(b)

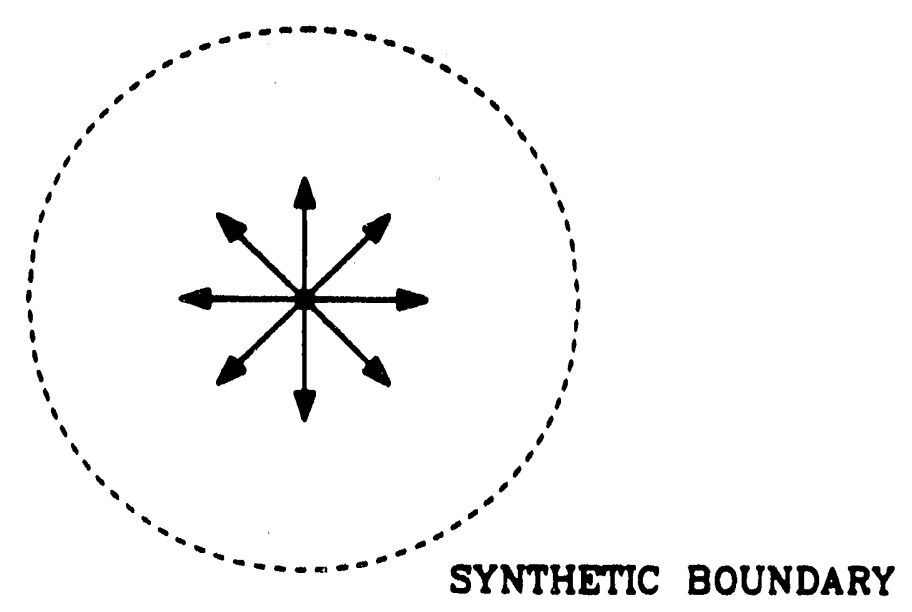

(c)

Figure 2. Cross-sectional views of displacements with (a) planar symmetry, (b) cylindrical symmetry, and (c) spherical symmetry. The respective synthetic boundaries are a plane, a cylindrical shell, and a spherical shell. 
Table 1. Three Types of Synthetic Boundary Conditions

\begin{tabular}{|c|c|c|}
\hline TYPE & CONDITION & MEANING \\
\hline DIRICHLET & $S=S_{\infty}$ & $S$ has reached its asymptotic value \\
NEUMANN & $\frac{\partial S}{\partial \xi}=0$ & $S$ has attained some final state downstream \\
ROBIN & $\frac{\partial S}{\partial \xi}=K\left(S-S_{\infty}\right)$ & $S$ tends to $S_{\infty}$ at the asympiotic rate \\
\hline
\end{tabular}

dispersive terms familiar to engineers. A different combination of the equation of continuity and Darcy's law of each phase yields in dimensional form

$$
\phi \frac{\partial S}{\partial t}+q f^{\prime}(S) \frac{\partial S}{\partial x}=\frac{\partial}{\partial x}\left[D(S) \frac{\partial S}{\partial x}\right]
$$

where

$$
f^{\prime}(S) \equiv \frac{d f}{d S} \equiv \frac{d}{d S}\left[\frac{k_{r w}}{M k_{r o}+k_{r w}}\right]
$$

is the derivative of the fractional flow of water $f$ and

$$
D(S) \equiv-\frac{k}{\mu_{w}} \frac{M k_{r o} k_{r w}}{M k_{r o}+k_{r w}} \frac{d P_{c}}{d S}
$$

is the capillary dispersion coefficient. Evaluating the saturation-dependent functions at the value of saturation far downstream, i.e. $S_{\infty}$, gives the linear equation

$$
\phi \frac{\partial S}{\partial t}+q f^{\prime}\left(S_{\infty}\right) \frac{\partial S}{\partial x}=D\left(S_{\infty}\right) \frac{\partial^{2} S}{\partial x^{2}}
$$

which is to be solved with the single condition

$$
S(\infty, t)=S_{\infty}
$$

Defining a translating coordinate $X \equiv x-q f^{\prime}\left(S_{\infty}\right) t / \phi$ leads to the dispersion equation

$$
\phi \frac{\partial S}{\partial t}=D\left(S_{\infty}\right) \frac{\partial^{2} S}{\partial X^{2}}
$$

which can be solved. with the single boundary condition (17) [using the combination of variables $\mathrm{X} / \sqrt{4 D\left(S_{\infty}\right) t / \phi}$ (Carslaw and Jaeger, 1959)] to relate $S$ and its gradient at locations far downstream of regions of change:

$$
\frac{\partial S}{\partial x}=-\frac{\exp \left[\frac{-\left(x-q f^{\prime}\left(S_{\infty}\right) t / \phi\right)^{2}}{\sqrt{4 D\left(S_{\infty}\right) t / \phi}}\right]}{\sqrt{\pi D\left(S_{\infty}\right) t / \phi} \operatorname{erfc}\left[\frac{x-q f^{\prime}\left(S_{\infty}\right) t / \phi}{\sqrt{4 D\left(S_{\infty}\right) t / \phi}}\right]} \times\left[S-S_{\infty}\right] .
$$


In terms of the units of length, time, and pressure given in Eqs. (3)-(5), the non-dimensional form of Eq. (19a) is

$$
\frac{\partial S}{\partial \xi}=-\frac{\exp \left[\frac{-\left(\xi-f^{\prime}\left(S_{\infty}\right) \tau\right)^{2}}{\sqrt{4 D^{*}\left(S_{\infty}\right) \tau}}\right]}{\sqrt{\pi D^{*}\left(S_{\infty}\right) \tau} \operatorname{erfc}\left[\frac{\xi-f^{\prime}\left(S_{\infty}\right) \tau}{\sqrt{4 D^{*}\left(S_{\infty}\right) \tau}}\right]} \times\left[S-S_{\infty}\right]
$$

where the non-dimensional capillary dispersion coefficient is

$$
D^{*}(S) \equiv-\frac{M k_{r o} k_{r w}}{M K_{r o}+k_{r w}} \frac{d P_{c}^{*}}{d S}
$$

Equation (19) is a Robin-type condition that can be used at a downstream synthetic boundary. Manipulating Eqs. (6) and (7) gives the same result, but only after several more steps.

An appropriate location of the synthetic boundary can be obtained from Eq. (19a). The constant of proportionality in Eq. (19a) is insensitive to moving the boundary farther downstream if the argument of the complementary error function is larger than some number $H$, for which 3 or 4 is typically suitable. Smaller values of $H$ yield boundaries that are too close to the region of change for the asymptotic solution to be adequate there, and larger values of $H$ increase the extent of the domain beyond the size necessary for accurate and efficient computation. A justifiable location $x_{S}$ of the downstream synthetic boundary is thus

$$
x_{S}=q f^{\prime}\left(S_{\infty}\right) t / \phi+H \sqrt{4 D\left(S_{\infty}\right) t / \phi}
$$

or, in non-dimensional form,

$$
\xi_{S}=f^{\prime}\left(S_{\infty}\right) \tau+H \sqrt{4 D^{*}\left(S_{\infty}\right) \tau}
$$

The terms on the right side are the contributions of convection and dispersion, respectively, to the motion of the advancing region of change. In this sense, $H$ weighs the influence of dispersion on $\xi_{S}$. As $\tau$ increases, the downstream synthetic boundary must be moved farther downstream in order to retain the same level of accuracy at all $\tau$. The position of the synthetic boundary can be advanced in time according to Eq. (21), but in our solutions we set $\xi_{S}$ to its value at the maximum time desired.

Equation (19) is a Robin boundary condition that is appropriate to one-dimensional planar flow, such as accornpanies uniform injection of fluid into a porous medium through an ofen face (see Figure 2a). Another common flow pattern, injection through a well (Figure 2b), is cylindrically symmetric. A third, less common, flow scheme is injection through a point (Figure 2c), which is spherically symmetric. Table 2 lists the dimensional convection-dispersion equations that are valid far downstream of the region of greatest change in the cases of flows with planar, cylindrical, and spherical symmetries. 
Table 2

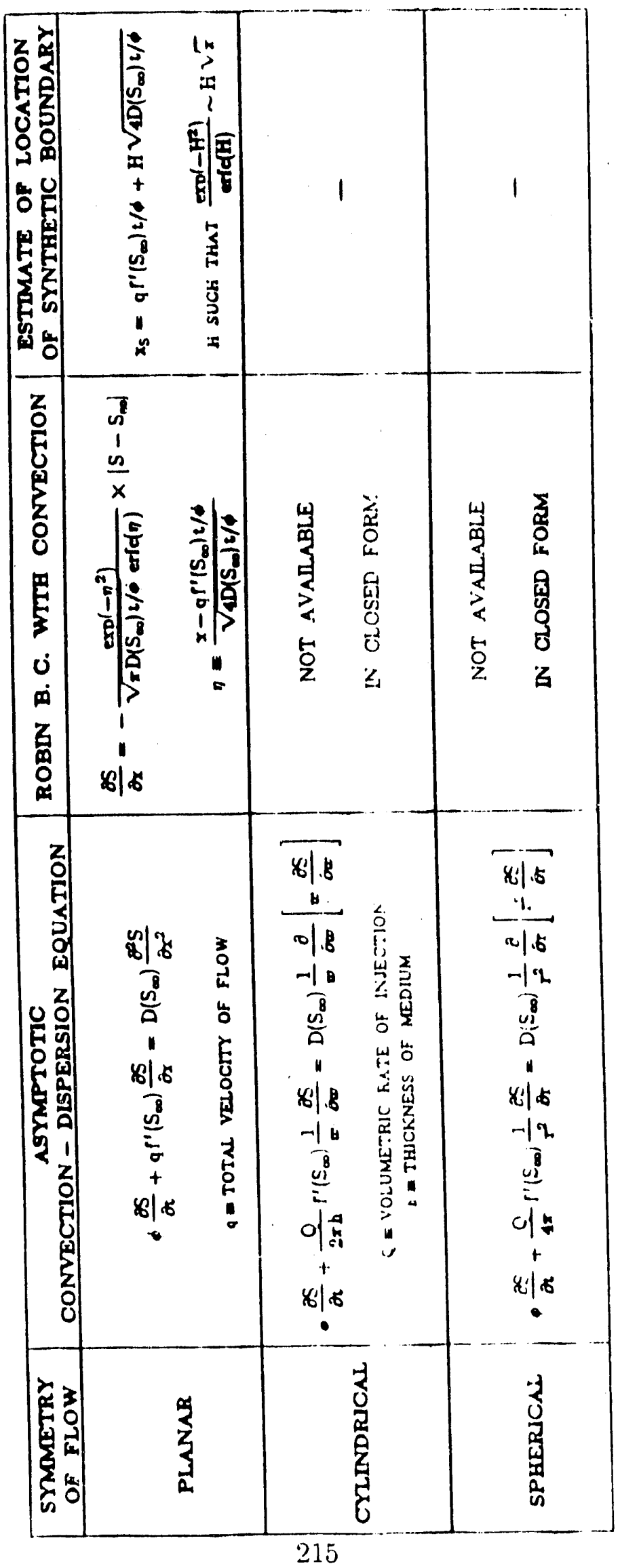


To our knowledge, only the planar case admits a closed-form solution, and so allows us to write both a Robin boundary condition and an estimate of the location of the synthetic boundary. Under certain conditions, however, the influence of convection relative to that of dispersion can be ignored far downstream.

In the case of planar flow, the convection-dispersion equation (13) can be written

$$
\phi \frac{\partial S}{\partial t}+\frac{g f^{\prime}\left(S_{\infty}\right)}{x_{S}} \frac{\partial S}{\partial \chi}=\frac{D\left(S_{\infty}\right)}{x_{S}^{2}} \frac{\partial^{2} S}{\partial \chi^{2}}
$$

where $\chi$ is clistance measured in units of $x_{S}$, i.e. the length of the domain of interest. Should $\partial S / \partial \chi$ and $\partial^{2} S / \partial \chi^{2}$ be of the same order of magnitude, the criterion of negligible convection would be

$$
\frac{q f^{\prime}\left(S_{\infty}\right) x_{S}}{D\left(S_{\infty}\right)} \ll 1
$$

The non-dimensional group is a Peclet number. This criterion, however, conflicts with the idea that moving the synthetic boundary farther downstream (increasing $x_{S}$ ) should improve both the validity of the boundary condition there and thus the quality of the solution in the region of interest. Increasing $x_{S}$ leads to a violation of (23), whatever the values of $q, f^{\prime}\left(S_{\infty}\right)$, and $D\left(S_{\infty}\right)$. Convection cannot be neglected far downstream if it is important in the region of interest. Fortunately, we can solve the convection-dispersion equation and thus derive a Robin boundary condition that includes convection in the case of planar flow.

In the cases of cylindrically and spherically symmetric flows, in which $\varpi$ and $r$ denote radial distance, the velocity of flow falls as $\varpi$ and $r^{2}$ respectively, and there are useful criteria of negligible convection. The convection-dispersion equation in the case of cylindrically symmetric flow (see Table 2) can be written

$$
\phi \frac{\partial S}{\partial t}+\frac{Q}{2 \pi h} \frac{f^{\prime}\left(S_{\infty}\right)}{\varpi_{S}^{2}} \frac{1}{\chi} \frac{\partial S}{\partial \chi}=\frac{D\left(S_{\infty}\right)}{\varpi_{S}^{2}} \frac{1}{\chi} \frac{\partial}{\partial \chi}\left[\chi \frac{\partial S}{\partial \chi}\right]
$$

where $\chi$ is distance measured in units of $\varpi_{S}$. Should the gradient and Laplacian of $S$ be of the same order of magnitude, the criterion of negligible convection would be

$$
1 \gg \frac{f^{\prime}\left(S_{\infty}\right)}{D\left(S_{\infty}\right)} \frac{Q}{2 \pi h}
$$

which is independent of the location of the synthetic boundary. Following the same procedure in the case of spherically symmetric flow gives the criterion

$$
r_{S} \gg \frac{f^{\prime}\left(S_{\infty}\right)}{D\left(S_{\infty}\right)} \frac{Q}{4 \pi}
$$

Here, moving the synthetic boundary farther downstream eventually allows a convectionfree description regardless of the values of $Q, f^{\prime}\left(S_{\infty}\right)$, and $D\left(S_{\infty}\right)$. Table 3 summarizes the 
Table 3. Criteria of Negligible Convection in Regions Far Downstream

\begin{tabular}{|c|c|}
\hline $\begin{array}{c}\text { SYMMETRY } \\
\text { OF FLOW }\end{array}$ & CRITERION \\
\hline PLANAR & $\frac{1}{x_{S}} \gg \frac{q f^{\prime}\left(S_{\infty}\right)}{D\left(S_{\infty}\right)}(\ddagger)$ \\
$q \equiv$ Total velocity of flow \\
CYLINDRICAL & $1 \gg \frac{f^{\prime}\left(S_{\infty}\right)}{D\left(S_{\infty}\right)} \frac{Q}{2 \pi h}$ \\
& $\begin{array}{c}Q \equiv \text { Volumetric rate of injection } \\
h \equiv \text { Thickness of medium } \\
\text { SPHERICAL }\end{array}$ \\
\hline
\end{tabular}

$(\ddagger)$ Violates the principle that enlarging the region of interest should improve the validity of a boundary condition derived from the situation far downstream. Use boundary condition of Table 2.

conditions under which the dispersion equation adequately describes transport in regions far downstream.

Where the influence of convection is slight in the cases of cylindrically and spherically symmetric flows, we can solve the dispersion equation to derive a Robin boundary condition. Tahle 4 gives (in dimensional form) the dispersion equation in the simple geometries and the associated Robin boundary conditions, which follow from solving the dispersion equation with combination of variables and applying the downstream condition (17).

One remaining issue is where to locate the synthetic boundary in cases where dispersion dominates convection far downstream. To get an idea of where to place the synthetic boundary at any particular time $t$, we superpose the distances that convection and dispersion carry the region of change. In the case of cylindrically symmetric flow, the argument in the Robin boundary condition that results from the dispersion equation (see Table 4), i.e.

$$
\nu=\frac{\varpi}{\sqrt{4 D\left(S_{\infty}\right) t / \phi}}
$$

becornes

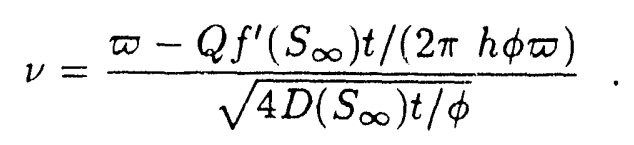


Table 4

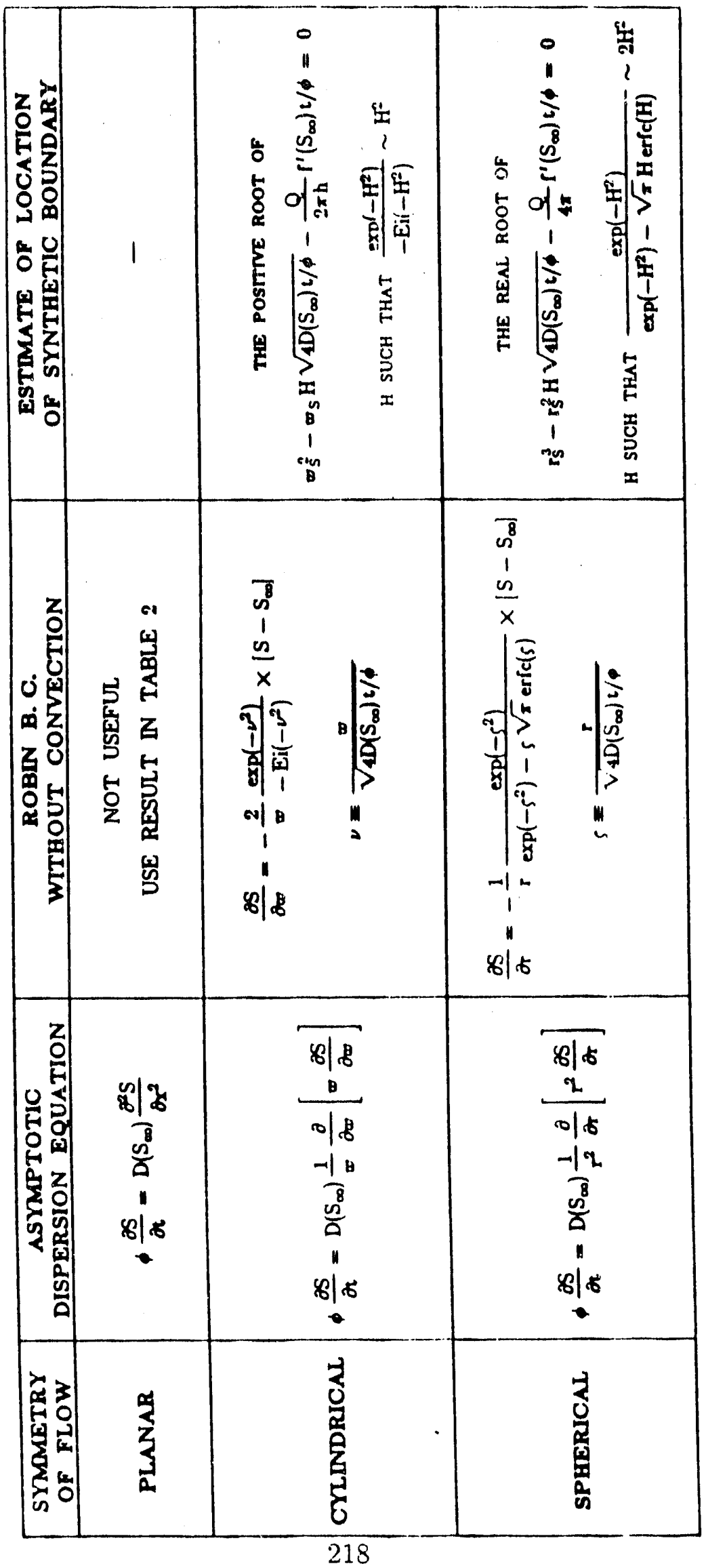


Following the reasoning that precedes Eq. (21), setting $\nu=H$ and solving the quadratic equation given in Table 4 , which has one positive root, yields $\varpi_{S}$. Similarly, superposing convection and dispersion in the case of spherically symmetric flow yields

$$
\zeta=\frac{r-Q f^{\prime}\left(S_{\infty}\right) t /\left(4 \pi \phi r^{2}\right)}{\sqrt{4 D\left(S_{\infty}\right) t / \phi}}
$$

Setting $\zeta=H$ and solving the cubic equation given in Table 4, which has one real root in cases of physically meaningful coefficients, gives $r_{S}$.

Although we report Robin boundary conditions in the cases of flows with planar, cylindrical, and spherical symmetries, our comparison of boundary conditions is restricted in this paper to planar flows. We believe that our conclusions appiy equally well to cylindrically and spherically symmetric flows.

Specification of an initial saturation profile completes the problem statement. One choice is a medium filled uniformly with oil, but resolving accurately the development of the saturation front near the injection face at small times would require special considerations that are not of interest here. Fortunately, Fokas and Yortsos (1982) and Yortsos and Fokas (1983) reported a closed-form solution to a one-dimensional, semi-infinite, immiscible displacement for particular but realistic relative permeability and capillary pressure functions. We use their solution not only to facilitate the comparison of our computer-aided solutions, but also to generate initial saturation profiles.

Equations (6) and (7) and their associated initial and boundary conditions we solved using Galerkin's method with piecewise quadratic finite element basis functions and the time integration scheme of Gresho, Lee, and Sani (1980). Most details of the solution technique are not crucial to this paper, but Strang and Fix (1973) explain well the mathematical basis of Galerkin's finite element method, and Zienkiewicz (1977), among others, describes its use.

One detail of the solution method that is important is that the Dirichlet-type boundary condition on $P_{a}^{*}$ at the synthetic boundary is enforced by replacing the pressure equation (Eq. 7) there by Eq. (12). The discarded information that the pressure equation represents (viz. incompressibility) can be used to write a 'pseudo-boundary condition' on $\mathrm{S}$ at the synthetic boundary:

$$
\nabla^{*} S=-\frac{1+\left(M k_{r o}+k_{r u^{\prime}}\right) \nabla^{*} P_{a}^{*}}{\frac{1}{2}\left(M k_{r o}-k_{r u}\right) d P_{c}^{*} / d S} .
$$

This is not a proper boundary condition because it provides no new constraint to single out a particular solution from a family of general solutions. The condition of incompressibility is used already at the injection face in the form of Eq. (11). In spite of this objection, however, we report below that enforcing this pseudo-boundary condition at the synthetic boundary sometimes works. Setting $\nabla^{*} S$ with Eq. (30) is in essence solving the prcblem minus one boundary condition. 


\section{Results and Discussion}

The first set of results compares the solutions with Dirichlet, Neumann, and Robin boundary conditions (see Table 1 ) in 12 cases that pair 4 different capillary numbers $C a$ and 3 different positions of the synthetic boundary as set with the parameter $H$ through Eq. (21b). The capillasy number measures the relative strengths of convection and dispersion; lower capillary numbers imply weaker convection or stronger dispersion and more upstream signalling (elliptic character). Thus, we expect the influence of the condition at the synthetic boundary to extend farther upstream the smaller the value of $\mathrm{Ca}$. We do not report the solutions of average pressure, because they do not alter the conclusions drawn from the saturation profiles alone. Also, at a given capillary number, the size of each finite element, and thus the discretization error of the solution, are the same among cases of different domain lengths.

Figur 3 shows the computed saturation profiles and closed-form solutions of all cases of $C a$ and $H$. Profiles in cases of higher capillary numbers have steeper gradients. In Figure 3(d), a solution satisfying a Dirichlet condition was beyond reach: the adaptivelysized time step became too small for the solution at $t=12 \mathrm{hr}$ to be attained with a reasonable amount of computational effort. This is symptomatic of solutions that vary irregularly from one time level to the next. In all cases, moving the synthetic boundary farther downstream gave more accurate solutions because whatever asymptotic behavior sets the boundary condition becomes more realistic. Larger domains entail greater costs, however, because more basis functions are needed to obtain a solution of given accuracy on an enlarged domain. The Dirichlet condition is unrealistic on the smaller domains because the region of change reaches the boundary, and the Robin condition appears to be superior to the Neumann condition.

Figures 4 and 5, which are plots of relative error along the domain, show that the Robin condition is most accurate. Visible are node-to-node oscillations, or 'wiggles,' in the convection-dominated case of $C a=10^{-6}$. Wiggles are caused by an inadequate discretization (Gresho and Lee, 19S1), which is more likely should an inappropriate boundary condition, such as the Dirichlet or even the Neumann, demand the formation of a boundary layer, or end layer, upstream of the outflow boundary. The minor, longer-range oscillations apparent in Figure 5(a,b,e,f,i,j) result from trying to resolve abrupt changes in saturation with the levels of discretization used. An adaptive discretization scheme, such as that of Benner et al. (1987) or, more recently, Yeckel and Scriven (1990), would concentrate nodes to resolve better these sharp changes. Clearly, the Robin boundary condition provides the most accurate solution at a given cost.

The second set of results compares the solutions with the Robin and 'pseudo' boundary conditions (Eqs. 19 and 30) in the two extreme cases of capillary number, i.e. $C a=10^{-6}$ and $10^{-9}$, and a single position of the synthetic boundary. Figure 6 shows the saturation prefiles. Both boundary conditions provide good solutions in the convection-dominated caise, even allowing the region of change to pass through the boundary with little error - 

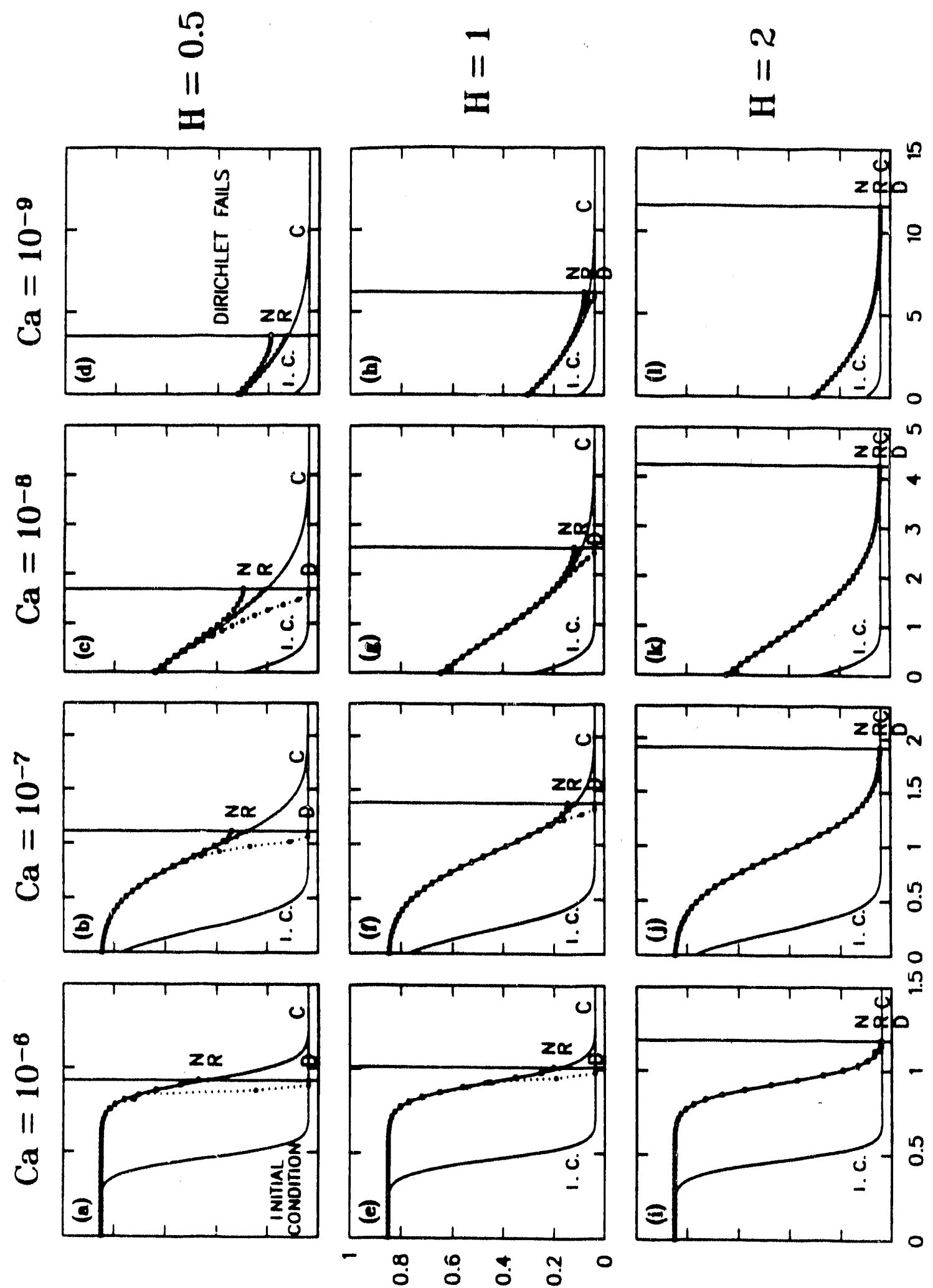

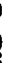

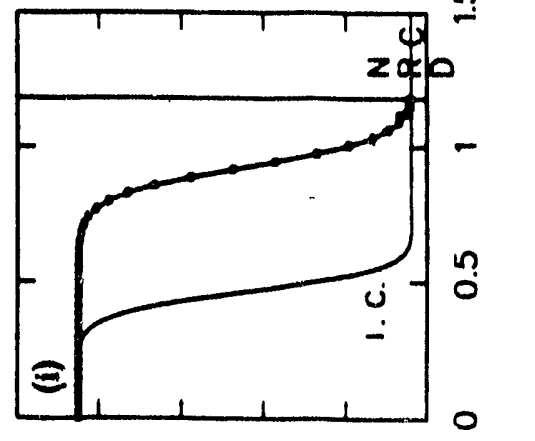

NOILHYC $L \forall S$ YGHUM

Figure 3. Computed saturation profiles at $t=12 \mathrm{hr}$ for the 12 cases with Dirichlet. (D), Neumann (N), and Robin (R) boundary conditions and the closed-form solution $(\mathrm{C})$. 

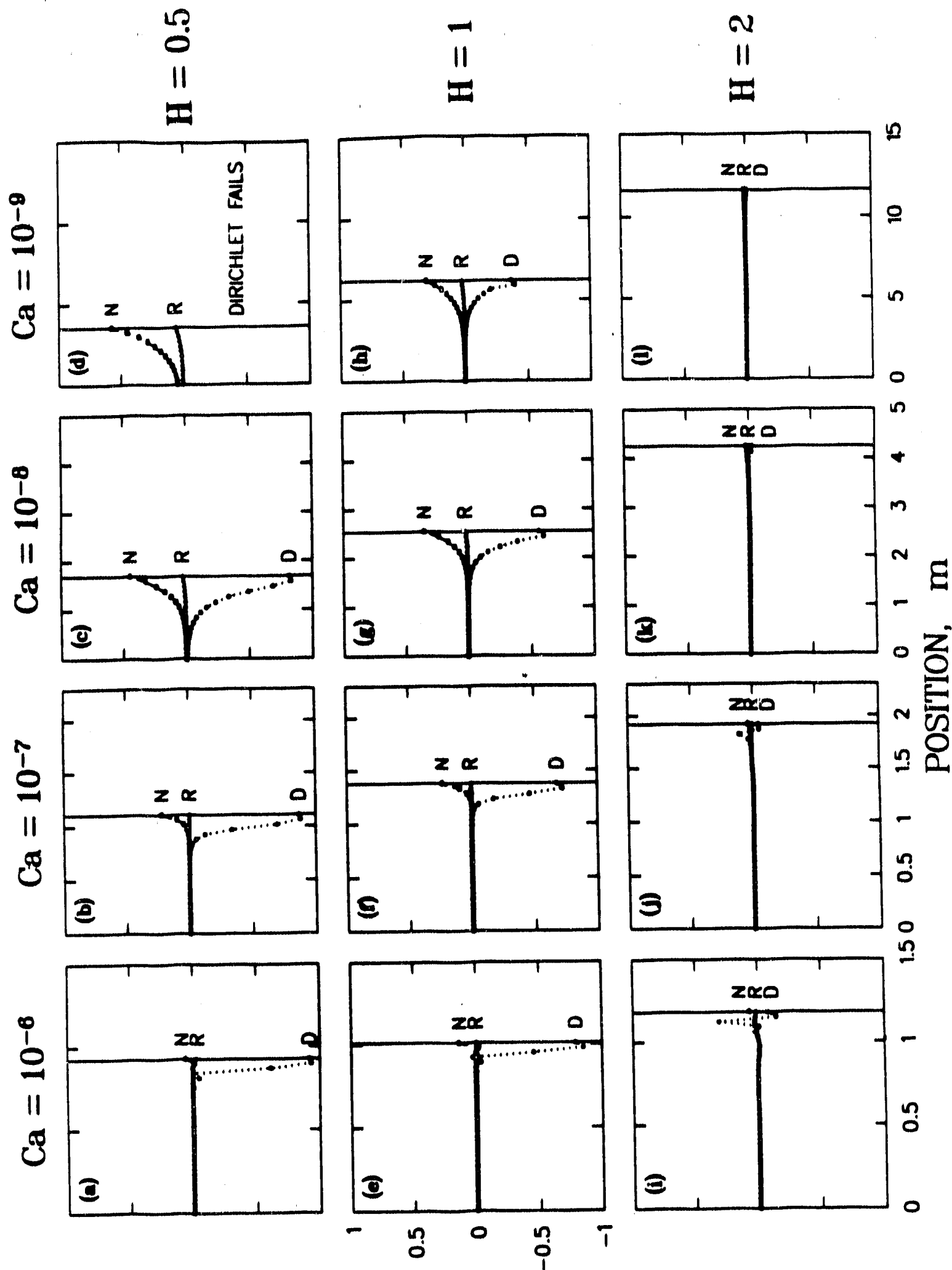

NOILVY

Figure 4. Profiles of the relative error $\left(S-S_{\text {closed form }}\right) / S_{\text {closed form }}$ for the cases of Figure 3. 

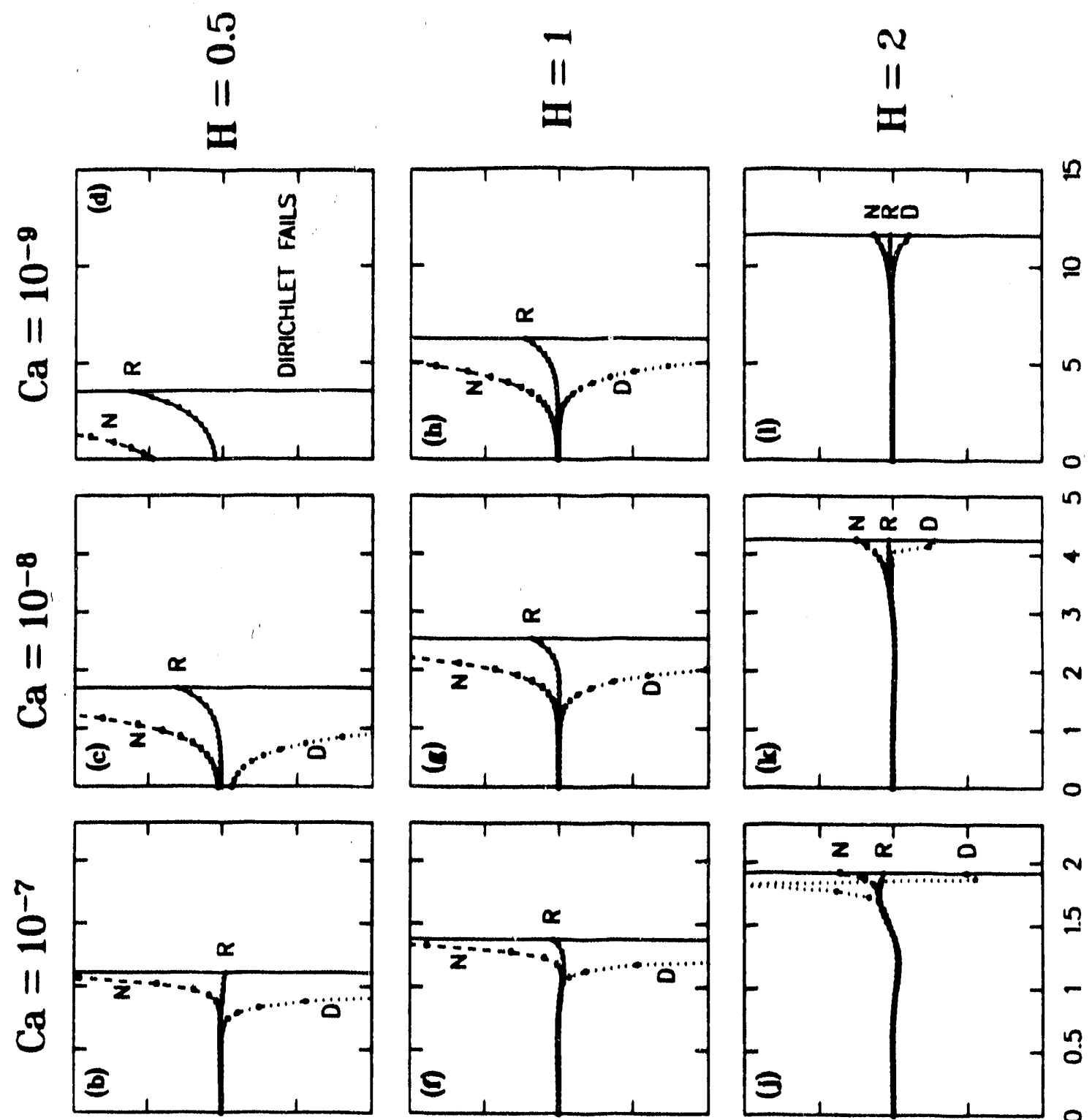

0

n
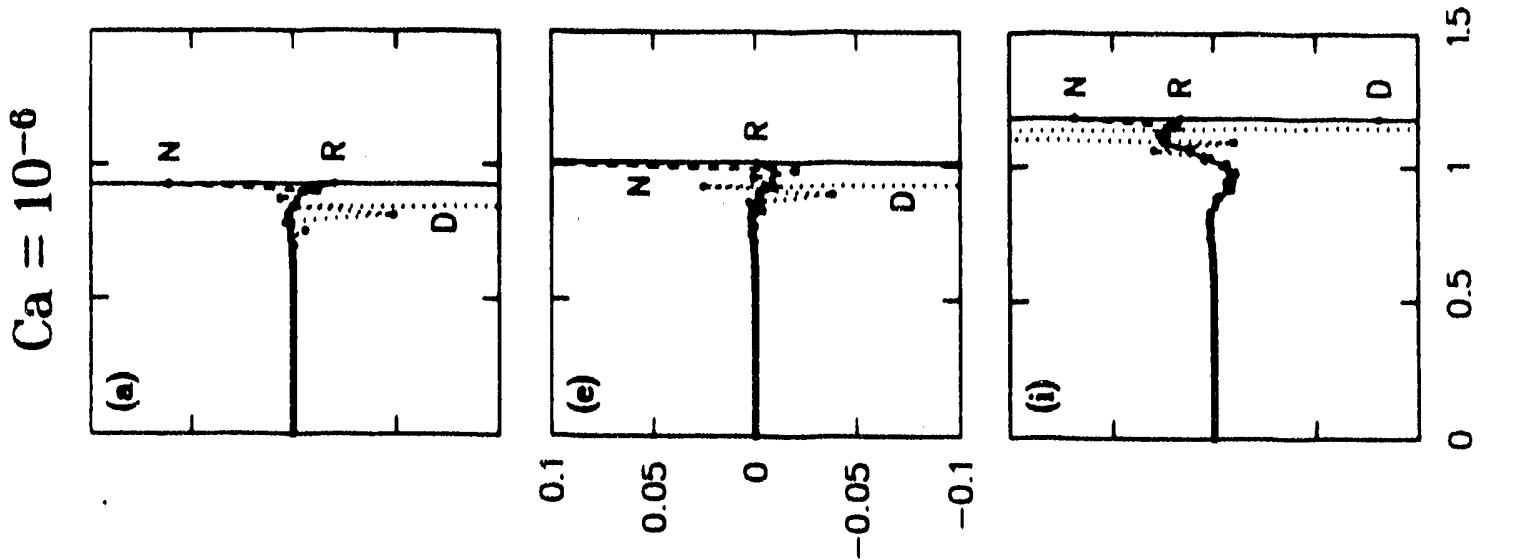

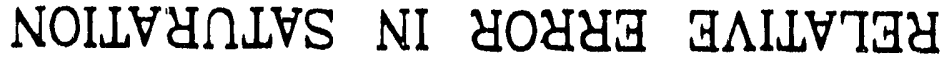

Figure 5. Profiles of the relative error in saturation on a magnified ordinate. 

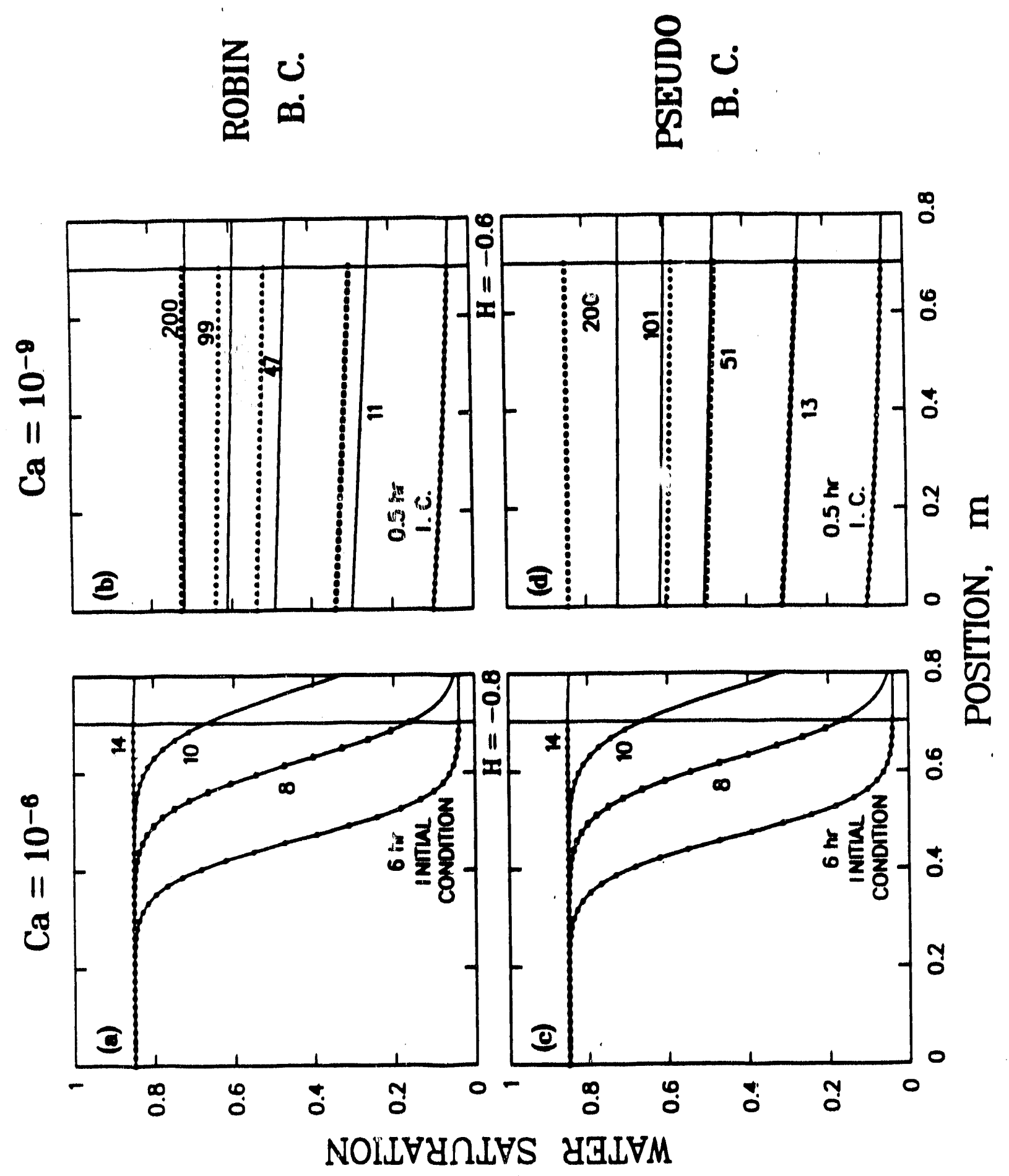

Figure 6. Computed saturation profiles for the two extreme capillary numbers with the Robin and 'pseudo' boundary conditions. Curves are the closed-form solutions. 
the mark of the ideal synthetic boundary condition. The pseudo condition gives slightly more accurate solutions than the Robin condition (see curves at $t=10 \mathrm{hr}$ ). In the dispersion-dominated case, however, the solution with the pseudo condition drifts off course badly; the Robin condition is inappropriate too, but this is to be expected in the light of the value of $H$ that corresponds to this position of the boundary $(H=-0.6)$.

Figure 7, which depicts the history of the relative error at the boundary, is a better indicator. In the convection-dominated case, the pseudo condition performs very well, but the kink just after $t=9 \mathrm{hr}$ contrasts with the smooth behavior of the Robin condition. Trouble with the pseudo condition is clear in Figure 7(b), the dispersion-dominated case. Just past $t=100 \mathrm{hr}$, the quality of its solution decays abruptly.

The behavior of the pseudo boundary condition follows from its not being, in fact, a proper boundary condition at all; that is, it leaves the equations with an infinity of solutions. This condition works to a degree because discretization and truncation errors are large enough to mask its redundancy. A symptom of the basic singularity, however, is the poor rate of convergence of the Newton iteration process used to solve the non-linear algebraic equations produced by the Galerkin finite element method. In work not shown here, we successively refined the discretization near the synthetic boundary, reducing the discretization error there, and the convergence of the Newton iterations and the quality of the solutions decayed - behavior opposite that of a well-posed problem.
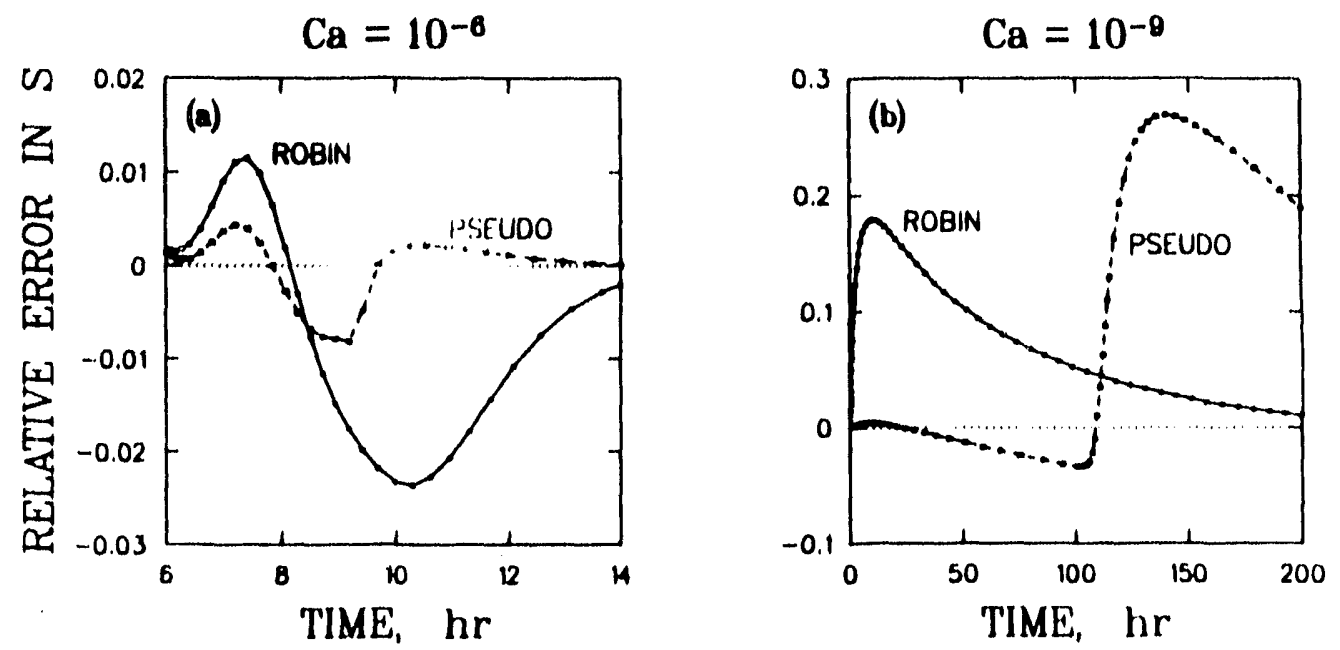

Figure 7 . History of relative error $\left(S-S_{\text {closed form }}\right) / S_{\text {closed form }}$ at the synthetic boundary with the Robin and 'pseudo' boundary conditions.

The pseudo boundary condition performs better for convection-dominated flows because its influence does not extend far upstream. The character of the solution upstream of the boundary asserts itself and keeps the entire solution near the desired branch. In contrast, the entire profiles of the dispersion-dominated case are largely controlled by the 
downstream boundary condition, and when the solution strays from the proper course, there is little influence from upstream to restore it.

Although the pseudo boundary condition can give smaller errors than the Robin boundary condition, it is more expensive computationally because of the poor rate of convergence of the Newton iterations. Even if cost re not a concern, the unpredictability of this boundary condition disqualifies it. Thus, our conclusion is that the Robin boundary condition is the best of those considered here for getting the most accurate solution at fixed cost. We infer that the Robin condition would also provide at the lowest cost a solution of desired accuracy.

\section{Conclusions}

An accurate mathematical description of any system depends on realistic boundary conditions, which describe the interaction of that system and its surroundings. The selection of boundary conditions in continuous-flow situations is often difficult because the boundaries of finite computational domains do not coincide with phase boundaries, and are thus synthetic.

Using the example of two-phase flow in a one-dimensional, semi-infinite porous medium, we have compared solutions that each satisfies one of four types of synthetic boundary conditions: Dirichlet (first kind), Neumann (second kind), Robin (third kind), and 'pseudo,' which is in essence none. The Robin boundary condition gives the most accurate solution at fixed computational cost, and, we believe, the lowest cost at fixed accuracy. Dirichlet and Neumann conditions require larger domains and are more apt to produce wiggles in solutions with little upstream signalling by creating non-physical boundary layers that may not be resolvable by the discretization adopted. The pseudo boundary condition provides satisfying results in systems with little upstream signalling, allowing fronts to pass through the synthetic boundary, but its redundant nature inhibits the convergence of the Newton iterations, which increases computational costs. The lack of reliability of the pseudo boundary condition makes it a poor choice in any event.

Although we examined the particular case of two-phase flow in porous media, our findings surely relate to a variety of situations in mass, heat, and momentum transport. We believe that Robin-type boundary conditions deserve more widespread use. 


\section{References}

Basaran, O.A., Electrohydrodynamics of Drops and Bubbles. Ph.D. Thesis, University of Minnesota, Minneapolis, MN (1984).

Benner, R.E. Jr., Davis, H.T. and Scriven, L.E., 1987, SIAM J. Sci. Stat. Comput. 8, 529-549 (1987).

Bischoff, K.B., Chem. Engng. Sci. 12, 69-70 (1960).

Bischoff, K.B., Chem. Engng. Sci. 16, 131-133 (1961).

Bixler, N.E., Stability of a Coating Flow. Ph.D. Thesis, University of Minnesota, Minneapolis, MN (1982).

Carslaw, H.S. and Jaeger, J.C., Conduction of Heat in Solids, 2nd ed., Clarendon Press, Oxford (1959) pp. 89-90.

Danckwerts, P.V., Chem. Engng. Sci. 2, 1-13 (1953).

de Marsily, G., Quantitative Hydrogeology - Groundwater Hydrology for Engineers. 4cadenic Press, Orlando, FL (1986) pp. 135-139.

Fokas, A.S. and Yortsos, Y.C., SIAM J. Appl. Math. 42, 318-332 (1982).

Fourier, J., Théorie analytique de la chaleur, Paris (1822). Translated from the French by A. Freeman, The Analytical Theory of Heat, University Press, Cambridge (1878).

Gresho, P.M., Lee, R.L. and Sani, R.L., in Recent Advances in Numerical Methods in Fluids (Edited by C. Taylor and K. Morgan), v. 1, Pineridge Press, Swansea, UK (1980) pp. 27-79.

Gresho, P.M. and Lee, R.L., Computers and Fluids 9, 223-253 (1981).

Iordanidis, K.I., J. Inst. Maths. Applics. 12, 91-96 (1973).

Novy, R.A., Davis, H.T. and Scriven, L.E., Chem. Engng. Sci. 45, 1515-1524 (1990).

Peaceman, D.W., Fundamentals of Numerical Reservoir Simulation. Elsevier, NY (1977).

Pearson, J.R.A., Chem. Engng. Sci. 10, 281-284 (1959).

Robin, G., Annales Scientifiques de l'Ecole Normale Supérieure 3, supplement, 58 pages (1886).

Strang, G. and Fix, G.J., An Analysis of the Finite Element Method. Prentice-Hall, Englewond Cliffs, NJ (1973).

Wehner, J.F. and Wilhelm, R.H., Chem. Engng. Sci. 6, 89-93 (1956).

Yeckel, A. and Scriven, L.E., private communication.

Yortsos, Y.C. and Fokas, A.S., Soc. Pet. Eng. J. 23, 115-123 (1983).

Zienkiewicz, O.C., The Finite Element Method, 3rd ed. McGraw-Hill, New York (1977). 


\section{DIRECT IMAGING OF SURFACTANT MICELLES, VESICLES, DISCS AND RIPPLE PHASE STRUCTURES BY CRYO-TRANSMISSION ELECTRON MICROSCOPY}

\section{Synopsis}

Surfactant microstructures in dilute aqueous solutions and dispersions - globular, swollen and cylindrical or wormlike micelles, discoid and ripple phase structures, and uniand multi-lamellar vesicles - can be seen at high resolution by cryo-transmission electron microscopy (cryo-TEM) of thin vitrified sample films. Sample films are prepared within a mber where temperature and chemical activities of the surrounding vapor are controlled, thereby preventing evaporation and temperature changes that could alter the microstructure in the labile systems. The thin liquid films are quenched by rapidly plunging them into liquid ethane. The resulting vitrified samples are mounted into a cold-stage and transferred into a TEM for direct observation. Monophasic solutions of cetyltrimethylammonium bromide (CTAB) show globular micelles that swell with added toluene or styrene to form swollen micelles. Wormlike micelles form in CTAB-NaBr solutions. Dilute mixtures of dipalmitoylphosphatidylcholine (DPPC) and diheptanoylphosphatidylcholine (DHPC) show discoid structures above the main transition temperature of DPPC and $\Lambda$ and $\Lambda / 2$ ripple structures of the $\mathrm{P}_{\beta^{\prime}}$ phase at temperatures below the main transition temperatlire. A new model is proposed for the $\Lambda$ structure and the ripple structures are shown to exist as single bilayers. Biphasic dispersions of sodium 4-(1'-heptylnonyl)benzenesulfonate (SHBS) show spheroidal and tubular vesicles, and complex encapsulated vesicles and coiled tubules. Vesicle-like microstructures of SHBS persist at $90^{\circ} \mathrm{C}$. At the relatively low SHBS concentrations studied there is no evidence of the constant spacing characteristic of the lamellar phase at higher concentrations, suggesting that the structures observed may result from unbinding fluctuations that disrupt lamellar phases.

\section{Introduction}

Surfactants in dilute aqueous solutions and in dispersions assemble into a variety of microstructures: micelles, microemulsions, vesicles and liquid crystalline structures. These microstructures are characterized by sheet-like surfactant regions (a few nanometers in thickness) across which composition changes rapidly. Such systems are of biological and technological importance, e.g., in detergency, drug delivery, catalysis, enhanced oil recovery, ultrafiltration, and nanoscale particle production. The macroscopic properties rheology, surface tension and conductivity - of these systems clepend markedly on the way the surfactant is organized in three dimensions. The microstructures are often difficult to elucidate because of size, composition, etc. Conventional techniques like radiation scattering, although useful, provide indirect evidence of microstructures and to interpret the results requires a model. 


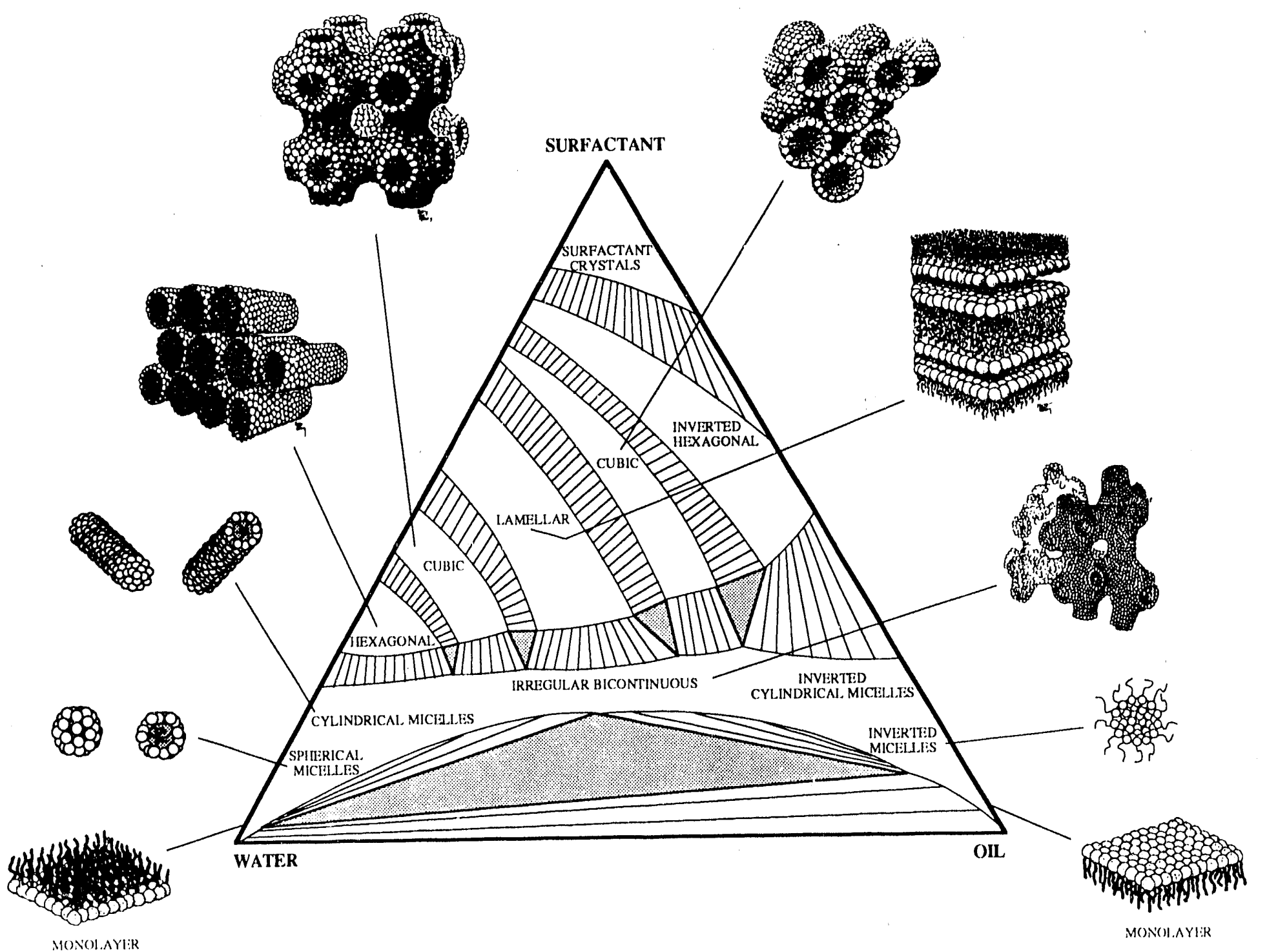

Figure 1. Schematic oil-water-surfactant phase diagram with artistic models of microstructures depicted. (Redrawn from Davis et al.1987 with two added models). 
Figure 1 is an idealized ternary phase diagram in which are illustrated severa the equilibrium fluid microstructures that have been identified in solutions of surfact with water and/or oil. Solutions of globular, or "spherical," and tubular, or "cylindric micelles and of their inverted analogs are well documented, as are lyotropic liquid crysta. phases with lamellar, hexagonal and cubic symmetries. However, the local shapes of sheet-like zones the aggregated surfactant forms and the role and importance of molec fluctuations and bending and stretching motions of the sheet-like zones in micellar solut and liquid crystals are unsettled issues that are the subjects of much current research Davis et al.1987). Here we describe how microstructures whose morphological scales : the range from the local curvature of the surfactant sheet-like zones in equilibrium mice microemulsions and non-equilibrium vesicles to the supramolecular organization of 1 assemblies in liquid and cubic crystalline phases, as well as the structural transitions occur between different microstructured states, can be imaged directly by means of rect developed electron microscopy techniques.

Transmission electron microscopy is an attractive way to study microstructure: cause it directly produces images at high resolution. However, surfactant microstruck cannot be easily imaged with TEM because: 1) the high vapor pressures of the surfac. solutions make them incompatible with the low pressures $\left(<10^{-6}\right.$ torr $)$ in TEM; 2 ). trons induce chemical reactions in surfactant solutions that can change microstructurel 3 ) often there is insufficient contrast between the microstructures themselves and theirroundings. Various sample preparation techniques, e.g., chemical fixation, staining, drying have been used to overcome these problems, but each technique has introd new problems, e.g., microstructural rearrangement due to composition changes, leag of membrane components, drying stresses, etc. Sample preparation affects the structuf the sample, often radically enough to obscure the original microstructure. A new sae preparation technique that solidifies the sample so quickly as to vitrify, rather than crylize, liquid water has overcome many of these limitations, and opened a window on $d$, high resolution images of virtually the original microstructure of surfactant systems.

Two important advances have been made. The first is the ability to cool belore glass transition temperature of the sample at atmospheric pressure so quickly that cris do not form. This is achieved by plunging thin films of water or other liquid or semi-id materials into cryogens like liquid ethane or propane at their freezing point (Adrit al.1984). The cooling rate is so fast $\left(10^{4}\right.$ to $10^{5} \mathrm{~K} / \mathrm{sec}$; Costello et al. 1982) thatter vitrifies, i.e., water molecules do not rearrange into a crystalline form as they id at slower cooling rates. Vitrification of water is an important indicator of microstural integrity. If water molecules do not rearrange appreciably during cooling, then $\mathrm{r}$ molecules like surfactants and supramolecular aggregates like micelles are not likely torrange appreciably. Vitrification avoids the artifacts of crystallization, diffaction cont, and excessive radiation damage seen in micrographs of CTAB micelles reported rey by Ness and Moth (1988), and allows a better view of the sizes, shapes and connecy of the microstructures. This fast-freezing, cryo-TEM technique was used by Dubt et al.(1984) to study droplet packing in water-diluted samples of so-called glass-foig 
microemulsions, and by Talmon (1986) to study microstructures in surfactant dispersions. The latter found, however, that preparing thin films of sample in the open air subjected them unavoidably to evaporation and temperature change, which induced artifacts - microstructure not present in the original liquid.

The second advance is the means for preparing the thin films in an environmental chamber where they can be kept at the controlled temperature and chemical activities of the surrounding vapor until a few milliseconds before they are vitrified (Bellare et al.1986a, 1988; Bellare 1988). In this way artifacts caused by temperature or concentration changes can be prevented, as demonstrated in studies of vesicles, spherical and cylindrical micelles, and hexagonal liquid and cubic crystals (Bellare et al.1986a\&b, Burns and Talmon 1987, Vinson 1988, Vinson et al.1989, Siegel et al.1989).

The results of the cryo-TEM study of surfactant microstructures in aqueous dispersions and solutions we report here illustrate the current capability of cryo-TEM. Sections 2 and 3 describe the sample preparation and systems studied. Section 4 presents a series of micrographs of vesicles and globular, cylindrical, and swollen micelles showing some structures that have never been seen previously.

\section{Sample preparation}

Samples were prepared in the controlled environment vitrification system, or CEVS (Figures 2, 3), which is described in detail elsewhere (Bellare et al.1988). In the CEVS, temperature was controlled to within $\pm 0.1^{\circ} \mathrm{C}$ by controlling the current to a 600 Watt halogen-quartz lamp. An insulated reservoir mounted on the outside rear wall of the CEVS could be charged with refrigerant if a heat sink was required. The chemical activities of the vapor were controlled by saturating the chamber with the volatile components present in the sample. This was accomplished either with porous sponges extending upward from liquid reservoirs or with a sparger system. The air inside the chamber was recirculated across the sponges or through the sparger to reduce temperature and composition gradients in the vapor.

In the studies reported here, thin films of sample were formed by placing a drop of the liquid on a holey polymer support film which had been coated with carbon and mounted on the surface of a standard TEM grid (Vinson 1987). The drop was blotted with filter paper so that thin $(\leq 1 \mu \mathrm{m})$ films of the sample remained, and these spanned the holes in the support film. The entire assembly was then vitrified by rapidly plunging it through a synchronous shutter at the bottom of the environmental chamber and into liquid ethane situated immediately beneath.

The vitrified samples were examined in a JEOL 100CX analytical electron microscope, operated at $100 \mathrm{kV}$ in the conventional TEM mode. To do this they were transferred from liquid ethane to liquid nitrogen, in which they were then transported, and mounted onto a 


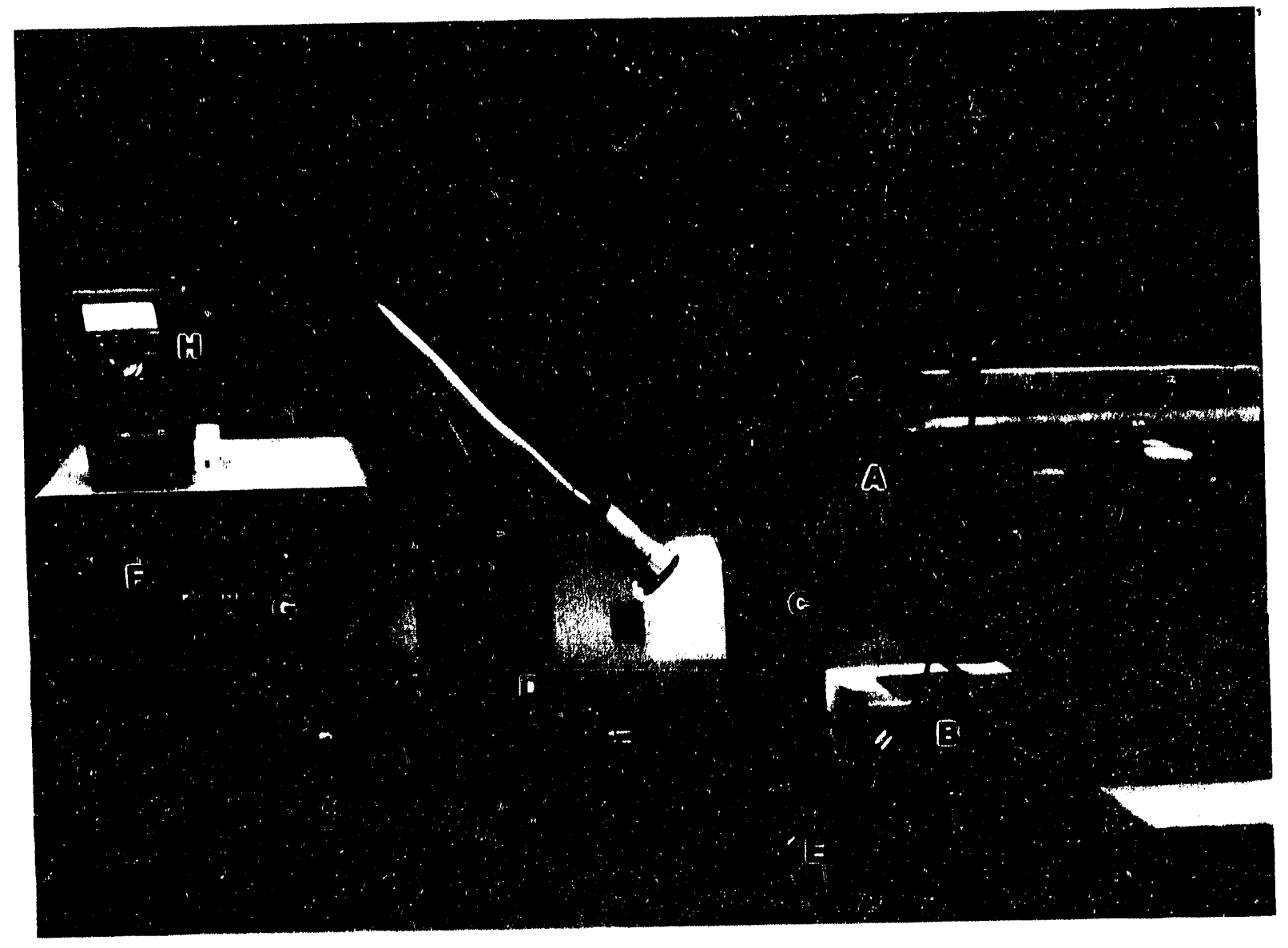

Figure 2 . The controlled environment vitrification system (CEVS) used to prepare samples for cryo-transmission electron microscopy. Parts of the system are labelled as follows: (A) environmental chamber; (B) cryogen reservoir; (C) stereomicroscope; (D) illuminator; (E) double-cable release; $(\mathrm{F})$ digital thermometer; $(\mathrm{G})$ temperature controller; and $(\mathrm{H})$ meter to display relative humidity measured by a sensor located in the envirommental chamber. 


\section{CRYO-TRANSMISSION ELECTRON MICROSCOPY WITH \\ CONTROLLED ENVIRONMENT VITRIFICATION}

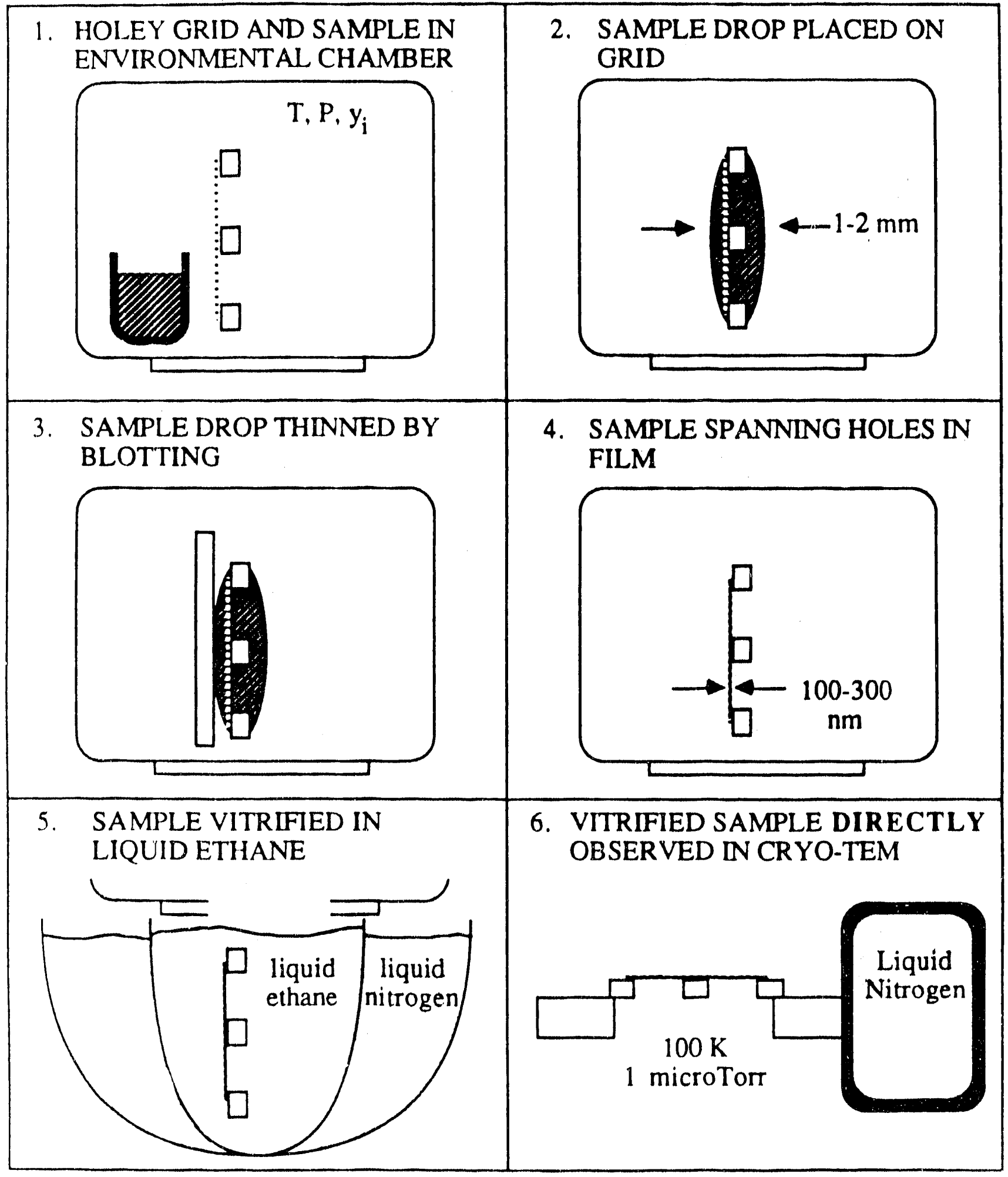

Figure 3. Schematic of sample preparation procedure with the CEVS. 
modified JEOT, EM-Specimen Cooling Holder using a cold-stage transfer module (Perlov et al.1983). The sample holder temperature was maintained below $-165^{\circ} \mathrm{C}$ during imaging. Images were recorded on Kodak SO-163 film and developed in Kodak D-19 developer (undiluted) for $12 \mathrm{~min}$. Images were recorded at $3.9 \mu \mathrm{m}$ underfocus of the microscope objective lens to provide phase contrast, which is mainly responsible for gradients of optical density in the images. Images were recorded at 20,000-33,000x $( \pm 5 \%)$ and further enlarged threefold to tenfold when printed. Prints were made on Agfa Brovira Speed paper of contrast grades 3 to 5 . Unsharp masking, a form of background subtraction to compensate for the relatively narrow dynamic range of printing papers (Guettler et al.1987), was used to print negatives that had a large range of optical densities.

\section{Systems studied}

Cetyltrimethylammonium bromide, or CTAB, was obtained from Fluka (Ronkonkoma, NY) and used without further purification. Dipalmitoylphosphatidylcholine (DPPC) and diheptanoylphosphatidylcholine (DHPC) were obtained from Avanti Polar Lipids, Inc. (Birmingham, AL). Dry mixtures of the lipids were prepared by removing the organic solvent (chloroform) under a stream of nitrogen gas followed by evacuation to $50 \mu \mathrm{m} \mathrm{Hg}$ for 12 hours. Sodium 4-(1'-heptylnonyl)benzenesulfonate, or SHBS, was synthesized at the University of Texas, Austin by Dr. W. H. Wade and purified as described by Franses (1979). The surfactants were dissolved or dispersed in distilled water further purified by ion-exchange. Solutions and dispersions were made by pouring water into weighed quantities of surfactants, and repeatedly inverting the container by hand at $0.5 \mathrm{~Hz}$ for about 10 minutes. Swollen micelles were prepared by adding the swelling agent (toluene or styrene) to the surfactant solution and stirring for $10 \mathrm{~h}$ on a magnetic stirrer rotating, at about $2 \mathrm{~Hz}$.

Cryo-TEM samples of monophasic CTAB solutions at temperatures ranging from 24 to $26^{\circ} \mathrm{C}$ were prepared at concentrations below $(0.01 \mathrm{wt} \%)$, at and above $(1.0 \mathrm{wt} \%)$ the critical micelle concentration range, or cmc, which is about 0.036 wt $\%$ at $25^{\circ} \mathrm{C}$ (Ekwall et al.1971). Micelles of CTAB (1 wt $(\%)$ were swollen with toluene $(0.4 \mathrm{wt} \%)$ and cryo-TEM samples were prepared from the solution at $27^{\circ} \mathrm{C}$. Swollen CTAB (1 wt \%) micelles were also formed by adding styrene $(0.6 \mathrm{wt} \%)$ that contained $0.1 \mathrm{wt} \%$ azoisobutyronitrile (AIBN), a polymerization initiator. Cryo-TEM samples were prepared at $27^{\circ} \mathrm{C}$ from solutions that had not been exposed to ultraviolet irradiation and from solutions that had been exposed to $254 \mathrm{~nm}$ ultraviolet irradiation for 1 hour. Cylindrical micelles formed in CTAB $(0.37$ wt $\%$ )-sodium bromide $(3.0 \mathrm{wt} \%)$ solutions at $35^{\circ} \mathrm{C}$ were examined. Aqueous mixtures of the lipids were prepared at $20 \mathrm{mM} \mathrm{DPPC}(1.47 \mathrm{wt} \%)$ and $5 \mathrm{mM} \mathrm{DHPC}(0.24 \mathrm{wt}(\%)$ and examined at $45^{\circ} \mathrm{C}$ and $28^{\circ} \mathrm{C}$. Aqueous dispersions of SHBS were examined at $2 \mathrm{wt} \%$ and $25^{\circ} \mathrm{C}$, where the phase diagram (Franses et al.1980) shows the system to be biphasic, and at $0.1 \mathrm{wt} \%$ and $90^{\circ} \mathrm{C}$, where the plase diagram shows the system to be monophasic. 


\section{Results and discussion}

Contrast in unstained surfactant samples comes mainly from gradients in the phase of electron waves traversing samples that have spatially varying electron-refractive indices. The phase gradients arise because electrons are scattered by different amounts in different parts of the sample. The objective lens of the microscope can be defocused to add a contribution to the phase shift of the scattered electrons. This allows the microscopist control over interference of scattered electrons with unscattered electrons, 'which is the basis of phase contrast. Because the phase shifts of the electrons also depend on the structure from which they are scattered, some spatial frequencies are enhanced by the interference while others are attenuated. A nominal defocus of $4 \mu \mathrm{m}$ accentuates spatial frequencies corresponding to about $5 \mathrm{~nm}$, but attenuates spatial frequencies corresponding to smaller real space distances. Therefore, this defocus is appropriate for imaging supramolecular structures like micelles and bilayers that are typically $4-5 \mathrm{~nm}$ in diameter or thickness, as computer simulations of phase contrast images indicate (Bellare 1988). In general, the optimal defocus is determined by the object size or periodicity being imaged. Objects containing a wide range of spacings may require several images to be taken at different amounts of defocus.

Amplitude, or mass thickness, contrast is a second, but often negligible contribution to the contrast of surfactant microstructures. Amplitude contrast arises when scattered electrons are prevented from contributing to the image by the objective aperture. Regions of the image from which more electrons are removed appear dark. Total electron scattering depends on the atomic numbers of the elements encountered and the sample thickness traversed. Amplitude contrast from unstained surfactant microstructures is usually negligible because the regions of surfactant typically sampled by the electrons are small (a few nanometers), and the majority of the elements $(\mathrm{C}, \mathrm{H}, \mathrm{O})$ present in the microstructure do not differ significantly in atomic number from the aqueous or oleic matrix.

Figure $4 \mathrm{a}$ is a micrograph of monophasic CTAB $(0.01 \mathrm{wt} \%)$ solution below the cmc. As expected, inspection of Figure 4a shows no discernible microstructure within the vitrified film. The surfactant is in molecular solution or submicellar aggregates which cannot be resolved. The structured appearance of the surrounding polymer film (seen more readily in Figures 6,10 and 11) is the result of electron-bcam induced free radical reactions at the polymer/vitreous ice interface (Talmon 1987). The dark round objects (marked $C^{C}$ in Figure 4a) are crystalline ice formed from moisture that has condensed onto the surface of the sample. The crystalline ice appears dark because the crystal planes diffract electrons at angles large enough that the electrons are removed by the objective aperture and do not contribute to the image. The ice crystal is surrounded by a bright fringe called a Fresnel fringe. This fringe stems from interference between unscattered electrons and electrons scattered from the discontinuity that exists at the periphery of the ice crystal where it meets the vitreous ice, i.e., at the vacuum-ice crystal-vitreous ice line of contact. The appearance and intensity of the fringe are determined by the defocus condition and the local contact angle the ice crystal makes with the vitreous ice. 

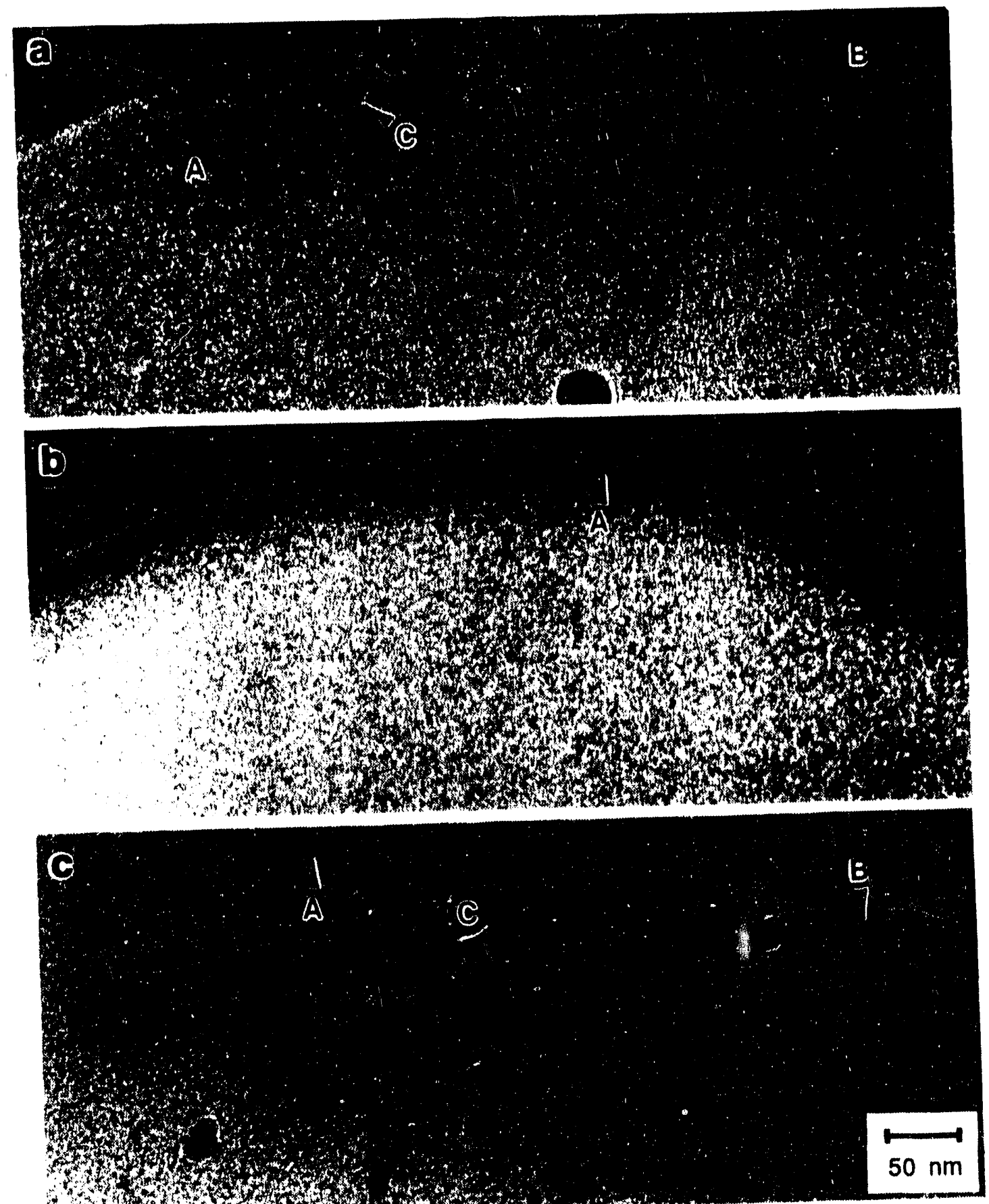

Figure 4 (a). Monophasic solutions of CTAB (0.01 wt $\%)$ below the cme show no discernible microstructure within the vitrified film (A) of solution spanning the hole in the polymer support film (B). The dark spots (C) are surface contamination. (b) CTAB $(0.03 \mathrm{wt} \%)$ solutions near the cme show a few structures (A) that may be micelles or sub-micellar structures. (c) CTAB (1.0 wt, solutions above the cme show globular micelles (A) of 5-7 nm diameter. Chain-like structures that appear to comprise several spherical micelles are infrequently seen (B). Again, contamination (C) can be secn on the sample surface. 
Figure $4 \mathrm{~b}$ is of an aqueous $\operatorname{CTAB}(0.03 \mathrm{wt} \%)$ solution near the cme and shows a few micelles 5-6 nm in diameter and also smaller, possibly submicellar, aggregates. The surfactant structures in this micrograph, and also in Figures 5, 6, 10 and 11 are found near the surrounding polymer film. Surface tension makes the sample film, which spans a hole in the support film, take on a biconcave shape. The sample thickness near the center of a hole can range from stbmicellar dimensions to hundreds of nanometers depending upon the size of the hole in the polymer film and the amount of sample that remains after blotting. The thickness, which can be as much as a micrometer, is greatest near the edge of a hole. The thickness gradient can cause microstructures to segregate by size. Size-segregation is commonly observed when imaging larger vesicular structures as in Figure 10. However, micelles may be absent from the central portion of the biconcave sample film of micellar solution as a result of micellar partitioning driven by the film thickness dependence of the disjoining potential.

Aqueous solutions containing 1 wt $\%$ CTAB are above the cme and show globular, perhaps spherical, micelles 5-7 nm in diameter as seen in Figure 4c. This is in agreement with the hydrodynamic radius of $3.2 \mathrm{~nm}$ reported by Dorshow et al.(1982) from dynamic light scattering at low ionic strengths and $25^{\circ} \mathrm{C}$. The image is a two-dimensional projection of a three-dimensional sample; therefore, the measured sizes can be affected by overlapping projections from two or more micelles. Because the TEM has a large depth of field and the sample thickness can be much greater than the diameter of a micelle, the spacings between micelles in the projected images are small. Figure $4 \mathrm{c}$ demonstrates that the micelles are not confined to the edge of the biconcave film if the sample is sufficiently thick.

Figure 5a shows CTAB (1 wt\%) micelles swollen with toluene (0.4 wt\%). Diameters of the swollen micelles measured from the micrographs range from 6-9 nm. The aggregation number of the micelles rises to accommodate the larger surface area of the swollen micelle. Higher resolution images would provide better size estimates which can be used to calculate aggregation numbers for comparison with aggregation numbers measured by other experimental techniques, e.g., fluorescence quenching, light scattering, etc.

Figure 5b shows CTAB (1 wt\%) micelles swollen with styrene (0.6 wt\%) that contained $0.1 \mathrm{wt} \%$ AIBN. Measured diameters of the swollen micelles range from 9-10 nm. Micrographs of samples prepared from solutions that were exposed to ultraviolet light (254 $\mathrm{nm}$ ) to initiate polymerization show swollen micelles with diameters from 8-9 nm as seen in Figure 5c. The difference between the diameters of the unpolymerized and polymerized micelles may be attributable to the $10 \%$ volume reduction of styrene upon polymerization. However, the experimentally measured size changes of the micelles are not larger than the resolution (about $2 \mathrm{~nm}$ ) in the micrographs. Although further experiments are needed, it appears that polymerization of the swollen micelles in the absence of emulsified styrene preserved the styrene inventory within the micelles without the latter coalescing or aggregating. 

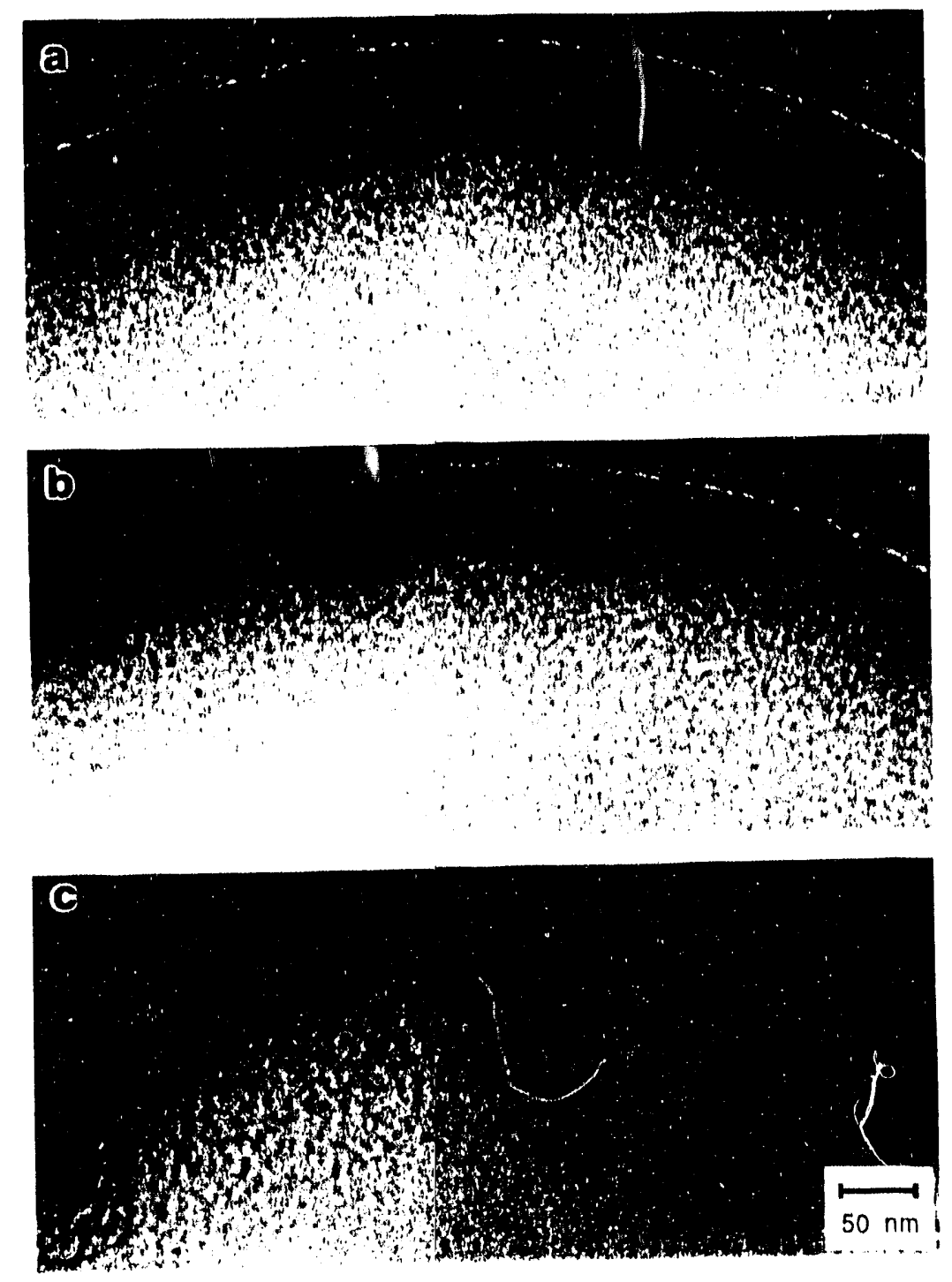

Figure 5 (a) CTAB (1.0 wt\%) micelles) swollen with toluene $(0.4 \mathrm{wt} \%)$ are $6-8 \mathrm{~nm}$ in diameter. Note size increarer unswollen nicelles (Figure 4c). (b) CTAB (1.0 wt \%) micelles swollen wilyrene $(0.6 \mathrm{wt} \%)$ are $9-10 \mathrm{~mm}$ in diameter. (c) Swollen CTAB (1.0 wt\%) mic with a polymerized styrene $(0.6 \%)$ corc have diameters ranging from 8-9 nompare with Figure 5b).

Figure 6 shows that aqueous CTAl37 wt\%) solutions with arlded $\mathrm{NaBr}(3.0 \mathrm{wt} \%)$ contain cylindrical or wormlike micellesh diameters of about $5 \mathrm{~nm}$ and lengths of several micrometers. The micelles changm spherical to cylindrical because the added electrolyte alters the surface charge der and reduces head-group repulsions (Lindman and Wennerstrom 1980, Missel et al.1; this change decreases the offective area per head group and allows the geometricarefered structure of lower mean curvature to form. The cylindrical micolles may fom entanglenent network; they are being further investigated with stereomicroscop obtain threc-clinensional information abont 
the shape of the micelles and the internicellar interactions. It is interesting to note that a branched micolle has been observed only once (cf. Vinson 1088). In Fignee 6 a loole is sech in the central portion of the semple film, where the sample thickness is so small that the micelles have been pushed toward the edge of the vitrified film. Also seen is a tear in the sample film extending from the hole to the sample elges. Ten's may arise from mechanical damage that occurs during sample transfer and then grow into holes as the vitreous matrix flows to relieve stresses when it is irradiated by electrons.

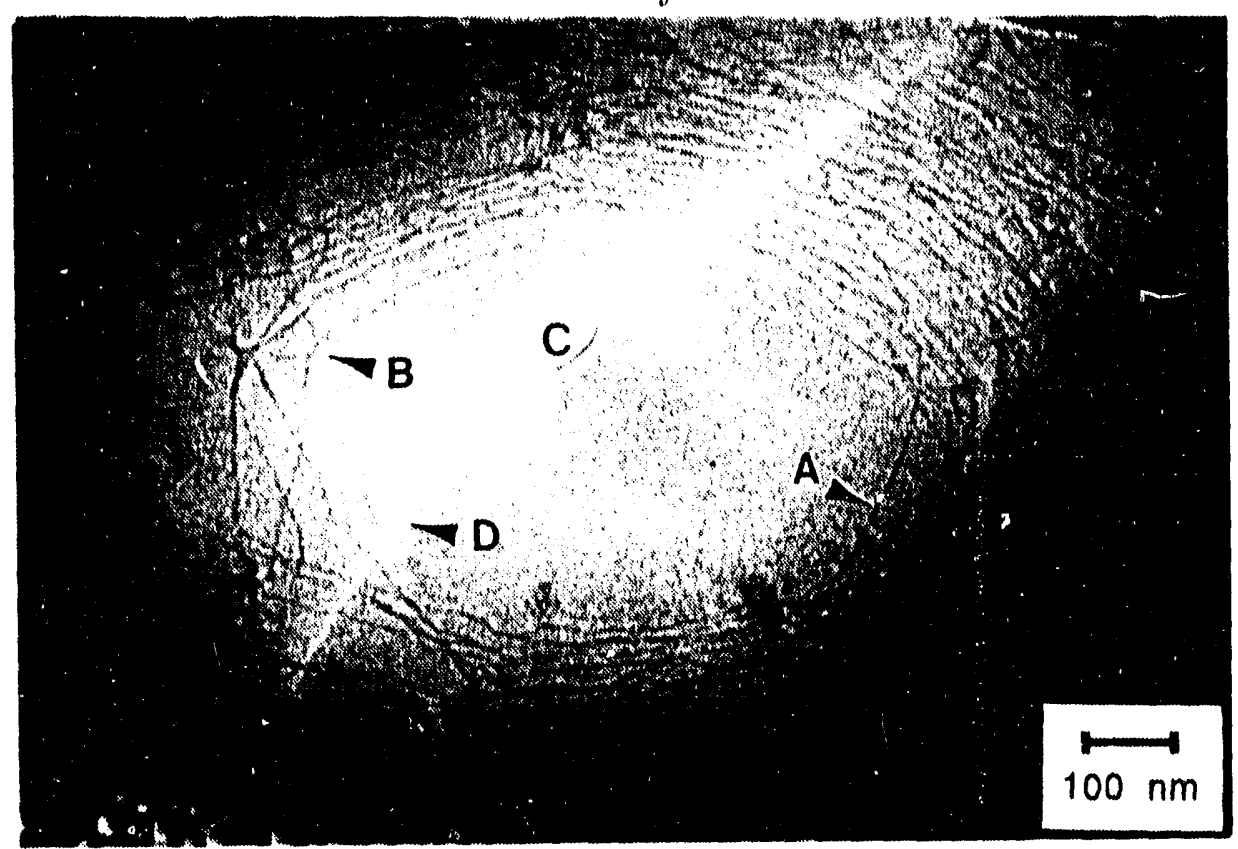

Figure 6. Wormlike micelles (A) with diameters of $5 \mathrm{~nm}$ and lengths of several micrometers are seen in $\mathrm{CTAB}(0.37 \mathrm{wt} \%)$ solutions containing added electrolyte ( $\mathrm{NaBr}, 3.0$ wt\%). That the micelles interact is eviclent from the area marked $B$, where two micelles are intertwined. Holes (C) and tears (D) are occasionally seen in the vitrified sample films. Prepared from $35^{\circ} \mathrm{C}$. Reproduced from Proc. 1988 EMSA, vol. 46, (C) 1989 San Francisco Press, Inc., by permission.

Figure 7 shows discoidal structures, thought to be oblate spheroids, in aqueous mixtures containing $20 \mathrm{mM} \mathrm{DPPC}(1.47 \mathrm{wt} \%)$ and $5 \mathrm{mM} \mathrm{DHPC}(0.24 \mathrm{wt} \%)$ prepared from $45^{\circ} \mathrm{C}$. The DHPC, which forms spherical micelles in the absence of DPPC, may be more concentrated at the edges of the discs where the mean curvature is higher. The discoidal structures are also seen when DPPC is replaced by sphingomyelin (Vinson 1990). The discs are seen at various orientations with respect to the electron beam. When the axis of revolution is parallel to the beam the projection of the structure is a circular image about 20 to $30 \mathrm{~nm}$ in diameter with weak contrast. When the axis of revolution is perpendicular to the: beam the projected image is a clark line about 5-7 11 wide. The line appears dark because its width is in the range accentuated by phase contrast. The contribution from anplitude contrast is larger than normal becanse the electrons travel a longer distance (2)-30 nm) through the discs than in the parallel orientation $(\sim 5-7$ 111n). However, phase contrast is still the major contributor, as is indicated by the lack of contrast, between the rucls and 
middle of a line; contrast is expected if ample contrast is the major contributor. At orientations between parallel and 1nomnl, the ected innges appear somewhat elliptical as expected. Disclike models have been prop for bile salt-lecithin mixed micelles by Small (1067) based largely on X-rny diffractictudies of the hexagonal liquid crystalline phase, and by Mazer et al.(1980) based on ielastic light-scattering. Fromherz and Rüppel (1985) have inferred the formation ocs about $3.3 \mathrm{~nm}$ thick by $26 \mathrm{~nm}$ in diameter from negativcly stained and dried TEecimens of mixtures of egg lecithin and taurochenodesoxycholine. However, this spec preparation technique has been shown to give rise to artifacts and can lead to unreliamages of surfactant microstructures (cf. Tulmon 1983, Kilpatrick et al.1985, Vinson a'almon 1989).

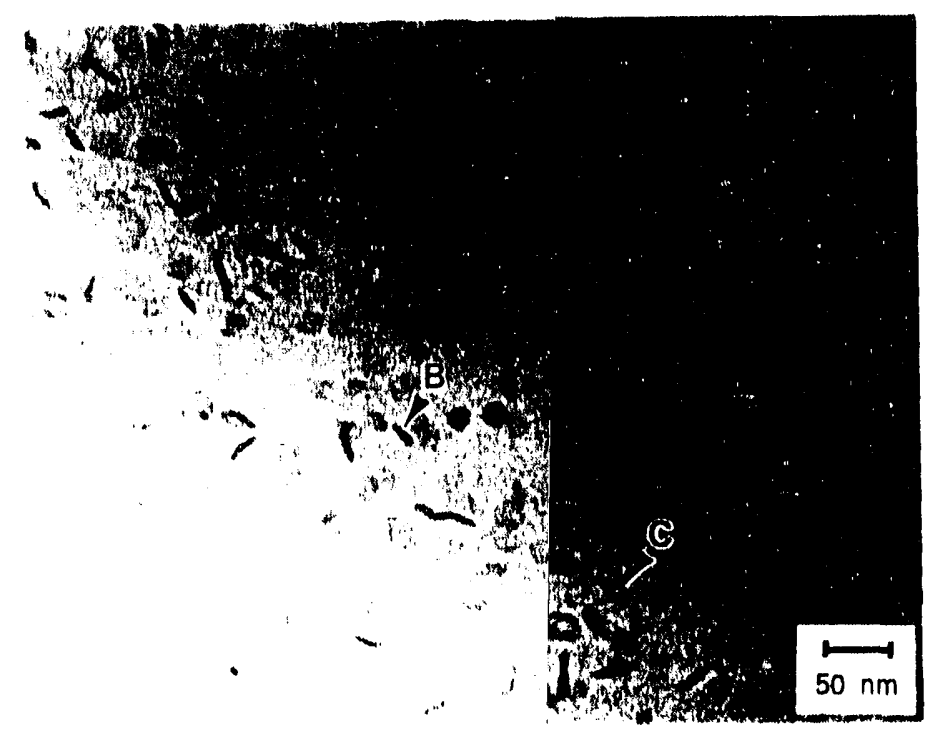

Figure 7. DPPC and DHPC mixtures at $45^{\circ}$ low discoid structures. The discs appear to be oblate spheroids and are see various orientations with respect to the electron beam: (A) Axis of revolutiarallel to beam gives circular image 20-30 $\mathrm{nm}$ in cliameter, (B) Axis of revoluperpendicular to beam gives line 5-6 nm in diameter, and (C) Axis of revolu at other angles gives elliptical shapes.

When the temperature of the DPPC/Dy mixture is dropped to $28^{\circ} \mathrm{C}$ (below the chain melting or main transition temperatu large rippled bilayers, as seen in Figure 8, coexist with the discoidal structures ane evidently formed from lipid previously contained within the disss. It is not know rhe bilayers are equilibrium structures at this composition and temperature. The rip structures are bilayers of the $\mathrm{P}_{\beta^{\prime}}$ phase, which is known to exist in the DPPC-watcrem at temperatures between the $\mathrm{L}_{\alpha}$ and $\mathrm{L}_{\beta^{\prime}}$ phases. X-ray diffraction studies indicatat the lipid hydrocarbon chains pack into it regular hexagonal lattice (Janiak et al.107

Sackmann et al.(1980) found that the phase exhibits two characteristic ripple structures, called the $\Lambda$ and $\Lambda / 2$ structures. $\Lambda / 2$ structure has a short wavelength and a single ripple amplitude, whereas the $\Lambda$ strre has a longer wavelength and two ripple 


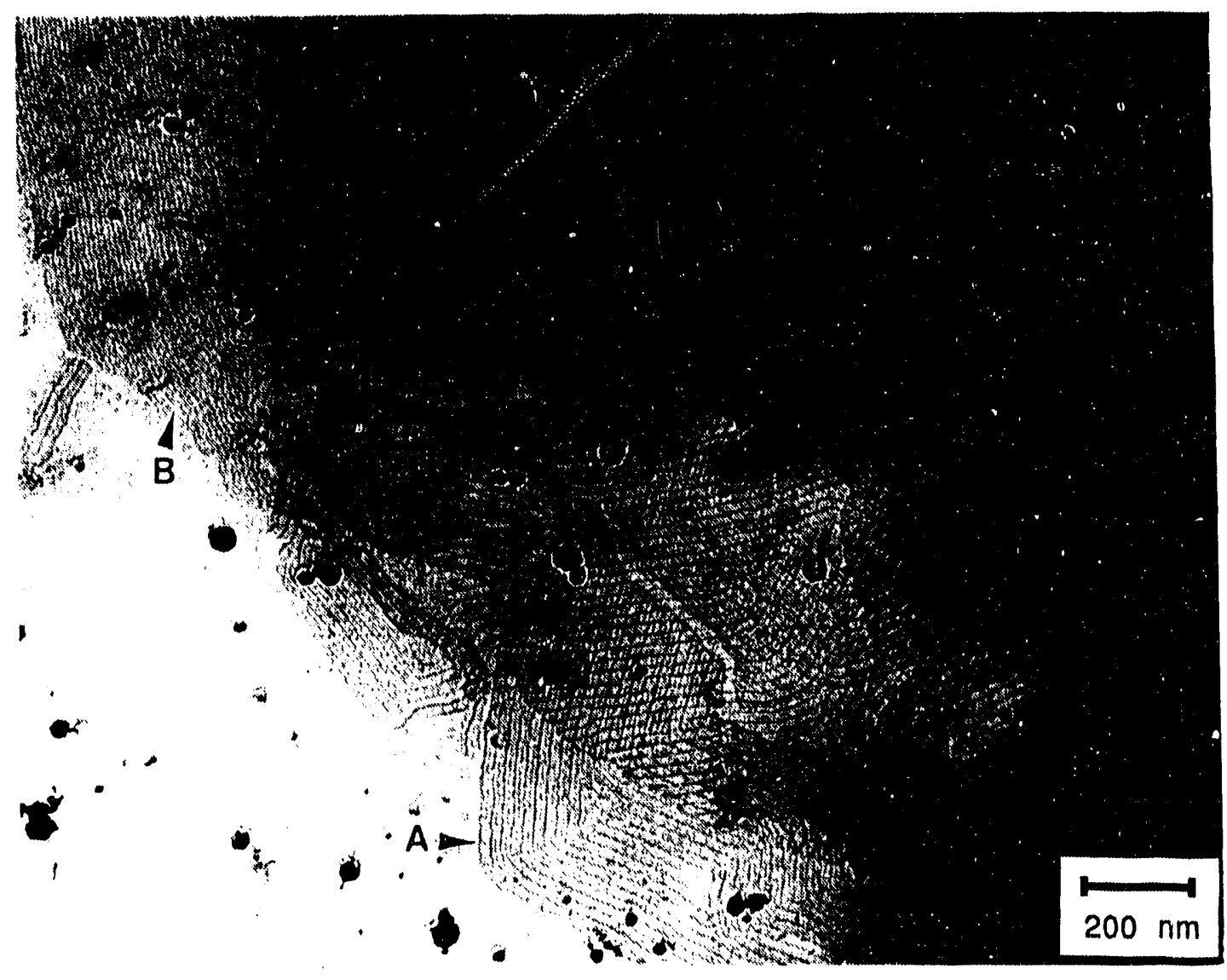

Figure 8. DPPC and DHPC nixtures at $28^{\circ} \mathrm{C}$ show $\Lambda$ (A) and $\Lambda / 2$ (B) ripple structures of the $P_{\beta^{\prime}}$ phasse. 
amplitudes. Freeze-fracture transmission electron microscopy of dimyristoylphosphatidylcholine (DMPC) in water revealed an asymmetric modulation of 12-13 nm wavelength in the $\Lambda / 2$ structure and a symmetric modulation which produces line spacing periodicities of 22-24 $\mathrm{mm}$ in the $\Lambda$ structure (Sackmann et al.1980, Rüppel and Sackmann 1983, Zasadzinski and Schneider 1987, Zasadzinski et al.1988). Zasadzinski and Schneider determined that the width of the large and small ripples of the $\Lambda$ structure are $18 \mathrm{~nm}$ and $4 \mathrm{~nm}$, respectively. From the shadowing of freeze-fracture replicas, Zasadzinski and Schneider estimated that the amplitudes of the large and small ripples are at least $9 \mathrm{~nm}$ and $2 \mathrm{~nm}$, respectivelv. Zasadzinski et al.(1988) estimated the ripple amplitude of the $\Lambda / 2$ structure at $4.5 \mathrm{~nm}$ from scannirg tunneling microscopy of freeze-fracture replicas.

DPPC ripple structures have been studied less than their LMPC counterparts. Freezefracture results of Pinto da Silva (1971) show ripple spacings broadening from approximately 20 to $38 \mathrm{~nm}$ within a liposome. Luna and $\mathrm{McC}$ connell (1977) found periodicities of 13 to $17 \mathrm{~nm}$ in DPPC liposomes. Low-angle x-ray diffraction data of Janiak et al.(1976) suggest that the ripple wavelength shortens with increasing water concentration, from 162 $\AA$ at $21 \%$ water to a minimum of $140 \AA$ at $25 \%$ water. Watts et al.(1978) have reported line spacing periodicities in freeze-fracture images of dimyristoylphosphatidylglycerol and dipalmitoylphosphatidylglycerol of $215 \pm 20 \AA$ and $295 \pm 20 \AA$, respectiveiy.

The structures imaged in Figures 8 and 9 are probably single bilayers (as determined from their contrast) rather than stacks of bilayers (liposomes) as in the previous freezefracture and $x$-ray studies. This is the first strong evidence that these rippled structures can exist as individual bilayers. The bilayers are relatively rigid because the temperature is below the hydrocarbon chain melting temperature. So they probably do not easily bend during sample preparation and, in the thin liquid sample, orierit nearly parallel to the grid (perpendicular to the electron beam). This circumstance is advantageous because it eliminates the need to compensate for bilayer tilt.

Figure 8 displays both the $\Lambda / 2$ and $\Lambda$ structures, the $\Lambda$ structure being more prominent. The line spacing periodicities of the $\Lambda / 2$ and $\Lambda$ structures measured from Figure 8 are 11-12 $\mathrm{nm}$ and 21-24 $\mathrm{nm}$, respectively. The $\Lambda$ structure produces an alternating pattern of parallel thick (4-6 nm) dark lines and thin faint lines superimposed on a lighter background. The distance separating two dark lines is $17-18 \mathrm{~nm}$. These measurements are in accord with the periodicities determined in DMPC ripple structures by freeze-fracture transmission electron microscopy (Zasadzinski and Schneider 1987, Zasadzinski et al.1988).

Figure 9 shcws images and schematics of defects found in ripple structures. Figure 9 a shows a $+1 / 2$ disclination in a $\Lambda$ structure. In this image the line width changes from the dark $4 \mathrm{~nm}$ line to the thin faint linc, suggesting that the large and small peaks merge around the point defect. Figure $9 \mathrm{~b}$ shows a $120^{\circ}$ bend, which is a fingerprint of a $-1 / 2$ disclination. This is a common defect owing to the hexagonal packing of the hydrocarbon chains. 

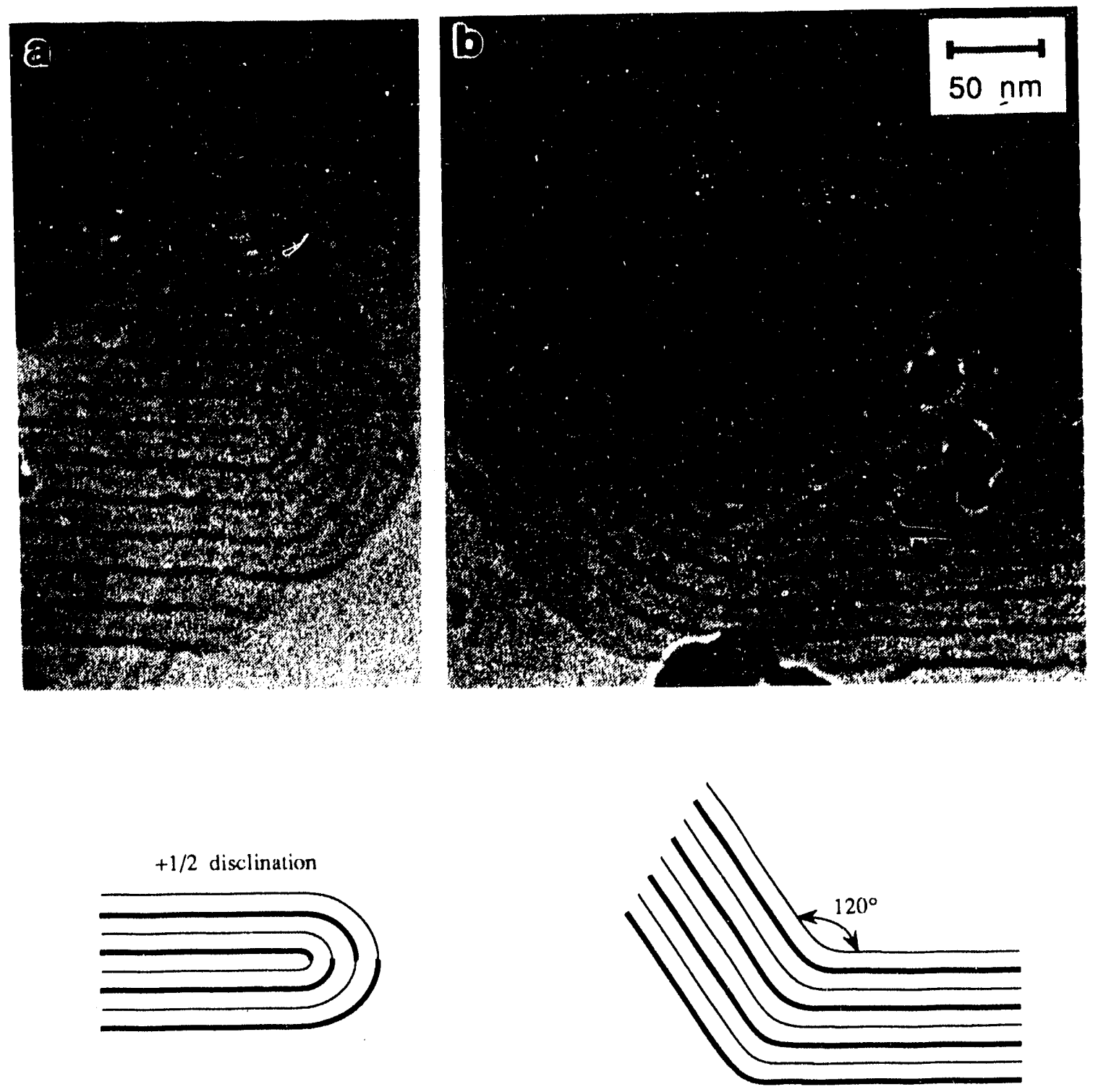

Figure 9. Micrographs and schematics of defects in $\Lambda$ ripple structures in DPPC and DHPC mixtures at $28^{\circ} \mathrm{C}$. (a) $\mathrm{A}+1 / 2$ disclination which shows merging of large and small amplitude ripples. (b) $120^{\circ}$ bend. 
As discussed above, the phase contrast produced by a defocus of $4 \mu \mathrm{m}$ enhances sizes and periodicities of about $5 \mathrm{~nm}$. Phase contrast requires that there be gradients in the projection of the potential distribution of the specimen onto a plane perpendicular to the electron beam, $\mathbf{r}=(x, y)$, i.e. that the integral of the potential distribution in the $z$ directi , be nonuniform in the ( $x, y$ )-plane (Lenz 1971). This means that there must be composition gradients, thickness gradients, or both. In an underfocused condition such as that used here, domains of higher potentials appear darker than domains of lower potentials. The mean potentials for water, the lecithin tail-group, and the lecithin headgroup are 5.81, 6.98, and 7.65-9.08 volts, respectively (Bellare 1989). This means that regions appear darker where electrons traverse greater lengths of the bilayer.

We propose the structure diagrammed in Figure 10a to explain the phase contrast produced by the $\Lambda$ structure. This is a sinusoidal arrangement with an extra peak, $4 \mathrm{~nm}$ in width, superimposed at each maximum and minimum. In the model shown in Figure $10 \mathrm{a}$, the $4 \mathrm{~nm}$ peak in the $\Lambda$ structure produces a single dark line. The small peak could give rise to two dark lines at less underfocus, but at $4 \mu \mathrm{m}$ defocus the information from the two sides of the $4 \mathrm{~nm}$ peak, where the electron path through the bilayer is longest, is not resolved and images as a single line. The two lines of inflection points on either side of the large peak are the loci of greatest inclination and produce faint lines because these are where the electron path through the bilayer is greatest. The wavelength or period of this model is twice the line spacing periodicity, or about $42-48 \mathrm{~nm}$. This model does account correctly for the pattern seen in freeze-fracture images, provided the amplitude of the large peak is great enough to prevent shadowing of the small peak in the trough region. An alternative model that produces the correct phase contrast is similar to the model shown in Figure 10a, but has the small peaks turned away from the midplane of the lamellae rather than toward the midplane. However, this model can be discounted because it does not account correctly for the pattern in freeze-fracture images.

A model previously suggested for the $\Lambda$ structure as seen in freeze-fracture replicas is "W" shaped, i.e., a small peak in the center of a deep trough (Sackmann et al.1980, Zasadzinski and Schneider 1987). Such a structure would not produce the phase contrast seen in Figures 8 and 9. Were its minima and maxima rounded, it would instead produce a pattern in which two adjacent dark lines are separated by two thin faint lines (Figure 10b). Furthermore, if the features of the " $\mathrm{W}$ " model were unrounded, i.e. angular (cf. Figure 11 of Sackmann et al.1980), and the incline of the bilayer everywhere were the same, then the model would not produce any contrast because there would not be any gradients in the projected specimen potential. If the incline of one peak were greater than that of the other, the image produced by phase contrast would show parallel dark lines superimposed on a uniform background as shown in Figure 10c.

The model proposed in Figure 10a is more symmetric than the "W" models in Figures $10 \mathrm{~b}$ and $10 \mathrm{c}$, in which there is an extra peak only at each minimum.

Figure 11 shows spheroidal and tubular vesicles, and complex encapsulated vesicles 

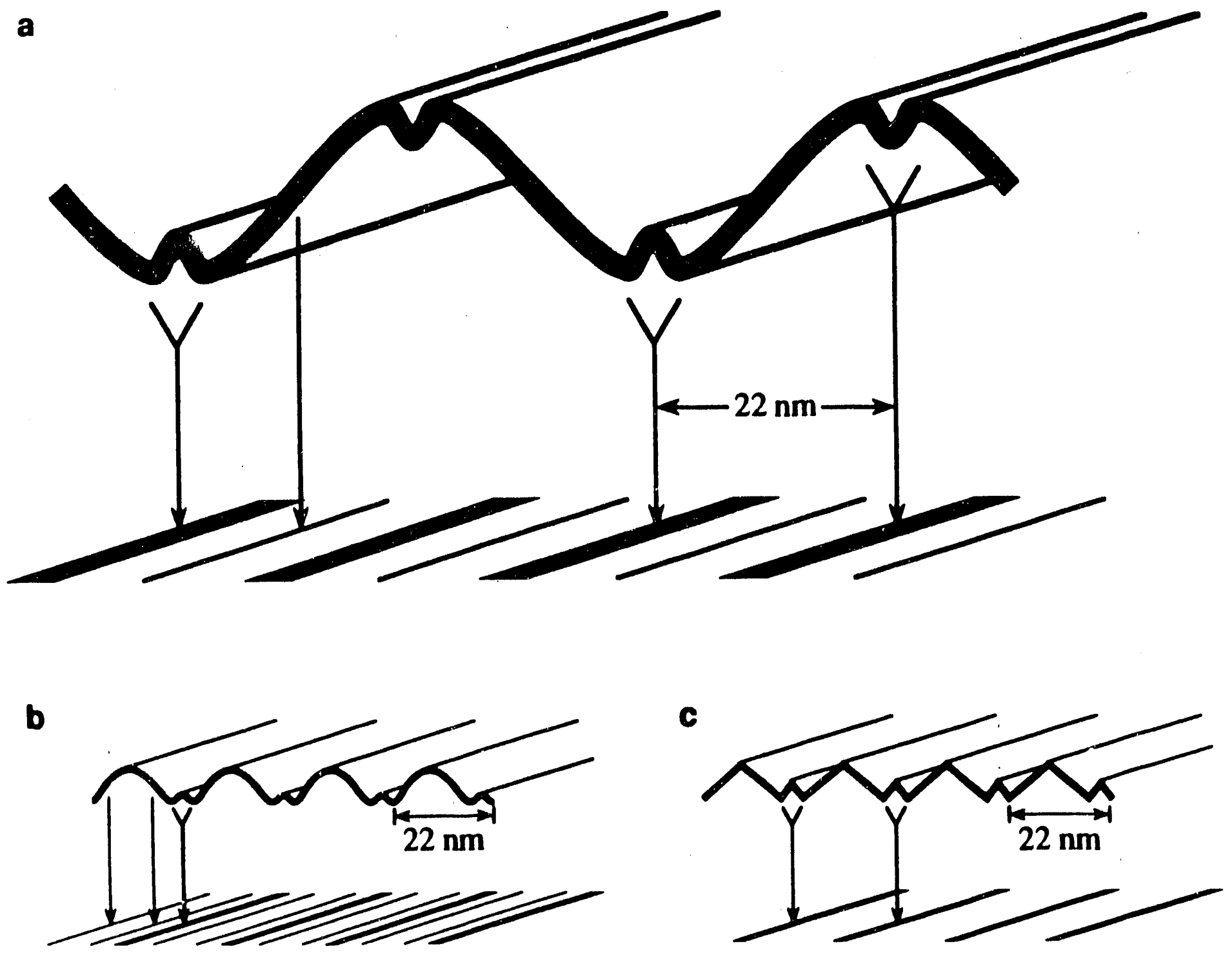

Figure 10. (a) Proposed model for $\Lambda$ ripple structure imaged in Figures 8 and 9 . The small peaks give rise to dark lines and the inflection points of the large peaks produce faint lines. (b) and (c) "W" shaped models with rounded and angular features, respectively. These models were suggested on the basis of freeze-fracture results, but do not produce the phase contrast observed in Figures 8 and 9. 


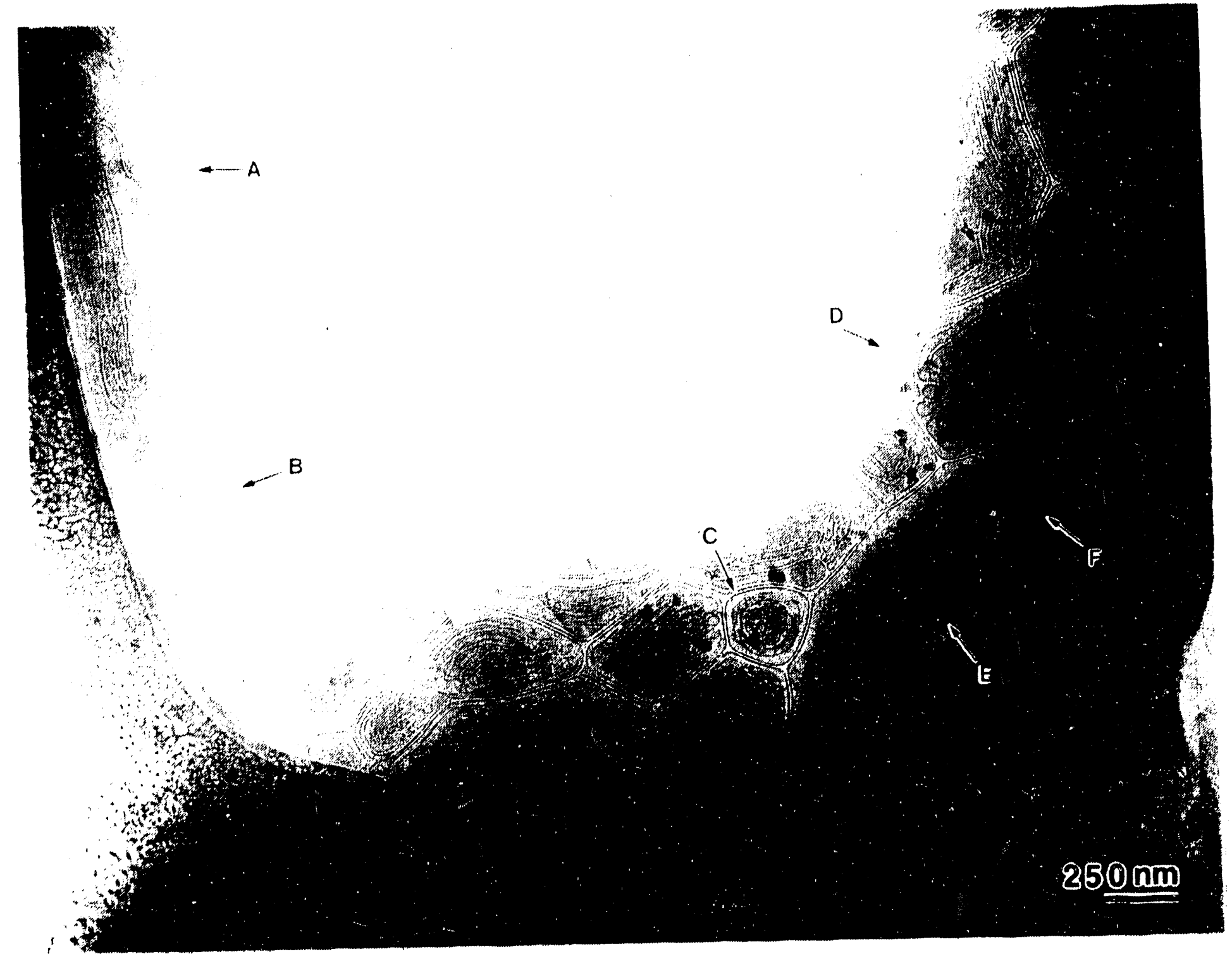

Figure 11. Biphasic dispersions of SHBS $(2 \mathrm{wt} \%)$ prepared from $25^{\circ} \mathrm{C}$ show (A) spherical and (B) tubular vesicles, and (C) complex encapsulated vesicles and (D) coiled tubes within larger vesicles. Also seen are tubules frozen apparently while undulating (E). 
coiled tubules in samples prepared from SHBS (2 wt\%) dispersions at $25^{\circ} \mathrm{C}$. The neters of the tubes vary somewhat regularly along their length, giving direct visual lence of membrane undulation. Over the size range imaged by this technique (5-2000 there is no evidence for the existence of lamellar phase with the bilayer spacing $30 \AA$ ) characteristic of more concentrated dispersions (>10 wt\%, Miller et al.1984). vesicular structures seen here may be characteristic of the unbinding fluctuations that upt lamellar phases (Lipowsky and Leibler 1986).

Aqueous SHBS (0.1 wt\%) mixtures held at elevated temperatures $\left(90^{\circ} \mathrm{C}\right)$ for one week tain a variety of microstructures. Inspection of Figure 12 shows small ( 10 nm ) cles, nodular tubes, and tubes of more complex topography, including 'wart'-like proions from tubes of varying diameter. The thickness of the wart membrane in some is appears to be less than that of the tube membrane. Perhaps the warts exist due iteric repulsive forces strong enough at this high temperature to disrupt the bilayer orm monolayers. An alternative explanation is that the apparent membrane thickness ends on the curvature components of the membrane along the viewing direction. The Ictures have the appearance of a foam in certain regions. These structures were not orted in previous high temperature studies (Franses et al.1980).

That swollen and wormlike micelles can be imaged is an encouragement to microemul1 research. Although microemulsions have been imaged recently by fast-freezing followed fracturing and replication (Jahn and Strey 1988; Bodet et al.1988), direct cryo-TEM iges have been elusive. We have found that radiation damage by the electron beam cures the structure of microemulsions that contain about equal volumes of oil and wa-

Low-dose imaging techniques with high voltage electron microscopes may prevent ;rostructural changes and allow reliable imaging of midrange microemulsions. We are rently trying this proce lure.

\section{mmary}

Direct, high resolution images of surfactant microstructures have been obtained by o-transmission electron microscopy of vitrified hydrated samples that had not been mically stained, dried, or replicated. The labile microstructures were imaged without deleterious effects of phase-change or radiation damage. Surfactant solutions below, $r$, and above the critical micelle concentration range $(\mathrm{cmc})$ have been investigated as 1 as systems containing swollen micelles. Wormlike, or "cylindrical" micelles that form on addition of electrolyte were also imaged. These form what appear to be entanglement works; however, stereomicroscopy must be brought to bear on visualizing the threerensional structure.

Aqueous mixtures of short and long chain lipids show the first reliable images of disdal microstructures at temperatures above the hydrocarbon chain melting temperature. low the chain melting temperature single bilayers exhibiting the $\Lambda$ and $\Lambda / 2$ structures 


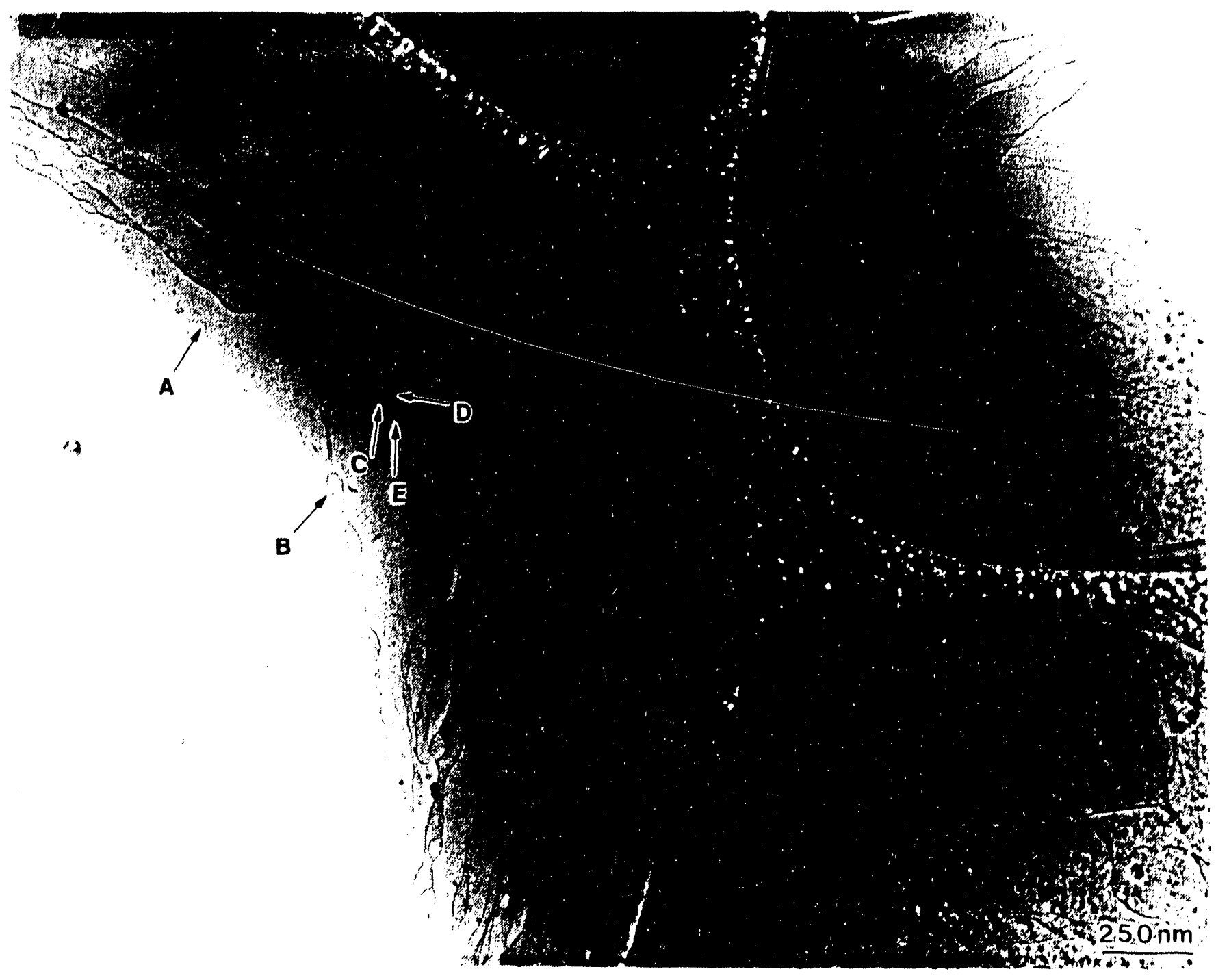

Figure 12. SHBS $(0.1 \mathrm{wt} \%)$ at $90^{\circ} \mathrm{C}$ shows small $(\sim 10 \mathrm{~nm})$ vesicles $(\mathrm{A})$, nodular tubes (B), and tubes of more complex topology, including 'wart'-like protrusions from tubes of varying diameter. The thickness of the wart membrane in some areas $(\mathrm{C}, \mathrm{D})$ appears to be less than that of the tube membrane (E). Reproduced from J. Electron Microsc. Tech., vol. 10, C1988 Alan R. Liss, Inc., by permission. 
of the $\mathrm{P}_{\beta^{\prime}}$ phase were observed. A new model has been proposed for the $\Lambda$ structure to explain the image contrast.

Dilute biphasic dispersions showed uni- and multi-lamellar vesicles. The multi-lamellar vesicles imaged did not have the constant lamellar spacing characteristic of the lamellar phase at higher SHBS concentrations. Vesicular tubules were imaged that appear to have been captured while undulating to form spheroidal vesicles. Complex microstructures seen in dilute SHBS dispersions at $90^{\circ} \mathrm{C}$ indicate that a high temperature state of vesicle-like aggregation exists even though the solution appears to be monophasic by other techniques (Franses et al.1980). The observation of surfactant microstructures, as illustrated here, affirms the importance of direct visualization in colloidal research.

\section{References}

Adrian, M., Dubochet, J., Lepault, J., McDowall, and A. W., Nature 308, 32-38 (1984). Bellare, J. R., Davis, H. T., Scriven, L. E., and Talmon, Y., "Proc. XIth Int. Cong. on Electron Microscopy" (T. Imura, S. Maruse, and T. Suzuki, Eds.), Vol. 1, pp. 367-368. The Japanese Society of Electron Microscopy, held in Kyoto, Japan, August 31-September 7, 1986a.

Bellare, J.R., H. T. Davis, L.E. Scriven and Y. Talmon, in "Proc. XIth Int. Cong. on Electron Microscopy" (T. Imura, S. Maruse, and T. Suzuki, Eds.), Vol. 1, pp. 369-370. The Japanese Society of Electron Microscopy, held in Kyoto, Japan, August 31-September 7, 1986b.

Bellare, J. R., Doctoral dissertation, University of Minnesota, 1988.

Bellare, J. R., Davis, H. T., Scriven, L. E., and Talmon, Y., J. Electron Microsc. Tech. 10, 87 (1988)

Bodet, J.-F., Bellare, J. R., Davis, H. T., Scriven, L. E., and Miller, W. G., J. Phys. Chem. 92, 1898-1902 (1988).

Burns, J., and Talmon, Y., in "Proc. of the 45th Annual Meeting of the Electron Microscopy Society of America" (G. W. Bailey, Ed.), pp. 500-501. San Francisco Press, San Francisco, held in Baltimore, Maryland, August 2-7, 1987.

Costello, M. J., Fetter, R., and Corless, J. M., in "Science of Biological Specimen Preparation" (O. Johari, Ed.), pp. 105-115. SEM Inc. AMF O'Hare Chicago, IL 60666, 1982.

Davis, H. T., Bodet, J. F., Scriven, L. E., and Miller, W. G., in "Physics of Amphiphilic Layers" (J. Meunier, D. Langevin, and N. Boccara, Eds.), Vol. 21, pp. 310-327. Springer Proceedings in Physics, Springer Verlag, Berlin, 1987.

Dorshow, R., Briggs, J., Bunton, C. A., and Nicoli, D. F., J. Phys. Chem. 86, 2388-2395 (1982).

Dubochet, J., Adrian, M., Teixeira, J., Alba, C. M., Kadiyala, R. K., MacFarlane, D. R., and Angell, C. A., J. Phys. Chem. 88, 6727-6732 (1984).

Ekwall, P., Mandell, L., and Solyom, P., J. Colloid Interface Sci. 35, 519 (1971).

Franses, E. I., Puig, J. E., Talmon, Y., Miller, W. G., Scriven, L. E., and Davis, H. T., J. Phys. Chem. 84, 1547 (1980). 
Franses, E. I., Doctoral dissertation, University of Minnesota, 1979.

Fromherz, P., and Rüppel, D., FEBS (Fed. Eur. Biochem. Soc.) Lett. 179, 155-159 (1985).

Guettler, R. D., Bellare, J. R., Davis, H. T., and Scriven, L. E., in "Proc. of the 45th Annual Meeting of the Electron Microscopy Society of America" (G. W. Bailey, Ed.), pp. 750-751. San Francisco Press, San Francisco, held in Baltimore, Maryland, August 2-7, 1987.

Jahn, W., and Strey, R., J. Phys. Chem. 92, 2294-2301 (1988).

Janiak, M. J., Small, D. M., and Shipley, G. G., Biochemistry 15, 4575-4580 (1976).

Janiak, M. J., Small, D. M., and Shipley, G. G., J. Biol. Chem. 254; 6068-6078 (1979).

Kilpatrick, P. K., Miller, W. G., and Talmon, Y., J. Colloid Interface Sci. 107, 146-158 (1985).

Lenz, F. A., in "Electron Microscopy in Materials Science" (U. Valdrè, Ed.), pp. 541-569. Academic Press, New York, 1971.

Lindman, B., and Wennerström, H., Micelles. Topics in Current Chemistry 1 (1980)

Lipowsky, R., and Leibler, S., Phys. Rev. Lett. 56, 2541 (1986).

Luna, E. J., and McConnell, H. M., Biochim. Biophys. Acta 466, 381-392 (1977).

Mazer, N. A., Benedek, G. B., and Carey, M. C., Biochemistry 19, 601-615 (1980).

Miller, W. G., Blum, F. D., Davis, H. T., Franses, E. I., Kaler, E. W., Kilpatrick, P. K., Nietering, K. E., Puig, J. E., and Scriven, L. E., in "Surfactants in Solution" (K. L. Mittal and JB. Lindman, Eds.), Vol. 1, pp. 175-191. Plenum Publishing Corp., 1984.

Missel, P. J., Mazer, N. A., Benedek, G. B., and Young, C. Y., J. Phys. Chem. 84, 1044-1057 (1980).

Ness, J. N., and Moth, D. K., J. Colloid Interface Sci. 123, 546-547 (1988).

Perlov, G., Talmon, Y., and Falls, A. H., Ultramicroscopy 11, 283-288 (1983).

Pinto da Silva, P., J. Microscopie 12, 185-192 (1971).

Rüppel, D., and Sackmann, E., J. Physique 44, 1025-1034 (1983).

Sackmann, E., Rüppel, D., and Gebhardt, C., in "Springer Series in Chemical Physics" (W. Helfrich and G.Heppke, Eds.), 11, pp. 309-326. Springer-Verlag, Berlin, 1980.

Siegel, D. P., Burns, J. L., Chestnut, M. H., and Talmon, Y., Biophys. J. 56, 161-169 (1989).

Sinall, D.M., Gastroenerology 52, 607-610 (1967).

Talmon, Y., J. Colloid Interface Sci. 93, 366-382 (1983).

Talmon, Y., Colloids and Surfaces 19, 237-248 (1986).

Talmon, Y., in "Cryotechniques in Biological Electron Microscopy" (R. A. Steinbrecht and K. Zierold, Eds.), pp. 64-84. Springer-Verlag, Berlin, 1987.

Vinson, P. K., in "Proc. of the 45th Annual Meeting of the Electron Microscopy Society of America" (G. W. Bailey, Ed.), pp. 644-645. San Francisco Press, San Francisco, held in Baltimore, Maryland, August 2-7, 1987.

Vinson, P. K., in "Proc. of the 46th Ann. Meeting of the Electron Microscopy Society of America" (G. W. Bailey, Ed.), pp. 112-113. San Francisco Press, San Francisco, held in Milwaukee, Wisconsin, August 7-12, 1988.

Vinson, P. K., Talinon, Y., and Walter, A., Biophys. J. 56, 669-681 (1989).

Vinson, P. K., and Talmon, Y., J. Colloid Interfuce Sci. 133, 288-289 (1989). 
Vinson, P. K., Doctoral dissertation, University of Minnesota, 1990.

Watts, A., Harlos, K., Maschke, W., and Marsh, D., Biochim. Biophys. Acta 510, 63-74 (1978).

Zasadzinski, J. A. N., and Schneider, M. B., J. Physique 48, 2001-2011 (1987).

Zasadzinski, J. A. N., Schneir, J., Gurley, J., Elings, V., and Hansma, P. K., Science 239, 1013-1015 (1988). 


\section{A LOW COST ANIMATION SYSTEM APPLIED TO RAY TRACING IN LIQUID CRYSTALS}

\section{Synopsis}

Animated movies of scientic graphics can be recorded on film with the low cost system of hardware and softwere described here. The hardware consists of a $16 \mathrm{~mm}$ camera, a stepper motor, and a simple camera-motor controller. The software is designed to produce bitmaps from graphical data, combine bitmaps into composite frames, and record frames onto film. The camera is fully controlled by the same graphics workstation that is used to display the images, so fades and dissolves can be performed in software with a camera not equipped for such special effects. The graphical clata, generated on a supercomputer, is subsequently transferred to the workstation where it is stored and recorded frame by frame according to a configuration file. A variant of the software, which operates across local and wide area networks, makes use of network computing software to send computationally intensive tasks to a remote supercomputer or to other workstations in a distributed computing environment. We have used the system to simulate polarized light microscope images of liquid crystals according to a single-scattering ray-tracing theory.

\section{Introduction}

Scientific research today often involves simulation of time dependent phenomena wherein the dynamical equations underlying the physical process are solved by supercomputers. Examples include studies of fluid flow in porous media, molecular dynamics of rigid rods, diffusion and aggregation of colloids, and chemical reaction dynamics. The results of such simulations are often best presented as a sequence of graphical output cast in the form of an animated movie.

Equally amenable to animation are results of simulating a process where it is not time but some other parameter that is progressing. The parameter may be a rotation angle, a reaction extent, a volume fraction or mean curvature. In this chapter the simulation of interest is image formation in polarized light microscopy of liquid crystals. Animation of the images, as objects rotate and deform, is valuable as a guide to image interpretation. We first review the theory of image formation in optical microscopy and then describe the animation system we developed to present the results as a movie.

\section{Ray Tracing in Liquid Crystals}

A microscope image is the intensity distribution on a viewing plane of a light beam transmitted through the specimen. Thus, simulation of microscope images is equivalent to ray tracing through the specimen. In polarized light microscopy a plane polarized 
beam of light passes through the specimen and then through an analyzer, after which it forms an image on a viewing screen. Polarized light microscopy is widely used to study the microstructure of crystals, liquid crystaly and other optically anisotropic specimens. Ray tracing through ordinary crystals is straight forward because Maxwell's equations describing the transmission of light through such media can be solved with relative ease. Ray tracing through liquid crystals is not easy because their symmetries are complex and the interaction of light with their varied shapes results in complex equations and boundary conditions.

We have recently shown (Bellare, et al.1989) that with a single scattering approximation the intensity distribution $\psi(x, y)$ of a beam of unit incident intensity that has passed through a polarizer with polarization direction $\mathbf{e}_{p}$, through a specimen of thickness $\left(z_{f a r}-z_{n e a r}\right)$ and of local electric susceptibility $\mathbf{X}$, and through an analyzer with analyzer direction $\mathbf{e}_{a}$ is

$$
\psi(x, y)=\left[\int_{z_{\text {near }}}^{z / a r}\left(\mathbf{I}+C_{2} \mathbf{X}\right): \mathbf{e}_{p} \mathbf{e}_{a} d z\right]^{2}
$$

where $\mathbf{I}$ is the unit dyadic and $\mathbf{X}$, the electric susceptibility, is a dyadic whose eigenvalues $X_{i}=\left(n_{i}^{2}-1\right), i=1,2,3$ depend on the three principal refractive indices $n_{i}$ of the specimen.

The equilibrium microstructure of lamellar lianid crystals consisting of elongated molecules is the structure with the lowest free energy. The free energy is lowest when the lamellae are a parallel family of surfaces with zero mean curvature or equivalently, a family of parallel planes of infinite extent. Although there are numerous zero mean curvature surfaces that are multiply connected and curved,(Anderson et al.1989) they do not form parallel families. Lamellae of finite size have edges, i.e., crystalline boundaries, which have higher free energy, and so such materials must have closed forms without edges if the edge energy is to be minimized. The competition between zero mean curvature and zero edge energy leads to a compromise between planes and closed forms. Local regions of a material with large mean curvatures are called defects. Defects are not favored because they contribute relatively large free energy per unit volume of the material. Maxwell (1869) showed that there is only one family of parallel surfaces without surface defects. This is the family of Dupin cyclides. Such a family has two line defects: one is an ellipse or parabola, the other a confocal hyperbola or parabola. A surface of a Dupin cyclide is the envelope of a variable sphere tangent to three fixed spheres (Dupin 1822).

Our goal was to calculate images from liquid crystals in the shape of Dupin cyclides, spheroids and ellipsoids, and to illustrate how the images change as the objects rotate in space relative to the polarizer and analyzer. The images change when the objects rotate because the orientation of the electric susceptibility, i.e., its eigenvectors, rotate with respect to the laboratory viewing frame in which Eq. (1) is defined. Thus our simulation evaluated Eq. (1) for the various shapes as they were rotated. Details of the simulation technique are given in (Bellare et al. 1989). The simulation results were gray-scale graphical images that were animated into a movie with the system described 
next.

\section{The Animation System}

Although there has been much progress in video based animation systems for recording scientific graphics, (Johnston, et al, 1087: Jolnston, et al, 1988) we chose film as a recording medium. The chief deterrent to film-based animation has been the high cost of commercial film recorders. Hence we elected to build a low-cost system based on a standard $16 \mathrm{~mm}$ camera coupled to a simple controller. The iclea was to aim a tripod-mounted camera at a graphics workstation screen, clisplay a bitmap to be animated and shoot one or more frames of film. The movie was made by shooting a sequence of bitmaps. The camera and computer screen were enclosed in a light-1, roof chamber assembled from an adjustable frame covered by dark material. The system was built from standard components for $\$ 1500$, including a camera, controller, light-proof chamber, and tripod. The only additional hardware required was the personal computer or workstation on which the graphics were displayed.

\section{Why Film?}

Even with the current electronic imaging revolution, traditional silver halide based film remains superior to video as a recording medium. Whereas video has advantages of instant gratification and reusability of media, film beats video on four counts: resolution, dynamic range, single-frame indexing, and longevity. Resolution is the ability to distinguish fine detail. Video tape in the popular NTSC format is limited in resolution to about 300 lines, whereas film can resolve more than 3000 lines. Most graphics workstations have $1024^{2}$ pixels, so they can display more detailed images than video can record. Dynamic range is the ratio of maximum to minimum intensities that can be faithfully recorded. A large dynamic range is desirable because it is possible to record tonal variations both at low and high intensities. Video is limited to a dynamic range of $2^{3}$ whereas film has a dynamic range of $2^{7}$. A graphics workstation with 24 bits per pixel (eight bits per primary color) has a dynamic range of $2^{8}$. While there are better video formats in vogue (and newer ones are being planned), none yet exceed the resolution or dynamic range of film. Furthermore, the equipment for the better video formats is priced beyond the budget of many researchers. Because standard video recording equipment has no means of indexing to a single frame, special expensive equipment is required for frame-by-frame animation. Film is invariably sprocketed; so it is routinely exposed frame-by-frame in every camera. Videotape is not an archival recording material because its magnetic fields fade with time. When properly processed, film is archival: it is generally expected to last over a hundred years without special care.

Once recorded on film, a movie is easily transferred to video if required for distribution. This indirect route to video-a standard technique in commercial studios for quality TV 
programming - is superior to direct recording on tape because film compresses the dynamic range of the original graphics so that subsequent transfer to tape does not as greatly exceed its dynamic range. Moreover, $16 \mathrm{~mm}$ cameras are easily available for a fraction of the cost of a video tape recorder with a single frame controller. New and used $16 \mathrm{~mm}$ cameras are reviewed and advertised for sale in the publications American Cinematographer and Shutterbug.

\section{Animation Hardware}

The animation system consists of a $16 \mathrm{~mm}$ movie camera, a stepper motor, and a camera-motor controller. We used a Bolex H16 camera. This camera is designed to be driven by an internal spring, but it also has a shaft extending to the exterior that can be driven by a motor. The shaft not only advances the film but it also opens and closes the shutter. As the shaft rotates at constant angular velocity, the shutter opens, stays open for a certain time interval, then closes, and the film advances. One clockwise turn $\left(360^{\circ}\right)$ of the shaft advances and exposes eight frames. If the shaft is rotated in the opposite direction, the film is exposed as it is rewound, and so if double exposure is desired it can easily be accomplished.

The shaft is connected to a stepper motor (Vexta model PH265-02B) which rotates by $360^{\circ}$ when 200 state transitions are applied to its windings in a particular order linown as the drive sequence (Fig. 1). Thus 25 state transitions expose and advance one frame of the film. If the drive sequence is reversed, the motor rotates in the opposite direction. The motor is driven by a camera-motor controller, which in turn is driven by the graphics workstation. The film is advanced or rewound by toggling the state of Data Terminal Ready (DTR, pin 20) on the RS-232C serial port of the workstation. The camera-motor controller is edge triggered. A transition from off to on moves the camera motor one step, and a transition from on to off also moves the motor one step. Whether the film advances or rewinds depends on the logic state of Data Set Ready (DSR, pin 6). If DSR is off, the film advances; if on, it rewinds. To summarize, DTR from the workstation is connected to the camera-motor controller, which converts each DTR transition into a drive sequence to rotate the stepper motor connected to the camera.

The camera-motor controller (CMC) consists of electronic components that 1) condition the input DTR and DSR signals; 2) produce a drive sequence for the motor by means of control logic; and 3) switch the motor coils. Major componenis of the CMC are a line receiver (Motorola, Phoenix, AZ, Part Number MC1489P), a binary up-down counter (Texas Instruments, Dallas, TX, Part Number SN74191N), an exclusive-OR gate (National Semiconductor, Santa Clara, CA, Part Number DM7486N), a buffer (National Semiconductor DM7404N), and four very high gain power Darlington transistors (General Electric, Auburn, NY, Part Number D40C7) used as coil drivers. These are all connected as shown in Fig. 1. 


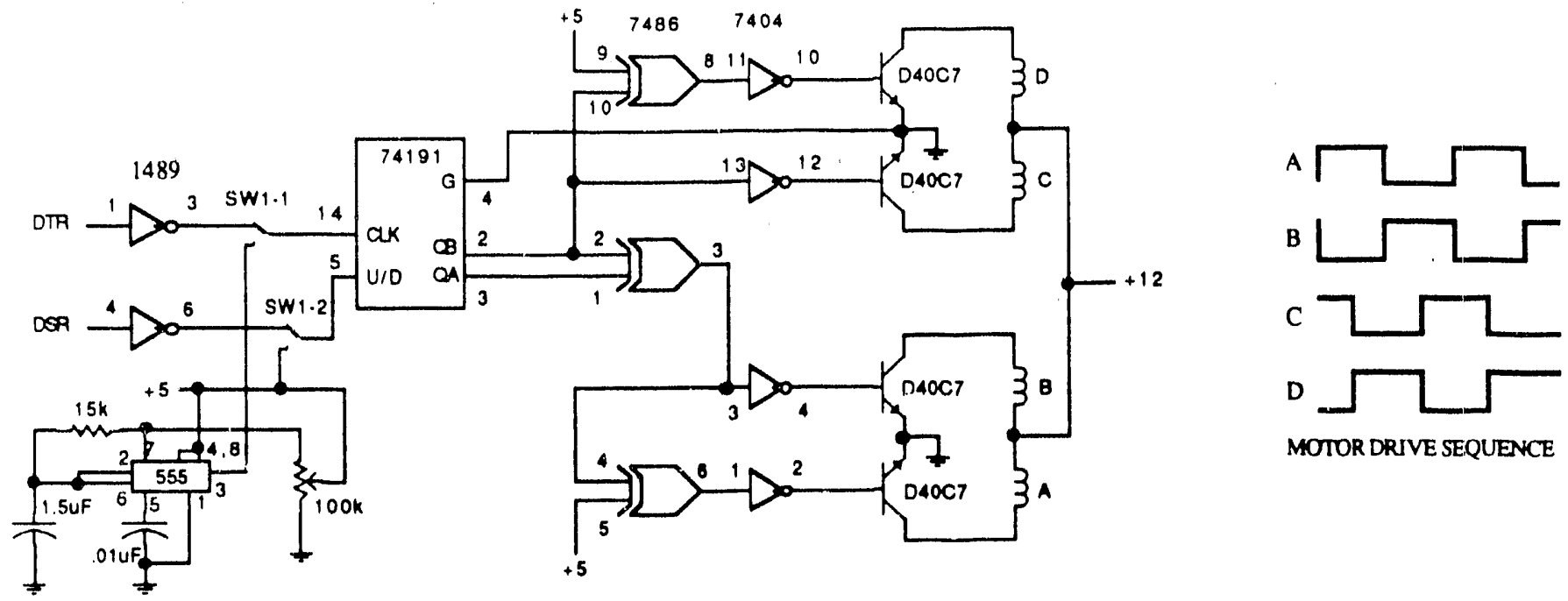

Figure 1. (a) Circu't diagram of the camera-motor controller and the step sequence it applies to the stepper motor windings.

The DTR signal from the workstation is connected to the line receiver of the CMC, which converts the RS-232C voltage levels to TTL. The control logic is provided by the binary up-down counter, which outputs a bidirectional four step sequence. By enabling DSR, which is connected to the down-up input of the counter, the step sequence and the motor direction are reversed. The exclusive-OR gates decode each of the four states into a drive sequence which is buffered to provide the output current capability required to toggle the coil drivers. When coils $\mathrm{A}$ and $\mathrm{C}$ of the motor are energized, they attract the nurth pole of the permanent magnet rotor. Since current through coils B and D is in the opposite direction, they attract the south pole of the rotor. As the drive sequence progresses, the coils are sequentially energized and the rotor tuins in steps. An astable timer (National Semiconductor, Part Number LM555CN) is uied in place of the DTR signal to provide a drive signal for mechanically advancing the motor independent of the workstation. Manual-mechanical advancement is useful while lrading and unloading film, and while advancing film between scenes.

Power is provided by a precision regulated 13.8 VDC power suppiv (Tripp Lite Division 


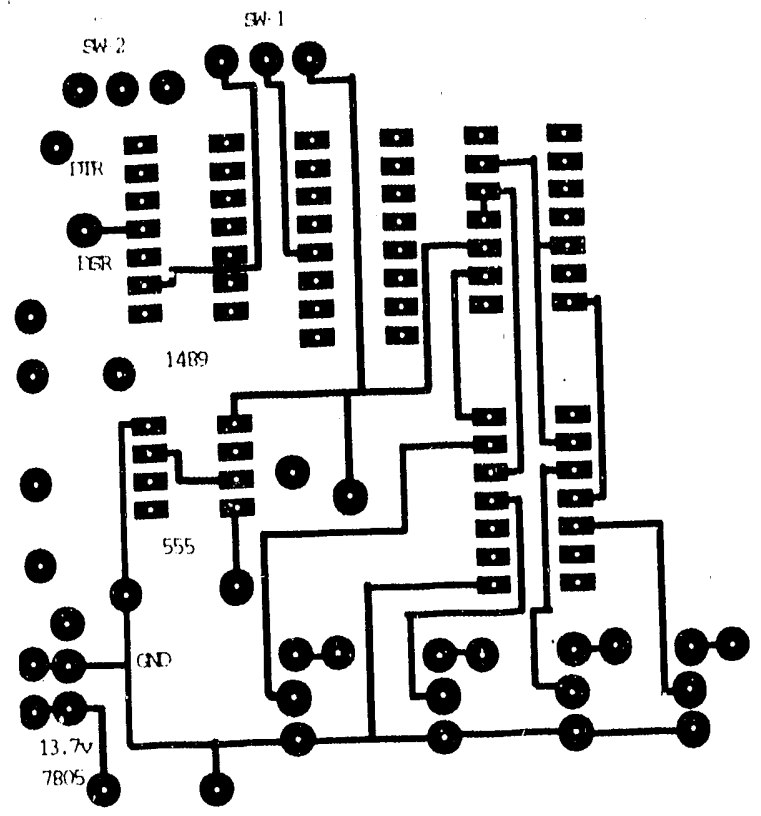

TOP

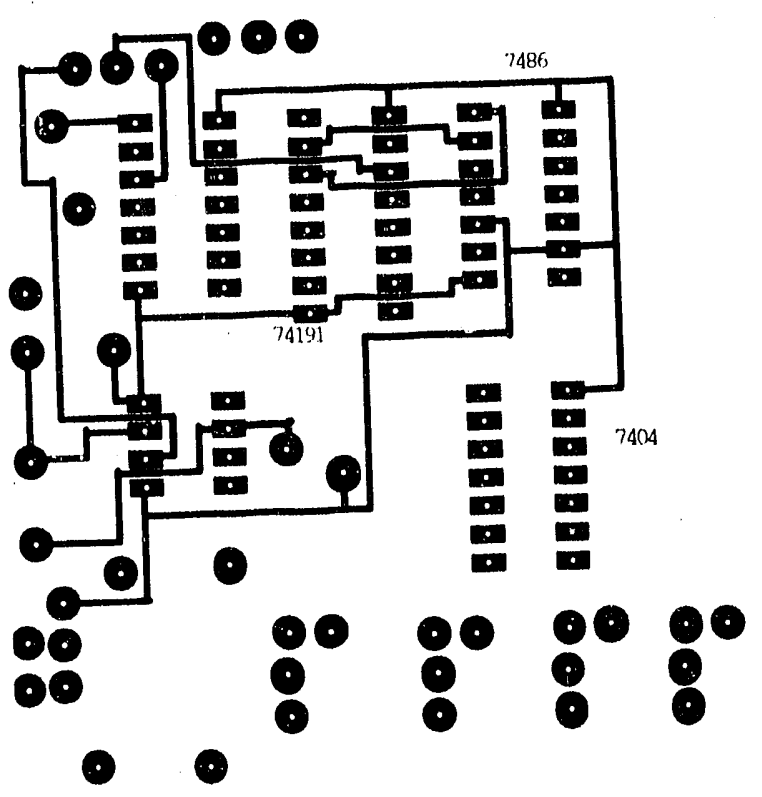

BO'TTOM

Figure 1. (b) Printed circuit layout for the controller. The top and bottom conducting foils of the double-sided board are shown in black.

of Trippe Manufacturing Company, Chicago, IL, model PR-3a) modified to +5 VDC for the control logic and $+12 \mathrm{VDC}$ for the motor coils. The +5 volts is obtained by the addition of a series voltage regulator (National Semiconductor, Part Number LM7805C).

To expose one frame, eight DTR transitions, $7 \mathrm{~ms}$ apart, are output to the CMC. This moves the camera drive train enough to open the camera shutter from a starting position, but not enough to advance the film. The film is exposed for the desired amount of time by suppressing DTR transitions for that period. Then 17 DTR transitions are output. This closes the shutter and advances the film to the next frame. Thus, the speed of film movement, the direction of film travel, and the exposure of the film are under direct control of the workstation. This permits animation software to fade scenes in and out, to dissolve between scenes, to animate periodic events from one period, and to effect slow motion, time lapse and time reversal.

\section{Animation Software}

The animation hardware can be used with any workstation or computer that can control signals on an output port via software, because all the $\mathrm{CMC}$ needs is at least one signal that can be switched on and off. The signal can come from a RS-232C serial port as described above, or it can come from a parallel port or from a bidirectional port. We have even used a Commodore 64 computer successfully to make animations by controlling a pin 
on its user port. However, our principal animation software was developed for the Apollo family of graphics workstations. The computer animation system software has three main programs:

1. Make_Bitmap, converts a numerical-data representation of an image into a bitmap compatible with Apollo Computer's DN580 or DN500 color workstations

2. Make Frame, creates the image captured on film by merging text and bitmaps created by Make Bitmap into a 1280 by 1024 frame buffer.

3. Umn. Movie, uses the bitmaps created by Make Frame or Make Bitmap to make a movie scene by repeatedly placing bitmaps on the computer screen and signaling the movie camera to advance one or more frames.

\section{Make_Bitmap}

Make Bitmap opens a data file that describes an image, creates a bitmap, and then saves the bitmap to disk. Currently two different data-file formats are supported. The first format represents a two-dimensional array of gray-scale data. The first line contains the $\mathrm{X}$ and $Y$ dimensions of the input array (which are also the dimensions of the output bitmap). The rest of the file is a list of numbers between 0 (black) and 1.0 (white) representing the two-dimensional array stored in row major form. The second format is an SRL file produced by Movie.byu (Christiansen, et al., 1987) The first line of an SRL file contains the background color's red, green, and blue components. The remaining lines contain scanline descriptions with the following format: $\mathrm{Y}$-value, starting $\mathrm{X}$-value, ending $\mathrm{X}$-value, starting red value, ending red value, starting green value, ending green value, starting blue value, and ending blue value. The red, green, and blue values are linearly interpolated across the given $\mathrm{X}$ interval. Make Bitmap takes as input a file containing data-file types ( 0 for grayscale and 1 for SRL files), data file names, and bitmap file names each on a line by themselves. For example, an input file that reads two grayscale data files, two SRL files, and creates four bitmap files (saved as bitmapl through bitmap4) looks like this:

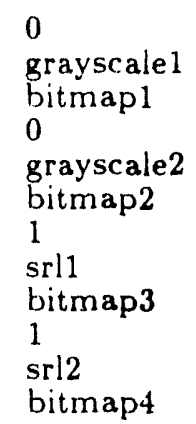

The following is the general algorithm in a pseudo-pascal syntax: program make_bitmap;

BEGIN

open(input file);

initialize graphics mode;

while not end of file(input file) do

read(input file, data file _type);

read(input file, data file name);

read (input file, bitmap file name); 


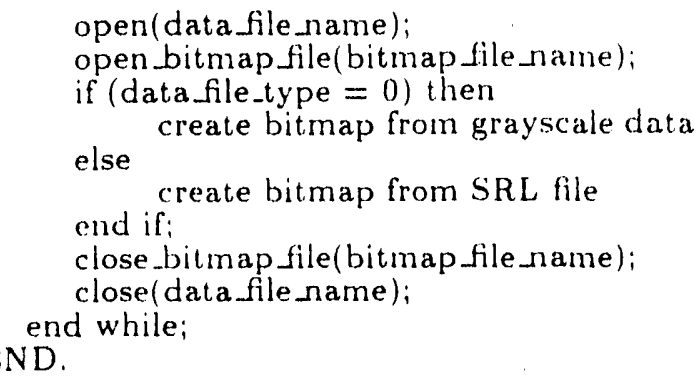

\section{Make_Frame}

This program takes the bitmaps saved by Make_bitmap and places them on the screen at coordinates supplied by the user. The user can then interactively add text anywhere in the frame buffer by using the mouse and keyboard. At the end of the program the screen is saved on the disk drives as a 1280 by 1024 bitmap file. Make Frame opens and reads a configuration file which describes the bitmap locations, and defines several constants used to fill the color map. Line One contains the red, green, and blue components of the background color, Line Two contains the maximum and minimum color map indices, and the maximum and minimum intensities, Line Three has the number of bitmaps to place on the screen, and the rest of the file has the name of the bitmap on a line by itself followed by the $\mathrm{X}$ and $\mathrm{Y}$ components of the upper left corner of the bitmap. The algorithm for this program is as follows:

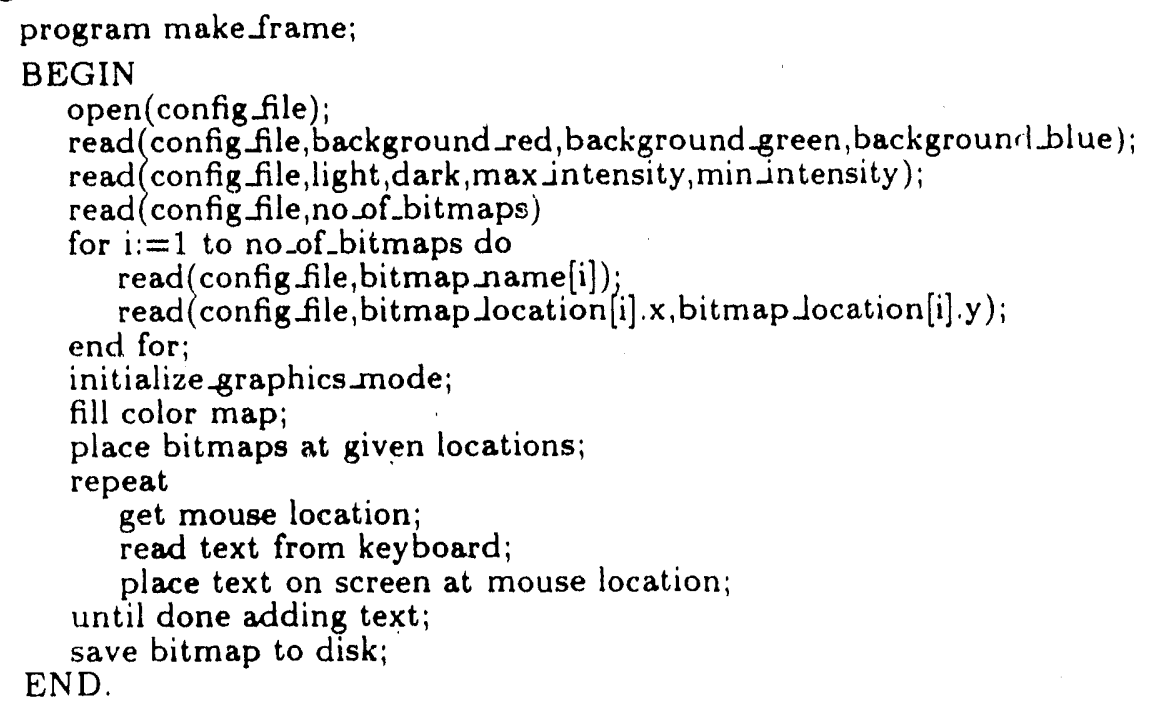

\section{Umn.Movie}

Umn. Movie is a program to capture bitmaps produced by Make Frame or Make.Bitmap on $16 \mathrm{~mm}$ film. Currently this program optionally fades in on a display, shoots a series film frames, then optionally fades out. As do the other programs, this one reads configuration data from an input file of the following format: Line One contains the number of frames 
to fade in (can be zero), the number of frames to expose for this bitmap, the number of frames to fade out (also can be zero), the length of time (in seconds) to expose each frame, the background red, green, and blue components, the maximum and minimum gray indices (between 0 and 255), the maximum and minimum intensities (between 0 and 1.0), and the number of bitmaps used to create the display. The rest of the file is made up of bitmap file names on a line by themselves with the next line containing the $X$ and $Y$ coordinates and an origin flag $(-1$ means $X$ and $Y$ are the center of the bitmap and 0 means $X$ and $Y$ are the upper left corner).

Consider the following Umn.Movie example: you have four 200 by 200 pixel bitmaps (called b1, b2, b3, b4) that you want to fade in from black, display for ten seconds, then fade out to black. The following configuration file will do this with 5 fade in frames, 240 full intensity frames, 5 fade out frames, an exposure time of 1.0 second, a blue background, gray levels ranging from 255 to 0 , maximum intensity of 1.0 and a minimum intensity of 0 :

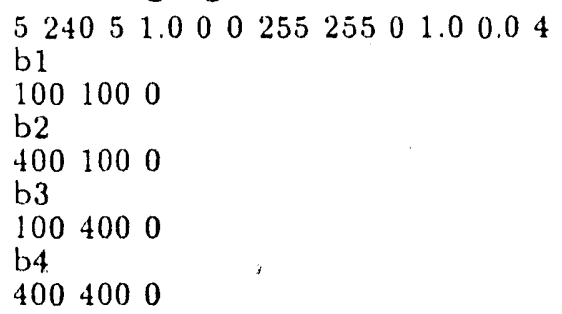

In summary, Umn. Movie puts a picture on the screen, opens the camera shutter by cycling DTR on the serial port, leaves the shutter open for the exposure length of time, and then closes the shutter by cycling DTR. A shell script can be used to call Umn.Movie with different configuration files to make a movie scene. The general algorithm is:

program umn.movie;

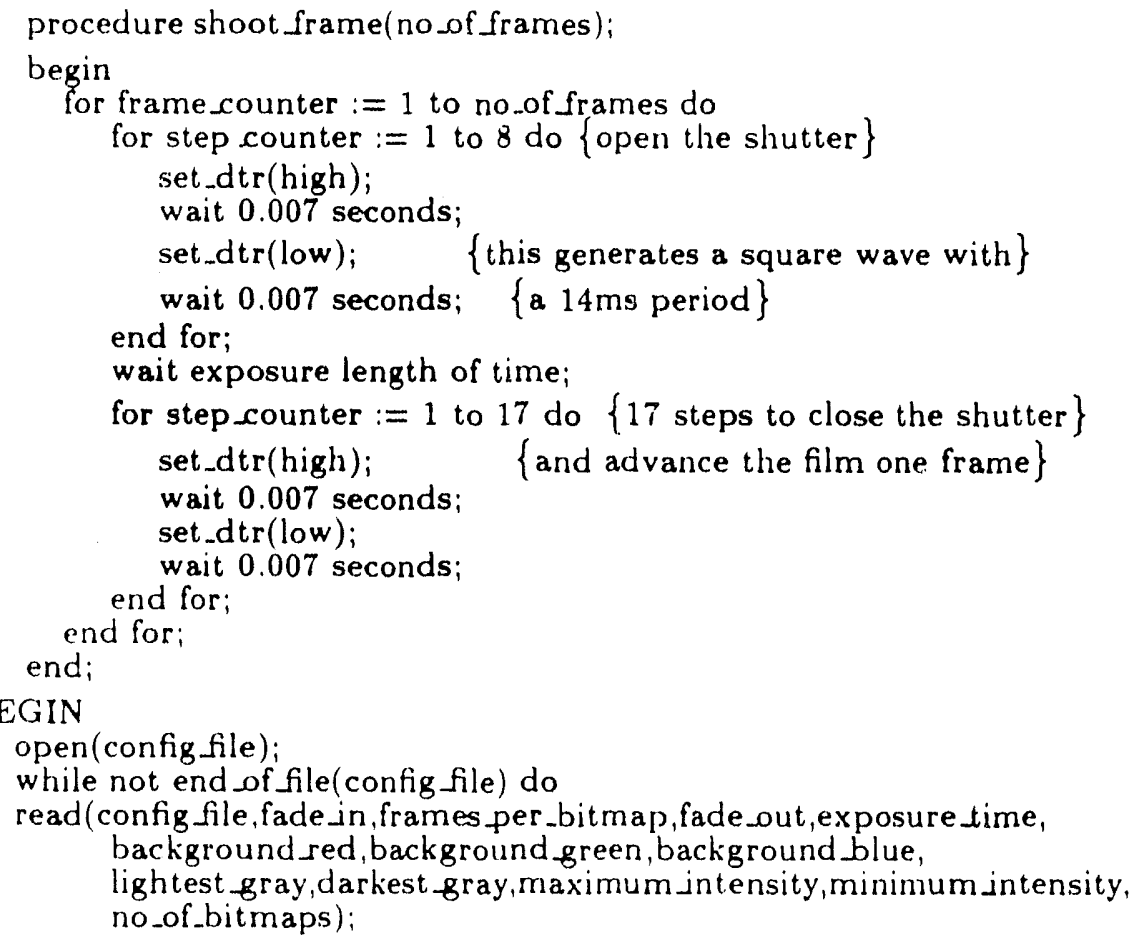




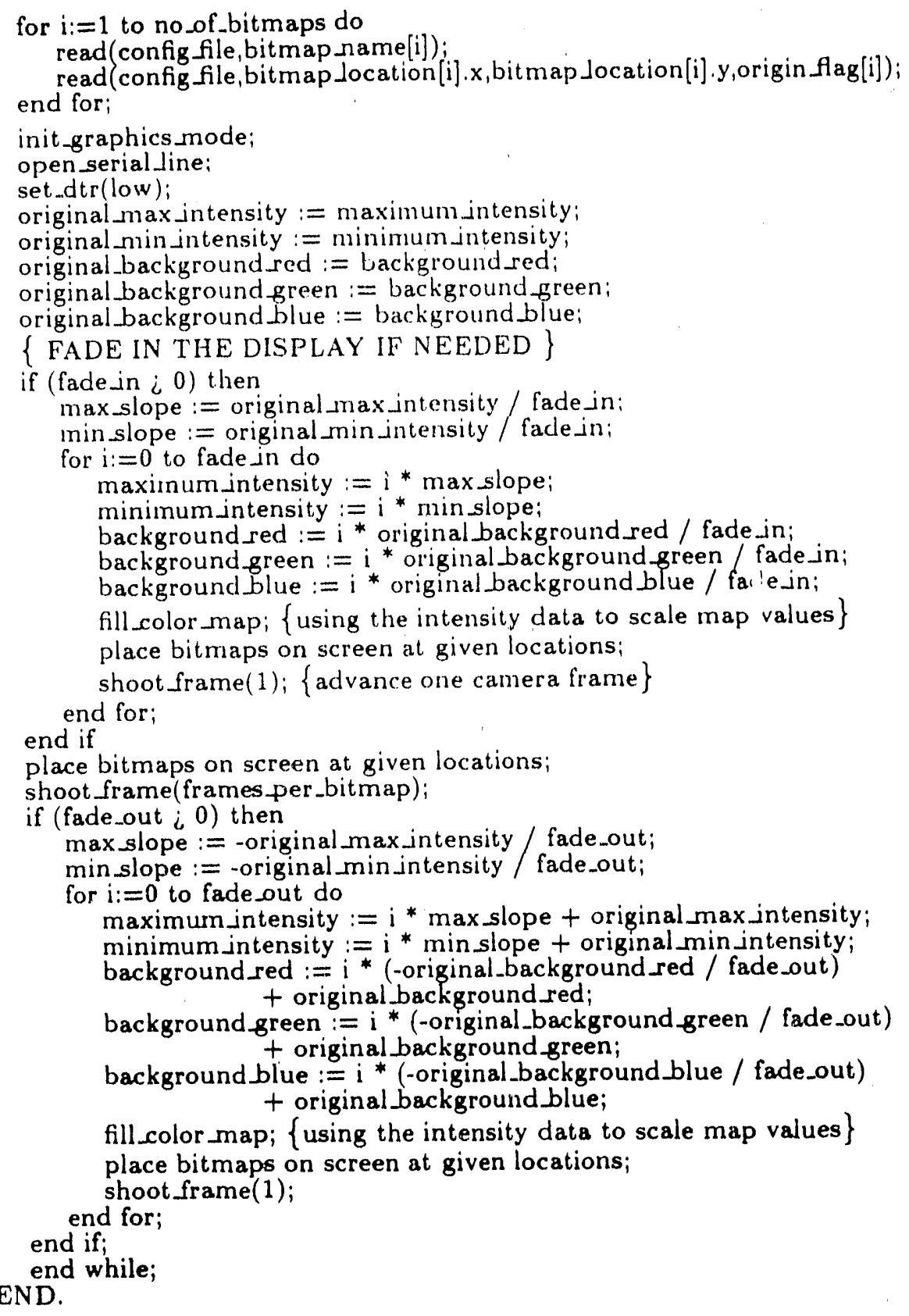

An animated movie is made by generating a series of frames and calling Umn.Movie to film the series in sequence. Each frame can consist of one or more bitmaps. Several sequences ("scenes") can be specified in one configuration file with fades and dissolves. Thus, one call to Umn.Movie can film the entire movie specified in a configuration file without any need for further human intervention. 


\section{Network Computing}

The animation system described above has one major limitation. Each bitmap is a 1280-by-1024 array of 24-bit integers (3.75 Mb. of data). So one second of film (at 24 frames per second and 3 frames per bitmap) can require as much as $30 \mathrm{Mb}$. of disk storage. This is a worst-case example and storage requirements can be reduced by not saving the background data or by using various data compression techniques (Johnston, et al.1088). To have a system which makes a movie of arbitrary length without humai intervention would require an unreasonable amount of clisk storage. A solution to this problem was found by using Apollo Computer's Network Computing System (NCS) (Apollow Computer Inc. 1987). NCS is made up of two components: the network interface definition language (NIDL) compiler, and the network computing kernel (NCK). An interface definition is written in NIDL syntax and passed through the NIDL compiler to create several include files and stub procedures for distributed applications. Stub procedures are the routines that make remote procedure calls look almost local. With NIDL, programers do not have to handle details like data format conversions across a heterogeneous network of computers. $\mathrm{NCK}$ is the run-time library that finds resources on the network by using a location broker.

Without NCS, the computationally intensive procedures (which create the numericaldata representations of an image) are done on a supercomputer and saved on magnetic tape. The tapes are then restored on the Apollo disk drives until available free space is exhausted. That portion of the data is run through Make_Bitmap, Make-Frame, and Umn.Movie and thus recorded on film. The old bitmaps and data files are then deleted and replaced with data for the next run (this step requires human intervention).

With NCS a remote procedure call is made to the supercomputer to generate the data needed for one workstation display. Those data are then processed by Make_Bitmap, Make Frame, and Umn.Movie and the whole process repeated for the next display. In this way very few data files and bitmaps need to be saved (and can be saved as temporary files).

\section{Results and Discussion}

Figure 2 shows one frame of the animated movie, which was recorded on Kodak 7292 with a $25 \mathrm{~mm}$ f $/ 1.4$ Switar lens exposed for $2 \mathrm{sec}$ at $\mathrm{f} / 16$ per frame and 3 frames per computer image. The Apollo version of the software and the movie (recorded in color on $16 \mathrm{~mm}$ film and $3 / 4^{\prime \prime}$ U-matic (NTSC) or $1 / 2^{\prime \prime}$ VHS (NTSC) videotape format), are available for loan from the authors. The figure shows polarized microscope images from a variety of objects selected to illustrate different possible shapes of liquid crystals: oblate spheroids, prolate spheroids, ellipsoids, fused spheres of equal diameters, fused spheres of unequal diameters, ring torus, spindle torus, and three types of Dupin cyclides. The movie clearly depicts the changes in the images as the objects are rotated, thereby providing a guide for interpreting images from polarized light microscopy. 


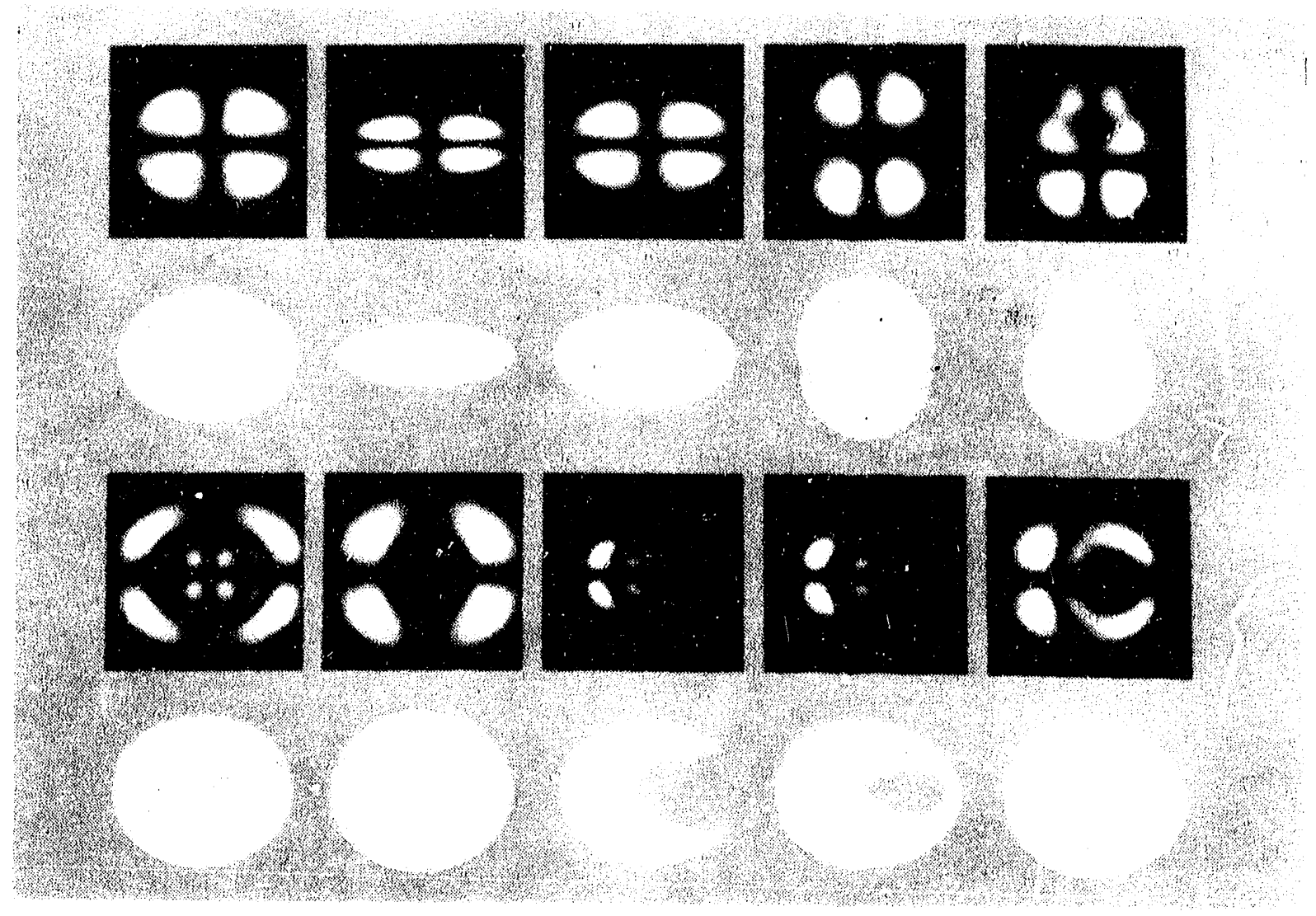

Figure 2. One frame of the movie generated using the animation system. It depicts liquid crystals of different shapes and the results of a special case of ray tracing through them. The special case is that of orthogonal polarizer and analyzer, which makes the ray tracing results simulations of cross-polarized optical microscope images of the objects. The objects are shown in perspective view along the same viewing direction as the images depicted below the object. The objects are (in left-toright and top-to-bottom order) oblate spheroid, prolate spheroid, ellipsoid, fused spheres of equal diameters, fused spheres of unequal diameters, ring torus, spindle torus, horned Dupin cyclide, ring Dupin cyclide, and spindle Dupin cyclide. 
The animation system permits recording of graphics at high resolution on film with equipment that costs about an order of magnitude less than equivalent video equipment. The system is so flexible that it can be used with any computer that can toggle an output pin. Because the speed and direction of film transport can be controlled by the software, special effects can be generated easily. The success of the system is, we believe, clearly demonstrated in the movie we made. When used in a distributed computing environment with network computing software, it becomes a powerful yet affordable tool to produce animations of supercomputer simulations efficiently.

\section{References}

Anderson, D. M., Davis, H. T., Nitsche, J. C. C. and Scriven, L. E., Advances in Chemical Physics 77, 377 (1990).

Apollo Computer Inc. (1987), Network Computing System Reference, Order No. 010200 Revision 00, Chelmsford, MA 01824.

Bellare, J. R., Davis, H. T., Miller, W. G. and Scriven, L. E. (1989), J. Colloid and Interf. Sci., 136 , 305 (1990).

Christiansen, H., Stephenson, M., Nay, B. and Grimsrud, A. (1987), Movie.byu Training Text, 2nd edition, Bookcrafters.

Dupin, Charles (1822), Applications de geometrie et de mechanique, a la marine, aux ponts et chaussees, etc., pour faire suite aux developpements de geometrie, Paris: Batchelier.

Johnston, W. E., Hall, D. E., Renema, F. and Robertson, D. (1987), Principles and techniques for low cost computer generated video movies, LBL-22330, Univ. of California, Lawrence Berkeley Laboratory, Berkeley, CA.

Johnston, W. E., Hall, D. E., Huang, J., Rible, M. and Robertson, D. (1988), Distributed scientific video movie making, LBL-24996, Univ. of California, Lawrence Berkeley Laboratory, Berkeley, CA.

Maxwell, J. C. (1869), On the cyclide, Quart. J. Pure and Appl. Math., 9, 111-126. 


\section{SURFACES OF CONSTANT MEAN CURVATURE WITH PRESCRIBED CONTACT ANGLE}

\section{Synopsis}

A Galerkin weighted resiclual formulation of the Surface Divergence Theorem is used with finite element basis functions to compute geometric moclels of foam and emulsion structure. The models are surfaces of constant mean curvature arranged on a simple cubic lattice so that they meet the bounclary planes of the unit cells at prescribed contact angles. Surfaces are computed for a variety of contact angles and mean curvatures. With contact angle between $5^{\circ}$ and $133^{\circ}$, the structure inverts as the mean curvature decreases, whereas with contact between $134^{\circ}$ and $180^{\circ}$, the structure fills the unit cell as the mean curvature decreases, passes through a turning point, and then increases. The models have implications to mercury porosimetry.

\section{Introduction}

The relationships between the structures and properties of foams and emulsions are not yet fully understood. An emulsion or a foam is clefined in general terms as a reasonably stable dispersion of one fluid phase in another, immiscible, fluid phase. Various additives may prevent the dispersion from separating for a period of time yet it is not thermodynamically stable. The relationships between physical structures and physical properties of such dispersions are of practical as well as theoretical interest. These relationships can be computed from three-dimensional models of the structures. At volume fractions below some critical value, there is an 'internal' phase that is dispersed in a continuous 'external' phase and the former's form can be modeled as undistorted spheres. At volume fractions approaching unity, the 'internal' phase forms polyhedral structures and the 'external' phase is limited to thin films between polyhedral faces and channels at the polyhedral edges. At intermediate volume fractions, there are structures that are not well characterized, ruch less well understood. Physical properties camnot be accurately simulated without adequate model structures.

Lissant and coworkers (1066, 1970, 1973, and 1974) modeled monodispersed emulsions with simple geometric structures. Sphcres were packed in unit cells of regular lattices: simple cubic, tetrakaidecahedral, and rhomboiclal dodecahedral. The spheres were undistorted until the volume fraction of the internal phase reached the critical volume fraction, namely that at which the spheres first touched their neighbors. As the volume fraction was raised further, the spheres were either rearrangerl to form a more relaxed packing on a different lattice or they were distorted at constant volume. The distortion was characterized by the point of contact between the spheres growing to form a circular disk of thin film. The remaining curved surfaces were assumed to retain spherical shapes as the volume fraction of the internal phase increased. Sucil an assumption required the contact angle between 
the curved surface and the film to change as the volume fraction of the internal phase was expanded and the film was compressed.

Princen (1070) and Princen, et al. (10S(0) recognized that the curved surface would have constant mean curvature, the contact nugle between it and the thin film would not change as the volume fraction is raised, and the thin films would not be circular. Princen (1980) suggested that advanced numerical techniques might be employed to find constant mean curvature surfaces that meet unit cell boundaries at a specified contact angle.

The situation of a constant mean ('urvature surface separating two immiscible fluids and contacting boundary planes at a contact angle other than zero arises elsewhere than in foum and emulsion structures. Mercury porosimetry is a technique by which a distribution of pore sizes is deduced from the anount of mercury that enters an evacuated porous medium as a function of pressure applied to the mercury. Mercury makes contact angles with solids in the range of $130--140^{\circ}$ (Heinenz, 1086) and is therefore a partially wetting liquicl. A complex three-dimensional pore structure is often modeled as an idealized array of cylindrical capillaries of various diancters. The curvature of the mercury-gas surface, and therefore the diameter of the capillary invaded, is proportional to the injection pressure relative to ambient, $\Delta P$, according to the Young-Laplace equation, $\Delta P=2 H v$, where $H$ is the mean curvature (the reciprocal of capillary diameter in the model), and $\sigma$ is the surface tension. Real pore space is not a bundle of simple tubes and the model cloes not account for such events as mercury being forced into grooves and corners without necessarily invading new pores as the pressure is increased. When a porous medium is occupied by two fluid phases, the grooves and corners, regions of high wall curvature, are the first to fill with wetting liquid when its content is increased. The flow channels these regions form when they are connected are the chief routes by which more of the wetting liquid can enter or, alternatively, can leave as the content of wetting liquid is decreased.

Examples that have been exanined include wetting fluid being displaced by nonwetting fluid in toroidal pores with grooves in the wall (Roof 1970), in square capillaries (Lenormand et al. 1984, Arriola et al. 1983, Legait 1983, and Gauglitz et al. 1987), and in triangular capillaries (Singhal and Somerton 1970). To a first approximation, the connected channels are covered by menisci of constant mean curvature. To a second approximation, they have sinall gradients of mean curvature and often can be approximated by the assumption that curvature in the axial direction is small (Ransohoff and Radke 1988).

Anderson et al. (1990) developed a method to construct smooth constant mean curvature surfaces that are periodic in three dimensions, i.e., that have the symmetries of a space group. The method consists of computing the surface in a single primitive cell of the corresponding point group, the walls of which are symmetry planes and so they are necessarily intersected by the surface at right angles. The full surface can then be assembled from rotated, reflected, and translated replicas of the primitive cell.

In this work, the same method was used to construct constant mean curvature surfaces 
of simple cubic symmetry that meet the walls of the unit cell, a cube, at a prescribed contact angle. The algorithm developed by Anderson et al. (1090), see also Anderson (1986), was modified, as described below, to compute the surface in the primitive cell, one of the walls of which is not, in general, a symmetry planc.

\section{Computational method}

Within a single unit cube, the constant menn curvature surface divides space into an internal domain and an external domain (see Figure 1). The internal domain lies between the center of the unit cell and the curved surface; the external domain lies between the surface and the edges and corners of the cube. On each wall of the cube, there is a region contained in the interior of the surface. These regions are referred to as flat films because in a physical system the liquid occupying the internal domain forms a fllm along the cube wall.

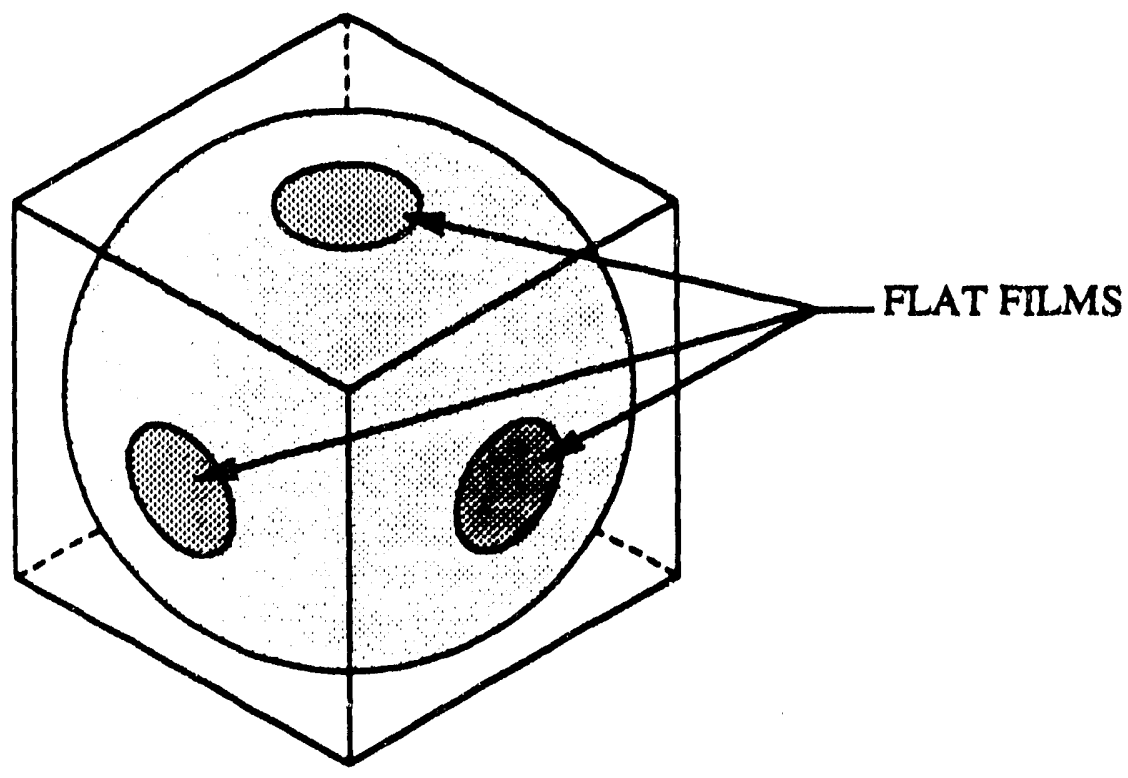

Figure 1. Single simple cubic unit cell. Flat films are shown on the boundary planes of the unit cell.

The simple cubic unit cell was subdivided by its planes of reflective symmetry into forty-eight primitive cells, tetrahedrons, as shown in Figure 2a. The portion of the constant mean curvature surface contained within a primitive cell was mapped onto a twodimensional domain. The desired surface was then computed as a solution to a form of the Surface Divergence Theorem in which the surface itself is unknown. Surface area and volume fractions were computed from the solution. The surface was drawn in threedimensional representation by the reverse process: the two-dimensional 'computational' 
domain was mapped into the primitive cell, the primitive cell was reflected in the symmetry planes to recreate the anit cell, and, when desired, the corresponding periodic surface was created by translations of the mit coll.

CUBIC UNTT CELL

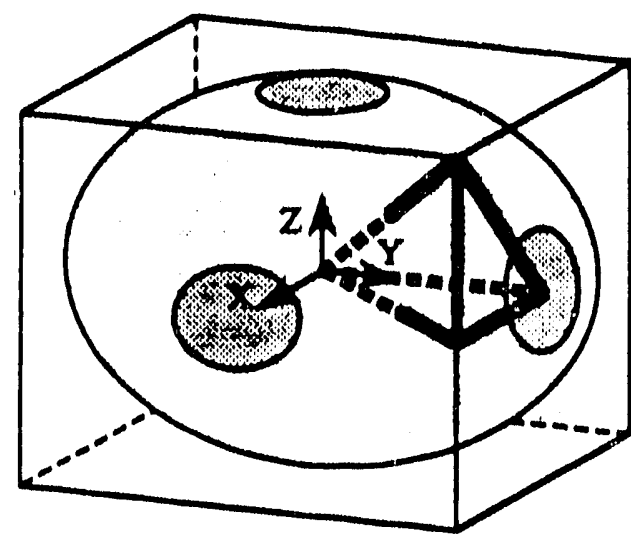

TETRAHEDRAL CELL

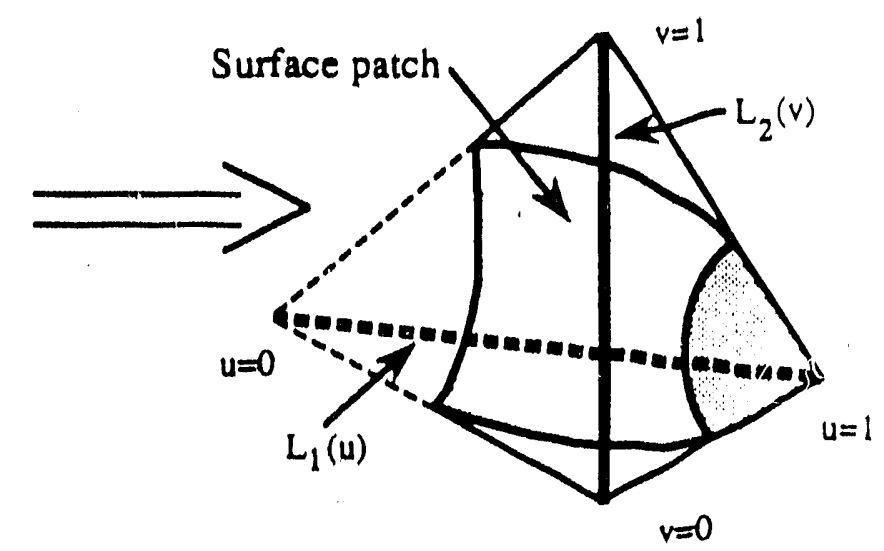

Figure 2. Reduction of unit cell to tetrahedral primitive cell.

The surface patch within a single tetrahedron was mapped onto a two-dimensional domain in the way adopted by Anderson (1986) to take advantage of the geometry of the tetrahedron (see Figure 2): two opposed edges, $\mathrm{L}_{1}$ and $\mathrm{L}_{2}$, of the tetrahedron are orthogonal and never intersect. $\mathbf{L}_{1}$ is a segment of a line connecting the center of the unit cell to the center of a neighboring cell. $L_{2}$ is a segment of an edge of the unit cell. The variable $u$ was clefined to vary from zero to one on the length of $L_{1}$; the second variable, $v$, was defined to vary from zero to one on the length of $\mathrm{L}_{2}$. In cartesian coordinates, the line segments were $\mathrm{L}_{1}(0, u / 2,0)$ and $\mathrm{L}_{2}\left(1 / 2,1 / 2, u^{\prime} / 2\right)$. The surface is the locus of inten sections of the surface and line segments drawn from $\mathrm{L}_{1}(u)$ to $\mathrm{L}_{2}(v)$ :

$$
\mathbf{X}(u, v)=\mathbf{L}_{1}(u)+w(u, v)\left(\mathbf{L}_{2}(v)-\mathbf{L}_{1}(u)\right) .
$$

The variable $w(u, v)$ is simply the fractional clistance from $\mathbf{L}_{1}(u)$ to the intersection Thus, each point on the surface maps onto a unique point on the two-dimensional $(u, v)$ domain provided the line segment $\mathrm{L}_{2}(v)-\mathrm{L}_{1}(u)$ intersects the surface once and only once; otherwise, the mapping fails.

With this representation, the unknown surface $\mathbf{X}$ was computed from the surface 
divergence theorem, in which it oc'uss us an unlanown (Weatherburn 1027):

$$
\iint_{S} \nabla_{S^{\prime}} \cdot \mathbf{F} d A=\oint_{\partial S^{\prime}} \mathbf{V} \cdot \mathbf{F} d s-\iint_{S} 2 H \mathbf{N} \cdot \mathbf{F} d \cdot A
$$

Here $\partial S$ is the curve that bounds the surface $S, \nabla_{s^{\prime}}$ is the surface divergence operator, $\mathbf{F}$ is an arbitrary vector field, $V$ is the unit vector locally nomal wo $\partial S$ outwardly pointing, and tangent to the surface, and $\mathrm{N}$ is the unit vector normal to the surface, and $H$ is the mean curvature. The mean curvature was used as a constant in this work but can be chosen as an arbitrary function of position. Anderson's (1086) choice of the vector field is the key: $\mathbf{F}=\frac{\partial \mathbf{X}}{\partial u^{\prime}} \phi^{i}(u, v)=\left(\mathbf{L}_{2}(v)-\mathbf{L}_{1}(u)\right) \phi^{i}(u, u)$, where $o^{i}(u, u)$ is any member of the set of basis functions in which the unknown surface is to represcnted. The scalar product $V \cdot F$ is a function of the angle between the suffuce mal, in succession, the boundary planes of the primitive cell. On the symmetry planes it vanishes and on the plane that is a wall of the unit cell it is

$$
\mathbf{V} \cdot \mathbf{F}=\mathbf{V} \cdot \frac{\partial \mathbf{X}}{\partial w} \phi^{i}=\left|\mathbf{L}_{2}(u)-\mathbf{L}_{1}(u)\right| \phi^{i} \cos \gamma \sin \eta
$$

where $\gamma$ is the specified contact angle and $\eta$ is the computed angle between the surface tangent vector and $\mathbf{F}$.

Eq. 2 was solved by the Galerkin method with finite element basis functions. The two-dimensional $(u, v)$ domain was subdivided into $20 \times 20$ square elements. The unknown variable $w$ was approximated by 441 bilinear basis functions, i.e., $w=\sum_{j} \alpha_{j} \phi^{j}(u, v)$. With $\mathbf{F}$ chosen as above, the surface divergence theorem then yields 441 weighted residual equations, $i=1$ to 441 , for the 441 unlinown coefficients, $\alpha_{j}$ :

$$
R_{\mathbf{i}}=\iint_{S} \nabla_{S} \cdot \frac{\partial \mathbf{X}}{\partial w} \phi^{i} d A-\oint_{\partial S^{\prime}}\left|\frac{\partial \mathbf{X}}{\partial w}\right| \phi^{i} \cos \gamma \sin \eta d s+\iint_{S} 2 H \mathbf{N} \cdot \frac{\partial \mathbf{X}}{\partial w} \phi^{i} d A=0
$$

The finite element representation of the surface enters through the derivatives of $\mathbf{X}$ with respect to $u\left(\mathbf{X}_{u}\right)$ and $v\left(\mathbf{X}_{v}\right)$, and their appearance in $\nabla_{S}, d A, d s$, and $\mathbf{N}$ :

$$
\begin{gathered}
\nabla_{S}=\frac{\left(\mathbf{X}_{v} \cdot \mathbf{X}_{v}\right) \mathbf{X}_{u}-\left(\mathbf{X}_{u} \cdot \mathbf{X}_{v}\right) \mathbf{X}_{v}}{\left|\mathbf{X}_{u} \times \mathbf{X}_{v}\right|^{2}} \frac{\partial}{\partial u}+\frac{-\left(\mathbf{X}_{u} \cdot \mathbf{X}_{v}\right) \mathbf{X}_{u}+\left(\mathbf{X}_{u} \cdot \mathbf{X}_{u}\right) \mathbf{X}_{v}}{\left|\mathbf{X}_{u} \times \mathbf{X}_{v}\right|^{2}} \frac{\partial}{\partial v} \\
d A=\left|\mathbf{X}_{u} \times \mathbf{X}_{v}\right| d u d v \\
d s=\left|\mathbf{X}_{u}\right| d u+\left|\mathbf{X}_{v}\right| d v
\end{gathered}
$$

and

$$
\mathbf{N}=\frac{\mathbf{X}_{u} \times \mathbf{X}_{v}}{\left|\mathbf{X}_{u} \times \mathbf{X}_{v}\right|}
$$

The system of nonlinear algebraic equations, Eq. 4, was solved for the unknown $\alpha_{j}$ 's by Newton's method. 'The first surface computed was the Schwarz surface of zero mean 
curvature and $90^{\circ}$ contact angle. The Schwarz surface divides space evenly, one-half of space on one sicle, one-half of space on the other. The initial estimate of $\alpha_{j}=0.5$ for all $j$ was used for the Schwarz surface. Initial estimates were generated by first-order continuation in mean curvature when families of surfaces with the same contact angle were being computed. The first-order continuation was used as described by Anderson (1986). Zeroth-order continuation in contact angle was used when families of surfaces with the same mean curvature but different contact angles were being computed. Newton's method was iterated until the solution, $w$, was within as specified tolerance: $\left\|w^{k+1}-w^{k}\right\|<10^{-6}$ wh e $k$ is the number of iterations. The computations were performed on a Cray-2 supercomputer.

The total surface area that was computed was the area of the curved constant mean curvature surface plus the area of the six flat films at the walls of the cube (see Figure 1):

$$
A=48 \int_{0}^{1} \int_{0}^{1}\left|\frac{\partial \mathrm{X}}{\partial i i} \times \frac{\partial \mathrm{X}}{\partial v}\right| d u d v+48 \int_{0}^{1} \frac{w^{2}}{8} d v .
$$

The volume fraction was clefined as the ratio of internal domain volume to the volume of the unit cube:

$$
V=48 \int_{0}^{w} \int_{0}^{1} \int_{0}^{1} \frac{\partial(x, y, z)}{\partial(u, v, i v)} d u d v d w=48 \int_{0}^{1} \int_{0}^{1} \frac{1}{8}\left(\frac{w^{2}}{2}-\frac{w^{3}}{3}\right) d u d v .
$$

Eq.s 9 anc 10 were computed from the solution of Eq. 4 by Gaussian quadrature with four quadrature points per element.

The unit of length throughout the computations was the edge length $a$ of the cubic unit cell. Thus the reported mean curvatures are dimensionless products of mean curvature and edge length. Likewise, surface area are in units of $a^{2}$.

\section{Results and discussion}

Surfaces of broad ranges of mean curvature and contact angle were computed. The algorithm was first tested by computing simple surfaces for which surface area and volume fraction can be found from mensuration formulas.

A sphere centered in a cube intersects the walls if its diameter exceeds the cube's edge length. The angle at which the portion of spherical surface within the cube contacts the walls is $\gamma=\cos ^{-1}(-H / 2)$. The mean curvature, $H$, of the spher al surface is simply the reciprocal of the sphere radius. The total surface area within the cube is the surface area of the sphere less that of the six caps that lie outside plus that of the six flat circular films on the walls:

$$
\text { Total Area }=\frac{-4 \pi}{H^{2}}\left(2-\frac{3}{2} H\right)+6\left(\frac{\pi}{4} \tan ^{2} \gamma\right)
$$

The fraction of the cube volume that lies within that surface is

$$
\text { Volume fraction }=\frac{\pi}{3} \frac{1}{H^{3}}(9 H-8)-\frac{\pi}{4} .
$$


A comparison of surface areas computed by mensuration formula of Eq. 11 to those computed by Eq. 9 from solutions of Eq. 4 by Galerkin's method with 441 bilinear basis functions is listed in Table 1. A comparison of volume fractiotis computed by mensuration formula of Eq. 12 to those computed by Eq. 10 from solutions of Eq. 4 by Galerkin's method with 441 bilinear basis functions is listed in Table 2 .

The limiting case is the inscribed splere, which has mean curvature 2 and makes $180^{\circ}$ with the walls at the points of contact: the surface area is $\pi$ volume fraction is $\pi / 6$. The surface area computed by Eq. 0 is in error by $8.65 \%$; the volume fraction computed by Eq. 10 is in error by $12.9 \%$. The sphere is tangential to the walls of the unit cube; the edge of the surface at the cube wall is a single point. The primitive tetrahedral cell (Figure 4.2) used in the mapping of surfaces contains a three-sided surface patch. As a consequence, the mapping of $\mathrm{Eq}$. 4.1, which mips a .uur-sided surface patch to a twodimensional computational region, fails.

The computed surface with $170^{\circ}$ contact angle and $H=1.9696$ has error in total surface area of $4.33 \%$ and error in volune fraction error of $6.3 \%$. The mapping of this surface is close to failing because the edge of the surface at the cube wall is very small compared to the orher three surface edges in the primitive tetrahedron. The radii of the circular films on the cube walls is $0.09 a$ where a is the edge length of the unit cube. At $180^{\circ}$ contact angle, the surface edges on the other three walls of the primitive tetrahedral cell have radii of $0.5 a$.

The length of the surface edge at the cube walls increases as contact angle decreases. The radius, $r$, of the circular films is related to contact angle as $r=-0.5 a \tan \gamma$. The errors in computed total surface area of surfaces with contact angle between $160^{\circ}$ and $140^{\circ}$ were less than $0.1 \%$. The errors in computed volume fraction of the surface with $160^{\circ}$ contact angle was $0.13 \%$ while the errors of surfaces with contact angle between $155^{\circ}$ and $140^{\circ}$ were $0.04 \%$ or less. The accuracy of the computations increased as the contact angle was decreased because the length of the surface edges at the cube walls increased. Spherical surfaces that make contact angles with the unit cube walls less than $135^{\circ}$ are disjoint within the cube, i.e., they are confined to the eight cube conners. Again, the primitive tetrahedral cell $\mathrm{cos}$ ains three-sided surface patches and the mapping at Eq. 1 fails.

Two other surfaces were used for tests. One surface is a polyhedral structure composed of squares at the six walls of the unit cube and a total of eight equilateral triangles connecting the squares (see Figure 4.5 lower right). This surface has $125.26^{\circ}$ contact angle with the cube walis and $H=0$. The surface area is $\sqrt{3}$ and the volume fraction of the internal domain is $5 / 6$. The computed surface area is 1.7245 , in error by $0.44 \%$; the computed volume fraction is 0.8345 , in error by $0.14 \%$ The Schwarz surface has zero mean curvature and makes $90^{\circ}$ contact angle with the walls (Schwarz 1865; see Figures 4.6 upper and lower right). The surface area, 2.3453, and volume fraction, 0.5000 , computed by this method were withn $0.01 \%$ and $0.00 \%$, respectiveiy, of the values 2.3451 and 0.5000 reported by Schoen (1970). 
Table 1

\begin{tabular}{c|c|ccc}
\hline$H$ & $\gamma$ & $\begin{array}{c}\text { TOR.IULA } \\
\text { AREA }\end{array}$ & $\begin{array}{c}\text { COMP AREA } \\
\text { AREA }\end{array}$ & $\begin{array}{c}\text { ERROR } \\
\%\end{array}$ \\
\hline 2.0000 & 180.0 & 3.1416 & 3.4132 & 8.65 \\
1.9696 & 170.0 & 3.2381 & 3.3782 & 4.33 \\
1.8794 & 160.0 & 3.5384 & 3.5371 & 0.04 \\
1.8126 & 155.0 & 3.7744 & 3.7768 & 0.06 \\
1.7320 & 150.0 & 4.0760 & 4.0784 & 0.06 \\
1.6383 & 145.0 & 4.4522 & 4.4543 & 0.05 \\
1.5321 & 140.0 & 4.9140 & 4.9156 & 0.03 \\
\hline
\end{tabular}

Table 2

\begin{tabular}{|c|c|c|c|c|}
\hline \multirow[b]{2}{*}{$H$} & \multirow[b]{2}{*}{$\gamma$} & \multicolumn{3}{|c|}{ VOLUME FRACTION } \\
\hline & & $\begin{array}{c}\text { FORMULA } \\
\text { VOL. F. }\end{array}$ & $\begin{array}{c}\text { COMPUTED } \\
\text { VOL. F. }\end{array}$ & $\begin{array}{c}\text { ERROR } \\
\%\end{array}$ \\
\hline $2.000 C$ & 180.0 & 0.5236 & 0.5910 & 12.87 \\
\hline 1.9696 & 170.0 & 0.5476 & 0.5821 & 6.30 \\
\hline 1.8794 & 160.0 & 0.6200 & 0.6201 & 0.13 \\
\hline 1.8126 & 155.0 & 0.6764 & 0.6767 & 0.04 \\
\hline 1.7320 & 150.0 & 0.7439 & 0.7442 & 0.04 \\
\hline 1.6383 & 145.0 & 0.8208 & 0.8211 & 0.04 \\
\hline 1.5321 & 140.0 & 0.9002 & 0.9004 & 0.02 \\
\hline
\end{tabular}


Comparisons of surface areas and volume fractions computed by mensuration formulas to those computed by Galerkin's method with different numbers of bilinear basis functions are listed in Table 3. The error in total surface area of the computed surfaces with $180^{\circ}$ contact angle increased from $7.09 \%$ for 121 basis functions to $9.01 \%$ for 961 basis functions. The error in volume fraction of these same surfaces increased from $10.26 \%$ for 121 basis functions to $13.50 \%$ for 961 basis functions. The use of more basis functions actually decreased the computational accuracy. The crror in total surface area of the computed surfaces with $150^{\circ}$ contact angle decreased from $0.25 \%$ for 121 basis functions to $0.02 \%$ for 961 basis functions. The error in rolume fraction of these same surfaces decreased from $0.70 \%$ for 121 basis functions to $0.01 \%$ for 961 basis functions. The use of more basis functions increased the computational accuracy. 441 basis functions were used in subsequent calculations.

Table 3

\begin{tabular}{c|c|c|cc|cc}
\hline $\mathrm{H}$ & $\gamma$ & $\begin{array}{c}\text { BASIS } \\
\text { FUNCTIONS }\end{array}$ & $\begin{array}{c}\text { TOTAL } \\
\text { AREA }\end{array}$ & $\begin{array}{c}\text { ERROK } \\
\%\end{array}$ & $\begin{array}{c}\text { VOLUME } \\
\text { FRACT. }\end{array}$ & $\begin{array}{c}\text { ERROR } \\
\%\end{array}$ \\
\hline 2.0000 & \multirow{2}{*}{$180.0^{\circ}$} & FORMULA & 3.1416 & $* * * * *$ & 0.5236 & $* * * * *$ \\
& & 121 & 3.3643 & 7.09 & 0.5773 & 10.26 \\
& & 256 & 3.3999 & 8.22 & 0.5873 & 12.17 \\
& & 441 & 3.4132 & 8.65 & 0.5910 & 12.87 \\
& & 676 & 3.4204 & 8.87 & 0.5930 & 13.25 \\
& & 961 & 3.4248 & 9.01 & 0.5943 & 13.50 \\
\hline 1.7320 & \multirow{2}{*}{$150.0^{\circ}$} & FORMULA & 4.0760 & $* * * * *$ & 0.7439 & $* * * * *$ \\
& & 36 & 4.1161 & 0.98 & 0.7491 & 0.70 \\
& & 1.21 & 4.0862 & 0.25 & 0.7452 & 0.17 \\
& & 256 & 4.0804 & 0.11 & 0.7445 & 0.08 \\
& & 441 & 4.0784 & 0.06 & 0.7442 & 0.04 \\
& & 676 & 4.0775 & 0.04 & 0.7441 & 0.03 \\
& & 961 & 4.0770 & 0.02 & 0.7440 & 0.01
\end{tabular}

A series of surfaces with $180^{\circ}$ contact angle were computed. Four surfaces with $180^{\circ}$ contact angle are shown in Figure 3. The surface with $H=2$ and $180^{\circ}$ contact angle corresponds to an undistorted sphere. As the curvature is increased, the point of contact between the sphere and the cube wall flattens to form a circular film. However, as the curvature is increased further, the film loses its circular shape and the curved surfaces are no longer spherical. The surface distorts to fill into the edges of the cube. Consequently, the volume fraction of the internal domain approaches one and the total surface area approaches six, the surface area of the cube. During this distortion, the surface remains continuous, which means the external clomain also remains continuous as narrow channels along the cube edges. Surfaces of $180^{\circ}$ contact angle were computed with mean curvature up to $H=100$. The surfare areas of the curved constant mean curvature surfaces as 
functions of mean curvature and contact angle are shown in Figure 9. Volume fractions of the internal domain are shown as functions of mean curvature and contact angle in Figure 10. Surface areas and volune fractions of constant mean curvature surfaces are listed in tables of Appendix $B$.

Another series of surfaces was computed but with $150^{\circ}$ contact angle. Four surfaces with $150^{\circ}$ contact angle are shown in Figure 4 . Again, the surface clistorts to fill the unit cell as the mean curvature is increased from the curvature of a sphere. In this case, the sphere had a mean curvature of 1.732 and extended beyond the walls of the cube. Surfaces of $150^{\circ}$ contact angle were computed with mean curvature up to $H=100$. As the mean curvature is decreased from 1.732, the volume fraction of the internal domain decreases from 0.742 (Figure 10). At $H=1.691$, a turning point is encountered and the curvature then increases while the volume fraction continues to clecrease to a minimum of 0.436 $H=2$. The volume fraction increases as $H$ increases until $H=2.035$ where the surface begins to self-intersect. That is to say, for the surface to satisfy Eq.4, it passes through or intersects itself. At the point of self-intersection, the mapping of Eq. 1 fails. The surface area of the curved constant mean curvature surface increases from 2.77 at $H=1.732$, reaches a local maximum of 2.78 at $H=1.75$, clecreases to 2.74 at $H=1.94$, and then increases to 3.04 at $H=2.04$ (Figure 4.9 )

Surfaces with contact angle between $180^{\circ}$ to $134^{\circ}$ distort to fill the cube as mean curvature is increased. Surfaces with contact angle less than or equal to $133^{\circ}$ do not distort to fill the unit cube. Three surfaces with $120^{\circ}$ contact angle are shown in Figure 5. The surface with $120^{\circ}$ contact angle and $H=2.04$, self-intersects. The internal domain volume fraction decreases as the mean curvature is decreased from $H=2.04$ until a minimum volume fraction is reached at $H=1.9$. The volume fraction increases as mean curvature is decreased further until the surface becomes disjoint at $H=-0.415$; the surface is confined to the corners of the unit cube and is no longer continuous along the cube edges. When the surface becomes disjoint, the mapping of $\mathrm{Eq} .1$ fails. As mean curvature decreases from $H=2.04$ to $H=-0.415$, the surface area of the curved surface decreases from 3.0 to a local minimum of 2.34 at $H=1.72$, increases to a local maximum of 2.45 at $H=1$, decreases to a local minimum of 1.66 at $H=-0.25$, and increases to 1.92 at $H=-0.415$.

Anderson (1986) computed a series of surfaces with $90^{\circ}$ contact angle. Four surfaces with $90^{\circ}$ contact angle are shown in Figure 6. At $H=2$ and $H=-2$, the surfaces selfintersect and the mapping of Eq. 4.1 fails. Anderson (1986) reported that surfaces with $90^{\circ}$ contact angle invert as the mean curvature is changed from positive to negative. As mean curvature is decreased from $H=2$ to $H=-2$, the volume fraction decreases from 0.465 to a minimum of 0.25 at $H=1.8$. increases to a maximum of 0.75 at $H=-1.8$, and decreases to 0.535 at $H=-2$. The area of the curved surface decrcases from 2.9 at $H=2$ to a minimum of 2.0 at $H=2$ (below a turning point at $H=2.13$ ), increases to a 

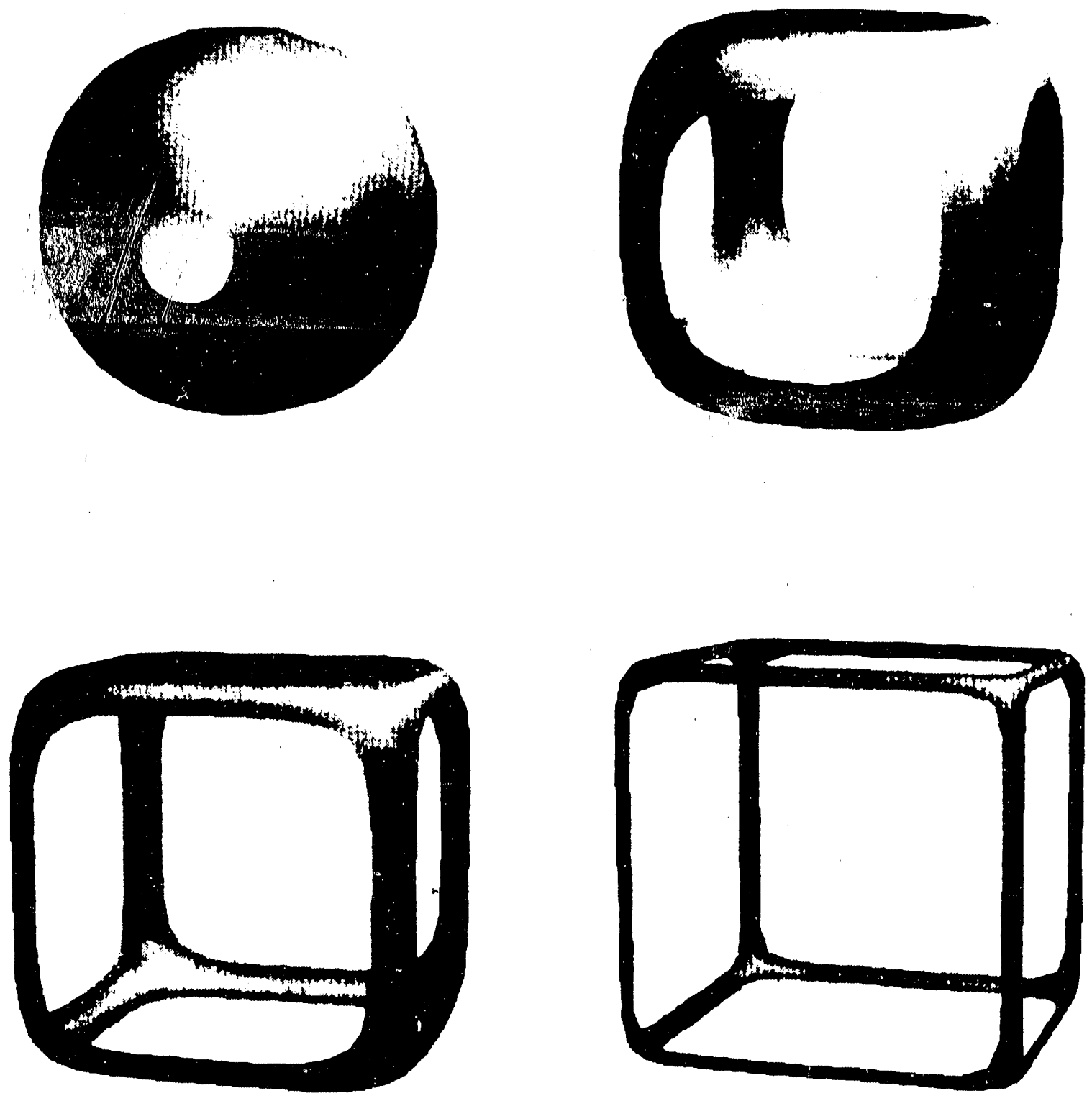

Figure 3. Four surfaces with $180^{\circ}$ contact angle, (upper left) $H=2$, (upper right) $H=3$, (lower left) $H=5$, and (lower left) $H=10$. 

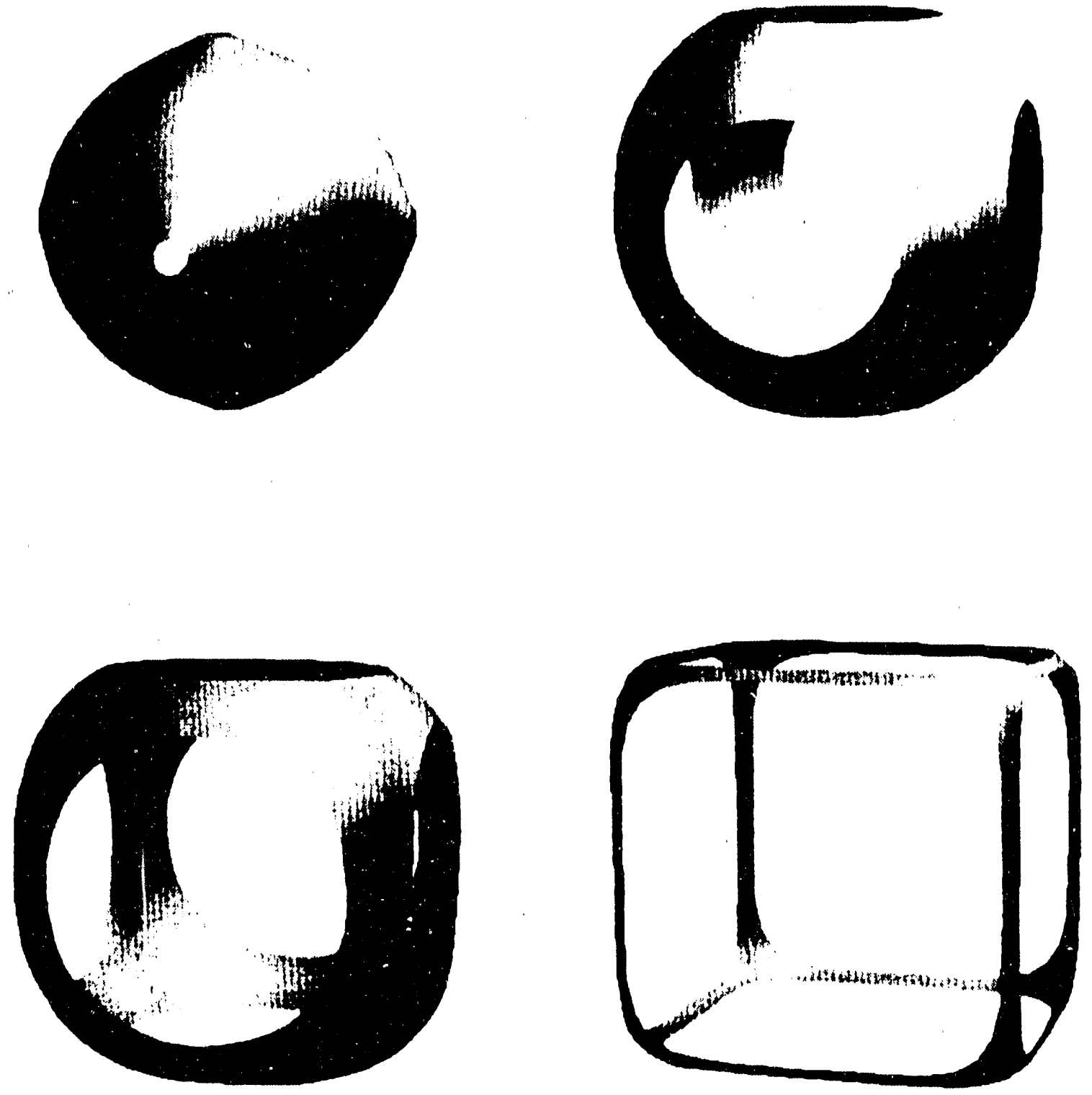

Figure 4. Four surfaces with $150^{\circ}$ contact angle, (upper left) $H=2$, (upper right) $H=1.7$, (lower left) $H=2$, and (lower left) $H=5$. 

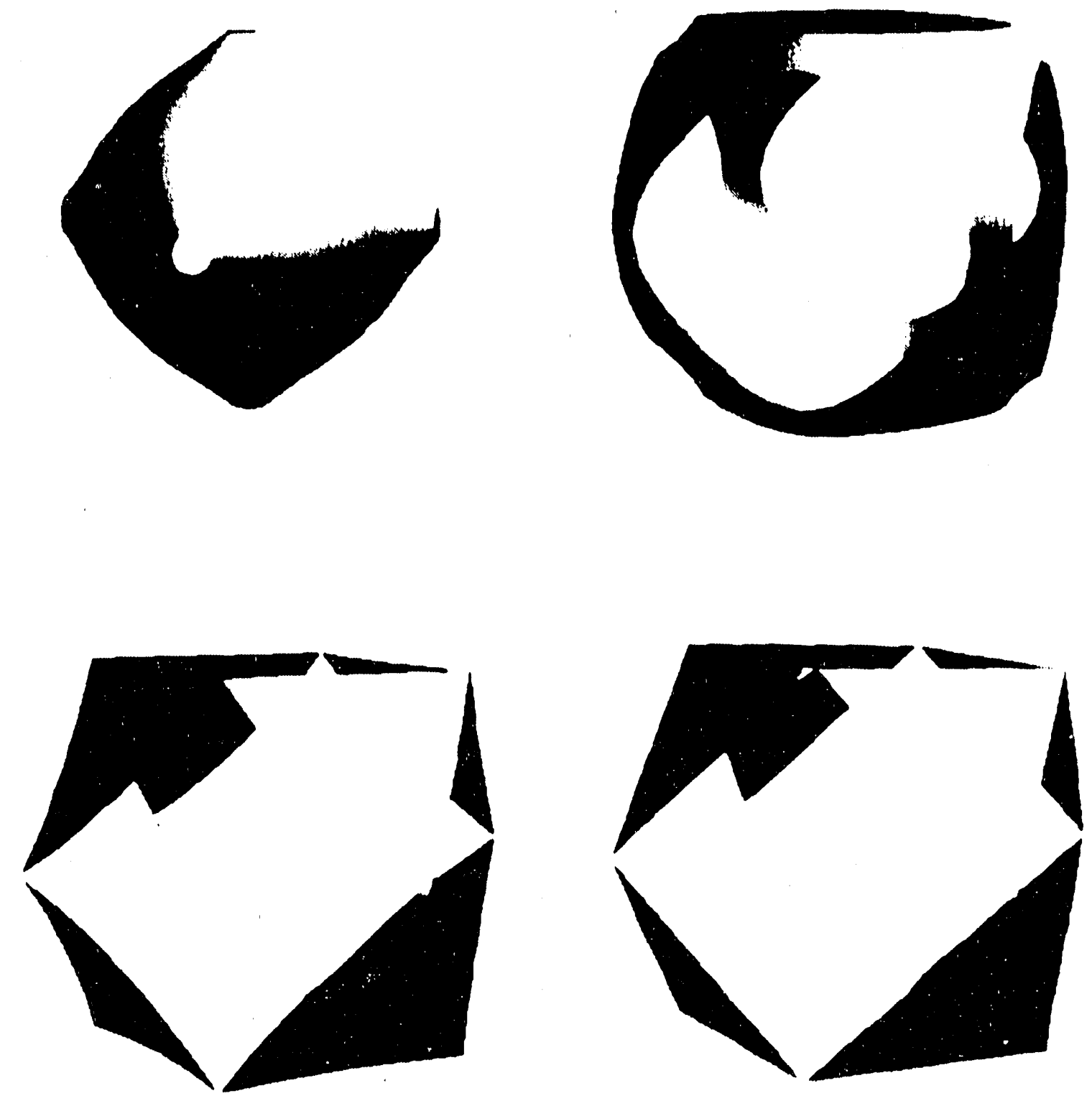

Figure 5. Three surfaces with $120^{\circ}$ contact angle, (upper left) $H=2$, (upper right) $H=0$, (lower left) $H=-0.4$. Shown in (lower right) is the surface with contact of $125.26^{\circ}$ and $H=0$, which corresponds to portions of octahedra centered at the corners of the unit cell. 

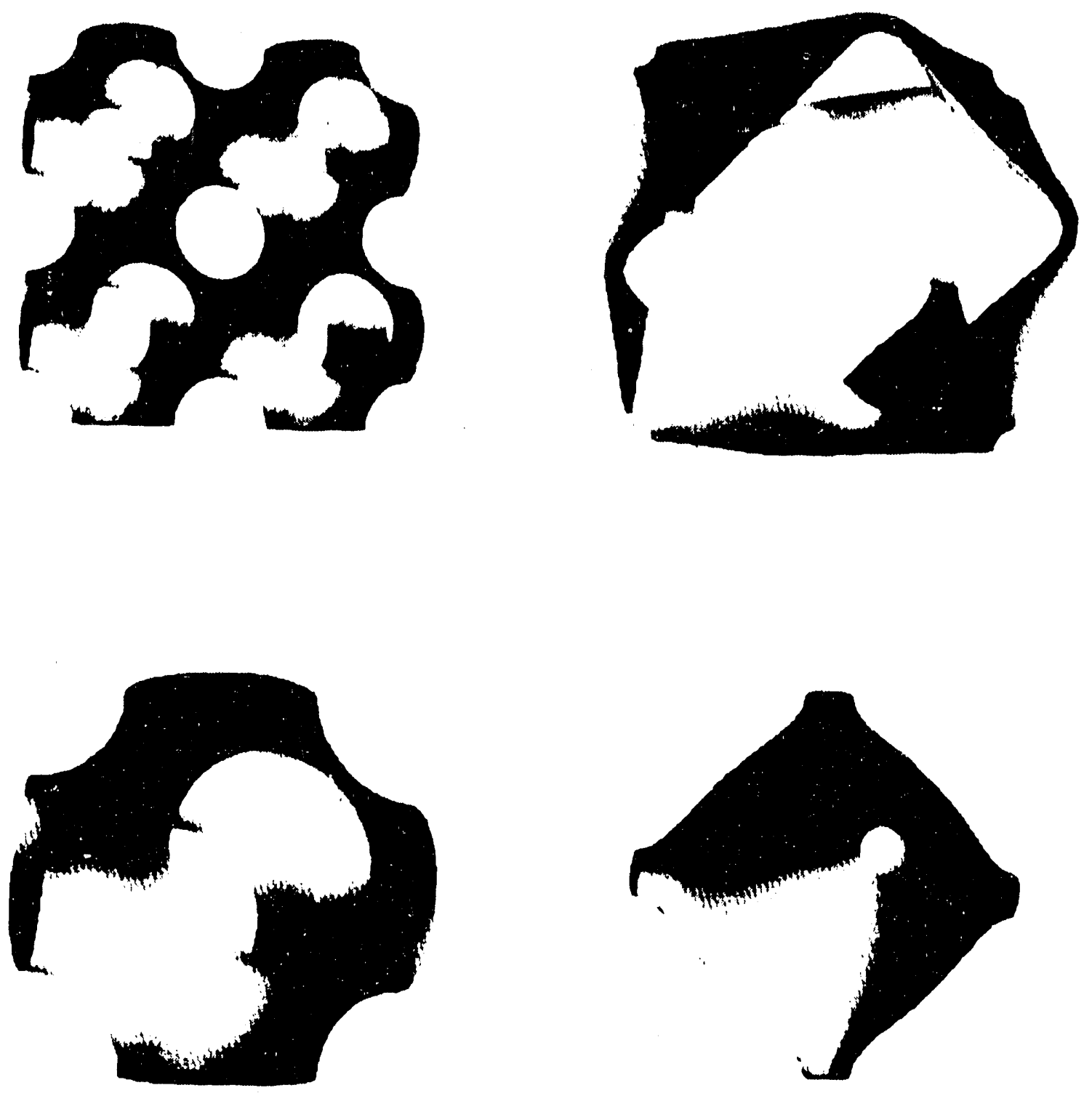

Figure 6. Four surfaces with $90^{\circ}$ contact angle, (upper left) $H=2$, (upper right) $H=0$ the Schwarz surface, (lower left) $H=-2$. Shown in (lower right) are four unit cells connected to illustrate the periodicity of the three-dimensional strurture. 

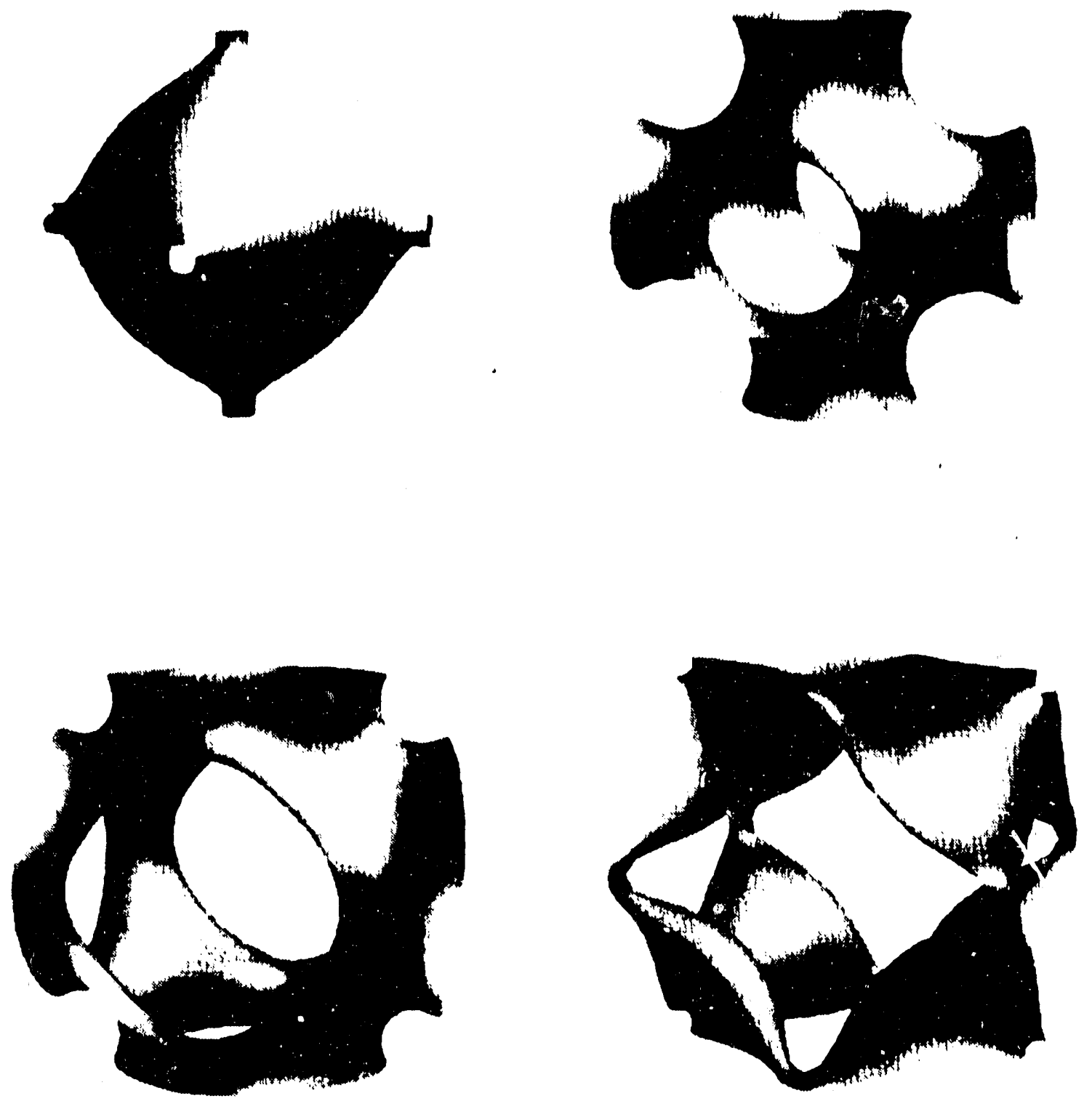

Figure 7. Four surfaces with $60^{\circ}$ contact angle, (upper left) $H=2.2$, (upper right) $H=0$, (lower left) $H=-2$, and (lower right) $H=-3.2$. 

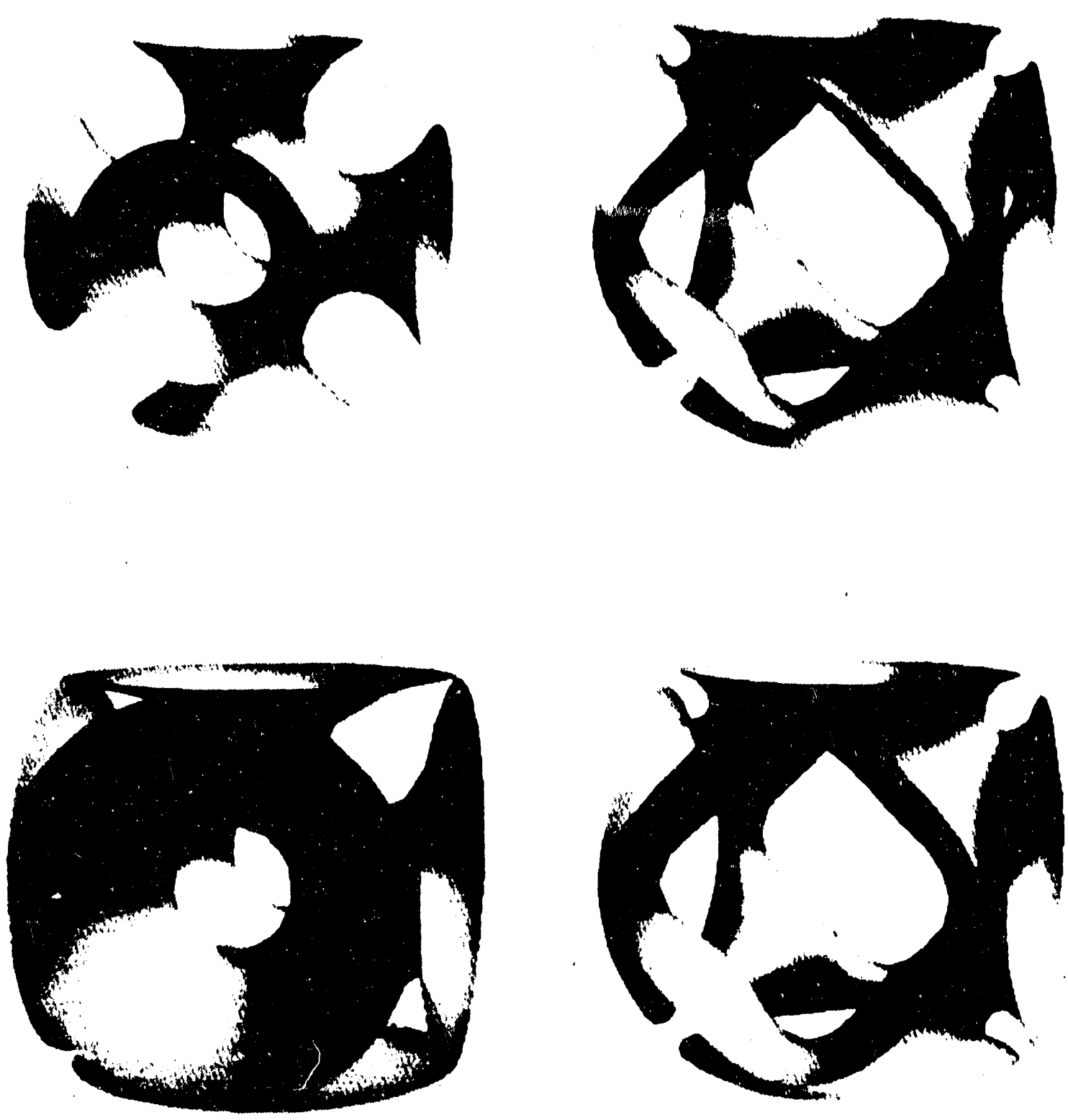

Figurc 8. Two surfaces with $30^{\circ}$ contact angle, (upper left) $H=0$, (upper right) $H=-3$; and two surfaces with $5^{\circ}$ contact angle, (lowei left) $H=-0.4$, and (lower right) $H=-3$. 


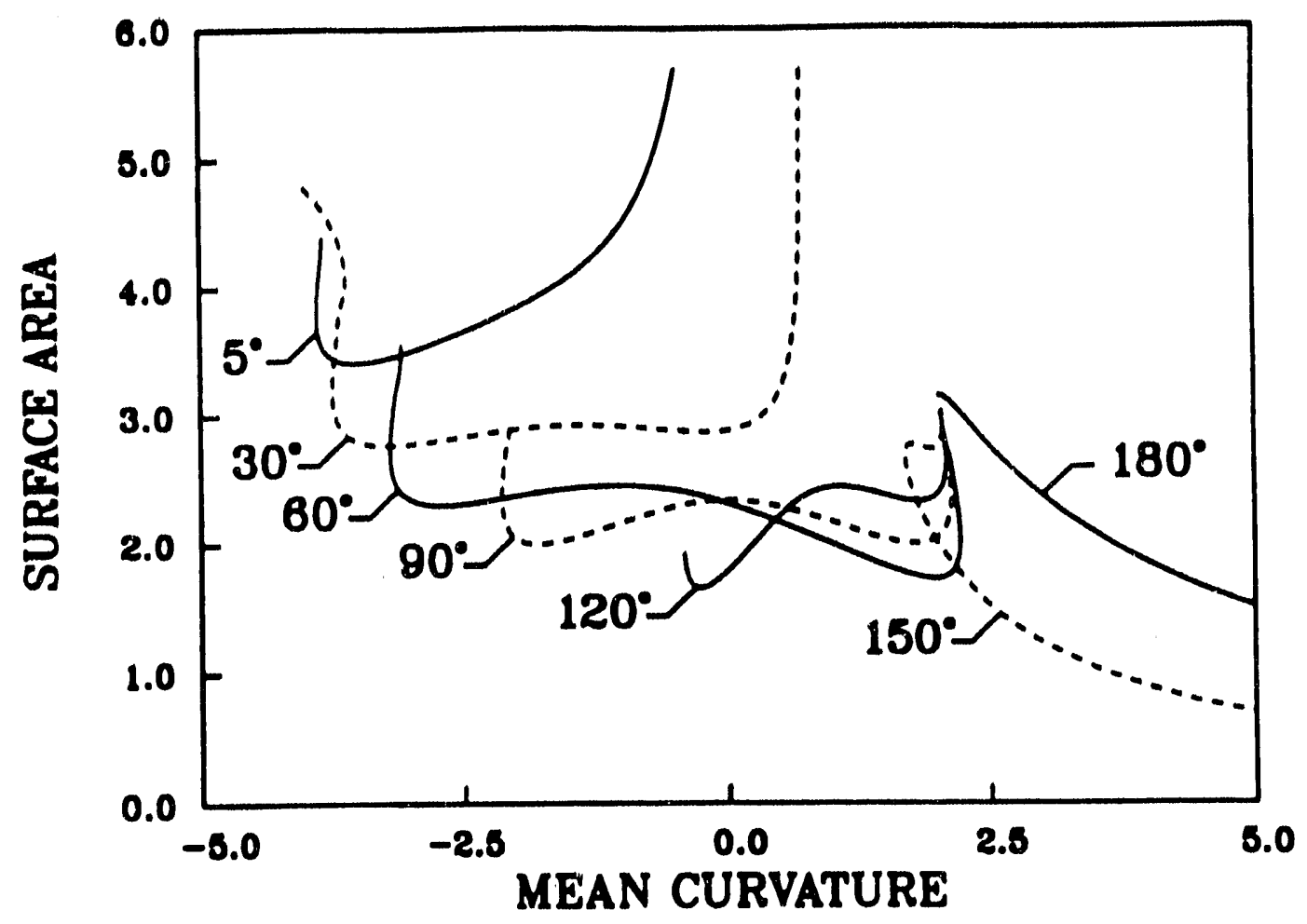

Figure 9. Area of curved constant mean curvature surface as a function of mean curvature for contact angles of $180^{\circ}, 150^{\circ}, 120^{\circ}, 90^{\circ}, 60^{\circ}, 30^{\circ}$, and $5^{\circ}$.

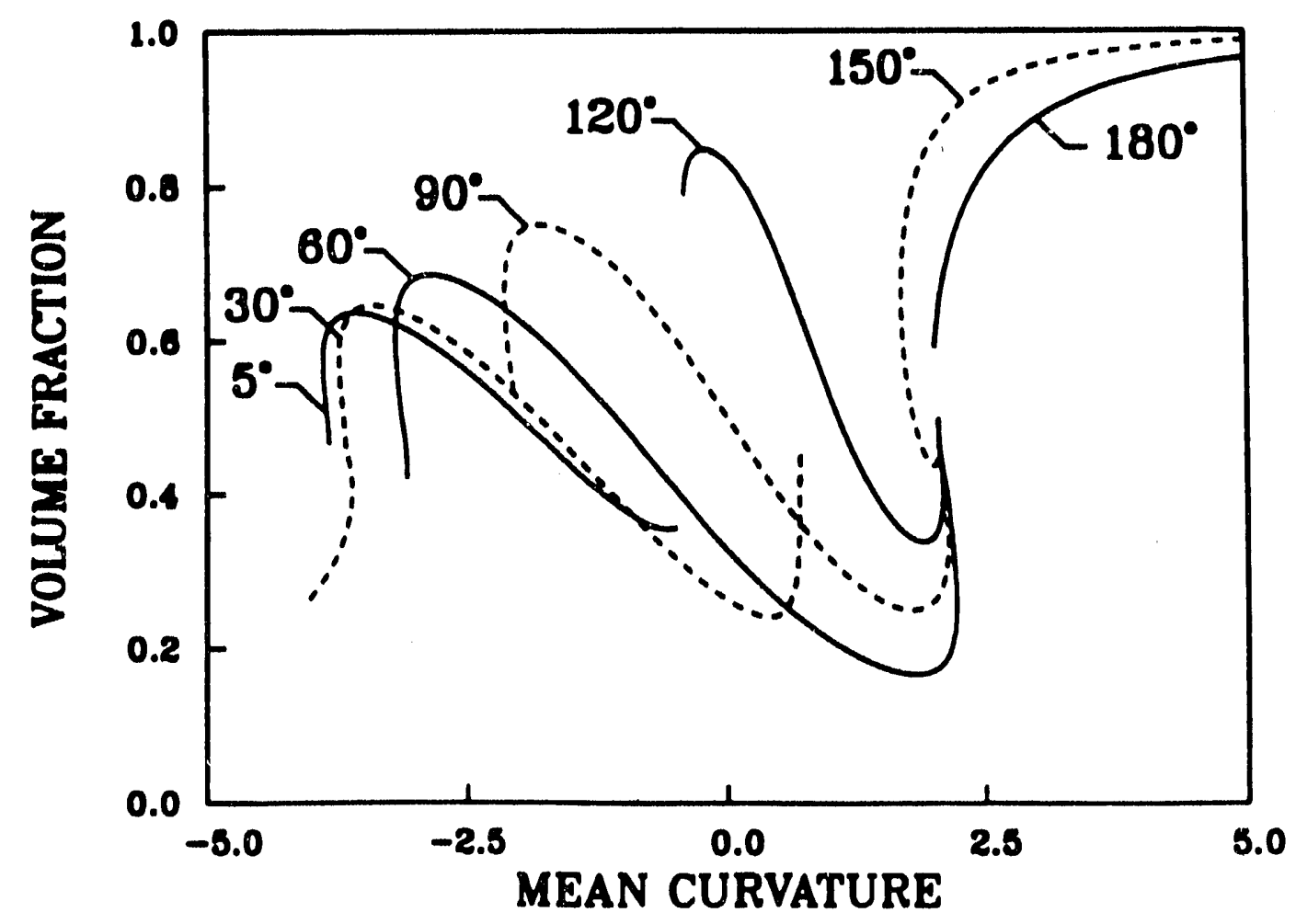

Figure 10. Volume fraction of internal domain as a function of mean curvature for contact angles of $180^{\circ}, 150^{\circ}, 1.20^{\circ}, 90^{\circ}, 60^{\circ}, 30^{\circ}$, and $5^{\circ}$. 


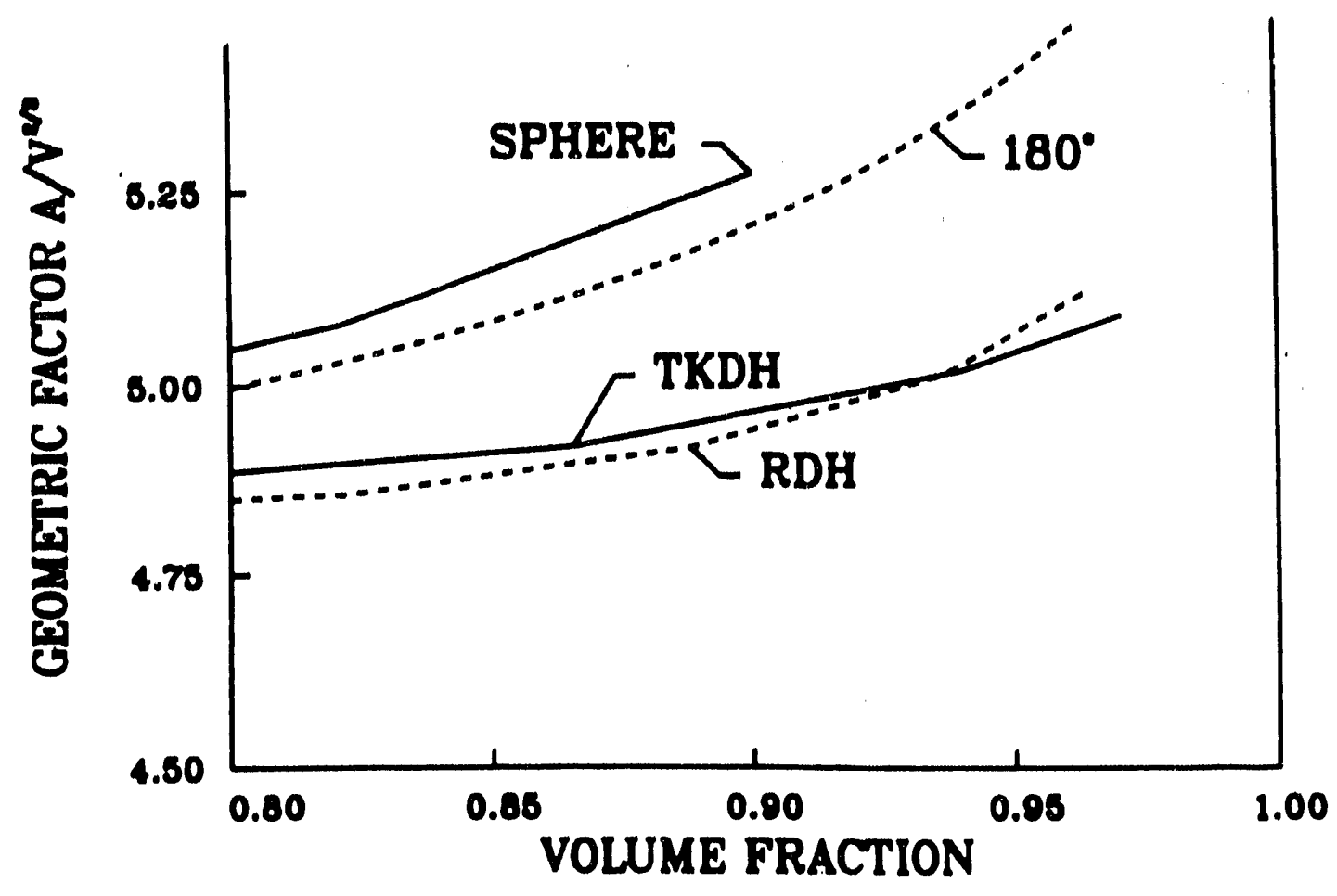

Figure 11. Geometric factor $\left(A / V^{2 / 3}\right)$ as function of volume fraction of deformed spheres in simple cubic $(\mathrm{SC})$, rhomboidal dodecahedral $(\mathrm{RDH})$, and tetrakaidecal edral (TKDH) packings as well as constant mean curvatures surfaces of $180^{\circ}$ contact angle.

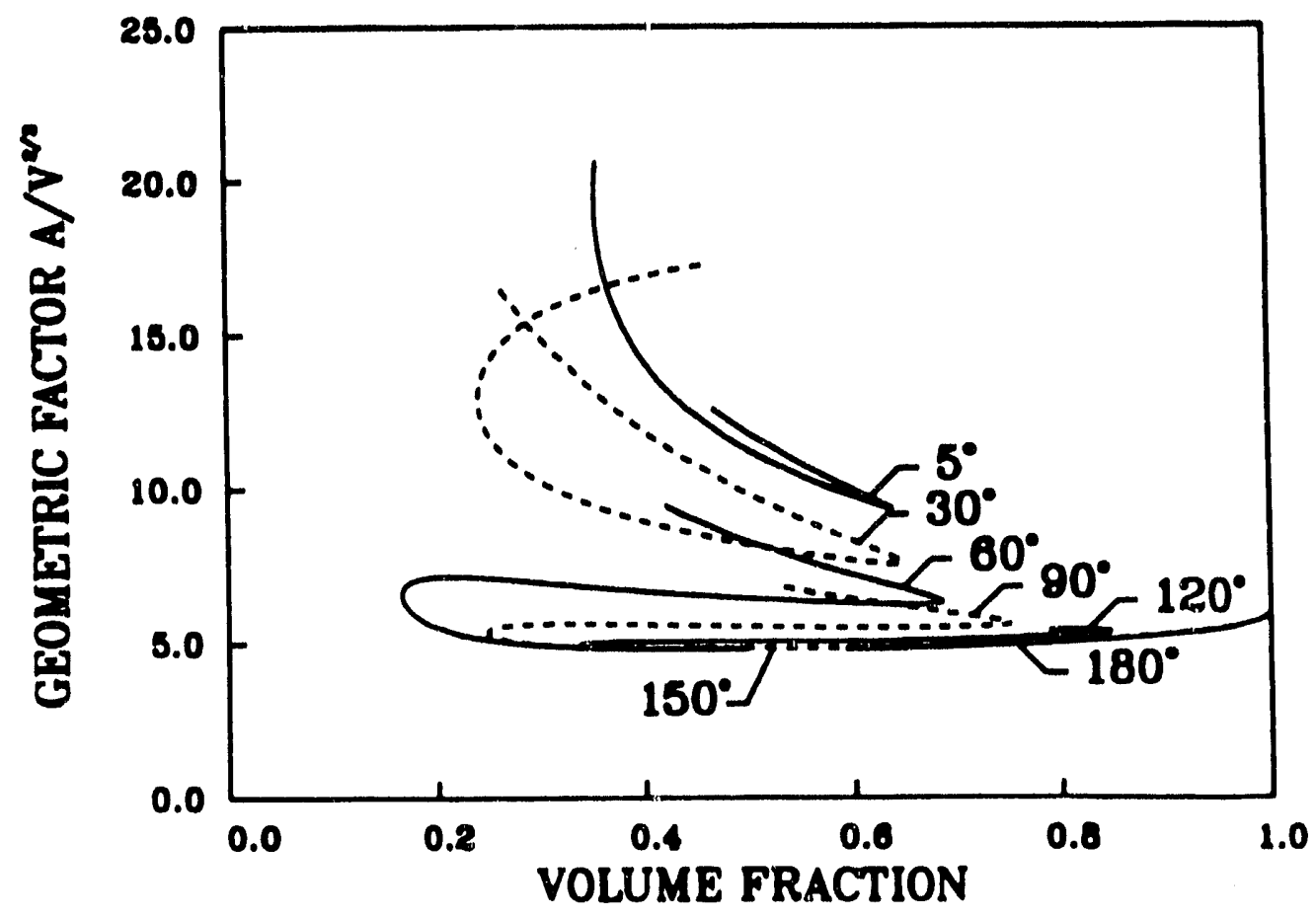

Figure 12. Geometric factor $\left(A / V^{2 / 3}\right)$ as function of volume fraction for constant mean curvature surfaces of $180^{\circ}, 150^{\circ}, 120^{\circ}, 90^{\circ}, 60^{\circ}, 30^{\circ}$, and $5^{\circ}$. 
local maximum of 2.34 at $H=0$, decreases to 2.0 at $H=2$, and increases to 2.9 at $H=2$ (above a turning point at $H=-2.13$ ).

A series of surfaces of $60^{\circ}$ contact angle were computed between the limits of $H=2.06$ and $H=-3.09$. Four surfaces of $60^{\circ}$ contact angle are shown in Figure 7 . The surface with $60^{\circ}$ contact angle and $H=2.06$, self-intersects. The internal domain volume fraction decreases from 0.453 as the mean curvature is increased to a turning point at $H=2.21$ and then decreased to $H=1.85$ where a minimum volume fraction of 0.166 is reached. The volume fraction increases to 0.686 mean curvature is clecreased further until $H=-2.85$. As the mran curvature is decreased further, the volume fraction decreases to 0.420 at $H=-3.09$ where the surface self-intersects. As mean curvature is decreased from $H=2.06$ to $H=-2.85$, the area of the curved surface clecreases from 2.86 to a minimum of 1.73 at $H=2$, increases to a local maximum of 2.46 at $H=-1.1$, decreases to 2.30 at $H=2.7$, and increases to 3.55 at $H=-3.09$.

A series of surfaces of $30^{\circ}$ contact angle were computed between the limits of $H=0.70$ and $H=-4.02$. Two surfaces of $30^{\circ}$ contact angle are shown in Figures 4.8 upper left and right. The surface with $30^{\circ}$ contact angle and $H=0.70$, self-intersects. The internal domain volume fraction decreases from 0.46 at $H=0.7$ to 0.24 at $H=0.40$, increases to a maximum of 0.65 at $H=-3.4$, and clecreases to 0.26 at $H=-4$ where the surface selfintersects. As $H$ is decreased from 0.7 to -4 , the area of the curved surface decreases from 5.68 to 2.77 at $H=-3.2$, and increases to 4.8 at $H=-4$ where the surface self-intersects.

A series of surfaces of $5^{\circ}$ contact angle were computed between the limits of $H=-0.49$ and -3.8. Surfaces with $H=-0.49$ and $H=-3.8$ self-intersect. Two surfaces of $5^{\circ}$ contact angle are shown in Figures 4.8 lower left and right. The internal domain volume fraction increases from 0.26 at $H=-0.49$ to 0.64 at $H=-3.6$, and decreases to 0.46 at $H=-3.8$. As $H$ is decreased from -0.49 to -3.8 , the area of the curved surface decreases from 5.7 to a minimum of 3.4 at $H=-3.5$, and increases to 4.4 at $H=-3.8$.

Lissant (1966) defined a dimensionless gcometric factor as the total surface area of the internal domain divided by the two-thirds power of the internal domain volume. This geometric factor removes particle size dependence from the surface area. A filled uni $i$ cube has a geometric factor of $A / V^{2 / 3}=6$. Lissant calculated geometric factors of rhomboidal dodecahedral $(\mathrm{RDH})$ and tetrakaidecahedral (TKDH) surfaces. These geometric factors as functions of volume fraction are shown in Figure 4.11 along with the geometric factors from simple cubic spheres and surfaces of corstant mean curvature and $180^{\circ}$ contact angle. For a given volume fraction, the surface with the lowest geometric factor has the lowest surface area. Spheres of RDH or TKDH arrangements pack more tightly than do spheres of simple cubic arrangements. Packed simple cubic spheres occupy $52 \%$ of volume, packed TKDH spheres occupy $68 \%$ of volume, and packed RDH spheres occupy $74 \%$ of volume. The geometric factor of RDH spheres, is lowest, that of TKDH spheres are next, followed by the.t of simple cubic spheres. As the curvature of the spheres is increased, the spheres deform but the ranking of the geometric factors remains the same until volume fraction 
of $94 \%$ when the geometric factors of RDH and TKDH switch ranks. The constant mean curvature surfaces of $180^{\circ}$ contact angle have lower geometric factors than deformed simple cubic spheres of the same volume fraction, i.e., the $180^{\circ}$ surfaces have less surface area than deformed simple cubic spheres of the same volume fraction. However, constant mean curvature surfaces of $180^{\circ}$ contact angle have higher geometric factors and surface areas than deformed RDH and TKDH spheres of the same volume fraction. Geometric factors of constant mean curvature surfaces as functions of contact angle and mean curvature are shown in Figure 4.12. Surface with contact angle of $180^{\circ}$ have the lowest geometric factor for a specified volume fraction. Surfaces of contact angle less than $180^{\circ}$ have higher surface areas and, therefore, higher geometric factors.

\section{Summary}

A Galerkin weighted residual formulation of the Surface Divergence Theorem is used with finite element basis functions to compute geometric models of foam and emulsion structure. The models are surfaces of constant mean curvature arranged on a simple cubic lattice so that they meet the boundary planes of the unit cells at prescribed contact angles. Surfaces are computed for a variety of contact angles and mean curvatures. With contact angle between $5^{\circ}$ and $133^{\circ}$, the surface inverts as the mean curvature decreases, whereas with contact angle between $134^{\circ}$ and $180^{\circ}$, the surface fills the unit cell as the mean curvature decreases, passes through a turning point, and then increases.

\section{References}

Anderson, D. M., Studies in the microstructure of microemulsion, $\mathrm{Ph}$. D. Thesis, University of Minnesota 1986.

Anderson, D. M., H. T. Davis, J. C. C. Nitsche, and L. E. Scriven, Periodic surfaces of prescribed mean curvature, Adv. in Chem. Phys., 77, 3371990.

Arriola, A., G. P. Willhite, and D. W. Green, Trapping of oil drops in a noncircular pore throat and mobilization upon contact with a surfactant, Soc. of Pet. Eng. J. 23, 99-114 1983.

Gauglitz, P. A., C. M. St. Laurent, and C. J. Radke, Snap-off of gas bubbles in smoothly constricted noncircular capillaries, Soc. of Pet. Eng. J.27, 753-765 1987.

Heimenz, P. C., Principles of Colloid and Surface Chemistry, Dekker, 337,1986.

Legait, B., Laminer flow of two phases through a capillary tube with variable square cross-section, J. of Coll. and Int. Sci. 96, 28-38, 1983.

Lenormand, R., C. Zarcone, and A. Sarr, Mechanisms of the displacement of one fluid by another in a network of capillary ducts, J. of Fluid Mech., (135), 337-353 1984.

Lissant, K. J., The geometry of high-internal-phase-ratio emulsions, J. of Coll. and Int. Sci., 22, 462-468, 1966.

Lissant, K. J., Geometry of emulsions, J. Soc. Cosmetic Chemists, 21, 141-154, 1970.

Lissant, K. J., K. G. Mayhan A study of medium and high internal phase ratio wa- 
ter/polymer emulsions, J. of Coll. and Int. Sci., 42, 201-208 1973.

Lissant, K. J., B. W. Peace, S. H. Wu, and I. G. Mayhan Structure of high-internal-phaseratio emulsions, J. of Coll. and Int. Sci., 47, 416-423, 1974.

Princen, H. M., Highly concentrated emulsion. I. Cylindrical systems, J. of Coll. and Int. Sci., 71, 55-66, 1979.

Princen, H. M., M. P. Aronson, and J. C. Moser,Highly concentrated emulsions. II. Real systems. The effect of film thickness and contact angle on the volume fraction in creamed emulsions, J. of Coll. and Int. Sci., 75, 246-270, 1980.

Ransohoff, T. C., and C. J. Radke, Laminar flow of a wetting liquid along the corners of a predominantly gas-occupied noncircular pore, J. of Coll. and Int. Sci., 121, 392-401, 1988.

Roof, J. G., Snap-off of oil droplets in water-wet pores, Soc. of Pet. Eng. J. 10, 85-89, 1970.

Schoen, A. H., Infinite periodic minimal surfaces without self- intersections, NASA Technical Note, TND-5541, 1970.

Schwarz, H. A., Monatsberichte der Koniglichen Akademie der Wissenschaften zu Berlin, Jahrgang 1865, 149-153, 1865.

Singhal, A. K. and Somerton, W. H., Two-phase flow through a non-circular capillary at low Reynolds numbers, J. of Can. Pet. Tech., 9, 197-205, 1970.

Weatherburn, C. E., Differential Geometry of Three Dimensions, Cambridge University Press, London, 239, 1927. 


\section{THESIS TITLES}

Gregory L. Deitrick, Ph.D. Thesis, University of Minnesota, 1990. Efficient molecular simulation of chemical potential a new measurement and control system for the surface forces apparatus.

John G. Sheehan, M.S. Thesis, University of Minnesota, 1990. High resolution cryogenic scanning electron microscopy.

Phillip K. Vinson, Ph.D. Thesis, University of Minneosta, 1990. Cryo-electron microscopy of microstructures in complex liquids.

Min Xie, M.S. Thesis, University of Minneosta, 1990. Structure and dynamics of microemulsions using nuclear magnetic resonance.

David S. Bohlen, Ph.D. Thesis, University of Minesota, 1990. A small-angle $\mathrm{x}$-ray scattering exploration of nonionic microemulsion structure. 


\section{LIST OF PUBLICATIONS}

1. Anderson, D.M., H. T. Davis and L. E. Scriven, J. Chem. Phys. 91, 3246-3251 (1989).

2. Bellare, J.R., H. T. Davis, W. G. Miller, and L.E. Scriven, J. Colloid and Inter. Sci. 136, No. 2, 305-326, 1990.

3. Bitsanis,I., S. Somers, H. T. Davis and M. Tirrell, J. Chem. Phys., September 1990.

4. Chapela, G.A., H. T. Davis and L.E . Scriven, Chemical Physics 129, 201-207 (1989).

5. Chapella, G., L. E. Scriven and H. T. Davis, J. Chem. Phys.91, 4307-4313 (1989).

6. Davis, H.T., Europhysics Letters 8, 629-632 (1989).

7. Davis, H.T., J. F. Bodet, L. E. Scriven and W. G. Miller, Physics A 157, 470-481 (1989).

8. Davis, H.T. Chem. Eng. Sci. 44, 1709-1805 (1989).

9. Novy, R.A., P. G. Toledo, H. T. Davis and L. E. Scriven, Chem. Eng. Sci, 44, 1785-1797 (1989).

10. Novy, R.A., H.T. Davis, and L.E. Scriven, Chem. Eng. Sci. 45, NO. 6, 1515-1524, 1990.

11. Novy, R.A., H.T. Davis and L.E. Scriven, Chem. Engng. Sci. (submitted 20 October 1989).

12. Toledo, P. G., R. A. Novy, H. T. Davis and L. E. Scriven, Soil Sci. Soc. Am. J., 54: 673-679 (1990).

13. Toledo, P. G., L.E. Scriven, and H.T. Davis, SPE \#19618, Oct. 8-11, 1989, San Antonio, Texas.

14. Suh, S.-H., L. Mier Y Teran, H.S. White, H.T. Davis, J.Chem. Phys. 142, 203-211 (1990).

15. Vanderlick, T.K., H. T. Davis and J. K. Percus, J. Chem. Phys., 91(11), 7136-7145,1 December 1989.

16. Vanderlick, T. K., L. E. Scriven and H. T. Davis, J. Chem. Phys. 90, 2422 (1989). 
17. Vinson, P. K. , Y. Talmon and A. Walter, Biophysical Journal 56, 669 (1989).

18. Vinson, P. K. , Y. Talmon, J. Colloid and Interface Sci. 133, 288 (1989). 

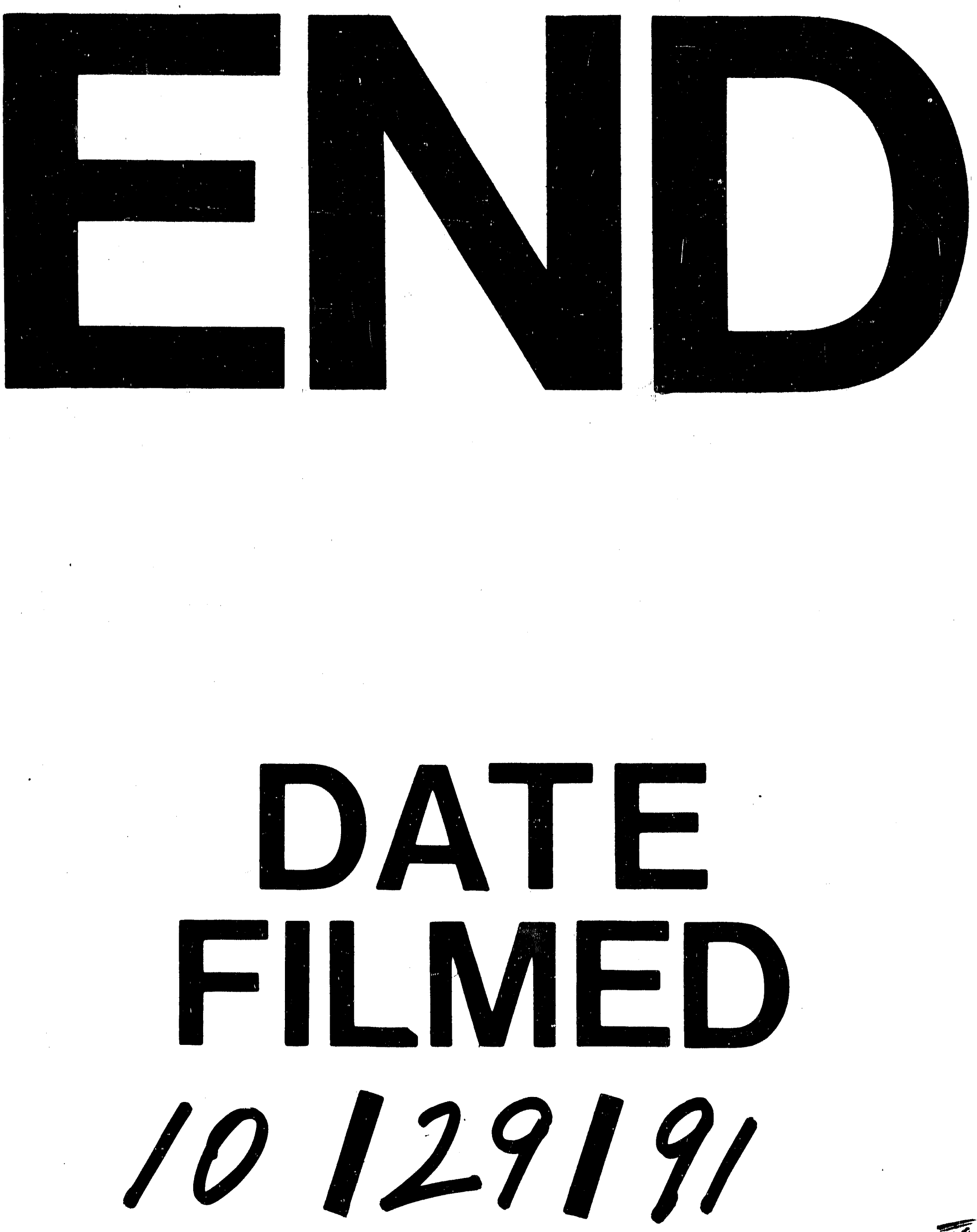
